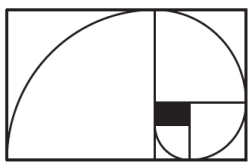

\title{
Grupos de difeomorfismos del círculo
}

\section{Andrés Navas}

\begin{abstract}
Group actions on the circle is a broad and active area in mathematics having many relations with geometry, topology, 1-dimensional dynamics, geometric group theory, and rigidity theory. This book covers most of the classical as well as some recent aspects of the theory, mostly under smoothness assumptions for the action. Some of the topics included here are the recent generalizations of Denjoy and Sacksteder Theorems in regularity smaller than $\mathrm{C}^{2}$, and several algebraic obstructions for smooth group actions on the circle.
\end{abstract}





\section{Índice}

$\begin{array}{ll}\text { Introducción } & 7\end{array}$

$\begin{array}{ll}\text { Notaciones y Definiciones } & 11\end{array}$

1 Ejemplos de Grupos que actúan sobre el Círculo 13

1 El grupo de rotaciones . . . . . . . . . . . . . . . . 13

2 El grupo de traslaciones y el grupo afín . . . . . . . . . . . 14

$3 \quad$ El grupo $\operatorname{PSL}(\mathbf{2}, \mathbb{R}) \ldots \ldots \ldots$

$3.1 \operatorname{PSL}(\mathbf{2}, \mathbb{R})$ y las transformaciones de Möbius . . . . . 15

3.2 $\operatorname{PSL}(\mathbf{2}, \mathbb{R})$ y la corriente geodésica de Liouville . . . 18

$3.3 \operatorname{PSL}(\mathbf{2}, \mathbb{R})$ y la propiedad de convergencia . . . . . 19

4 Acciones de grupos de Lie . . . . . . . . . . . . . . . . . . 20

5 Los grupos de Thompson . . . . . . . . . . . . . . 22

5.1 La realización de Thurston . . . . . . . . . . . . 24

5.2 La realización de Ghys y Sergiescu . . . . . . . . . . 27

2 Sobre la Dinámica de Grupos de Homeomorfismos 31

1 Invariantes minimales . . . . . . . . . . . . . . . . . 31

2 Algunos resultados combinatorios . . . . . . . . . . . . . . 37

2.1 La teoría de Poincaré . . . . . . . . . . . . . . 37

2.2 Número de rotación y medidas invariantes . . . . . . 42

2.3 Acciones efectivas sobre la recta . . . . . . . . . . 44

2.4 Acciones libres . . . . . . . . . . . . . . 47

2.5 Número de traslación y medidas quasi-invariantes . . 52

$2.6 \quad$ Una aplicación: grupos promediables ordenables . . 59

3 Medidas invariantes y grupos libres . . . . . . . . . . . . . 64

3.1 La alternativa de Tits débil . . . . . . . . . . . . 64

3.2 Una interpretación probabilística . . . . . . . 69

3 Dinámica de Grupos de Difeomorfismos de Clase C ${ }^{2} \quad 78$

1 El teorema de Denjoy . . . . . . . . . . . . . 78

2 El teorema del punto fijo hiperbólico de Sacksteder . . . . . 87

$2.1 \quad$ La versión clásica en clase $\mathrm{C}^{\mathbf{1}+\text { lip }}$. . . . . . . . . . . 88 
2.2 La versión $\mathrm{C}^{\mathbf{1}}$ para pseudo-grupos . . . . . . . . . . 92

2.3 Una versión $\mathrm{C}^{\mathbf{1}}$ vía exponentes de Lyapunov . . . . 98

3 Primer teorema de Duminy: no existencia de minimal ex-

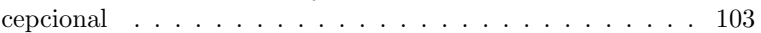

3.1 Presentación del resultado . . . . . . . . . . . 103

3.2 Una aplicación de primer retorno dilatante . . . . 105

3.3 Prueba del teorema . . . . . . . . . . . . 109

4 Segundo teorema de Duminy: el espacio de fines de órbitas semi-excepcionales ... . . . . . . . . . . . . 111

4.1 Presentación del resultado . . . . . . . . . . . . . 111

4.2 Un criterio para distinguir dos fines diferentes . . . . 115

$4.3 \quad$ Fin de la demostración . . . . . . . . . . . . . 118

5 Dos problemas abiertos . . . . . . . . . . . . 121

5.1 Acciones minimales . . . . . . . . . . . . . . 121

5.2 Acciones con un minimal excepcional . . . . . . . . . 130

$6 \quad$ Conjugación diferenciable entre grupos de difeomorfismos . 135

6.1 Linearización de Sternberg y conjugaciones $\mathrm{C}^{\mathbf{1}}$. . . 136

6.2 El caso de las conjugaciones bilipschitzianas . . . . . 141

4 Estructura Algebraica y Rigidez: Métodos Dinámicos 145

1 Grupos abelianos de difeomorfismos . . . . . . . . . . . . . 145

1.1 El lema de Kopell . . . . . . . . . . . . . . . . . . 145

$1.2 \quad$ El teorema de Szekeres . . . . . . . . . . . . 150

1.3 La clasificación . . . . . . . . . . . . . . . 156

1.4 Contra-ejemplos de Denjoy . . . . . . . . . . . 156

1.5 Rigidez en regularidad intermedia . . . . . . . . . 166

2 Grupos nilpotentes de difeomorfismos . . . . . . . . . . . . 174

2.1 Los teoremas de Plante y Thurston . . . . . . . . . . 174

2.2 Crecimiento de grupos de difeomorfismos . . . . . . 176

2.3 Nilpotencia, crecimiento y regularidad intermedia . . 182

3 Grupos solubles de difeomorfismos . . . . . . . . . . . . . . 188

3.1 Ejemplos y formulación de resultados . . . . . . . . 188

3.2 El caso metabeliano . . . . . . . . . . . . . . 192

3.3 El caso de la recta . . . . . . . . . . . . . . 196

4 Un paréntesis en la clasificación . . . . . . . . . . . . . . 199

5 Estructura Algebraica y Rigidez: Métodos Cohomológicos 201

1 El teorema de estabilidad de Thurston . . . . . . . . . . . . 201

2 Rigidez para grupos de Kazhdan . . . . . . . . . . . . . . 206

2.1 La propiedad (T) de Kazhdan . . . . . . . . . . . . 206

2.2 Enunciado del resultado . . . . . . . . . . . . . . . 211

2.3 Demostración del teorema . . . . . . . . . . . . . . 214

2.4 Propiedad (T) relativa y propiedad de Haagerup . . 220

3 Supra-rigidez para redes de rango superior . . . . . . . . . . 221

$3.1 \quad$ Presentación del resultado . . . . . . . . . . . . . . 221 
ÍNDICE

3.2 Supra-rigidez cohomológica . . . . . . . . . . . 224

3.3 Supra-rigidez para acciones sobre el círculo . . . . . 228

Apéndice

Algunos conceptos básicos de álgebra . . . . . . . . . . . . . . 233

Medidas invariantes y promediabilidad . . . . . . . . . . . 234

Bibliografía

238 



\section{Introducción}

La teoría clásica de los sistemas dinámicos comprende el estudio cualitativo y cuantitativo de las órbitas de una aplicación o de un campo de vectores. En el caso en que la aplicación considerada es invertible, o que el campo en cuestión es regular, lo anterior puede ser interpretado como una acción del grupo $(\mathbb{Z},+)$ o de $(\mathbb{R},+)$ respectivamente. Desde este punto de vista, la dinámica clásica puede ser pensada como una rama particular de la teoría general de acciones de grupos.

Desde un punto de vista más algebraico, el estudio de grupos de difeomorfismos puede ser entendido como una extensión al caso "no lineal" de la teoría de representaciones. A través de este estudio se procura entender la estructura interna de un grupo mediante sus acciones sobre variedades diferenciables. Aparecen entonces nuevas nociones algebraicas, y diversos aspectos topológicos y/o analíticos del espacio en consideración cobran relevancia. Esta visión de la teoría ha sido muy fructífera en los últimos años, ya que gracias a ella se ha podido establecer (de manera a veces sorprendente) diversos resultados de naturaleza puramente algebraica mediante métodos esencialemente dinámicos.

Sin duda alguna resulta ambicioso (y tal vez imposible) considerar directamente la teoría general de acciones de grupos. Es natural entonces restringir el campo de estudio, ya sea limitando la clase de grupos que intervienen o bien el tipo de espacios sobre los que ellos actúan. En estas notas seguiremos el segundo camino, y nos concentraremos en el estudio de las acciones de grupos sobre la más simple de las variedades compactas: el círculo. Veremos que, a pesar de la aparente simplicidad del problema, éste resulta sorprendentemente interesante y complejo.

En la literatura existe ya un completo y elegante tratado sobre la teoría de acciones por homeomorfismos del círculo debido a Ghys [72]. Es por esta razón que hemos orientado estas notas principalmente a la teoría de acciones por difeomorfismos, la cual es sensiblemente diferente en diversos aspectos. Sin embargo, aún dentro del contexto de los difeomorfismos, este texto resulta incompleto. Nos hubiese gustado incluir al menos una pequeña sección introductoria a la "teoría de los pequeños denominadores", expandirnos en torno al teorema de Sacksteder introduciéndonos en la "teoría de niveles", desarrollar en parte la teoría cohomológica de los 
grupos de difeomorfismos del círculo estudiando el cociclo de Bott-VirasoroThurston (o clase de Godbillon-Vey), y por sobre todo haber añadido dos capítulos centrados respectivamente en los grupos de difeomorfismos realanalíticos y en las representaciones de grupos fundamentales y modulares de superficies. Las inexorables restricciones de tiempo y espacio nos impidieron continuar nuestro trabajo en tales direcciones...

Este texto comienza con una breve sección en la que fijamos las notaciones y damos las definiciones de los conceptos básicos a utilizar. Ciertos conceptos más elaborados, como por ejemplo el de promediabilidad, son tratados en el apéndice.

En el capítulo 1 estudiamos algunos ejemplos de grupos sencillos que actúan sobre el círculo. Luego de detenernos en el estudio del grupo de rotaciones, del grupo afín y el de Möbius, tratamos el caso general de los grupos de Lie, para finalizar centrándonos en los grupos de Thompson.

En el capítulo 2 estudiamos algunos resultados fundamentales de la dinámica de grupos de homeomorfismos. En la primera parte del capítulo analizamos ciertos aspectos combinatorios que aparecen de manera natural en este estudio. Comenzamos haciendo un recuento de los resultados más relevantes de la teoría de Poincaré para acciones de $(\mathbb{Z},+)$. Posteriormente, estudiamos la relación entre el número de rotación y las medidas invariantes, para luego tratar el caso de las acciones efectivas y libres por homeomorfismos del círculo y de la recta. Como una aplicación de lo anterior daremos una demostración puramente dinámica de un resultado reciente de Witte, el cual establece la indicabilidad local de los grupos promediables y ordenables. Debemos señalar que un tópico que no trataremos en estas notas, pese a estar muy relacionado con los temas precedentes, es el de la clase de Euler, tanto en cohomología usual como en cohomología acotada. Las razones para no haber incluido este tópico son, en primer lugar, el que se encuentra excelentemente desarrollado en [72], y en segundo lugar el hecho que, si bien juega un rol esencial en la caracterización de acciones por homeomorfismos del círculo, su importancia en la teoría diferenciable es bastante menor. En la segunda parte del capítulo 2 abordamos esencialmente un resultado debido a Margulis, el cual establece una versión débil de la alternativa de Tits para grupos de homeomorfismos del círculo. Para este resultado no daremos la prueba original de Margulis, sino que desarrollaremos la demostración alternativa propuesta por Ghys, la cual se presta para una reinterpetación probabilística dentro del contexto de la teoría de caminatas aleatorias sobre grupos.

En el capítulo 3 hemos reunido una serie de teoremas de índole dinámico para los cuales una cierta hipótesis de diferenciabilidad (en general $\mathrm{C}^{2}$ ) es esencial. Comenzamos con el más importante de ellos, a saber, el teorema de Denjoy. Estudiamos luego otros resultados relacionados, como lo son los teoremas de Sacksteder y de Duminy. Luego de discutir algunos problemas abiertos importantes para la teoría, finalizaremos tratando el problema de la diferenciabilidad de la conjugación entre grupos de difeomorfismos (para 
casos muy particulares). Hubiese sido natural estudiar también en este capítulo algunas propiedades específicas a clases de diferenciabilidad superiores a $\mathrm{C}^{2}$. Sin embargo, no nos extenderemos en torno a esta promisoria vertiente de investigación, ya que hasta hoy no se vislumbra ninguna forma sistemática para abordar el asunto (vea sin embargo [32, 43, 202]).

Los capítulos 4 y 5 corresponden a una tentativa de descripción de los grupos de difeomorfismos de variedades unidimensionales sobre la base de informaciones algebraicas relevantes. Comenzamos analizando el caso de los grupos abelianos y nilpotentes valiéndonos en parte del famoso "lema de Kopell". Estudiamos después los grupos solubles de difeomorfismos, y explicamos las dificultades que se presentan al momento de tratar el caso de los grupos promediables. Finalmente, nos concentramos en torno a las acciones de grupos que satisfacen propiedades cohomológicas especiales. Después de revisar el ya clásico teorema de estabilidad de Thurston, estudiamos los teoremas de rigidez para las acciones de grupos de Kazhdan y de supra-rigidez para las acciones de redes de rango superior. Estos últimos pueden ser pensados como una culminación natural (aunque tal vez no definitiva) de una serie de resultados de obstrucciones para acciones unidimensionales de redes de grupos de Lie simples y semisimples de rango superior.

Nos hemos esforzado en hacer estas notas lo más autocontenidas posible. Si bien muchos de los resultados presentados son bastante recientes, las técnicas empleadas son en general elementales y no requieren mayores conocimiento previos. Hemos añadido también una serie de ejercicios complementarios con el objetivo de ampliar el panorama de cada uno de los resultados y de indicar algunas referencias relevantes. Advertimos sin embargo al lector que estos "ejercicios" pueden variar abruptamente en nivel de dificultad. De hecho, los (mini-)resultados a veces allí expuestos en muchas ocasiones no figuran en la literatura. Éste también es el caso de tres secciones completas del texto, a saber las secciones 4, 5.1 y 6.2. La primera de ellas merece especial atención, pues contiene la demostración original de un teorema probado por Duminy (hace ya casi 30 años) sobre la existencia de infinitos fines para hojas semi-excepcionales de foliaciones de codimensión 1 y transversalmente de clase $C^{2}$. La necesidad de publicar la extraordinaria prueba de Duminy de este notable resultado (para el cual una referencia alternativa es [40]) fue una de las motivaciones para la confección de este texto.

La versión original de estas notas fue escrita para ser presentada, en forma de un minicurso, en el $2^{\circ}$ Workshop de Sistemas Dinámicos de la Universidad Católica del Norte en Agosto del 2001. El texto que aquí se presenta corresponde a una versión ligeramente remozada de una segunda edición preparada para ser presentada -también en la forma de minicursosdurante el verano del 2006 en el Instituto Nacional de Matemática Pura e Aplicada IMPA, y en Diciembre del 2006 en el Instituto de Matemáticas 
y Ciencias Afines IMCA. Queda consignado el reconocimiento del autor a estas instituciones por sus respectivas invitaciones, así como al PBCT y a CONICYT por su respaldo mediante el Proyecto "Anillo en Sistemas Dinámicos de Baja Dimensión". Sin embargo, a nivel institucional se debe destacar por sobre todo el apoyo del laboratorio UMPA de la École Normale Supérieure de Lyon, donde comenzó a gestarse el proyecto de escribir estas notas, así como del Institut des Hautes Études Scientifiques, donde este texto comenzó a tomar su forma definitiva.

Este trabajo se ha visto enormemente enriquecido gracias a la discusión con muchos colegas. Diversas observaciones a lo largo del texto, así como el contenido de algunos ejemplos y ejercicios, han resultado de (algunas veces breves pero siempre) fructíferas conversaciones, y resulta grato para el autor constatar aquí su agradecimiento para con Carlos Moreira (ejercicio 3.4), Juan Rivera-Letelier (ejemplo 3.65), Albert Fathi (ejercicio 5.55), JeanChristophe Yoccoz (ejercicios 1.16 y 5.35), Sylvain Crovisier (proposición 4.64), Tsachik Gelander (ejercicio 5.56), Victor Kleptsyn (ejercicio 3.68), Adolfo Guillot (observación 3.40), Takashi Tsuboi (ejercicio 3.82), y Dave Witte (proposición 5.31). Pero por sobre todos, los agradecimientos son para Étienne Ghys por su constante motivación a trabajar en el tema de los grupos de difeomorfismos del círculo a través de numerosas y apasionantes explicaciones de sus posibles ramificaciones y de sus relaciones con diversas áreas de la matemática. Como el lector constatará a lo largo de la lectura, Ghys es uno de los principales inspiradores de muchos de los desenvolvimientos de la teoría que pasamos a revisar. 


\section{Notaciones y Definiciones}

El círculo será siempre denotado por $\mathrm{S}^{1}$. Sobre él consideraremos la orientación en contra de los punteros del reloj. Designaremos por $] a, b[$ al intervalo abierto desde $a$ hasta $b$ según esta orientación. Notemos que si $b$ pertenece a $] a, c[$ entonces $c \in] b, a[$ y $a \in] c, b[$. A veces denotaremos estas relaciones simplemente por $a<b<c<a$. De manera análoga se definen los intervalos $[a, b],[a, b[\mathrm{y}] a, b]$. distancia entre $a$ y $b$ es la más pequeña de las longitudes de los intervalos $] a, b[\mathrm{y}] b, a[$. Denotaremos esta distancia por $\operatorname{dist}(a, b)$ o $|a-b|$, según sea conveniente. De manera análoga, la longitud de un intervalo $I$ (ya sea del círculo o de la recta $\mathbb{R}$ ) será designada por $|I|$. La medida de Lebesgue de un boreliano $A$ de $\mathrm{S}^{1}$ o de $\mathbb{R}$ será denotada simplemente por $\operatorname{Leb}(A)$.

A lo largo de estas notas, y salvo mención explícita de lo contrario, sólo consideraremos homeomorfismos y difeomorfismos directos, es decir, que preservan orientación. Denotaremos por $\mathrm{Homeo}_{+}\left(\mathrm{S}^{1}\right)$ al grupo de los homeomorfismos del círculo. Para $k \in \mathbb{N} \cup\{\infty\}$, el grupo de los difeomorfismos de clase $\mathrm{C}^{k}$ del círculo será denotado por Difeo ${ }_{+}^{k}\left(\mathrm{~S}^{1}\right)$. Consideraremos también el grupo Difeo ${ }_{+}^{1+\tau}\left(\mathrm{S}^{1}\right)$ de los difeomorfismos del círculo que poseen una derivada Hölder continua de exponente $\tau$. Para $0<\tau<1$, la $\tau$-norma de Hölder de la derivada de $f \in \operatorname{Difeo}_{+}^{1+\tau}\left(\mathrm{S}^{1}\right)$ será denotada por $\left|f^{\prime}\right|_{\tau}$, es decir,

$$
\left|f^{\prime}\right|_{\tau}=\sup _{x \neq y} \frac{\left|f^{\prime}(x)-f^{\prime}(y)\right|}{|x-y|^{\tau}} .
$$

El grupo de los difeomorfismos del círculo de derivada lipschitziana será denotado Difeo ${ }_{+}^{1+\operatorname{lip}}\left(\mathrm{S}^{1}\right)$. Por su parte, Difeo ${ }_{+}^{\mathrm{w}}\left(\mathrm{S}^{1}\right)$ designará al subgrupo de Difeo ${ }_{+}^{\infty}\left(\mathrm{S}^{1}\right)$ formado por los difeomorfismos real-analíticos. Finalmente, la notación Difeo $\left(\mathrm{S}^{1}\right)$ será utilizada en el caso en que el grado de diferenciabilidad (que supondremos al menos $\mathrm{C}^{1}$ ) se subentienda del contexto o sea irrelevante.

En algunas situaciones pensaremos la recta real como siendo el recubrimiento universal del círculo ya sea a través de la aplicación $x \mapsto e^{i x}$, o bien por la aplicación $x \mapsto e^{2 \pi i x}$, dependiendo de si parametrizamos el círculo por $[0,2 \pi]$ o por $[0,1]$. Salvo mención de lo contrario, consideraremos la primera de estas parametrizaciones. Por abuso de notación, 
para cada homeomorfismo directo $f$ del círculo, comúnmente denotaremos también por $f$ a cada uno de sus levantamientos a la recta. De esta forma, $f: \mathbb{R} \rightarrow \mathbb{R}$ es una función continua estrictamente creciente que para todo $x \in \mathbb{R}$ verifica las igualdades $f(x+2 \pi)=f(x)+2 \pi$ ó $f(x+1)=f(x)+1$, según la parametrización escogida. Al grupo formado por los levantamientos a la recta de homeomorfismos directos del círculo lo denotaremos $\widetilde{H o m e o}_{+}\left(\mathrm{S}^{1}\right)$. La rotación de ángulo $\theta$ del círculo será designada por $R_{\theta}$. Observe nuevamente que $\theta$ es un ángulo en $[0,2 \pi]$ o en $[0,1]$, dependiendo de la parametrización considerada.

En muchas ocasiones nos concentraremos directamente en los subgrupos de $\mathrm{Homeo}_{+}\left(\mathrm{S}^{1}\right)$ o de $\mathrm{Difeo}_{+}\left(\mathrm{S}^{1}\right)$. Sin embargo, nos interesaremos también en las representaciones de un grupo topológico $\Gamma$ en el grupo de los homeomorfismos o difeomorfismos directos de $\mathrm{S}^{1}$ (a las que, abusando del lenguaje, llamaremos a veces acciones). De manera más precisa, trabajaremos con homomorfismos (continuos) $\Phi$ de $\Gamma$ sobre $\mathrm{Homeo}_{+}\left(\mathrm{S}^{1}\right) \mathrm{o}$ Difeo $_{+}\left(\mathrm{S}^{1}\right)$. Por simplicidad, a menudo identificaremos el elemento $g \in \Gamma$ con la transformación $\Phi(g)$. Sin embargo, para evitar confusiones, denotaremos $I d$ a la aplicación identidad de $\mathrm{S}^{1}$, y denotaremos $i d$ al elemento neutro de un grupo que actúa, ya sea sobre el círculo u otro espacio. Recordemos que, en general, una acción $\Phi$ de un grupo $\Gamma$ sobre un espacio M es efectiva si para todo $g \neq i d$ la aplicación $\Phi(g)$ no es la identidad en M. La acción es libre si para todo $g \neq i d$ se tiene $\Phi(g)(x) \neq x$ para todo $x \in \mathrm{M}$. Recordemos finalmente que dos homeomorfismos $f, g$ en $\mathrm{Homeo}_{+}\left(\mathrm{S}^{1}\right)$ son topológicamente conjugados si existe un homeomorfismo directo $h$ de $\mathrm{S}^{1}$ tal que $h \circ f=g \circ h$ (en general, llamaremos conjugado de $f$ por $h$ al homeomorfismo $h f h^{-1}$ ). Análogamente, diremos que dos acciones $\Phi_{1}$ y $\Phi_{2}$ de un grupo $\Gamma$ por homeomorfismos directos de $S^{1}$ son topológicamente conjugadas si existe $h \in$ Homeo $_{+}\left(\mathrm{S}^{1}\right)$ tal que $h \Phi_{1}(g)=\Phi_{2}(g) h$ para todo $g \in \Gamma$ (a menudo suprimiremos el símbolo de la composición de aplicaciones). El grupo libre (no abeliano) a $n$ generadores será designado por $\mathbb{L}_{n}$. Para evitar confusiones, denotaremos por $\mathbb{T}^{1}$ al toro unidimensional, enfatizando la estructura de grupo subyacente en él. Esta estructura se identifica con la del grupo $(\mathbb{R}$ mód $1,+$ ), o con la del grupo de rotaciones $\operatorname{SO}(2, \mathbb{R})$. 


\section{Capítulo 1}

\section{Ejemplos de Grupos que actúan sobre el Círculo}

\section{El grupo de rotaciones}

El grupo $\mathrm{SO}(2, \mathbb{R})$ de las rotaciones de $\mathrm{S}^{1}$ es el más sencillo de los grupos que actúan transitivamente por homeomorfismos del círculo. Módulo conjugación topológica, puede ser caracterizado como el grupo de los homeomorfismos de $\mathrm{S}^{1}$ que preservan una medida de probabilidad con propiedades similares a las de la medida de Lebesgue. Diremos que una medida $\mu$ es de soporte total si la medida de todo conjunto abierto y no vacío es positiva.

Proposición 1.1. Si $\Gamma$ es un subgrupo de $\mathrm{Homeo}_{+}\left(\mathrm{S}^{1}\right)$ que preserva una medida de probabilidad de soporte total y sin átomos, entonces $\Gamma$ es topológicamente conjugado a un subgrupo de $\mathrm{SO}(2, \mathbb{R})$.

Demostración. La medida $\mu$ sobre $\mathrm{S}^{1}$ dada por la hipótesis induce de manera natural una medida $\sigma$-finita $\tilde{\mu}$ en $\mathbb{R}$ que verifica $\tilde{\mu}([x, x+2 \pi])=1$ para todo $x \in \mathbb{R}$. Definamos $\varphi: \mathbb{R} \rightarrow \mathbb{R}$ por $\varphi(x)=2 \pi \tilde{\mu}([0, x])$ si $x>0$, y por $\varphi(x)=-2 \pi \tilde{\mu}([x, 0])$ si $x<0$. Sea $g$ un elemento arbitrario de $\Gamma$. Fijemos un levantamiento a la recta $\tilde{g}$ tal que $\tilde{g}(0)>0$. Para $y>0$ tenemos

$$
\begin{aligned}
\varphi \tilde{g} \varphi^{-1}(y) & =2 \pi \tilde{\mu}\left(\left[0, \tilde{g} \varphi^{-1}(y)\right]\right)=2 \pi \tilde{\mu}([0, \tilde{g}(0)])+2 \pi \tilde{\mu}\left(\left[\tilde{g}(0), \tilde{g} \varphi^{-1}(y)\right]\right) \\
& =2 \pi \tilde{\mu}([0, \tilde{g}(0)])+2 \pi \tilde{\mu}\left(\left[0, \varphi^{-1}(y)\right]\right),
\end{aligned}
$$

por lo que

$$
\varphi \tilde{g} \varphi^{-1}(y)=2 \pi \tilde{\mu}([0, \tilde{g}(0)])+y .
$$

Un argumento análogo prueba que esta última igualdad vale también para $y \leq 0$. Luego, $\varphi \tilde{g} \varphi^{-1}$ es la traslación de longitud $2 \pi \tilde{\mu}([0, \tilde{g}(0)])$. La aplicación $\varphi$ es $2 \pi$-periódica, por lo que induce un homeomorfismo de $\mathrm{S}^{1}$. 
Conjugado con este homeomorfismo, cada $g \in \Gamma$ es la rotación de ángulo $2 \pi \tilde{\mu}([0, g(0)])$ mód $2 \pi$.

Los grupos compactos verifican la hipótesis de la proposición anterior. En efecto, si $\Gamma$ es un grupo compacto, podemos considerar sobre él la medida de Haar $d g$. Si $\Gamma$ actúa sobre el círculo por homeomorfismos, definimos una medida de probabilidad $\mu$ sobre los borelianos de $\mathrm{S}^{1}$ por

$$
\mu(A)=\int_{\Gamma} \operatorname{Leb}(g A) d g .
$$

Esta medida $\mu$ es invariante por $\Gamma$, no posee átomos y es de soporte total. Concluimos así lo siguiente.

Proposición 1.2. Todo subgrupo compacto de $\mathrm{Homeo}_{+}\left(\mathrm{S}^{1}\right)$ es conjugado a un subgrupo de $\mathrm{SO}(2, \mathbb{R})$.

\section{$2 \quad$ El grupo de traslaciones y el grupo afín}

Si un grupo actúa sobre el círculo fijando un punto entonces él actúa de manera obvia sobre la recta (identificando dicho punto fijo con el infinito). Luego, para comprender las acciones de grupos sobre el círculo es necesario comprender también las acciones sobre la recta.

Un grupo interesante de homeomorfismos de la recta es el grupo afín $\mathrm{Af}_{+}(\mathbb{R})$. Al elemento $g \in \mathrm{Af}_{+}(\mathbb{R})$ dado por $g(x)=a x+b, a>0$, le asociamos la matriz

$$
\left(\begin{array}{ll}
a & b \\
0 & 1
\end{array}\right) \in \mathrm{GL}_{+}(2, \mathbb{R}) .
$$

Vía esta correspondencia, el grupo $\mathrm{Af}_{+}(\mathbb{R})$ se identifica a un subgrupo de $\mathrm{GL}_{+}(2, \mathbb{R})$.

Recordemos que una medida de Radon es una medida no trivial definida sobre los borelianos de un espacio topológico que es finita sobre los compactos. Por ejemplo, la medida de Lebesgue es de Radon. Como esta medida es preservada módulo un factor multiplicativo por los elementos de $\mathrm{Af}_{+}(\mathbb{R})$, tiene sentido la siguiente definición.

Definición 1.3. Sean $v$ una medida de Radon sobre la recta y $\Gamma$ un subgrupo de $\mathrm{Homeo}_{+}(\mathbb{R})$. Decimos que $v$ es quasi-invariante por $\Gamma$ si para cada $g \in \Gamma$ existe un real positivo $\kappa(g)$ tal que $g^{*}(v)=\kappa(g) \cdot v$ (es decir, para todo boreliano $A \subset \mathbb{R}$ se cumple $v(g(A))=\kappa(g) \cdot v(A)$.)

Análogamente a la proposición 1.1, tenemos la siguiente caracterización del grupo afín.

Proposición 1.4. Sea $\Gamma$ un subgrupo de $\operatorname{Homeo}_{+}(\mathbb{R})$. Si $\Gamma$ deja quasiinvariante una medida de Radon de soporte total y sin átomos, entonces $\Gamma$ es conjugado a un subgrupo del grupo afín. 
Demostración. Definamos $\varphi: \mathbb{R} \rightarrow \mathbb{R}$ por $\varphi(x)=v([0, x])$ si $x \geq 0$, y por $\varphi(x)=-v([x, 0])$ si $x<0$. Es fácil verificar que $\varphi$ es un homeomorfismo. Además, si $g \in \Gamma$ y $x \geq 0$ son tales que $g(x) \geq 0$ y $g(0) \geq 0$, entonces

$$
\begin{aligned}
\varphi(g(x)) & =v([0, g(x)])=\kappa(g) v\left(\left[g^{-1}(0), x\right]\right) \\
& =\kappa(g) v([0, x])+\kappa(g) v\left(\left[g^{-1}(0), 0\right]\right)=\kappa(g) \varphi(x)-\kappa(g) \varphi\left(g^{-1}(0)\right),
\end{aligned}
$$

Por lo que

$$
\varphi g \varphi^{-1}(x)=\kappa(g) x-\kappa(g) \varphi\left(g^{-1}(0)\right) .
$$

De hecho, no es difícil ver que esta igualdad se cumple de manera general para todo $x \in \mathbb{R}$ y todo $g \in \Gamma$, lo cual concluye la demostración.

El grupo afín contiene al grupo de las traslaciones de la recta, y el argumento de la demostración de la proposición 1.1 muestra lo siguiente.

Proposición 1.5. Sea $\Gamma$ un subgrupo de $\mathrm{Homeo}_{+}(\mathbb{R})$. Si $\Gamma$ deja invariante una medida de Radon de soporte total y sin átomos, entonces $\Gamma$ es topológicamente conjugado a un subgrupo del grupo de traslaciones.

Ejercicio 1.6. La derivada logarítmica de un difeomorfismo $f: I \subset \mathbb{R} \rightarrow J \subset \mathbb{R}$ de clase $\mathrm{C}^{2}$ es definida por $D l(f)(x)=\left(\log \left(f^{\prime}\right)\right)^{\prime}(x)$. Pruebe que $D l(f) \equiv 0$ si y sólo si $f$ es la restricción de un elemento de $\mathrm{Af}_{+}(\mathbb{R})$. A partir de la igualdad

$$
\log \left((f \circ g)^{\prime}\right)(x)=\log \left(g^{\prime}\right)(x)+\log \left(f^{\prime}\right)(g(x)),
$$

pruebe la siguiente relación de cociclo

$$
D l(f \circ g)(x)=D l(g)(x)+g^{\prime}(x) \cdot D l(f)(g(x)) .
$$

\section{El grupo $\operatorname{PSL}(2, \mathbb{R})$}

\section{1 $\operatorname{PSL}(2, \mathbb{R})$ y las transformaciones de Möbius}

Denotaremos por $\mathbb{D}$ al disco disco de Poincaré, es decir, al disco unitario abierto dotado de la métrica hiperbólica

$$
\frac{d u}{2\left(1-|u|^{2}\right)}, \quad \text { donde } \quad u \in \mathbb{D} \text {. }
$$

El grupo de los difeomorfismos (no necesariamente directos) de $\mathbb{D}$ que preservan esta métrica coincide con el grupo de los difeomorfismos conformes de $\mathbb{D}$, y contiene al grupo de Möbius como subgrupo de índice 2 . Lo anterior significa que los únicos difeomorfismos directos $g: \mathbb{D} \rightarrow \mathbb{D}$ que preservan orientación y verifican en todo punto $u \in \mathbb{D}$ y todo vector $\zeta \in T_{u}(\mathbb{D}) \sim \mathbb{R}^{2}$ la igualdad

$$
\frac{\|D g(u)(\zeta)\|}{2\left(1-|g(u)|^{2}\right)}=\frac{\|\zeta\|}{2\left(1-|u|^{2}\right)},
$$


son aquéllos que en notación compleja se escriben de la forma

$$
g(z)=e^{i \theta} \cdot \frac{z-a}{1-\bar{a} z}, \quad \text { donde } \quad \theta \in[0,2 \pi], \quad a \in \mathbb{C}, \quad|a|<1, \quad z \in \mathbb{D} .
$$

El grupo de Möbius es denotado por $\mathcal{M}$. Cada una de las aplicaciones de este grupo induce un difeomorfismo real-analítico del círculo en sí mismo.

Consideremos ahora la aplicación $\varphi(z)=(z+i) /(1+i z)$. Observe que $\varphi\left(\mathrm{S}^{1}\right)=\mathbb{R} \cup\{\infty\}$; además, la imagen de $\mathbb{D}$ por $\varphi$ es el semiplano superior de $\mathbb{R}^{2}$, el cual dotado con la métrica inducida es conocido como plano hiperbólico y denotado por $\mathbb{H}^{2}$. En notación compleja, la acción de cada elemento de $\mathcal{M}$ sobre $\mathbb{H}^{2}$ es de la forma $z \mapsto\left(a_{1} z+a_{2}\right) /\left(a_{3} z+a_{4}\right)$ para ciertos $a_{1}, a_{2}, a_{3}, a_{4}$ en $\mathbb{R}$ tales que $a_{1} a_{4}-a_{2} a_{3}=1$. Por lo tanto, si $a_{3}=0$ entonces obtenemos una transformación afín, lo cual muestra que $\mathrm{Af}_{+}(\mathbb{R})$ es un subgrupo de $\mathcal{M}$.

Asociemos ahora a cada elemento $z \mapsto\left(a_{1} z+a_{2}\right) /\left(a_{3} z+a_{4}\right)$ de $\mathcal{M}$ la matriz

$$
\left(\begin{array}{ll}
a_{1} & a_{2} \\
a_{3} & a_{4}
\end{array}\right) \in \mathrm{SL}(2, \mathbb{R}) .
$$

Un cálculo sencillo muestra que la matriz asociada a la composición de dos elementos de $\mathcal{M}$ es igual al producto de las matrices asociadas a estos elementos. Por otra parte, dos matrices $M_{1}, M_{2}$ de $\operatorname{SL}(2, \mathbb{R})$ definen el mismo elemento de $\mathcal{M}$ si y sólo si ellas coinciden o bien $M_{1}=-M_{2}$. De esta forma, el grupo de Möbius se identifica al grupo proyectivo $\operatorname{PSL}(2, \mathbb{R})$.

El grupo $\operatorname{PSL}(2, \mathbb{R})$ verifica una propiedad notable de transitividad y rigidez: dados dos triples cíclicamente ordenados de puntos del círculo $(a, b, c)$ y $\left(a^{\prime}, b^{\prime}, c^{\prime}\right)$, existe un único elemento $g \in \operatorname{PSL}(2, \mathbb{R})$ que envía $a$, $b$ y $c$ en $a^{\prime}, b^{\prime}$ y $c^{\prime}$ respectivamente (en particular, si $g$ fija tres puntos entonces $g=I d$ ).

Los elementos de $\mathcal{M} \sim \operatorname{PSL}(2, \mathbb{R})$ pueden ser clasificados según el número de puntos fijos sobre $\mathrm{S}^{1} \subset \overline{\mathbb{D}}$. Observe que, para hallar dichos puntos fijos en el modelo del semiplano superior, debemos resolver la ecuación

$$
\frac{a_{1} z+a_{2}}{a_{3} z+a_{4}}=z .
$$

Un análisis simple muestra que pueden darse tres casos:

(i) $\left|a_{1}+a_{4}\right|<2$. En este caso las soluciones de (1.2) son dos puntos conjugados (y distintos) del plano complejo. Luego, en el modelo de Poincaré, la aplicación $g$ no fija ningún punto sobre el círculo. Dejamos al lector la tarea de demostrar que $g$ es conjugado a una rotación.

(ii) $\left|a_{1}+a_{4}\right|=2$. En este caso las dos soluciones de (1.2) coinciden y se hallan sobre la recta real. De esta forma, en el modelo de Poincaré, $g$ fija un único punto sobre el círculo.

(iii) $\left|a_{1}+a_{4}\right|>2$. En este caso existen dos soluciones distintas de (1.2) sobre el eje real. Así, la aplicación fija dos puntos sobre el círculo, uno de ellos atractor y el otro repulsor. 
La expresión $\left|a_{1}+a_{4}\right|$ corresponde al valor absoluto de la traza de la matriz respectiva (si bien la función $M \mapsto a_{1}+a_{4}$ no está bien definida en $\operatorname{PSL}(2, \mathbb{R})$, su valor absoluto sí lo está). Las figuras 1,2 y 3 a continuación ilustran los casos (i), (ii) y (iii) respectivamente. En el caso (i) decimos que la aplicación es elíptica, en (ii) decimos que ella es parabólica siempre que no sea la identidad, mientras que en (iii) decimos que es hiperbólica.

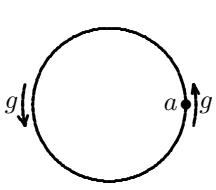

Figura 1

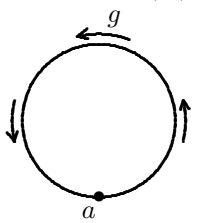

Figura 2

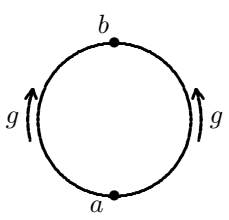

Figura 3

Ejercicio 1.7. Pruebe que dos elementos hiperbólicos cualesquiera de $\operatorname{PSL}(2, \mathbb{R})$ son topológicamente conjugados. ¿Vale lo mismo para las aplicaciones parabólicas y/o elípticas?

Si bien la dinámica de cada elemento del grupo $\operatorname{PSL}(2, \mathbb{R})$ es muy particular, la estructura completa de sus subgrupos no queda determinada completamente por cada una de estas dinámicas. Más precisamente, si un subgrupo $\Gamma$ de $\mathrm{Homeo}_{+}\left(\mathrm{S}^{1}\right)$ es tal que cada uno de sus elementos es topológicamente conjugado a un elemento de $\operatorname{PSL}(2, \mathbb{R})$, esto no implica que $\Gamma$ sea conjugado a un subgrupo de $\operatorname{PSL}(2, \mathbb{R})$, incluso si las órbitas de $\Gamma$ son densas. Ejemplos de subgrupos que satisfacen estas curiosas propiedades fueron dados por Kovačević en [119]. No es muy difícil mejorar la construcción de uno de ellos para obtener un ejemplo de un grupo de difeomorfismos real-analíticos de $\mathrm{S}^{1}$ que verifica las propiedades anteriormente mencionadas [144].

Ejercicio 1.8. Dado un difeomorfismo $f: I \subset \mathbb{R} \rightarrow J \subset \mathbb{R}$ de clase $\mathrm{C}^{3}$, su derivada schwarziana es definida por

$$
S(f)=\frac{f^{\prime \prime \prime}}{f^{\prime}}-\frac{3}{2}\left(\frac{f^{\prime \prime}}{f^{\prime}}\right)^{2} .
$$

Verifique que la derivada schwarziana de un difeomorfismo entre abiertos de la recta es nula si y sólo si dicho difeomorfismo es la restricción de una transformación de Möbius. Pruebe la siguiente relación de cociclo

$$
S(f \circ g)(x)=S(g)(x)+\left(g^{\prime}(x)\right)^{2} \cdot S(f)(g(x)) .
$$

Ejercicio 1.9. Pruebe la siguientes fórmulas para la derivada schwarziana de difeomorfismos de clase $\mathrm{C}^{3}$ :

$$
\begin{gathered}
S(g)(y)=6 \lim _{x \rightarrow y}\left[\frac{g^{\prime}(x) g^{\prime}(y)}{(g(x)-g(y))^{2}}-\frac{1}{(x-y)^{2}}\right]=6 \lim _{x \rightarrow y} \frac{\partial^{2}}{\partial y \partial x} \log \left(\frac{g(x)-g(y)}{x-y}\right), \\
-\frac{1}{2 \sqrt{d g / d x}} S(g)=\frac{d^{2}}{d x^{2}}\left(\frac{1}{\sqrt{d g / d x}}\right) .
\end{gathered}
$$




\section{2 $\operatorname{PSL}(2, \mathbb{R})$ y la corriente geodésica de Liouville}

Recordemos que cada geodésica del disco de Poincaré está determinada por sus puntos extremos en el círculo, los cuales son necesariamente diferentes. De esta manera, el espacio de las geodésicas no orientadas del plano hiperbólico se identifica al cuociente de $S^{1} \times S^{1} \backslash \Delta$ por la relación de equivalencia que identifica a los puntos $(s, t)$ y $(t, s)$, donde $t \neq s$. Una corriente geodésica es una medida de Radon sobre dicho espacio de las geodésicas, y puede ser pensada como una medida $L$ definida sobre los borelianos de $\mathrm{S}^{1} \times \mathrm{S}^{1}$ disjuntos de la diagonal $\Delta$, que es finita sobre los compactos de $\mathrm{S}^{1} \times \mathrm{S}^{1} \backslash \Delta$, y que verifica la condición de simetría

$$
L([a, b] \times[c, d])=L([c, d] \times[a, b]), \quad a<b<c<d<a .
$$

Proposición 1.10. La acción diagonal de $\operatorname{PSL}(2, \mathbb{R})$ sobre $\mathrm{S}^{1} \times \mathrm{S}^{1} \backslash \Delta$ preserva la corriente geodésica

$$
L v=\frac{d s d t}{4 \operatorname{sen}^{2}\left(\frac{s-t}{2}\right)} .
$$

Demostración. Recordemos en primer lugar que la razón cruzada de cuatro puntos $e^{i a}, e^{i b}, e^{i c}, e^{i d}$ en $\mathrm{S}^{1}$ está definida por

$$
\left[e^{i a}, e^{i b}, e^{i c}, e^{i d}\right]=\frac{\left(e^{i a}-e^{i c}\right)\left(e^{i b}-e^{i d}\right)}{\left(e^{i a}-e^{i d}\right)\left(e^{i c}-e^{i b}\right)} .
$$

Un cálculo simple muestra que la razón cruzada es invariante por transformaciones de Möbius. Recíprocamente, si un homeomorfismo de $\mathrm{S}^{1}$ preserva la razón cruzada entonces pertenece al grupo de Möbius. Notemos ahora que, para $a<b<c<d<a$, la medida $L v([a, b] \times[c, d])$ es igual a

$$
\begin{aligned}
\int_{c}^{d} \int_{a}^{b} \frac{d s d t}{4 \operatorname{sen}^{2}\left(\frac{s-t}{2}\right)} & =\int_{c}^{d}\left[-\frac{\cos \left(\frac{s-t}{2}\right)}{2 \operatorname{sen}\left(\frac{s-t}{2}\right)}\right]_{s=a}^{s=b} d t \\
& =\int_{c}^{d} \frac{1}{2}\left[\cot \left(\frac{a-t}{2}\right)-\cot \left(\frac{b-t}{2}\right)\right] d t \\
& =\log \left(\left|\frac{\operatorname{sen}\left(\frac{b-d}{2}\right) \operatorname{sen}\left(\frac{a-c}{2}\right)}{\operatorname{sen}\left(\frac{b-c}{2}\right) \operatorname{sen}\left(\frac{a-d}{2}\right)}\right|\right) .
\end{aligned}
$$

Puesto que $\left|\operatorname{sen}\left(\frac{x-y}{2}\right)\right|=\frac{\left|e^{i x}-e^{i y}\right|}{2}$, obtenemos finalmente

$$
L v([a, b] \times[c, d])=\log \left(\left|\left[e^{i a}, e^{i b}, e^{i c}, e^{i d}\right]\right|\right)=\log \left(\left[e^{i a}, e^{i b}, e^{i c}, e^{i d}\right]\right),
$$

donde la última igualdad se justifica por el hecho que la razón cruzada de cuatro puntos cíclicamente ordenados sobre el círculo es un número real positivo. Como $\operatorname{PSL}(2, \mathbb{R})$ actúa sobre $\mathrm{S}^{1}$ preservando la razón cruzada, la medida $L v$ es preservada. 
La medida $L v$, llamada medida de Liouville, satisface la igualdad

$$
e^{-L v([a, b] \times[c, d])}+e^{-L v([b, c] \times[d, a])}=1
$$

para todo $a<b<c<d<a$. En efecto,

$$
\begin{aligned}
e^{-L v([a, b] \times[c, d])}+e^{-L v([b, c] \times[d, a])} & =\frac{1}{\left[e^{i a}, e^{i b}, e^{i c}, e^{i d}\right]}+\frac{1}{\left[e^{i b}, e^{i c}, e^{i d}, e^{i a}\right]} \\
& =\frac{\left(e^{i a}-e^{i d}\right)\left(e^{i b}-e^{i c}\right)}{\left(e^{i a}-e^{i c}\right)\left(e^{i b}-e^{i d}\right)}+\frac{\left(e^{i b}-e^{i a}\right)\left(e^{i c}-e^{i d}\right)}{\left(e^{i b}-e^{i d}\right)\left(e^{i c}-e^{i a}\right)} \\
& =\frac{\left(e^{i a}-e^{i d}\right)\left(e^{i b}-e^{i c}\right)-\left(e^{i b}-e^{i a}\right)\left(e^{i c}-e^{i d}\right)}{\left(e^{i a}-e^{i c}\right)\left(e^{i b}-e^{i d}\right)} \\
& =1 .
\end{aligned}
$$

Veremos a continuación que, en cierto sentido, la propiedad (1.7) caracteriza a la medida de Liouville.

Para una corriente geodésica cualquiera $L$ denotaremos por $\Gamma_{L}$ al grupo de los homeomorfismos del círculo que preservan $L$. Por ejemplo, es fácil verificar que $\Gamma_{L v}=\operatorname{PSL}(2, \mathbb{R})$. En general, el grupo $\Gamma_{L}$ es muy pequeño, la mayoría de las veces trivial. Sin embargo, existe una condición que asegura que dicho grupo sea topológicamente conjugado a $\operatorname{PSL}(2, \mathbb{R})$. El siguiente resultado podría ser considerado como un análogo para el grupo de Möbius de las proposiciones 1.1, 1.4 ó 1.5. Vea [14] para su demostración, así como el ejercicio 1.16 para una versión alternativa.

Proposición 1.11. Si L es una corriente geodésica verificando la propiedad (1.7), entonces $\Gamma_{L}$ es conjugado a $\operatorname{PSL}(2, \mathbb{R})$ por un homeomorfismo que transforma la corriente geodésica $L$ en la corriente de Liouville.

Ejercicio 1.12. Utilizando la invariancia de la corriente de Liouville para transformaciones de Möbius, pruebe que si $f: I \rightarrow \mathbb{R}$ es un difeomorfismo local de clase $\mathrm{C}^{1}$ cuya derivada satisface

$$
f^{\prime}(x) f^{\prime}(y)=\frac{(f(x)-f(y))^{2}}{(x-y)^{2}}
$$

para todo $x \neq y$ en $I$, entonces $f$ es de la forma $x \mapsto(a x+b) /(c x+d)$ para ciertos reales $a, b, c, d$ (compare con (1.4)).

\section{3 $\operatorname{PSL}(2, \mathbb{R})$ y la propiedad de convergencia}

Sea $\Gamma$ un subgrupo de $\mathrm{Homeo}_{+}\left(\mathrm{S}^{1}\right)$. Diremos que una sucesión $\left(g_{n}\right)$ de elementos en $\Gamma$ posee la propiedad de convergencia si contiene una subsucesión $\left(g_{n_{k}}\right)$ de $\left(g_{n}\right)$ que satisface una de las afirmaciones siguientes:

(i) existen $a, b$ en $\mathrm{S}^{1}$ (no necesariamente diferentes) tales que $g_{n_{k}}$ converge a $b$ puntualmente en $\mathrm{S}^{1}-\{a\}$ y $g_{n_{k}}^{-1}$ converge puntualmente al punto $a$ en $\mathrm{S}^{1} \backslash\{b\}$; 
(ii) existe $g \in$ Homeo $_{+}\left(\mathrm{S}^{1}\right)$ tal que $g_{n_{k}}$ converge a $g$ y $g_{n_{k}}^{-1}$ converge a $g^{-1}$ puntualmente en todo el círculo.

Diremos que $\Gamma$ posee la propiedad de convergencia si toda sucesión en $\Gamma$ posee dicha propiedad.

Observe que la propiedad de convergencia es invariante por conjugación topológica. Por otra parte, se comprueba fácilmente que todo subgrupo de $\operatorname{PSL}(2, \mathbb{R})$ posee la propiedad de convergencia (vea los ejercicios $1.15 \mathrm{y}$ 1.16). Recíprocamente, un teorema de difícil demostración debido esencialmente a Casson, Jungreis, Gabai, Hinkkanen y Tukia [45, 68, 70, 95, 204] establece que la propiedad de convergencia caracteriza (módulo conjugación topológica) a los subgrupos de $\operatorname{PSL}(2, \mathbb{R})$.

Teorema 1.13. Un subgrupo de $\mathrm{Homeo}_{+}\left(\mathrm{S}^{1}\right)$ es topológicamente conjugado a un subgrupo de $\operatorname{PSL}(2, \mathbb{R})$ si y sólo si satisface la propiedad de convergencia.

Es posible probar que en el caso de subgrupos discretos de $\mathrm{Homeo}_{+}\left(\mathrm{S}^{1}\right)$, la propiedad de convergencia es equivalente a la condición de que la acción del grupo sea libre y propiamente discontinua en el espacio de las ternas ordenadas de puntos del círculo [204].

Ejercicio 1.14. Pruebe directamente a partir de la definición que si $\Gamma$ posee la propiedad de convergencia y $g \in \Gamma$ fija tres puntos del círculo, entonces $g=I d$.

Ejercicio 1.15. Un homeomorfismo $g$ de $\mathrm{S}^{1}$ es $C$-quasi-simétrico si para todo $a<b<c<d<a$ tales que $[a, b, c, d]=2$ se tiene $1 / C \leq[g(a), g(b), g(c), g(d)] \leq C$. Pruebe que si $\Gamma$ es un subgrupo uniformemente quasi-simétrico de $\mathrm{Homeo}_{+}\left(\mathrm{S}^{1}\right)$, es decir, tal que todos sus elementos son $C$-quasi-simétricos (respecto a la misma constante $C$ ), entonces $\Gamma$ verifica la propiedad de convergencia. Concluya que $\Gamma$ es topológicamente conjugado a un subgrupo de $\operatorname{PSL}(2, \mathbb{R})$.

Observación. De acuerdo a un resultado de Markovic [131], bajo las hipótesis precedentes el grupo $\Gamma$ es quasi-simétricamente conjugado a un subgrupo de $\operatorname{PSL}(2, \mathbb{R})$

Ejercicio 1.16. Sea $L$ una corriente geodésica que verifica $L([a, a] \times[b, c])=0$ para todo $a<b \leq c<a$ y $L([a, b[\times] b, c])=\infty$ para todo $a<b<c<a$ (observe que la corriente de Liouville satisface estas propiedades). Pruebe que $\Gamma_{L}$ posee la propiedad de convergencia (vea [143] si tiene problemas para ello). Concluya que $\Gamma_{L}$ es topológicamente conjugado a un subgrupo de $\operatorname{PSL}(2, \mathbb{R})$. Note sin embargo que esta conjugación topológica no transforma necesariamente $L$ en la corriente de Liouville, pues la propiedad (1.7) es invariante por conjugación y no es satisfecha a priori por los elementos de $\Gamma_{L}$.

\section{Acciones de grupos de Lie}

El objetivo de esta sección es poner en evidencia el hecho que los grupos localmente compactos que pueden admitir acciones novedosas por homeo- 
morfismos de $\mathrm{S}^{1}$ son los grupos discretos (pensamos los grupos discretos como grupos de Lie de dimensión cero). Es por ello que, en muchísimas ocasiones, nos centraremos sólo en el estudio de las acciones de tales grupos. Para lograr nuestro objetivo utilizaremos un teorema profundo de Montgomery y Zippin [138], el cual establece que un grupo topológico localmente compacto es un grupo de Lie si y sólo si existe una vecindad de la identidad que no contiene ningún subgrupo compacto no trivial.

Proposición 1.17. Sea $\Gamma$ un grupo topológico localmente compacto. Para toda representación $\Phi$ de $\Gamma$ en $\mathrm{Homeo}_{+}\left(\mathrm{S}^{1}\right)$, la imagen $\Phi(\Gamma)$ (dotada de la topología inducida) es un grupo de Lie.

Demostración. El grupo $\Phi(\Gamma)$, dotado de la topología inducida, es localmente compacto. El conjunto

$$
V=\{g \in \Phi(\Gamma): \operatorname{dist}(x, g(x))<2 \pi / 3\}
$$

es una vecindad de $I d$ en $\Phi(\Gamma)$. Probaremos que $V$ no contiene ningún grupo compacto no trivial, lo cual -gracias al teorema de Montgomery y Zippin- implica la proposición.

Supongamos que $\Gamma_{0}$ es un subgrupo no trivial de Homeo $+\left(\mathrm{S}^{1}\right)$ contenido en $V$ y que $\Gamma_{0}$ es compacto según la topología inducida. Para cada $f \in \Gamma$ sea $\tilde{f} \in \widehat{\mathrm{Homeo}_{+}}\left(\mathrm{S}^{1}\right)$ el (único) levantamiento de $f$ tal que $\operatorname{dist}(\tilde{f}(x), x)<2 \pi / 3$ para todo $x \in \mathbb{R}$. Si $g$, $h$ están en $\Gamma_{0}$ entonces es fácil verificar que $\widetilde{g h}=\tilde{g} \tilde{h}$. Luego, $\Gamma_{0}$ se inyecta en el grupo $\widetilde{\mathrm{Homeo}_{+}}\left(\mathrm{S}^{1}\right)$. Observe que $\widetilde{\mathrm{Homeo}_{+}}\left(\mathrm{S}^{1}\right)$ no posee elementos de torsión. Sin embargo, la proposición 1.2 muestra que todo subgrupo compacto no trivial de $\mathrm{Homeo}_{+}\left(\mathrm{S}^{1}\right)$ posee elementos de torsión. Esta contradicción prueba la proposición.

Ejercicio 1.18. Es possible dar una prueba de la proposición precedente que no se apoya sobre la proposición 1.2. Para ello basta con utilizar la versión unidimensional del siguiente lema debido a Newman [152] (vea también [117]), el cual invitamos al lector a demostrar: si $f$ es un homeomorfismo no trivial y de orden finito de la esfera $n$-dimensional $\mathrm{S}^{n}$ de diámetro 1 , entonces existe $i \in \mathbb{N}$ tal que $\operatorname{dist}\left(f^{i}, I d\right)>1 / 2$.

Sugerencia. Si la desigualdad contraria es satisfecha para todo $i$ entonces cada órbita queda contenida en un hemisferio de la esfera. En tal caso podemos definir una aplicación continua bar $: \mathrm{S}^{n} \rightarrow \mathrm{S}^{n}$ haciendo corresponder a cada $x$ el "baricentro" bar $(x)$ de su órbita dentro del hemisferio correspondiente. Esta aplicación satisface $\operatorname{bar}(f(x))=\operatorname{bar}(x)$ para todo $x$, de donde se deduce fácilmente que el grado topológico de $f$ es un múltiplo de su orden. Sin embargo, siendo $f$ homotópico a la identidad, su grado topológico es igual a 1.

Observación. De la afirmación demostrada se deduce casi inmediatamente que

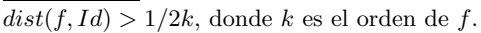

No es difícil obtener la clasificación de las acciones transitivas de grupos de Lie conexos sobre variedades unidimensionales [72]. Módulo conjugación topológica la lista completa está formada por: 
(i) la acción de $(\mathbb{R},+)$ por traslaciones en la recta;

(ii) la acción del grupo $\mathrm{SO}(2, \mathbb{R})$ por rotaciones del círculo;

(iii) la acción del grupo afín $\mathrm{Af}_{+}(\mathbb{R})$ sobre la recta;

(iv) la acción del grupo $\operatorname{PSL}_{k}(2, \mathbb{R})$ cuyos elemento son los levantamientos de los elementos de $\operatorname{PSL}(2, \mathbb{R})$ al recubrimiento $\hat{\mathrm{S}}^{1}$ a $k$ hojas del círculo, con $k \geq 1$ (observe que $\hat{\mathrm{S}}^{1}$ es topológicamente un círculo);

(v) la acción del grupo $\widetilde{\mathrm{PSL}}(2, \mathbb{R})$ cuyos elementos son los levantamientos a la recta de los elementos de $\operatorname{PSL}(2, \mathbb{R})$.

De cierta manera, esta clasificación indica que existen tres tipos de geometría en variedades unidimensionales: euclideana, afín y proyectiva [130]. La clasificación de las acciones efectivas no transitivas de grupos de Lie conexos se desprende de la anterior. En efecto, las órbitas de una acción de este tipo son puntos o intervalos. Luego, considerando el conjunto Fix $(\Gamma)$ de los puntos fijos globales de la acción, sobre cada componente conexa del complemento de $\operatorname{Fix}(\Gamma)$ obtenemos una acción dada por una sobreyección de $\Gamma$ sobre $(\mathbb{R},+), \operatorname{SO}(2, \mathbb{R}), \mathrm{Af}_{+}(\mathbb{R}), \operatorname{PSL}_{k}(2, \mathbb{R})$ ó $\widetilde{\operatorname{PSL}}(2, \mathbb{R})$.

\section{Los grupos de Thompson}

Para mayor simplicidad, en esta sección trabajaremos con la parametrización del círculo por el intervalo $[0,1]$ vía la aplicación $x \mapsto e^{2 \pi i x}$. Consideremos el grupo de los homeomorfismos $\tilde{f}: \mathbb{R} \rightarrow \mathbb{R}$ tales que:

(i) $\tilde{f}(0)=0$;

(ii) existe una sucesión $\ldots x_{-1}<x_{0}<x_{1}<\ldots$ (divergente en ambos sentidos) de racionales diádicos tal que cada restricción $\left.\tilde{f}\right|_{\left[x_{i}, x_{i+1}\right]}$ es afín y su derivada es una potencia entera de 2 ;

(iii) $\tilde{f}(x+1)=\tilde{f}(x)+1$ para todo $x \in \mathbb{R}$.

Cada $\tilde{f}$ induce un homeomorfismo $f$ del intervalo [0,1] dado por $f(1)=1$ y $f(s)=\tilde{f}(s)$ mód 1 para $s \in[0,1]$. Obtenemos de esta manera un grupo de homeomorfismos de $[0,1]$, el cual es llamado grupo de Thompson y denotado por $\mathrm{F}$.

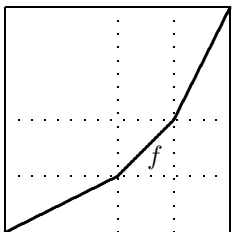

Figura 4

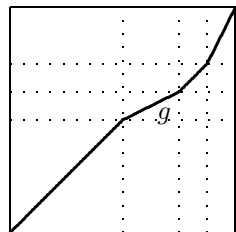

Figura 5 
El grupo F posee muchas propiedades notables, las cuales no son siempre fáciles de demostrar. En primer lugar, F admite la presentación finita

$$
\mathrm{F}=\left\langle f, g:\left[f g^{-1}, f^{-1} g f\right]=\left[f g^{-1}, f^{-2} g f^{2}\right]=i d\right\rangle,
$$

donde $[\cdot, \cdot]$ denota el conmutador de dos elementos y $f, g$ son los homeomorfismos graficados en las figuras 4 y 5 respectivamente.

Puesto que todo homeomorfismo no trivial del intervalo tiene orden infinita, F es un grupo sin torsión. De la demostración del teorema 1.19 resultará evidente que $\mathrm{F}$ posee subgrupos abelianos libres de índice infinito. Por otra parte, el cuociente abelianizado $\mathrm{F} /[\mathrm{F}, \mathrm{F}]$ es isomorfo a $\mathbb{Z} \times \mathbb{Z}$. Para probar esto basta considerar, para cada $[h] \in \mathrm{F} /[\mathrm{F}, \mathrm{F}]$, el valor de la derivada de $h$ en 0 y en 1 . Dichos valores no dependen del representante $h$, pues $[f, g]^{\prime}(0)=[f, g]^{\prime}(1)=1$ para todo $f, g$ en F. Tomando luego el logaritmo en base 2 de dichos valores, obtenemos un homomorfismo de $\mathrm{F} /[\mathrm{F}, \mathrm{F}]$ en $\mathbb{Z} \times \mathbb{Z}$. Finalmente, es posible probar que éste es en realidad un isomorfismo, aunque para ello es necesario utilizar el hecho (no sencillo de demostrar) que el grupo [F, F] es simple [37].

Más información sobre el grupo de Thompson $\mathrm{F}$ puede ser encontrada por ejemplo en [24, 37] y [78]. En particular, en la primera de estas referencias se aborda el problema de saber si $\mathrm{F}$ es un grupo promediable (vea el apéndice). Una de las dificultades de este problema radica en que $\mathrm{F}$ no posee subgrupos libres a dos generadores (vea el ejercicio 5.65). Esto es un corolario de un resultado obtenido por Brin y Squier en [28] y que nosotros reproducimos a continuación. En el caso en que F no fuese promediable entonces sería el primer ejemplo de un grupo de presentación finita, sin torsión, no promediable y que no contiene a $\mathbb{L}_{2}$, lo cual respondería definitivamente a una pregunta dejada por Von Newman. Señalemos que un ejemplo de un grupo de presentación finita no promediable y que no contiene a $\mathbb{L}_{2}$ ha sido construido por Olshanski y Sapir en [160].

Teorema 1.19. El grupo $\mathrm{AfP}_{+}([0,1])$ de los homeomorfismos afines por partes de $[0,1]$ no contiene subgrupos libres a dos generadores.

Demostración. Supongamos por contradicción que dos elementos $f$ y $g$ de $\mathrm{AfP}_{+}([0,1])$ generan un subgrupo libre. Para cada $h \in \mathrm{AfP}_{+}([0,1])$ denotemos por $\operatorname{sop}_{0}(h)$ al "soporte abierto" de $h$, es decir, al conjunto de los puntos de $[0,1]$ no fijos por $h$. El conjunto $I=\operatorname{sop}_{0}(f) \cup s o p_{0}(g)$ puede ser expresado como la unión de un número finito de intervalos abiertos $I_{1}, \ldots, I_{n}$. Observe que el cierre del conjunto $\operatorname{sop}_{0}([f, g])$ está contenido en $I$, pues en una vecindad de cada punto extremo $x_{0}$ de cada $I_{i}$ las aplicaciones $f$ y $g$ son de la forma $x \mapsto \lambda\left(x-x_{0}\right)+x_{0}$, y por lo tanto conmutan.

De entre los elementos no triviales $h$ de $\langle f, g\rangle$ tales que $\overline{s o p_{0}(h)}$ está contenido en $I$, escojamos uno, digamos $h_{0}$, tal que el número de componentes de $I$ que intersectan a $\overline{s o p_{0}\left(h_{0}\right)}$ sea minimal. Sea $] a, b[$ una de las componentes de intersección y sea $[c, d]$ un intervalo contenido en el interior de 
]$a, b\left[\right.$ y que contiene a $\left.\operatorname{sop}_{0}\left(h_{0}\right) \cap\right] a, b[$. Si $x$ pertenece a $] a, b[$ entonces la órbita de $x$ por el grupo generado por $f$ y $g$ está contenida en $] a, b[$, y el supremo de dicha órbita es un punto fijo por $f$ y $g$, por lo que coinciden con el punto $b$. Se deduce entonces la existencia de un elemento $\bar{h} \in\langle f, g\rangle$ que envía el intervalo $[c, d]$ hacia la derecha de $d$. En particular, las restricciones de $h_{0}$ y $\bar{h} h_{0} \bar{h}^{-1}$ al intervalo $[a, b]$ conmutan, generando así un subgrupo isomorfo a $\mathbb{Z} \times \mathbb{Z}$. Por otra parte, $h_{0}$ y $\bar{h} h_{0} \bar{h}^{-1}$ no conmutan en $\langle f, g\rangle \sim \mathbb{L}_{2}$, pues en caso contrario generarían un subgrupo isomorfo a $\mathbb{Z}$. El conmutador entre $h_{0}$ y $\bar{h} h_{0} \bar{h}^{-1}$ es entonces un elemento no trivial cuyo soporte abierto no intersecta a $[a, b]$, por lo que intersecta menos componentes de $I$ que el soporte abierto de $h_{0}$. Sin embargo, esto contradice la elección de $h_{0}$.

Si consideramos los homeomorfismos $\tilde{f}$ de la recta que satisfacen sólo las propiedades (ii) y (iii) de las correspondientes a los levantamientos de los elementos de $\mathrm{F}$, y tales que $\tilde{f}(0)$ es un racional diádico, entonces al pasar al cuociente obtenemos un grupo de homeomorfismos de $\mathrm{S}^{1}$, el cual es denotado por G. Este grupo posee la propiedad remarcable de ser a la vez infinito, de presentación finita y simple. De hecho, G fue el primer ejemplo de un grupo satisfaciendo simultáneamente estas tres propiedades.

\subsection{La realización de Thurston}

Para comprender un poco mejor los grupos de Thompson daremos otras dos definiciones de ellos. La primera se basa en el trabajo original de Thompson, mientras que la segunda está basada en una idea de Thurston. Comencemos con algunas definiciones.

Un árbol diádico (no trivial) $\mathcal{T}$ es una colección finita de aristas cerradas (es decir, que incluyen sus puntos extremos o vértices) tales que:

(i) existe un vértice marcado llamado la raíz del árbol y denotado por $\sigma$;

(ii) cada vértice diferente de la raíz es el punto final ya sea de una o de tres aristas, mientras que la raíz es el punto final de ninguna o de dos aristas;

(iii) $\mathcal{T}$ es conexo.

Si un vértice es un punto final de tres aristas, ellas pueden etiquetarse por $\Upsilon_{a}, \Upsilon_{i}$ y $\Upsilon_{d}$. En relación a este etiquetaje, las aristas deben ser pensadas como aquélla que apunta hacia abajo, a izquierda y a derecha respectivamente. Las aristas que parten de $\sigma$ pueden etiquetarse por $\Upsilon_{i}$ y $\Upsilon_{d}$. Un vértice $v \neq \sigma$ que es un punto final de una única arista es llamado una hoja del árbol. El conjunto de hojas de $\mathcal{T}$ será denotado por $h j(\mathcal{T})$. Notemos que existe un orden cíclico natural entre las hojas de cada árbol diádico. Respecto a este orden cíclico, la noción de primera hoja se define de manera evidente.

Dados un árbol $\mathcal{T}$ y una hoja $p \in h j(\mathcal{T})$, diremos que un árbol $\mathcal{T}^{\prime}$ es un árbol germinado de $\mathcal{T}$ a partir de $p$ si $\mathcal{T}^{\prime}$ es igual a la unión de $\mathcal{T}$ y dos 
aristas cerradas partiendo de $p$. Notemos que el número de hojas de un árbol germinado es igual a 1 más el número de hojas del original.

Consideremos ahora dos árboles $\mathcal{T}_{1}$ et $\mathcal{T}_{2}$ que poseen el mismo número de hojas. Diremos que una transfomación $h j\left(\mathcal{T}_{1}\right) \rightarrow h j\left(\mathcal{T}_{2}\right)$ es G-admisible si preserva el orden cíclico de las hojas, y diremos que ella es F-admisible si además envía la primera hoja de $\mathcal{T}_{1}$ sobre la primera hoja de $\mathcal{T}_{2}$. Vamos a definir una relación de equivalencia entre transformaciones admisibles. Daremos la definición explícita sólo para el caso de aplicaciones G-admisibles, pues el caso de transformaciones F-admisibles es análogo.

Fijemos una aplicación G-admisible $g: h j\left(\mathcal{T}_{1}\right) \rightarrow h j\left(\mathcal{T}_{2}\right)$ y una hoja $p$ de $\mathcal{T}_{1}$. Consideremos ahora los árboles $\mathcal{T}_{1}^{\prime}$ y $\mathcal{T}_{2}^{\prime}$ germinados de $\mathcal{T}_{1}$ y $\mathcal{T}_{2}$ a partir de $p$ y $g(p)$ respectivamente. Definamos la aplicación $g^{\prime}: h j\left(\mathcal{T}_{1}^{\prime}\right) \rightarrow h j\left(\mathcal{T}_{2}^{\prime}\right)$ por $g^{\prime}(q)=g(q)$ si $q$ es una hoja de $\mathcal{T}_{1}$ diferente de $p$, y por $g^{\prime}\left(p_{1}\right)=p_{1}^{\prime}$ y $g^{\prime}\left(p_{2}\right)=p_{2}^{\prime}$, donde $p_{1} \neq p$ y $p_{2} \neq p$ son los vértices de las aristas $\Upsilon_{i}$ y $\Upsilon_{d}$ que parten desde $p$ respectivamente (y de manera análoga para $p_{1}^{\prime}$ y $p_{2}^{\prime}$ en relación a $g(p))$. La aplicación $g^{\prime}$ será llamada una germinación de $g$.

En general, dadas dos aplicaciones G-admisibles $g: h j\left(\mathcal{R}_{1}\right) \rightarrow h j\left(\mathcal{S}_{1}\right)$ y $h: h j\left(\mathcal{R}_{2}\right) \rightarrow h j\left(\mathcal{S}_{2}\right)$, diremos que $g$ es G-equivalente respectivamente a $h$ si existe una secuencia finita $g_{0}=g, g_{1}, \ldots, g_{n}=h$ de aplicaciones Gadmisibles tal que, para cada $k \in\{1, \ldots, n\}$, ya sea $g_{k}$ es una germinación de $g_{k-1}$, o bien $g_{k-1}$ es una germinación de $g_{k}$. Denotemos por $G$ al conjunto de las aplicaciones G-admisibles módulo esta relación de equivalencia.

Procederemos ahora a definir una estructura de grupo en $F$ y $G$. Nuevamente, daremos la definición explícita sólo para el caso de $G$, pues el caso de $F$ es análogo. Fijemos entonces dos elementos $f, g$ de $G$. No es difícil verificar que existen árboles diádicos $\mathcal{R}, \mathcal{S}$ y $\mathcal{T}$ tales que en la clase de $f$ y en la de $g$ existen aplicaciones (que denotaremos también por $f$ y $g$ respectivamente) que verifican $g: h j(\mathcal{R}) \rightarrow h j(\mathcal{S})$ y $f: h j(\mathcal{S}) \rightarrow h j(\mathcal{T})$. Definimos entonces el elemento $f g \in G$ como la clase de la aplicación

$$
f g: h j(\mathcal{R}) \rightarrow h j(\mathcal{T}) .
$$

El lector verificará sin dificultad que esta definición no depende de los representantes elegidos, y que dotado de este producto, $G$ es un grupo. Un ejemplo de composición de elementos de $G$ aparece en la figura 6 . Notemos que el elemento neutro es la clase de la transformación que envía la raíz (pensada como la única hoja de un árbol trivial) sobre sí misma.

Explicaremos ahora la relación entre los grupos $G$ y $F$ que acabamos de definir y aquéllos que actúan sobre el círculo y el intervalo respectivamente. Para esto, a cada vértice de un árbol diádico asociaremos un subintervalo de $[0,1]$ del tipo $\left[i / 2^{n},(i+1) / 2^{n}\right]$ de la manera siguiente:

(i) a la raíz le asociamos el intervalo $[0,1]$;

(ii) si al vértice $p$ le hemos asociado el intervalo $[a, b]$ y $p$ no es una hoja, entonces a $p_{1}$ y $p_{2}$ les asociamos los intervalos $[a,(a+b) / 2]$ y $[(a+b) / 2, b]$ respectivamente, donde $p_{1} \neq p$ y $p_{2} \neq p$ son los vértices finales de las aristas $\Upsilon_{i}$ y $\Upsilon_{d}$ que nacen desde $p$. 


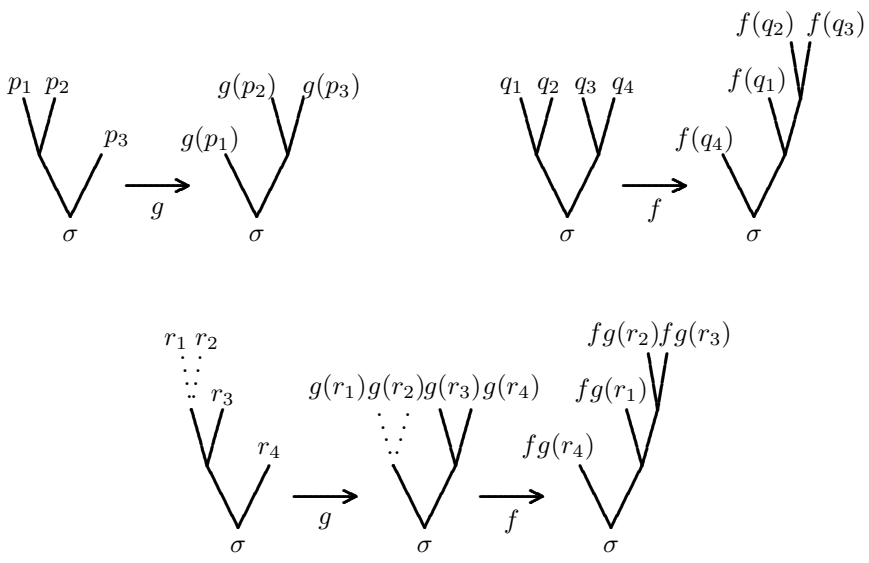

Figura 6

Para cada $g \in G$ escogemos un representante $g: h j\left(\mathcal{T}_{1}\right) \rightarrow h j\left(\mathcal{T}_{2}\right)$ y le asociamos el homeomorfismo $\mathrm{S}^{1} \rightarrow \mathrm{S}^{1}$ que envía el intervalo correspondiente a cada hoja $p$ de $\mathcal{T}_{1}$ de manera afín sobre el intervalo correspondiente a la hoja $g(p)$. No es difícil convencerse de que esta asociación no depende del representante escogido. Obtenemos así un homomorfismo del grupo $G$ recientemente definido y el grupo $\mathrm{G}$ que actúa sobre el círculo, y es fácil ver que este homomorfismo es de hecho un isomorfismo.

Para finalizar esta sección probaremos que $\mathrm{G}$ es isomorfo a un subgrupo de Difeo ${ }_{+}^{1+l i p}\left(\mathrm{~S}^{1}\right)$. La demostración que presentaremos se basa en una idea de Thurston. Siguiendo una construcción de Ghys y Sergiescu, probaremos en la sección siguiente que un resultado más fuerte es válido: G es topológicamente conjugado a un subgrupo del grupo de los difeomorfismos de clase $\mathrm{C}^{\infty}$ del círculo.

La idea de Thurston consiste en considerar particiones finitas de $\mathrm{S}^{1}$ dadas por la sucesión de Farey en lugar de particiones en intervalo diádicos. En otras palabras, a cada vértice de un árbol diádico le asociamos un subintervalo de $[0,1]$ de la manera siguiente:

(i) a la raíz le asociamos el intervalo $[0,1]$;

(ii) si al vértice $p$ le hemos asociado el intervalo $[a / b, c / d]$ y $p$ no es una hoja, entonces a $p_{1}$ y $p_{2}$ les asociamos los intervalos $[a / b,(a+b) /(c+d)]$ y $[(a+b) /(c+d), c / d]$ respectivamente, donde $p_{1} \neq p$ y $p_{2} \neq p$ son los vértices finales de las aristas $\Upsilon_{i} \mathrm{y} \Upsilon_{d}$ que parten de $p$.

Así, para cada $g \in G$ escogemos un representante $g: h j\left(\mathcal{T}_{1}\right) \rightarrow h j\left(\mathcal{T}_{2}\right)$ y le hacemos corresponder el homeomorfismo del círculo que envía el intervalo 
asociado a cada hoja $p$ de $\mathcal{T}_{1}$ sobre el intervalo correspondiente a la hoja $g(p)$ por una aplicación de $\operatorname{PSL}(2, \mathbb{Z})$. Como en el caso anterior, todo está bien definido módulo la relación de equivalencia que define al grupo $G$. Se puede además explicitar las transformaciones de $\operatorname{PSL}(2, \mathbb{Z})$ usadas en la definición. En efecto, no es difícil verificar por inducción que si a un vértice se le ha asociado el intervalo $[a / b, c / d]$ entonces $b c-a d=1$. Por lo tanto, la única aplicación de $\operatorname{PSL}(2, \mathbb{Z})$ que envía $I=[a / b, c / d]$ sobre $J=\left[a^{\prime} / b^{\prime}, c^{\prime} / d^{\prime}\right]$ es $\gamma_{I, J}=\gamma_{J} \circ \gamma_{I}^{-1}$, donde

$$
\gamma_{I}(x)=\frac{(c-a) x+a}{(d-b) x+b}, \quad \gamma_{J}(x)=\frac{\left(c^{\prime}-a^{\prime}\right) x+a^{\prime}}{\left(d^{\prime}-b^{\prime}\right) x+b^{\prime}} .
$$

Notemos que $\gamma_{I}^{\prime}(x)=1 /((d-b) x+b)^{2}$, y por lo tanto

$$
\gamma_{I, J}^{\prime}\left(\frac{a}{b}\right)=\left(\frac{b}{b^{\prime}}\right)^{2}, \quad \gamma_{I, J}^{\prime}\left(\frac{a^{\prime}}{b^{\prime}}\right)=\left(\frac{d}{d^{\prime}}\right)^{2} .
$$

Estas igualdades muestran que para cada $g \in G$ el homeomorfismo $\operatorname{PSL}(2, \mathbb{Z})$ por partes asociado es de clase $\mathrm{C}^{1+\mathrm{lip}}$, pues los valores de las derivadas a izquierda y a derecha en los puntos de quiebre coinciden.

Hemos construido así una nueva acción de G sobre el círculo, esta vez por difeomorfismos de clase $\mathrm{C}^{1+\text { lip }}$. No es muy difícil probar que este nuevo grupo de transformaciones $\operatorname{PSL}(2, \mathbb{Z})$ por partes y aquél afín y diádico por partes son topológicamente conjugados.

\subsection{La realización de Ghys y Sergiescu}

Una propiedad remarcable (y a primera vista sorprendente) del grupo de Thompson $\mathrm{G}$ es la posibilidad de ser realizado como un grupo de difeomorfismos de clase $\mathrm{C}^{\infty}$ del círculo. Mencionemos que en general se desconoce cuáles son los subgrupos de $\mathrm{AfP}_{+}\left(\mathrm{S}^{1}\right)$ que verifican esta propiedad; de entre estos grupos, aquéllos considerados por Stein en [191] resultan muy interesantes, tanto del punto de vista algebraico como dinámico.

Siguiendo esencialmente (una parte de) [78], asociaremos una representación de $\mathrm{G}$ en $\mathrm{Homeo}_{+}\left(\mathrm{S}^{1}\right)$ a cada homeomorfismo $H: \mathbb{R} \rightarrow \mathbb{R}$ satisfaciendo las propiedades siguientes:

(i) para cada $x \in \mathbb{R}$ se tiene $H(x+1)=H(x)+2$,

(ii) $H(0)=0$.

Observe que la función $H(x)=2 x$ verifica estas dos propiedades: la representación asociada corresponderá a la inclusión canónica de G en el grupo de los homeomorfismos afines por pedazos del círculo.

Para la construcción fijemos algunas notaciones. Primeramente, $\mathbb{Q}_{2}(\mathbb{R})$ designará el grupo de los números diádicos (pensado como un subgrupo del grupo de traslaciones). Por $\mathrm{Af}_{+}\left(\mathbb{Q}_{2}, \mathbb{R}\right)$ designaremos el grupo de las transformaciones afines de la recta que preservan el conjunto de los racionales 
diádicos, mientras que por $\operatorname{AfP}_{+}\left(\mathbb{Q}_{2}, \mathbb{R}\right)$ designaremos el grupo de los homeomorfismos de la recta que son $\mathrm{Af}_{+}\left(\mathbb{Q}_{2}, \mathbb{R}\right)$ por partes. De manera análoga, $\mathbb{Q}_{2}\left(\mathrm{~S}^{1}\right)$ denotará el grupo de las rotaciones diádicas del círculo, y $\operatorname{AfP}_{+}\left(\mathbb{Q}_{2}, \mathrm{~S}^{1}\right)$ denotará el grupo de los homeomorfismos diádicamente afines por partes de $\mathrm{S}^{1}$. Recuerde finalmente que, para cada $a \in \mathbb{R}$, la traslación de desplazamiento $a$ es denotada $T_{a}$.

Lema 1.20. La correspondencia $\Phi_{H}: \mathbb{Q}_{2}(\mathbb{R}) \rightarrow$ Homeo $_{+}(\mathbb{R})$ dada por $p / 2^{q} \mapsto H^{-q} T_{p} H^{q}$ está bien definida y es un homomorfismo de grupos.

Demostración. Para probar que la definición no es contradictoria debemos verificar que para todo par de enteros $p$ y $q \geq 0$ se cumple la igualdad $\Phi_{H}\left(p / 2^{q}\right)=\Phi_{H}\left(2 p / 2^{q+1}\right)$, es decir,

$$
H^{-q} T_{p} H^{q}=H^{-(q+1)} T_{2 p} H^{q+1} .
$$

Para ello observe que esta igualdad equivale a $H(x+p)=H(x)+2 p$, por lo que resulta inmediatamente de la propiedad (i). Para verificar que $\Phi_{H}$ es un homomorfismo observe que

$\Phi_{H}\left(\frac{p}{2^{q}}+\frac{p^{\prime}}{2^{q}}\right)=H^{-q} T_{p+p^{\prime}} H^{q}=H^{-q} T_{p} H^{q} H^{-q} T_{p^{\prime}} H^{q}=\Phi_{H}\left(\frac{p}{2^{q}}\right) \Phi_{H}\left(\frac{p^{\prime}}{2^{q}}\right)$.

Lema 1.21. El homomorfismo $\Phi_{H}$ del lema precedente se extiende a un homomorfismo de $\mathrm{Af}_{+}\left(\mathbb{Q}_{2}, \mathbb{R}\right)$ en $\mathrm{Homeo}_{+}(\mathbb{R})$ mediante la correspondencia

$$
\left(\begin{array}{cc}
2^{n} & p / 2^{q} \\
0 & 1
\end{array}\right) \longmapsto \Phi_{H}\left(\frac{p}{2^{q}}\right) \circ H^{n} .
$$

Demostración. La afirmación resulta inmediatamente de la igualdad

$$
H \circ \Phi_{H}\left(\frac{p}{2^{q+1}}\right)=\Phi_{H}\left(\frac{p}{2^{q}}\right) \circ H .
$$

La extensión del homomorfismo $\Phi_{H}$ a $\mathrm{Af}_{+}\left(\mathbb{Q}_{2}, \mathbb{R}\right)$ también será denotada $\Phi_{H}$. Su definición para cada $g \in \operatorname{AfP}_{+}\left(\mathbb{Q}_{2}, \mathbb{R}\right)$ es un poco más delicada. Fijemos una sucesión $\left(a_{n}\right)_{n \in \mathbb{Z}}$ de racionales diádicos que sea estrictamente creciente y que no admita puntos de acumulación, así como una sucesión de elementos $h_{n} \in \mathrm{Af}_{+}\left(\mathbb{Q}_{2}, \mathbb{R}\right)$, de modo que para todo $n \in \mathbb{Z}$ se tenga

$$
\left.g\right|_{\left[a_{n}, a_{n+1}\right]}=\left.h_{n}\right|_{\left[a_{n}, a_{n+1}\right]} .
$$

Si definimos $b_{n}=\Phi_{H}\left(a_{n}\right)(0)$, entonces es fácil ver que la sucesión $\left(b_{n}\right)_{n \in \mathbb{Z}}$ también es estrictamente creciente y no admite puntos de acumulación. Con estas notaciones vale la siguiente proposición. 
Proposición 1.22. Si a cada $g \in \operatorname{AfP}_{+}\left(\mathbb{Q}_{2}, \mathbb{R}\right)$ asociamos la transformación que sobre cada intervalo $\left[b_{n}, b_{n+1}\left[\right.\right.$ coincide con $\Phi_{H}\left(h_{n}\right)$, entonces obtenemos un homomorfismo de $\mathrm{AfP}_{+}\left(\mathbb{Q}_{2}, \mathbb{R}\right)$ en $\mathrm{Homeo}_{+}\left(\mathrm{S}^{1}\right)$ que extiende $a \Phi_{H}$.

Demostración. El que la transformación asociada a cada $g$ está bien definida (i.e., no depende de la elección de los $a_{n}$ ) se deduce rápidamente de la definición, al igual que el hecho que la transformación asociada a cada $g \in \mathrm{Af}_{+}\left(\mathbb{Q}_{2}, \mathbb{R}\right)$ coincide con $\Phi_{H}(g)$. Para comprobar que la aplicación asociada a cada $g \in \operatorname{AfP}_{+}\left(\mathbb{Q}_{2}, \mathbb{R}\right)$ es un homeomorfismo, debemos verificar la continuidad en cada punto $b_{n}$, lo cual se reduce a probar que

$$
\Phi_{H}\left(h_{n}\right)\left(b_{n}\right)=\Phi_{H}\left(h_{n-1}\right)\left(b_{n}\right) .
$$

Observe que lo anterior es equivalente a

$$
\Phi_{H}\left(h_{n} T_{a_{n}}\right)(0)=\Phi_{H}\left(h_{n-1} T_{a_{n}}\right)(0)
$$

es decir,

$$
\Phi_{H}\left(T_{-a_{n}} h_{n-1}^{-1} h_{n} T_{a_{n}}\right)(0)=0 .
$$

Ahora bien, como $g$ es continua tenemos $h_{n}\left(a_{n}\right)=h_{n-1}\left(a_{n}\right)$, por lo que $T_{-a_{n}} h_{n-1}^{-1} h_{n} T_{a_{n}}$ es un elemento de $\mathrm{Af}_{+}\left(\mathbb{Q}_{2}, \mathbb{R}\right)$ que fija el origen, esto es, una aplicación $f$ de la forma $x \mapsto 2^{k} x$. La igualdad (1.8) que buscábamos verificar se deduce entonces de $\Phi_{H}(f)=H^{k}$ y del hecho que, por la propiedad (ii), $H$ fija el origen.

Observe que de la propiedad (i) se desprende que $\Phi_{H}(p)=T_{p}$ para todo entero $p$. De esta forma, $\Phi_{H}$ induce un homomorfismo inyectivo (que denotaremos también por $\left.\Phi_{H}\right)$ de $\mathrm{G}$ en $\mathrm{Homeo}_{+}\left(\mathrm{S}^{1}\right)$.

Proposición 1.23. Supongamos que, para cierto entero positivo $r$ o para $r=\infty$, la aplicación $H$ sea un difeomorfismo de clase $\mathrm{C}^{r}$ satisfaciendo la siguiente condición:

(iii) $)_{r} H^{\prime}(0)=1$ y $H^{(i)}(0)=0$ para todo $i \in\{2, \ldots, r\}$.

Entonces la imagen $\Phi_{H}(\mathrm{G})$ está contenida en el grupo de los difeomorfismos de clase $\mathrm{C}^{r}$ del círculo.

Demostración. Retomando las notaciones de la proposición precedente, debemos verificar que para cada $i \in\{1, \ldots, r\}$ se tiene

$$
\Phi_{H}\left(h_{n}\right)^{(i)}\left(b_{n}\right)=\Phi_{H}\left(h_{n-1}\right)^{(i)}\left(b_{n}\right) .
$$

Sin embargo, esto se deduce del hecho que el deasrrollo de Taylor al orden $r$ de la aplicación $\Phi_{H}\left(T_{-a_{n}} h_{n-1}^{-1} h_{n} T_{a_{n}}\right)=H^{k}$ coincide con el de la identidad. 
Observe que la propiedad (iii) $r$ no puede ser satisfecha para $r=\infty$ por ningún difeomorfismo real-analítico. De hecho, los grupos F y G no pueden actuar de manera efectiva por difeomorfismos real-analíticos del círculo (como G es un grupo simple, lo anterior implica que toda acción de G por difeomorfismos real-analíticos del círculo es trivial). Esto puede ser probado de diversas maneras, pero aparecerá como un hecho evidente tras la lectura de la sección 3: el grupo F contiene subgrupos solubles de orden de solubilidad tan grande como se quiera, mientras que todo grupo soluble de difeomorfismos real-analíticos del intervalo es metabeliano.

En la sección 1 retomaremos algunos aspectos dinámicos de la construcción precedente. Señalemos para concluir que el carácter diádico de lo que precede no es exclusivo: para cada entero $m \geq 2$ una construcción análoga puede ser realizada comenzando con una transformación $H$ satisfaciendo $H(x+1)=H(x)+m$ para todo real $x$. El grupo así obtenido puede ser pensado como un grupo de Thompson m-ádico. Desde el punto de vista algebraico el caso $m=2$ es especial en lo que respecta por ejemplo al grupo de automorfismos: referimos al lector a [26] y [25] para más información en torno a esto. Para una panorámica completa sobre los avances recientes (especialmente en aspectos cohomológicos) en torno a los grupos de Thompson recomendamos la lectura de [184]. 


\section{Capítulo 2}

\section{Sobre la Dinámica de Grupos de Homeomorfismos}

\section{Invariantes minimales}

Un subconjunto $\Lambda$ de $\mathrm{S}^{1}$ es invariante por un subgrupo $\Gamma$ de Homeo+ $\left(\mathrm{S}^{1}\right)$ si $g(x) \in \Lambda$ para todo $x \in \Lambda$ y todo $g \in \Gamma$. Un compacto invariante $\Lambda$ es minimal si los únicos subconjuntos cerrados e invariantes de $\Lambda$ son el conjunto vacío y el propio $\Lambda$.

Teorema 2.1. Si $\Gamma$ es un subgrupo de $\mathrm{Homeo}_{+}\left(\mathrm{S}^{1}\right)$, entonces se cumple una (y sólo una) de las afirmaciones siguientes:

(i) existe al menos una órbita finita;

(ii) todas las órbitas son densas;

(iii) existe un compacto invariante minimal homeomorfo al conjunto de Cantor, el cual es único y está contenido en la adherencia de toda órbita.

Demostración. La familia de los subconjuntos cerrados, invariantes y no vacíos de $\mathrm{S}^{1}$ está ordenada por inclusión, y como la intersección de compactos encajados es no vacía, el lema de Zorn es aplicable. Existe entonces un cerrado invariante no vacío y minimal $\Lambda$. Como la frontera $\partial \Lambda$ y el conjunto $\Lambda^{\prime}$ de los puntos de acumulación de $\Lambda$ son cerrados invariantes contenidos en $\Lambda$, por la minimalidad de $\Lambda$ se tienen las siguientes posibilidades:

(i) $\Lambda^{\prime}$ es vacío: en tal caso $\Lambda$ es una órbita finita;

(ii) $\partial \Lambda$ es vacío: en tal caso $\Lambda=\mathrm{S}^{1}$, por lo que todas las órbitas son densas;

(iii) $\Lambda=\Lambda^{\prime}$ y $\partial \Lambda=\Lambda$, es decir, $\Lambda$ es un conjunto cerrado de interior vacío tal que todos sus puntos pertenecen a su adherencia (en otras palabras, $\Lambda$ es un conjunto de Cantor). 
Probaremos que, en el último de los casos, $\Lambda$ está contenido en la adherencia de toda órbita, lo cual obviamente implica su unicidad. Sean $x \in \mathrm{S}^{1}$ e $y \in \Lambda$ puntos arbitrarios. Debemos probar que existe una sucesión $\left(g_{n}\right)$ de elementos de $\Gamma$ tal que $g_{n}(x)$ converge a $y$. Si $x \in \Lambda$, esto se desprende de la minimalidad de $\Lambda$. Si $x \in \mathrm{S}^{1} \backslash \Lambda$, consideramos el intervalo $\left.I=\right] a, b[$ contenido en $\mathrm{S}^{1} \backslash \Lambda$ tal que $a, b \in \Lambda$ y $x \in I$. Como la órbita de $a$ es densa en el conjunto $\Lambda$, el cual no posee puntos aislados, debe existir una sucesión $\left(g_{n}\right)$ en $\Gamma$ tal que $g_{n}(a)$ converja a $y$ de modo que los intervalos $g_{n}(I)$ sean disjuntos. La longitud de $g_{n}(I)$ debe entonces tender a cero, por lo que $g_{n}(x)$ converge a $y$.

Ejercicio 2.2. Pruebe que si un grupo de homeomorfismos del círculo posee órbitas finitas, entonces la cardinalidad de todas ellas es la misma.

Ejercicio 2.3. Dé ejemplos de grupos numerables de homeomorfismos de la recta que no admitan conjuntos cerrados invariantes minimales (y no vacíos).

En el caso en que todas las órbitas son densas, diremos que la acción es minimal. Si existe un Cantor minimal invariante, dicho conjunto será llamado un minimal excepcional. Para comprender mejor este último caso es conveniente introducir la siguiente terminología.

Definición 2.4. Dados dos homeomorfismos $f$ y $g$ de $\mathrm{S}^{1}$, decimos que $f$ es semiconjugado a $g$ si existe una aplicación continua y de grado uno $\varphi: \mathrm{S}^{1} \rightarrow \mathrm{S}^{1}$ cuyos levantamientos a $\mathbb{R}$ son funciones no decrecientes y tal que $\varphi f=g \varphi$. De manera similar, decimos que una acción $\Phi_{1}$ de un grupo $\Gamma$ por homeomorfismos de $S^{1}$ es semiconjugada a otra acción $\Phi_{2}$ si existe $\varphi$ con las propiedades anteriores y tal que $\varphi \Phi_{1}(g)=\Phi_{2}(g) \varphi$ para todo $g \in \Gamma$.

La aplicación $\varphi$ puede ser no inyectiva; en el caso en que ella es inyectiva, la semiconjugación es en realidad una conjugación. Observe que si un grupo $\Gamma$ actúa sobre $\mathrm{S}^{1}$ admitiendo un minimal excepcional $\Lambda$, entonces al reemplazar la clausura de cada componente conexa de $S^{1} \backslash \Lambda$ por un punto se obtiene un círculo topológico $\mathrm{S}_{\Lambda}^{1}$ sobre el cual $\Gamma$ actúa de manera natural por homeomorfismos: la acción original resulta entonces semiconjugada a esta acción minimal inducida sobre $\mathrm{S}_{\Lambda}^{1}$.

Observación 2.5. La relación de semiconjugación no es una relación simétrica. La relación de equivalencia minimal generada por ella es llamada equivalencia monótona. De manera más precisa, dos acciones $\Phi_{1}$ y $\Phi_{2}$ son monótamente equivalentes si existe una acción $\Phi$ que es semiconjugada tanto a $\Phi_{1}$ como a $\Phi_{2}$. El lector interesado hallará más información al respecto en [36].

No es difícil construir ejemplos de homeomorfismos de $\mathrm{S}^{1}$ que admiten un minimal excepcional. En efecto, cualquier conjunto de Cantor de $\mathrm{S}^{1}$ puede ser fácilmente exhibido como el minimal excepcional de un homeomorfismo del círculo (vea por ejemplo la sección 2.1). A continuación ve- 
remos un ejemplo sencillo de un grupo de difeomorfismos real-analíticos de $\mathrm{S}^{1}$ que admite un minimal excepcional. Dicho grupo está generado por dos transformaciones de Möbius $f$ y $g$. El difeomorfismo $f$ es simplemente la rotación $R_{2 \pi / 3}$ de ángulo $2 \pi / 3$ (centrada en el origen $O$ ). El difeomorfismo $g$ es una rotación hiperbólica de ángulo $\pi$ centrada en un punto $P \in \mathbb{D}$ situado a distancia euclideana de $O$ mayor que $2-\sqrt{3}$ (vea la figura 7 ). Equivalentemente, $g$ es la reflexión (hiperbólica) con respecto a la geodésica hiperbólica que pasa por $P$ y es perpendicular a la geodésica que une a dicho punto con $O$. La razón de la restricción sobre la distancia entre $O$ y $P$ radica en que si ella es exactamente $2-\sqrt{3}$, entonces las órbitas del grupo generado por $f$ y $g$ son densas.

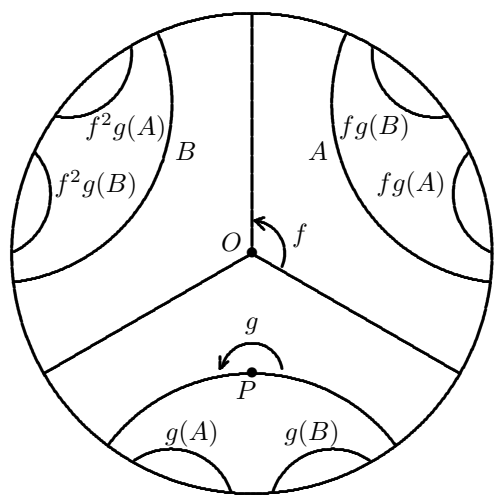

Figura 7

Señalemos que el grupo que actúa en el ejemplo precedente puede ser presentado algebraicamente de la forma $\Gamma=\left\langle f, g: g^{2}=f^{3}=i d\right\rangle$. Este grupo es conocido con el nombre de grupo modular. Su inyección canónica en $\operatorname{PSL}(2, \mathbb{R})$ se obtiene al identificar $f$ con $R_{2 \pi / 3}$ y $g$ con la rotación de ángulo $\pi$ centrada en el punto $(\sqrt{3}-2,0) \in \mathbb{D}$. Vía esta inyección, el grupo modular se identifica a $\operatorname{PSL}(2, \mathbb{Z})$, y la acción correspondiente es minimal.

Una perturbación de una "versión afín por partes" del ejemplo anterior permite obtener una acción por difeomorfismos de clase $\mathrm{C}^{\infty}$ de modo que el minimal excepcional sea el conjunto de Cantor terciario tradicional. Basta considerar un difeomorfismo $h$ de $\mathrm{S}^{1}$ cuya restricción a los intervalos $[0, \pi / 6],[\pi / 3, \pi / 2]$ y $[\pi, 3 \pi / 2]$ sea afín, con derivada 3,1 y $1 / 3$ respectivamente, tal que $h(x)=x+2 \pi / 3$ para $x \in[\pi / 3, \pi / 2]$, y tal que $h$ esté definido de manera coherente en los restantes intervalos de modo que $(h g)^{3}=I d$, donde $g$ denota la rotación de ángulo $\pi$ (vea la figura 8). Definiendo $f=h g$ obtenemos la acción deseada. 
Resulta muy interesante constatar que, si bien el ejemplo de la figura 7 databa de antiguos trabajos de Klein y Poincaré, durante algún tiempo este tipo de ejemplos fue "olvidado" por los especialistas. Es así como el "primer ejemplo" de un grupo de difeomorfismos (de clase $\mathrm{C}^{\infty}$ ) con un minimal excepcional es atribuido a Sacksteder [180]; dicho ejemplo corresponde a (una ligera variación de) aquél ilustrado más abajo...

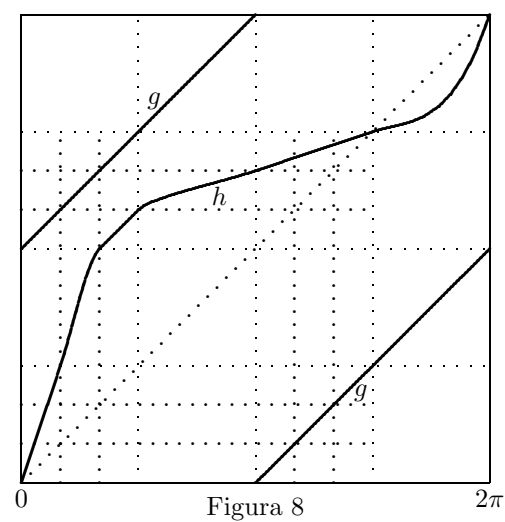

Para el caso de grupos fuchsianos (es decir, para subgrupos discretos de $\operatorname{PSL}(2, \mathbb{R})$ ), la nomenclatura en relación al teorema 2.1 es especial. Si existe una órbita finita entonces el grupo es llamado elemental. En el caso en que las órbitas son densas, el grupo es de primera especie. Finalmente, en el caso de un minimal excepcional, el grupo es de segunda especie [109]. Ejemplos importantes de grupos de segunda especie son los llamados grupos de Schottky. Éstos son grupos generados por dos elementos hiperbólicos $g_{0}$ y $g_{1}$ de $\operatorname{PSL}(2, \mathbb{R})$ para los cuales existen intervalos disjuntos $I_{0}, I_{1}, J_{0}, J_{1}$ en $\mathrm{S}^{1}$ tales que, para $i \in\{0,1\}$,

$$
g_{i}\left(I_{i} \cup J_{i} \cup J_{1-i}\right) \subset I_{i} \quad \text { y } \quad g_{i}^{-1}\left(J_{i} \cup I_{i+1} \cup J_{1-i}\right) \subset J_{i} .
$$

En la figura 9 se muestra el diagrama combinatorio respectivo. No es difícil ver que $\left\langle g_{0}, g_{1}\right\rangle$ actúa sobre $\mathrm{S}^{1}$ admitiendo un minimal excepcional, a saber,

$$
\Lambda=\bigcap_{g \in\left\langle g_{0}, g_{1}\right\rangle} g\left(\overline{I_{0}} \cup \overline{I_{1}} \cup \overline{J_{0}} \cup \overline{J_{1}}\right) .
$$

Ejercicio 2.6. Dé ejemplos de subgrupos finitamente generados de $\operatorname{Difeo}+\left(\mathrm{S}^{1}\right)$ que admitan un minimal excepcional de modo que todas las órbitas del complemento de dicho minimal sean densas. 
Observación. Sobre la base de un resultado no publicado debido a Hector es posible demostrar que si $\Gamma$ es un grupo (no necesariamente finitamente generado) de difeomorfismos real-analíticos del círculo con un minimal excepcional, entonces ninguna de sus órbitas es densa (vea por ejemplo [144])

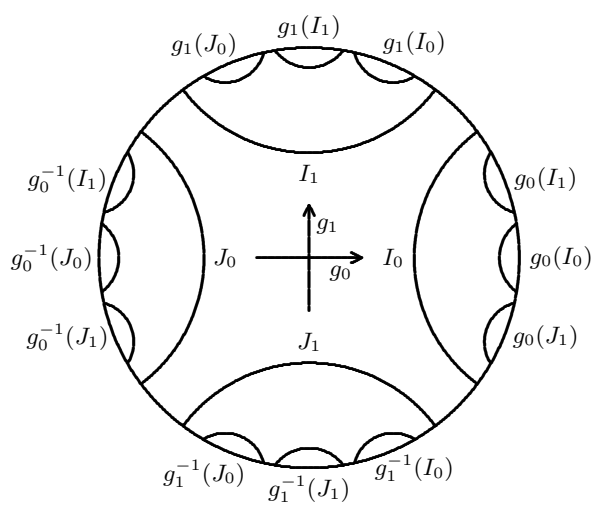

Figura 9

Ejercicio 2.7. Dé un ejemplo de un grupo $\Gamma$ (no finitamente generado) de difeomorfismos real-analíticos del círculo actuando de manera minimal y tal que cada uno de sus subgrupos finitamente generados admita un minimal excepcional. Análogamente, dé un ejemplo de un subgrupo de Difeow+ ${ }_{+}^{w}\left(\mathrm{~S}^{1}\right)$ actuando de manera minimal y cuyos subgrupos finitamente generados admitan órbitas finitas (si tiene problemas con esto último vea el ejemplo 3.7 ).

Concluimos esta sección con otro importante ejemplo de un grupo que actúa por difeomorfismos de clase $\mathrm{C}^{\infty}$ del círculo admitiendo un minimal excepcional: el grupo de Thompson G. En efecto, a cada homeomorfismo $H: \mathbb{R} \rightarrow \mathbb{R}$ satisfaciendo ciertas propiedades (i) y (ii) hemos asociado en la sección 5.2 un homomorfismo $\Phi_{H}: \mathrm{G} \rightarrow \mathrm{Homeo}_{+}\left(\mathrm{S}^{1}\right)$, el cual toma valores en Difeo $_{+}^{r}\left(\mathrm{~S}^{1}\right)$ si $H$ es un difeomorfismo de clase $\mathrm{C}^{r}$ que satisface la condición (iii) $)_{r}$. Resulta bastante claro que todos estos grupos $\Phi_{H}(\mathrm{G})$ son topológicamente semiconjugados a (la inclusión canónica de) $\mathrm{G}$ (en $\mathrm{Homeo}_{+}\left(\mathrm{S}^{1}\right)$ ); dejamos la verificación de esto al lector. Sin embargo, algunos de ellos no son conjugados entre sí, de acuerdo a la siguiente proposición.

Proposición 2.8. Si el homeomorfismo $H: \mathbb{R} \rightarrow \mathbb{R}$ satisface las propiedades (i) y (ii) de la sección 5.2 y posee al menos dos puntos fijos, entonces el grupo $\Phi_{H}(\mathrm{G})$ admite un minimal excepcional. 
Demostración. Si $a$ y $b$ son puntos fijos de $H$ entonces la condición (i) implica que $a$ y $b$ están en un mismo dominio fundamental. En otras palabras, el intervalo abierto de la recta de extremidades $a$ y $b$ se proyecta inyectivamente en un intervalo abierto $I$ del círculo que satisface $\bar{H}^{n}(I)=I$ para todo $n \geq 0$ (donde $\bar{H}$ denota la aplicación de grado 2 de $\mathrm{S}^{1}$ en sí mismo inducida por $H$ ). Por otra parte, para cada $n \geq 0$ el conjunto $\bar{H}^{-n}(I)$ es la unión de una familia de $2^{n}$ intervalos abiertos y disjuntos, y $\bar{H}^{-n}(I) \subset \bar{H}^{-m}(I)$ para todo $m \geq n$. Luego, el conjunto $\cup_{n>0} \bar{H}^{-n}(I)$ es un abierto invariante por $\bar{H}$ de complemento no vacío. No es difícil concluir entonces que dicho conjunto es invariante por $\Phi_{H}(\mathrm{G})$ (vea el ejercicio 2.12). Por lo tanto, no todas las órbitas de $\Phi_{H}(\mathrm{G})$ son densas, y puesto que $\Phi_{H}(\mathrm{G})$ no posee órbitas finitas, necesariamente debe admitir un minimal excepcional.

Utilizando las técnicas dinámicas del capítulo 3, no es difícil probar que todas las acciones de $\mathrm{G}$ por difeomorfismos de clase $\mathrm{C}^{2}$ del círculo son semiconjugadas a la acción estándar afín por partes (el lector interesado hallará una prueba de esta afirmación en [78]). Sin embargo, no nos extenderemos en torno a esto, pues al parecer la hipótesis de regularidad es superflua, y toda acción de $\mathrm{G}$ por homeomorfismos del círculo debiese ser semiconjugada a la acción estándar [123].

Ejercicio 2.9. Usando los teoremas 1.19 y 2.77, pruebe que toda acción de F por homeomorfismos del círculo admite un punto fijo global.

Ejercicio 2.10. Pruebe que si la aplicación $H$ es dilatante, es decir, si para todo par de puntos $x, y$ de la recta se tiene $\operatorname{dist}(H(x), H(y))>\operatorname{dist}(x, y)$, entonces todas las órbitas del grupo $\Phi_{H}(\mathrm{G})$ son densas.

Ejercicio 2.11. Pruebe que $\mathrm{G}$ admite acciones por homeomorfismos afines por partes del círculo que preservan un minimal excepcional.

Ejercicio 2.12. Fijado el homeomorfismo $H$ satisfaciendo las condiciones (i) y (ii) de la sección 5.2, considere la relación de equivalencia de la aplicación inducida $\bar{H}$ sobre el círculo. Pruebe que las clases de equivalencia de esta relación coinciden con las órbitas del grupo $\Phi_{H}(\mathrm{G})$.

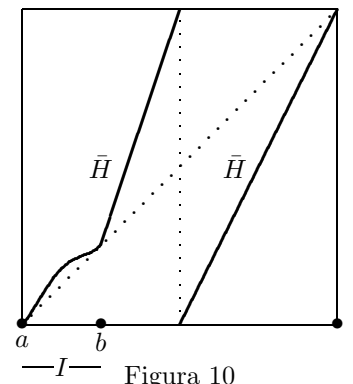




\section{Algunos resultados combinatorios}

\subsection{La teoría de Poincaré}

En esta sección abordaremos algunos tópicos clásicos en torno al más importante de los invariantes dinámicos para homeomorfismos del círculo: el número de rotación. Comenzamos con un lema elemental.

Lema 2.13. Sea $\left(a_{n}\right)_{n \in \mathbb{Z}}$ una sucesión de números reales. Suponga que existe una constante $C \in \mathbb{R}$ tal que

$$
\left|a_{m+n}-a_{m}-a_{n}\right| \leq C
$$

para todo $m, n$ en $\mathbb{Z}$. Entonces existe un único $\rho \in \mathbb{R}$ tal que la sucesión $\left(\left|a_{n}-n \rho\right|\right)_{n \in \mathbb{Z}}$ es acotada. Dicho valor es igual al límite de la sucesión $\left(a_{n} / n\right)$ cuando $n$ tiende a $\pm \infty$ (en particular, dicho límite existe).

Demostración. Por simplicidad, daremos la demostración sólo para el caso de sucesiones $\left(a_{n}\right)_{n \in \mathbb{N}}$ que satisfacen (2.1). El caso general se demuestra usando las mismas ideas, pero trabajando cuidadosamente con el signo de cada término de la sucesión.

Para cada $n \in \mathbb{N}$ consideramos el intervalo $I_{n}=\left[\left(a_{n}-C\right) / n,\left(a_{n}+C\right) / n\right]$. Afirmamos que $I_{m n}$ está contenido en $I_{n}$ para todo $m, n$ en $\mathbb{N}$. En efecto, de $(2.1)$ se obtiene $\left|a_{m n}-m a_{n}\right| \leq(m-1) C$, de donde se concluye que $a_{m n}+C \leq m a_{n}+m C$, y por lo tanto

$$
\frac{a_{m n}+C}{m n} \leq \frac{a_{n}+C}{n} .
$$

Análogamente se prueba que

$$
\frac{a_{m n}-C}{m n} \geq \frac{a_{n}-C}{n},
$$

y estas dos últimas desigualdades implican que $I_{m n} \subset I_{n}$.

Una aplicación simple de la propiedad de intersección finita muestra que la intersección $I=\cap_{n \in \mathbb{N}} I_{n}$ es no vacía. Si $\rho$ pertenece a $I$ entonces $\rho$ está en cada uno de los intervalos $I_{n}$, de donde se concluye fácilmente que

$$
\left|a_{n}-n \rho\right| \leq C \text {. }
$$

Luego, $\rho$ satisface la afirmación del lema. Si $\rho^{\prime} \neq \rho$ entonces

$$
\left|a_{n}-n \rho^{\prime}\right|=\left|\left(a_{n}-n \rho\right)+n\left(\rho-\rho^{\prime}\right)\right| \geq n\left|\rho-\rho^{\prime}\right|-C,
$$

por lo que $\left|a_{n}-n \rho^{\prime}\right|$ tiende al infinito. Finalmente, de (2.2) se deduce que $\left|\rho-a_{n} / n\right| \leq C / n$ para todo $n$, por lo que $\rho=\lim _{n \rightarrow \infty}\left(a_{n} / n\right)$.

Consideraremos la parametrización de $\mathrm{S}^{1}$ por $[0,1]$. Dado un homeomorfismo $f$ de $\mathrm{S}^{1}$, designamos por $F: \mathbb{R} \rightarrow \mathbb{R}$ a un levantamiento cualquiera de $f$ a la recta. 
Proposición 2.14. Para cada $x \in \mathbb{R}$ existe el límite

$$
\lim _{n \rightarrow \pm \infty} \frac{1}{n}\left[F^{n}(x)-x\right],
$$

$y$ dicho límite es independiente de $x$.

Demostración. En primer lugar, notemos que para todo $x, y$ en $\mathbb{R}$ y todo $m \in \mathbb{Z}$ tenemos

$$
|| F^{m}(x)-x|-| F^{m}(y)-y|| \leq 2 .
$$

En efecto, puesto que $F^{m}(x+n)=F^{m}(x)+n$ para todo $n \in \mathbb{Z}$, para demostrar (2.3) podemos suponer que $x$ e $y$ pertenecen a [0, 1[. En tal caso, la desigualdad (2.3) es inmediata de verificar. Fijemos $x \in \mathbb{R}$ y denotemos $a_{n}=F^{n}(x)-x$. Tenemos

$$
a_{m+n}=F^{m+n}(x)-x=\left[F^{m}\left(F^{n}(x)\right)-F^{n}(x)\right]+\left[F^{n}(x)-x\right],
$$

por lo que

$$
\begin{aligned}
\left|a_{m+n}-a_{m}-a_{n}\right| & =\left|F^{m+n}(x)-x-\left(F^{m}(x)-x\right)-\left(F^{n}(x)-x\right)\right| \\
& =\left|F^{m}\left(F^{n}(x)\right)-F^{n}(x)-\left(F^{m}(x)-x\right)\right| \leq 2 .
\end{aligned}
$$

Por el lema anterior, la expresión $\left[F^{n}(x)-x\right] / n$ converge, y la desigualdad (2.3) permite probar que el límite correspondiente no depende de $x$.

Si consideramos dos levantamientos del homeomorfismo $f$, los límites dados por la proposición anterior difieren por un número entero. Definimos entonces el número de rotación de $f$ por

$$
\rho(f)=\lim _{n \rightarrow \pm \infty} \frac{1}{n}\left[F^{n}(x)-x\right] \quad \text { mód } 1 .
$$

Por ejemplo, es fácil verificar que para la rotación de ángulo $\theta \in[0,1[$ se tiene $\rho\left(R_{\theta}\right)=\theta$. Además, para todo homomorfismo $f$, todo $x \in \mathrm{S}^{1}$ y todo $m \in \mathbb{Z}$, se tiene

$$
\rho\left(f^{m}\right)=\lim _{n \rightarrow \pm \infty} \frac{1}{n}\left[F^{m n}(x)-x\right]=m \cdot \lim _{n \rightarrow \pm \infty} \frac{1}{m n}\left[F^{m n}(x)-x\right],
$$

de donde se concluye que $\rho\left(f^{m}\right)=m \rho(f)$.

Si $f$ posee un punto periódico entonces $\rho(f)$ es un número racional. En efecto, si $f^{p}(x)=x$ entonces para cada levantamiento $F$ de $f$ tenemos $F^{p}(x)=x+q$ para cierto $q \in \mathbb{Z}$ ( $x$ denota a la vez un punto de $\mathrm{S}^{1}$ y uno de sus levantamientos a $\mathbb{R})$. Obtenemos así

$$
\lim _{n \rightarrow \pm \infty} \frac{1}{p n}\left[F^{p n}(x)-x\right]=\lim _{n \rightarrow \pm \infty} \frac{1}{p n}[(x+n q)-x]=\frac{q}{p},
$$

de donde se concluye que

$$
\rho(f)=\frac{q}{p} \quad \text { mód } 1 .
$$

Recíprocamente, tenemos el siguiente resultado. 
Proposición 2.15. Si $f \in \mathrm{Homeo}_{+}\left(\mathrm{S}^{1}\right)$ tiene número de rotación racional, entonces $f$ posee al menos un punto periódico.

Demostración. Dada la igualdad $\rho\left(g^{m}\right)=m \rho(g)$, basta demostrar que todo homeomorfismo del círculo de número de rotación nulo posee al menos un punto fijo. Supongamos entonces que $f \in \mathrm{Homeo}_{+}\left(\mathrm{S}^{1}\right)$ no tenga puntos fijos, y sea $F: \mathbb{R} \rightarrow \mathbb{R}$ un levantamiento de $f$ tal que $F(0) \in] 0,1[$. Observe que la función $x \mapsto F(x)-x$ no se anula en ningún punto de la recta. Luego, por continuidad y periodicidad, existe una constante $\delta \in] 0,1[$ tal que para todo $x \in \mathbb{R}$ se tiene

$$
\delta \leq F(x)-x \leq 1-\delta .
$$

Colocando $x=F^{i}(0)$ en esta desigualdad, sumando desde $i=0$ hasta $n-1$ y dividiendo por $n$, obtenemos

$$
\delta \leq \frac{F^{n}(0)}{n} \leq 1-\delta .
$$

Tomando el límite cuando $n$ tiende a $+\infty$ en esta última desigualdad obtenemos $\delta \leq \rho(f) \leq 1-\delta$. Luego, si $f \in$ Homeo $_{+}\left(\mathrm{S}^{1}\right)$ carece de puntos fijos, entonces $\rho(f) \neq 0$.

Dejamos al lector la tarea de probar que si el número de rotación de $f$ es racional, entonces el periodo de todos sus puntos periódicos debe ser el mismo.

Proposición 2.16. Los homeomorfismos topológicamente conjugados del círculo tienen el mismo número de rotación.

Demostración. Debemos probar que para todo $f, g$ en $\mathrm{Homeo}_{+}\left(\mathrm{S}^{1}\right)$ vale la igualdad $\rho(f)=\rho\left(g f g^{-1}\right)$. Sean $F$ y $G$ levantamientos a la recta de $f$ y $g$ respectivamente tales que $F(0)$ y $G(0)$ pertenecen a $[0,1[$. Es claro que $G^{-1}$ es un levantamiento de $g^{-1}$. Para probar la proposición, debemos estimar el valor de la expresión

$$
\left|\left(G F G^{-1}\right)^{n}(x)-F^{n}(x)\right|=\left|G F^{n} G^{-1}(x)-F^{n}(x)\right| .
$$

No es difícil verificar que $|G(x)-x|<2 \quad$ y $\left|G^{-1}(x)-x\right|<2$ para todo $x \in \mathbb{R}$. Además, si $|x-y|<2$ entonces $\left|F^{n}(x)-F^{n}(y)\right|<3$. Concluimos entonces que

$\left|G F^{n} G^{-1}(x)-F^{n}(x)\right| \leq\left|G F^{n} G^{-1}(x)-F^{n} G^{-1}(x)\right|+\left|F^{n} G^{-1}(x)-F^{n}(x)\right|<5$,

de donde obtenemos, para todo $x \in \mathbb{R}$,

$$
\frac{\left|\left(G F G^{-1}\right)^{n}(x)-F^{n}(x)\right|}{n}<\frac{5}{n},
$$

lo cual implica que $\rho(f)=\rho\left(g f g^{-1}\right)$. 
La proposición anterior puede ser extendida para homeomorfismos semiconjugados. Dejamos la prueba de ello al lector.

Como consecuencia de lo anterior, la dinámica de un homeomorfismo $f$ de número de rotación racional $p / q$ queda determinada por dicho número y por la "dirección" de la dinámica de $f^{q}$ sobre cada una de las componentes conexas del complemento del conjunto de los puntos periódicos de $f$.

El caso de número de rotación irracional es más interesante. Puesto que las órbitas de toda rotación de ángulo $\theta \notin \mathbb{Q}$ son densas, podría pensarse en la existencia de un único modelo dinámico en este caso. Sin embargo, no es difícil fabricar ejemplos de homeomorfismos de $\mathrm{S}^{1}$ de número de rotación irracional que no son topológicamente conjugados a la rotación respectiva. En efecto, consideremos una rotación cualquiera de ángulo $\theta$ irracional. Fijemos un punto $x \in \mathrm{S}^{1}$ y reemplacemos cada punto $f^{i}(x), i \in \mathbb{Z}$, por un intervalo de longitud $1 / 2^{|i|}$. Obtenemos entonces un círculo $\mathrm{S}_{x}^{1}$ de longitud mayor, y la rotación $R_{\theta}$ induce un homeomorfismo $R_{\theta, x}$ de $\mathrm{S}_{x}^{1}$ al extender $R_{\theta}$ de manera afín a cada intervalo añadido al círculo. Las aplicaciones $R_{\theta} \mathrm{y}$ $R_{\theta, x}$, vistas como homeomorfismos del círculo, son semiconjugadas. Como consecuencia, el número de rotación de $R_{\theta, x}$ es igual a $\theta$. Sin embargo, ninguna órbita de $R_{\theta, x}$ es densa. En particular, $R_{\theta, x}$ no es topológicamente conjugado a la rotación $R_{\theta}$.

Pese a lo anterior, existe un único modelo módulo semiconjugación topológica.

Teorema 2.17. Si el número de rotación $\rho(f)$ de $f \in \mathrm{Homeo}_{+}\left(\mathrm{S}^{1}\right)$ es irracional, entonces $f$ es semiconjugado a la rotación de ángulo $\rho(f)$. La semiconjugación es una conjugación si y sólo si todas las órbitas de $f$ son densas.

Demostración. Sea $F: \mathbb{R} \rightarrow \mathbb{R}$ un levantamiento de $f$ a la recta tal que $F(0) \in[0,1[$. Por el lema 2.13 y la demostración de la proposición 2.14, para cada $x \in \mathbb{R}$ el valor de

$$
\varphi(x)=\sup _{n \in \mathbb{Z}}\left(F^{n}(x)-n \rho(F)\right)
$$

es finito. La aplicación $\varphi: \mathbb{R} \rightarrow \mathbb{R}$ verifica las siguientes propiedades:

(i) $\varphi$ es creciente y continua a izquierda;

(ii) $\varphi(x+1)=\varphi(x)+1$ para todo $x \in \mathbb{R}$;

(iii) $\varphi(F(x))=\varphi(x)+\rho(F)$ para todo $x \in \mathbb{R}$.

A partir de estas propiedades vemos claramente que para demostrar que $\varphi$ es una semiconjugación debemos probar que $\varphi$ es continua. Para esto comencemos observando que, para cada $x \in \varphi(\mathbb{R})$, el conjunto $\varphi^{-1}(x)$ es ya sea un punto o bien un intervalo no degenerado. Designemos entonces por $\widetilde{\operatorname{Plan}}(F)$ la unión del interior de estos últimos intervalos, y por $\widetilde{\operatorname{Salt}}(F)$ la unión de los interiores de los intervalos del complemento de $\varphi(\mathbb{R})$. Los 
conjuntos $\widetilde{\operatorname{Plan}}(F)$ y $\widetilde{\operatorname{Salt}}(F)$ son invariantes por las traslaciones enteras de la recta, por lo que inducen subconjuntos Plan $(f)$ y Salt $(f)$ del círculo. Es fácil ver que Salt $(f)$ es invariante por la rotación de ángulo $\rho(f)$. Puesto que $\rho(f)$ es irracional, Salt $(f)$ debe ser vacío, lo cual implica que $\varphi$ es continua e induce entonces una semiconjugación entre $f$ y $R_{\rho(f)}$. Finalmente, observe que Plan $(f)$ es invariante por $f$. Luego, si las órbitas por $f$ son densas entonces Plan $(f)$ es vacío; en tal caso $\varphi$ es inyectiva, por lo que induce una conjugación entre $f$ y $R_{\rho(f)}$.

El estudio de la dinámica combinatoria de un homeomorfismo de número de rotación irracional se reduce entonces al de la rotación respectiva. Para $\theta \in[0,1] \backslash \mathbb{Q}$ definimos por inducción los enteros positivos

$$
q_{1}=1, \quad q_{n+1}=\min \left\{q>q_{n}: \operatorname{dist}(q \theta, \mathbb{N})<\operatorname{dist}\left(q_{n} \theta, \mathbb{N}\right)\right\}
$$

Es bien conocido que la sucesión $\left(q_{n}\right)_{n \in \mathbb{N}}$ es tal que

$$
\operatorname{dist}\left(q_{n} \theta, \mathbb{N}\right)=\left\{q_{n} \theta\right\} \text { si y sólo si } \operatorname{dist}\left(q_{n+1} \theta, \mathbb{N}\right)=1-\left\{q_{n+1} \theta\right\},
$$

donde $\{a\}=a-[a]$ denota la parte fraccionaria de $a$ (vea por ejemplo [89]). Si proyectamos sobre el círculo y continuamos denotando por $q_{n} \theta$ los puntos respectivos de $S^{1}$, la condición (2.4) significa que ya sea se tienen las relaciones $-q_{n} \theta<q_{n+1} \theta<0<-q_{n+1} \theta<q_{n} \theta<-q_{n} \theta$, o bien se verifica $q_{n} \theta<-q_{n+1} \theta<0<q_{n+1} \theta<-q_{n} \theta<q_{n} \theta$. Designamos por $I_{n}$ el intervalo de $\mathrm{S}^{1}$ de puntos extremos 0 y $q_{n} \theta$, cerrado en 0 y abierto en $q_{n} \theta$. El intervalo abierto de puntos extremos $-q_{n} \theta$ y $q_{n} \theta$ será designado por $J_{n}$. Observe por otra parte que $\left(q_{n}+q_{n+1}\right) \theta$ pertenece a $J_{n}$ (vea las figuras 11 y 12).

Afirmamos ahora que los intervalos $R_{j \theta}\left(I_{n}\right), j \in\left\{0,1, \ldots, q_{n+1}-1\right\}$, son dos a dos disjuntos. En efecto, si se verificara $R_{j \theta}\left(I_{n}\right) \cap R_{k \theta}\left(I_{n}\right) \neq \emptyset$ para ciertos $0 \leq j<k<q_{n+1}$, entonces se tendría $R_{(k-j) \theta}\left(I_{n}\right) \cap I_{n} \neq \emptyset$, lo cual implicaría $\operatorname{dist}((k-j) \theta, 0)<\operatorname{dist}\left(q_{n} \theta, 0\right)$. Como $k-j<q_{n+1}$, esto contradiría la definición de $q_{n+1}$.

A partir de lo anterior es fácil ver que los intervalos $R_{j \theta}\left(J_{n}\right)$, con $j$ en $\left\{0,1, \ldots, q_{n+1}-1\right\}$, recubren al círculo, y cada punto $x \in \mathrm{S}^{1}$ está contenido en a lo más dos de ellos. Reemplazando $\theta$ por $-\theta$ obtenemos dos sucesiones $I_{-n}$ y $J_{-n}$ de intervalos tales que $J_{n}=I_{n} \cup I_{-n}=J_{-n}$. Vemos así que cada punto del círculo está en a lo más dos intervalos de la forma $R_{j \theta}\left(J_{n}\right)$, donde $j \in\left\{-\left(q_{n+1}-1\right),-\left(q_{n+1}-2\right), \ldots, 0\right\}$.

Las notaciones introducidas en los últimos párrafos son relativamente estándar y serán utilizadas más adelante. 


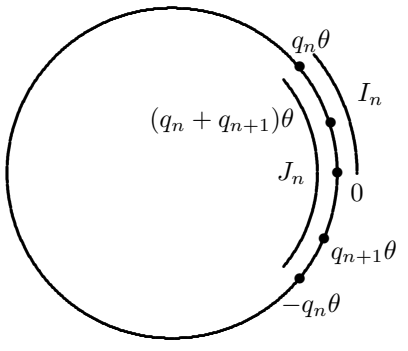

Figura 11

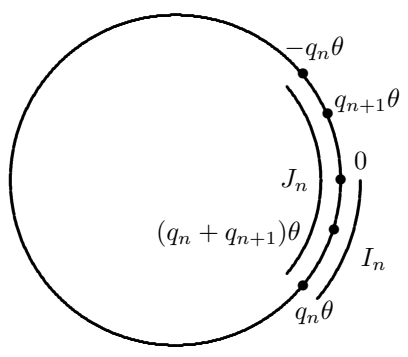

Figura 12

Ejercicio 2.18. Pruebe que para todo $f \in \mathrm{Homeo}_{+}\left(\mathrm{S}^{1}\right)$ existe un ángulo $\theta$ en $[0,1]$ tal que el número de rotación de $R_{\theta} \circ f$ es no nulo.

Ejercicio 2.19. Dados dos parámetros $\lambda_{1}>0$ y $\lambda_{2}>0$, de los cuales al menos uno es diferente de 0 , y tales que $\left(\lambda_{1}-1\right)\left(\lambda_{2}-1\right) \leq 0$, definamos $a=a\left(\lambda_{1}, \lambda_{2}\right)$ como siendo el único real que verifica $\lambda_{1} a+\lambda_{2}(1-a)=1$. Considere el (único) homeomorfismo afín por partes $f=f_{\lambda_{1}, \lambda_{2}}: \mathrm{S}^{1} \rightarrow \mathrm{S}^{1}$ que satisface $f(a)=0 \mathrm{y}$ cuya derivada es idénticamente igual a $\lambda_{1}$ sobre $] 0, a\left[\right.$ y a $\lambda_{2}$ sobre $] a, 1[$. Nuestro objetivo es calcular el número de rotación de $f_{\lambda_{1}, \lambda_{2}}$ (este ejemplo es debido a Boshernitzan [16]).

(i) Para $\sigma=\lambda_{1} / \lambda_{2}$, considere el homeomorfismo $h_{\sigma}$ del intervalo $[0,1]$ definido por $h_{\sigma}(x)=\left(\sigma^{x}-1\right) /(\sigma-1)$. Verifique que $h_{\sigma}^{-1} \circ f_{\lambda_{1}, \lambda_{2}} \circ h_{\sigma}$ coincide con la rotación $R_{\rho}$, donde $\rho$ satisface la igualdad $\sigma^{\rho}=\lambda_{1}$.

(ii) Concluya que $\rho\left(f_{\lambda_{1}, \lambda_{2}}\right)=\log \left(\lambda_{1}\right) /\left(\log \left(\lambda_{1}\right)-\log \left(\lambda_{2}\right)\right)$.

Ejercicio 2.20. Fijemos ahora un real positivo $\sigma \neq 1$, y para cada $\rho \in[0,1]$ consideremos el homeomorfismo del círculo $g_{\sigma, \rho}=h_{\sigma} \circ R_{\rho} \circ h_{\sigma}^{-1}$.

(i) Verifique que $g_{\sigma, \rho}$ coincide con $f_{\lambda_{1}, \lambda_{2}}$, donde $\lambda_{1}=\sigma^{\rho}$ y $\lambda_{2}=\sigma^{\rho-1}$.

(ii) Concluya que dentro del grupo de los homeomorfismos afines por partes del círculo, existen incrustaciones continuas del grupo de las rotaciones que no vienen dadas por conjugaciones por homeomorfismos afines por partes (para más información en torno a este interesantísimo tema el lector puede consultar [135, $136])$.

\subsection{Número de rotación y medidas invariantes}

Sea $f$ un homeomorfismo del círculo. Por el teorema de Bogolioubov y Krylov, existe al menos una medida de probabilidad $\mu$ sobre $\mathrm{S}^{1}$ invariante por $f$ (vea el apéndice). Observe que el valor de $\mu([x, f(x)[)$ es independiente de $x \in \mathrm{S}^{1}$. En efecto, si $y$ es otro punto del círculo entonces

$\mu([y, f(y)[)=\mu([y, f(x)[)+\mu([f(x), f(y)[)=\mu([y, f(x)[)+\mu([x, y[)=\mu([x, f(x)[)$.

Denotaremos dicho valor por $\rho_{\mu}(f)$. 
Teorema 2.21. Se tiene la igualdad $\rho(f)=\rho_{\mu}(f)$.

Demostración. La medida $\mu$ se levanta en una medida $\sigma$-finita $\tilde{\mu}$ sobre $\mathbb{R}$. Fijemos un punto $x \in \mathrm{S}^{1}$ y consideremos una preimagen de dicho punto en $\mathbb{R}$, a la que denotaremos también por $x$. Observe que cualquier levantamiento $F$ de $f$ preserva $\tilde{\mu}$. Además, $\tilde{\mu}([x, x+k[)=k$ para todo $k \in \mathbb{N}$. Luego, si $F^{n}(x) \in[x+k, x+k+1[$ entonces

$$
F^{n}(x)-x-1 \leq k \leq \tilde{\mu}\left(\left[x, F^{n}(x)[) \leq k+1 \leq F^{n}(x)-x+1 .\right.\right.
$$

Concluimos que

$$
\lim _{n \rightarrow \infty} \frac{F^{n}(x)-x}{n}=\lim _{n \rightarrow \infty} \frac{\tilde{\mu}\left(\left[x, F^{n}(x)[)\right.\right.}{n}=\lim _{n \rightarrow \infty} \frac{1}{n} \sum_{i=0}^{n-1} \tilde{\mu}\left(\left[F^{i}(x), F^{i+1}(x)[),\right.\right.
$$

y como para todo $i \in \mathbb{N}$ se tiene $\tilde{\mu}\left(\left[F^{i}(x), F^{i+1}(x)[)=\tilde{\mu}([x, F(x)[)\right.\right.$,

$$
\lim _{n \rightarrow \infty} \frac{F^{n}(x)-x}{n}=\tilde{\mu}([x, F(x)[) .
$$

Por lo tanto, $\rho(f)=\mu\left(\left[x, f(x)[)=\rho_{\mu}(f)\right.\right.$.

Resulta útil analizar el soporte de una medida de probabilidad $\mu$ invariante por un homeomorfismo $f$ de $\mathrm{S}^{1}$. Si $\rho(f)$ es racional entonces $f$ posee puntos periódicos y $\mu$ está soportada en dichos puntos. Si $\rho(f)$ es irracional entonces pueden darse dos casos: si $f$ admite un minimal excepcional $\Lambda$ entonces el soporte de $\mu$ es $\Lambda$ y $\mu$ no posee átomos, mientras que si las órbitas de $f$ son densas entonces el soporte de $\mu$ es todo el círculo y $\mu$ tampoco posee átomos.

Para cada $\mu \in \operatorname{Prob}\left(\mathrm{S}^{1}\right)$ designaremos por $\Gamma_{\mu}$ al grupo de los homeomorfismos de $\mathrm{S}^{1}$ que preservan $\mu$. Observe que para todo $f, g$ en $\Gamma_{\mu}$ se tiene

$$
\rho_{\mu}(f g)=\mu\left(\left[x, f g(x)[)=\mu\left(\left[x, g(x)[)+\mu\left(\left[g(x), f g(x)[)=\rho_{\mu}(f)+\rho_{\mu}(g) .\right.\right.\right.\right.\right.\right.
$$

Como consecuencia, la función número de rotación restricta a $\Gamma_{\mu}$ es un homomorfismo sobre $\mathbb{T}^{1}$.

Si $\Gamma$ es un subgrupo promediable de $\mathrm{Homeo}_{+}\left(\mathrm{S}^{1}\right)$, entonces existe una medida de probabilidad sobre $S^{1}$ invariante por $\Gamma$ (vea el apéndice). Como consecuencia de lo anterior obtenemos la siguiente proposición (vea [72] para una demostración alternativa usando cohomología acotada).

Proposición 2.22. La restricción de la función número de rotación a todo subgrupo promediable de $\mathrm{Homeo}_{+}\left(\mathrm{S}^{1}\right)$ es un homomorfismo sobre $\mathbb{T}^{1}$.

Ejercicio 2.23. Pruebe que la única medida de probabilidad invariante por una rotación de ángulo irracional es la de Lebesgue. Concluya que si $\Gamma$ es un subgrupo de $\mathrm{Homeo}_{+}\left(\mathrm{S}^{1}\right)$ cuyos elementos conmutan con un homeomorfismo minimal del círculo, entonces $\Gamma$ es topológicamente conjugado a un grupo de rotaciones. 
Ejercicio 2.24. Dé ejemplos de subgrupos $\Gamma$ de Homeo $+\left(S^{1}\right)$ que no preserven ninguna medida de probabilidad del círculo y tales que la restricción de la función número de rotación a $\Gamma$ sea un homomorfismo sobre $\mathbb{T}^{1}$.

Observación. Tras la lectura de la sección 3.2, el lector debiese poder probar que para todo grupo $\Gamma$ verificando las propiedades pedidas la imagen $\rho(\Gamma)$ es finita.

\subsection{Acciones efectivas sobre la recta}

En esta sección veremos que el hecho que un grupo actúe efectivamente sobre la recta está relacionado con la posibilidad de "ordenar" dicho grupo.

Definición 2.25. Una relación de orden $\preceq$ en un grupo $\Gamma$ es invariante $a$ izquierda (resp. a derecha) si para todo $g, h$ en $\Gamma$ tales que $g \preceq h$ se tiene $f g \preceq f h$ (resp. $g f \preceq h f$ ) para todo $f \in \Gamma$. La relación es bi-invariante si ella es invariante a izquierda y a derecha simultáneamente.

Si $\preceq$ es una relación de orden en $\Gamma$ diremos que $f \in \Gamma$ es positivo si $f \succ i d$, y diremos que un elemento $f$ está entre $g$ y $h$ si $g \prec f \prec h$ ó $h \prec f \prec g$. Para simplificar la nomenclatura, diremos que un grupo $\Gamma$ es ordenable (resp. bi-ordenable) si admite un orden total e invariante a izquierda (resp. bi-invariante).

Ejercicio 2.26. Pruebe que un grupo $\Gamma$ es ordenable si y sólo si contiene un subsemigrupo $\Gamma_{+}$de modo que $\Gamma \backslash\{i d\}$ es la unión disjunta de $\Gamma_{+}$con el semigrupo $\Gamma_{-}=\left\{g: g^{-1} \in \Gamma_{+}\right\}$.

Ejercicio 2.27. Verifique que $\{(m, n): m>0$ ó $m=0$ y $n>0\}$ corresponde al conjunto de los elementos positivos de un orden total e invariante a izquierda en $\mathbb{Z}^{2}$ (llamado orden lexicográfico).

Ejercicio 2.28. Pruebe que el grupo libre $\mathbb{L}_{2}$ es bi-ordenable siguiendo las indicaciones dadas a continuación.

(i) Considere el anillo (no abeliano) $\mathbb{A}=\mathbb{Z}\langle\langle X, Y\rangle\rangle$ de las series de potencias formales con coeficientes enteros en dos variables independientes (no conmutantes) $X$ e $Y$. Denotando por $o(k)$ el subconjunto de $\mathbb{A}$ de los elementos cuyos términos poseen grado mayor o igual a $k$, verifique que $L=1+o(1)=\{1+S: S \in o(1)\}$ es un subgrupo multiplicativo de $\mathbb{A}$.

(ii) Si $f, g$ son los generadores de $\mathbb{L}_{2}$, pruebe que la aplicación $\phi$ que asocia a $f$ (resp. $g$ ) el elemento $1+X$ (resp. $1+Y$ ) de $\mathbb{A}$ se extiende de manera única a un homomorfismo inyectivo $\phi: \mathbb{L}_{2} \rightarrow L$.

(iii) Defina un orden de tipo lexicográfico en $L$ bi-invariante bajo multiplicación por elementos de $L$ (observe que este orden no será invariante bajo multiplicaciones por elementos de $\mathbb{A}$ ). Usando el homomorfismo $\phi$, induzca un orden total y bi-invariante en $\mathbb{L}_{2}$.

Observación. La técnica precedente, debida a Magnus, permite probar fácilmente que $\mathbb{L}_{2}$ es residualmente nilpotente (compare con el ejercicio 2.33). Para esto basta con verificar inductivamente que $\Phi\left(\Gamma_{i}^{\text {nil }}\right)$ está contenido en $1+o(i+1)$ para todo $i \geq 0$. 
El siguiente teorema da una caracterización dinámica de los grupos ordenables.

Teorema 2.29. Si $\Gamma$ es un grupo numerable, entonces las afirmaciones siguientes son equivalentes:

(i) $\Gamma$ actúa de manera efectiva sobre la recta por homeomorfismos que preservan orientación,

(ii) $\Gamma$ admite un orden total invariante a izquierda.

Demostración. Supongamos que $\Gamma$ actúa de manera efectiva sobre la recta por homeomorfismos que preservan orientación. Consideremos una sucesión $\left(x_{n}\right)$ densa en $\mathbb{R}$, y definamos $g \preceq h$ si $g=h$ o si el menor índice $n$ para el cual $g\left(x_{n}\right) \neq h\left(x_{n}\right)$ es tal que $g\left(x_{n}\right)<h\left(x_{n}\right)$. No es difícil probar que $\preceq$ es una relación de orden total e invariante a izquierda.

Supongamos ahora que $\Gamma$ admite un orden total e invariante a izquierda $\preceq$. Escojamos una numeración $\left(g_{i}\right)$ de $\Gamma$, hagamos $t\left(g_{0}\right)=0$ y supongamos que $t\left(g_{0}\right), \ldots, t\left(g_{i}\right)$ ya han sido definidos. Si $g_{i+1}$ es mayor (resp. menor) que $g_{0}, \ldots, g_{i}$, entonces definimos $t\left(g_{i+1}\right)=\max \left\{t\left(g_{0}\right), \ldots, t\left(g_{i}\right)\right\}+1$ (resp. $\left.\min \left\{t\left(g_{0}\right), \ldots, t\left(g_{i}\right)\right\}-1\right)$. Finalmente, si $g_{m} \prec g_{i+1} \prec g_{n}$ para ciertos $m, n$ en $\{0, \ldots, i\}$, y si $g_{j}$ no está entre $g_{m}$ y $g_{n}$ para ningún $0 \leq j \leq i$, entonces definimos $t\left(g_{i+1}\right)=\left(t\left(g_{m}\right)+t\left(g_{n}\right)\right) / 2$.

Observe que $\Gamma$ actúa de manera natural sobre $t(\Gamma)$ por $g\left(t\left(g_{i}\right)\right)=t\left(g g_{i}\right)$, y esta acción se extiende continuamente a la clausura de $t(\Gamma)$. Finalmente, extendemos la acción a toda la recta de modo que cada aplicación $g$ sea afín sobre cada intervalo del complemento del cierre de $t(\Gamma)$.

Fijado un orden total e invariante a izquierda $\preceq$ en un grupo numerable $\Gamma$, además de una numeración $\left(g_{i}\right)$ de éste, llamaremos realización dinámica a la acción construida en la demostración del teorema precedente. Es fácil comprobar que (si $\Gamma$ es no trivial entonces) dicha realización no admite puntos fijos globales. Otra propiedad interesante e igualmente fácil de verificar es el hecho que si $f$ es un elemento de $\Gamma$ cuya realización admite dos puntos fijos $a<b$ (que pueden ser iguales a $\pm \infty$ ) de modo que $] a, b[$ no contiene puntos fijos de $f$, entonces necesariamente existen puntos de la forma $t(g)$ dentro de $] a, b[$.

Ejercicio 2.30. Dé ejemplos explícitos de grupos libres de homeomorfismos de la recta para concluir que $\mathbb{L}_{n}$ es ordenable para todo $n \geq 2$ (compare con el ejercicio 2.28; vea también la proposición 4.5 de [72]).

Para más información en torno a la teoría de los grupos ordenables recomendamos la lectura de [116]. Ejemplos relevantes de grupos no (totalmente) ordenables a izquierda son los subgrupos de índice finito de $\operatorname{SL}(n, \mathbb{Z})$ para $n \geq 3$, como lo muestra el siguiente resultado debido a Witte [208].

Teorema 2.31. Si $n \geq 3$ y $\Gamma$ es un subgrupo de índice finito de $\operatorname{SL}(n, \mathbb{Z})$, entonces $\Gamma$ no admite ningún orden total invariante a izquierda. 
Demostración. Daremos la demostración sólo para el caso $n=3$, pero el argumento de la prueba es fácilmente modificable para $n>3$ (considere la inyección canónica de $\operatorname{SL}(3, \mathbb{Z})$ en $\operatorname{SL}(n, \mathbb{Z}))$.

Supongamos que $\preceq$ sea un orden total invariante a izquierda en $\Gamma$. Como $\Gamma$ es de índice finito en $\operatorname{SL}(3, \mathbb{Z})$, para $k \in \mathbb{N}$ suficientemente grande los siguientes elementos están en $\Gamma$ :

$$
\begin{aligned}
g_{1}=\left(\begin{array}{lll}
1 & k & 0 \\
0 & 1 & 0 \\
0 & 0 & 1
\end{array}\right), & g_{2}=\left(\begin{array}{lll}
1 & 0 & k \\
0 & 1 & 0 \\
0 & 0 & 1
\end{array}\right), & g_{3}=\left(\begin{array}{lll}
1 & 0 & 0 \\
0 & 1 & k \\
0 & 0 & 1
\end{array}\right), \\
g_{4}=\left(\begin{array}{lll}
1 & 0 & 0 \\
k & 1 & 0 \\
0 & 0 & 1
\end{array}\right), & g_{5}=\left(\begin{array}{lll}
1 & 0 & 0 \\
0 & 1 & 0 \\
k & 0 & 1
\end{array}\right), & g_{6}=\left(\begin{array}{lll}
1 & 0 & 0 \\
0 & 1 & 0 \\
0 & k & 1
\end{array}\right) .
\end{aligned}
$$

Es fácil verificar que para cada $i \in \mathbb{Z} / 6 \mathbb{Z}$ valen las siguientes relaciones:

$$
g_{i} g_{i+1}=g_{i+1} g_{i}, \quad\left[g_{i-1}, g_{i+1}\right]=g_{i}^{k} .
$$

Para $g \in \Gamma$ definimos $|g|=g$ si $g \succeq i d$, y hacemos $|g|=g^{-1}$ en caso contrario. Definimos también la relación $\gg$ por $g \gg h$ si $g \succ h^{n}$ para todo $n \geq 1$.

Fijemos ahora un índice $i$ y consideremos nuestra relación $\succeq$ en el grupo generado por $g_{i-1}, g_{i}$ y $g_{i+1}$. Es posible elegir tres elementos $a, b$ y $c$ superiores (según $\succ$ ) a $i d$, tales que ya sea $a=g_{i-1}^{ \pm 1}, b=g_{i+1}^{ \pm 1}, c=g_{i}^{ \pm k}$, o bien $a=g_{i+1}^{ \pm 1}, b=g_{i-1}^{ \pm 1}, c=g_{i}^{ \pm k}$, y de modo que

$$
a c=c a, \quad b c=c b, \quad a b a^{-1} b^{-1}=c^{-1} .
$$

Afirmamos que $a \gg c$ o $b \gg c$. Para probar esto, suponemos que para cierto $n \geq 1$ se tenga $c^{n} \succ a$ y $c^{n} \succ b$. Sea $d_{m}=a^{m} b^{m}\left(a^{-1} c^{n}\right)^{m}\left(b^{-1} c^{n}\right)^{m}$. Puesto que $d_{m}$ es un producto de elementos positivos, $d_{m}$ es positivo. Por otra parte, no es difícil verificar que $d_{m}=c^{-m^{2}+2 m n}$, por lo que $d_{m} \prec i d$ para $m$ suficientemente grande, lo cual es una contradicción.

La afirmación anterior nos permite concluir que ya sea $\left|g_{i}\right| \ll\left|g_{i-1}\right|$ o bien $\left|g_{i}\right| \ll\left|g_{i+1}\right|$. Si asumimos por ejemplo que $\left|g_{1}\right| \ll\left|g_{2}\right|$, entonces obtenemos $\left|g_{1}\right| \ll\left|g_{2}\right| \ll\left|g_{3}\right| \ll\left|g_{4}\right| \ll\left|g_{5}\right| \ll\left|g_{6}\right| \ll\left|g_{1}\right|$, lo cual es una contradicción. El caso $\left|g_{1}\right| \gg\left|g_{2}\right|$ es análogo.

Un importante teorema debido a Margulis implica en particular que, para $n \geq 3$, todo subgrupo normal de un subgrupo de índice finito de $\operatorname{SL}(n, \mathbb{Z})$ es ya sea finito o bien de índice finito [129]. Obtenemos así la versión fuerte del teorema de Witte.

Teorema 2.32. Para $n \geq 3$, toda acción de un subgrupo de índice finito de $\mathrm{SL}(n, \mathbb{Z})$ por homeomorfismos de la recta es trivial. 
Ejercicio 2.33. Pruebe directanente que todo grupo nilpotente, finitamente generado y sin torsión es bi-ordenable. De manera más general, pruebe que lo mismo vale para todo grupo residualmente nilpotente $\Gamma$ para el cual los cuocientes (abelianos) sucesivos $\Gamma_{i}^{\text {nil }} / \Gamma_{i+1}^{\text {nil }}$ no poseen torsión (vea el apéndice).

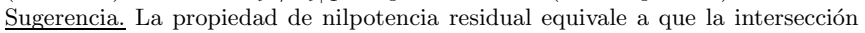
$\cap_{i \geq 0} \Gamma_{i}^{\text {nil }}$ se reduce al elemento neutro.

Ejercicio 2.34. Sea $\mathrm{N}_{n}$ el grupo de las matrices triangulares superiores de orden $n \times n$ a coeficientes enteros y tales que todo coeficiente de la diagonal sea igual a 1. Pruebe que $\mathrm{N}_{n}$ es ordenable utilizando su acción natural sobre $\mathbb{Z}^{n}$ y el orden lexicográfico sobre este último conjunto.

Observación. Es fácil verificar que $\mathrm{N}_{n}$ es nilpotente y sin torsión. Por otra parte, un teorema debido a Malcev [172] establece que todo grupo nilpotente, finitamente generado y sin torsión, es un subgrupo de algún $\mathrm{N}_{n}$. Esto permite reobtener indirectamente el primer resultado del ejercicio anterior.

\subsection{Acciones libres}

Hemos visto que el problema de hallar acciones efectivas sobre la recta es equivalente a un problema de ordenamiento del grupo en cuestión. Las proposiciones siguientes, debidas a Hölder, muestran que el problema de hallar acciones libres sobre la recta es equivalente a un problema de ordenamiento arquimediano del grupo.

Definición 2.35. Una relación de orden total $\preceq$ en un grupo $\Gamma$ es arquimediana si para todo $g, h$ en $\Gamma$, con $g \neq i d$, existe $n \in \mathbb{Z}$ tal que $g^{n} \succ h$.

Proposición 2.36. Si $\Gamma$ es un grupo que actúa libremente por homeomorfismos de la recta, entonces $\Gamma$ admite un orden total bi-invariante $y$ arquimediano.

Demostración. Consideremos el orden $\preceq$ en $\Gamma$ según el cual $g \prec h$ si $g(x)<h(x)$ para algún $x \in \mathbb{R}$ (equivalentemente, para todo $x \in \mathbb{R}$ ). Esta relación de orden es total. Además, como la acción es libre, $\preceq$ es una relación bi-invariante y arquimediana. Dejamos los detalles al lector.

La recíproca a la proposición anterior será una consecuencia de la proposición siguiente, la cual implica que los únicos grupos que pueden actuar libremente por homeomorfismos de la recta son los subgrupos de $(\mathbb{R},+)$.

Proposición 2.37. Si $\Gamma$ admite un orden total bi-invariante y arquimediano, entonces $\Gamma$ es isomorfo a un subgrupo de $(\mathbb{R},+)$.

Demostración. Supongamos que $\Gamma$ sea no trivial y fijemos un elemento positivo $f$ en $\Gamma$. Para cada $g \in \Gamma$ y cada $p \in \mathbb{N}$ consideremos el único entero $q=q(p)$ tal que $f^{q} \preceq g^{p} \prec f^{q+1}$.

Primera parte: existe el límite

$$
\lim _{p \rightarrow \infty}\left\{\frac{q}{p}: f^{q} \preceq g^{p} \prec f^{q+1}\right\} .
$$


En efecto, si $f^{q\left(p_{1}\right)} \preceq g^{p_{1}} \prec f^{q\left(p_{1}\right)+1}$ y $f^{q\left(p_{2}\right)} \preceq g^{p_{2}} \prec f^{q\left(p_{2}\right)+1}$ entonces

$$
f^{q\left(p_{1}\right)+q\left(p_{2}\right)} \preceq g^{p_{1}+p_{2}} \prec f^{q\left(p_{1}\right)+q\left(p_{2}\right)+2},
$$

de donde se concluye que $q\left(p_{1}\right)+q\left(p_{2}\right) \leq q\left(p_{1}+p_{2}\right) \leq q\left(p_{1}\right)+q\left(p_{2}\right)+1$. La afirmación resulta entonces del lema 2.13.

Denotaremos $\phi(g)$ al límite dado por la expresión (2.5). Observe que el valor de $\phi(g)$ es finito. En efecto, para $g \in \Gamma$ existe $n \in \mathbb{Z}$ tal que $f^{n} \preceq g \prec f^{n+1}$. Luego, $f^{n p} \preceq g^{p} \prec f^{(n+1) p}$, de donde se concluye que

$$
n=\lim _{p \rightarrow \infty} \frac{n p}{p} \leq \phi(g) \leq \lim _{p \rightarrow \infty} \frac{(n+1) p-1}{p}=n+1 .
$$

Segunda parte: la aplicación $\phi: \Gamma \rightarrow(\mathbb{R},+)$ es un homomorfismo.

Sean $g_{1}, g_{2}$ elementos arbitrarios de $\Gamma$. Supongamos que $g_{1} g_{2} \preceq g_{2} g_{1}$ (el caso $g_{2} g_{1} \preceq g_{1} g_{2}$ es análogo). Si $f^{q_{1}} \preceq g_{1}^{p} \prec f^{q_{1}+1} \quad$ y $\quad f^{q_{2}} \preceq g_{2}^{p} \prec f^{q_{2}+1}$ entonces, por la bi-invariancia de $\preceq$, se tiene

$$
f^{q_{1}+q_{2}} \preceq g_{1}^{p} g_{2}^{p} \preceq\left(g_{1} g_{2}\right)^{p} \preceq g_{2}^{p} g_{1}^{p} \prec f^{q_{1}+q_{2}+2} .
$$

De esto se concluye que

$\phi\left(g_{1}\right)+\phi\left(g_{2}\right)=\lim _{p \rightarrow \infty} \frac{q_{1}+q_{2}}{p} \leq \phi\left(g_{1} g_{2}\right) \leq \lim _{p \rightarrow \infty} \frac{q_{1}+q_{2}+1}{p}=\phi\left(g_{1}\right)+\phi\left(g_{2}\right)$,

y por lo tanto $\phi\left(g_{1} g_{2}\right)=\phi\left(g_{1}\right)+\phi\left(g_{2}\right)$.

Tercera parte: el homomorfismo $\phi$ es inyectivo.

Observe que $\phi$ preserva orden, es decir, si $g_{1} \preceq g_{2}$ entonces $\phi\left(g_{1}\right) \leq \phi\left(g_{2}\right)$. Observe además que $\phi(f)=1$. Sea $h$ un elemento de $\Gamma$ tal que $\phi(h)=0$. Supongamos que $h \neq i d$. Existe entonces un entero $n$ tal que $h^{n} \succeq f$. De lo anterior se concluye que $0=n \phi(h)=\phi\left(h^{n}\right) \geq \phi(f)=1$, lo cual es absurdo. Luego, si $\phi(h)=0$ entonces $h=i d$, lo cual prueba que $\phi$ es inyectiva.

Si $\Gamma$ es un grupo no trivial que actúa libremente sobre la recta, fijamos sobre él la relación de orden dada por la proposición 2.36. Este orden permite construir un isomorfismo $\phi$ entre $\Gamma$ y un subgrupo de $(\mathbb{R},+)$. Si $\phi(\Gamma)$ es isomorfo a $(\mathbb{Z},+)$, entonces la acción de $\Gamma$ es conjugada a la acción por traslaciones enteras de la recta. En caso contrario, el grupo $\phi(\Gamma)$ es denso en $(\mathrm{R},+)$. Para cada punto $x$ de la recta definimos

$$
\varphi(x)=\sup \{\phi(h) \in \mathbb{R}: h(0) \leq x\} .
$$

Es fácil ver que $\varphi$ es una aplicación no decreciente. Además, ella verifica la igualdad $\varphi(h(x))=\varphi(x)+\phi(h)$ para todo $x \in \mathbb{R}$ y todo $h \in \Gamma$. Finalmente, $\varphi$ es continua, pues en caso contrario el conjunto $\mathbb{R} \backslash \varphi(\mathbb{R})$ sería un abierto no vacío e invariante por las traslaciones de $\phi(\Gamma)$. 
En resumen, si $\Gamma$ es un grupo que actúa libremente por homeomorfismos de la recta, entonces su acción es semiconjugada a una acción por traslaciones.

Ejercicio 2.38. Si $\Gamma$ admite un order total invariante a izquierda y arquimediano, entonces dicho orden es necesariamente bi-invariante; en particular, la hipótesis de bi-invariancia en la proposición 2.37 es superflua. Para probar esta afirmación (debida a Conrad) desarrolle los ítemes a continuación.

(i) Pruebe que una relación de orden total e invariante a izquierda $\prec$ es biinvariante si y sólo si su cono positivo $\Gamma_{+}=\{g \in \Gamma: g \succ i d\}$ es estable por conjugación, es decir, si $h g h^{-1}$ pertenece a $\Gamma_{+}$para todo $g \in \Gamma_{+}$y todo $h \in \Gamma$ (vea el ejercicio 2.26).

(ii) Sea $\prec$ un orden total, invariante a izquierda y arquimediano en un grupo $\Gamma$. Supongamos que $g \in \Gamma_{+}$y $h \in \Gamma_{-}$sean tales que $h g h^{-1} \notin \Gamma_{+}$. Considere el menor entero positivo tal que $h^{-1} \prec g^{n}$. Usando la relación $h g h^{-1} \prec i d$ verifique que $h^{-1} \prec g^{-1} h^{-1} \prec h^{n-1}$, lo cual contradice la definición de $n$. Concluya que $\Gamma_{+}$es estable bajo conjugación por elementos de $\Gamma_{-}$.

(iii) Suponga ahora que $g \in \Gamma_{+}$y $h \in \Gamma_{-}$sean tales que $h g h^{-1} \notin \Gamma_{+}$. En tal caso $h g^{-1} h^{-1} \succ i d$, y como $h^{-1} \in \Gamma_{-}$, por el ítem (ii) se tiene $h^{-1}\left(h g^{-1} h^{-1}\right) h \in \Gamma_{+}$, es decir, $g^{-1} \in \Gamma_{+}$, lo cual es absurdo.

Ejercicio 2.39. De manera alternativa al argumento esbozado en el ejercicio precedente, pruebe que la acción sobre la recta de la realización dinámica de todo orden total, invariante a izquierda y arquimediano en un grupo numerable es libre.

Consideremos ahora un grupo $\Gamma$ que actúa libremente por homeomorfismos del círculo. La imagen inversa $\tilde{\Gamma}$ de $\Gamma$ en $\widetilde{\mathrm{Homeo}_{+}}\left(\mathrm{S}^{1}\right)$ actúa libremente sobre la recta. Si repetimos los argumentos de la demostración de la proposión anterior, considerando la traslación $x \mapsto x+1$ como el elemento $f$ de la segunda parte, entonces se obtiene que $\tilde{\Gamma}$ es isomorfo a un subgrupo de $(\mathbb{R},+)$, y este isomorfismo desciende en un isomorfismo entre $\Gamma$ y un subgrupo de $\mathrm{SO}(2, \mathbb{R})$. Para referencia futura, expresamos esto como un teorema.

Teorema 2.40. Si $\Gamma$ es un grupo que actúa libremente por homeomorfismos de $\mathrm{S}^{1}$, entonces $\Gamma$ es isomorfo a un subgrupo del grupo de rotaciones.

De manera análoga al caso de la recta, bajo las hipótesis anteriores la acción de $\Gamma$ es semiconjugada a la del grupo de rotaciones correspondiente.

Ejercicio 2.41. Pruebe que todo subgrupo finitamente generado de Homeo+ $\left(\mathrm{S}^{1}\right)$ cuyos elementos son todos de torsión es cíclico y finito.

Ejercicio 2.42. Sea $\Gamma$ un subgrupo de $\operatorname{PSL}(2, \mathbb{R})$ cuyos elementos son todos elípticos. Pruebe que $\Gamma$ es conjugado a un grupo de rotaciones del círculo. 
Ejercicio 2.43. Sea $\Gamma$ un subgrupo del grupo de los homeomorfismos de la recta. Suponga que exista un intervalo $[a, b]$ tal que, para todo $g \in \Gamma$, los puntos fijos de $g$ están contenidos en $[a, b]$. Pruebe que $\Gamma$ es abeliano.

Ejercicio 2.44. Dé una demostración alternativa a la proposición 1.2 usando el teorema de Hölder.

Los resultados expuestos anteriormente muestran que el hecho de actuar libremente sobre el círculo o la recta caracteriza (módulo semiconjugación topológica) a los grupos de rotaciones y traslaciones respectivamente. Observe por otra parte que los elementos no triviales del grupo afín fijan a lo más un punto de la recta. Veremos a continuación que, salvo una excepción evidente, esta propiedad caracteriza módulo semiconjugación a dicho grupo. El resultado siguiente es debido a Solodov.

Teorema 2.45. Sea $\Gamma$ un subgrupo de $\mathrm{Homeo}_{+}(\mathbb{R})$ tal que cada elemento no trivial de $\Gamma$ fija a lo más un punto de la recta. Suponga que no existe ningún punto invariante por todos los elementos de $\Gamma$. Entonces $\Gamma$ es semiconjugado a un subgrupo del grupo afín.

Observe que en el caso que descartamos en que existe un punto $x_{0}$ que es fijo por todos los elementos de $\Gamma$, las acciones de $\Gamma$ sobre $]-\infty, x_{0}[\mathrm{y}$ ]$x_{0}, \infty[$ son libres, $\mathrm{y}$ por lo tanto semiconjugadas a acciones de grupos de traslaciones. Sin embargo, la acción de $\Gamma$ sobre la recta no es necesariamente semiconjugada a la acción de un subgrupo del estabilizador de un punto de $\mathbb{R}$ por el grupo afín, pues $\Gamma$ podría contener elementos para los cuales $x_{0}$ es un punto fijo de tipo parabólico.

Demostración del teorema 2.45. Supondremos en lo que sigue que la acción de $\Gamma$ no es libre (el caso de una acción libre está cubierto por el teorema de Hölder). Bajo esta suposición, $\Gamma$ no puede ser abeliano. En efecto, en caso contrario la órbita de un punto fijo $x_{0}$ de un elemento no trivial $g$ de $\Gamma$ estaría contenida en el conjunto de los puntos fijos de $g$. Luego, $x_{0}$ sería un punto fijo global de $\Gamma$, lo cual es una contradicción.

Primera parte: afirmamos que si $\Gamma_{0}$ es un subgrupo normal de $\Gamma$ que contiene un elemento no trivial con un punto fijo, entonces $\Gamma_{0}$ posee un elemento con un punto fijo atractor.

En efecto, sea $h_{0} \in \Gamma_{0}$ un elemento no trivial tal que $h_{0}\left(x_{0}\right)=x_{0}$ para cierto punto $x_{0} \in \mathbb{R}$. Por hipótesis, existe un elemento $g$ de $\Gamma$ tal que $x_{1}=g\left(x_{0}\right) \neq x_{0}$. Cambiando $g$ por $g^{-1}$ si fuese necesario, podemos suponer que $x_{1}>x_{0}$. Definamos $h_{1}=g h_{0} g^{-1} \in \Gamma_{0}$. Supongamos que $x_{0}$ sea un punto fijo parabólico de $h_{0}$. Reemplazando $h_{0}$ por su inverso en caso de necesidad, tenemos $h_{0}(y)>y$ para $y \neq x_{0}$. Sea $h=h_{0} h_{1}^{-1} \in \Gamma_{0}$. Es fácil verificar que $h\left(x_{0}\right)<x_{0}$ y $h\left(x_{1}\right)>x_{1}$. Luego, $h$ posee un punto fijo repulsor en $] x_{0}, x_{1}[$, lo cual demuestra la afirmación.

Segunda parte: definición de una relación de orden. 
Dados $g, h$ en $\Gamma$, escribimos $g \preceq h$ si existe $x \in \mathbb{R}$ tal que $g(y) \leq h(y)$ para todo $y \geq x$. Es fácil verificar que ésta es una relación de orden total y bi-invariante. Afirmamos que ella satisface la siguiente versión débil de la propiedad arquimediana: si $f \in \Gamma$ posee un punto fijo repulsor y $g \in \Gamma$, entonces existe $n \in \mathbb{N}$ tal que $g \preceq f^{n}$. En efecto, si $x_{0}$ es el punto fijo de $f$ fijemos $x_{-}, x_{+}$tales que $x_{-}<x_{0}<x_{+}$. Para $n \in \mathbb{N}$ suficientemente grande tenemos $f^{n}\left(x_{-}\right)<g\left(x_{-}\right)$y $f^{n}\left(x_{+}\right)>g\left(x_{+}\right)$, por lo que $g^{-1} f^{n}$ posee un punto fijo en el intervalo $] x_{-}, x_{+}\left[\right.$. Como $g^{-1} f^{n}\left(x_{+}\right)>x_{+}$, esto implica que $f^{n}(x)>g(x)$ para todo $x \geq x_{+}$, por lo que $g \preceq f^{n}$.

Tercera parte: un homomorfismo hacia los reales.

Fijemos un elemento $f \in \Gamma$ con un punto fijo repulsor. Al igual que en la demostración del teorema de Hölder, para $g \in \Gamma$ tal que $i d \preceq g$ definimos

$$
\phi(g)=\lim _{p \rightarrow \infty}\left\{\frac{q}{p}: f^{q} \preceq g^{p} \prec f^{q+1}\right\},
$$

y para $g \preceq i d$ definimos $\phi\left(g^{-1}\right)=-\phi(g)$. Obtenemos así un homomorfismo $\phi: \Gamma \rightarrow(\mathbb{R},+)$ tal que $\phi(f)=1$. Observe que si $\Gamma_{0}$ es un subgrupo normal de $\Gamma$ que contiene un elemento no trivial con un punto fijo, entonces por la primera parte existe $h \in \Gamma_{0}$ con un punto fijo repulsor. Por la segunda parte tenemos $f \preceq h^{n}$ para $n \in \mathbb{N}$ suficientemente grande, por lo que $\phi(h) \geq 1 / n$. En particular, $\phi\left(\Gamma_{0}\right) \neq\{0\}$.

Cuarta parte: la acción de $[\Gamma, \Gamma]$ sobre la recta es libre.

En efecto, puesto que $[\Gamma, \Gamma]$ es normal en $\Gamma$, si $\Gamma$ posee un elemento no trivial con un punto fijo entonces $\phi([\Gamma, \Gamma]) \neq 0$, lo cual es absurdo, pues $[\Gamma, \Gamma]$ está contenido en el núcleo de $\phi$.

Tenemos entonces que $[\Gamma, \Gamma]$ es semiconjugado a un grupo de traslaciones, es decir, existen un homomorfismo $\phi_{0}:[\Gamma, \Gamma] \rightarrow(\mathbb{R},+)$ y un homeomorfismo $\varphi$ de la recta tales que $\varphi(h(x))=\varphi(x)+\phi_{0}(h)$ para todo $x \in \mathbb{R}$ y todo $h \in[\Gamma, \Gamma]$. Afirmamos que $\phi_{0}([\Gamma, \Gamma])$ no es discreto. En efecto, en caso contrario es fácil ver que las conjugaciones por elementos de $\Gamma$ deberían preservar al generador de $\phi_{0}([\Gamma, \Gamma]) \sim \mathbb{Z}$, por lo que $[\Gamma, \Gamma]$ estaría contenido en el centro de $\Gamma$. Sin embargo, esto es imposible, dado que $\Gamma$ contiene elementos con (exactamente) un punto fijo.

Quinta parte: fin de la demostración.

Por lo anterior, la imagen $\phi_{0}([\Gamma, \Gamma])$ es densa en $\mathbb{R}$. Es fácil ver entonces que la conjugación $\varphi$ de la cuarta parte es única módulo post-conjugación con un elemento del grupo afín. Como $[\Gamma, \Gamma]$ es normal en $\Gamma$, para cada $g \in \Gamma$ se tiene que $\varphi \circ g \circ \varphi^{-1}$ es un elemento del grupo afín.

Enfatizamos nuevamente que los teoremas de Hölder y Solodov no pueden ser extendidos de manera razonable al caso de grupos (a órbitas densas) de homeomorfismos del círculo cuyos elementos no triviales fijan a lo más dos puntos [119], incluso en el caso real-analítico [144]. 


\subsection{Número de traslación y medidas quasi-invariantes}

De manera análoga al caso del círculo, para cada medida de Radon $v$ sobre los borelianos de la recta podemos considerar el grupo $\Gamma_{v}$ de los homeomorfismos que preservan $v$. Para $g \in \Gamma_{v}$ definimos el número de traslación de $g$ respecto a $v$ por $\tau(g)=v([x, g(x)[)$ si $g(x) \geq x$, y por $\tau(g)=-v([g(x), x[)$ si $g(x)<x$. Es fácil ver que dicho valor no depende de la elección de $x \in \mathbb{R}$.

El número de traslación satisface propiedades análogas a las del número de rotación de homeomorfismos de $\mathrm{S}^{1}$. Por ejemplo, para $g \in \Gamma_{v}$ se cumple

$$
\tau_{v}(g)=0 \text { si y sólo si } g \text { posee al menos un punto fijo. }
$$

En efecto, si $\operatorname{Fix}(g)=\emptyset$ entonces la órbita de cada punto $x$ de la recta es no acotada en ambas direcciones. Fijemos $x \in \mathbb{R}$ y supongamos (cambiando $g$ por $g^{-1}$ si es necesario) que $g(x)>x$. Si $\tau_{v}(g)=0$ entonces de las igualdades $v\left(\left[x, g^{n}(x)[)=v\left(\left[g^{-n}(x), x[)=0\right.\right.\right.\right.$, donde $n \in \mathbb{N}$, se concluye al hacer tender $n$ al infinito que $v(]-\infty,+\infty[)=0$, lo cual es absurdo. Luego, si $\operatorname{Fix}(g)=\emptyset$ entonces $\tau_{v}(g) \neq 0$. Recíprocamente, si $x \in \operatorname{Fix}(g)$ entonces $\tau_{v}(g)=v([x, g(x)[)=v([x, x[)=0$.

Notemos que una propiedad más fuerte para elementos $g \in \Gamma_{v}$ es válida:

$$
\text { si } \operatorname{Fix}(g) \neq \emptyset \text { entonces } \operatorname{sop}(v) \subset \operatorname{Fix}(g) \text {. }
$$

En efecto, en caso contrario existiría un boreliano $A$ tal que $v(A)>0$ y $A \cap g(A)=\emptyset$. Al menos uno de los conjuntos $\cup_{n \in \mathbb{N}} g^{n}(A)$ o $\cup_{n \in \mathbb{N}} g^{-n}(A)$ debe ser acotado, y por lo tanto su medida finita. Sin embargo, las medidas de estos conjuntos son iguales a $\sum_{n \in \mathbb{N}} v(A)=\infty$.

Note que la aplicación $\tau_{v}: \Gamma_{v} \rightarrow \mathbb{R}$ es un homomorfismo de $\Gamma_{v}$ sobre $(\mathbb{R},+)$. Esta observación será fundamental al tratar problema de la unicidad (módulo un factor multiplicativo) de la medida invariante.

Lema 2.46. Si $v_{1} y v_{2}$ son dos medidas de Radon invariantes por un subgrupo $\Gamma$ de Homeo $_{+}(\mathbb{R})$, entonces existe $\kappa>0$ tal que los homomorfismos $\tau_{v_{1}} y \tau_{v_{2}}$ verifican la relación $\tau_{v_{1}}=\kappa \tau_{v_{2}}$.

Demostración. Debido a (2.6), los núcleos de $\tau_{v_{1}}$ y $\tau_{v_{2}}$ coinciden con $\Gamma_{0}=\{g: \operatorname{Fix}(g) \neq \emptyset\}$. Obtenemos así dos homomorfismos $\tau_{1}$ y $\tau_{2}$ de $\Gamma / \Gamma_{0}$ sobre $(\mathbb{R},+)$. Fijemos un punto $x_{0}$ en $\operatorname{Fix}\left(\Gamma_{0}\right)$, cuya existencia está garantizada por (2.7). El grupo $\Gamma / \Gamma_{0}$ actúa libremente sobre la órbita $\Gamma\left(x_{0}\right)$, por lo que admite una relación de orden bi-invariante y arquimediana $\prec$ dada por $g_{1} \Gamma_{0} \prec g_{2} \Gamma_{0}$ si $g_{1}\left(x_{0}\right)<g_{2}\left(x_{0}\right)$. Si fijamos un elemento $f \in \Gamma$ tal que $\Gamma_{0} \prec f \Gamma_{0}$, es fácil ver que para todo $g \in \Gamma$ se tiene

$$
\tau_{i}\left(g \Gamma_{0}\right)=\tau_{i}\left(f \Gamma_{0}\right) \cdot \lim _{p \rightarrow \infty}\left\{\frac{q}{p}: f^{q} \Gamma_{0} \preceq g^{p} \Gamma_{0} \prec f^{q+1} \Gamma_{0}\right\} .
$$

Luego, $\tau_{2}(f) \cdot \tau_{1}=\tau_{1}(f) \cdot \tau_{2}$, lo cual concluye la demostración. 
Si $\Gamma$ preserva $v$ y $\tau_{v}(\Gamma)$ es trivial o isomorfo a $\mathbb{Z}$, no podemos esperar la unicidad (módulo un factor positivo) de la medida invariante $v$. Diferente es el caso en que $\tau_{v}(\Gamma)$ es denso en $\mathbb{R}$.

Proposición 2.47. Si $v_{1} y v_{2}$ son dos medidas de Radon invariantes por un subgrupo $\Gamma$ de $\mathrm{Homeo}_{+}(\mathbb{R})$ tales que $\tau_{v_{1}}(\Gamma)$ y $\tau_{v_{2}}(\Gamma)$ son densos en $\mathbb{R}$, entonces existe $\kappa>0$ tal que dichas medidas verifican la relación $v_{1}=\kappa v_{2}$.

Demostración. Por el lema anterior, podemos suponer que $\tau_{v_{1}}=\tau_{v_{2}}$. Probaremos entonces que $v_{1}=v_{2}$. Para esto, primero notemos que ninguna de las medidas en cuestión posee átomos. En efecto, si $v_{i}\left(\left\{x_{0}\right\}\right)>0$ entonces todo elemento positivo y no trivial de $\tau_{v_{i}}(\Gamma)$ sería mayor o igual que $v_{i}\left(\left\{x_{0}\right\}\right)>0$, contradiciendo la hipótesis de densidad de $\tau_{v_{i}}(\Gamma)$. Dejamos al lector la tarea de probar que esta hipótesis implica también que la acción de $\Gamma$ sobre los soportes de $v_{1}$ y $v_{2}$ es minimal (i.e., sus órbitas son densas).

Probaremos ahora que los soportes $\operatorname{sop}\left(v_{1}\right)$ y $\operatorname{sop}\left(v_{2}\right)$ son iguales. En efecto, en caso de desigualdad existiría un punto $x \in \operatorname{sop}\left(v_{i}\right) \backslash \operatorname{sop}\left(v_{i+1}\right)$ (la indexación es considerada módulo 2). Por la densidad de las órbitas sobre los soportes, podríamos escoger $g \in \Gamma$ tal que $g(x) \neq x$ y tal que la medida $v_{i+1}$ del intervalo de puntos extremos $x$ y $g(x)$ sea nula. Esto implicaría que $\tau_{v_{i+1}}(g)=0$ y $\tau_{v_{i}}(g) \neq 0$, lo cual es absurdo.

Para finalizar la prueba de la igualdad de la medidas $v_{1}$ y $v_{2}$, debemos probar que ellas otorgan la misma masa a intervalos cuyos extremos son puntos del soporte. Si $[x, y]$ es un intervalo de este tipo podemos escoger $g_{n} \in \Gamma$ tal que $g_{n}(x)$ converja a $y$. Obtenemos así

$$
\begin{aligned}
v_{1}([x, y]) & =\lim _{n \rightarrow \infty} v_{1}\left(\left[x, g_{n}(x)\right]\right)=\lim _{n \rightarrow \infty} \tau_{v_{1}}\left(g_{n}\right)=\lim _{n \rightarrow \infty} \tau_{v_{2}}\left(g_{n}\right) \\
& =\lim _{n \rightarrow \infty} v_{2}\left(\left[x, g_{n}(x)\right]\right)=v_{2}([x, y]),
\end{aligned}
$$

lo cual concluye la demostración.

Por todo lo anterior, resulta interesante conocer a priori cuáles son las condiciones que garantizan la existencia de una medida de Radon invariante. El resultado siguiente, debido a Plante [167], es un importante aporte en tal dirección.

Teorema 2.48. Si $\Gamma$ es un subgrupo finitamente generado y virtualmente nilpotente de $\mathrm{Homeo}_{+}(\mathbb{R})$, entonces $\Gamma$ preserva una medida de Radon sobre la recta.

Enunciamos inmediatamente un corolario de este teorema a ser utilizado más adelante.

Corolario 2.49. Si $\Gamma$ es un subgrupo finitamente generado y virtualmente nilpotente de $\mathrm{Homeo}_{+}(\mathbb{R})$, entonces todo elemento del grupo de los conmutadores $[\Gamma, \Gamma]$ fija al menos un punto de la recta. 
En efecto, si $v$ es una medida invariante dada por el teorema de Plante, entonces el número de traslación de todo elemento de $[\Gamma, \Gamma]$ respecto a $v$ es nulo. La afirmación del corolario se concluye entonces de (2.6).

La demostración original de Plante para el teorema 2.48 involucra ideas muy interesantes relacionadas con el crecimiento de grupos (sección 2.2, capítulo 4), la noción de pseudo-grupo (sección 2, capítulo 3), y la promediabilidad (vea el apéndice), las que se aplican en muchas otras situaciones. Sin embargo, presentaremos a continuación una demostración muchísimo más directa y sencilla inspirada en los métodos de la sección 1 de este capítulo. Ella se basa en el hecho que los grupos nilpotentes no contienen semigrupos libres.

Definición 2.50. Dos elementos $f, g$ de un grupo $\Gamma$ generan un semigrupo libre si los elementos de la forma $f^{n} g^{m_{r}} f^{n_{r}} \cdots g^{n_{1}} f^{n_{1}} g^{m}$, con $n_{j}$ y $m_{j}$ enteros positivos, $m \geq 0$ y $n \geq 0$, son dos a dos distintos para elecciones diferentes de los exponentes.

Ejercicio 2.51. Pruebe que ningún grupo virtualmente nilpotente contiene semigrupos libres a dos generadores.

Sugerencia. Reduzca el caso general al caso nilpotente, y razone por inducción sobre el grado de nilpotencia.

Para encontrar semigrupos libres dentro de grupos actuando por homeomorfismos de variedades unidimensionales, la siguiente noción será de utilidad.

Definición 2.52. Decimos que dos homeomorfismos de la recta están entrecruzados sobre un intervalo $] a, b[$ si uno de ellos fija $] a, b$ [ pero no tiene puntos fijos en el interior, mientras que el otro envía $a$ o $b$ dentro de $] a, b[$.

Recalquemos que, en la definición anterior, $a$ (resp. $b$ ) puede ser igual a $-\infty$ (resp. $+\infty$ ). El siguiente criterio elemental para obtener semigrupos libres a dos generadores es bastante conocido.

Lema 2.53. Todo subgrupo $\Gamma$ de $\mathrm{Homeo}_{+}(\mathbb{R})$ que posee elementos entrecruzados contiene semigrupos libres a dos generadores.

Demostración. Supongamos que existan $f, g$ en $\Gamma$ y un intervalo $[a, b]$ tales que $\operatorname{Fix}(f) \cap[a, b]=\{a, b\}$ y $g(a) \in] a, b[$ (el caso en que $g(b) \in] a, b[$ es análogo). Cambiando $f$ por su inversa si es necesario, podemos suponer que $f(x)<x$ para todo $x \in] a, b[$. Definamos $c=g(a) \in] a, b[$ y fijemos un punto $\left.d^{\prime} \in\right] c, b\left[\right.$. Como $g f^{n}(a)=c$ para todo $n \in \mathbb{N}$ y como $g f^{n}\left(d^{\prime}\right)$ converge a $c$ cuando $n$ tiende al infinito, para $n \in \mathbb{N}$ suficientemente grande la aplicación $g f^{n}$ posee un punto fijo sobre $] a, d^{\prime}[$. Fijemos un tal $n \in \mathbb{N}$ y sea $d>c$ el ínfimo de los puntos fijos de $g f^{n}$ en $] a, b[$. Para $m \in \mathbb{N}$ suficientemente grande tenemos $f^{m}(d)<c$, por lo que el lema del ping-pong positivo aplicado a las restricciones de $f^{m}$ y $g f^{n}$ a $[a, b]$ prueba que el semigrupo generado por tales elementos es libre (vea el ejercicio 2.85). 
De acuerdo al ejercicio 2.51 y al lema 2.53, el siguiente resultado constituye una generalización del teorema 2.48 (compare con $[11,190]$ ).

Teorema 2.54. Sea $\Gamma$ un grupo finitamente generado de homeomorfismos de la recta. Si $\Gamma$ no posee elementos entrecruzados, entonces $\Gamma$ preserva una medida de Radon sobre los borelianos de $\mathbb{R}$.

Demostración. Si $\Gamma$ admite puntos fijos globales entonces la afirmación es obvia: la medida de Dirac soportada en cualquiera de estos puntos es invariante por la acción.

Supongamos en lo que sigue que no hay puntos fijos globales para la acción, y fijemos un sistema finito $\left\{f_{1}, \ldots, f_{k}\right\}$ de generadores para $\Gamma$. Comenzamos afirmando que (al menos) uno de estos generadores no admite puntos fijos. Para verificar esto razonamos por contradicción suponiendo que cada uno de los $f_{i}$ posee puntos fijos. Sea $x_{1} \in \mathbb{R}$ un punto fijo de $f_{1}$. Si $f_{2}$ fija $x_{1}$ entonces haciendo $x_{2}=x_{1}$ tenemos que $x_{2}$ es fijado tanto por $f_{1}$ como por $f_{2}$. En caso contrario, escojamos un punto fijo $x_{2}$ para $f_{2}$ de modo que $f_{2}$ no posea ningún otro punto fijo entre $x_{1}$ y $x_{2}$. Como $f_{1}, f_{2}$ no se entrecruzan sobre ningún intervalo (y lo mismo ocurre para $f_{1}^{-1} \mathrm{y}$ $f_{2}$ ), el elemento $f_{1}$ debe fijar también $x_{2}$. Si $f_{3}$ fija $x_{2}$ hacemos $x_{3}=x_{2}$; si no, consideramos un punto $x_{3}$ que sea fijado por $f_{3}$ de modo que $f_{3}$ no posea ningún punto fijo entre $x_{2}$ y $x_{3}$. El argumento usado anteriormente permite nuevamente probar que $f_{1}, f_{2}$ y $f_{3}$ fijan $x_{3}$. Continuando de esta manera podemos hallar un punto fijo común para todos los generadores $f_{i}$, es decir, un punto fijo global para la acción de $\Gamma$, lo cual contradice nuestra suposición.

Afirmamos ahora que existe un conjunto cerrado no vacío invariante y minimal para la acción de $\Gamma$. Para probar esto consideremos un generador $f=f_{i}$ sin puntos fijos, fijemos un punto arbitrario $x_{0}$ de la recta, y denotemos por $I$ al intervalo $\left[x_{0}, f\left(x_{0}\right)\right]$ (resp. $\left[f\left(x_{0}\right), x_{0}\right]$ ) si $f\left(x_{0}\right)>x_{0}$ (resp. si $\left.f\left(x_{0}\right)<x_{0}\right)$. En la familia $\mathcal{F}$ de conjuntos cerrados no vacíos e invariantes por $\Gamma$ consideremos la relación de orden $\preceq$ definida por $\Lambda_{1} \succeq \Lambda_{2}$ si $\Lambda_{1} \cap I \subset \Lambda_{2} \cap I$. Como $f$ no tiene puntos fijos, toda órbita de $\Gamma$ debe intersectar al intervalo $I$, por lo que $\Lambda \cap I$ es un compacto no vacío para todo $\Lambda \in \mathcal{F}$. Podemos así aplicar el lema de Zorn, el cual nos brinda un elemento maximal para el orden $\preceq$. Dicho elemento maximal no es otra cosa que la intersección con $I$ de un conjunto $\Lambda$ invariante por $\Gamma$, cerrado, no vacío y minimal.

Observe ahora que tanto la frontera $\partial \Lambda$ de $\Lambda$ como el conjunto $\Lambda^{\prime}$ de los puntos de acumulación de $\Lambda$ son también cerrados e invariantes por $\Gamma$. Debido a la minimalidad de $\Lambda$, sólo tres casos pueden presentarse.

(i) $\Lambda^{\prime}=\emptyset$.

En este caso $\Lambda$ es discreto, es decir, $\Lambda$ coincide con el conjunto de los puntos de una sucesión $\left(y_{n}\right)_{n \in \mathbb{Z}}$ satisfaciendo $y_{n}<y_{n+1}$ para todo $n$ y que carece de puntos de acumulación. Resulta sencillo verificar entonces que la medida de Radon $v=\sum_{n \in \mathbb{Z}} \delta_{y_{n}}$ es invariante por $\Gamma$. 
(ii) $\partial \Lambda=\emptyset$.

En este caso $\Lambda$ coincide con toda la recta. Afirmamos que la acción de $\Gamma$ es libre. En efecto, si éste no fuera el caso entonces existirían un elemento $g \in \Gamma$ y un intervalo $] a, b[$ estrictamente contenido en la recta de modo que $g$ fija $] a, b[$ pero no posee ningún punto fijo en su interior. Como la acción es minimal, debe existir un elemento $h \in \Gamma$ que envía $a$ o $b$ dentro de $] a, b[$; sin embargo, esto implica que $g$ y $h$ se entrecruzan en $] a, b[$, lo cual viola nuestra hipótesis. Ahora bien, como la acción de $\Gamma$ en la recta es libre, el teorema de Hölder implica que $\Gamma$ es topológicamente conjugado a un grupo de traslaciones. La preimagen de la medida de Lebesgue por la conjugación resulta ser entonces una medida de Radon invariante por la acción de $\Gamma$.

(iii) $\partial \Lambda=\Lambda^{\prime}=\Lambda$.

En este caso $\Lambda$ es "localmente" un conjunto de Cantor. Colapsando a un punto la clausura de cada una de las componentes conexas del complemento de $\Lambda$, obtenemos una nueva "recta topológica" con una acción de $\Gamma$ inducida por semiconjugación. Como en el caso (ii), se verifica sin dificultad que esta nueva acción es libre, y por lo tanto preserva una medida de Radon. La preimagen de esta medida por la semiconjugación resulta ser entonces una medida de Radon invariante por la acción original de $\Gamma$.

Ejercicio 2.55. Sea $\Gamma$ un grupo (no necesariamente finitamente generado) de homeomorfimos de la recta que no contiene elementos entrecruzados.

(i) Pruebe directamente (i.e., sin usar el teorema 2.54) que el conjunto $\Gamma_{0}$ de los elementos de $\Gamma$ que poseen puntos fijos constituye un subgrupo normal de $\Gamma$.

(ii) Pruebe que el grupo $\Gamma / \Gamma_{0}$ admite una relación de orden total, bi-invariante y arquimediana.

(iii) Usando (i) y (ii) dé una demostración alternativa al teorema 2.54.

El lector verificará sin mayor dificultad que la hipótesis de generación finita es necesaria para el teorema 2.54 (vea además [166]). Sin embargo, a lo largo de la demostración dicha hipótesis fue utilizada sólo para hallar un elemento sin puntos fijos. Por lo tanto, si esta última condición es asumida como hipótesis entonces el teorema continúa siendo válido (vea también el ejercicio 2.62). Para referencia futura, enunciamos esto como una proposición para el caso de grupos conmutativos.

Proposición 2.56. Todo subgrupo abeliano de Homeo+ $(\mathbb{R})$ que posee al menos un elemento sin puntos fijos preserva una medida de Radon sobre la recta.

Ejercicio 2.57. Pruebe la validez de la proposición precedente considerando la acción inducida sobre el círculo topológico obtenido al cuocientar la recta por la acción del elemento sin puntos fijos.

El teorema 2.48 concierne los grupos nilpotentes, y cabe preguntarse qué ocurre en el caso de los grupos solubles. Observe para comenzar que si $\theta \neq 0$ y $\kappa \neq 1$, entonces el subgrupo de $\mathrm{Af}_{+}(\mathbb{R})$ generado por los elementos 
$f(x)=x+\theta$ y $g(x)=\kappa x$ no preserva ninguna medida de Radon sobre la recta. Sin embargo, puesto que los elementos del grupo afín preservan la medida de Lebesgue módulo un factor multiplicativo, teniendo en mente la proposición 1.4 es natural preguntarse bajo qué condiciones un subgrupo soluble de $\mathrm{Homeo}_{+}(\mathbb{R})$ deja quasi-invariante una medida de Radon de la recta. Nuevamente consideraremos sólo grupos finitamente generados, pues de acuerdo al ejercicio 2.63 existen grupos abelianos no finitamente generados de homeomorfismos de la recta que no admiten ninguna medida de Radon quasi-invariante no trivial.

Los grupos solubles son construidos a partir de grupos abelianos por extensiones sucesivas. Por ello, comenzamos nuestro estudio con una observación elemental: si $\Gamma_{0}$ es un subgrupo normal de un subgrupo $\Gamma$ de $\mathrm{Homeo}_{+}(\mathbb{R})$ que preserva una medida de Radon $v$, entonces para todo $g \in \Gamma$ la medida $g(v)$ también es invariante por $\Gamma_{0}$. En efecto, para todo $h \in \Gamma_{0}$ tenemos

$$
\left(g h g^{-1}\right)(g(v))=g(h(v))=g(v) .
$$

Por el lema 2.46, existe una constante $\kappa(g)=\kappa_{v}(g)$ tal que $\tau_{g(v)}=\kappa(g) \tau_{v}$. Los tres lemas siguientes tratan el problema de probar la existencia de una medida quasi-invariante para la acción de un grupo a partir de la quasiinvariancia de una medida para uno de sus subgrupos normales.

Lema 2.58. Sean $\Gamma_{0}$ un subgrupo normal de un subgrupo $\Gamma$ de $\mathrm{Homeo}_{+}(\mathbb{R})$ y $v$ una medida de Radon invariante por $\Gamma_{0}$. Suponga que $\tau_{v}\left(\Gamma_{0}\right) \neq\{0\}$. Si $\kappa(\Gamma)=\{1\}$ y $\Gamma / \Gamma_{0}$ es promediable, entonces existe una medida de Radon invariante por $\Gamma$.

Demostración. Si $\tau_{v}\left(\Gamma_{0}\right)$ es denso en $\mathbb{R}$ entonces la proposición 2.47 muestra que $v$ es invariante por $\Gamma$. Supongamos en lo que sigue que $\tau_{v}\left(\Gamma_{0}\right)$ es cíclico y normalicemos $v$ de manera que $\tau_{v}\left(\Gamma_{0}\right)$ sea igual a $\mathbb{Z}$. Sea $h_{0} \in \Gamma_{0}$ tal que $\tau_{v}\left(h_{0}\right)=1$. Consideremos el núcleo $\Gamma_{0}^{*}$ de $\tau_{v}$. El conjunto $\operatorname{Fix}\left(\Gamma_{0}\right)$ es no vacío (pues contiene al soporte de $v$ ) y es invariante por $\Gamma_{0}$, por lo que es no acotado en ambas direcciones. Además, $\Gamma_{0}^{*}=\left\{h \in \Gamma_{0}: \operatorname{Fix}(h) \neq \emptyset\right\}$, lo cual muestra que $\Gamma_{0}^{*}$ es normal en $\Gamma$.

Utilizaremos ahora una idea semejante a la de la prueba de la proposición 2.56. Afirmamos en primer lugar que $\Gamma_{0} / \Gamma_{0}^{*}$ está contenido en el centro del grupo $\Gamma / \Gamma_{0}^{*}$. Para probar esto debemos mostrar que para todo $g \in \Gamma \mathrm{y}$ todo $x \in \operatorname{Fix}\left(\Gamma_{0}^{*}\right)$ se tiene $g^{-1} h_{0} g(x)=h_{0}(x)$. Fijemos la medida $v_{1}$ de masa igual a 1 en cada punto del conjunto $\left\{h_{0}^{n}(x), n \in \mathbb{Z}\right\}$. Observe que las restricciones a $\Gamma_{0}$ de los homomorfismos $\tau_{v}$ y $\tau_{v_{1}}$ coinciden. Es fácil ver entonces que $\kappa_{v}=\kappa_{v_{1}}$. De la hipótesis $\kappa(\Gamma)=1$ concluimos que

$$
v_{1}\left(\left[x, g^{-1} h_{0} g(x)[)=\tau_{v_{1}}\left(g^{-1} h_{0} g\right)=\tau_{g_{*}\left(v_{1}\right)}\left(h_{0}\right)=v_{1}\left(\left[x, h_{0}(x)[),\right.\right.\right.\right.
$$

lo cual implica que $g^{-1} h_{0} g(x) \leq h_{0}(x)$. Por otro lado, considerando la medida $v_{2}$ de masa 1 en cada punto $g^{-1} h_{0}^{n} g(x)$ y cambiando $h_{0}$ por $g^{-1} h_{0} g$, el mismo argumento muestra que $h_{0}(x) \leq g^{-1} h_{0} g(x)$. 
Fijemos $x_{0} \in \operatorname{Fix}\left(\Gamma_{0}^{*}\right)$. Observe que el grupo $\left(\Gamma / \Gamma_{0}^{*}\right) /\left(\Gamma_{0} / \Gamma_{0}^{*}\right)=\Gamma / \Gamma_{0}$ actúa sobre el espacio cuociente $\operatorname{Fix}\left(\Gamma_{0}^{*}\right) /\left\langle h_{0}\right\rangle\left(x_{0}\right)$, el cual es compacto. Por la hipótesis de promediabilidad, $\Gamma / \Gamma_{0}$ preserva una medida de probabilidad sobre este espacio, la cual se levanta en una medida de Radon sobre $\operatorname{Fix}\left(\Gamma_{0}^{*}\right)$ invariante por $\Gamma$.

Lema 2.59. Sean $\Gamma_{0}$ un subgrupo normal de un subgrupo $\Gamma$ de $\mathrm{Homeo}_{+}(\mathbb{R})$ y $v$ una medida de Radon invariante por $\Gamma_{0}$. Suponga que $\tau_{v}\left(\Gamma_{0}\right) \neq\{0\}$. Si $\kappa(\Gamma) \neq\{1\}$ entonces $v$ es quasi-invariante por $\Gamma$.

Demostración. Sean $h \in \Gamma_{0}$ y $g \in \Gamma$ tales que $\tau_{v}(h) \neq 0$ y $\kappa(g) \neq 1$. De la igualdad (válida para todo $m, n$ en $\mathbb{Z}$ )

$$
\tau_{v}\left(g^{-n} h^{m} g^{n}\right)=\tau_{g_{*}^{n}(v)}\left(h^{m}\right)=m \kappa(g)^{n} \tau_{v}(h)
$$

se deduce fácilmente que $\tau_{v}(\Gamma)$ es denso en $\mathbb{R}$. La proposición 2.47 muestra entonces que $v$ es quasi-invariante por $\Gamma$.

Lema 2.60. Sean $\Gamma_{0}$ un subgrupo normal de un subgrupo $\Gamma$ de $\mathrm{Homeo}_{+}(\mathbb{R})$ y $v$ una medida de Radon quasi-invariante por $\Gamma_{0}$. Sea $\Gamma_{0}^{*}$ el subgrupo formado por los $h \in \Gamma_{0}$ tales que $h_{*}(v)=v$. Si $\tau_{v}\left(\Gamma_{0}^{*}\right) \neq\{0\}$ y $\kappa\left(\Gamma_{0}\right) \neq\{1\}$, entonces $v$ es quasi-invariante por $\Gamma$.

Demostración. Como $\tau_{v}\left(\Gamma_{0}^{*}\right) \neq\{0\}$ y $\kappa\left(\Gamma_{0}\right) \neq\{1\}$, es fácil ver que un elemento $h \in \Gamma_{0}$ pertenece a $\Gamma_{0}^{*}$ si y sólo si $\operatorname{Fix}(h)=\emptyset$ o si $\operatorname{Fix}(h)$ es no acotado en ambas direcciones. Ambas condiciones son invariantes por conjugación, de lo cual se concluye que $\Gamma_{0}^{*}$ es normal en $\Gamma$. Es claro además que $\kappa(\Gamma) \neq\{1\}$. La afirmación resulta entonces del lema precedente.

Estamos ahora preparados para abordar el problema de la existencia de una medida de Radon quasi-invariante para una familia amplia de subgrupos solubles de $\mathrm{HomeO}_{+}(\mathbb{R})$.

Teorema 2.61. Sea $\Gamma$ un subgrupo soluble de Homeo $(\mathbb{R})$. Supongamos que $\Gamma$ admita una cadena de subgrupos $\{i d\}=\Gamma_{n} \triangleleft \Gamma_{n-1} \triangleleft \ldots \triangleleft \Gamma_{0}=\Gamma$ tal que cada $\Gamma_{i}$ sea finitamente generado y cada cuociente $\Gamma_{i-1} / \Gamma_{i}$ sea abeliano. Entonces existe una medida de Radon invariante por $\Gamma$.

Demostración. Supondremos que $\Gamma$ no preserva ninguna medida de Radon. Tenemos $\operatorname{Fix}(\Gamma)=\emptyset$, pues en caso contrario la delta de Dirac con masa en un punto fijo sería una medida invariante. Sea $j>0$ el índice más pequeño para el cual $\operatorname{Fix}\left(\Gamma_{j}\right) \neq \emptyset$. El grupo abeliano y finitamente generado $\Gamma_{j-1} / \Gamma_{j}$ actúa sobre el conjunto cerrado y no acotado $\operatorname{Fix}\left(\Gamma_{j}\right)$ por homeomorfismos que preservan orden. Dejamos al lector la tarea sencilla de probar, a partir del teorema de Plante, la existencia de una medida de Radon invariante para esta acción. Esta medida induce evidentemente una medida de Radon sobre la recta invariante por $\Gamma_{j-1}$ cuyo soporte está contenido en $\operatorname{Fix}\left(\Gamma_{j}\right)$. Note que $\tau_{v}\left(\Gamma_{j-1}\right) \neq\{0\}$, pues $\operatorname{Fix}\left(\Gamma_{j-1}\right)=\emptyset$. 
Sea $k>0$ el índice más pequeño para el cual $\Gamma_{k}$ preserva una medida de Radon. Abusando de la notación, designemos dicha medida también por $v$. Es fácil ver que necesariamente se tiene $\tau_{v}\left(\Gamma_{k}\right) \neq\{0\}$. De acuerdo al lema 2.58 tenemos $\kappa\left(\Gamma_{k-1}\right) \neq\{1\}$, y por el lema 2.59 esto implica que $v$ es quasi-invariante por $\Gamma_{k-1}$. El lema 2.60 permite probar inductivamente que $v$ es quasi-invariante por $\Gamma_{k-2}, \Gamma_{k-3}$, etc. Se concluye así que $v$ es quasi-invariante por $\Gamma$.

La hipótesis de generación finita de cada $\Gamma_{i}$ es satisfecha por una clase importante de grupos, a saber, los grupos policíclicos [209]. Por otro lado, ella puede ser debilitada, tal como lo indica el ejercicio siguiente.

Ejercicio 2.62. La dinámica de un subgrupo $\Gamma$ de $\mathrm{Homeo}_{+}(\mathbb{R})$ es dicha acotadamente generada si existe un sistema de generadores $\mathcal{G}$ de $\Gamma$ y un punto $x_{0} \in \Gamma$ tales que $\left\{h\left(x_{0}\right): h \in \mathcal{G}\right\}$ es un subconjunto acotado de la recta. Pruebe que los teoremas $2.48,2.54$ y 2.61 son válidos si la hipótesis de generación finita es reemplazada por una hipótesis de dinámica acotadamente generada.

Ejercicio 2.63. Dé un ejemplo de un subgrupo abeliano numerable no finitamente generado de $\mathrm{Homeo}_{+}(\mathbb{R})$ sin medida de Radon quasi-invariante (alternativamente, vea [166]).

\subsection{Una aplicación: grupos promediables ordenables}

En esta sección utilizaremos las ideas desarrolladas precedentemente para dar una "demostración dinámica" del siguiente resultado de carácter algebraico (recomendamos la lectura previa del apéndice para el concepto de promediabilidad de un grupo; vea además el ejemplo que postcede al ejercicio 5.4).

Teorema 2.64. Todo grupo ordenable, promediable, finitamente generado e infinito admite un homomorfismo no trivial sobre $(\mathbb{R},+)$.

Este teorema, que responde a una vieja interrogante en el ámbito de los grupos ordenables, ha sido demostrado recientemente por Witte. En nuestra exposición seguiremos esencialmente la misma (brillante) idea de [207], pero evitaremos utilizar los resultados de la teoría de los grupos $C$ ordenables, la cual juega un rol relativamente importante en dicho trabajo.

El primer ingrediente de la prueba se remonta a una idea de Ghys y Sikora, y consiste en la introducción del espacio de los órdenes asociado a un grupo ordenable, así como de una acción del grupo sobre el mismo. De manera más precisa, si $\Gamma$ es un grupo finitamente generado y ordenable, denotaremos por $\mathcal{O}(\Gamma)$ al conjunto de todos los órdenes totales e invariantes a izquierda en $\Gamma$. Si fijamos un sistema finito $\mathcal{G}$ de generadores de $\Gamma$, entonces podemos definir una distancia entre dos elementos distintos $\leq$ $\mathrm{y} \preceq \operatorname{de} \mathcal{O}(\Gamma)$ haciendo $\operatorname{dist}(\leq, \preceq)=e^{-n}$, donde $n$ es el máximo entero no negativo tal que los órdenes $\leq \mathrm{y} \preceq$ coinciden sobre la bola $B_{\mathcal{G}}(n)$ de 
radio $n$ en $\Gamma$ (vea el apéndice para el concepto de bola dentro de un grupo). En otras palabras, $n$ es el mayor entero no negativo tal que para todo $g, h$ en $B_{\mathcal{G}}(n)$ se tiene $g \leq h$ si y sólo $g \preceq h$. Haciendo $\operatorname{dist}(\preceq, \preceq)=0$ para todo orden total e invariante a izquierda $\preceq$, es fácil comprobar que la función dist así definida es una métrica (que depende de $\mathcal{G}$ ) en el espacio $\mathcal{O}(\Gamma)$. De hecho, el espacio resultante verifica la propiedad ultramétrica y es compacto.

Observación 2.65. El estudio de la estructura del espacio de órdenes de un grupo ordenable es un tópico interesante en sí mismo. Un elegante resultado de Tararin [116] describe los grupos ordenables que admiten un número finito de órdenes (totales e invariantes a izquierda). Si un grupo admite infinitos órdenes, entonces admite necesariamente una cantidad no numerable [121, 141]. Para grupos abelianos de rango superior, para grupos nilpotentes no abelianos sin torsión, y también para grupos libres no abelianos, los espacios de órdenes son homeomorfos al conjunto de Cantor (vea [189] y [141] respectivamente). Sin embargo, existen grupos cuyos espacios de órdenes son infinitos (no numerables) y poseen puntos aislados [58, 141]. De acuerdo a Linnell [121], uno de los intereses de lo anterior dice relación con la estructura del semigrupo formado por los elementos positivos respecto a un orden específico, tal como queda consignado en el ejercicio siguiente.

Ejercicio 2.66. Pruebe que si $\preceq$ no es un punto aislado del espacio de órdenes de un grupo finitamente generado y ordenable, entonces el conjunto de los elementos positivos según $\preceq$ no es finitamente generado como semigrupo.

El grupo $\Gamma$ actúa (continuamente) sobre $\mathcal{O}(\Gamma)$ por multiplicación a derecha: dados un orden $\preceq$ y un elemento $f \in \Gamma$, la imagen de $\preceq$ bajo $f$ es el orden $\preceq_{f}$ cuyos elementos positivos son los conjugados por $f$ de los elementos positivo según $\preceq$. En otras palabras, se tiene $g \preceq_{f} h$ si y solamente si $g f^{-1} \preceq h f^{-1}$.

Diremos que una relación de orden total e invariante a izquierda $\preceq$ es recurrente a derecha si para todo par de elementos $f, h$ en $\Gamma$ tales que $f \succ i d$ existe $n \in \mathbb{N}$ satisfaciendo $f h^{n} \succ h^{n}$. Por ejemplo, todo orden total y biinvariante es recurrente a derecha. La siguiente proposición constituye el paso fundamental en la prueba de Witte.

Proposición 2.67. Si $\Gamma$ es un grupo finitamente generado, promediable $y$ ordenable a izquierda, entonces $\Gamma$ admite (al menos) un orden (total, invariante a izquierda y) recurrente a derecha.

Para probar esta proposición necesitamos la siguiente forma débil del teorema de recurrencia de Poincaré (vea el ejercicio 2.69 para su versión fuerte).

Teorema 2.68. Si $T$ es una transformación que preserva una medida de probabilidad $\mu$ sobre un espacio de medida $\mathrm{M}$, entonces para todo subconjunto medible $A$ de $\mathrm{M}$ y $\mu$-casi todo punto $x \in A$ existe $n \in \mathbb{N}$ tal que $T^{n}(x)$ pertenece a $A$. 
Demostración. El conjunto de los puntos de $A$ que no vuelven a caer en $A$ bajo iteraciones de $T$ coincide con $B=A \backslash \cup_{n \in \mathbb{N}} T^{-n}(A)$. Se verifica fácilmente los conjuntos de la forma $T^{-i}(B)$, con $i>0$, son dos a dos disjuntos. Puesto que $T$ preserva $\mu$, dichos conjuntos tienen la misma medida, y como la masa total de $\mu$ es 1 , la única posibilidad es que dicha medida sea igual a cero. Por lo tanto, $\mu(B)=0$, es decir, $\mu$-casi todo punto de $A$ vuelve a caer en $A$ por algún iterado de $T$.

Ejercicio 2.69. Pruebe que, bajo las hipótesis del teorema precedente, $\mu$-casi todo punto de $A$ vuelve infinitas veces al conjunto $A$ bajo iteraciones de $T$.

Demostración de la proposición 2.67. Por definición, si $\Gamma$ es un grupo finitamente generado y promediable, entonces su acción sobre $\mathcal{O}(\Gamma)$ preserva una medida de probabilidad $\mu$. Probaremos que $\mu$-casi todo elemento de $\mathcal{O}(\Gamma)$ es recurrente a derecha. Para esto consideremos, para cada $g \in \Gamma$, el subconjunto $A_{g}$ de $\mathcal{O}(\Gamma)$ formado por todos los órdenes (totales e invariantes a izquierda) $\preceq$ tales que $g \succ i d$. Por el teorema de recurrencia de Poincaré, para todo $f \in \Gamma$ el conjunto $B_{g}(f)=A_{g} \backslash \cup_{n \in \mathbb{N}} f^{n}\left(A_{g}\right)$ tiene $\mu$-medida nula. Luego, la medida de $B_{g}=\cup_{f \in \Gamma} B_{g}(f)$ también es nula, así como la del conjunto $B=\cup_{g \in \Gamma} B_{g}$. Consideremos un elemento cualquiera $\preceq$ del conjunto (de $\mu$-medida total) $A=\mathcal{O}(\Gamma) \backslash B$. Dados $g \succ i d$ y $f \in \Gamma$, de la inclusión $B_{g}(f) \subset B$ deducimos que $\preceq$ no pertenece a $B_{g}(f)$, por lo que existe $n \in \mathbb{N}$ tal que $\preceq$ pertenece a $f^{n}\left(A_{g}\right)$. En otras palabras, se tiene $g \succ_{f^{-n}} i d$, es decir, $g f^{n} \succ f^{n}$. Puesto que $g \succ i d$ y $f \in \Gamma$ eran arbitrarios, esto demuestra la recurrencia a derecha de $\preceq$.

La condición de recurrencia a derecha para un orden invariante a izquierda tiene una consecuencia dinámica, tal como queda estipulado en la siguiente proposición.

Proposición 2.70. Sea $\Gamma$ un grupo numerable que admite un orden (total, invariante a izquierda y) recurrente a derecha $\preceq$. Si $\left(g_{n}\right)_{n \geq 0}$ es cualquier numeración de $\Gamma$, entonces la realización dinámica de $\Gamma$ asociada al orden $\preceq$ y a dicha numeración es un subgrupo de $\mathrm{Homeo}_{+}(\mathbb{R})$ sin elementos entrecruzados.

Demostración. La afirmación es obvia si $\Gamma$ es trivial, por lo que en lo que sigue supondremos que $\Gamma$ es infinito. Inspirándonos en la demostración de la proposición 2.53, supongamos que existan $f, g$ en $\Gamma$ y un intervalo $[a, b]$ tales que (para sus realizaciones dinámicas se cumpla) $\operatorname{Fix}(f) \cap[a, b]=\{a, b\}$ y $g(a) \in] a, b[$ (el caso en que $g(b)$ pertenece a $] a, b[$ es análogo). Cambiando $f$ por su inversa si es necesario, podemos suponer que $f(x)<x$ para todo $x \in] a, b[$. Como ya observamos tras el teorema 2.29, necesariamente debe existir $g_{i} \in \Gamma$ tal que $t\left(g_{i}\right)$ pertenece al intervalo $] a, b[$. Sea $j \geq 0$ el índice para el cual $g_{j}=i d$. Conjugando $f$ y $g$ con el elemento $g_{j} g_{i}^{-1}$ si es necesario, podemos suponer que $t\left(g_{j}\right)=t(i d)$ está en $] a, b[$. Además, cambiando $g$ por $f^{-n} g$ para $n$ suficientemente grande, podemos suponer que 
$g(a)>t\left(g_{j}\right)$. Definamos $\left.c=g(a) \in\right] t\left(g_{j}\right), b[$, y fijemos un punto $d \in] c, b[$. Como $g f^{n}(a)=c$ para todo $n \in \mathbb{N}$ y como $g f^{n}(d)$ converge a $c<d$ cuando $n$ tiende a infinito, si $n \in \mathbb{N}$ es suficientemente grande entonces la aplicación $h_{n}=g f^{n}$ verifica $\left.h_{n}(a)>a, h_{n}(d)<d, \operatorname{Fix}\left(h_{n}\right) \cap\right] a, d\left[\subset\left[c_{n}, c_{n}^{\prime}\right] \subset\right] c, h_{n}(d)[$ y $\left\{c_{n}, c_{n}^{\prime}\right\} \subset \operatorname{Fix}\left(h_{n}\right)$ para ciertas sucesiones de puntos $c_{n}$ y $c_{n}^{\prime}$ convergiendo a $c$ por la derecha (vea la figura 13 a continuación ${ }^{1}$ ). Observe que cada $h_{n}$ satisfaciendo las propiedades precedentes es un elemento positivo de $\Gamma$, pues de $h_{n}\left(t\left(g_{j}\right)\right)>h_{n}(a)=c>t\left(g_{j}\right)$ se concluye que $t\left(h_{n}\right)>t(i d)$, y por la construcción de la realización dinámica esto implica que $h_{n} \succ i d$.

Fijemos $m>n$ suficientemente grandes de modo que las propiedades anteriores se verifiquen para $h_{m}$ y $h_{n}$ y se cumpla además $\left.\left[c_{m}, c_{m}^{\prime}\right] \subset\right] c, c_{n}[$. Fijemos $k \in \mathbb{N}$ suficientemente grande de modo que $h_{n}^{k}(a)>h_{m}\left(c_{n}\right)$, y definamos $h=h_{m}^{k}$. Para todo $i \in \mathbb{N}$ se tiene $\left.h^{i}\left(t\left(g_{j}\right)\right) \in\right] h_{m}\left(c_{n}\right), c_{n}[$, por lo que $h_{m} h^{i}\left(t\left(g_{j}\right)\right)<h_{m}\left(c_{n}\right)<h(a)<h\left(t\left(g_{j}\right)\right)$. Luego, $h_{m} h^{i} \prec h \prec h^{i}$ para todo $i \in \mathbb{N}$. Sin embargo, esto viola la hipótesis de recurrencia a derecha para $\preceq$.

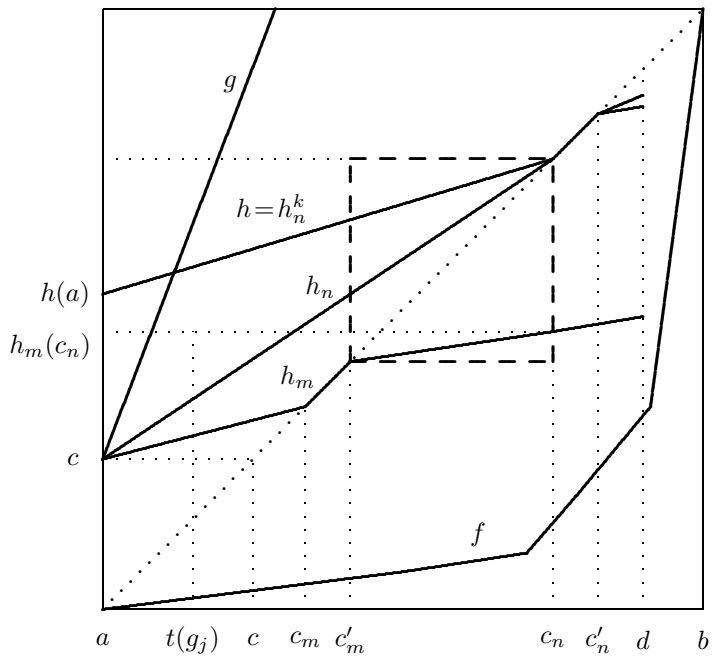

Figura 13

\footnotetext{
${ }^{1}$ En la figura 13 los homeomorfismos han sido esbozados como si fuesen afines por partes. Sin embargo, en general las realizaciones dinámicas de grupos ordenables no son subgrupos de $\mathrm{AfP}_{+}(\mathbb{R})$. El bosquejo es entonces una mera simplificación de lo que realmente ocurre.
} 
El lector habrá notado que en la demostración precedente no usamos la propiedad de recurrencia a derecha en toda su magnitud. En efecto, sólo utilizamos el hecho que, para todo par de elementos positivos $f, h$ en $\Gamma$, existe un entero positivo $n$ tal que $f h^{n} \succ h$. Esta última se conoce con el nombre de propiedad de Conrad, y los grupos que admiten un orden (total, invariante a izquierda y) que satisface dicha propiedad son llamados $C$ ordenables. Existe una interesante literatura en torno a esta noción con una perspectiva esencialmente algebraica (vea $[48,116]$ y también la observación 5.7 del último capítulo). Lo que precede añadido al ejercicio 2.71 indica que dicha literatura tiene, al menos para grupos numerables, una traducción dinámica natural: de manera un poco imprecisa, la condición de Conrad es el equivalente algebraico de la condición de no existencia de elementos entrecruzados para las correspondientes acciones sobre la recta [141].

Debemos señalar además que la noción de elementos entrecruzados corresponde, en el lenguaje de la teoría de foliaciones, a la noción de hojas resorte [39]. Esta última es sin embargo más general, pues se aplica también a pseudo-grupos de homeomorfismos de variedades unidimensionales (vea la definición 3.14). Finalmente, en el lenguaje de los sistemas dinámicos, los elementos entrecruzados corresponden a versiones unidimensionales de las herraduras de Smale [163].

Ejercicio 2.71. Sean $\left(x_{n}\right)$ una sucesión densa de puntos de la recta y $\Gamma$ un subgrupo de $\mathrm{Homeo}_{+}(\mathbb{R})$. Pruebe que si $\Gamma$ no posee elementos entrecruzados, entonces la relación de orden inducida por $\left(x_{n}\right)$ y construida en la demostración del teorema 2.29 satisface la propiedad de Conrad.

Ejercicio 2.72. Sea $\preceq$ una relación de orden (total e invariante a izquierda) en un grupo $\Gamma$.

(i) Pruebe que si $\preceq$ satisface la condición de Conrad, entonces para todo par de elementos positivos $f, h$ de $\Gamma$ se tiene $f h^{2} \succ h$.

Sugerencia. Siguiendo [141], suponga lo contrario y verifique que para los elementos positivos $f$ y $g=f h$ de $\Gamma$ se tiene $f g^{n} \prec g$ para todo $n \in \mathbb{N}$.

(ii) De manera más general, pruebe que para todo entero positivo $n$ se tiene $f h^{n} \succ h^{n-1}$.

(iii) Pruebe que $\preceq$ puede ser recurrente a derecha aunque contenga elementos positivos $f, h$ tales que $f h^{2} \prec h^{2}$.

Ejercicio 2.73. Dé ejemplos de grupos $C$-ordenables que no admitan órdenes (totales, invariantes a izquierda y) recurrentes a derecha (alternativamente, vea el ejemplo 4.5 de [207]).

Ejercicio 2.74. Verifique que el grupo $\Gamma$ de presentación $\left\langle f, g: f g f^{-1}=g^{-1}\right\rangle$ admite órdenes (totales, invariantes a izquierda y) recurrentes a derecha, pero no admite órdenes bi-invariantes.

Demostración del teorema 2.64. Si $\Gamma$ es promediable, finitamente generado y ordenable a izquierda, la proposición 2.67 nos da la existencia de un orden (total, invariante a izquierda y) recurrente a derecha en $\Gamma$. 
Por la proposición 2.70, la realización dinámica asociada a dicho orden y a cualquier numeración $\left(g_{n}\right)_{n \geq 0}$ de $\Gamma$ tal que $g_{0}=i d$ es un subgrupo de $\mathrm{Homeo}_{+}(\mathbb{R})$ (isomorfo a $\Gamma$ y) sin elementos entrecruzados. Por el teorema 2.54, $\Gamma$ preserva una medida de Radon $v$ sobre la recta. Recordemos ahora que si $\Gamma$ no se reduce al elemento neutro, entonces todas sus realizaciones dinámicas son grupos sin puntos fijos globales. Por lo tanto, la función número de traslación respecto a $v$ es un homomorfismo no trivial sobre $(\mathbb{R},+)$ (vea $(2.6)$ y el inicio de la demostración del teorema 2.54).

Ejercicio 2.75. Pruebe que el grupo de los homeomorfismos afines por pedazos del intervalo es bi-ordenable, y que sus subgrupos finitamente generados admiten homomorfismos no triviales sobre $(\mathbb{R},+)$.

Observación. Recuerde que, de acuerdo al teorema 1.19, el grupo $\mathrm{AfP}_{+}([0,1])$ (al igual que cualquier grupo promediable) no contiene subgrupos libres a dos generadores. Junto con lo anterior esto torna natural la siguiente pregunta, cuya eventual respuesta afirmativa constituiría una generalización importante del teorema 2.64: ¿los subgrupos finitamente generados de $\mathrm{Homeo}_{+}([0,1])$ sin subgrupos libres a dos generadores admiten homomorfismos no triviales sobre $(\mathbb{R},+)$ ? Un resultado interesante en esta dirección aparece en [122].

\section{Medidas invariantes y grupos libres}

\subsection{La alternativa de Tits débil}

Un notable teorema de Tits establece que todo subgrupo finitamente generado de $\operatorname{GL}(n, \mathbb{R})$ contiene un subgrupo libre a dos generadores o bien es virtualmente soluble (vea $[19,20,21]$ para versiones modernas de este resultado). Esta dicotomía, conocida como la alternativa de Tits, no es válida para grupos de homeomorfismos del círculo, tal como lo muestra el siguiente ejercicio.

Ejercicio 2.76. Demuestre que el grupo de Thompson F no es virtualmente soluble (recuerde sin embargo que, por el teorema 1.19, F no contiene subgrupos libres a dos generadores).

Sin embargo, una versión débil de la alternativa puede ser establecida. El siguiente resultado fue conjeturado por Ghys y demostrado por Margulis en [128]. Nosotros desarrollaremos sin embargo una demostración dada posteriormente por el propio Ghys en [72], la cual se presta para una interpretación probabilística que será abordada en la sección 3.2 de este capítulo.

Teorema 2.77. Si $\Gamma$ es un subgrupo de $\mathrm{Homeo}_{+}\left(\mathrm{S}^{1}\right)$ entonces existe una medida de probabilidad sobre $\mathrm{S}^{1}$ invariante por $\Gamma$ o bien $\Gamma$ contiene un subgrupo libre a dos generadores. 
Toda acción sobre $\mathrm{S}^{1}$ de un grupo virtualmente soluble admite una medida invariante, pues todo grupo virtualmente soluble es promediable (vea el ejercicio 5.67). De esta forma, el teorema anterior es realmente una versión débil del teorema de Tits. Sin embargo, en este caso la alternativa no es excluyente. En efecto, de acuerdo a la sessión 2.3 de este capítulo, el grupo libre a dos generadores admite una acción (efectiva) sobre el intervalo, la cual induce una acción sobre el círculo con un punto fijo global, y por lo tanto con una medida de probabilidad invariante...

Para probar el teorema de Margulis comenzamos observando que, de acuerdo con el teorema 2.1, existen tres posibilidades según la naturaleza de los conjuntos cerrados invariantes minimales.

En el caso (i) en que $\Gamma$ posee una órbita finita existe evidentemente una medida de probabilidad invariante, a saber, el promedio de las delta de Dirac con masa en los puntos de dicha órbita. Afirmamos a continuación que el caso (iii) en que existe un minimal excepcional se reduce al caso (ii) en que todas las órbitas son densas. En efecto, supongamos que $\Gamma$ actúa sobre el círculo admitiendo un minimal excepcional $\Lambda$. La acción asociada sobre el círculo topológico $\mathrm{S}_{\Lambda}^{1}$ es minimal, es decir, todas sus órbitas son densas. $\mathrm{Si}$ existe una medida invariante para esta acción entonces esta medida no posee átomos y tiene soporte total, por lo que induce una medida invariante sobre $\mathrm{S}^{1}$ de soporte $\Lambda$. Por otra parte, si dos elementos de $\Gamma$ actuando sobre $\mathrm{S}_{\Lambda}^{1}$ generan un grupo libre, estos elementos también generan un grupo libre al actuar en el círculo original.

De esta forma, para probar el teorema de Margulis debemos considerar sólo el caso minimal, es decir, cuando todas las órbitas son densas.

Definición 2.78. Sea $\Gamma$ un grupo actuando sobre $\mathrm{S}^{1}$ por homeomorfismos que preservan orientación.

(i) Decimos que la acción es equicontinua si para todo $\delta>0$ existe $\varepsilon>0$ tal que si $\operatorname{dist}(x, y)<\varepsilon$ entonces $\operatorname{dist}(g(x), g(y))<\delta$ para todo $g \in \Gamma$.

(ii) Decimos que la acción es expansiva si para cada $x \in \mathrm{S}^{1}$ existen un intervalo abierto $I$ conteniendo $x$ y una sucesión $\left(g_{n}\right)$ de elementos de $\Gamma$ tales que la longitud de los intervalos $g_{n}(I)$ converge a cero.

Lema 2.79. Si $\Gamma$ actúa por homeomorfismos del círculo a órbitas densas, entonces la acción es equicontinua o expansiva.

Demostración. Si la acción no es equicontinua entonces existe $\delta>0$ tal que para todo $n \in \mathbb{N}$ existen $x_{n}, y_{n}$ en $\mathrm{S}^{1}$ y $g_{n}$ en $\Gamma$ tales que $\left.\mid\right] x_{n}, y_{n}[\mid$ tiende a cero y $\operatorname{dist}\left(g_{n}\left(x_{n}\right), g_{n}\left(y_{n}\right)\right) \geq \delta$. Pasando a una subsucesión podemos suponer que $g_{n}\left(x_{n}\right)$ tiende a un punto $a \in \mathrm{S}^{1}$ y $g_{n}\left(y_{n}\right)$ tiende a $b \in \mathrm{S}^{1}$. Observe que $\operatorname{dist}(a, b) \geq \delta$. Por la compacidad de $\mathrm{S}^{1}$, y puesto que la acción es minimal, existen $h_{1}, \ldots, h_{k} \in \Gamma$ tales que $\mathrm{S}^{1}=\cup_{i=1}^{k} h_{i}(] a, b[)$. Sea $\varepsilon>0$ el número de Lebesgue de este cubrimiento. Si dist $(x, y)<\varepsilon$ entonces existe $j \in\{1, \ldots, k\}$ tal que $x$ e $y$ pertenecen a $h_{j}(] a, b[)$. Los puntos $g_{n}^{-1} h_{j}^{-1}(x)$ y 
$g_{n}^{-1} h_{j}^{-1}(y)$ pertenecen entonces al intervalo $] x_{n}, y_{n}$ [ para $n$ suficientemente grande, por lo que la distancia entre ellos tiende a cero. Esto prueba que la $\varepsilon$-vecindad de todo punto del círculo es "contraible" a un punto por elementos de $\Gamma$, por lo que la acción es expansiva.

Si $\Gamma$ actúa en $\mathrm{S}^{1}$ de manera equicontinua entonces, de acuerdo con el teorema de Ascoli-Arzelá, el cierre de $\Gamma$ en $\mathrm{Homeo}_{+}\left(\mathrm{S}^{1}\right)$ es compacto. Luego, por la proposición 1.2, existe una medida de probabilidad sobre $\mathrm{S}^{1}$ que es invariante por $\Gamma$ y que permite conjugar $\Gamma$ a un grupo de rotaciones del círculo. De esta forma, para finalizar la prueba del teorema de Margulis, es suficiente demostrar la siguiente proposición.

Proposición 2.80. Si $\Gamma$ es un grupo que actúa por homeomorfismos del círculo de manera minimal y expansiva, entonces $\Gamma$ contiene un subgrupo libre a dos generadores.

La idea de Margulis consiste en probar directamente (una afirmación equivalente a) esta proposición valiéndose del "lema de ping-pong" de Klein. La prueba de Ghys también usa este argumento, pero en ella se efectúa un estudio preliminar del "dominio maximal de contracción" que no sólo permite probar la proposición, sino que también entrega una información más precisa sobre la acción.

Definición 2.81. Si la acción de $\Gamma$ es minimal y expansiva, entonces diremos que ella es fuertemente expansiva si, para todo intervalo abierto $I$ cuyo cierre no es todo el círculo, existe una sucesión de elementos $g_{n} \in \Gamma$ tal que la longitud $\left|g_{n}(I)\right|$ converge a cero.

No toda acción (minimal y) expansiva es fuertemente expansiva: considere por ejemplo los subgrupos de $\operatorname{PSL}_{k}(2, \mathbb{R})$ para $k \geq 2$. En lo que sigue veremos que estos "recubrimientos finitos" son las únicas obstrucciones a la expansividad fuerte para el caso expansivo.

Lema 2.82. Si la acción de un subgrupo $\Gamma$ de $\mathrm{Homeo}_{+}\left(\mathrm{S}^{1}\right)$ es minimal y expansiva, entonces existe un homeomorfismo $R: \mathrm{S}^{1} \rightarrow \mathrm{S}^{1}$ de orden finito y que conmuta con todos los elementos de $\Gamma$, de modo que la acción de $\Gamma$ sobre el círculo cuociente $\mathrm{S}^{1} / \sim$ obtenido como espacio de órbitas de $R$ es fuertemente expansiva.

Demostración. Para cada $x \in \mathrm{S}^{1}$ definamos $R(x) \in \mathrm{S}^{1}$ como siendo el "supremo" de los puntos $y$ para los cuales el intervalo ] $x, y$ [ es "contraible", i.e., existe una sucesión $\left(g_{n}\right)$ de elementos de $\Gamma$ tal que la longitud |] $g_{n}(x), g_{n}(y)[\mid$ converge a cero. El que $R$ conmuta con todos los elementos de $\Gamma$ resulta inmediatamente de la definición. Además, $R$ es una función monótona y "de grado 1". Afirmamos que $R$ es un homeomorfismo de orden finito.

Para verificar que $R$ es estrictamente monótona razonamos por contradicción, y consideramos el conjunto (no vacío) Plan $(R)$ formado por 
la unión de los interiores de los intervalos sobre los cuales $R$ es constante. Como $R$ centraliza a $\Gamma$, este conjunto es invariante bajo la acción. Luego, por minimalidad, Plan $(R)$ es todo el círculo, por lo que $R$ es una aplicación constante, lo cual es absurdo. Para verificar que $R$ es continua se puede usar un argumento análogo utilizando la unión (no vacía) $\operatorname{Salt}(R)$ de los interiores de los intervalos que son evitados por la imagen de $R$ (compare con la prueba del teorema 2.17).

El número de rotación de $R$ no puede ser irracional. En efecto, si lo fuera y $R$ admitiera un minimal excepcional entonces, como $R$ conmuta con $\Gamma$, dicho conjunto sería invariante por $\Gamma$, lo cual contradice la minimalidad de la acción de $\Gamma$. Si por el contrario $\rho(R)$ fuese irracional y las órbitas de $R$ fuesen densas, entonces la única medida de probabilidad invariante por $R$ sería invariante por $\Gamma$; este grupo sería por lo tanto topológicamente conjugado a un grupo de rotaciones (vea el ejercicio 2.23), lo cual contradice la expansividad de la acción.

El homeomorfismo $R$ es de orden finito. En efecto, siendo su número de rotación racional, él admite puntos periódicos. El conjunto $\operatorname{Per}(R)$ de tales puntos es entonces un cerrado no vacío, el cual es invariante por $\Gamma$ (pues $R$ y $\Gamma$ conmutan). De la minimalidad de $\Gamma$ deducimos que $\operatorname{Per}(R)$ coincide con todo el círculo.

Para concluir notemos que, por la definición de $R$, la acción de $\Gamma$ sobre $\mathrm{S}^{1} / \sim$ es fuertemente expansiva.

En el contexto del lema precedente, el orden del homeomorfismo $R$ será llamado el grado de $\Gamma$ y será denotado por $d(\Gamma)$. Observe que $d(\Gamma)=1$ si y sólo si $R$ es la identidad, es decir, si la acción original de $\Gamma$ es fuertemente expansiva.

Volvamos ahora a la proposición 2.80. Como ya adelantamos, la estrategia de la demostración consiste en encontrar dos elementos cuya acción sobre el círculo (módulo un "recubrimiento finito") sea semejante a la acción de dos aplicaciones hiperbólicas de $\operatorname{PSL}(2, \mathbb{R})$ que engendran un grupo de Schottky. Dichas aplicaciones generan un grupo libre, de acuerdo al famoso lema de ping-pong de Klein reproducido a continuación.

Lema 2.83. Sean $\mathrm{M}$ un conjunto y $\Gamma$ un grupo de biyecciones de $\mathrm{M}$. Supongamos que existan dos subconjuntos no vacios y disjuntos $A_{0}$ y $A_{1}$ de $\mathrm{M} y$ dos elementos $g_{0}$ y $g_{1}$ en $\Gamma$ tales que $g_{0}^{n}\left(A_{1}\right) \subset A_{0}$ y $g_{1}^{n}\left(A_{0}\right) \subset A_{1}$ para todo $n \in \mathbb{Z} \backslash\{0\}$. Entonces $g_{0}$ y $g_{1}$ generan un grupo libre.

Demostración. Debemos probar que toda palabra $g=g_{0}^{i_{1}} g_{1}^{j_{1}} \cdots g_{0}^{i_{k}} g_{1}^{j_{k}}$ que es reducida (i.e., tal que los exponentes son no nulos a excepción posible del primero o del último) representa un elemento no trivial de $\Gamma$. Para ello basta observar que si $i_{1}=0$ y $j_{k} \neq 0$ (resp. $i_{1} \neq 0$ y $\left.j_{k}=0\right)$, entonces (el elemento representado por $g$ ) envía $A_{0}$ en $A_{1}$ (resp. $A_{1}$ en $A_{0}$ ), por lo que no puede ser la identidad. Si $i_{1}$ y $j_{k}$ son ambos no nulos entonces 
conjugando convenientemente $g$ se puede obtener una palabra para la cual el argumento precedente es aplicable.

Si $\Gamma$ actúa de manera expansiva sobre el círculo entonces consideramos la acción (fuertemente expansiva) sobre el círculo cuociente $\mathrm{S}^{1} / \sim$. Constatamos así la existencia de dos sucesiones de intervalos $L_{n}$ y $L_{n}^{\prime}$ en $\mathrm{S}^{1} / \sim$ convergiendo respectivamente a dos puntos $a$ y $b$ de modo que para cada $n \in \mathbb{Z}$ existe $g_{n} \in \Gamma$ tal que $f_{n}\left(\mathrm{~S}^{1} \backslash L_{n}\right)=L_{n}^{\prime}$. Podemos suponer que $a \neq b$, pues en caso contrario podemos reemplazar $f_{n}$ por $g f_{n}$ para cierto elemento $g \in \Gamma$ que no fije $a^{\prime}$ (dicho elemento existe por la minimalidad de la acción). Afirmamos que existe $f \in \Gamma$ tal que $a^{\prime}=f(a)$ y $b^{\prime}=f(b)$ son distintos tanto de $a$ como de $b$. Esto puede ser probado sin mayor dificultad (i.e., puede ser considerado como un ejercicio), pero también se desprende directamente del siguiente lema algebraico debido a Newmann.

Lema 2.84. Ningún grupo puede ser escrito como unión finita de clases laterales a izquierda de subgrupos de índice infinito.

Demostración. Supongamos que $\Gamma=S_{1}\left[g_{1}\right] \cup \ldots \cup S_{k}\left[g_{k}\right]$ sea una descomposición de un grupo en clases a izquierda $\left[g_{i}\right]$, donde cada conjunto $S_{i}$ es finito y las clases $\left[g_{i}\right]$ son dos a dos distintas. Probaremos por inducción en $k$ que una de las clases $\left[g_{i}\right]$ tiene índice finito en $\Gamma$.

Si $k=1$ no hay nada que demostrar. Supongamos entonces que la afirmación sea satisfecha para $k \leq n$ y consideremos una descomposición $\Gamma=S_{1}\left[g_{1}\right] \cup \ldots \cup S_{n+1}\left[g_{n+1}\right]$. Si $S_{1}\left[g_{1}\right]=\Gamma$ entonces $\left[g_{1}\right]$ tiene índice finito en $\Gamma$. En caso contrario, existe $g \in \Gamma$ tal que $g\left[g_{1}\right] \cap S_{1}\left[g_{1}\right]=\emptyset$. Tenemos entonces

$$
g\left[g_{1}\right] \subset S_{2}\left[g_{2}\right] \cup \ldots \cup S_{n+1}\left[g_{n+1}\right],
$$

por lo que

$$
S_{1}\left[g_{1}\right] \subset T_{2}\left[g_{2}\right] \cup \ldots \cup T_{n+1}\left[g_{n+1}\right],
$$

donde $T_{i}=S_{1} g^{-1} S_{i}$ es finito. Luego,

$$
\Gamma=\left(T_{2} \cup S_{2}\right)\left[g_{2}\right] \cup \ldots \cup\left(T_{n+1} \cup S_{n+1}\right)\left[g_{n+1}\right] .
$$

Por la hipótesis de inducción, existe $j \in\{2, \ldots, n+1\}$ tal que $\left[g_{j}\right]$ tiene índice finito en $\Gamma$.

Para concluir la demostración de la proposición 2.80, escojamos $n$ suficientemente grande de modo que los intervalos $L_{n}, L_{n}^{\prime}, f\left(L_{n}\right)$ y $f\left(L_{n}^{\prime}\right)$ sean disjuntos, y hagamos $g_{0}=f_{n}$ y $g_{1}=f g_{0} f^{-1}$. Si denotamos por $J_{0}, I_{0}, J_{1} \mathrm{e}$ $I_{1}$ respectivamente la unión de los intervalos en la preimagen por $R$ de $L_{n}$, $L_{n}^{\prime}, f\left(L_{n}\right), f\left(L_{n}^{\prime}\right)$, entonces se verifica fácilmente que para todo $k \in \mathbb{Z} \backslash\{0\}$ se tiene $g_{0}^{k}\left(I_{1} \cup J_{1}\right) \subset I_{0} \cup J_{0}$ y $g_{1}^{k}\left(I_{0} \cup J_{0}\right) \subset I_{1} \cup J_{1}$. Por el lema 2.83, $g_{0}$ y $g_{1}$ generan un grupo libre. 
Ejercicio 2.85. Pruebe la siguiente "versión positiva" del lema de Klein: si $g_{0}$ y $g_{1}$ son dos biyecciones de un conjunto M para el cual existen subconjuntos no vacíos y disjuntos $A_{0}$ y $A_{1}$ tales que $g_{0}^{n}\left(A_{1}\right) \subset A_{0}$ y $g_{1}^{n}\left(A_{0}\right) \subset A_{1}$ para todo entero positivo $n$, entonces $g_{0}$ y $g_{1}$ generan un semigrupo libre a dos generadores (vea la definición 2.50).

\subsection{Una interpretación probabilística}

Sean $\Gamma$ un grupo numerable de homeomorfismos del círculo y $p$ una medida de probabilidad no degenerada sobre $\Gamma$ (es decir, tal que su soporte genere a $\Gamma$ como semigrupo). Una medida de probabilidad $\mu$ sobre el círculo es dicha estacionaria (respecto a $p$ ) si $p * \mu=\mu$, es decir si para toda función continua $\psi: \mathrm{S}^{1} \rightarrow \mathbb{R}$ se tiene

$$
\int_{\mathrm{S}^{1}} \psi(x) d \mu(x)=\int_{\Gamma} \int_{\mathrm{S}^{1}} \psi(g(x)) d \mu(x) d p(g) .
$$

Para entender esta definición, consideremos el operador de difusión definido en el espacio de las funciones continuas por

$$
D \psi(x)=\int_{\Gamma} \psi(g(x)) d p(g) .
$$

Este operador actúa de manera dual sobre el espacio de las medidas de probabilidad, y esta acción dual corresponde precisamente a aquélla dada por $\nu \mapsto p * \nu$. La existencia de (al menos) una medida estacionaria $\mu$ sobre el círculo es una consecuencia directa del teorema de punto fijo de Kakutani [213]; obviamente, ella también puede ser deducida por un argumento simple de medias de Birkhoff (i.e., usando la técnica de Bogolioubov y Krylov; vea el apéndice).

Lema 2.86. Si las órbitas de $\Gamma$ son densas, entonces $\mu$ es de soporte total $y$ sin átomos. Si $\Gamma$ admite un conjunto de Cantor invariante y minimal, entonces este conjunto coincide con el soporte de $\mu, y \mu$ tampoco posee átomos.

Demostración. Verifiquemos primeramente que si $\mu$ posee átomos entonces $\Gamma$ admite órbitas finitas (en relación a este caso vea el ejercicio 2.95). En efecto, si $x$ es un punto de medida (positiva y) maximal, entonces a partir de la igualdad

$$
\mu(p)=\int_{\Gamma} \mu\left(g^{-1}(x)\right) d p(g),
$$

se concluye que $\mu\left(g^{-1}(x)\right)=\mu(x)$ para todo $g$ en el soporte de $p$. Como $p$ es no degenerada, esto sigue siendo verdad para todo elemento $g$ de $\Gamma$. Como la masa total de $\mu$ es finita, la única posibilidad es que la órbita de $x$ por $\Gamma$ sea finita. 
Si la acción de $\Gamma$ es minimal, entonces el soporte de $\mu$ es todo el círculo, pues dicho soporte es un cerrado invariante por la acción. Si $\Gamma$ admite un minimal excepcional $\Lambda$ entonces, como este conjunto es único, debe estar contenido en el soporte de $\mu$. De esta manera, para probar que $\Lambda$ y $\operatorname{sop}(\mu)$ coinciden, debemos verificar que $\mu(I)=0$ para toda componente conexa de $\mathrm{S}^{1} \backslash \Lambda$. Ahora bien, si éste no fuese el caso entonces escogiendo una componente tal $I$ de masa maximal se concluiría (por un argumento análogo a aquél del caso de existencia de átomos) que la órbita de $I$ es finita, lo cual es absurdo, pues las órbitas de las extremidades de $I$ son densas en $\Lambda$.

La existencia de medidas estacionarias permite establecer un resultado no evidente de regularidad. Mencionemos que, de acuerdo a [93], un resultado de este tipo sólo puede ser válido en dimensión 1 (vea sin embargo el ejercicio 2.89).

Proposición 2.87. Todo subgrupo numerable de Homeo $+\left(\mathrm{S}^{1}\right)$ (resp. de $\left.\mathrm{Homeo}_{+}([0,1])\right)$ es topológicamente conjugado a un grupo de homeomorfismos lipschitzianos.

Demostración. Consideremos primeramente el caso de un subgrupo numerable $\Gamma$ de $\mathrm{Homeo}_{+}\left(\mathrm{S}^{1}\right)$ cuyas órbitas son densas. Dotemos dicho grupo de una medida de probabilidad no degenerada y simétrica $p$, y consideremos una medida de probabilidad $\mu$ sobre el círculo que sea estacionaria respecto a $p$. Para cada intervalo $I \subset \mathrm{S}^{1}$ y cada elemento $g \in \operatorname{sop}(p)$ se tiene

$$
\mu(I)=\sum_{h \in \operatorname{sop}(p)} \mu\left(h^{-1}(I)\right) p(g) \geq \mu(g(I)) p\left(g^{-1}\right),
$$

por lo que

$$
\mu(g(I)) \leq \frac{1}{p(g)} \mu(I) .
$$

Tomemos ahora un homeomorfismo $\varphi$ del círculo en sí mismo que envíe $\mu$ sobre la medida de Lebesgue. Si $J$ es un intervalo cualquiera entonces, por (2.10), para todo $g \in \operatorname{sop}(p)$ se tiene

$$
\left|\varphi \circ g \circ \varphi^{-1}(J)\right|=\mu\left(g \circ \varphi^{-1}(J)\right) \leq \frac{1}{p(g)} \mu\left(\varphi^{-1}(J)\right)=\frac{1}{p(g)}|J| .
$$

Luego, para todo $g \in \operatorname{sop}(p)$ la transformación $\varphi \circ g \circ \varphi^{-1}$ es lipschitziana de constante de Lipschitz $1 / p(g)$. Como $p$ es una medida no degenerada, esto demuestra la proposición para el caso minimal.

Si $\Gamma$ es un subgrupo numerable cualquiera de $\mathrm{Homeo}_{+}\left(\mathrm{S}^{1}\right)$, entonces agregando una rotación irracional a un sistema de generadores y considerando el grupo generado, el problema se reduce al caso minimal. Los argumentos dados más arriba muestran que este nuevo grupo (y por lo tanto 
el grupo original $\Gamma$ ) es topológicamente conjugado a un grupo de homeomorfismos lipschitzianos. Finalmente, identificando las extremidades del intervalo $[0,1]$, cada subgrupo $\Gamma$ de $\mathrm{Homeo}_{+}([0,1])$ induce un grupo de homeomorfismos del círculo (con un punto fijo global "etiquetado"). Por lo tanto, si $\Gamma$ es numerable entonces este nuevo grupo es conjugado por un elemento $\varphi$ de $\mathrm{Homeo}_{+}\left(\mathrm{S}^{1}\right)$ a un grupo de homeomorfismos lipschitzianos del círculo. Para obtener una conjugación genuina en $\mathrm{Homeo}_{+}([0,1])$ basta componer $\varphi$ con una rotación de modo que el punto etiquetado del círculo sea enviado sobre sí mismo.

Ejercicio 2.88. Usando el argumento de la demostración precedente pruebe que, para todo $\varepsilon>0$, todo homeomorfismo del círculo o del intervalo es topológicamente conjugado a un homeomorfismo lipschitziano cuya derivada (bien definida en casi todo punto) es menor o igual que $1+\varepsilon$ (el lector debiese también poder demostrar esta afirmación por un método directo, es decir, sin la necesidad de utilizar la medida estacionaria).

Observación. Valiéndose de la desigualdad bien conocida $h_{\text {top }}(T) \leq d \log (\operatorname{Lip}(T))$ (válida para la entropía topológica de aplicaciones lipschitzianas $T$ de variedades compactas de dimensión $d$ ), la afirmación del ejercicio precedente da una demostración directa y conceptual del hecho que la entropía topológica de cualquier homeomorfismo del círculo o del intervalo es nula.

Ejercicio 2.89. Pruebe que todo grupo numerable de homeomorfismos de una variedad compacta es topológicamente conjugado a un grupo de homeomorfismos absolutamente continuos respecto a la medida de Lebesgue.

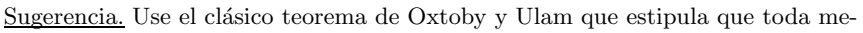
dida de soporte total y sin átomos de una variedad compacta es la imagen de la medida de Lebesgue por un homeomorfismo (vea [81] para una presentación concisa de este resultado). Use también el hecho que sobre toda variedad compacta existen acciones minimales de grupos finitamente generados (vea por ejemplo [65]).

Ejercicio 2.90. Una función continua $\psi: \mathrm{S}^{1} \rightarrow \mathbb{R}$ es dicha armónica si ella es invariante por la difusión, es decir, $D(\psi)=\psi$. Pruebe la siguiente versión del principio del máximo: si $\psi$ es armónica entonces el conjunto de puntos en los que $\psi$ asume su valor máximo es invariante por $\Gamma$. Pruebe que lo mismo vale para funciones supra-armónicas, esto es, para funciones $\psi$ satisfaciendo $D \psi \geq \psi$.

La definición de medida estacionaria se extiende a cualquier acción sobre un espacio métrico compacto $\mathrm{M}$ de un grupo numerable $\Gamma$ dotado de una probabilidad $p$. En tal generalidad designamos por $\Omega$ al espacio de las sucesiones $\left(g_{1}, g_{2}, \ldots\right) \in \Gamma^{\mathbb{N}}$ (dotado de la medida producto $\left.\mathbb{P}=p^{\mathbb{N}}\right)$. Si $\sigma$ es el desplazamiento sobre $\Omega$ (i.e., $\left.\sigma\left(g_{1}, g_{2}, \ldots\right)=\left(g_{2}, g_{3}, \ldots\right)\right)$, entonces se verifica fácilmente que una medida de probabilidad $\mu$ sobre el círculo es estacionaria respecto a $p$ si y sólo si la medida $\mathbb{P} \times \mu$ es invariante por la transformación producto cruzado $T: \Omega \times \mathrm{S}^{1} \rightarrow \Omega \times \mathrm{S}^{1}$ definida por

$$
T(\omega, x)=\left(\sigma(\omega), h_{1}(\omega)(x)\right)=\left(\sigma(\omega), g_{1}(x)\right), \quad \omega=\left(g_{1}, g_{2}, \ldots\right) .
$$


Siguiendo el trabajo seminal [67] de Furstenberg, para estudiar la evolución de las composiciones aleatorias consideramos el proceso inverso dado por $\bar{h}_{n}(\omega)=g_{1} \cdots g_{n}$. La razón de esto radica en la siguiente observación: si $\psi$ es una función continua definida sobre el círculo y $\mu$ es una medida estacionaria, entonces la sucesión de variables aleatorias

$$
\xi_{n}(\omega)=\int_{\mathrm{S}^{1}} \psi d\left(g_{1} \cdots g_{n}(\mu)\right)=\int_{\mathrm{S}^{1}} \psi d\left(\bar{h}_{n}(\omega)(\mu)\right)
$$

es una martingala. En efecto, para todo $g_{1}, \ldots, g_{n}$ en $\Gamma$ la igualdad (2.8) aplicada a la función $x \mapsto \psi\left(g_{1} \cdots g_{n}(x)\right)$ nos da

$$
\int_{\Gamma} \int_{\mathrm{S}^{1}} \psi d\left(g_{1} \cdots g_{n} g(\mu)\right) d p(g)=\int_{\mathrm{S}^{1}} \psi d\left(g_{1} \cdots g_{n}(\mu)\right),
$$

es decir, $\mathbb{E}\left(\xi_{n+1} \mid g_{n+1}\right)=\xi_{n}$. Por el teorema de convergencia de martingalas, la sucesión $\left(\xi_{n}(\omega)\right)$ converge para casi todo $\omega \in \Omega$. Si fijamos una familia numerable de funciones $\psi_{k}$ que sea densa en el espacio de funciones continuas, entonces lo que precede (añadido a la compacidad del espacio de medidas de probabilidad de M) muestra que, para un conjunto de probabilidad total $\Omega_{0}$ de $\Omega$, el límite siguiente (respecto a la topología débil) existe:

$$
\lim _{n \rightarrow \infty} g_{1} g_{2} \cdots g_{n}(\mu)=\lim _{n \rightarrow \infty} \bar{h}_{n}(\omega)(\mu)=\omega(\mu) .
$$

Además, la aplicación (bien definida en casi todo punto) $\omega \mapsto \omega(\mu)$ es medible (para mayores detalles vea la página 199 de [129]).

Proposición 2.91. Sea $\Gamma$ sea un subgrupo numerable de $\mathrm{Homeo}_{+}\left(\mathrm{S}^{1}\right)$ cuyas órbitas son densas. Si la propiedad de expansividad fuerte es satisfecha, entonces para casi toda sucesión $\omega \in \Omega_{0}$ la medida $\omega(\mu)$ es una delta de Dirac.

Demostración. Probaremos que para todo $\varepsilon \in] 0,1]$ existe un subconjunto de probabilidad total $\Omega_{\varepsilon}$ de $\Omega_{0}$ tal que para todo $\omega \in \Omega_{\varepsilon}$ existe un intervalo $I$ de longitud $|I| \leq \varepsilon$ tal que $\omega(\mu)(I) \geq 1-\varepsilon$. Esto permite concluir de manera evidente que, para todo $\omega$ contenido en el conjunto (de probabilidad total) $\Omega^{*}=\cap_{n \in \mathbb{N}} \Omega_{1 / n}$, la medida $\omega(\mu)$ es una delta de Dirac.

Fijemos entonces $\varepsilon>0$. Para cada $n \in \mathbb{N}$ designemos por $\Omega^{n, \varepsilon}$ el conjunto de las sucesiones $\omega \in \Omega_{0}$ tales que, para todo $m \geq 0$ y todo intervalo $I$ de $\mathrm{S}^{1}$ de longitud $|I| \leq \varepsilon$, se tiene $\bar{h}_{n+m}(\omega)(\mu)(I)<1-\varepsilon$. Debemos probar que la probabilidad de $\Omega^{n, \varepsilon}$ es nula. Para ello comenzaremos exhibiendo un subconjunto finito $\mathcal{G}_{\varepsilon}$ de $\operatorname{sop}(p)$, así como un entero $l \in \mathbb{N}$, tales que para todo $r \in \mathbb{N}$ y todo $\left(g_{1}, \cdots, g_{r}\right) \in \Gamma^{r}$ existen un intervalo $I$ de longitud $|I| \leq \varepsilon$, un entero $\ell \leq 2 l$, y elementos $f_{1}, \ldots, f_{\ell}$ de $\mathcal{G}_{\varepsilon}$ satisfaciendo

$$
g_{1} \cdots g_{r} f_{1} \cdots f_{\ell}(\mu)(I) \geq 1-\varepsilon .
$$


Fijemos dos puntos distintos $a$ y $b$ del círculo, así como un entero $q>1 / \varepsilon$, y tomemos $q$ puntos diferentes $a_{1}, \ldots, a_{q}$ de la órbita de $a$ por $\Gamma$. Para cada $i \in\{1, \ldots, q\}$ fijemos un elemento $h_{i} \in \Gamma$ y un intervalo abierto $U_{i}$ conteniendo $a_{i}$ de modo que los $U_{i}$ sean dos a dos disjuntos, $h_{i}(a)=a_{i}$ y $h_{i}(U)=U_{i}$ para alguna vecindad $U$ de $a$ que no contenga a $b$. Consideremos ahora una vecindad $V$ de $b$ disjunta de $U$ y tal que $\mu\left(\mathrm{S}^{1} \backslash V\right) \geq 1-\varepsilon$. Por la minimalidad de la acción y la propiedad de expansividad fuerte, existe $h \in \Gamma$ tal que $h\left(\mathrm{~S}^{1} \backslash V\right) \subset U$. Cada elemento de $\left\{h_{1}, \ldots, h_{q}, h\right\}$ puede ser escrito como un producto de elementos del soporte de $\mu$. Esto puede ser realizado de diversas maneras, pero si fijamos de una vez por todas una escritura tal para $h$ y cada $h_{i}$, entonces el conjunto $\mathcal{G}_{\varepsilon}$ de los elementos de $\operatorname{sop}(p)$ que son utilizados es finito. Sea $l$ el número maximal de elementos que aparecen en una de las escrituras precedentes. Para verificar (2.11), notemos que para $g=g_{1} \cdots g_{r}$ los intervalos $g\left(U_{i}\right)$ son dos a dos disjuntos, por lo que la longitud de al menos uno de ellos está mayorada por $\varepsilon$. Si fijamos un intervalo tal $I=g\left(U_{i}\right)$ entonces obtenemos

$$
g_{1} \cdots g_{r} h_{i} h(\mu)(I)=\mu\left(h^{-1}(U)\right) \geq \mu\left(\mathrm{S}^{1} \backslash V\right) \geq 1-\varepsilon,
$$

lo cual concluye la verificación de (2.11).

Hagamos $\rho=\min \left\{p(f): f \in \mathcal{G}_{\varepsilon}\right\}$ y definamos $\Omega_{n, m}^{\varepsilon}$ como el conjunto de $\operatorname{los} \omega \in \Omega_{0}$ tales que para todo intervalo $I$ de longitud $|I| \leq \varepsilon$ y todo $k \leq m$ se tiene $\bar{h}_{n+k}(\omega)(\mu)(I)<1-\varepsilon$. Debido a $(2.11)$ tenemos

$$
\mathbb{P}\left(\Omega_{n, 2 l t}^{\varepsilon}\right) \leq\left(1-\rho^{2 l}\right)^{t} .
$$

Por lo tanto, pasando al límite cuando $t$ tiende al infinito concluimos que $\mathbb{P}\left(\Omega^{n, \varepsilon}\right)=0$, lo cual permite acabar la demostración.

Valiéndose de un argumento bien conocido, la proposición 2.91 permite probar la unicidad de la medida estacionaria. Señalemos en todo caso que, de acuerdo a [53], este resultado es válido en el contexto muchísimo más general de las foliaciones de codimensión 1 (la noción de medida estacionaria en ese contexto es aquélla de Garnett; vea [38, 69]).

Teorema 2.92. Sea $\Gamma$ un grupo numerable de homeomorfismos del círculo dotado de una medida de probabilidad no degenerada $p$. Si $\Gamma$ no preserva ninguna medida de probabilidad del círculo, entonces la medida estacionaria (respecto a p) es única.

Demostración. Supongamos primeramente que la acción de $\Gamma$ sea minimal y satisfaga la propiedad de expansividad fuerte, y fijemos una medida de probabilidad $\mu$ sobre $\mathrm{S}^{1}$ que sea estacionaria respecto a $p$. Para cada $\omega \in \Omega$ tal que el límite $\lim _{n \rightarrow \infty} \bar{h}_{n}(\omega)(\mu)$ existe y es una medida de Dirac, designemos por $\varsigma_{\mu}(\omega)$ el átomo de la medida $\omega(\mu)$, i.e., el punto de $\mathrm{S}^{1}$ tal que $\omega(\mu)=\delta_{\varsigma_{\mu}(\omega)}$. La aplicación $\varsigma_{\mu}: \Omega \rightarrow \mathrm{S}^{1}$ queda así definida en casi 
todo punto y es medible. Afirmamos que las medidas $\mu$ y $\varsigma_{\mu}(\mathbb{P})$ coinciden. En efecto, por la estacionaridad de $\mu$,

$$
\mu=p^{* n} * \mu=\sum_{g \in \Gamma} p^{* n}(g) g(\mu)=\int_{\Omega} \bar{h}_{n}(\omega)(\mu) d \mathbb{P}(\omega) .
$$

Por lo tanto, pasando al límite cuando $n$ tiende al infinito (lo cual es posible gracias al teorema de convergencia dominada),

$$
\mu=\int_{\Omega} \lim _{n \rightarrow \infty} h_{n}(\omega)(\mu) d \mathbb{P}(\omega)=\int_{\Omega} \delta_{\varsigma_{\mu}(\omega)} d \mathbb{P}(\omega),
$$

es decir, $\mu=\varsigma_{\mu}(\mathbb{P})$.

Consideremos ahora dos probabilidades estacionarias $\mu_{1}$ y $\mu_{2}$. La medida de probabilidad $\mu=\left(\mu_{1}+\mu_{2}\right) / 2$ también es estacionaria, y la función $\varsigma_{\mu}$ verifica, para $\mathbb{P}$-casi todo $\omega \in \Omega$,

$$
\frac{\delta_{\varsigma_{\mu_{1}}(\omega)}+\delta_{\varsigma_{\mu_{2}}(\omega)}}{2}=\delta_{\varsigma_{\mu}(\omega)}
$$

Evidentemente, esto no es posible a menos que $\varsigma_{\mu_{1}}$ y $\varsigma_{\mu_{2}}$ coincidan casi ciertamente. Así, por la afirmación de la primera parte de la prueba,

$$
\mu_{1}=\varsigma_{\mu_{1}}(\mathbb{P})=\varsigma_{\mu_{2}}(\mathbb{P})=\mu_{2} .
$$

Supongamos ahora que $\Gamma$ actúe de manera minimal y expansiva (pero no fuertemente expansiva), y fijemos una probabilidad $\mu$ que sea estacionaria (respecto a $p$ ). De acuerdo con el lema 2.82, existe un homeomorfismo $R: \mathrm{S}^{1} \rightarrow \mathrm{S}^{1}$ de orden finito que conmuta con todos los elementos de $\Gamma$ y tal que la acción inducida sobre el círculo topológico $\mathrm{S}^{1} / \sim$ obtenido como espacio de órbitas de $R$ es (minimal y) fuertemente expansiva. Para cada $x \in \mathrm{S}^{1}$ notemos $\psi(x)=\mu([x, R(x)[)$. Como $\mu$ no posee átomos, la función $\psi$ es continua, y puesto que $R$ conmuta con todos los elementos de $\Gamma$, ella es armónica. En consecuencia, el conjunto de los puntos en que $\psi$ asume su valor máximo es invariante por $\Gamma$ (vea el ejercicio 2.90). Como las órbitas de $\Gamma$ son densas, $\psi$ es constante; en otras palabras, $\mu$ es invariante por $R$. Por otra parte, $\mu$ se proyecta sobre una medida de probabilidad estacionaria para la acción de $\Gamma$ sobre $\mathrm{S}^{1} / \sim$. Por la primera parte de la demostración, dicha medida es única, lo cual prueba la unicidad de $\mu$.

Si $\Gamma$ admite un conjunto de Cantor invariante y minimal, entonces dicho conjunto coincide con el soporte de $\mu$. Colapsando las componentes conexas del complemento de este conjunto se obtiene una acción minimal; si esta acción es expansiva, entonces los argumentos precedentes muestran la unicidad de la medida estacionaria. Para completar la demostración basta constatar que, en todos los casos que aún no han sido considerados, $\Gamma$ deja invariante una medida de probabilidad del círculo. 
Definamos el coeficiente de contracción contr $(h)$ de un homeomorfismo $h$ del círculo como el ínfimo de $\operatorname{los} \varepsilon>0$ tales que existen dos intervalos cerrados $I$ y $J$ del círculo de longitud a lo más $\varepsilon$ y tales que $h\left(\overline{\mathrm{S}^{1} \backslash I}\right)=J$. Esta definición permite dar una "versión topológica" de la proposición 2.91 (para las composiciones en el "orden natural").

Proposición 2.93. Bajo las hipótesis de la proposición 2.91, para casi todo $\omega=\left(g_{1}, g_{2}, \ldots\right) \in \Omega$ el coeficiente de contracción de $h_{n}(\omega)=g_{n} \cdots g_{1}$ converge a cero cuando $n$ tiende al infinito.

Demostración. Como $\mu$ no posee átomos y su soporte es total, existe un homeomorfismo $\varphi$ de $\mathrm{S}^{1}$ que envía $\mu$ sobre la medida de Lebesgue. Por lo tanto, dado que la afirmación a demostrar es invariante por conjugación topológica, podemos suponer que $\mu$ coincide con la medida de Lebesgue.

Denotemos por $\bar{p}$ la probabilidad sobre $\Gamma$ definida por $\bar{p}(g)=p\left(g^{-1}\right), \mathrm{y}$ notemos $\bar{\Omega}$ el espacio de probabilidad $\Gamma^{\mathbb{N}}$ dotado de la medida $\bar{p}^{\mathbb{N}}$. Sobre este espacio consideremos el proceso $\bar{h}_{n}(\bar{\omega})=g_{1} \cdots g_{n}$, donde $\bar{\omega}=\left(g_{1}, g_{2}, \ldots\right)$. Por la proposición 2.91, para casi todo $\bar{\omega} \in \Omega$ y todo $\varepsilon>0$ existe un entero positivo $n(\varepsilon, \bar{\omega})$ tal que si $n \geq n(\varepsilon, \bar{\omega})$ entonces existe un intervalo (cerrado) $I$ tal que $\mu(I) \leq \varepsilon$ y $\bar{h}_{n}(\bar{\omega})(\mu)(I) \geq 1-\varepsilon$. Si designamos por $J$ la cerradura de $\mathrm{S}^{1} \backslash g_{n}^{-1} \cdots g_{1}^{-1}(I)$, entonces se comprueba fácilmente que $|I|=\mu(I) \leq \varepsilon$,

$$
|J|=1-\left|g_{n}^{-1} \cdots g_{1}^{-1}(I)\right|=1-\mu\left(\bar{h}_{n}(\bar{\omega})^{-1}(I)\right)=1-\bar{h}_{n}(\bar{\omega})(\mu)(I) \leq \varepsilon
$$

y $g_{n}^{-1} \cdots g_{1}^{-1}\left(\overline{\mathrm{S}^{1} \backslash I}\right)=J$. En consecuencia, $\operatorname{contr}\left(g_{n}^{-1} \cdots g_{1}^{-1}\right) \leq \varepsilon$ para todo $n \geq n(\varepsilon, \bar{\omega})$. La demostración es completada entonces observando que la transformación $\left(g_{1}, g_{2}, \ldots\right) \mapsto\left(g_{1}^{-1}, g_{2}^{-1}, \ldots\right)$ identifica los espacios $\left(\bar{\Omega}, \bar{p}^{\mathbb{N}}\right)$ y $\left(\Omega, p^{\mathbb{N}}\right)$.

Remarquemos que el coeficiente de contracción siempre es realizado, en el sentido que para todo homeomorfismo $h$ del círculo existen intervalos cerrados $I$ y $J$ tales que $\max \{|I|,|J|\}=\operatorname{contr}(h)$ y $h\left(\overline{\mathrm{S}^{1} \backslash I}\right)=J$ (estos intervalos no son necesariamente únicos). En consecuencia, para casi todo $\omega \in \Omega$ podemos elegir dos sucesiones de intervalos cerrados $I_{n}(\omega)$ y $J_{n}(\omega)$ cuya longitud tiende a cero y tales que $h_{n}(\omega)\left(\overline{\mathrm{S}^{1} \backslash I_{n}(\omega)}\right)=J_{n}(\omega)$ para todo $n \in \mathbb{N}$. Para cualquiera de estas elecciones, los intervalos $I_{n}(\omega)$ convergen hacia el punto $\varsigma_{\mu}(\omega)$.

Ejercicio 2.94. Los resultados de esta sección fueron obtenidos por el autor en colaboración con Deroin y Kleptsyn en [54] (resultados parciales aparecen en [113]). Debemos señalar sin embargo la existencia de una referencia bastante anterior respecto a estos temas: se trata del trabajo [3] de Antonov, en el cual aparecen resultados "casi equivalentes" expresados en un lenguaje puramente probabilístico. Los ítemes a continuación corresponden a las etapas más importantes del trabajo de Antonov. 
(i) Fijada una medida de probabilidad $p$ sobre un sistema de generadores (no necesariamente finito) de un subgrupo $\Gamma$ de $\mathrm{Homeo}_{+}\left(\mathrm{S}^{1}\right)$ sin órbita finita, considere la probabilidad $\bar{p}$ dada por $\bar{p}(g)=p\left(g^{-1}\right)$ (compare con la proposición 2.93). Si $\bar{\mu}$ es una medida estacionaria sobre $\mathrm{S}^{1}$ (respecto a $\bar{p}$ ), verifique que para todo $x, y$ en $\mathrm{S}^{1}$ la sucesión de variables aleatorias

$$
\xi_{n}^{x, y}(\omega)=\bar{\mu}\left(\left[g_{n} \cdots g_{1}(x), g_{n} \cdots g_{1}(y)\right]\right)
$$

es una martingala. Esto implica en particular que esta sucesión converge casi ciertamente; lo que queremos probar es que el límite correspondiente es igual a 0 ó 1 (al menos en el caso que consideramos a seguir, a saber, cuando no existe ningún homeomorfismo no trivial del círculo que conmuta con todos los elementos de $\Gamma$ ).

(ii) Sea $\nu$ una medida estacionaria para la acción diagonal de $\Gamma$ sobre el toro $\mathrm{S}^{1} \times \mathrm{S}^{1}$. Verifique que la distribución (respecto a $\mathbb{P} \times \nu$ ) de $\xi_{n}^{x, y}$ coincide con la de $\xi_{n+1}^{x, y}$.

(iii) Utilizando la relación (válida para cualquier martingala de cuadrado integrable)

$$
\mathbb{E}\left(\xi_{n+1}^{2}\right)=\mathbb{E}\left(\xi_{n}^{2}\right)+\mathbb{E}\left(\left(\xi_{n+1}-\xi_{n}\right)^{2}\right),
$$

concluya que para $\nu$-casi todo punto $(x, y) \in \mathrm{S}^{1} \times \mathrm{S}^{1}$ se tiene $\xi_{n+1}^{x, y}=\xi_{n}^{x, y}$.

(iv) Definiendo $\psi((x, y))=\bar{\mu}([x, y])$ deduzca de (iii) que para $\nu$-casi todo punto $(x, y) \in \mathrm{S}^{1} \times \mathrm{S}^{1}$ y todo $g \in \Gamma$ se cumple $\psi((x, y))=\psi((g(x), g(y)))$.

(v) A partir de (iv) pruebe que el soporte de la medida $\nu$ está contenido en la diagonal.

Sugerencia. Sin pérdida de generalidad, considere el caso en que las órbitas de $\Gamma$ en $\mathrm{S}^{1}$ son densas. Suponga que $\left.\alpha \in\right] 0,1[$ es tal que el conjunto

$$
X_{\alpha, \varepsilon}=\left\{(x, y) \in \mathrm{S}^{1} \times \mathrm{S}^{1}: \psi((x, y)) \in[\alpha-\varepsilon, \alpha+\varepsilon]\right\}
$$

tiene $\nu$-medida positiva para todo $\varepsilon>0$. Concluya a partir de (iv) que la restricción normalizada de $\nu$ a $X_{\alpha, \varepsilon}$ es una medida estacionaria. Haciendo tender $\varepsilon$ a 0 y pasando a un límite débil, obtenga una medida estacionaria $\bar{\nu}$ sobre el toro concentrada en el conjunto

$$
\left\{(x, y) \in \mathrm{S}^{1} \times \mathrm{S}^{1}: \psi([x, y])=\alpha\right\} .
$$

La densidad de las órbitas permite entonces definir de manera única un homeomorfismo $R$ de $\mathrm{S}^{1}$ satisfaciendo $\psi((x, R(x)))=\alpha$. Compruebe que $R$ conmuta con todos los elementos de $\Gamma$, contradiciendo la hipótesis hecha al final del ítem (i).

(vi) Fijados $x, y$ en $\mathrm{S}^{1}$ considere la delta de Dirac $\delta_{(x, y)}$ concentrada en el punto $(x, y) \in \mathrm{S}^{1} \times \mathrm{S}^{1}$. Teniendo en cuenta que todo valor de adherencia de la sucesión de medidas

$$
\nu_{n}=\frac{1}{n} \sum_{j=0}^{n-1} p^{* n} * \delta_{(x, y)}
$$

es una medida estacionaria (la cual debe concentrarse en la diagonal según el ítem (v)), y considerando que $\xi_{n}^{x, y}$ converge casi ciertamente, concluya que el límite de $\xi_{n}^{x, y}$ es igual a 0 ó 1 . 
Ejercicio 2.95. Pruebe que si $\Gamma$ es un subgrupo de $\mathrm{Homeo}_{+}([0,1])$ sin punto fijo global en el interior y $p$ es una medida de probabilidad simétrica y no degenerada sobre $\Gamma$, entonces toda medida de probabilidad sobre $[0,1]$ que es estacionaria respecto a $p$ está soportada en los extremos de $[0,1]$ (señalemos que la hipótesis de simetría de $p$ es esencial para la validez de esta afirmación, como lo muestra por ejemplo [107]).

Sugerencia. Sea $\mu$ una medida estacionaria soportada en $] 0,1[$. Verifique primeramente que $\mu$ no posee átomos. Luego, colapsando las componentes conexas del complemento de su soporte y reparametrizando el intervalo, reduzca el caso general a aquél en que $\mu$ es la medida de Lebesgue. La estacionaridad nos da entonces, para todo $s \in] 0,1[$,

$$
s=\mu([0, s])=\int_{\Gamma} \mu\left(g^{-1}([0, s])\right) d p(g)=\int_{\Gamma} \frac{\mu(g([0, s]))+\mu\left(g^{-1}([0, s])\right)}{2} d p(g),
$$

por lo que

$$
s=\int_{\Gamma} \frac{g(s)+g^{-1}(s)}{2} d p(g) .
$$

Así, integrando entre 0 y un punto arbitrario $t \in] 0,1[$,

$$
t^{2}=\int_{\Gamma} \int_{0}^{t}\left(g(s)+g^{-1}(s)\right) d s d p(g)
$$

Observando la figura 14 concluya que para todo homeomorfismo $f$ del intervalo y todo $t \in[0,1]$ se tiene

$$
\int_{0}^{t}\left(f(s)+f^{-1}(s)\right) d s \geq t^{2},
$$

con la igualdad si y sólo si $f(t)=t$. Concluya que $t$ es un punto fijo global de la acción, contradiciendo la hipótesis (para una demostración alternativa usando el "teorema ergódico de Garnett" vea [54]).

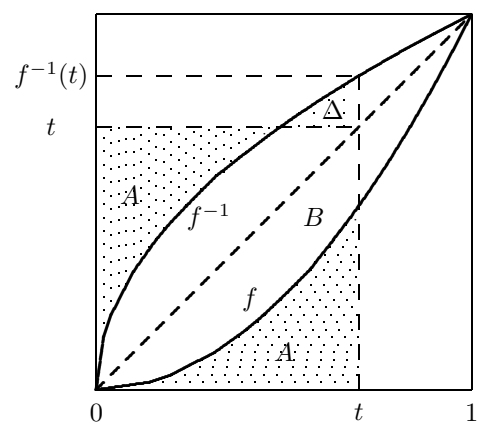

$$
\begin{gathered}
A=\int_{0}^{t} f(s) d s \\
B=\int_{0}^{t} f^{-1}(s) d s \\
\Downarrow \\
A+B=t^{2}+\Delta
\end{gathered}
$$

Figura 14 


\section{Capítulo 3}

\section{Dinámica de Grupos de Difeomorfismos de Clase $\mathrm{C}^{2}$}

\section{El teorema de Denjoy}

En la sección 2.1 del capítulo 2 vimos un ejemplo de un homeomorfismo de $\mathrm{S}^{1}$ de número de rotación irracional que admite un minimal excepcional. En esta sección veremos que dicho fenómeno no puede producirse para difeomorfismos de cierta regularidad, de acuerdo a un clásico resultado debido a Denjoy. Para enunciarlo denotaremos por Difeo ${ }_{+}^{1+v a}\left(\mathrm{~S}^{1}\right)$ al grupo de los difeomorfismos de clase $\mathrm{C}^{1}$ de $\mathrm{S}^{1}$ cuya derivada posee variación acotada. Diremos que un tal difeomorfismo es de clase $\mathrm{C}^{1+\mathrm{va}}$, y denotaremos $V(f)$ la variación total del logaritmo de su derivada, es decir,

$$
V(f)=\sup _{a_{0}<\ldots<a_{n}=a_{0}} \sum_{i=0}^{n-1}\left|\log \left(f^{\prime}\right)\left(a_{i+1}\right)-\log \left(f^{\prime}\right)\left(a_{i}\right)\right|=\operatorname{var}\left(\log \left(f^{\prime}\right) ; \mathrm{S}^{1}\right) .
$$

Si $I$ es un intervalo utilizaremos la notación $V(f ; I)=\operatorname{var}\left(\left.\log \left(f^{\prime}\right)\right|_{I}\right)$.

Teorema 3.1. Si $f$ es un difeomorfismo de clase $\mathrm{C}^{1+\mathrm{va}}$ del círculo con número de rotación irracional, entonces $f$ es topológicamente conjugado a la rotación de ángulo $\rho(f)$.

Todo difeomorfismo de clase $\mathrm{C}^{2}$ o $\mathrm{C}^{1+\text { lip }}$ de $\mathrm{S}^{1}$ pertenece a Difeo ${ }_{+}^{1+\mathrm{va}}\left(\mathrm{S}^{1}\right)$. Por ejemplo, para todo $f \in \operatorname{Difeo}_{+}^{2}\left(\mathrm{~S}^{1}\right)$ se tiene

$$
V(f)=\int_{\mathrm{S}^{1}}\left|\frac{f^{\prime \prime}(s)}{f^{\prime}(s)}\right| d s .
$$


De lo anterior se deduce que la conclusión del teorema de Denjoy es válida para difeomorfismos de clase $\mathrm{C}^{1+\text { lip }}$. Señalemos sin embargo que esto no es válido en clase $\mathrm{C}^{1+\tau}$ para ningún $\tau<1$, de acuerdo con el siguiente resultado debido a Herman [94] y que nosotros reproduciremos en la sección 1.4 del capítulo 4.

Teorema 3.2. Para todo ángulo irracional $\theta$, todo $\tau<1$ y toda vecindad de la rotación $R_{\theta}$ en Difeo $_{+}^{1+\tau}\left(\mathrm{S}^{1}\right)$, existe un elemento en dicha vecindad con número de rotación $\theta$ que no es topológicamente conjugado a $R_{\theta}$.

Antes de pasar a la demostración del teorema 3.1 quisiéramos dar una "prueba heurística" para un caso particular pero muy ilustrativo. Supongamos que $f$ sea un difeomorfismo de clase $\mathrm{C}^{1}$ del círculo de número de rotación irracional para el cual existe un conjunto de Cantor invariante y minimal $\Lambda$, de modo que la derivada de $f$ sobre $\Lambda$ sea constante e igual a 1. Siguiendo [92] probaremos por contradicción que, en tal caso, $f$ no puede ser de clase $\mathrm{C}^{1+\text { lip }}$ (compare con [155]; vea también 3.4). Para ello, fijemos una componente conexa $I$ del complemento de $\Lambda$, y para cada $n \in \mathbb{N}$ denotemos $I_{n}=g^{n}(I)$. Observe que $\left|I_{n+1}\right|=g^{\prime}(p)\left|I_{n}\right|$ para cierto $p \in I_{n}$, y como la derivada de $g$ en los extremos de $I_{n}$ es igual a 1 concluimos que

$$
\left|\frac{\left|I_{n+1}\right|}{\left|I_{n}\right|}-1\right|=\left|f^{\prime}(p)-1\right| \leq C\left|I_{n}\right|,
$$

donde $C$ es la constante de Lipschitz de la derivada de $f$. Tenemos entonces

$$
\left|I_{n+1}\right| \geq\left|I_{n}\right|\left(1-C\left|I_{n}\right|\right) .
$$

Sin pérdida de generalidad, podemos suponer que las longitudes de $I$ y de todos sus iterados (positivos) por $f$ son inferiores o iguales a $1 / 2 C$. La contradicción buscada resulta entonces del siguiente lema elemental haciendo $d=1$ (el caso $d>1$ aparecerá de manera natural en la sección 1.4 del capítulo 4).

Lema 3.3. Si $d \in \mathbb{N} y\left(\ell_{n}\right)$ es una sucesión de números reales positivos tal que $\ell_{n} \leq 1 / C^{d}(1+1 / d)^{d}$ y $\ell_{n+1} \geq \ell_{n}\left(1-C \ell_{n}^{1 / d}\right)$ para todo $n \in \mathbb{N}$ y cierta constante $C>0$, entonces existe una constante $A>0$ tal que $\ell_{n} \geq A / n^{d}$ para todo $n \in \mathbb{N}$. En particular, si $d=1$ entonces $S=\sum \ell_{n}$ diverge.

Demostración. La función $s \mapsto s\left(1-C s^{1 / d}\right)$ es creciente sobre el intervalo $\left[0,\left(\frac{1}{C(1+1 / d)}\right)^{d}\right]$. Usando este hecho la afirmación del lema se prueba fácilmente por inducción para la constante $A=\min \left\{\ell_{1}, d^{d} / 2^{d^{2}} C^{d}\right\}$. Dejamos la verificación de los detalles a cargo del lector.

Presentaremos dos demostraciones diferentes del teorema de Denjoy (claro está, ambas usarán de manera esencial las propiedades combinatorias de un homeomorfismo de número de rotación irracional). La primera prueba, 
debida al propio Denjoy, consiste en controlar la distorsión que produce el difeomorfismo respecto a la estructura afín de los intervalos del círculo. Como veremos más adelante, ésta es la idea esencial de la prueba de otros resultados de dinámica unidimensional de acciones de grupos, como por ejemplo los teoremas de Sacksteder y Duminy. En la segunda demostración estudiaremos la distorsión respecto a la estructura proyectiva del círculo. Esta idea es más novedosa y se ha revelado muy fructífera en los últimos años. Gracias a ella, Yoccoz extendió en [212] el teorema de Denjoy para homeomorfismos de clase $\mathrm{C}^{\infty}$ cuyos puntos críticos no son infinitamente planos, mientras que $\mathrm{Hu}$ y Sullivan obtuvieron en [97] resultados bastante finos en relación a la clase de diferenciabilidad "optimal" del teorema de Denjoy para difeomorfimos. Nosotros utilizaremos una variación de esta idea para obtener un teorema general de rigidez en la sección 2 del último capítulo.

Primera demostración del teorema 3.1. Debemos probar que si (el grupo generado por) $f$ admite un minimal excepcional $\Lambda$, entonces $f$ no puede ser de clase $\mathrm{C}^{1+\mathrm{va}}$. Supongamos lo contrario y fijemos una componente conexa $I$ de $\mathrm{S}^{1} \backslash \Lambda$. Sea $x_{0}$ un punto del interior de $I$ y sea $\varphi$ la semiconjugación de $f$ a $R_{\theta}$, con $\theta=\rho(f)$. Sin pérdida de generalidad, podemos suponer que $\varphi\left(x_{0}\right)=0$. Para $n \in \mathbb{N}$ consideremos los intervalos $I_{n}$ y $J_{n}$ de la construcción dada al final de la sección 2.1 del capítulo 2. Definamos $I_{n}(f)=\varphi^{-1}\left(I_{n}\right)$ y $J_{n}(f)=\varphi^{-1}\left(J_{n}\right)$. Observe que $I$ está contenido en cada $I_{n}(f)$. De las propiedades de $I_{n}$ y $J_{n}$ se deduce que:

(i) los intervalos en $\left\{f^{j}\left(J_{n}(f)\right): j \in\left\{0,1, \ldots, q_{n+1}-1\right\}\right\}$ recubren $\mathrm{S}^{1}, \mathrm{y}$ cada punto de $\mathrm{S}^{1}$ está en a lo más dos de ellos;

(ii) el intervalo $f^{q_{n+1}}\left(I_{n}(f)\right)$ está contenido en $J_{n}(f)$ para todo $n \in \mathbb{N}$; lo mismo es válido para $f^{-q_{n+1}}\left(I_{-n}(f)\right)$.

Afirmamos que para cada $x, y$ en $I$ y cada $n \in \mathbb{N}$ se tiene

$$
\left|\left(f^{q_{n+1}}\right)^{\prime}(x)\left(f^{-q_{n+1}}\right)^{\prime}(y)\right| \geq e^{-2 V(f)} .
$$

Para probar esta desigualdad, denotamos $\bar{y}=f^{-q_{n+1}}(y)$. Puesto que $I$ está contenido en $I_{-n}(f)$, por la propiedad (ii) tenemos $\bar{y} \in J_{n}(f)$. A partir de (1.1) y de la propiedad (i) obtenemos

$$
\begin{aligned}
\left|\log \left(\left(f^{q_{n+1}}\right)^{\prime}(x)\left(f^{-q_{n+1}}\right)^{\prime}(y)\right)\right| & =\left|\log \left(\frac{\left(f^{q_{n+1}}\right)^{\prime}(x)}{\left(f^{q_{n+1}}\right)^{\prime}(\bar{y})}\right)\right| \\
& \leq \sum_{k=0}^{q_{n+1}^{-1}}\left|\log \left(f^{\prime}\right)\left(f^{k}(x)\right)-\log \left(f^{\prime}\right)\left(f^{k}(\bar{y})\right)\right| \\
& \leq \sum_{k=0}^{q_{n+1}-1} V\left(f ; f^{k}\left(J_{n}(f)\right)\right) \\
& \leq 2 V(f),
\end{aligned}
$$

de donde se concluye fácilmente la desigualdad (3.3). 
Para finalizar, notemos que el valor de $\left|f^{q_{n+1}}(I)\right| \cdot\left|f^{-q_{n+1}}(I)\right|$ es igual a $\left(f^{q_{n+1}}\right)^{\prime}(x)\left(f^{-q_{n+1}}\right)^{\prime}(y) \cdot|I|^{2}$ para ciertos $x, y$ en $I$. De la desigualdad (3.3) se deduce que esta última expresión está inferiormente acotada por $\exp (-2 V(f)) \cdot|I|^{2}$. Sin embargo, esto es absurdo, pues los intervalos $f^{k}(I)$ son dos a dos disjuntos.

Para la segunda demostración utilizaremos la siguiente notación: dados un intervalo $I=[a, b]$ y un difeomorfismo $f$ definido sobre $I$, definimos (compare con (1.4))

$$
M(f ; I)=\frac{|f(b)-f(a)|}{|b-a| \sqrt{f^{\prime}(a) f^{\prime}(b)}} .
$$

Dejamos al lector la tarea de probar la relación (compare con (1.3))

$$
M(f \circ g ; I)=M(g ; I) \cdot M(f ; g(I)) .
$$

Observe por otra parte que de la existencia de un punto $c \in I$ tal que $|f(b)-f(a)|=f^{\prime}(c)|b-a|$ se concluye inmediatamente que

$|\log M(f ; I)| \leq \frac{1}{2}\left[\left|\log \left(f^{\prime}(c)\right)-\log \left(f^{\prime}(a)\right)\right|+\left|\log \left(f^{\prime}(c)\right)-\log \left(f^{\prime}(b)\right)\right|\right] \leq \frac{V(f ; I)}{2}$.

Segunda demostración del teorema 3.1. Supongamos nuevamente que $f$ admita un minimal excepcional $\Lambda$ y retomemos las notaciones introducidas al comienzo del la primera demostración. Para cada $n \in \mathbb{N}$ fijemos dos puntos $a \in f^{-\left(q_{n}+q_{n+1}\right)}(I)$ y $b \in f^{-q_{n+1}}(I)$ tales que

$$
\left(f^{q_{n+1}}\right)^{\prime}(a)=\frac{\left|f^{-q_{n}}(I)\right|}{\left|f^{-\left(q_{n}+q_{n+1}\right)}(I)\right|} \quad \text { y } \quad\left(f^{q_{n+1}}\right)^{\prime}(b)=\frac{|I|}{\mid f^{-q_{n+1}(I) \mid}} .
$$

El intervalo abierto $J$ de puntos extremos $a$ y $b$ está contenido en $J_{n}(f)$ (recuerde las propiedades combinatorias estudiadas al final de la sección 2.1 del capítulo 2 ; vea en particular las figuras 11 y 12). Por (3.4), (3.5) y la propiedad (i) usada en la demostración anterior,

$$
\left|\log M\left(f^{q_{n+1}} ; J\right)\right| \leq \sum_{k=0}^{q_{n+1}-1}\left|\log M\left(f, f^{k}(J)\right)\right| \leq \sum_{k=0}^{q_{n+1}-1} \frac{W\left(f ; f^{k}(J)\right)}{2} \leq V(f) .
$$

Obtenemos entonces

$$
\left(\frac{\left|f^{q_{n+1}}(J)\right|}{|J|}\right)^{2} \frac{1}{\left(f^{q_{n+1}}\right)^{\prime}(a)\left(f^{q_{n+1}}\right)^{\prime}(b)} \geq \exp (-2 V(f)),
$$

es decir,

$$
\left(\frac{\left|f^{q_{n+1}}(J)\right|}{|J|}\right)^{2} \frac{\left|f^{-\left(q_{n}+q_{n+1}\right)}(I)\right|}{\left|f^{-q_{n}}(I)\right|} \frac{\left|f^{-q_{n+1}}(I)\right|}{|I|} \geq \exp (-2 V(f)) .
$$


Como $\left|f^{q_{n+1}}(J)\right| \leq 1$ para todo $n \in \mathbb{N}$ y $\left|f^{-\left(q_{n}+q_{n+1}\right)}(I)\right|$ tiende a cero cuando $n$ tiende a infinito, esta desigualdad implica que, para todo $n$ suficientemente grande,

$$
\left|f^{-q_{n+1}}(I)\right| \geq\left|f^{-q_{n}}(I)\right| \text {. }
$$

Sin embargo, esto contradice el hecho que $\left|f^{-q_{n}}(I)\right|$ converge a cero cuando $n$ tiende al infinito.

Ejercicio 3.4. La siguiente demostración del teorema de Denjoy en clase $\mathrm{C}^{1+l i p}$ es de particular interés por el hecho de no utilizar ningún argumento de control de distorsión.

(i) Suponga que $f$ sea un contra-ejemplo de Denjoy de clase $\mathrm{C}^{1+\text { lip }}$ y denote por $I$ uno de sus intervalos errantes. Para cada $j \in \mathbb{Z}$ escoja un punto $x_{j} \in f^{j}(I)$ tal que $\left|f^{j+1}(I)\right|=f^{\prime}\left(x_{j}\right)\left|f^{j}(I)\right|$. Denotando por $C$ la constante de Lipschitz de $f^{\prime}$ y fijando $n \in \mathbb{N}$, de la desigualdad $\left|f^{\prime}\left(x_{j}\right)-f^{\prime}\left(x_{j-q_{n}}\right)\right| \leq C\left|x_{j}-x_{j-q_{n}}\right|$ concluya que, para cierta constante $\bar{C}>0$ independiente de $n$ y todo $j \in\left\{0, \ldots, q_{n}-1\right\}$,

$$
\frac{\left|f^{j+1}(I)\right| \cdot\left|f^{-q_{n}+j}(I)\right|}{\left|f^{j}(I)\right| \cdot\left|f^{-q_{n}+j+1}(I)\right|} \geq 1-\bar{C}\left|x_{j}-x_{j-q_{n}}\right| .
$$

(ii) Usando las propiedades combinatorias de la sección 2.1 del capítulo 2, concluya a partir de la desigualdad precedente que existe una constante $D>0$ tal que, para todo $n \in \mathbb{N}$,

$$
\frac{\left|f^{q_{n}}(I)\right| \cdot\left|f^{-q_{n}}(I)\right|}{|I|^{2}} \geq D e^{-D}
$$

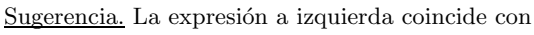

$$
\prod_{j=0}^{q_{n}-1} \frac{\left|f^{j+1}(I)\right| \cdot\left|f^{-q_{n}+j}(I)\right|}{\left|f^{j}(I)\right| \cdot\left|f^{-q_{n}+j+1}(I)\right|} .
$$

Utilice la desigualdad del ítem (i) para estimar cada factor de este producto para $j$ suficientement grande, y luego use la desigualdad $1-x \geq e^{-x / 2}$ (válida para $x>0$ suficientemente pequeño) además del hecho que los intervalos de extremos $x_{j}$ y $x_{j-q_{n}}$, con $j \in\left\{0, \ldots, q_{n}-1\right\}$, son dos a dos disjuntos

(iii) A partir de esta desigualdad del ítem (ii) obtenga una contradicción.

Para construir homeomorfismos diferenciables de $\mathrm{S}^{1}$ que admitan un minimal excepcional se pueden seguir dos caminos. El primero es aceptar la presencia de puntos críticos. En esta dirección, en [88] se construye un homeomorfismo de clase $\mathrm{C}^{\infty}$ de $\mathrm{S}^{1}$ que admite un minimal excepcional (por el resultado de Yoccoz mencionado anteriormente, un homeomorfismo con tales propiedades no puede ser real-analítico). El otro camino consiste en considerar difeomorfismos de clase inferior a $\mathrm{C}^{2}$. Nosotros estudiaremos algunos ejemplos de este tipo en la sección 1.4 del capítulo 4 .

En lo que se refiere a la naturaleza del minimal excepcional que puede aparecer en clase inferior a $\mathrm{C}^{2}$, mencionemos que cualquier conjunto de 
Cantor en $\mathrm{S}^{1}$ puede ser exhibido como el minimal excepcional de un homeomorfismo del círculo. Sin embargo, si se asume cierta regularidad del homeomorfismo en cuestión, entonces el conjunto de Cantor que aparece debe poseer ciertas propiedades. Por ejemplo, en [125] se demuestra que el Cantor terciario tradicional no puede ser el minimal excepcional de un difeomorfismo de clase $\mathrm{C}^{1}$ de $\mathrm{S}^{1}$ (vea también [154]). Por otra parte, en [156] se prueba que todo conjunto de Cantor afín puede aparecer como el minimal de un homeomorfismo lipschitziano de $\mathrm{S}^{1}$.

Desde la perspectiva de las acciones de grupos, el teorema de Denjoy puede ser reformulado diciendo que no existe ninguna acción de $(\mathbb{Z},+)$ por difeomorfismos de clase $\mathrm{C}^{1+\text { va }}$ del círculo admitiendo un minimal excepcional. En general, llamaremos grupos de Denjoy a los que cumplen esta propiedad. El problema de caracterizar estos grupos no parece sencillo. A continuación presentamos un modesto resultado en tal dirección.

Proposición 3.5. Sea $\Gamma$ un grupo finitamente generado de difeomorfismos de clase $\mathrm{C}^{1+l i p}$ del círculo. Si $\Gamma$ admite un minimal excepcional, entonces $\Gamma$ contiene un subgrupo libre generado por dos elementos para los cuales existe un juego de ping-pong asociado.

Demostración. Probaremos que $\Gamma$ actúa de manera expansiva en el círculo topológico $\mathrm{S}_{\Lambda}^{1}$ asociado al minimal excepcional $\Lambda$. Por (la demostración dada para) el teorema 2.77, esto implica que $\Gamma$ contiene un grupo libre generado por dos elementos para los cuales existe un juego de ping-pong asociado.

Supongamos que la acción (minimal) de $\Gamma$ sobre $\mathrm{S}_{\Lambda}^{1}$ no sea expansiva. Entonces ella es equicontinua, y existe una medida invariante de soporte total y sin átomos sobre $S_{\Lambda}^{1}$ que permite conjugar $\Gamma$ a un grupo de rotaciones de $\mathrm{S}_{\Lambda}^{1}$. Sean $g_{1}, \ldots, g_{n}$ los generadores de $\Gamma$. Si cada $g_{i}$ es de orden finito entonces cada órbita en $S_{\Lambda}^{1}$ es finita, lo cual contradice la minimalidad de $\Lambda$. Por otra parte, si cierto generador $g_{i}$ no es de orden finito en $\mathrm{S}_{\Lambda}^{1}$, entonces el número de rotación de $g_{i}$ es irracional. Como $g_{i}$ posee órbitas no densas (contenidas en $\Lambda$ ), esto contradice al teorema de Denjoy.

La proposición precedente permite concluir que todo grupo finitamente generado y promediable es un grupo de Denjoy (pues ningún grupo promediable contiene a $\mathbb{L}_{2}$ ). Sin embargo, esta última afirmación puede ser establecida directamente, sin hacer referencia al teorema de Margulis.

Ejercicio 3.6. Pruebe que todo grupo promediable y finitamente generado es un grupo de Denjoy.

Sugerencia. Suponga que $\Gamma$ es un grupo finitamente generado y promediable que actúa sobre $\mathrm{S}^{1}$ por difeomorfismos de clase $\mathrm{C}^{1+\text { va }}$ admitiendo un minimal excepcional $\Lambda$. Considere la acción de $\Gamma$ sobre el círculo topológico $S_{\Lambda}^{1}$ asociado a $\Lambda$ y sea $\mu$ una probabilidad sobre $\mathrm{S}_{\Lambda}^{1}$ invariante por esta acción. Pruebe que $\mu$ es de soporte total y no posee átomos. Concluya que $\Gamma$ es semiconjugado a un grupo de rotaciones y obtenga una contradicción utilizando el teorema de Denjoy. 
La proposición 3.5 es aún válida para grupos no finitamente generados cuando existe una constante $C$ tal que $V(g) \leq C$ para todo generador $g$. Sin embargo, ella no es válida para subgrupos arbitrarios de Difeo ${ }_{+}^{1+v a}\left(\mathrm{~S}^{1}\right)$ que admiten un minimal excepcional, como lo muestra el siguiente ejemplo, debido esencialmente a Hirsh [96] (vea también [104]).

Ejemplo 3.7. Sea $H: \mathbb{R} \rightarrow \mathbb{R}$ un homeomorfismo verificando las propiedades (i) y (ii) de la sección 5.2 (capítulo 1 ) y tal que $\operatorname{Fix}(H)=\{a, b\}$, con $a=0$ y $b>0$ pequeño. Este homeomorfismo $H$ induce una aplicación $\bar{H}$ de grado 2 del círculo en sí mismo (vea la figura 10). Designemos por $I$ al intervalo $] a, b[$ (visto sobre el círculo). Definamos $g_{1}: \mathrm{S}^{1} \rightarrow \mathrm{S}^{1}$ por $g_{1}(x)=y$ si $\bar{H}(x)=\bar{H}(y)$ y $x \neq y$. En general, para cada $n \in \mathbb{N}$ consideremos la aplicación $\bar{H}^{n}: \mathrm{S}^{1} \rightarrow \mathrm{S}^{1}$ de grado $2^{n}$, y definamos $g_{n}: \mathrm{S}^{1} \rightarrow \mathrm{S}^{1}$ por $g_{n}(x)=y$ si $\bar{H}^{n}(x)=\bar{H}^{n}(y)$ y $\bar{H}^{n}(x) \neq \bar{H}^{n}\left(y^{\prime}\right)$ para todo $\left.y^{\prime} \in\right] x, y[$.

El grupo $\Gamma=\Gamma_{H}$ generado por los $g_{n}$ es abeliano e isomorfo a $\mathbb{Q}_{2}\left(\mathrm{~S}^{1}\right)$. Su acción sobre $\mathrm{S}^{1}$ es semiconjugada a la de un grupo de rotaciones, siendo el conjunto $\Lambda=\mathrm{S}^{1} \backslash \cup_{n \in \mathbb{N}} \cup_{i=0}^{2^{n}-1} g_{n}^{i}(C)$ su minimal excepcional. Observe que si $H$ es un difeomorfismo real-analítico de la recta, entonces los elementos de $\Gamma_{H}$ son difeomorfismos real-analíticos del círculo. Note finalmente la similitud de esta construcción con la última de la sección 1 del capítulo 1: el grupo $\Gamma_{H}$ es un subgrupo (de la realización $\Phi_{H}(\mathrm{G})$ ) del grupo de Thompson (ipero $\Phi_{H}(\mathrm{G})$ no es un grupo de difeomorfismos real-analíticos de $\mathrm{S}^{1}$ !).

El teorema de Denjoy puede ser visto también como una consecuencia de la siguiente proposición, la cual sirve de base para el estudio del importante problema de la regularidad de la conjugación de difeomorfismos del círculo a rotaciones.

Proposición 3.8. Sean $\theta \in] 0,1[$ un irracional y $p / q$ una de las aproximaciones de $\theta$ por racionales (i.e., $|\theta-p / q| \leq 1 / q^{2}$ ). Si $f$ es un homeomorfismo de $\mathrm{S}^{1}$ de número de rotación $\theta$ y $\psi: \mathrm{S}^{1} \rightarrow \mathbb{R}$ es una función de variación acotada (no necesariamente continua), entonces denotando por $\mu$ la (única) medida de probabilidad sobre $\mathrm{S}^{1}$ invariante por $f$ se tiene, para todo $x \in \mathrm{S}^{1}$,

$$
\left|\sum_{i=0}^{q-1} \psi\left(f^{i}(x)\right)-q \int_{\mathrm{S}^{1}} \psi d \mu\right| \leq \operatorname{var}\left(\psi ; \mathrm{S}^{1}\right) .
$$

En particular, si $f$ es diferenciable y su derivada es de variación acotada, entonces para todo $x \in \mathrm{S}^{1}$ y todo $n \in \mathbb{N}$ se tiene

$$
\exp (-V(f)) \leq\left(f^{q_{n}}\right)^{\prime}(x) \leq \exp (V(f)) .
$$

Para probar esta proposición usaremos el siguiente lema combinatorio.

Lema 3.9. Si $\theta \in] 0,1[$ es irracional y $p, q$ son dos enteros relativamente primos tales que $q \neq 0$ y $|\theta-p / q| \leq 1 / q^{2}$, entonces para cada $i \in\{0, \ldots, q-1\}$ el intervalo $] i / q,(i+1) / q[$ contiene un único punto del conjunto $\{j \theta(\bmod 1): j \in\{1, \ldots, q\}\}$. 
Demostración. Por hipótesis tenemos

$$
0<\theta-\frac{p}{q}<\frac{1}{q^{2}} \quad \text { o bien } \quad-\frac{1}{q^{2}}<\theta-\frac{p}{q}<0 .
$$

Consideremos sólo el primer caso, pues el segundo es análogo. Tenemos entonces, para todo $j \in\{1, \ldots, q\}$,

$$
0<j \theta-\frac{j p}{q}<\frac{j}{q^{2}} \leq \frac{1}{q}
$$

por lo que $j \theta \bmod 1$ pertenece al intervalo $] j p / q,(j p+1) / q[$. La afirmación del lema resulta entonces del hecho que los intervalos $[i / q,(i+1) / q]$ recubren al círculo y tienen longitud $1 / q$.

Demostración de la proposición 3.8. Resulta evidente que el enunciado de la proposición es equivalente a que la desigualdad

$$
\left|\sum_{i=1}^{q} \psi\left(f^{i}(x)\right)-q \int_{\mathrm{S}^{1}} \psi d \mu\right| \leq \operatorname{var}\left(\psi ; \mathrm{S}^{1}\right)
$$

es válida para todo $x \in \mathrm{S}^{1} \mathrm{y}$ toda función continua $\psi$. Para probar esto último consideremos la aplicación del círculo sobre sí mismo definida por

$$
\varphi(y)=\int_{x}^{y} d \mu \bmod 1
$$

De la igualdad $\varphi \circ f=R_{\theta} \circ \varphi$ se deduce que, si definimos $x_{j}=f^{j}(x)$, entonces $\varphi\left(x_{j}\right)=j \theta$ mód 1 . Por el lema precedente, si para cada $j \in\{1, \ldots, q\}$ escogemos un intervalo $\left.I_{j}=\right] i_{j} / q,\left(i_{j}+1\right) / q$ [ conteniendo $\varphi\left(x_{j}\right)$, entonces los intervalos que aparecen son dos a dos disjuntos. Así, denotando $J_{j}$ el intervalo $\varphi^{-1}\left(\bar{I}_{j}\right)$ (cuya masa según $\mu$ es igual a $1 / q$ ), de la igualdad

$$
\left|\sum_{i=1}^{q} \psi\left(f^{i}(x)\right)-q \int_{\mathrm{S}^{1}} \psi d \mu\right|=\left|\sum_{j=1}^{q}\left(\psi\left(f^{j}(x)\right)-q \int_{J_{j}} \psi d \mu\right)\right|
$$

se concluye que

$$
\begin{aligned}
\left|\sum_{i=1}^{q} \psi\left(f^{i}(x)\right)-q \int_{\mathrm{S}^{1}} \psi d \mu\right| & \leq q \sum_{j=1}^{q} \int_{J_{j}}\left|\psi\left(f^{j}(x)\right)-\psi\right| d \mu \\
& \leq \sum_{j=1}^{q} \sup _{y \in J_{j}}\left|\psi\left(f^{j}(x)\right)-\psi(y)\right| \leq \operatorname{var}\left(\psi, \mathrm{S}^{1}\right) .
\end{aligned}
$$

La segunda parte de la proposición resulta de la primera parte aplicada a la función $\psi(x)=\log \left(f^{\prime}(x)\right)$, teniendo en cuenta la igualdad

$$
\int_{\mathrm{S}^{1}} \log \left(f^{\prime}\right) d \mu=0 .
$$


Señalemos sin embargo que esta última igualdad no es del todo evidente (vea el ejercicio 3.12). Para verificarla notemos que a partir de (3.6) se deduce que, para todo $x \in \mathrm{S}^{1}$,

$$
\exp (-V(f)) \leq \frac{\left(f^{q_{n}}\right)^{\prime}(x)}{\exp \left(q_{n} \int_{\mathrm{S}^{1}} \log \left(f^{\prime}\right) d \mu\right)} \leq \exp (V(f)) .
$$

Integrando respecto a la medida de Lebesgue concluimos que

$$
\exp (-V(f)) \leq \frac{1}{\exp \left(q_{n} \int_{\mathrm{S}^{1}} \log \left(f^{\prime}\right) d \mu\right)} \leq \exp (V(f)),
$$

lo cual puede satisfacerse para todo $n \in \mathbb{N}$ si y sólo si (3.8) se cumple.

La relación (3.6) (resp. (3.7)) es conocida como la desigualdad de DenjoyKoksma (resp. desigualdad de Denjoy). Consignemos que ambas desigualdades, al igual que el teorema de Denjoy, valen aún para homeomorfismos afines por partes de $\mathrm{S}^{1}$, siendo los argumentos de demostración esencialmente los mismos que los del caso $\mathrm{C}^{1+\text { va }}$ (obviamente, en el caso afín por partes consideramos una derivada lateral en lugar de la usual). Por ejemplo, la igualdad (3.8) sigue cumpliéndose...

Observación 3.10. Para difeomorfismos $f$ del círculo de clase $\mathrm{C}^{2+\tau}$ y número de rotación irracional vale una versión más fuerte de la proposición 3.8: respecto a la topología $\mathrm{C}^{1}$ se tiene la convergencia de $f^{q_{n}}$ a la identidad, donde $p_{n} / q_{n}$ designa la $n$-ésima aproximación racional de $\rho(f)$ (vea $[94,110,211])$.

Ejercicio 3.11. Pruebe que validez de la desigualdad (3.7) para todo $n \in \mathbb{N}$ implica la validez del teorema de Denjoy.

Ejercicio 3.12. Demuestre la igualdad (3.8) para todo difeomorfismo de clase $\mathrm{C}^{1}$ del círculo de número de rotación irracional usando el teorema ergódico de Birkhoff y la ergodicidad única de $f$ (vea [126]).

Observemos que el teorema de Denjoy implica que si $\Gamma$ es un subgrupo de Difeo ${ }_{+}^{1+v a}\left(\mathrm{~S}^{1}\right)$ que admite un minimal excepcional, entonces cada uno de sus elementos posee puntos periódicos. En efecto, si $g \in \Gamma$ no posee puntos periódicos entonces su número de rotación es irracional, lo cual implica la densidad de las órbitas por $g$ (y por lo tanto de las órbitas por $\Gamma$ ). Una consecuencia interesante de este hecho es la racionalidad del número de rotación de los elementos del grupo de Thompson G. En efecto, en la sección 1 del capítulo 2 se construyó una acción de G por difeomorfismos de clase $\mathrm{C}^{\infty}$ del círculo que es semiconjugada a la acción estándar y que admite un minimal excepcional. Así, el que $\rho(g)$ pertenezca a $\mathbb{Q}$ para cada $g \in \mathrm{G}$ resulta de la observación precedente y de la invariancia del número de rotación por semiconjugación. Siguiendo [124], invitamos a continuación al lector a desarrollar una demostración "directa" usando sólo la versión de la desigualdad de Denjoy para homeomorfismos afines por partes (demostraciones alternativas aparecen en [33] y [112]). 
Ejercicio 3.13. Dado $g \in \mathrm{G}$ definamos $M_{n} \in \mathbb{N}$ y $N_{n} \in \mathbb{N} \cup\{0\}$ de modo que para cada $n \in \mathbb{N}$ se tenga $g^{n}(0)=M_{n} / 2^{N_{n}}$, con $M_{n}$ impar o nulo. Supongamos que 0 no sea un punto periódico. En tal caso, se tiene $M_{n} \neq 0$ para todo $n \in \mathbb{N}$. (i) Pruebe por contradicción que $N_{n}$ tiende al infinito junto con $n$.

Sugerencia. Observe que para todo $N \in \mathbb{N}$, el conjunto de racionales diádicos con denominador inferior o igual a $2^{N}$ es finito.

(ii) A partir de la convergencia de $N_{n}$ al infinito concluya que $\lim _{n \rightarrow \infty}\left(g^{n}\right)^{\prime}(0)=0$ (consideramos la derivada a derecha de la aplicación en cuestión).

Sugerencia. Escriba $g(x)=2^{\lambda(x)}+M(x) / 2^{N(x)}$ para ciertas funciones $\lambda, M$ y $N$ a valores enteros y uniformemente acotadas sobre $\mathrm{S}^{1}$. Verifique entonces que para $n \in \mathbb{N}$ suficientemente grande se tiene

$$
M_{n+1}=M_{n}+2^{N_{n}-\lambda\left(g^{n}(0)\right)-N\left(g^{n}(0)\right)} M\left(g^{n}(0)\right), \quad N_{n+1}=N_{n}-\lambda\left(g^{n}(0)\right) .
$$

(iii) Suponga que el número de rotación de $g$ sea irracional y obtenga una contradicción aplicando la desigualdad de Denjoy.

\section{El teorema del punto fijo hiperbólico de Sacksteder}

Comencemos recordando la noción de pseudo-grupo de homeomorfismos.

Definición 3.14. Una familia $\Gamma=\{g: \operatorname{dom}(g) \rightarrow \operatorname{ran}(g)\}$ de homeomorfismos locales de un espacio topológico $\mathrm{M}$ es un pseudo-grupo si las siguientes propiedades son satisfechas:

- el dominio $\operatorname{dom}(g)$ y el rango $\operatorname{ran}(g)$ de cada $g \in \Gamma$ son conjuntos abiertos; - si $g, h$ pertenecen a $\Gamma$ y $\operatorname{ran}(h) \subset \operatorname{dom}(g)$, entonces $g h$ pertenece a $\Gamma$;

- si $g \in \Gamma$ entonces $g^{-1} \in \Gamma$;

- la identidad de $\mathrm{M}$ es un elemento de $\Gamma$;

- si $g$ pertenece a $\Gamma$ y $A \subset \operatorname{dom}(g)$ es un conjunto abierto, entonces la restricción $\left.g\right|_{A}$ de $g$ a $A$ es un elemento de $\Gamma$;

- si $g: \operatorname{dom}(g) \rightarrow \operatorname{ran}(g)$ es un homeomorfismo local de $\mathrm{M}$ tal que para todo $x \in \operatorname{dom}(g)$ existe una vecindad $V_{x}$ tal que $\left.h\right|_{V_{x}}$ es un elemento de $\Gamma$, entonces $g$ pertenece a $\Gamma$.

Las nociones de órbita y conjunto invariante para un pseudo-grupo se definen de manera evidente. Diremos que $\Gamma$ es finitamente (resp. contablemente) generado si existe un subconjunto finito (resp. contable) $\mathcal{G}$ de $\Gamma$ tal que todo elemento de $\Gamma$ se escribe como la restricción a su dominio de un producto de elementos de $\mathcal{G}$. Una medida $\mu$ sobre los borelianos de $\mathrm{M}$ es invariante por $\Gamma$ si para todo boreliano $A$ y todo $g \in \Gamma$ se tiene $\mu(A \cap \operatorname{dom}(g))=\mu(g(A) \cap \operatorname{ran}(g))$. Finalmente, para $a \in \mathrm{M}$ denotamos $\Gamma(a)$ la órbita de $a$ por $\Gamma$, y para cada $p \in \Gamma(a)$ definimos su orden por

$$
\operatorname{ord}(p)=\min \left\{n \in \mathbb{N}: \text { existen } h_{i_{j}} \in \mathcal{G} \text { tales que } h_{i_{n}} \cdots h_{i_{1}}(a)=p\right\} .
$$




\subsection{La versión clásica en clase $\mathrm{C}^{1+l i p}$}

En esta sección probaremos la versión clásica de un un teorema debido a Sacksteder [179] sobre la existencia de puntos fijos hiperbólicos para elementos de un pseudo-grupo de difeomorfismos unidimensionales (en las secciones siguientes trataremos su versión moderna en clase $\mathrm{C}^{1}$ ).

Teorema 3.15. Sea $\Gamma$ un pseudo-grupo finitamente generado de difeomorfismos de clase $\mathrm{C}^{1+l i p}$ de una variedad compacta unidimensional. Supongamos que cada generador $h_{i}$ se extienda a un difeomorfismo de clase $\mathrm{C}^{1+l i p}$ definido en la clausura de dom $\left(h_{i}\right)$. Supongamos además que exista un conjunto de Cantor $\Lambda$ invariante por $\Gamma$ tal que para una componente conexa $I=] a, b[$ del complemento de $\Lambda$, ya sea el punto a es un punto de acumulación de la órbita $\Gamma(a)$, o bien $b$ es un punto de acumulación de $\Gamma(b)$. Entonces existen $p \in \Lambda$ y $g \in \Gamma$ tales que $g(p)=p$ y $g^{\prime}(p)<1$.

Un conjunto $\Lambda$ que verifica las hipótesis precedentes (salvo eventualmente aquélla que concierne la regularidad) es llamado conjunto excepcional local. Para probar el teorema 3.15, una herramienta técnica fundamental es el lema que presentamos a continuación, cuya versión en clase $\mathrm{C}^{1+\text { lip }}$ es debida esencialmente a Schwartz [182]. Daremos inmediatamente la versión general en clase $\mathrm{C}^{1+\tau}$ para referencia futura; una versión ligeramente más fina en clase $\mathrm{C}^{1+\text { lip }}$ será presentada en la sección 4.2 de este capítulo.

Lema 3.16. Sea $\Gamma$ un pseudo-grupo de difeomorfismos de clase $\mathrm{C}^{1+\tau}$ (resp. $\left.\mathrm{C}^{1+l i p}\right)$ de una variedad unidimensional compacta. Supongamos que existan un subconjunto finito $\mathcal{G}$ de $\Gamma$, una constante $S \in[1, \infty[$, y un intervalo abierto $I$, de modo que a cada elemento $g \in \mathcal{G}$ sea posible asociar un intervalo compacto $L_{g}$ contenido en un dominio abierto de definición de $g$, y tales que para cada $m \in \mathbb{N}$ exista $g_{i_{m}} \in \mathcal{G}$ tal que para todo $n \in \mathbb{N}$ el elemento $g_{i_{n}} \cdots g_{i_{1}}$ de $\Gamma$ verifica las propiedades siguientes:

- si $g_{i_{k}}=g$ entonces el intervalo $g_{i_{k-1}} \cdots g_{i_{1}}(I)$ está contenido en $L_{g}$ (convenimos en que $g_{i_{k-1}} \cdots g_{i_{1}}$ es la identidad para $k=1$ );

- se tiene la desigualdad

$$
\sum_{k=0}^{n-1}\left|g_{i_{k}} \cdots g_{i_{1}}(I)\right|^{\tau} \leq S \quad\left(\text { resp. } \sum_{k=0}^{n-1}\left|g_{i_{k}} \cdots g_{i_{1}}(I)\right| \leq S\right) .
$$

Entonces existe una constante positiva $\ell=\ell(\tau, S,|I| ; \mathcal{G})$ tal que si para algún $n \in \mathbb{N}$ el intervalo $h_{n}(I)$ está contenido en una $\ell$-vecindad de $I$ y no intersecta el interior de $I$, entonces la aplicación $h_{n}$ posee un punto fijo hiperbólico (el cual está próximo de la extremidad correspondiente de I).

Demostración. Para simplificar consideraremos los casos $\tau \in] 0,1[$ (hölderiano) y $\tau=1$ (lipschitziano) de manera simultánea. Sea $\varepsilon>0$ una cons- 
tante tal que cada $g \in \mathcal{G}$ está definido en una $2 \varepsilon$-vecindad de $L_{g}$. Fijemos $C>0$ tal que para todo $g \in \mathcal{G}$ y todo $x, y$ en la $\varepsilon$-vecindad de $L_{g}$ se tenga

$$
\left|\log \left(g^{\prime}(x)\right)-\log \left(g^{\prime}(y)\right)\right| \leq C|x-y|^{\tau} .
$$

Probaremos que la afirmación del lema es satisfecha para

$$
\ell=\min \left\{\frac{|I|}{2 \exp \left(2^{\tau} C S\right)}, \frac{|I| \varepsilon}{2 S^{1 / \tau} \exp \left(2^{\tau} C S\right)}\right\} .
$$

Denotemos por $J$ la $2 \ell$-vecindad de $I$, y notemos $I^{\prime}$ (resp. $I^{\prime \prime}$ ) la componente conexa de $J \backslash I$ a la derecha (resp. a izquierda) de $I$. Probaremos por inducción que las propiedades siguientes son simultáneamente satisfechas:

(i) ${ }_{k} \quad g_{i_{k}} \cdots g_{i_{1}}\left(I^{\prime}\right)$ está contenido en la $\varepsilon$-vecindad de $L_{g}$;

(ii) ${ }_{k} \quad\left|g_{i_{k}} \cdots g_{i_{1}}\left(I^{\prime}\right)\right| \leq\left|g_{i_{k}} \cdots g_{i_{1}}(I)\right|$;

(iii) $)_{k} \quad \sup _{x, y \in I \cup I^{\prime}} \frac{\left(g_{i_{k}} \cdots g_{i_{1}}\right)^{\prime}(x)}{\left(g_{i_{k}} \cdots g_{i_{1}}\right)^{\prime}(y)} \leq \exp \left(2^{\tau} C S\right)$.

La condición (iii) $)_{0}$ es satisfecha de manera trivial, mientras que (i) $)_{0}$ y (ii) $)_{0}$ se desprenden de la hipótesis $\left|I^{\prime}\right|=2 \ell \leq|I|$. Supongamos que (i) ${ }_{j}$, (ii) ${ }_{j}$ y (iii $_{j}$ sean válidas para todo $j \in\{0, \ldots, k-1\}$. En tal caso tenemos, para todo $x, y$ en $I \cup I^{\prime}$,

$$
\begin{aligned}
\left|\log \left(\frac{\left(g_{i_{k}} \cdots g_{i_{1}}\right)^{\prime}(x)}{\left(g_{i_{k}} \cdots g_{i_{1}}\right)^{\prime}(y)}\right)\right| & \leq \sum_{j=0}^{k-1}\left|\log \left(g_{i_{j+1}}^{\prime}\left(g_{i_{j}} \cdots g_{i_{1}}(x)\right)\right)-\log \left(g_{i_{j+1}}^{\prime}\left(g_{i_{j}} \cdots g_{i_{1}}(y)\right)\right)\right| \\
& \leq C \sum_{j=0}^{k-1}\left|g_{i_{j}} \cdots g_{i_{1}}(x)-g_{i_{j, n}} \cdots g_{i_{1}}(y)\right|^{\tau} \\
& \leq C \sum_{j=0}^{k-1}\left(\left|g_{i_{j}} \cdots g_{i_{1}}(I)\right|+\left|g_{i_{j}} \cdots g_{i_{1}}\left(I^{\prime}\right)\right|\right)^{\tau} \\
& \leq C 2^{\tau} S .
\end{aligned}
$$

Esta desigualdad prueba (iii) $k$. En lo que respecta a (i) $k$ y (ii) $)_{k}$, observemos que existen $x \in I$ e $y \in I^{\prime}$ tales que

$\left|g_{i_{k}} \cdots g_{i_{1}}(I)\right|=|I| \cdot\left(g_{i_{k}} \cdots g_{i_{1}}\right)^{\prime}(x) \quad$ y $\quad\left|g_{i_{k}} \cdots g_{i_{1}}\left(I^{\prime}\right)\right|=\left|I^{\prime}\right| \cdot\left(g_{i_{k}} \cdots g_{i_{1}}\right)^{\prime}(y)$.

Luego, por (iii) $k$,

$$
\frac{\left|g_{i_{k}} \cdots g_{i_{1}}\left(I^{\prime}\right)\right|}{\left|g_{i_{k}} \cdots g_{i_{1}}(I)\right|}=\frac{\left(g_{i_{k}} \cdots g_{i_{1}}\right)^{\prime}(x)}{\left(g_{i_{k}} \cdots g_{i_{1}}\right)^{\prime}(y)} \cdot \frac{\left|I^{\prime}\right|}{|I|} \leq \exp \left(2^{\tau} C S\right) \frac{\left|I^{\prime}\right|}{|I|},
$$

lo cual prueba $(\mathrm{i})_{k}$ y $(\mathrm{ii})_{k}$ dada la definición de $\ell$. Obviamente, propiedades análogas valen al reemplazar $I^{\prime}$ por $I^{\prime \prime}$.

Supongamos ahora que $h_{n}(I)$ esté contenido en la $\ell$-vecindad del intervalo $I$ y no intersecte el interior de $I$. La propiedad (ii) $)_{n}$ nos da entonces $h_{n}(J) \subset J$. Además, si $h_{n}(I) \subset J$ está a derecha (resp. a izquierda) de $I$, 
entonces $h_{n}\left(I \cup I^{\prime}\right) \subset I^{\prime}$ (resp. $\left.h_{n}\left(I^{\prime \prime} \cup I\right) \subset I^{\prime \prime}\right)$. Como ambos casos son análogos, consideraremos solamente el primero de ellos. Evidentemente, $h_{n}$ posee al menos un punto fijo $x$ en $I^{\prime}$. Resta verificar que se trata de un punto fijo hiperbólico y contractante. Ahora bien, escogiendo $y \in I$ tal que $h_{n}^{\prime}(y)=\left|h_{n}(I)\right| /|I| \leq \ell /|I|$ vemos que, por $(\text { iii })_{n}$

$$
h_{n}^{\prime}(x) \leq h_{n}^{\prime}(y) \exp \left(2^{\tau} C S\right) \leq \frac{\ell \exp \left(2^{\tau} C S\right)}{|I|} \leq \frac{1}{2},
$$

lo cual concluye la demostración.

Una desigualdad del tipo $\sum_{k \geq 0}\left|g_{i_{k}} \cdots g_{i_{1}}(I)\right| \leq S$ es válida por razones evidentes cuando los intervalos $g_{i_{k}} \cdots g_{i_{1}}(I)$ son dos a dos disjuntos. En tal caso, la sutileza del lema precedente radica en la posibilidad de controlar la distorsión para las composiciones sucesivas sobre la $2 \ell$-vecindad de $I$, a pesar de que las imágenes de esta vecindad no son necesariamente disjuntas.

Demostración del teorema 3.15. Fijemos $\varepsilon>0$ tal que para todo $x$ en el dominio de $g_{i} \in \mathcal{G}$ se tenga $] x-\varepsilon, x+\varepsilon\left[\subset \operatorname{dom}\left(h_{i}\right)\right.$. Supongamos que $b$ sea un punto de acumulación de $\Gamma(b)$ (el caso en que $a$ es punto de acumulación de $\Gamma(a)$ es análogo $)$. Sea $\left(h_{n}\right)=\left(g_{i_{n}} \cdots g_{i_{1}}\right)$ una sucesión en $\Gamma$ tal que $h_{n}(b)$ converja a $b$, tal que $h_{n}(b) \neq b$ para todo $n \in \mathbb{N}$, y tal que el orden del punto $h_{n}(b) \in \Gamma(b)$ sea realizado por $h_{n}$, i.e., el número mínimo de composiciones necesarias para llegar a $h_{n}(b)$ es $n$ (dejamos al lector la verificación de la existencia de tal sucesión). Para cada $n \in \mathbb{N}$ denotemos $I_{n}$ la componente conexa del complemento de $\Lambda$ que tiene por uno de sus extremos al punto $h_{n}(b)$. Puesto que la serie $\sum_{n \in \mathbb{N}}\left|I_{n}\right|$ converge, para $N$ suficientemente grande debe tenerse $\left|I_{n}\right|<\varepsilon$ para todo $n \geq N$, por lo que las hipótesis del lema precedente son satisfechas para $I=I_{N}$ y toda constante $S \geq 1$ mayor a la longitud total de la variedad unidimensional subyacente. Concluimos así que, para $n$ suficientemente grande, la aplicación $h_{n} \in \Gamma$ contrae (hiperbólicamente) sobre sí mismo el intervalo $I^{\prime}$ de longitud $\ell$ y situado inmediatamente a derecha de $I$. Por lo tanto, $h_{n}$ posee un único punto fijo en $I$ (a saber, el punto $p=\bigcap_{k \in \mathbb{N}}\left(h_{n}\right)^{k}\left(I^{\prime}\right)$ ), el cual es hiperbólico (vea la figura 15). Observe finalmente que $p$ pertenece a $\Lambda$, pues $I^{\prime}$ contiene puntos de $\Lambda$ y $\Lambda$ es un compacto invariante por $\Gamma$.

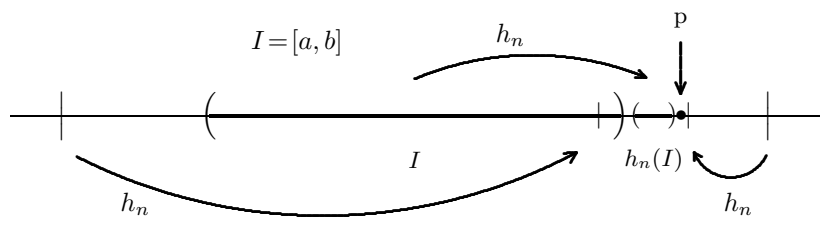

Figura 15

De lo anterior se deduce inmediatamente lo siguiente. 
Corolario 3.17. Sea $\Gamma$ un subgrupo finitamente generado de $\operatorname{Difeo}{ }_{+}^{1+l i p}\left(\mathrm{~S}^{1}\right)$. Si $\Gamma$ actúa admitiendo un minimal excepcional $\Lambda$, entonces existen $p \in \Lambda$ y $g \in \Gamma$ tales que $g(p)=p \quad$ y $g^{\prime}(p)<1$.

Observe que el teorema de Denjoy para difeomorfismos de clase $\mathrm{C}^{1+l i p}$ puede ser obtenido directamente de este corolario (esto no es extraño, pues el propio Sacksteder obtuvo su resultado pensando en generalizar el teorema de Denjoy para grupos de difeomorfismos y foliaciones de codimensión 1). Sin embargo, a juicio del autor éste no es un buen punto de vista. En efecto, la prueba del teorema de Denjoy utiliza en parte la estructura combinatoria de la dinámica de un homeomorfismo de número de rotación irracional, la cual es muy particular. Por otro lado, para pseudo-grupos que actúan sin probabilidad invariante, la dinámica combinatoria (al menos localmente) es completamente diferente. En la sección siguiente desarrollaremos esta idea en profundidad con el fin de obtener resultados más finos que los de esta sección. Como "aperitivo" veremos inmediatamente cómo el teorema de Sacksteder puede ser extendido para grupos actuando de manera minimal y que no son conjugados a grupos de rotaciones. Comenzamos con una astuta observación debida a Ghys que aparece en [60].

Proposición 3.18. Sea $\Gamma$ un pseudo-grupo de difeomorfismos de clase $\mathrm{C}^{1+l i p}$ del círculo o de un intervalo acotado cuyas órbitas son densas. Supongamos que exista un elemento $f \in \Gamma$ que fija un único punto sobre un intervalo no degenerado (no necesariamente abierto) conteniendo dicho punto. Entonces existe un elemento en $\Gamma$ con un punto fijo hiperbólico.

Demostración. Sean $a$ el punto fijo dado por la hipótesis y $a^{\prime}>a$ tal que la restricción de $f$ a un intervalo $\left[a, a^{\prime}\right]$ no tenga punto fijo a excepción de $a$ y tal que, cambiando $f$ por $f^{-1}$ si es necesario, se tenga $f(y)<y$ para todo $y \in] a, a^{\prime}$ ] (si tal punto $a^{\prime}$ no existe entonces se puede considerar un intervalo del tipo $\left[a^{\prime}, a\right]$ con propiedades análogas). Como las órbitas de $\Gamma$ son densas, existe $h \in \Gamma$ tal que $h(a) \in] a, a^{\prime}[$. Fijemos $n \in \mathbb{N}$ suficientemente grande de modo que $\left[a, f^{n}\left(a^{\prime}\right)\right] \subset \operatorname{dom}(h)$ y $\left.\left.h\left(\left[a, f^{n}\left(a^{\prime}\right)\right]\right) \subset\right] a, a^{\prime}\right]$.

Consideremos los intervalos $A_{0}=\left[a, f^{n}\left(a^{\prime}\right)\right]$ y $B_{0}=h\left(\left[a, f^{n}\left(a^{\prime}\right)\right]\right)$, y definamos inductivamente los conjuntos $A_{j+1}=f^{n}\left(A_{j} \cup B_{j}\right)$ y $B_{j+1}=h\left(A_{j+1}\right)$. Para $\epsilon>0$ pequeño considere el pseudo-grupo generado por las restricciones de $f^{n}$ y $h$ a los intervalos $] a-\varepsilon, h f^{n}\left(a^{\prime}\right)+\varepsilon[\mathrm{y}] a-\varepsilon, f^{n}\left(a^{\prime}\right)+\varepsilon[$ respectivamente. Respecto a este pseudo-grupo, el conjunto de Cantor $\Lambda=\cap_{j \in \mathbb{N}}\left(A_{j} \cup B_{j}\right)$ satisface las hipótesis del teorema de Sacksteder, de donde se obtiene el resultado.

Observe que, por el teorema de Hölder, un grupo de homeomorfismos del círculo que no es semiconjugado a un grupo de rotaciones contiene siempre un elemento $f$ que satisface la hipótesis de la proposición precedente. Puesto que toda semiconjugación de un grupo cuyas órbitas son densas a un grupo de rotaciones es necesariamente una conjugación, como corolario de lo anterior obtenemos lo siguiente. 
Corolario 3.19. Sea $\Gamma$ un subgrupo de Difeo ${ }_{+}^{1+l i p}\left(\mathrm{~S}^{1}\right)$. Suponga que las órbitas de $\Gamma$ son densas y que $\Gamma$ no es conjugado a un grupo de rotaciones (equivalentemente, suponga que $\Gamma$ actúa de manera minimal y no preserva ninguna medida de probabilidad del círculo). Entonces existen $p \in \Lambda$ y $g \in \Gamma$ tales que $g(p)=p$ y $g^{\prime}(p)<1$.

En la sección siguiente veremos que este corolario vale aún en clase $\mathrm{C}^{1}$.

Ejercicio 3.20. El hecho que una holonomía no trivial sea hiperbólica resulta relevante cuando se estudian propiedades de estabilidad y rigidez (vea por ejemplo la sección 6.1 de este capítulo). Sin embargo, el conocimiento de que cierta holonomía es no trivial (a pesar de que no sea necesariamente hiperbólica) también puede resultar de interés. La proposición siguiente engloba una lúcida observación debida a Hector. Antes de pasar a la demostración, el lector debiese reflexionar un momento testeando su veracidad para los ejemplos que ya hemos estudiado (grupo modular, grupo G de Thompson, etc).

Proposición 3.21. Sea $\Gamma$ un grupo finitamente generado de difeomorfismos de clase $\mathrm{C}^{1+l i p}$ del círculo que admite un minimal excepcional $\Lambda$. Si p es la extremidad de una de las componentes conexas $I$ de $\mathrm{S}^{1} \backslash \Lambda$, entonces existe $g \in \Gamma$ que fija $p$ y cuya restricción a $I \cap V$ es no trivial para toda vecindad $V$ de $p$.

Sugerencia. Suponga lo contrario y contradiga el hecho que para toda vecindad de $p$ existen elementos de $\Gamma$ con puntos fijos hiperbólicos repulsivos en ella.

\subsection{La versión $\mathrm{C}^{1}$ para pseudo-grupos}

El objetivo principal de esta sección y la siguiente consiste en formular (y demostrar) diversas generalizaciones del teorema de Sacksteder en clase $\mathrm{C}^{1}$. Algunas de estas nuevas versiones fueron obtenidas por Hurder en [99, 100, $101,102]$ vía métodos dinámicos ligados a la teoría de Pesin. Sin embargo, las versiones optimales y (posiblemente) definitivas aparecen en [54], donde son obtenidas gracias a la introducción de métodos probabilísticos.

Teorema 3.22. Si $\Gamma$ es un pseudo-subgrupo de difeomorfismos de clase $\mathrm{C}^{1}$ de una variedad unidimensional compacta sin medida de probabilidad invariante, entonces $\Gamma$ posee elementos con puntos fijos hiperbólicos.

Para probar este teorema, inspirándonos en la demostración de la proposición 3.18 supongamos que $\Gamma$ contenga dos elementos $f$ y $h$ que verifican: (i) el dominio de definición de $f$ contiene un intervalo $\left[a, a^{\prime}[\right.$ de modo que $f(a)=a \quad$ y $f$ contracta topológicamente hacia el punto fijo $a$;

(ii) $h$ está definido en una vecindad de este punto fijo y $h(a) \in] a, a^{\prime}[$.

Hagamos $c=h(a)$ y fijemos $\left.d^{\prime} \in\right] c, a^{\prime}$. Reemplazando $f$ por $f^{n}$ para $n \in \mathbb{N}$ suficientemente grande si es necesario, podemos suponer que $f\left(d^{\prime}\right)<c$, que $f\left(d^{\prime}\right)$ pertenece al dominio de definición de $h$, y que $\left.h f\left(d^{\prime}\right) \in\right] c, d^{\prime}[$. Esta última condición implica en particular que $h f$ posee puntos fijos en $] c, d^{\prime}[$. 
Sea $d$ el primer punto fijo $h f$ a la derecha de $c$, y sea $b=f(d)$. El intervalo abierto $I=] b, c[$ corresponde al "primer gap" (i.e., a la componente conexa "central" del complemento) de un conjunto de Cantor $\Lambda$ invariante por $f$ y $g=h f$ (vea la figura 16).

Proposición 3.23. Con las notaciones precedentes, el pseudo-grupo generado por $f$ y $g$ contiene elementos con puntos fijos hiperbólicos pertenecientes al conjunto $\Lambda$.

La demostración de esta proposición es particularmente sencilla en clase $\mathrm{C}^{1+\tau}$ (para $\tau>0$ ). Además, ella permite ilustrar de manera nítida el aporte de los métodos probabilísticos en la teoría. El caso general (en clase $\mathrm{C}^{1}$ ) será tratado más adelante. Para una demostración alternativa y "determinística" de un resultado estrechamente relacionado vea [108].
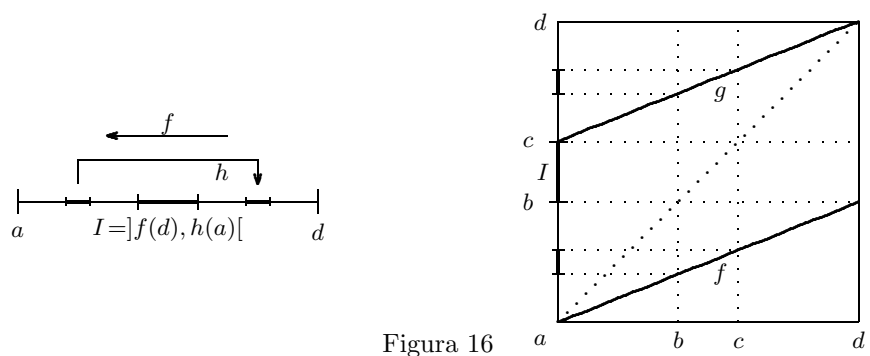

Figura 16

Demostración de la proposición 3.23 en clase $C^{1+\tau}$. Sobre el espacio $\Omega=\{f, g\}^{\mathbb{N}}$ consideremos la medida de Bernoulli $\mathbb{P}$ de peso $1 / 2$ para cada "variable aleatoria" $f$ ó $g$. Dados $\omega=\left(g_{1}, g_{2}, \ldots\right) \in \Omega$ y $n \in \mathbb{N}$ notemos $h_{n}(\omega)=g_{n} \cdots g_{1}$, y hagamos $h_{0}(\omega)=i d$. Siendo los intervalos de la familia $\left\{g_{n} \cdots g_{1}(I): n \geq 0,\left(g_{1}, \ldots, g_{n}\right) \in\{f, g\}^{n}\right\}$ dos a dos disjuntos, tenemos

$$
\sum_{n \geq 0} \sum_{\left(g_{1}, \ldots, g_{n}\right) \in\{f, g\}^{n}}\left|g_{n} \cdots g_{1}(I)\right| \leq d-a<\infty .
$$

Por otra parte, el teorema de Fubini nos da

$$
\begin{aligned}
\sum_{n \geq 0} \sum_{\left(g_{1}, \ldots, g_{n}\right) \in\{f, g\}^{n}}\left|g_{n} \cdots g_{1}(I)\right| & =\sum_{n \geq 0} 2^{n}\left(\int_{\Omega}\left|h_{n}(\omega)(I)\right| d \mathbb{P}(\omega)\right) \\
& =\int_{\Omega}\left(\sum_{n \geq 0} 2^{n}\left|h_{n}(\omega)(I)\right|\right) d \mathbb{P}(\omega) .
\end{aligned}
$$

Concluimos así que para $\mathbb{P}$-casi toda sucesión aleatoria $\omega \in \Omega$ la serie $\sum 2^{n}\left|h_{n}(\omega)(I)\right|$ converge. Para cada $B>0$ consideremos el conjunto

$$
\Omega(B)=\left\{\omega \in \Omega:\left|h_{n}(\omega)(I)\right| \leq B / 2^{n} \text { para todo } n \geq 0\right\} .
$$


La probabilidad $\mathbb{P}[\Omega(B)]$ converge a 1 cuando $B$ tiende al infinito; en particular, podemos fijar $B$ de modo que $\mathbb{P}[\Omega(B)]>0$. Observemos que si $\omega$ pertenece a $\Omega(B)$ entonces

$$
\sum_{n \geq 0}\left|h_{n}(\omega)(I)\right|^{\tau} \leq B^{\tau} \sum_{n \geq 0} \frac{1}{2^{n \tau}}=S<\infty .
$$

Consideremos el intervalo $J^{\prime}=[b-\ell, c+\ell]$ conteniendo al intervalo errante $I$, donde $\ell=\ell(\tau, S,|I| ;\{f, g\})$ es la constante que aparece en el lema 3.16. Si $N \in \mathbb{N}$ es suficientemente grande entonces $f^{N} g$ y $g^{N} f$ envían todo el intervalo $[0,1]$ sobre $J^{\prime} \backslash I$. Una aplicación directa del lema de Borel-Cantelli nos da entonces $\mathbb{P}\left[h_{n}(\omega)(I) \subset J^{\prime} \backslash I\right.$ infinitas veces $]=1$. Si $\omega \in \Omega(B)$ y $n \in \mathbb{N}$ satisfacen $h_{n}(\omega)(I) \subset J^{\prime} \backslash I$, entonces el lema 3.16 muestra que $h_{n}(\omega)$ posee un punto fijo hiperbólico. Finalmente, puesto que $\Lambda$ es invariante por el pseudo-grupo y el punto fijo hallado atrae por $h_{n}(\omega)$ a una parte de este conjunto, dicho punto fijo debe pertenecer a $\Lambda$.

La prueba de la versión general (en clase $\mathrm{C}^{1}$ ) de la proposición 3.23 necesita de pequeñas mejoras técnicas para hallar puntos fijos hiperbólicos en ausencia de control de distorsión. La idea esencial consiste en notar que, cuando se sabe a priori que la dinámica es (diferenciablemente) contractante en alguna parte, simples aplicaciones del teorema del valor medio permiten verificar que la contracción persiste sobre un dominio mayor.

Conservando las notaciones de la demostración precedente, fijemos de una vez por todas una constante $\varepsilon \in] 0,1 / 2$. Sabemos que para $\mathbb{P}$-casi todo $\omega \in \Omega$ existe $B=B(\omega) \geq 1$ tal que

$$
\left|h_{n}(\omega)(I)\right| \leq \frac{B}{2^{n}} \quad \text { para todo } \quad n \geq 0 .
$$

Lema 3.24. Existe una constante $\bar{C}$ que depende sólo de $f$ y $g$ tal que, si $\omega=\left(g_{1}, g_{2}, \ldots\right) \in \Omega$ satisface $(3.10)$, entonces para todo $x \in I$ y todo entero $n \geq 0$ se tiene

$$
h_{n}(\omega)^{\prime}(x) \leq \frac{B \bar{C}}{(2-\varepsilon)^{n}} .
$$

Demostración. Fijemos $\varepsilon_{0}>0$ suficientemente pequeño de modo que para todo par de puntos $y, z$ de $[a, d]$ a distancia menor o igual que $\varepsilon_{0}$ se tenga

$$
\frac{f^{\prime}(y)}{f^{\prime}(z)} \leq \frac{2}{2-\varepsilon} \quad \text { y } \quad \frac{g^{\prime}(y)}{g^{\prime}(z)} \leq \frac{2}{2-\varepsilon} .
$$

Es fácil verificar la existencia de $N \in \mathbb{N}$ tal que, para todo $\omega \in \Omega$ y todo $i \geq 0$, la longitud del intervalo $h_{N+i}(\omega)(I)$ es menor o igual que $\varepsilon_{0}$. Afirmamos que (3.11) se cumple para $\bar{C}=\max \{A, \bar{A}\}$, donde

$$
A=\sup _{x \in I, n \leq N, \omega \in \Omega} \frac{h_{n}(\omega)^{\prime}(x)(2-\varepsilon)^{n}}{B}, \bar{A}=\sup _{x, y \in I, \omega \in \Omega} \frac{h_{N}(\omega)^{\prime}(x)}{h_{N}(\omega)^{\prime}(y)|I|}\left(\frac{2-\varepsilon}{2}\right)^{N} .
$$


En efecto, si $n \leq N$ entonces (3.11) se cumple debido a la condición $\bar{C} \geq A$. Para $n>N$ fijemos $y=y(n) \in I$ tal que $\left|h_{n}(\omega)(I)\right|=h_{n}(\omega)^{\prime}(y)|I|$. Para $x \in I$ la distancia entre los puntos $h_{N+i}(\omega)(x)$ y $h_{N+i}(\omega)(y)$ es menor o igual que $\varepsilon_{0}$ para todo $i \geq 0$. Luego, por (3.12),

$$
\begin{aligned}
\frac{h_{n}(\omega)^{\prime}(x)}{h_{n}(\omega)^{\prime}(y)} & =\frac{h_{N}(\omega)^{\prime}(x)}{h_{N}(\omega)^{\prime}(y)} \frac{g_{N+1}^{\prime}\left(h_{N}(\omega)(x)\right)}{g_{N+1}^{\prime}\left(h_{N}(\omega)(y)\right)} \cdots \frac{g_{n}^{\prime}\left(h_{n-1}(\omega)(x)\right)}{g_{n}^{\prime}\left(h_{n-1}(\omega)(y)\right)} \\
& \leq \frac{h_{N}(\omega)^{\prime}(x)}{h_{N}(\omega)^{\prime}(y)}\left(\frac{2}{2-\varepsilon}\right)^{n-N},
\end{aligned}
$$

por lo que

$$
\begin{aligned}
h_{n}(\omega)^{\prime}(x) & \leq \frac{h_{N}(\omega)^{\prime}(x)}{h_{N}(\omega)^{\prime}(y)} \frac{\left|h_{n}(\omega)(I)\right|}{|I|}\left(\frac{2}{2-\varepsilon}\right)^{n-N} \\
& \leq \frac{h_{N}(\omega)^{\prime}(x)}{h_{N}(\omega)^{\prime}(y)} \frac{B}{|I| 2^{n}}\left(\frac{2}{2-\varepsilon}\right)^{n-N} \leq \frac{B \bar{C}}{(2-\varepsilon)^{n}},
\end{aligned}
$$

donde la última desigualdad se desprende de la condición $\bar{C} \geq \bar{A}$.

Para verificar la persistencia de la contracción diferenciable más allá del intervalo $I$ haremos uso de un argumento "dual" al precedente. Fijemos una constante $\varepsilon_{1}>0$ suficientemente pequeña de modo que para todo $y, z$ en $[a, d]$ a distancia menor o igual que $\varepsilon_{1}$ se tenga

$$
\frac{f^{\prime}(y)}{f^{\prime}(z)} \leq \frac{2-\varepsilon}{2-2 \varepsilon} \quad \text { y } \quad \frac{g^{\prime}(y)}{g^{\prime}(z)} \leq \frac{2-\varepsilon}{2-2 \varepsilon} .
$$

Lema 3.25. Sean $C \geq 1, \omega=\left(g_{1}, g_{2}, \ldots\right) \in \Omega y x \in[a, d]$ tales que

$$
h_{n}(\omega)^{\prime}(x) \leq \frac{C}{(2-\varepsilon)^{n}} \quad \text { para todo } \quad n \geq 0 .
$$

Si $y \in[a, d]$ es tal que $\operatorname{dist}(x, y) \leq \varepsilon_{1} / C \quad$ y $n \geq 0$ entonces

$$
h_{n}(\omega)^{\prime}(y) \leq \frac{C}{(2-2 \varepsilon)^{n}} .
$$

Demostración. Verificaremos la desigualdad (3.15) por inducción. Para $n=0$ ella tiene lugar debido a la condición $C \geq 1$. Admitamos que ella sea válida para todo $j \in\{0, \ldots, n\}$, y hagamos $y_{j}=h_{j}(\omega)(y)$ y $x_{j}=h_{j}(\omega)(y)$. Supongamos que $y \leq x$ (el caso $y \geq x$ es análogo). Cada punto $y_{j}$ pertenece entonces al intervalo $h_{j}(\omega)\left(\left[x-\varepsilon_{1} / C, x\right]\right)$. Por la hipótesis de inducción,

$$
\left|h_{j}(\omega)\left(\left[x-\varepsilon_{1} / C, x\right]\right)\right| \leq \frac{C}{(2-2 \varepsilon)^{j}}\left|\left[x-\varepsilon_{1} / C, x\right]\right| \leq C \frac{\varepsilon_{1}}{C}=\varepsilon_{1} .
$$


De la definición de la constante $\varepsilon_{1}$ se concluye que para todo $j \leq n$ se tiene $g_{j+1}^{\prime}\left(y_{j}\right) \leq g_{j+1}^{\prime}\left(x_{j}\right)\left(\frac{2-\varepsilon}{2-2 \varepsilon}\right)$. Por lo tanto, debido a la hipótesis $(3.14)$,

$$
\begin{aligned}
h_{n+1}(\omega)^{\prime}(y) & =g_{1}^{\prime}\left(y_{0}\right) \cdots g_{n+1}^{\prime}\left(y_{n}\right) \leq g_{1}^{\prime}\left(x_{0}\right) \cdots g_{n+1}^{\prime}\left(x_{n}\right)\left(\frac{2-\varepsilon}{2-2 \varepsilon}\right)^{n+1} \\
& \leq \frac{C}{(2-\varepsilon)^{n+1}}\left(\frac{2-\varepsilon}{2-2 \varepsilon}\right)^{n+1} \leq \frac{C}{(2-2 \varepsilon)^{n+1}},
\end{aligned}
$$

lo cual concluye la verificación por inducción de (3.15).

Estamos ahora en condiciones de completar la demostración de la proposición 3.23. Para ello notemos que, por el lema 3.24, si $C$ es suficientemente grande entonces la probabilidad del conjunto

$$
\Omega(C, \varepsilon)=\left\{\omega \in \Omega: h_{n}(\omega)^{\prime}(x) \leq \frac{C}{(2-\varepsilon)^{n}} \text { para todo } n \geq 0 \text { y todo } x \in I\right\}
$$

es positiva. Fijemos un tal $C \geq 1$, hagamos $\ell=\min \left\{\varepsilon_{1} / 2 C,|I| / 2\right\}$, y designemos por $J$ la $2 \ell$-vecindad de $I$. El lema 3.25 implica que para todo $\omega \in \Omega(C, \varepsilon)$, todo $n \geq 0$ y todo $y \in J$,

$$
h_{n}(\omega)^{\prime}(y) \leq \frac{C}{(2-2 \varepsilon)^{n}} .
$$

Si $J^{\prime}$ designa la $\ell$-vecindad del intervalo $I$ entonces la probabilidad del conjunto $\left\{\omega: h_{n}(\omega)(I) \subset J^{\prime} \backslash I\right.$ infinitas veces $\}$ es igual a 1 . En consecuencia, y dado que $\varepsilon<1 / 2$, existen $\omega \in \Omega(C, \varepsilon)$ y $m \in \mathbb{N}$ tales que $h_{m}(\omega)(I) \subset J^{\prime} \backslash I$ y $(2-2 \varepsilon)^{m}>C$. Por (3.16), y puesto que $\ell \leq|I| / 2$, esto implica que $h_{m}(\omega)$ envía $J$ sobre una de las dos componentes conexas de $J \backslash I$ de manera tal que $h_{m}(\omega)$ posee un punto fijo en dicha componente, el cual es hiperbólico (y pertenece a $\Lambda$ ). La prueba de la proposición 3.23 queda así concluida.

A partir de lo que precede vemos que para probar el teorema 3.22 basta con verificar que todo pseudo-grupo de difeomorfismos unidimensionales y de clase $\mathrm{C}^{1} \sin$ probabilidad invariante posee elementos $f$ y $h$ que satisfacen las propiedades (i) y (ii) del comienzo de esta sección (compare con la proposición 2.54). Si bien esto es verdad incluso para pseudo-grupos de homeomorfismos unidimensionales, la demostración involucra técnicas que no queremos introducir en estas notas para no oscurecer la idea esencial de la prueba: el lector interesado puede hallar la demostración completa en [54]. En todo caso, para el caso de grupos actuando sobre el círculo sin probabilidad invariante, la obtención de tales elementos es relativamente sencilla: para acciones minimales ella se deduce directamente del teorema de Hölder, mientras que para acciones con un minimal excepcional se deduce de este mismo teorema aplicado a la acción sobre el círculo topológico obtenido tras colapsar las componentes conexas del complemento de dicho 
minimal. En la sección siguiente veremos sin embargo que para grupos de difeomorfismos de clase $\mathrm{C}^{1}$ del círculo vale una versión considerablemente más fuerte del teorema: dichos grupos possen elementos cuyos puntos fijos (existen y) son todos hiperbólicos.

Ejercicio 3.26. Anticipando el resultado principal de la sección siguiente, pruebe que todo grupo $\Gamma$ de difeomorfismos de clase $C^{1+l i p}$ del círculo sin probabilidad invariante contiene elementos con al menos $2 d(\Gamma)$ puntos fijos hiperbólicos, la mitad de ellos contractantes y la otra mitad dilatantes.

Sugerencia. Considere el caso minimal y fuertemente expansivo (el caso general se reduce sin mayor problema a éste). Pruebe que $\Gamma$ contiene dos elementos $f$ y $g$ de modo que para cierto intervalo $I=[a, b]$ se tiene $g(I) \cup g^{-1}(I) \subset \mathrm{S}^{1} \backslash I$, $\lim _{n \rightarrow \infty} f^{n}(x)=b$ y $\lim _{n \rightarrow \infty} f^{-n}(x)=a$ para todo $x \in \mathrm{S}^{1} \backslash I$. Para $\varepsilon>0$ suficientemente pequeño aplique (una ligera modificación de) la técnica de Schwartz a las sucesiones de composiciones $\left(f^{n} g\right)$ y $\left(g^{-1} f^{-n}\right)$ sobre los intervalos $[a, a+\varepsilon]$ y $g^{-1}([b-\varepsilon, b])$.

Notemos que la hipótesis de no existencia de medida de probabilidad invariante asumida a lo largo de esta sección es necesaria para la validez del teorema 3.22. Un contra-ejemplo "poco interesante" lo constituye (el grupo generado por) un difeomorfismo con puntos fijos indiferentes. Contra-ejemplos mucho más interesantes vienen dados por difeomorfismos de clase $\mathrm{C}^{1}$ (o mejor aún de clase $\mathrm{C}^{1+\tau}$ ) del círculo con número de rotación irracional y órbitas no densas (en tal caso el soporte de la medida invariante coincide con conjunto de Cantor invariante; vea la sección 1.4 del capítulo 4 para la construcción de tales difeomorfismos). Estos últimos ejemplos muestran que el enunciado del teorema 3.15 no es válido para grupos de difeomorfismos de clase $\mathrm{C}^{1+\tau}$ del círculo. El caso $\mathrm{C}^{1+\text { va }}$ es sin embargo especial. Es muy probable que la proposición a seguir sea válida de manera más general para pseudo-grupos de difeomorfismos de clase $\mathrm{C}^{1+v a}$ y que preservan un minimal excepcional (compare con [59]).

Proposición 3.27. Si $\Gamma$ es un grupo finitamente generado de difeomorfismos de clase $\mathrm{C}^{1+\mathrm{va}}$ del círculo que preserva un minimal excepcional, entonces $\Gamma$ posee elementos con puntos fijos hiperbólicos.

Demostración. Basta probar que el grupo $\Gamma$ no puede preservar una medida de probabilidad, pues esto permite aplicar el teorema 3.22. Ahora bien, si $\Gamma$ preserva una medida de probabilidad, entonces es semiconjugado a un grupo de rotaciones. Como suponemos que $\Gamma$ es finitamente generado, al menos uno de sus generadores debiese tener número de rotación irracional. Sin embargo, la existencia de dicho elemento está en contradicción con el teorema de Denjoy.

Como ya adelantamos, una consecuencia del resultado a ser presentado en la sección a venir es que, bajo las hipótesis de la proposición 3.27, $\Gamma$ posee elementos cuyos puntos fijos (existen y) son todos hiperbólicos. 


\subsection{Una versión $\mathrm{C}^{1}$ vía exponentes de Lyapunov}

Para grupos de difeomorfismos de clase $\mathrm{C}^{1}$ del círculo es posible dar una versión global y optimal del teorema de Sacksteder. Recordemos que cada grupo $\Gamma$ de homeomorfismos del círculo sin probabilidad invariante tiene naturalmente asociado un grado $d(\Gamma) \in \mathbb{N}$ (vea la sección 3.1, capítulo 2).

Teorema 3.28. Si $\Gamma$ es un subgrupo finitamente generado de Difeo ${ }_{+}^{1}\left(\mathrm{~S}^{1}\right)$ sin medida de probabilidad invariante, entonces $\Gamma$ posee elementos cuyos puntos fijos son todos hiperbólicos. De manera más precisa, $\Gamma$ contiene elementos con $2 d(\Gamma)$ puntos fijos, la mitad de ellos hiperbólicamente contractantes y la otra mitad hiperbólicamente dilatantes.

Para la prueba de este teorema los métodos probabilísticos serán nuevamente muy útiles. Sea $\Gamma$ un subgrupo finitamente generado de Difeo ${ }_{+}^{1}\left(\mathrm{~S}^{1}\right)$ provisto de una medida de probabilidad $p$ no degenerada y de soporte finito. Sea $\mu$ una probabilidad estacionaria (respecto a $p$ ) sobre $\mathrm{S}^{1}$, y consideremos la transformación $T$ de $\Omega \times \mathrm{S}^{1}$ dada por

$$
T(\omega, x)=\left(\sigma(\omega), h_{1}(\omega)(x)\right) .
$$

Como $T$ preserva $\mathbb{P} \times \mu$, una aplicación directa del teorema ergódico de Birkhoff [126] a la función $(\omega, x) \mapsto \log \left(h_{1}(\omega)^{\prime}(x)\right)$ muestra que, para $\mu$ casi todo punto $x \in \mathrm{S}^{1}$ y para $\mathbb{P}$-casi todo camino aleatorio $\omega$ en $\Omega$, el límite siguiente existe:

$$
\lambda_{(\omega, x)}(\mu)=\lim _{n \rightarrow \infty} \frac{\log \left(h_{n}(\omega)^{\prime}(x)\right)}{n} .
$$

Dicho valor será llamado exponente de Lyapunov de $(\omega, x)$. El siguiente teorema ergódico aleatorio de Kakutani será esencial en lo que sigue (el lector puede consultar [66] ó [111] para mayores detalles).

Teorema 3.29. Si la medida de probabilidad estacionaria $\mu$ es ergódica, es decir, si ella no puede ser escrita como una combinación convexa no trivial de dos medidas estacionarias distintas, entonces la transformación $T$ es ergódica respecto a $\mathbb{P} \times \mu$.

Demostración. Sea $\psi: \Omega \times \mathrm{S}^{1} \rightarrow \mathbb{R}$ una función integrable e invariante por $T$. Debemos probar que $\psi$ es casi ciertamente constante. Para cada $n \geq 0$ denotemos $\psi_{n}$ la esperanza condicional de $\psi$ dadas las primeras $n$ entradas de $\omega \in \Omega$. Tenemos entonces

$$
\psi_{0}(\omega, x)=\mathbb{E}\left(\psi_{1}\right)(\omega, x) .
$$

Como $\psi$ es invariante por $T$, para cada $n \in \mathbb{N}$ tenemos

$$
\psi(\omega, x)=\psi\left(\sigma^{n}(\omega), h_{n}(\omega)(x)\right),
$$


de donde se deduce fácilmente que

$$
\psi_{n}(\omega, x)=\psi_{n-1}\left(\sigma(\omega), h_{1}(\omega)(x)\right)=\ldots \ldots=\psi_{0}\left(\sigma^{n}(\omega), h_{n}(\omega)(x)\right) .
$$

Concluimos entonces que

$$
\psi_{0}(\omega, x)=\mathbb{E}\left(\psi_{0}\right)\left(\sigma(\omega), h_{1}(\omega)(x)\right) .
$$

Ahora bien, puesto que la función $\psi_{0}(\omega, x)$ no depende de $\omega$, podemos escribir $\psi_{0}(\omega, x)=\bar{\psi}(x)$, y la igualdad anterior nos indica que $\bar{\psi}$ es invariante por el operador de difusión. Afirmamos que esto implica que la función $\bar{\psi}$ es (casi ciertamente) constante (respecto a $\mu$ ). Antes de verificar esto notemos que, debido a (3.17), esta afirmación implica que todas las funciones $\psi_{n}$ son constantes, por lo que la función límite $\psi$ también es constante, concluyendo así la prueba de la ergodicidad de $T$.

Para probar que $\bar{\psi}$ es constante supongamos lo contrario y escojamos $c \in \mathbb{R}$ de modo que los conjuntos $S_{+}=\{\bar{\psi} \geq c\}$ y $S_{-}=\{\bar{\psi}<c\}$ tengan $\mu$-medida positiva. La invariancia por difusión de $\bar{\psi}$ y de $\mu$ permiten verificar sin mayor dificultad que dichos conjuntos son invariantes por la acción. Ello se traduce en el hecho que $\mu=\mu \mathcal{X}_{S_{+}}+\mu \mathcal{X}_{S_{-}}$es una descomposición convexa no trivial de la medida estacionaria $\mu$, contradiciendo nuestra hipótesis.

Si $T$ es ergódica entonces el exponente de Lyapunov es $\mathbb{P} \times \mu$-casi constante e igual a

$$
\lambda(\mu)=\int_{\Omega} \int_{\mathrm{S}^{1}} \log \left(h_{1}(\omega)^{\prime}(x)\right) d \mu(x) d \mathbb{P}(\omega)=\int_{\Gamma} \int_{\mathrm{S}^{1}} \log \left(g^{\prime}(x)\right) d \mu(x) d p(g) .
$$

Notemos por otra parte que si $p$ es simétrica y la medida estacionaria $\mu$ es invariante por la acción de $\Gamma$, entonces el exponente de Lyapunov $\lambda_{(\omega, x)}(\mu)$ es nulo para casi todo $(\omega, x)$. En efecto, si $\mu$ es invariante entonces la transformación de $\Omega \times \mathrm{S}^{1}$ definida por $(\omega, x) \mapsto\left(\omega, h_{1}(\omega)^{-1}(x)\right)$ preserva $\mathbb{P} \times \mu$. Dada la simetría de $p$, esto implica que $\lambda(\mu)$ coincide con

$$
\begin{aligned}
& \int_{\Omega} \int_{\mathrm{S}^{1}} \log \left(h_{1}(\omega)^{\prime}(x)\right) d \mu(x) d \mathbb{P}(\omega)=\int_{\Omega} \int_{\mathrm{S}^{1}} \log \left(h_{1}(\omega)^{\prime}\left(h_{1}(\omega)^{-1}(x)\right)\right) d \mu(x) d \mathbb{P}(\omega) \\
& =-\int_{\Omega} \int_{\mathrm{S}^{1}} \log \left(\left(h_{1}(\omega)^{-1}\right)^{\prime}(x)\right) d \mu(x) d \mathbb{P}(\omega)=-\int_{\Gamma} \int_{\mathrm{S}^{1}} \log \left(\left(g^{-1}\right)^{\prime}(x)\right) d \mu(x) d p(g),
\end{aligned}
$$

es decir, $\lambda(\mu)=-\lambda(\mu)$. En conclusión, si $\mu$ es invariante y ergódica, entonces su exponente de Lyapunov es nulo. El caso general se deduce por un argumento estándar de descomposición ergódica [66, 111].

La proposición siguiente (la cual admite una versión general para foliaciones de codimensión 1; vea [53]) puede ser vista como una suerte de recíproca de la observación precedente. 
Proposición 3.30. Si p es (no degenerada, a soporte finito y) simétrica y $\Gamma$ no preserva ninguna medida de probabilidad sobre el círculo, entonces el exponente de Lyapunov de la única medida estacionaria es negativo.

Antes de dar la prueba de esta proposición explicaremos cómo obtener el teorema 3.28 a partir de ella. Supongamos por ejemplo que la acción de $\Gamma$ sea minimal y verifique la propiedad de expansividad fuerte (i.e., $d(\Gamma)=1$; vea la sección 3.1 del capítulo 2). En tal caso, sabemos que para todo $\omega$ perteneciente a un subconjunto $\Omega^{*}$ de probabilidad total de $\Omega$, el coeficiente de contracción contr $\left(h_{n}(\omega)\right)$ converge a 0 cuando $n$ tiende al infinito. Esto se traduce por el hecho que para todo $\omega \in \Omega^{*}$ existen dos intervalos cerrados $I_{n}(\omega)$ y $J_{n}(\omega)$ cuyas longitudes tienden a 0 y $h_{n}(\omega)\left(\overline{\mathrm{S}^{1} \backslash I_{n}(\omega)}\right)=J_{n}(\omega)$. Además, $I_{n}(\omega)$ converge a un punto $\varsigma_{+}(\omega)$, de modo que la aplicación $\varsigma_{+}: \Omega^{*} \rightarrow \mathrm{S}^{1}$ así definida es medible. Si $I_{n}(\omega)$ y $J_{n}(\omega)$ son disjuntos entonces los puntos fijos de $h_{n}(\omega)$ están en el interior de estos dos intervalos. Para demostrar la unicidad y la hiperbolicidad de al menos el punto fijo situado en $J_{n}(\omega)$ (i.e., del punto fijo contractante), es natural tratar de usar la negatividad del exponente de Lyapunov. Sin embargo, se presentan dos dificultades técnicas: los intervalos $I_{n}(\omega)$ y $J_{n}(\omega)$ no son necesariamente disjuntos, y la "rapidez" de contracción local depende del punto inicial $(\omega, x)$. Para soslayar el primer problema basta observar que los "tiempos" $n \in \mathbb{N}$ para los cuales $I_{n}(\omega) \cap J_{n}(\omega) \neq \emptyset$ son muy esporádicos (la densidad de este conjunto de enteros es genéricamente igual a 0$)$. La segunda dificultad es superada usando el hecho que la transformación $T$ es ergódica, lo cual implica que casi todo punto inicial $(\omega, x)$ caerá en cierto momento sobre un punto para el cual la rapidez de contracción está bien controlada. Remarquemos finalmente que, para probar la unicidad e hiperbolicidad del punto fijo dilatante, es necesario aplicar un argumento análogo al que precede a las composiciones de los inversos y en el orden opuesto (compare con el ejercicio 3.26). Para ello se puede pasar a las distribuciones en tiempo finito. En efecto, como la probabilidad $p$ es supuesta simétrica, las distribuciones de ambos procesos iterativos aleatorios coinciden para todo tiempo finito.

Si la acción es minimal y $d(\Gamma)>1$ entonces los argumentos precedentes pueden ser aplicados "pasando a un recubrimiento finito". Finalmente, si hay un minimal excepcional, entonces podemos razonar de manera análoga "sobre dicho minimal". La formalización de estas ideas no representa mayor dificultad técnica, y no desarrollaremos en detalle la prueba para no oscurecer nuestra presentación: el lector interesado puede consultar [54]. Señalemos en todo caso que de los argumentos contenidos en dicho artículo se desprende que un enunciado conciso del teorema queda plasmado en la afirmación siguiente: si para $d \in \mathbb{N}$ designamos por $\mathrm{D}_{d}\left(\mathrm{~S}^{1}\right)$ al conjunto de los difemorfismos de clase $\mathrm{C}^{1}$ del círculo que poseen exactamente $2 d$ puntos periódicos y todos ellos son hiperbólicos, entonces

$$
\mathbb{P}\left[\lim _{n \rightarrow \infty} \frac{1}{N} \operatorname{card}\left\{n \in\{1, \ldots, N\}: h_{n}(\omega) \in \mathrm{D}_{d(\Gamma)}\left(\mathrm{S}^{1}\right)\right\}=1\right]=1 .
$$


Ejercicio 3.31. Siguiendo (parte de) los argumentos precedentes, pruebe que todo grupo $\Gamma$ de homeomorfismos de $\mathrm{S}^{1}$ sin probabilidad invariante contiene elementos con al menos $2 d(\Gamma)$ puntos fijos. Dé un ejemplo de un tal grupo de modo que, para todo elemento que admita puntos fijos, el número de tales puntos sea estrictamente mayor a $2 d(\Gamma)$.

Observación. Ignoramos si el grupo de los homeomorfismos afines por partes del círculo contiene ejemplos como los pedidos anteriormente.

Pasemos ahora a la demostración de la proposición 3.30. Notemos

$$
\psi(x)=\int_{\Gamma} \log \left(g^{\prime}(x)\right) d p(g)
$$

y supongamos por contradicción que $\lambda(\mu) \geq 0$, es decir

$$
\int_{\mathrm{S}^{1}} \psi(x) d \mu(x) \geq 0 .
$$

Probaremos en tal caso que $\Gamma$ preserva una medida de probabilidad, contradiciendo así nuestra hipótesis. Para esto nos valdremos de un lema inspirado de la teoría de los ciclos foliados de Sullivan [195] (vea también [74]). Recordemos que el laplaciano $\Delta \zeta$ de un función real y continua $\zeta$ es definido por $\Delta \zeta=D \zeta-\zeta$, donde $D$ denota el operador de difusión (2.9). La función $\psi$ es armónica si su laplaciano es idénticamente nulo (compare con el ejercicio 2.90).

Lema 3.32. Bajo la hipótesis (3.18), existe una sucesión de funciones continuas $\zeta_{n}$ definidas sobre el círculo tales que, para todo entero $n \in \mathbb{N}$ y todo punto $x \in \mathrm{S}^{1}$,

$$
\psi(x)+\Delta \zeta_{n}(x) \geq-\frac{1}{n}
$$

Demostración. Notemos $C\left(\mathrm{~S}^{1}\right)$ el espacio de las funciones continuas sobre el círculo. Sea $E$ el subespacio formado por las funciones que aparecen como el laplaciano de alguna función de $C\left(\mathrm{~S}^{1}\right)$, y sea $C_{+}$el cono convexo de las funciones no negativas. Debemos probar que si $\psi$ satisface (3.18), entonces su imagen por la proyección $\pi: C\left(\mathrm{~S}^{1}\right) \rightarrow C\left(\mathrm{~S}^{1}\right) / \bar{E}$ está contenida en $\pi\left(C_{+}\right)$. Supongamos que éste no sea el caso. En tal situación, el teorema de separación de Hahn-Banach muestra la existencia de un funcional lineal continuo $\overline{\mathrm{I}}: C\left(\mathrm{~S}^{1}\right) / \bar{E} \rightarrow \mathbb{R}$ tal que $\overline{\mathrm{I}}(\pi(\psi))<0 \leq \overline{\mathrm{I}}(\pi(\eta))$ para toda $\eta \in C_{+}$. Evidentemente, $\overline{\mathrm{I}}$ induce un funcional continuo I $: C\left(\mathrm{~S}^{1}\right) \rightarrow \mathbb{R}$ idénticamente nulo sobre $E$ y tal que $\mathrm{I}(\psi)<0 \leq \mathrm{I}(\eta)$ para toda $\eta \in C_{+}$. Afirmamos que existe una constante $c \in \mathbb{R}$ tal que $\mathrm{I}=c \mu$ (estamos identificando las medidas de probabilidad a los funcionales lineales que ellas definen sobre el espacio de las funciones continuas por integración). Para probar esto comencemos observando que, como I es nulo sobre $E$, para toda $\zeta \in C\left(\mathrm{~S}^{1}\right)$ se tiene

$$
\langle D \mathrm{I}, \zeta\rangle=\langle\mathrm{I}, D \zeta\rangle=\langle\mathrm{I}, \Delta \zeta+\zeta\rangle=\langle\mathrm{I}, \zeta\rangle,
$$


es decir, I es invariante por (el operador dual a) la difusión. Supongamos que la descomposición de Hahn de I pueda ser expresada de la forma $\mathrm{I}=\alpha \nu_{1}-\beta \nu_{2}$, donde $\nu_{1}$ y $\nu_{2}$ son medidas de probabilidad a soportes disjuntos, $\alpha>0$ y $\beta>0$. En tal caso la igualdad $D I=$ I y la unicidad de la descomposición de Hahn de DI muestran que $\nu_{1}$ y $\nu_{2}$ también son invariantes por la difusión. En consecuencia, $\nu_{1}=\nu_{2}=\mu$, lo cual contradice el hecho que los soportes de $\nu_{1}$ y $\nu_{2}$ son disjuntos. El funcional I se expresa entonces de la forma $\mathrm{I}=c \nu$ para alguna medida de probabilidad $\nu$ del círculo, y la igualdad $\mathrm{I}=D \mathrm{I}$ implica que $\nu=\mu$.

Observemos ahora que, como

$$
0>\mathrm{I}(\psi)=c \mu(\psi)=c \int_{\mathrm{S}^{1}} \psi(x) d \mu(x),
$$

la hipótesis (3.18) implica que $\mu(\psi)>0$ y $c<0$. Por otro lado, la función constante e igual a 1 pertenece a $C_{+}$, por lo que $c=\mathrm{I}(1) \geq 0$. Esta contradicción concluye la demostración.

Volviendo a la prueba de la proposición 3.30 comencemos observando que, sumando una constante $c_{n}$ a cada $\zeta_{n}$ si es necesario, podemos suponer que la integral de $\exp \left(\zeta_{n}\right)$ es igual a 1 para todo $n \in \mathbb{N}$. Consideremos las medidas de probabilidad $\nu_{n}$ sobre el círculo definidas por

$$
\frac{d \nu_{n}(s)}{d s}=\exp \left(\zeta_{n}(s)\right)
$$

y fijemos una subsucesión $\nu_{n_{i}}$ que converja a una medida de probabilidad $\nu$ sobre $\mathrm{S}^{1}$. Probaremos que esta medida $\nu$ es invariante por $\Gamma$.

Verifiquemos primeramente que $\nu$ es una medida estacionaria. Para ello, notemos que si designamos por $J a c_{n}(g)$ la derivada de Radon-Nikodym de $g \in \Gamma$ respecto a $\nu_{n}$, entonces la relación (3.19) implica, para todo $x \in \mathrm{S}^{1}$,

$$
\begin{aligned}
\int_{\Gamma} \log \left(J a c_{n}(g)(x)\right) d p(g) & =\int_{\Gamma} \log \left(g^{\prime}(x)\right) d p(g)+\int_{\Gamma}\left[\zeta_{n}(g(x))-\zeta_{n}(x)\right] d p(g) \\
& =\psi(x)+\Delta \zeta_{n}(x) \geq-\frac{1}{n} .
\end{aligned}
$$

Observemos ahora que, puesto que el (dual del) operador de difusión actúa continuamente en el espacio de las medidas de probabilidad del círculo, la sucesión de medidas $D \nu_{n_{i}}$ converge débilmente hacia la medida $D \nu$. Ahora bien, la difusión de $\nu_{n}$ es una medida absolutamente continua respecto a $\nu_{n}$ cuya densidad se expresa de la forma

$$
\frac{d D \nu_{n}(x)}{d \nu_{n}(x)}=\int_{\Gamma} J a c_{n}\left(g^{-1}\right)(x) d p(g)=\int_{\Gamma} J a c_{n}(g)(x) d p(g) .
$$

Por la concavidad de la función logaritmo tenemos

$$
\frac{d D \nu_{n_{i}}(x)}{d \nu_{n_{i}}(x)} \geq \exp \left(\int_{\Gamma} \log \left(J a c_{n_{i}}(g)(x)\right) d p(g)\right) \geq \exp \left(-1 / n_{i}\right),
$$


es decir, $D \nu_{n_{i}} \geq \exp \left(-1 / n_{i}\right) \nu_{n_{i}}$. Pasando al límite obtenemos $D \nu \geq \nu, \mathrm{y}$ como $\nu$ y $D \nu$ tienen la misma masa total (finita), ellas son iguales: $\nu$ es por lo tanto estacionaria.

Estamos ahora en condiciones de completar la prueba de la invariancia de $\nu$. Para ello, fijemos un intervalo $J$ tal que $\nu(J)>0$ y consideremos las funciones $\left.\left.\psi_{n, J}: \Gamma \rightarrow\right] 0,1\right]$ definidas por $\psi_{n, J}(g)=\nu_{n}(g(J))$. De las igualdades

$\Delta \log \left(\psi_{n, J}\right)(i d)=\int_{\Gamma} \log \left(\frac{\nu_{n}(g(J))}{\nu_{n}(J)}\right) d p(g)=\int_{\Gamma} \log \left(\int_{J} J a c_{n}(g)(x) \frac{d \nu_{n}(x)}{\nu_{n}(J)}\right) d p(g)$

se deduce que

$$
\begin{aligned}
\Delta \log \left(\psi_{n, J}\right)(i d) & \geq \int_{\Gamma}\left(\int_{J} \log \left(J a c_{n}(g)(x)\right) \frac{d \nu_{n}(x)}{\nu_{n}(J)}\right) d p(g) \\
& =\int_{J}\left(\int_{\Gamma} \log \left(J a c_{n}(g)(x)\right) d p(g)\right) \frac{d \nu_{n}(x)}{\nu_{n}(J)} \geq-\frac{1}{n} .
\end{aligned}
$$

Notemos que esto es válido para todo intervalo $J$ satisfaciendo $\nu(J)>0$. Debido a las relaciones $\psi_{n, J}(g f)=\psi_{n, f(J)}(g)$, esto implica que el laplaciano de $\log \left(\psi_{n, J}\right)$ está minorado por $-1 / n$ en todo elemento de $\Gamma$. Por lo tanto, si $\psi_{J}$ es el límite de la sucesión de funciones $\psi_{n_{i}, J}$, es decir, $\psi_{J}(g)=\nu(g(J))$, entonces la función $\log \left(\psi_{J}\right)$ es supra-armónica (i.e su laplaciano es no negativo). Por otra parte, puesto que $p$ es simétrica, $\psi_{J}$ es armónica. En conclusión, para todo elemento $f \in \Gamma$ las desigualdades que figuran más abajo deben ser igualdades:

$\log \left(\psi_{J}\right)(f) \leq \int_{\Gamma} \log \left(\psi_{J}\right)(g f) d p(g) \leq \log \left(\int_{\Gamma} \psi_{J}(g f) d \mu(g)\right)=\log \left(\psi_{J}\right)(f)$.

Lo anterior implica que la función $\psi_{J}$ es constante. Ahora bien, como el intervalo verificando $\nu(J)>0$ era arbitrario, deducimos que la medida $\nu$ es invariante por todos los elementos de $\Gamma$. La proposición 3.30 queda así demostrada.

\section{Primer teorema de Duminy: no existencia de minimal excepcional}

\subsection{Presentación del resultado}

Los subgrupos de un grupo no discreto generados por elementos cercanos a la identidad tienden a tener una dinámica sencilla. Un resultado clásico que ilustra este fenómeno es el lema de Zazenhäus, el cual estipula que en todo grupo de Lie existe una vecindad de la identidad tal que todo grupo discreto generado por elementos de dicha vecindad es nilpotente. 
Otro teorema bien conocido en la misma dirección es la desigualdad de Jørgensen, según la cual si $f$ y $g$ son elementos de $\operatorname{PSL}(2, \mathbb{R})$ que generan un grupo de segunda especie entonces

$$
\left|\operatorname{tr}^{2}(f)-4\right|+|\operatorname{tr}([f, g])-2|>1,
$$

donde $\operatorname{tr}$ es la traza de la matriz respectiva [10, 109]. En esta misma dirección, Marden [127] probó que existe una constante universal $\varepsilon_{0}>0$ tal que para todo grupo fuchsiano no elemental $\Gamma$, todo sistema de generadores $\mathcal{G}$ de $\Gamma$ y todo punto $u$ del disco de Poincaré, existe $g \in \mathcal{G}$ satisfaciendo $\operatorname{dist}(u, g(u)) \geq \varepsilon_{0}$. Este resultado fue posteriormente extendido por Margulis a los grupos discretos de isometrías de espacios hiperbólicos de dimensión arbitraria (la desigualdad de Margulis continúa siendo válida para el caso de espacios a curvatura negativa más generales: vea [8]).

Para el caso de grupos de difeomorfismos se dispone de una gama de resultados análogos interesantes, especialmente en el caso real-analítico [15, $56,76,173,174]$. Una de las fuentes de inspiración para ellos es un hermoso teorema obtenido por Duminy alrededor del año 1977, y cuya demostración nunca fue publicada por dicho autor.

Teorema 3.33. Existe una constante universal $V_{0}>0$ que verifica la siguiente propiedad: si $\Gamma$ es un subgrupo de Difeo ${ }_{+}^{1+\mathrm{va}}\left(\mathrm{S}^{1}\right)$ generado por un conjunto $\mathcal{G}$ (no necesariamente finito) de difeomorfismos tales que al menos uno de ellos tenga un número finito de puntos periódicos, y tales que $V(g)<V_{0}$ para todo $g \in \mathcal{G}$, entonces $\Gamma$ no admite minimal excepcional.

Observe que la condición $V(g)<V_{0}$ significa que las aplicaciones de $\mathcal{G}$ están cercanas a rotaciones: la igualdad $V(g)=0$ es verificada si y sólo si $g$ es una rotación. En cuanto a la hipótesis de existencia de un generador con puntos periódicos aislados, señalemos al menos que ella es "genéricamente" satisfecha [133]. Es muy probable que en realidad se trate sólo de una hipótesis técnica y que el teorema siga siendo válido sin ella; esto ocurre por ejemplo en el caso real-analítico (vea el ejercicio 3.39).

Recordemos que si $f$ es un difeomorfismo de clase $\mathrm{C}^{1+\text { va }}$ e $I$ es un intervalo, entonces $V(f ; I)$ designa la variación total del logaritmo de la derivada de la restricción de $f$ a $I$. Es importante insistir en que para todo $f$ y $g$ de clase $\mathrm{C}^{1+\text { va }}$ se tiene la desigualdad

$$
V(f \circ g ; I) \leq V(g ; I)+V(f ; g(I)),
$$

así como la igualdad

$$
V\left(f^{-1} ; I\right)=V\left(f ; f^{-1}(I)\right) .
$$

En particular, esta última relación implica $V(g)=V\left(g^{-1}\right)$. Por ello, no hay pérdida de generalidad si para la prueba del teorema 3.33 se supone 
que $\mathcal{G}$ es simétrico (es decir, $g^{-1}$ pertenece a $\mathcal{G}$ para todo $g \in \mathcal{G}$ ). Por otra parte, para todo par de intervalos $I_{1}$ e $I_{2}$ contenidos en $I$ se tiene

$$
\frac{\left|f\left(I_{2}\right)\right|}{\left|I_{2}\right|} e^{-V(f ; I)} \leq \frac{\left|f\left(I_{1}\right)\right|}{\left|I_{1}\right|} \leq \frac{\left|f\left(I_{2}\right)\right|}{\left|I_{2}\right|} e^{V(f ; I)} .
$$

Observemos también que si $f$ es un difeomorfismo del círculo entonces existe necesariamente un punto $p \in \mathrm{S}^{1}$ tal que $f^{\prime}(p)=1$, a partir de lo cual se concluye fácilmente que

$$
\inf _{x \in \mathrm{S}^{1}} f^{\prime}(x) \geq e^{-V(f)} \quad \mathrm{y} \quad \sup _{x \in \mathrm{S}^{1}} f^{\prime}(x) \leq e^{V(f)} .
$$

Notemos finalmente que de (3.1) y (3.23) se deduce que si $g \in \operatorname{Difeo}_{+}^{2}\left(\mathrm{~S}^{1}\right)$ satisface $\left|g^{\prime \prime}(x)\right|<\delta$ para todo $x \in \mathrm{S}^{1}$ entonces $V(g)<2 \pi \delta \exp (-\delta)$. Esto indica que la conclusión del teorema 3.33 se aplica para cierta constante universal $\delta_{0}$ a los subgrupos de Difeo ${ }_{+}^{2}\left(\mathrm{~S}^{1}\right)$ generados por elementos $g$ satisfaciendo $\left|g^{\prime \prime}(x)\right| \leq \delta_{0}$ para todo $x \in \mathrm{S}^{1}$ (al menos cuando uno de los generadores tiene un número finito de puntos periódicos). De la misma manera, el lector no debiese tener problemas en adaptar los argumentos dados a continuación para grupos de homeomorfismos afines por partes del círculo generados por elementos cercanos a rotaciones.

\subsection{Una aplicación de primer retorno dilatante}

La demostración del teorema de Duminy está basada en un trabajo de dicho autor sobre las órbitas semi-excepcionales de foliaciones de codimensión 1 que nosotros abordaremos más adelante. El lema que presentamos a continuación ha sido transcrito de un manuscrito no publicado de Duminy (vea sin embargo [147]).

Lema 3.34. Sean $a, b, b^{\prime}, c$ puntos de la recta tales que $a<c<b$ y $a<b^{\prime}<b$. Sean $f:[a, c] \rightarrow[a, b]$ y $g:[c, b] \rightarrow\left[a, b^{\prime}\right]$ dos difeomorfismos de clase $\mathrm{C}^{1+\mathrm{va}}$ tales que $f(x)>x$ para todo $x \neq a$ y $g(x)<x$ para todo $x$ (vea la figura 17). Supongamos que para ciertos $m, n$ en $\mathbb{N} y[u, v] \subset[c, b]$ la aplicación $\bar{H}=g^{-n} \circ f^{-m}$ está definida en todo $[u, v]$. Entonces se tiene la desigualdad

$$
\frac{\bar{H}(v)-\bar{H}(u)}{v-u} \leq \frac{\bar{H}(v)-f^{-1}(\bar{H}(v))}{v-f^{-1}(v)}\left(1-\frac{1}{\sup _{x \in[a, c]} f^{\prime}(x)}\right) e^{V(f ;[a, c])+V(g ;[c, b])} .
$$

Demostración. Denotemos por comodidad $\bar{f}=f^{-1}$ y $\bar{g}=g^{-1}$ (vea la figura 18). Recordemos que $V(\bar{f} ;[a, b])=V(f ;[a, c])$ y $V\left(\bar{g} ;\left[a, b^{\prime}\right]\right)=V(g ;[c, b])$. Debemos entonces probar la desigualdad

$$
\frac{\bar{H}(v)-\bar{H}(u)}{v-u} \leq \frac{\bar{H}(v)-\bar{f}(\bar{H}(v))}{v-\bar{f}(v)}\left(1-\inf _{x \in[a, b]} \bar{f}^{\prime}(x)\right) e^{V(\bar{f} ;[a, b])+V\left(\bar{g} ;\left[a, b^{\prime}\right]\right)} .
$$


Para este efecto, notemos en primer lugar que

$$
V\left(\bar{f}^{m} ;[\bar{f}(v), v]\right) \leq \sum_{k=0}^{m-1} V\left(\bar{f} ;\left[\bar{f}^{k-1}(v), \bar{f}^{k}(v)\right]\right) \leq V(\bar{f} ;[a, b]) .
$$

Como $\bar{f}(v) \leq c \leq u<v$, tenemos

$$
\begin{aligned}
\frac{\bar{f}^{m}(v)-\bar{f}^{m}(u)}{v-u} & \leq \frac{\bar{f}^{m}(v)-\bar{f}^{m+1}(v)}{v-\bar{f}(v)} e^{V\left(\bar{f}^{m} ;[\bar{f}(v), v]\right)} \\
& \leq \frac{\bar{f}^{m}(v)-\bar{f}^{m+1}(v)}{v-\bar{f}(v)} e^{V(\bar{f} ;[a, b])} .
\end{aligned}
$$

Además,

$$
\bar{f}^{m+1}(v)-a=\bar{f}\left(\bar{f}^{m}(v)\right)-\bar{f}(a) \geq\left(\bar{f}^{m}(v)-a\right) \cdot \inf _{x \in[a, b]} \bar{f}^{\prime}(x),
$$

de donde se obtiene

$$
\bar{f}^{m}(v)-\bar{f}^{m+1}(v) \leq\left(\bar{f}^{m}(v)-a\right)\left(1-\inf _{x \in[a, b]} \bar{f}^{\prime}(x)\right) .
$$

Se deduce entonces que

$$
\frac{\bar{f}^{m}(v)-\bar{f}^{m}(u)}{v-u} \leq \frac{\bar{f}^{m}(v)-a}{v-\bar{f}(v)}\left(1-\inf _{x \in[a, b]} \bar{f}^{\prime}(x)\right) e^{V(\bar{f} ;[a, b])} .
$$

Puesto que $a \leq \bar{f}^{m}(u)<\bar{f}^{m}(v) \leq c=\bar{g}(a)$ y $\bar{g}^{n}$ está definida en todo el intervalo $\left[\bar{f}^{m}(u), \bar{f}^{m}(v)\right]$, argumentos análogos muestran que

$$
\frac{\bar{g}^{n}\left(\bar{f}^{m}(v)\right)-\bar{g}^{n}\left(\bar{f}^{m}(u)\right)}{\bar{f}^{m}(v)-\bar{f}^{m}(u)} \leq \frac{\bar{g}^{n}\left(\bar{f}^{m}(v)\right)-\bar{g}^{n}(a)}{\bar{f}^{m}(v)-a} e^{V\left(\bar{g} ;\left[a, b^{\prime}\right]\right)} .
$$

De (3.26) y (3.27) se concluye que $(\bar{H}(v)-\bar{H}(u)) /(v-u)$ está acotado por

$$
\frac{\bar{H}(v)-\bar{f}(\bar{H}(v))}{v-\bar{f}(v)} \frac{\bar{H}(v)-\bar{g}(a)}{\bar{H}(v)-\bar{f}(\bar{H}(v))}\left(1-\inf _{x \in[a, b]} \bar{f}^{\prime}(x)\right) e^{V(\bar{f} ;[a, b])+V\left(\bar{g} ;\left[a, b^{\prime}\right]\right)} .
$$

La desigualdad (3.25) se desprende inmediatamente de lo anterior, gracias a que $\bar{f}(\bar{H}(v)) \leq c=\bar{g}(a)$.

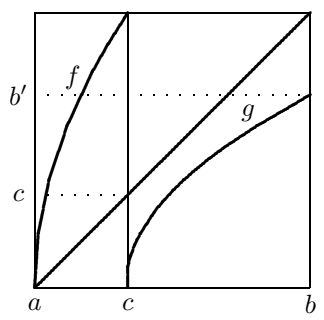

Figura 17

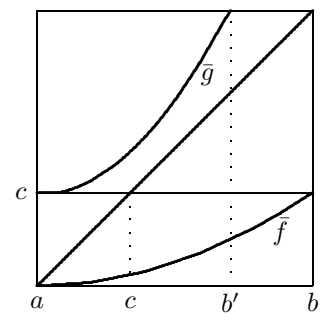

Figura 18 
Dadas dos aplicaciones $f, g$ como las del lema 3.34, para cada $x \in] c, b]$ existe un entero positivo $n=n(x)$ tal que $\left.\left.g^{n-1}(x) \in\right] c, b\right]$ y $\left.\left.g^{n}(x) \in\right] a, c\right]$. Análogamente, para $y \in] a, c]$ existe $m=m(y)$ tal que $\left.\left.f^{m-1}(y) \in\right] a, c\right]$ y $\left.\left.f^{m}(y) \in\right] c, b\right]$. Definimos la aplicación de primer retorno $\left.\left.H:[c, b] \rightarrow\right] c, b\right]$ por

$$
H(x)=f^{m\left(g^{n(x)}(x)\right)} \circ g^{n(x)}(x) .
$$

Observe que el conjunto de los puntos de discontinuidad de esta aplicación es $\left\{g^{-1}\left(f^{-j}(c)\right): j \in \mathbb{N}\right\}$. En particular, para todo $\varepsilon>0$, la intersección de este conjunto con $[c+\varepsilon, b]$ es finita. Por comodidad denotamos

$$
C(f)=\frac{\sup _{x \in[c, b]}(x-\bar{f}(x))}{\inf _{x \in[c, b]}(x-\bar{f}(x))} .
$$

Proposición 3.35. Bajo las hipótesis del lema 3.34, suponga además que

$$
\left(1-\frac{1}{\sup _{x \in[a, c]} f^{\prime}(x)}\right) e^{V(f ;[a, c])+V(g ;[c, b])}<1 .
$$

Entonces para todo $\kappa>1$ existe $N \in \mathbb{N}$ tal que $\left(H^{N}\right)^{\prime}(x)>\kappa$ para todo punto $x$ perteneciente al conjunto de puntos de diferenciabilidad de $H^{N}$.

Demostración. Sea $N \in \mathbb{N}$ tal que

$$
\left(1-\frac{1}{\sup _{x \in[a, c]} f^{\prime}(x)}\right)^{N} e^{N(V(f ;[a, c])+V(g ;[c, b]))}<\frac{1}{\kappa \cdot C(f)} .
$$

Afirmamos que cada rama de la aplicación $H^{N}$ es $\kappa$-dilatante. Para probar esto, sea $x$ un punto de una de las componentes conexas del dominio de diferenciabilidad de $H^{N}$. Fijemos un intervalo $[x-\varepsilon, x]$ contenido en esta componente, hagamos $\left[u_{0}, v_{0}\right]=[x-\varepsilon, x]$, y para $j \in\{1, \ldots, N-1\}$ denotemos $\left[u_{j}, v_{j}\right]=H^{j}\left(\left[u_{0}, v_{0}\right]\right)$. La desigualdad (3.24) aplicada a $\bar{H}=H^{-1}$ muestra que

$$
\frac{v_{j}-u_{j}}{v_{j+1}-u_{j+1}} \leq \frac{v_{j}-f^{-1}\left(v_{j}\right)}{v_{j+1}-f^{-1}\left(v_{j+1}\right)}\left(1-\frac{1}{\sup _{x \in[a, c]} f^{\prime}(x)}\right) e^{V(f ;[a, c])+V(g ;[c, b])} .
$$

Tomando el producto desde $j=0$ hasta $j=N-1$ obtenemos

$$
\frac{v_{0}-u_{0}}{v_{N}-u_{N}} \leq \frac{v_{0}-f^{-1}\left(v_{0}\right)}{v_{N}-f^{-1}\left(v_{N}\right)}\left(1-\frac{1}{\sup _{x \in[a, c]} f^{\prime}(x)}\right)^{N} e^{N(V(f ;[a, c])+V(g ;[c, b]))} .
$$

De (3.30) se concluye que $\left(H^{N}(x)-H^{N}(x-\varepsilon)\right) / \varepsilon>\kappa$, y como esta desigualdad vale para todo $\varepsilon>0$ pequeño, se deduce que $\left(H^{N}\right)^{\prime}(x)>\kappa$. 
Proposición 3.36. Si f y g satisfacen las hipótesis de la proposición 3.35, entonces todas las órbitas del pseudo-grupo generado por estas aplicaciones son densas en $] a, b[$.

Demostración. Supongamos lo contrario y consideremos una órbita del pseudo-grupo que no sea densa en $] a, b[$. Es fácil verificar entonces que los puntos $a, c$ y $b$ pertenecen al cierre de dicha órbita. Luego, si escogemos una componente conexa $] u, v[$ del complemento de dicho cierre contenida en ]$c, b[$ y de longitud maximal, entonces la proposición precedente establece que, para $N$ suficientemente grande $H^{N}(] u, v[)$ posee una longitud aún mayor, lo cual es absurdo.

A primera vista, las estimaciones anteriores podrían parecer demasiado técnicas. Para comprenderlas un poco mejor se puede considerar el caso particular en que las aplicaciones $f$ y $g$ son afines, digamos $f(x)=\lambda x$, con $\lambda>1$, y $g(x)=\eta(x-1 / \lambda)$. En tal caso, para cada $n \in \mathbb{N}$ la restricción de la aplicación de primer retorno $H:[1 / \lambda, 1] \rightarrow[1 / \lambda, 1]$ al intervalo $g^{-1}\left(\left[1 / \lambda^{n+1}, 1 / \lambda^{n}\right]\right)$ viene dada por $H(x)=f^{n} \circ g(x)$. Puesto que para todo $x \in g^{-1}\left(\left[1 / \lambda^{n+1}, 1 / \lambda^{n}\right]\right)$ se tiene

$$
\frac{1}{\lambda^{n}} \geq \eta\left(1-\frac{1}{\lambda}\right) \geq \frac{1}{\lambda^{n+1}}
$$

se deduce que

$$
H^{\prime}(x)=\eta \lambda^{n} \geq \frac{1}{\lambda-1}=\frac{1}{\lambda(1-1 / \lambda)} .
$$

Notemos por otra parte que $C(f)=\lambda$. La desigualdad (3.31) puede entonces ser escrita de la forma

$$
H^{\prime}(x) \geq \frac{1}{C(f)\left(1-\frac{1}{\sup _{x \in[0,1 / \lambda]} f^{\prime}(x)}\right)} .
$$

Como los valores de $V(f)$ y $V(g)$ son nulos en el caso afín, la similitud entre esta última desigualdad y aquéllas que han aparecido a lo largo de esta sección es evidente.

Ejercicio 3.37. Enuncie y demuestre una proposición englobando el principio siguiente: si la derivada de $f$ está cercana a 1 y la variación $V(f ;[a, c])$ es pequeña, entonces la constante $C(f)$ definida por (3.28) está cercana a 1.

Ejercicio 3.38. Mediante variaciones del segundo ejemplo de la sección 1 (capítulo 2) muestre la "optimalidad" de la proposición 3.36 cuando se impone a priori la condición $V(g)=0$; verifique que en tal caso el parámetro límite es (un múltiplo entero de) $\log (2)$. Modifique luego este ejemplo para mostrar la optimalidad de la misma proposición bajo la imposición $V(f)=V(g)$; muestre que en este caso el parámetro límite es (un múltiplo entero de) el logaritmo del número de oro. Para mayores detalles vea [147]. 


\subsection{Prueba del teorema}

Para ilustrar la idea de la demostración del teorema 3.33, supongamos que un grupo actúe sobre el círculo preservando un minimal excepcional $\Lambda$ y que dos generadores $f$ y $g$ estén relacionados como en la figura 17 sobre un subintervalo $[a, b]$ de $\mathrm{S}^{1}$ cuyo interior intersecta a $\Lambda$. En tal caso, razonando como en la prueba del corolario 3.36 concluimos que debe satisfacerse la desigualdad contraria a (3.29), es decir

$$
\left(1-\frac{1}{\sup _{x \in[a, c]} f^{\prime}(x)}\right) e^{V(f ;[a, c])+V(g ;[c, b])} \geq 1 .
$$

Por (3.23), esta desigualdad implica

$$
\left(1-e^{-V(f)}\right) e^{V(f ;[a, c])+V(g ;[c, b])} \geq 1 .
$$

Si $V(f)<V_{0}$ y $V(g)<V_{0}$ entonces $\left(1-e^{-V_{0}}\right) e^{2 V_{0}}>1$, es decir,

$$
e^{2 V_{0}}-e^{V_{0}}-1>0
$$

lo cual es imposible si $V_{0} \leq \log ((\sqrt{5}+1) / 2)$ (la aparición del número de oro no es misteriosa, pues esta constante estaría al parecer relacionada a la constante optimal del teorema: compare con el ejercicio 3.38).

Lamentablemente, la prueba para el caso general presenta ciertos problemas técnicos, pues no existen necesariamente dos generadores para los cuales el argumento anterior sea aplicable. Por ejemplo, hemos visto que el grupo modular puede actuar sobre $\mathrm{S}^{1}$ admitiendo un minimal excepcional, y los generadores de tal grupo son de orden finito... Es por esta razón que la hipótesis de un generador con puntos periódicos aislados se nos hace necesaria para nuestra demostración.

Demostración del teorema 3.33. Supongamos que $\Gamma$ admita un minimal excepcional $\Lambda$. Por el teorema de Denjoy, el conjunto de los puntos periódicos de cada elemento de $\Gamma$ es no vacío. Por hipótesis, existe un generador $g \in \mathcal{G}$ cuyos puntos periódicos son aislados. Denotemos $\operatorname{Per}_{*}(g)$ al conjunto de tales puntos, y definamos $\mathrm{P}(g)=\operatorname{Per}_{*}(g) \cap \Lambda$. Observe que el conjunto $\mathrm{P}(g)$ no es vacío. En efecto, si los puntos periódicos de $g$ son de orden $k$ y $p_{*} \in \Lambda$ no es punto fijo de $g^{k}$, entonces $\lim _{j \rightarrow \infty} g^{j k}\left(p_{*}\right)$ y $\lim _{j \rightarrow \infty} g^{-j k}\left(p_{*}\right)$ son puntos fijos de $g^{k}$ contenidos en $\Lambda \ldots$ Definamos $G=g^{k} \in \Gamma$.

Afirmamos que existe un punto $p \in \mathrm{P}(g)$ y un elemento $f \in \mathcal{G}$ tales que $f(p) \in \mathrm{S}^{1} \backslash \mathrm{P}(g)$. En efecto, en caso contrario el conjunto $\mathrm{P}(g)$ sería invariante por $\Gamma$, contradiciendo la minimalidad de $\Lambda$.

Designemos por $u$ y $v$ los puntos periódicos de $g$ a izquierda y derecha de $f(p)$ respectivemente. La aplicación $F=f \circ g^{k} \circ f^{-1}$ posee un punto fijo en $[u, v]$, a saber, $f(p)$. Sean $a$ el punto fijo de esta aplicación a izquierda 
de $v$, y $q$ el punto fijo a derecha de $a$. Reemplazando $G$ por $G^{-1}$ y $F$ por $F^{-1}$ si es necesario, podemos suponer que $G(x)<x \quad$ y $\quad F(x)>x$ para todo $x \in] a, v[$ (vea la figura 19).

Afirmamos ahora que, si $V_{0}$ es suficientemente pequeño, entonces el punto $b=F\left(G^{-1}(a)\right)$ pertenece al intervalo $] a, v[$. Para probar esto observamos en primer lugar que

$$
\begin{aligned}
V\left(F^{-1} ;[a, q]\right) & =V(F ;[a, q]) \leq \sum_{j=0}^{k-1} V\left(f \circ g \circ f^{-1} ; f \circ g^{j} \circ f^{-1}([a, q])\right) \\
& \leq V\left(f \circ g \circ f^{-1}\right)<3 V_{0} .
\end{aligned}
$$

De la misma manera se obtiene $V\left(G^{-1} ;[u, v]\right)=V(G ;[u, v])<V_{0}$. Sean $\left.x_{0} \in\right] a, v\left[\right.$ e $y_{0} \in(a, q)$ tales que

$$
\left(F^{-1}\right)^{\prime}\left(x_{0}\right)=\frac{F^{-1}(v)-a}{v-a} \quad \text { y } \quad\left(F^{-1}\right)^{\prime}\left(y_{0}\right)=1 .
$$

Evidentemente tenemos $\left|\log \left(F^{-1}\right)^{\prime}\left(y_{0}\right)-\log \left(F^{-1}\right)^{\prime}\left(x_{0}\right)\right| \leq V\left(F^{-1} ;[a, q]\right)$, $\mathrm{y}$ por lo tanto

$$
F^{-1}(v)-a>e^{-3 V_{0}}(v-a) .
$$

Por un argumento análogo se prueba que

$$
v-G^{-1}(a)>e^{-V_{0}}(v-a) .
$$

Si $b$ no estuviese en $] a, v$ [ entonces se tendría $F^{-1}(v) \leq G^{-1}(a)$. Esto implicaría, por (3.33) y (3.34),

$$
v-a \geq\left(F^{-1}(v)-a\right)+\left(v-G^{-1}(a)\right)>\left(e^{-V_{0}}+e^{-3 V_{0}}\right)(v-a),
$$

y por lo tanto $e^{3 V_{0}}-e^{2 V_{0}}>1$, lo cual es imposible si $V_{0} \leq \log (1.46557)$.

Los elementos $F$ y $G$ de $\Gamma$ están entonces relacionados sobre el intervalo $[a, b]$ como en la figura 17 . Obsérvese ahora que para todo $x \in[a, q]$ se tiene

$$
\left|\log \left(F^{\prime}\right)(x)-\log \left(F^{\prime}\right)\left(y_{0}\right)\right| \leq V(F ;[a, q])<3 V_{0},
$$

por lo que

$$
\sup _{x \in[a, c]} F^{\prime}(x)<e^{3 V_{0}} .
$$

Para $c=G^{-1}(a)$, la desigualdad (3.32) applicada a $F$ y $G$ muestra que $3 V_{0}+V_{0}>V(F ;[a, c])+V(G ;[c, b]) \geq \log \left(\frac{1}{1-\frac{1}{\sup _{x \in[a, c]} F^{\prime}(x)}}\right)>\log \left(\frac{1}{1-e^{-3 V_{0}}}\right)$.

Luego, $e^{4 V_{0}}-e^{V_{0}}>1$, lo cual es imposible si $V_{0} \leq \log (1.22074)$. 


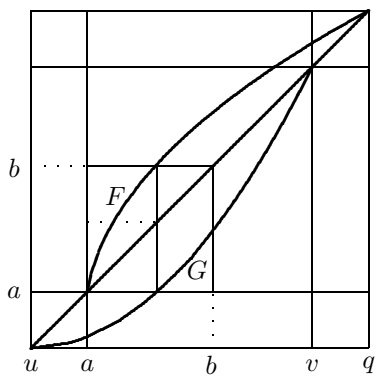

Figura 19

Ejercicio 3.39. Pruebe que para todo grupo $\Gamma$ de homeomorfismos del círculo que no es semiconjugado a un grupo de rotaciones y todo sistema de generadores $\mathcal{G}$ existe un elemento en la bola $B_{\mathcal{G}}(4)$ de radio 4 que no es semiconjugado a una rotación. Usando esto concluya que el teorema de Duminy vale para grupos de difeomorfismos analíticos generados por elementos $h$ satisfaciendo $V(h)<V_{0}$, $\sin$ importar si la hipótesis de existencia de un generador a puntos periódicos aislados es o no satisfecha (vea [147] para mayores detalles).

Ejercicio 3.40. Pruebe que para todo $g \in \operatorname{PSL}(2, \mathbb{R})$ se tiene

$$
V(g)=4 \operatorname{dist}(O, g(O)),
$$

donde $O$ denota el origen (i.e., el punto $(0,0))$ del disco de Poincaré. Concluya que, para subgrupos de $\operatorname{PSL}(2, \mathbb{R})$, la afirmación del teorema de Duminy se deduce del teorema de Marden o bien de la desigualdad de Margulis discutidos al inicio de la sección 3.1.

Observación. Mediante aplicaciones sucesivas de la desigualdad de Jørgensen, y utilizando algunos resultados de clasificación de grupos fuchsianos, Yamada determinó el valor de la constante optimal para el teorema de Marden. Gracias a la igualdad (3.35), una lectura atenta de [210] permite entonces verificar que el valor optimal para la constante $V_{0}$ del teorema de Duminy para subgrupos de $\operatorname{PSL}(2, \mathbb{R})$ es $4 \log (5 / 3)$, y que este valor es crítico para las acciones de $\operatorname{PSL}(2, \mathbb{Z})$ respecto a su sistema de generadores canónico.

\section{Segundo teorema de Duminy: el espacio de fines de órbitas semi-excepcionales}

\subsection{Presentación del resultado}

El objetivo de esta sección es presentar otro resultado extraordinario de Duminy sobre la estructura "al infinito" para ciertas órbitas de pseudogrupos de difeomorfismos en dimensión 1 . Sin duda alguna, la versión más conocida de este teorema es aquélla que concierne las foliaciones de codimensión 1: si la regularidad transversa es al menos $\mathrm{C}^{1+\text { lip }}$, entonces toda 
hoja semi-excepcional posee una infinidad de fines. Una prueba de este resultado aparece en [40]; nosotros daremos la prueba original (y lamentablemente no publicada) de Duminy con el objetivo de "rescatar del olvido" tan notable demostración.

Comencemos recordando algunas nociones básicas. Si M es un espacio topológico, el espacio de fines de $\mathrm{M}$ es el límite inverso de los espacios de las componentes conexas del complemento de las partes compactas (cada uno dotado de la topología discreta). Para espacios métricos localmente compactos y separables, esta definición puede hacerse más concreta. Fijemos una sucesión $\left(K_{n}\right)$ de partes compactas de M tales que $K_{n} \subset K_{n+1}$ para todo $n \in \mathbb{N}$ y $\mathrm{M}=\cup_{n \in \mathbb{N}} K_{n}$, y consideremos una sucesión de componentes conexas $C_{n}$ de $\mathrm{M} \backslash K_{n}$ tales que $C_{n+1} \subset C_{n}$ para todo $n \in \mathbb{N}$. Si $\left(K_{n}^{\prime}\right)$ y $\left(C_{n}^{\prime}\right)$ verifican estas mismas propiedades, entonces decimos que $\left(C_{n}\right)$ es equivalente a $\left(C_{n}^{\prime}\right)$ si para cada $n \in \mathbb{N}$ existe $m \in \mathbb{N}$ tal que $C_{n} \subset C_{m}^{\prime}$ y $C_{n}^{\prime} \subset C_{m}$. El espacio de fines de $\mathrm{M}$ se identifica entonces al conjunto de clases de equivalencia de esta relación. Se trata de un espacio topológico compacto y metrizable: fijada la sucesión $\left(K_{n}\right)$, la distacia entre (los fines determinados por) $\left(C_{n}^{1}\right)$ y $\left(C_{n}^{2}\right)$ es igual a $\exp (-k)$, donde $k$ es el ínfimo de los valores para el cual se tiene $C_{k}^{1} \neq C_{k}^{2}$.

Sea $\Gamma$ un pseudo-grupo de transformaciones de un espacio y $p$ un punto en él. Fijemos un sistema de generadores (simétrico) $\mathcal{G}$ para $\Gamma$. El grafo de Cayley (respecto a $\mathcal{G}$ ) de la órbita $\Gamma(p)$ es aquél cuyos vértices son los puntos de dicha órbita, de modo que dos de estos vértices $p^{\prime}$ y $p^{\prime \prime}$ están conectados por una arista (de longitud 1) si y sólo si existe $g \in \mathcal{G}$ tal que $g\left(p^{\prime}\right)=p^{\prime \prime}$. Definimos el espacio de fines $\mathcal{F}(\Gamma(p))$ de la órbita de $p$ como siendo el espacio de fines de dicho grafo $\operatorname{Cay}_{\mathcal{G}}(\Gamma(p))$. No es difícil comprobar que si bien el grafo de Cayley depende de $\mathcal{G}$, el espacio de fines asociado es independiente de la elección del sistema (finito) de generadores. Por simplicidad, en lo que sigue omitiremos la referencia a $\mathcal{G}$ cuando esto no cause confusión, y para cada $n \in \mathbb{N}$ designaremos por $B(n, p)$ la bola de centro $p$ y radio $n$ en $\operatorname{Cay}(\Gamma(p))$ (compare con la sección 2.5, capítulo 2).

Una órbita de un pseudo-grupo de homeomorfismos unidimensionales es dicha semi-excepcional si coincide con la órbita de un punto $p$ perteneciente a un minimal excepcional local $\Lambda$, de modo que $p$ es una extremidad de una de las componentes conexas del complemento $\Lambda^{c}$ de $\Lambda$. El segundo teorema de Duminy puede ser enunciado de la siguiente manera.

Teorema 3.41. Toda órbita semi-excepcional de un pseudo-grupo finitamente generado de difeomorfismos de clase $\mathrm{C}^{1+l i p}$ de una variedad unidimensional compacta posee una infinidad de fines.

Se desconoce si este resultado es válido para todas las órbitas de un minimal excepcional local $\Lambda$ de clase $\mathrm{C}^{1+\text { lip }}$ (se sabe sin embargo que esto ocurre en el caso real-analítico, de acuerdo a un resultado debido a Hector). Para el tratamiento de las órbitas semi-excepcionales la idea de base consiste en 
asociar, a cada punto de dicha órbita, la componente conexa de $\Lambda^{c}$ que tiene a este punto como una de sus extremidades. Para formalizar esta idea denotemos $\mathcal{G}^{*}=\cup_{k \in \mathbb{N}} \mathcal{G}^{k}$, y a cada $\hat{g}=\left(h_{1}, \ldots, h_{k}\right) \in \mathcal{G}^{*}$ asociemos el elemento $g=h_{k} \cdots h_{1}$. Si $I$ es una componente conexa de $\Lambda^{c}$ sobre la cual $g$ está definido, denotamos

$$
S(\hat{g} ; I)=|I|+\sum_{i=1}^{k}\left|h_{i} \cdots h_{1}(I)\right| .
$$

Si $p^{\prime}$ y $p^{\prime \prime}$ pertenecen a una misma órbita semi-excepcional $\Gamma(p)$ designamos por $\mathcal{G}^{*}\left(p^{\prime}, p^{\prime \prime}\right)$ al conjunto de todos los $\hat{g} \in \mathcal{G}^{*}$ tales que $g\left(p^{\prime}\right)=p^{\prime \prime}$. Consideramos entonces la componente conexa $I$ de $\Lambda^{c}$ que tiene a $p^{\prime}$ como extremidad, y definimos

$$
S\left(p^{\prime}, p^{\prime \prime}\right)=\inf _{\substack{\hat{g} \in \mathcal{G}^{*}\left(p^{\prime}, p^{\prime \prime}\right), I \subset \operatorname{dom}(g)}} S(\hat{g} ; I) .
$$

En el caso en que para ningún $\hat{g} \in \mathcal{G}^{*}\left(p^{\prime}, p^{\prime \prime}\right)$ se tenga $I \subset \operatorname{dom}(g)$, hacemos $S\left(p^{\prime}, p^{\prime \prime}\right)=\infty$.

Lema 3.42. Si $p^{\prime} y p^{\prime \prime}$ son puntos de una órbita semi-excepcional $\Gamma(p)$ para los que existe un elemento $f \in \Gamma$ tal que $f\left(p^{\prime}\right) \neq p^{\prime}$ y $f\left(p^{\prime \prime}\right) \neq p^{\prime \prime}$, entonces cada una de las sucesiones de puntos $p_{n}^{\prime}=f^{n}\left(p^{\prime}\right)$ y $p_{n}^{\prime \prime}=f^{n}\left(p^{\prime \prime}\right)$ determina un fin de $\Gamma(p)$, y dichos fines coinciden si y sólo si $\lim _{n \rightarrow \infty} S\left(p_{n}^{\prime}, p_{n}^{\prime \prime}\right)=0$.

Demostración. Puesto que $f\left(p^{\prime}\right) \neq p^{\prime}$, los puntos $f^{n}\left(p^{\prime}\right)$ son dos a dos distintos. Luego, la sucesión $\left(f^{n}\left(p^{\prime}\right)\right)$ escapa de todos los compactos de $\operatorname{Cay}(\Gamma(p))$, por lo que determina un fin de la órbita de $p$. Análogamente, $\left(f^{n}\left(p^{\prime \prime}\right)\right)$ determina un punto en $\mathcal{F}(\Gamma(p))$.

Fijemos ahora una constante $\delta>0$ tal que si $h \in \mathcal{G}$ y $x \in \operatorname{dom}(h) \cap \Lambda$, entonces la $2 \delta$-vecindad de $x$ está contenida en el dominio de $h$. Fijemos también $m_{0} \in \mathbb{N}$ de modo que $B\left(m_{0}, p\right)$ contenga todos los puntos de $\Gamma(p)$ que son extremidades de componentes conexas de $\Lambda^{c}$ de longitud mayor o igual que $\delta$. Para cada $n \in \mathbb{N}$ denotemos $I_{n}^{\prime}$ (resp. $I_{n}^{\prime \prime}$ ) la componente conexa de $\Lambda^{c}$ cuyo cierre contiene a $p_{n}^{\prime}$ (resp. $\left.p_{n}^{\prime \prime}\right)$. De las hipótesis $f\left(p^{\prime}\right) \neq p^{\prime}$ y $f\left(p^{\prime \prime}\right) \neq p^{\prime \prime}$ se deduce que los intervalos $I_{n}^{\prime}$ (resp. $\left.I_{n}^{\prime \prime}\right)$ son dos a dos disjuntos, y que por lo tanto la suma de sus longitudes es finita (en particular, $\left|I_{n}^{\prime}\right|$ y $\left|I_{n}^{\prime \prime}\right|$ tienden a 0 cuando $n$ tiende al infinito). Como el espacio de fines no depende del sistema (finito) de generadores, sin pérdida de generalidad podemos suponer que $f$ pertenece a $\mathcal{G}$.

Supongamos que, para todo $n \in \mathbb{N}$ suficientemente grande, cada camino $\hat{g}$ uniendo $p_{n}^{\prime}$ con $p_{n}^{\prime \prime}$ (i.e., tal que $g\left(p_{n}^{\prime}\right)=p_{n}^{\prime \prime}$ ) satisface $I_{n} \not \subset d o m(g)$. En este caso dichos caminos deben pasar por $B\left(m_{0}, p\right)$, de donde se deduce que los fines determinados por $\left(p_{n}^{\prime}\right)$ y $\left(p_{n}^{\prime \prime}\right)$ son diferentes. Luego, de la definición 
se concluye de manera obvia que

$$
\lim _{n \rightarrow \infty} S\left(p_{n}^{\prime}, p_{n}^{\prime \prime}\right)=\infty .
$$

Supongamos en lo que sigue que para una infinidad de enteros $n \in \mathbb{N}$ existe $\hat{g} \in \mathcal{G}^{*}\left(p_{n}^{\prime}, p_{n}^{\prime \prime}\right)$ tal que $I_{n} \subset \operatorname{dom}(g)$. Se comprueba entonces que lo mismo es válido para todo $n$ suficientemente grande. Así, un argumento de concatenación nos da, para todo $m \geq n$ suficientemente grandes,

$$
S\left(p_{m}^{\prime}, p_{m}^{\prime \prime}\right) \leq S\left(p_{n}^{\prime}, p_{n}^{\prime \prime}\right)+\sum_{j=n}^{m}\left(\left|I_{j}^{\prime}\right|+\left|I_{j}^{\prime \prime}\right|\right) .
$$

Esta desigualdad implica obviamente que la sucesión $\left(S\left(p_{n}^{\prime}, p_{n}^{\prime \prime}\right)\right)$ es de Cauchy, y que por lo tanto converge (a un límite finito).

Fijemos $\varepsilon>0$ arbitrario y notemos $S$ la suma de las longitudes de las componentes conexas de $\Lambda^{c}$. Obviamente, podemos escoger un conjunto finito $\mathcal{I}_{\varepsilon}$ de tales componentes cuya suma de longitudes sea mayor que $S-\varepsilon$. Sea $m_{\varepsilon} \in \mathbb{N}$ tal que $B\left(m_{\varepsilon}, p\right)$ contiene todos los puntos de $\Gamma(p)$ situados en el cierre de algún intervalo de $\mathcal{I}_{\varepsilon}$. Si los fines determinados por $\left(p_{n}^{\prime}\right)$ y $\left(p_{n}^{\prime \prime}\right)$ coinciden, entonces para todo $n$ suficientemente grande existe un camino $\hat{g} \in \mathcal{G}^{*}$ que une $p_{n}^{\prime}$ con $p_{n}^{\prime \prime}$ y que evita $B\left(m_{\varepsilon}, p\right)$. Esto implica evidentemente que $\sigma\left(p_{n}^{\prime}, p_{n}^{\prime \prime}\right) \leq \sigma\left(\hat{g} ; I_{n}^{\prime}\right) \leq \varepsilon$. Como $\varepsilon>0$ era arbitrario, concluimos que $\lim _{n \rightarrow \infty} S\left(p_{n}^{\prime}, p_{n}^{\prime \prime}\right)=0$.

Si por el contrario los fines en cuestión son distintos, entonces existe un entero $m_{0}^{\prime}$ tal que, para todo $n \in \mathbb{N}$ suficientemente grande, cada camino que une $p_{n}^{\prime}$ con $p_{n}^{\prime \prime}$ pasa por $B\left(m_{0}^{\prime}, p\right)$. Designando por $\varepsilon_{0}$ la longitud mínima entre las componentes conexas de $\Lambda^{c}$ que tienen un vértice de $B\left(m_{0}^{\prime}, p\right)$ como extremidad, concluimos que $S\left(p_{n}^{\prime}, p_{n}^{\prime \prime}\right)>\varepsilon_{0}$ para todo $n$ suficientemente grande. Por lo tanto, $\lim _{n \rightarrow \infty} S\left(p_{n}^{\prime}, p_{n}^{\prime \prime}\right)>0$.

Para probar el teorema 3.41 razonaremos por contradicción. Suponiendo que los fines determinados por dos sucesiones como las del lema precedente coinciden, interpretaremos la condición equivalente $\lim _{n \rightarrow \infty} S\left(p_{n}^{\prime}, p_{n}^{\prime \prime}\right)=0$ de manera dinámica: existen caminos $\hat{g}_{n}$ uniendo $p_{n}^{\prime}$ y $p_{n}^{\prime \prime}$ con distorsión arbitrariamente pequeña. Concluiremos entonces la demostración aplicando el corolario 3.36 a $f^{-1}$ y $g_{n}^{-1}$. Consignamos sin embargo que, en el curso de esta demostración, tendremos que soslayar dificultades técnicas no del todo sencillas.

Observación 3.43. Las órbitas asociadas a una acción libre de $\mathbb{Z}$ (resp. $\mathbb{Z}^{d}$ ) tienen evidentemente dos fines (resp. un fin). Es posible crear acciones libres no minimales de $\mathbb{Z}^{d}$ por difeomorfismos de clase $\mathrm{C}^{1+\tau}$ del círculo (para ciertos valores positivos de $\tau$ : vea la sección 1.4 del capítulo 4). Esto muestra que la hipótesis de regularidad $\mathrm{C}^{1+l i p}$ es esencial para la validez del segundo teorema de Duminy (para entender por qué aparecen sólo 1 ó 2 fines en los casos degenerados el lector debiese consultar el brillante artículo [75]). Ignoramos sin embargo si el teorema es válido en clases de diferenciabilidad inferiores cuando se asume $a$ priori que no existe ninguna medida de probabilidad invariante. 


\subsection{Un criterio para distinguir dos fines diferentes}

Para retomar notaciones, en lo que sigue consideramos una órbita semiexcepcional $\Gamma(p)$ asociada a un pseudo-grupo finitamente generado $\Gamma$ de difeomorfismos de clase $\mathrm{C}^{1+\text { lip }}$. Denotamos $\Lambda$ el minimal excepcional local, fijamos un sistema (simétrico) de generadores $\mathcal{G}$ de $\Gamma$, y denotamos $C$ una constante de lipschitzianidad simultánea para el logaritmo de la derivada de cada elemento $h \in \mathcal{G}$ sobre una $\delta$-vecindad de $\Lambda \cap \operatorname{dom}(h)(\operatorname{con} \delta>0)$.

Por el teorema de Sacksteder, existe un elemento $f \in \Gamma$ con un punto fijo $a \in \Lambda$ de modo que $f^{\prime}(a)<1$. Puesto que la órbita de $p$ intersecta a todo intervalo abierto conteniendo puntos de $\Lambda$, podemos fijar ya sea $b>a$ o bien $b<a$ de modo que $\Gamma(p) \cap] a, b[\neq \emptyset$ о $\Gamma(p) \cap] b, a[\neq \emptyset$ respectivamente. En lo que sigue consideraremos sólo el primer caso, pues el segundo es análogo. Podemos suponer que $|b-a|$ es pequeño de tal suerte que

$$
0<\inf _{x \in[a, b]} f^{\prime}(x)=m(f) \leq M(f)=\sup _{x \in[a, b]} f^{\prime}(x)<1 .
$$

Dado $\varepsilon>0$ podemos escoger $\left.b_{\varepsilon} \in\right] a, b[$ suficientemente cercano al punto $a$ de modo que

$$
\frac{C\left(b_{\varepsilon}-a\right)}{1-M(f)} \leq \frac{\varepsilon}{2}
$$

Para todo $n \in \mathbb{N}$ lo anterior implica

$$
V\left(f^{n} ;\left[a, b_{\varepsilon}\right]\right) \leq C \sum_{k=0}^{n-1}\left|f^{k}\left(\left[a, b_{\varepsilon}\right]\right)\right| \leq C\left(b_{\varepsilon}-a\right) \sum_{k=0}^{n-1} M(f)^{k} \leq \frac{\varepsilon}{2}
$$

Proposición 3.44. Sea $\varepsilon>0$ tal que $\varepsilon \leq \frac{1}{2} \log \left(\frac{1}{1-m(f)}\right)$. Supongamos que existan dos componentes conexas $] x, y[y] u, v[$ del complemento de $\Lambda$ tales que:

(i) $a<x<y<u<v<f\left(b_{\varepsilon}\right)$,

(ii) $\{x, u\}$ (resp. $\{y, v\}$ ) está contenido en $\Gamma(p)$,

(iii) se tienen las desigualdades

$$
\frac{f^{-1}(u)-u}{f^{-1}(u)-x} \exp (2 \varepsilon) \leq \frac{v-u}{y-x} \leq \frac{u-a}{x-a} \exp (-2 \varepsilon) .
$$

Entonces las sucesiones $\left(f^{n}(x)\right)$ y $\left(f^{n}(u)\right)$ (resp. $\left(f^{n}(y)\right)$ y $\left.\left(f^{n}(v)\right)\right)$ determinan dos fines diferentes de $\Gamma(p)$.

Para probar esta proposición necesitaremos refinar para el caso $\mathrm{C}^{1+l i p}$ las estimaciones de Schwartz presentadas en la sección 2.1 de este capítulo. 
Lema 3.45. Sean I una componente conexa del complemento de $\Lambda$ y $J$ un intervalo que contiene a I. Supongamos que para ciertos $\lambda>1$ y $n \in \mathbb{N}$ existe $\hat{g}=\left(h_{1}, \ldots, h_{n}\right) \in \mathcal{G}^{n}$ tal que

$$
S(\hat{g} ; I) \leq \inf \left\{\frac{\log (\lambda)}{\lambda C}, \frac{\delta}{\lambda}\right\} \quad y \quad \frac{|J|}{|I|} \leq \lambda \exp (-\lambda C S(\hat{g} ; I)) .
$$

Entonces se cumple:

(i) $J \subset \operatorname{dom}(g)$,

(ii) $V(g ; J) \leq \lambda C S(\hat{g} ; I)$,

(iii) $|g(J)| \leq \lambda|g(I)|$.

Demostración. La prueba es por inducción. Para $n=1$ tenemos $\hat{g}=g \in \mathcal{G}$ y $S(\hat{g} ; I)=|I|$. La propiedad (i) resulta del hecho que $J$ contiene a $I$ y $|J| \leq \lambda|I|=\lambda S(\hat{g} ; I) \leq \delta$. Para (ii) observe que $V(g ; J) \leq C|J| \leq \lambda C S(\hat{g} ; I)$. En cuanto a (iii) note que, por hipótesis y (3.22),

$$
\frac{|g(J)|}{|g(I)|} \leq \frac{|J|}{|I|} e^{V(g ; J)} \leq \lambda .
$$

Supongamos ahora que la afirmación del lema sea válida al orden $k$ y consideremos $\hat{g}=\left(h_{1}, \ldots, h_{k+1}\right)$ en $\mathcal{G}^{k+1}$ satisfaciendo las hipótesis. Si (i), (ii) y (iii) valen para $\left(h_{1}, \ldots, h_{k}\right) \in \mathcal{G}^{k}$ entonces:

- la propiedad (i) vale para $\hat{g}$, pues $h_{k} \cdots h_{1}$ está definido sobre $J$, el intervalo $h_{k} \cdots h_{1}(J)$ intersecta a $\Lambda$ y

$$
\left|h_{k} \cdots h_{1}(J)\right| \leq \lambda\left|h_{k} \cdots h_{1}(I)\right|<\lambda S(\hat{g} ; I) \leq \delta ;
$$

- la propiedad (ii) vale para $\hat{g}$, pues por la hipótesis inductiva tenemos

$$
\begin{aligned}
V(g ; J) & \leq V\left(h_{k+1} ; h_{k} \cdots h_{1}(J)\right)+V\left(h_{k} \cdots h_{1} ; J\right) \\
& \leq C\left|h_{k} \cdots h_{1}(J)\right|+\lambda C S\left(\left(h_{k}, \ldots, h_{1}\right) ; I\right) \leq \lambda C S(\hat{g} ; I)
\end{aligned}
$$

- la propiedad (iii) vale para $\hat{g}$, pues del ítem precedente y de la hipótesis del lema se deduce que

$$
\frac{|g(J)|}{|g(I)|} \leq \frac{|J|}{|I|} \exp (V(g ; J)) \leq \lambda .
$$

Observe que las hipótesis del lema precedente hacen intervenir de manera simultánea los parámetros $S(\hat{g} ; I)$ y $\lambda$. En particular, si $S(\hat{g} ; I)$ es pequeño entonces la afirmación del lema indica que la distorsión está controlada en una vecindad de $I$ cuyo tamaño relativo (respecto a $|I|$ ) es grande. Esta lúcida observación debida a Duminy será fundamental en lo que sigue.

Demostración de la proposición 3.44. Daremos la prueba sólo para el caso en que $\{x, u\}$ está contenido en $\Gamma(p)$, pues el otro caso es análogo. 
Supongamos por contradicción que los fines determinados por las sucesiones $\left(f^{n}(x)\right)$ y $\left(f^{n}(u)\right)$ sean los mismos, y denotemos $x_{n}=f^{n}(x), y_{n}=f^{n}(y)$, $u_{n}=f^{n}(u)$ y $v_{n}=f^{n}(v)$. Por el lema $3.42, S_{n}=S\left(x_{n}, u_{n}\right)$ converge a 0 cuando $n$ tiende al infinito. Luego, por definición, para cada $n \in \mathbb{N}$ existe $\hat{g}_{n} \in \mathcal{G}^{*}$ tal que $g_{n}\left(x_{n}\right)=u_{n}$ y $S\left(\hat{g}_{n} ;\left[x_{n}, y_{n}\right]\right) \leq 2 S_{n}$. Haciendo $\lambda_{n}=1 / \sqrt{S_{n}}$ vemos que para $n$ suficientemente grande se tiene $\lambda_{n}>1$ y

$$
2 S_{n} \leq \min \left\{\frac{\log \left(\lambda_{n}\right)}{\lambda_{n} C}, \frac{\delta}{\lambda_{n}}\right\}
$$

Para tales enteros $n$ hagamos

$\alpha_{n}=\lambda_{n} \exp \left(-2 \lambda_{n} C S_{n}\right), \quad x_{n}^{\prime}=x_{n}-\alpha_{n}\left(y_{n}-x_{n}\right), \quad y_{n}^{\prime}=y_{n}+\alpha_{n}\left(y_{n}-x_{n}\right)$.

Observe que $\alpha_{n}$ tiende al infinito junto con $n$. Por el lema 3.45, $g_{n}$ está definido sobre todo el intervalo $\left[x_{n}^{\prime}, y_{n}^{\prime}\right]$, con

$$
V\left(g_{n} ;\left[x_{n}^{\prime}, y_{n}^{\prime}\right]\right) \leq 2 \lambda_{n} C S_{n}
$$

Afirmación (i): existe un entero $N_{1}$ tal que si $n \geq N_{1}$ entonces $\left[a, f^{-1}\left(u_{n}\right)\right]$ está contenido en $\left[x_{n}^{\prime}, y_{n}^{\prime}\right]$.

En efecto, por (3.36),

$$
\frac{x_{n}-a}{y_{n}-x_{n}}=\frac{f^{n}(x)-f^{n}(a)}{f^{n}(y)-f^{n}(x)} \leq \frac{x-a}{y-x} \exp \left(V\left(f^{n} ;\left[a, b_{\varepsilon}\right]\right)\right) \leq \frac{x-a}{y-x} \exp (\varepsilon / 2) .
$$

Esto implica que si escogemos $n$ de modo que $\alpha_{n} \geq e^{\varepsilon / 2}(x-a) /(y-x)$ entonces $\left(x_{n}-a\right) /\left(y_{n}-x_{n}\right) \leq \alpha_{n}$, es decir, $x_{n}^{\prime} \leq a$. Análogamente, si $n$ es tal que $\alpha_{n} \geq e^{\varepsilon / 2}\left(f^{-1}(u)-y\right) /(y-x)$, entonces la desigualdad

$$
\frac{f^{-1}\left(u_{n}\right)-y_{n}}{y_{n}-x_{n}} \leq \frac{f^{-1}(u)-y}{y-x} \exp (\varepsilon / 2)
$$

implica $y_{n}^{\prime} \geq f^{-1}\left(u_{n}\right)$.

Afirmación (ii): existe un entero $N_{2} \geq N_{1}$ tal que si $n \geq N_{2}$ entonces $g_{n}(t) \geq t$ para todo $t \in\left[a, f^{-1}\left(u_{n}\right)\right]$.

Para verificar esto notemos que de (3.36) y de la hipótesis (iii) de la proposición se obtiene fácilmente

$$
\frac{f^{-1}\left(u_{n}\right)-u_{n}}{f^{-1}\left(u_{n}\right)-x_{n}} \exp (\varepsilon) \leq \frac{v_{n}-u_{n}}{y_{n}-x_{n}} \leq \frac{u_{n}-a}{x_{n}-a} \exp (-\varepsilon) .
$$

De (3.37) se concluye que $V\left(g_{n} ;\left[x_{n}^{\prime}, y_{n}^{\prime}\right]\right)$ tiende a 0 . Tomemos $N_{2} \geq N_{1}$ de modo que $V\left(g_{n} ;\left[x_{n}^{\prime}, y_{n}^{\prime}\right]\right) \leq \varepsilon / 2$ para todo $n \geq N_{2}$. Para un tal $n$ y todo $t \in\left[x_{n}^{\prime}, y_{n}^{\prime}\right]$ tenemos

$$
g_{n}^{\prime}(t) \leq \frac{v_{n}-u_{n}}{y_{n}-x_{n}} \exp (\varepsilon / 2)<\frac{u_{n}-a}{x_{n}-a},
$$




$$
g_{n}^{\prime}(t) \geq \frac{v_{n}-u_{n}}{y_{n}-x_{n}} \exp (-\varepsilon / 2)>\frac{f^{-1}\left(u_{n}\right)-u_{n}}{f^{-1}\left(u_{n}\right)-x_{n}} .
$$

Por el teorema del valor medio, la primera de estas desigualdades implica que $g_{n}(t)>t$ para todo $t \in\left[a, x_{n}\right]$, mientras que la segunda implica que $g_{n}(t)>t$ para todo $t \in\left[x_{n}, f^{-1}\left(u_{n}\right)\right]$ (vea la figura 20).

Para concluir fijemos $n \geq N_{2}$. Observe que de $g_{n}(a)<g_{n}\left(x_{n}\right)<u_{n}$ se deduce $f^{-1} g_{n}(a)<f^{-1}\left(u_{n}\right)<b_{\varepsilon}$. Consideremos las restricciones de $f$ y $g_{n}$ al intervalo $\left[a, f^{-1} g_{n}(a)\right]$. Puesto que

$$
\begin{aligned}
V\left(f ;\left[a, f^{-1} g_{n}(a)\right]\right)+V\left(g_{n} ;\left[a, g_{n}^{-1} f^{-1} g_{n}(a)\right]\right) & \leq V\left(f ;\left[a, b_{\varepsilon}\right]\right)+V\left(g_{n} ;\left[x_{n}^{\prime}, y_{n}^{\prime}\right]\right) \\
& \leq \varepsilon<\log \left(\frac{1}{1-m(f)}\right),
\end{aligned}
$$

aplicando el corolario 3.36 a las restricciones de $f^{-1}$ y $g_{n}^{-1}$ al intervalo $\left[a, f^{-1} g_{n}(a)\right]$ obtenemos una contradicción.

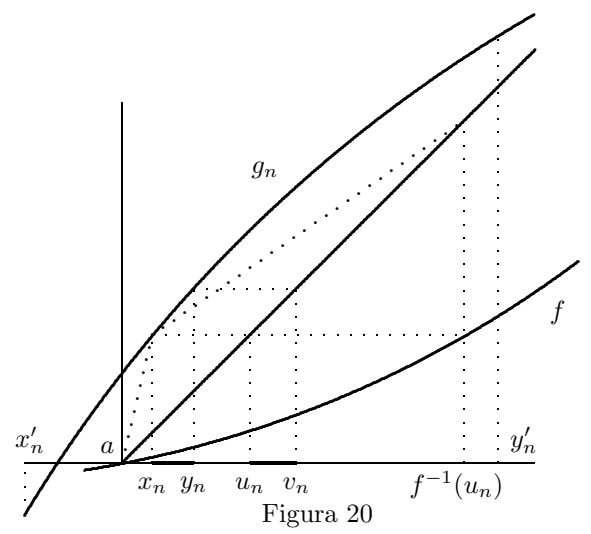

\subsection{Fin de la demostración}

Para completar la demostración del teorema 3.41 debemos hallar un par de intervalos $] x, y[\mathrm{y}] u, v[$ satisfaciendo las hipótesis de la proposición 3.44. Advertimos al lector que esto no es del todo elemental, por lo que resulta muy ilustrativo seguir las etapas finales (i.e., las afirmaciones (iii) y (iv)) de la demostración presentada a continuación para el caso del pseudogrupo ilustrado en la figura 16. Continuaremos considerando una constante positiva $\varepsilon \leq \frac{1}{2} \log \left(\frac{1}{1-m(f)}\right)$.

Afirmación (i): existen $\left.c_{\varepsilon} \in\right] a, f\left(b_{\varepsilon}\right)\left[\right.$ y $g_{\varepsilon} \in \Gamma$ tales que $\left[a, c_{\varepsilon}\right] \subset \operatorname{dom}\left(g_{\varepsilon}\right)$, $g_{\varepsilon}\left(c_{\varepsilon}\right)=c_{\varepsilon}, g_{\varepsilon}(t)>t$ para todo $t \in\left[a, c_{\varepsilon}\left[\right.\right.$ y $V\left(g_{\varepsilon} ;\left[a, c_{\varepsilon}\right]\right) \leq \varepsilon$. 
En efecto, puesto que la órbita de $a \in \Lambda$ intersecta al intervalo $] a, f\left(b_{\varepsilon}\right)[$, podemos escoger $g \in \Gamma$ y $c \in] a, f\left(b_{\varepsilon}\right)[$ de modo que $[g(a), g(c)] \subset] a, f\left(b_{\varepsilon}\right)[$ y $V(g ;[a, c]) \leq \varepsilon / 2$. Para $k$ suficientemente grande tenemos $f^{k} g(c)<c, \mathrm{y}$ puesto que $f^{\bar{k}} g(a)>a$, la aplicación $g_{\varepsilon}=f^{k} g$ posee puntos fijos en $] a, c[$. Si denotamos $c_{\varepsilon}$ el primero de esos puntos fijos a la derecha de $a$, entonces de $(3.36)$ se deduce $V\left(g_{\varepsilon} ;\left[a, c_{\varepsilon}\right)\right] \leq V\left(g ;\left[a, c_{\varepsilon}\right]\right)+V\left(f^{k} ;\left[a, b_{\varepsilon}\right]\right) \leq \varepsilon$.

Afirmación (ii): se tiene la desigualdad $f^{\prime}(a)+g_{\varepsilon}^{\prime}\left(c_{\varepsilon}\right) \leq e^{\varepsilon}$.

Para verificar esto, notemos primeramente que

$$
V\left(f ;\left[a, c_{\varepsilon}\right]\right)+V\left(g_{\varepsilon} ;\left[a, c_{\varepsilon}\right]\right) \leq \frac{3 \varepsilon}{2}<\log \left(\frac{1}{1-m(f)}\right) .
$$

Como las órbitas de $\Lambda$ no son densas en $\Lambda \cap] a, c_{\varepsilon}[$, aplicando el corolario 3.36 a $f^{-1}$ y $g_{\varepsilon}^{-1}$ concluimos que $f\left(c_{\varepsilon}\right)<g_{\varepsilon}(a)$, lo cual implica evidentemente que $m(f)+m\left(g_{\varepsilon}\right)<1 \quad$ (donde $m\left(g_{\varepsilon}\right)=\inf _{x \in\left[a, c_{\varepsilon}\right]} g_{\varepsilon}^{\prime}(x)$ ). Deducimos así que

$$
\begin{aligned}
f^{\prime}(a)+g_{\varepsilon}^{\prime}\left(c_{\varepsilon}\right) & \leq m(f) \exp \left(V\left(f ;\left[a, c_{\varepsilon}\right]\right)\right)+m\left(g_{\varepsilon}\right) \exp \left(V\left(g_{\varepsilon} ;\left[a, c_{\varepsilon}\right]\right)\right) \\
& \leq e^{\varepsilon}\left(m(f)+m\left(g_{\varepsilon}\right)\right)<e^{\varepsilon} .
\end{aligned}
$$

En lo que sigue denotaremos $\alpha=f^{\prime}(a)$ y $\beta=g_{\varepsilon}^{\prime}\left(c_{\varepsilon}\right) \quad$ (de modo que $\alpha+\beta<1$ ), e impondremos a $\varepsilon>0$ la condición suplementaria (válida si $\varepsilon>0$ es suficientemente pequeño)

$$
1-\alpha e^{\varepsilon}>\left(e^{\varepsilon}-\alpha\right)^{2} .
$$

Afirmación (iii): para todo $n \in \mathbb{N}$ se puede elegir $\kappa(\varepsilon, n)>0$ de modo que $\lim _{\varepsilon \rightarrow 0} \kappa(\varepsilon, n)>0 \mathrm{y}$, para todo $i \in\{1, \ldots, n\}$ y todo $t \in\left[a, c_{\varepsilon}\right]$,

$$
f^{n-i} g_{\varepsilon} f^{i}(t)-f^{n-i+1} g_{\varepsilon} f^{i-1}(t) \geq\left(c_{\varepsilon}-a\right) \kappa(\varepsilon, n) .
$$

En efecto, de $\alpha e^{-\varepsilon} \leq f^{\prime}(t) \leq \alpha e^{\varepsilon}$ y $f(a)=a$ concluimos que, para todo $t \in\left[a, c_{\varepsilon}\right]$,

$$
a+\alpha e^{-\varepsilon}(t-a) \leq f(t) \leq a+\alpha e^{\varepsilon}(t-a) .
$$

Análogamente, de $\beta e^{-\varepsilon} g_{\varepsilon}^{\prime}(t) \leq \beta e^{\varepsilon}$ y $g_{\varepsilon}\left(c_{\varepsilon}\right)=c_{\varepsilon}$ se deduce que

$$
c_{\varepsilon}-\beta e^{\varepsilon}\left(c_{\varepsilon}-t\right) \leq g_{\varepsilon}(t) \leq c_{\varepsilon}-\beta e^{-\varepsilon}\left(c_{\varepsilon}-t\right) .
$$

Luego,

$$
\begin{aligned}
g_{\varepsilon} f(t)-f g_{\varepsilon}(t) & \geq g_{\varepsilon}\left(a+\alpha e^{-\varepsilon}(t-a)\right)-\left(a+\alpha e^{\varepsilon}\left(g_{\varepsilon}(t)-a\right)\right) \\
& \geq c_{\varepsilon}-\beta e^{\varepsilon}\left(c_{\varepsilon}-a-\alpha e^{-\varepsilon}(t-a)\right)-\left(a+\alpha e^{\varepsilon}\left(c_{\varepsilon}-\beta e^{-\varepsilon}\left(c_{\varepsilon}-t\right)-a\right)\right) \\
& =\left(c_{\varepsilon}-a\right)\left[1-\beta\left(e^{\varepsilon}-\alpha\right)-\alpha e^{\varepsilon}\right] \\
& >\left(c_{\varepsilon}-a\right)\left[1-\alpha e^{\varepsilon}-\left(e^{\varepsilon}-\alpha\right)^{2}\right] .
\end{aligned}
$$


Haciendo $\kappa(\varepsilon, n)=\left(\alpha e^{-\varepsilon}\right)^{n}\left[1-\alpha e^{\varepsilon}-\left(e^{\varepsilon}-\alpha\right)^{2}\right]>0$ concluimos entonces que, para todo $i \in\{1, \ldots, n\}$ y todo $t \in\left[a, c_{\varepsilon}\right]$,

$$
\begin{aligned}
f^{n-i} g_{\varepsilon} f^{i}(t)-f^{n-i+1} g_{\varepsilon} f^{i-1}(t) & \geq\left(\alpha e^{-\varepsilon}\right)^{n-i}\left[g_{\varepsilon} f\left(f^{i-1}(t)\right)-f g_{\varepsilon}\left(f^{i-1}(t)\right)\right] \\
& >\left(\alpha e^{-\varepsilon}\right)^{n-i}\left(\alpha e^{-\varepsilon}\right)^{n}\left(c_{\varepsilon}-a\right)\left[1-\alpha e^{\varepsilon}-\left(e^{\varepsilon}-\alpha\right)^{2}\right] \\
& \geq\left(c_{\varepsilon}-a\right) \kappa(\varepsilon, n) .
\end{aligned}
$$

Observe finalmente que

$$
\lim _{\varepsilon \rightarrow 0} \kappa(\varepsilon, n)=\alpha^{n}\left[1-\alpha-(1-\alpha)^{2}\right]>0 .
$$

Insistamos en que la construcción precedente puede ser llevada a cabo para todo $\varepsilon>0$ suficientemente pequeño. Fijemos entonces $n \in \mathbb{N}$ e impongamos a $\varepsilon>0$ las condiciones suplementarias

$$
1+\kappa(\varepsilon, n) \geq e^{2(n+2) \varepsilon} \quad \text { y } \quad 1-\kappa(\varepsilon, n) \leq e^{-2(n+2) \varepsilon} .
$$

Tomemos una componente conexa $I$ de $\Lambda^{c}$ contenida en $] a, c_{\varepsilon}[$, y para cada $i \in\{1, \ldots, n\}$ hagamos

$$
I_{i}=f^{n-i} g_{\varepsilon} f^{i}(I)
$$

Afirmación (iv): para todo $i<j$, los intervalos $] x, y\left[=I_{i} \mathrm{y}\right] u, v\left[=I_{j}\right.$ satisfacen las condiciones de la proposición 3.44.

Sólo la verificación de la condición (iii) representa una dificultad. Comencemos entonces notando que, para todo $k \in\{1, \ldots, n\}$,

$$
\left(\alpha e^{-\varepsilon}\right)^{n} \beta e^{-\varepsilon} \leq \frac{\left|I_{k}\right|}{|I|} \leq\left(\alpha e^{\varepsilon}\right)^{n} \beta e^{\varepsilon},
$$

por lo que

$$
e^{-2(n+1) \varepsilon} \leq \frac{\left|I_{i}\right|}{\left|I_{j}\right|} \leq e^{2(n+1) \varepsilon} .
$$

Luego, por (3.38) y (3.39)

$$
\begin{aligned}
\frac{u-a}{x-a} \cdot \frac{y-x}{v-u} & =\left(\frac{u-x}{x-a}+1\right) \frac{y-x}{v-u} \geq\left(\frac{\left(c_{\varepsilon}-a\right) \kappa(\varepsilon, n)}{x-a}+1\right) \frac{y-x}{v-u} \\
& \geq(1+\kappa(\varepsilon, n)) 2^{-2(n+1) \varepsilon} \geq e^{2 \varepsilon}
\end{aligned}
$$

es decir

$$
\frac{u-a}{x-a} \exp (-2 \varepsilon) \geq \frac{v-u}{y-x}
$$

Análogamente,

$$
\begin{aligned}
\frac{f^{-1}(u)-u}{f^{-1}(u)-x} \cdot \frac{y-x}{v-u} & =\left(1-\frac{u-x}{f^{-1}(u)-x}\right) \frac{y-x}{v-u} \leq\left(1-\frac{\left(c_{\varepsilon}-a\right) \kappa(\varepsilon, n)}{f^{-1}(u)-x}\right) \frac{y-x}{v-u} \\
& \leq(1-\kappa(\varepsilon, n)) e^{2(n+1) \varepsilon} \leq e^{-2 \varepsilon}
\end{aligned}
$$


por lo que

$$
\frac{f^{-1}(u)-u}{f^{-1}(u)-x} \exp (2 \varepsilon) \leq \frac{v-u}{y-x} .
$$

Esto concluye la verificación de la condición (iii) de la proposición 3.44, y por lo tanto cierra la demostración del segundo teorema de Duminy.

Ejercicio 3.46. Verifique directamente que, para el pseudo-grupo generado por las aplicaciones $f$ y $g$ de la figura 16, el grafo de Cayley asociado a las órbitas semi-excepcionales es un árbol. Usando esto concluya directamente que el espacio de órbitas correspondiente tiene infinitos fines. Para las órbitas contenidas en $\Lambda$ que no son semi-excepcionales muestre que, si bien ellas no tienen necesariamente una estructura de árbol, también poseen infinitos fines.

\section{Dos problemas abiertos}

En la teoría de grupos de difeomorfismos de clase $\mathrm{C}^{1+l i p}$ del círculo subsisten aún dos importantes problemas. En lo que sigue daremos una breve reseña de cada uno de ellos.

\subsection{Acciones minimales}

El primer problema concierne la ergodicidad de las acciones minimales.

Problema 1. Si $\Gamma$ es un subgrupo finitamente generado de $\operatorname{Difeo}{ }_{+}^{1+l i p}\left(\mathrm{~S}^{1}\right)$ cuyas órbitas son densas, ¿es la acción de $\Gamma$ sobre $\mathrm{S}^{1}$ ergódica respecto a la medida de Lebesgue? (recordemos que una acción es ergódica si los conjuntos medibles e invariantes tienen medida nula o total; en lo que sigue, la ergodicidad será siempre considerada en relación a la medida de Lebesgue).

En el contexto de grupos de gérmenes en el origen de difeomorfismos holomorfos del plano complejo, una interrogante análoga tiene respuesta afirmativa $[82,103]$. Ésta es una de las razones que hacen presumir que la respuesta a la pregunta anterior debiese también ser afirmativa. Tal es el caso por ejemplo cuando $\Gamma$ posee un elemento cuyo número de rotación es irracional, de acuerdo al siguiente resultado obtenido independientemente por Herman y Katok. Para la demostración retomaremos las notaciones introducidas al final de la sección 2.1 del capítulo 2 en el estudio de la combinatoria de una rotación de ángulo irracional.

Teorema 3.47. Si un difeomorfismo $g \in \mathrm{Difeo}_{+}^{1+\mathrm{va}}\left(\mathrm{S}^{1}\right)$ tiene número de rotación irracional, entonces la acción del grupo generado por $g$ sobre el círculo es ergódica. 
Demostración. Antes de comenzar la prueba, recordemos que un punto de densidad de un conjunto $A \subset \mathrm{S}^{1}$ es un punto $p$ tal que

$$
\frac{\operatorname{Leb}(A \cap[p-\varepsilon, p+\varepsilon])}{\operatorname{Leb}([p-\varepsilon, p+\varepsilon])}
$$

tiende a 1 cuando $\varepsilon$ tiende a cero. Un teorema clásico debido a Lebesgue estipula que si $\operatorname{Leb}(A)>0$ entonces casi todo punto de $A$ es de densidad.

Supongamos que $g$ preserve un conjunto de medida positiva $A$. Probaremos que $A$ tiene medida total. Para ello, fijemos un punto de densidad $x_{0}$ de $A$. Sea $\varphi$ una conjugación de $g$ a la rotación de ángulo $\theta=\rho(g)$ tal que $\varphi\left(x_{0}\right)=0$. Como en la demostración del teorema de Denjoy, definamos $I_{n}(g)=\varphi^{-1}\left(I_{n}\right)$ y $J_{n}(g)=\varphi^{-1}\left(J_{n}\right)$. Observe que $x_{0} \in J_{n}(g)$ y que $\left|J_{n}(g)\right|$ converge a cero cuando $n$ tiende al infinito. Además, los intervalos $g^{k}\left(J_{n}(g)\right), k \in\left\{0, \ldots, q_{n+1}-1\right\}$, recubren el círculo, y cada punto de $\mathrm{S}^{1}$ está contenido en a lo más dos de ellos. Utilizando la desigualdad (3.22) no es difícil concluir que, para todo $k \in\left\{0, \ldots, q_{n+1}-1\right\}$,

$$
\begin{aligned}
\frac{\operatorname{Leb}\left(g^{k}\left(J_{n}(g) \backslash A\right)\right)}{\operatorname{Leb}\left(g^{k}\left(J_{n}(g)\right)\right)} & \leq \exp \left(V\left(g^{k} ; J_{n}(g)\right)\right) \cdot \frac{\operatorname{Leb}\left(J_{n}(g) \backslash A\right)}{\operatorname{Leb}\left(J_{n}(g)\right)} \\
& \leq \exp (2 V(g)) \cdot \frac{\operatorname{Leb}\left(J_{n}(g) \backslash A\right)}{\operatorname{Leb}\left(J_{n}(g)\right)} .
\end{aligned}
$$

Luego, por la invariancia de $A$,

$$
\begin{aligned}
\operatorname{Leb}\left(\mathrm{S}^{1} \backslash A\right) & \leq \sum_{k=0}^{q_{n+1}-1} \operatorname{Leb}\left(g^{k}\left(J_{n}(g) \backslash A\right)\right) \\
& \leq \exp (2 V(g)) \frac{\operatorname{Leb}\left(J_{n}(g) \backslash A\right)}{\operatorname{Leb}\left(J_{n}(g)\right)} \sum_{k=0}^{q_{n+1}-1} \operatorname{Leb}\left(g^{k}\left(J_{n}(g)\right)\right) \\
& \leq 2 \operatorname{Leb}\left(\mathrm{S}^{1}\right) \exp (2 V(g)) \frac{\operatorname{Leb}\left(J_{n}(g) \backslash A\right)}{\operatorname{Leb}\left(J_{n}(g)\right)}
\end{aligned}
$$

Siendo $x_{0}$ un punto de densidad de $A$, el valor de $\operatorname{Leb}\left(J_{n}(g) \backslash A\right) / \operatorname{Leb}\left(J_{n}(g)\right)$ converge a 0 cuando $n$ tiende al infinito, de donde se concluye inmediatamente que $\operatorname{Leb}\left(\mathrm{S}^{1} \backslash A\right)=0$.

De acuerdo a la proposición 1.1, todo grupo de homeomorfismos del círculo que actúa de manera minimal y preserva una medida de probabilidad es topológicamente conjugado a un grupo de rotaciones. Además, si dicho grupo es finitamente generado, necesariamente (al menos) uno de sus generadores tiene número de rotación irracional. Del teorema 3.47 se deduce entonces lo siguiente. 
Corolario 3.48. Si $\Gamma$ es un subgrupo finitamente generado de $\operatorname{Difeo}{ }_{+}^{1+\mathrm{va}}\left(\mathrm{S}^{1}\right)$ con una medida de probabilidad invariante y órbitas densas, entonces la acción de $\Gamma$ es ergódica con respecto a la medida de Lebesgue.

La hipótesis de diferenciabilidad es necesaria para este resultado. En efecto, en [158] se construyen ejemplos de difeomorfismos de clase $\mathrm{C}^{1}$ del círculo de número de rotación irracional cuyas órbitas son densas pero que no son ergódicos. Es muy posible que refinando el método de construcción de [158] se puedan fabricar ejemplos análogos de clase $\mathrm{C}^{1+\tau}$ para todo $\tau<1$.

Debido al teorema 3.47 , para grupos de difeomorfismos de clase $\mathrm{C}^{1+\text { lip }}$ del círculo actuando de manera minimal el problema de la ergodicidad se presenta cuando el número de rotación de cada elemento del grupo que actúa es racional. Bajo esta hipótesis se desconoce la respuesta general al problema. Sin embargo, como veremos a continuación, existe un caso muy importante en el cual la ergodicidad de la acción está asegurada.

Definición 3.49. La acción de un subgrupo $\Gamma$ de Difeo ${ }_{+}^{1}\left(\mathrm{~S}^{1}\right)$ es diferenciablemente dilatante si para todo punto $p \in \mathrm{S}^{1}$ existe un elemento $g \in \Gamma$ tal que $g^{\prime}(p)>1$.

Una consecuencia directa del teorema 3.50 que probaremos más adelante es el hecho que si la acción de un subgrupo finitamente generado $\Gamma$ de Difeo $_{+}^{1+\tau}\left(\mathrm{S}^{1}\right)$ es diferenciablemente dilatante y minimal, con $\tau>0$, entonces dicha acción es ergódica. Daremos sin embargo una versión más general de este fenómeno debido a que, a priori, la condición de dilatación de la definición no es invariante por conjugación diferenciable.

Para enunciar la versión general del principio mencionado más arriba, consideremos un subgrupo finitamente generado $\Gamma$ de $\operatorname{Difeo}_{+}^{1}\left(\mathrm{~S}^{1}\right)$ y fijemos un sistema (finito y) simétrico de generadores $\mathcal{G}$. Para cada $n \geq 1$ consideremos el conjunto

$$
B_{\mathcal{G}}(n)=\left\{h \in \Gamma: h=h_{m} \cdots h_{1} \quad \text { para algún } h_{i} \in \mathcal{G} \text { y } m \leq n\right\},
$$

y para cada $x \in \mathrm{S}^{1}$ definamos

$$
\lambda(x)=\limsup _{n \rightarrow \infty}\left(\max _{h \in B_{\mathcal{G}}(n)} \frac{\log \left(h^{\prime}(x)\right)}{n}\right) .
$$

Observe que este número siempre es finito, ya que está mayorado por

$$
\sup _{h \in \mathcal{G}, y \in \mathrm{S}^{1}} \log \left(h^{\prime}(y)\right) .
$$

Para cada $\lambda>0$ hagamos $E_{\lambda}(\Gamma)=\left\{x \in \mathrm{S}^{1}: \lambda(x) \geq \lambda\right\}$. El conjunto exponencial de la acción $E(\Gamma)$ es definido como la unión de los $E_{\lambda}(\Gamma)$ con $\lambda>0$, 
mientras que su complemento $S(\Gamma)$ es llamado conjunto subexponencial. Observe que cada $E_{\lambda}(\Gamma)$, así como $E(\Gamma)$ y $S(\Gamma)$, son conjuntos borelianos. Por otro lado, es fácil verificar que la función $x \mapsto \lambda(x)$ es invariante por la acción de $\Gamma$. Por lo tanto, los conjuntos $E_{\lambda}(\Gamma), E(\Gamma)$ y $S(\Gamma)$ son invariantes por $\Gamma$. Finalmente, si $\Gamma$ es diferenciablemente dilatante entonces $\lambda(x)>0$ para todo $x \in \mathrm{S}^{1}$.

Teorema 3.50. Supongamos que $\Gamma$ sea un subgrupo de $\operatorname{Difeo}_{+}^{1+\tau}\left(\mathrm{S}^{1}\right)$ para algún $\tau>0$. Si la acción de $\Gamma$ es minimal y el conjunto exponencial posee medida de Lebesgue positiva, entonces dicha acción es ergódica.

Obtendremos este teorema como una consecuencia casi directa de la proposición siguiente.

Proposición 3.51. Sean $\tau>0$ una constante $y \Gamma$ un subgrupo finitamente generado de Difeo ${ }_{+}^{1+\tau}\left(\mathrm{S}^{1}\right)$. Si la acción de $\Gamma$ es minimal y $A$ es un conjunto (medible) invariante por $\Gamma$ tal que $\operatorname{Leb}(E(\Gamma) \cap A)>0$, entonces $A$ tiene medida de Lebesgue total.

La demostración de esta proposición se basa en una versión "no uniformemente hiperbólica" de un argumento hoy clásico y debido en gran parte a Sullivan (vea la sección 4 de [147]). La idea de este argumento consiste en "hacer explotar una vecindad arbitrariamente pequeña" de un punto de densidad $x$ del conjunto invariante $E(\Gamma) \cap A$ mediante composiciones sucesivas por elementos del grupo. El punto técnico esencial consiste en controlar la distorsión de estas composiciones, de modo que en el límite dicha vecindad se transforme en un intervalo del círculo al cual se le ha sustraído a lo más un conjunto de medida nula. Para lograr este control las hipótesis $\lambda(x)>0$ y $\tau>0$ serán esenciales. Técnicamente, la situación es más simple en el caso diferenciablemente dilatante; para el caso general recurriremos al siguiente lema combinatorio debido a Pliss [169].

Lema 3.52. Dados $\lambda \in \mathbb{R}, \varepsilon>0$ y $A>0$, existe $\delta=\delta(\lambda, \varepsilon, A)>0$ tal que para toda sucesión finita $a_{1}, \ldots, a_{N}$ de números reales satisfaciendo $\left|a_{k}\right| \leq A$ para todo $k \in\{1, \ldots, N\} y$

$$
\sum_{k=1}^{N} a_{k} \leq N \lambda
$$

existen $\ell \geq N \delta$ y $1 \leq n_{1}<\ldots<n_{\ell} \leq N$ tales que, para todo $k \in\left\{0, \ldots, n_{i}-1\right\}$ y todo $i \in\{1, \ldots, \ell\}$, se tiene

$$
\sum_{j=k+1}^{n_{i}} a_{i} \leq\left(n_{i}-k\right)(\lambda+\varepsilon) .
$$


Demostración. Para cada $k \in\{0, \ldots, N-1\}$ sea $s(k)=\sum_{j=k+1}^{N}\left(a_{j}-(\lambda+\varepsilon)\right)$. Consideremos el conjunto de (todos) los índices $0=n_{0}<n_{1}<\ldots<n_{\ell}$ para los cuales se satisface

$$
s(k) \leq s\left(n_{i}\right) \quad \text { para todo } k \in\left\{0, \ldots, n_{i}-1\right\} .
$$

Para $i \geq 1$ y $k<n_{i}$ se cumple

$$
\sum_{j=k+1}^{n_{i}} a_{j}=s(k)-s\left(n_{i}\right)+(\lambda+\varepsilon)\left(n_{i}-k\right) \leq(\lambda+\varepsilon)\left(n_{i}-k\right) .
$$

Resta entonces estimar el valor de $\ell$. Para ello, observe en primer lugar que $s\left(n_{i-1}\right) \geq s\left(n_{i}-1\right)$, pues en caso contrario existiría $k \in\left\{n_{i-1}+1, \ldots, n_{i}-1\right\}$ tal que $s(k) \geq s\left(k^{\prime}\right)$ para todo $k^{\prime} \in\left\{n_{i-1}+1, \ldots, n_{i}-1\right\}$ (y por lo tanto para todo $\left.k^{\prime} \in\left\{0, \ldots, n_{i}-1\right\}\right)$, lo cual contradice la definición de $n_{i}$. Se tiene entonces

$$
s\left(n_{i-1}\right) \geq s\left(n_{i}\right)+\left(a_{n_{i}}-(\lambda+\varepsilon)\right) \geq s\left(n_{i}\right)-(A+\lambda+\varepsilon),
$$

por lo que

$$
s\left(n_{0}\right) \geq s\left(n_{\ell}\right)-\ell(A+\lambda+\varepsilon) .
$$

Puesto que

$$
s\left(n_{0}\right)=s(0)=\sum_{j=1}^{N} a_{j}-N(\lambda+\varepsilon) \leq-N \varepsilon,
$$

teniendo en cuenta que $s\left(n_{\ell}\right) \geq s(N-1)=a_{N}-(\lambda+\varepsilon)$ (pues $n_{\ell}$ es el mayor de los índices que satisface (3.40)), a partir de (3.41) deducimos

$$
-N \varepsilon \geq a_{N}-(\lambda+\varepsilon)-\ell(A+\lambda+\varepsilon) \geq-(\ell+1)(A+\lambda+\varepsilon) .
$$

Por lo tanto, la afirmación del lema se verifica para $\delta \geq \varepsilon / 2(\varepsilon+\lambda+A)$.

Para probar la proposición 3.51 fijemos un punto de densidad $x$ del conjunto $E_{\lambda}(\Gamma) \cap A$, el cual es invariante por $\Gamma$. Como $x$ pertenece a $E_{\lambda}(\Gamma)$, para todo $n \in \mathbb{N}$ suficientemente grande podemos hallar una sucesión finita $h_{n, 1}, \ldots, h_{n, n}$ de elementos de $\mathcal{G}$ tales que para $g_{n}=h_{n, n} \cdots h_{n, 1}$ se tenga

$$
\log \left(g_{n}^{\prime}(x)\right) \geq \frac{\lambda n}{2} .
$$

Para $\lambda_{n, j}=\log \left(h_{n, j}^{\prime}\right)\left(h_{n, j-1} \cdots h_{n, 1}(x)\right)$ se cumple entonces

$$
\sum_{j=1}^{n}\left(\frac{\lambda}{2}-\lambda_{n, j}\right) \leq 0
$$

Por el lema de Pliss, existen $\delta>0$ y $1 \leq n_{1}<\ldots<n_{\ell} \leq n$ tales que $\ell \geq \delta n \mathrm{y}$

$$
\sum_{j=k+1}^{n_{i}}\left(\frac{\lambda}{2}-\lambda_{n_{i}, j}\right) \leq \frac{\lambda\left(n_{i}-k\right)}{3}
$$


para todo $k \in\left\{0, \ldots, n_{i}-1\right\}$ y todo $i \in\{1, \ldots, \ell\}$. En otras palabras,

$$
\sum_{j=k+1}^{n_{i}} \lambda_{n_{i}, j} \geq\left(n_{i}-k\right)\left(\frac{\lambda}{2}-\frac{\lambda}{3}\right)=\frac{\lambda\left(n_{i}-k\right)}{6}
$$

para todo $k \in\left\{0, \ldots, n_{i}-1\right\}$ y todo $i \in\{1, \ldots, \ell\}$. Observemos que $n_{\ell} \geq \delta n$. Un argumento sencillo de tipo diagonal de Cantor muestra la existencia de una sucesión de elementos $h_{j} \in \mathcal{G}$ tales que para la sucesión $\left(f_{k}\right)$ definida por $f_{k}=h_{k} \cdots h_{1}$ y $f_{0}=i d$, el número de tiempos hiperbólicos es infinito. De manera más precisa, existe una sucesión infinita $n_{1}<n_{2}<\ldots$ tal que para todo $i \in \mathbb{N}$ y todo $k \in\left\{0, \ldots, n_{i}-1\right\}$,

$$
\sum_{j=k+1}^{n_{i}} \log \left(h_{k}^{\prime}\right)\left(f_{k-1}(x)\right) \geq \frac{\lambda\left(n_{i}-k\right)}{6} .
$$

Fijemos uno de esos tiempos hiperbólicos $m=n_{i}$. Sea $\left.\varepsilon_{0} \in\right] 0,1 / 2[$ una constante tal que, para todo $y, z$ satisfaciendo $\operatorname{dist}(y, z) \leq \varepsilon_{0}$ y todo $h \in \mathcal{G}$,

$$
\frac{\left(h^{-1}\right)^{\prime}(z)}{\left(h^{-1}\right)^{\prime}(y)} \leq \exp \left(\frac{\lambda}{12}\right) \text {. }
$$

Sea $C>0$ una constante de $\tau$-hölderianidad para el logaritmo de la derivada de los elementos de $\mathcal{G}$, i.e., para todo $h \in \mathcal{G}$ y todo $y, z$ en $\mathrm{S}^{1}$,

$$
\left|\log \left(h^{\prime}\right)(y)-\log \left(h^{\prime}\right)(z)\right| \leq C \operatorname{dist}^{\tau}(y, z) .
$$

Para cada $k \in\{0, \ldots, n\}$ denotemos $x_{k}=f_{k}(x)$ e $y_{k}=h_{k+1}^{-1} \cdots h_{m}^{-1}\left(x+\varepsilon_{0}\right)$ (de modo que $\left.y_{m}=x_{m}+\varepsilon_{0}\right)$. Finalmente, sea $D=C \varepsilon_{0}^{\tau} /\left(e^{\frac{\lambda \tau}{12}}-1\right)$.

Lema 3.53. Con las notaciones precedentes, para todo $p, q$ en $\left[x, y_{0}\right]$ se tiene

Además,

$$
\frac{f_{m}^{\prime}(p)}{f_{m}^{\prime}(q)} \leq e^{D}
$$

$$
\frac{\varepsilon_{0}}{D f_{m}^{\prime}(x)} \leq \operatorname{dist}\left(x, y_{0}\right) \leq \frac{D \varepsilon_{0}}{f_{m}^{\prime}(x)} .
$$

Demostración. Afirmamos en primer lugar que para todo $k \in\{0, \ldots, m\}$ se tiene

$$
\operatorname{dist}\left(x_{k}, y_{k}\right) \leq \varepsilon_{0} \exp (-\lambda(m-k) / 12) .
$$

La verificación de esta afirmación es por inducción (descendente). Para $k=m$ ella resulta de la definición $y_{m}=x_{m}+\varepsilon_{0}$. Supongamos que sea válida para $k+1, \ldots, m$. Puesto que $\left[x_{k}, y_{k}\right]=h_{k+1}^{-1} \cdots h_{m}^{-1}\left(\left[x_{m}, y_{m}\right]\right)$, existe $z_{m} \in\left[x_{m}, y_{m}\right]$ tal que

$$
\left(h_{k+1}^{-1} \cdots h_{m}^{-1}\right)^{\prime}\left(z_{m}\right)=\frac{\left|\left[x_{k}, y_{k}\right]\right|}{\left|\left[x_{m}, y_{m}\right]\right|} .
$$


Haciendo $z_{i}=h_{i+1}^{-1} \cdots h_{m}^{-1}\left(z_{m}\right)$ para $i \in\{k+1, \ldots, m-1\}$, por la hipótesis de inducción se tiene, para todo $i \in\{k+1, \ldots, m\}$,

$$
\operatorname{dist}\left(x_{i}, z_{i}\right) \leq \operatorname{dist}\left(x_{i}, y_{i}\right) \leq \varepsilon_{0} \exp (-\lambda(m-i) / 12) \leq \varepsilon_{0} .
$$

Observe que, debido a (3.43),

$$
\left(h_{i}^{-1}\right)^{\prime}\left(z_{i}\right) \leq\left(h_{i}^{-1}\right)^{\prime}(x) \exp (\lambda / 12),
$$

por lo que

$$
\begin{aligned}
\left(h_{k+1}^{-1} \cdots h_{m}^{-1}\right)^{\prime}\left(z_{m}\right) & =\left(h_{k+1}^{-1}\right)^{\prime}\left(z_{k+1}\right) \cdots\left(h_{m}^{-1}\right)^{\prime}\left(z_{m}\right) \\
& \leq\left(h_{k+1}^{-1}\right)^{\prime}\left(x_{k+1}\right) \cdots\left(h_{m}^{-1}\right)^{\prime}\left(x_{m}\right) \exp (\lambda(m-k) / 12) .
\end{aligned}
$$

Por (3.42) y (3.46) lo anterior implica que

$\frac{\left|\left[x_{k}, y_{k}\right]\right|}{\left|\left[x_{m}, y_{m}\right]\right|} \leq \exp (-\lambda(m-k) / 6) \exp (\lambda(m-k) / 12)=\exp (-\lambda(m-k) / 12)$,

por lo que

$$
\left|\left[x_{k}, y_{k}\right]\right| \leq \varepsilon_{0} \exp (-\lambda(m-k) / 12),
$$

completando así la prueba de la afirmación.

Ahora bien, si $p$ y $q$ pertenecen a $\left[x, y_{0}\right]$ entonces $f_{k}(p)$ y $f_{k}(q)$ pertenecen a $\left[x_{k}, y_{k}\right]$ para todo $k \in\{0, \ldots, m\}$. Por lo tanto,

$$
\begin{aligned}
\left|\log \left(\frac{f_{m}^{\prime}(p)}{f_{m}^{\prime}(q)}\right)\right| & \leq \sum_{k=0}^{m-1}\left|\log \left(h_{k+1}^{\prime}\right)\left(f_{k}(p)\right)-\log \left(h_{k+1}^{\prime}\right)\left(f_{k}(q)\right)\right| \\
& \leq C \sum_{k=0}^{m-1} d i s t^{\tau}\left(f_{k}(p), f_{k}(q)\right) \\
& \leq C \varepsilon_{0}^{\tau} \sum_{k \geq 0}\left(e^{-\lambda(m-k) / 12}\right)^{\tau}=D,
\end{aligned}
$$

lo cual prueba (3.44). Finalmente, existe $\bar{y} \in\left[x, y_{0}\right]$ tal que

$$
f_{m}^{\prime}\left(\bar{y}_{0}\right)=\frac{\left|\left[x_{m}, y_{m}\right]\right|}{\left|\left[x, y_{0}\right]\right|}=\frac{\varepsilon_{0}}{\left|\left[x, y_{0}\right]\right|}
$$

por lo que (3.44) implica

$$
\frac{\varepsilon_{0}}{\operatorname{dist}\left(x, y_{0}\right)} \in\left[e^{-D} f_{m}^{\prime}(x), e^{D} f_{m}^{\prime}(x)\right]
$$

lo cual prueba (3.45).

Para completar la demostración de la proposición 3.51, consideremos nuevamente la sucesión (infinita) de tiempos hiperbólicos $1 \leq n_{1}<n_{2}<\ldots$, y para cada $i \in \mathbb{N}$ denotemos

$$
\bar{x}_{i}=f_{n_{i}}(x), \quad \bar{y}_{i}=\bar{x}_{i}+\varepsilon, \quad y_{0}^{i}=f^{-n_{i}}\left(\bar{y}_{i}\right) .
$$


Por el lemma 3.53 y (3.42) tenemos

$$
\frac{f_{n_{i}}^{\prime}(p)}{f_{n_{i}}^{\prime}(q)} \leq e^{D} \quad \text { para todo } p, q \text { en }\left[x, y_{0}^{i}\right]
$$

y

$$
\frac{\varepsilon_{0}}{D f_{n_{i}}^{\prime}(x)} \leq \operatorname{dist}\left(x, y_{0}^{i}\right) \leq \frac{D \varepsilon_{0}}{f_{n_{i}}^{\prime}(x)} \leq \frac{D \varepsilon_{0}}{e^{\lambda n_{i} / 6}} .
$$

Pasando a una subsucesión si es necesario, podemos asumir que $f_{n_{i}}(x)$ converge a un punto $\bar{x} \in \mathrm{S}^{1}$. Probaremos que

$$
\operatorname{Leb}\left(\left(\mathrm{S}^{1} \backslash A\right) \cap\left[\bar{x}, \bar{x}+\varepsilon_{0}\right]\right)=0 .
$$

Para ello, fijemos $\varepsilon>0$. Puesto que $y_{0}^{i}$ tiende a $x$ cuando $i$ tiende al infinito y $x$ es un punto de densidad de $A$, para $i$ suficientemente grande se tiene

$$
\frac{\operatorname{Leb}\left(\left(\mathrm{S}^{1} \backslash A\right) \cap\left[x, y_{0}^{i}\right]\right)}{\operatorname{Leb}\left(\left[x, y_{0}^{i}\right]\right)} \leq \frac{\varepsilon}{2 \varepsilon_{0} D} .
$$

Podemos suponer también que $\operatorname{dist}\left(\bar{x}, \bar{x}_{i}\right) \leq \varepsilon / 2$. Por $(3.47)$,

$$
\begin{aligned}
\operatorname{Leb}\left(\left(\mathrm{S}^{1} \backslash A\right) \cap\left[\bar{x}, \bar{x}+\varepsilon_{0}\right]\right) & \leq \frac{\varepsilon}{2}+\varepsilon_{0} \frac{\operatorname{Leb}\left(\left(\mathrm{S}^{1} \backslash A\right) \cap\left[\bar{x}_{i}, \bar{x}_{i}+\varepsilon_{0}\right]\right)}{\operatorname{Leb}\left(\left[\bar{x}_{i}, \bar{x}_{i}+\varepsilon_{0}\right]\right)} \\
& \leq \frac{\varepsilon}{2}+\varepsilon_{0} D \frac{\operatorname{Leb}\left(\left(\mathrm{S}^{1} \backslash A\right) \cap\left[x, y_{0}^{i}\right]\right)}{\operatorname{Leb}\left(\left[x, y_{0}^{i}\right]\right)},
\end{aligned}
$$

por lo que (3.48) implica

$$
\operatorname{Leb}\left(\left(\mathrm{S}^{1} \backslash A\right) \cap\left[\bar{x}, \bar{x}+\varepsilon_{0}\right]\right) \leq \varepsilon .
$$

Puesto que esto vale para todo $\varepsilon>0$, concluimos que

$$
\operatorname{Leb}\left(\left(\mathrm{S}^{1} \backslash A\right) \cap\left[\bar{x}, \bar{x}+\varepsilon_{0}\right]\right)=0 .
$$

Usando la minimalidad de la acción de $\Gamma$ obtenemos $\operatorname{Leb}\left(\mathrm{S}^{1} \backslash A\right)=0$, por lo que $\operatorname{Leb}(A)=1$, lo cual cierra la prueba de la proposición 3.51.

Demostración del teorema 3.50. Si $\operatorname{Leb}(E(\Gamma))>0$ entonces, para $A=E(\Gamma)$, la proposición 3.51 permite concluir que $\operatorname{Leb}(E(\Gamma))=1$. De hecho, por la invariancia de cada $E_{\lambda}(\Gamma)$, existe un parámetro $\lambda>0$ tal que para casi todo punto $x \in \mathrm{S}^{1}$ se tiene $\lambda(x)=\lambda$.

Sea ahora $A$ un conjunto medible e invariante por $\Gamma$ tal que $\operatorname{Leb}(\Gamma)>0$. Puesto que $\operatorname{Leb}(E(\Gamma))=1$, tenemos

$$
\operatorname{Leb}(E(\Gamma) \cap A)=\operatorname{Leb}(A)>0 .
$$

Nuevamente, la proposición 3.51 implica que $\operatorname{Leb}(A)=1$, y esto demuestra la ergodicidad de la acción. 
Observación 3.54. La hipótesis $\tau>0$ es necesaria para la validez del teorema 3.50 , pues existen subgrupos finitamente generados de Difeo ${ }_{+}^{1}\left(\mathrm{~S}^{1}\right)$ que actúan de manera minimal pero no ergódica (y que además no preservan ninguna medida de probabilidad). En efecto, en [171] se da un ejemplo de una aplicación dilatante de clase $\mathrm{C}^{1}$ del círculo sobre sí mismo cuyo grado topológico es $m \geq 10$. Usando la linearización de Müller y Tsuboi del ejercicio 5.11, podemos asumir que la derivada de esta aplicación en el origen es igual a 1. Esto permite llevar a efecto (una leve modificación de) la construcción de Ghys y Sergiescu de la sección 5.2 para así obtener un grupo de Thompson $m$-ádico de difeomorfismos de clase $\mathrm{C}^{1}$ del círculo cuya acción es minimal pero no ergódica (vea el ejercicio 2.12).

Para cerrar esta sección, utilizaremos el teorema 3.50 para probar que si un subgrupo de $\operatorname{Difeo}_{+}^{1+l i p}\left(\mathrm{~S}^{1}\right)$ verifica las hipótesis del primer teorema de Duminy y no posee órbitas finitas (de manera equivalente, actúa de manera minimal), entonces su acción es ergódica.

Sea $\Gamma$ un subgrupo de Difeo ${ }_{+}^{1+l i p}\left(S^{1}\right)$ cuyas órbitas son densas y que es generado por una familia $\mathcal{G}$ de difeomorfismos que verifican $V(f)<V_{0}$ para todo $f \in \mathcal{G}$, y tales que un elemento $g \in \mathcal{G}$ posee un número finito de puntos periódicos. Si denotamos $\operatorname{Per}(g)$ al conjunto de tales puntos, entonces existen un punto $p \in \operatorname{Per}(g)$ y un elemento $f \in \mathcal{G}$ tales que $f(p)$ pertenece al complemento de $\operatorname{Per}(g)$. En efecto, en caso contrario el conjunto $\operatorname{Per}(g)$ sería invariante por $\Gamma$, contradiciendo la hipótesis de minimalidad.

Usando los mismos argumentos de la parte central de la demostración del teorema 3.33, a partir de $f$ y $g$ es posible fabricar dos elementos $F, G$ en $\Gamma$ que están "enlazados" sobre un intervalo $[a, b]$ de $\mathrm{S}^{1}$ como las aplicaciones del lema 3.34 , y tales que $V(F ;[a, c])$ y $V(G ;[c, b])$ son pequeños, donde $c=G^{-1}(a)$. Fijemos $\varepsilon>0$ suficientemente pequeño y sean $\left\{I_{1}, \ldots, I_{n}\right\}$ una familia de intervalos que recubren al círculo y $h_{1}, \ldots, h_{n}$ elementos de $\Gamma$ tales que $h_{i}(x) \in[c+\varepsilon, b]$ para todo $x \in I_{i}$. Definamos la constante $C$ haciendo $C^{-1}=\inf \left\{h_{i}^{\prime}(x): x \in I_{1} \cup \ldots \cup I_{n}\right\}$, y escojamos un entero suficientemente grande $N$ de modo que cada rama de la aplicación de retorno $H^{N}$ inducida por $F$ y $G$ sea $C$-dilatante (vea la proposición 3.35). Para $g_{i}=H^{N} h_{i} \in \Gamma$ se tiene $g_{i}^{\prime}(x)>1$ para todo $x \in I_{i}$ (observe que consideramos la derivada a derecha de la aplicación $H^{N}$ ). Luego, la acción de $\Gamma$ es diferenciablemente dilatante. La ergodicidad resulta entonces como una consecuencia del teorema 3.50.

Ejercicio 3.55. Pruebe que la acción minimal canónica de $\operatorname{PSL}(2, \mathbb{Z})$ sobre $\mathrm{S}^{1}$ no es diferenciablemente dilatante. Demuestre que lo mismo vale para las acciones suaves y minimales del grupo de Thompson G construidas en la sección 5.2 del primer capítulo.

Observación. Es posible probar que, para las acciones en cuestión, el conjunto exponencial tiene medida nula. A pesar de esto, no es difícil modificar los argumentos de la demostración del teorema 3.50 para probar dichas acciones son ergódicas. Para el caso particular de $\operatorname{PSL}(2, \mathbb{Z})$ consignemos además que la acción de todo grupo fuchsiano cuyas órbitas son densas es ergódica (vea por ejemplo $[153])$. 
Ejercicio 3.56. Enuncie de manera precisa y demuestre un resultado del siguiente tipo: si $\Gamma$ admite una familia a un parámetro $\Phi_{t}$ de representaciones en Difeo ${ }_{+}^{1+v a}\left(S^{1}\right)$ de modo que (todas) las órbitas de $\Phi_{0}(\Gamma)$ son densas y para $t>0$ cada $\Phi_{t}(\Gamma)$ admite un minimal excepcional, entonces la acción de $\Phi_{0}(\Gamma)$ no es diferenciablemente dilatante. Explique por qué esto permite redemostrar lo pedido en el ejercicio precedente.

\subsection{Acciones con un minimal excepcional}

El segundo problema abierto dice relación con la medida de los minimales excepcionales.

Problema 2. Si $\Gamma$ es un subgrupo finitamente generado de $\operatorname{Difeo}{ }_{+}^{1+\operatorname{lip}}\left(\mathrm{S}^{1}\right)$ que admite un minimal excepcional $\Lambda$, ¿es la medida de Lebesgue de $\Lambda$ necesariamente nula?

Un problema estrechamente relacionado es el de la finitud del número de clases para la relación de equivalencia que identifica dos componentes conexas de $\mathrm{S}^{1} \backslash \Lambda$ si una de ellas es la imagen de la otra por un elemento de $\Gamma$ (si esto fuese siempre válido, ello constituiría una generalización del clásico teorema de finitud de Ahlfors [1]). Por otra parte, una conjetura de Dippolito sugiere que la acción de $\Gamma$ sobre $\Lambda$ debiera ser topológicamente conjugada a la acción de un grupo de homeomorfismos afines por partes [55].

La nulidad de la medida de Lebesgue de minimales excepcionales es válida para grupos fuchsianos [153], pero la demostración en este caso utiliza métodos muy particulares que difícilmente podrían generalizarse para el caso general. En lo que sigue nos concentraremos en una clase particular de dinámica, a saber, aquélla de los minimales excepcionales markovianos. En términos sencillos, sobre dichos conjuntos subyace una dinámica conjugada a un subdesplazamiento de tipo finito, lo cual simplifica significativamente su estudio. Siguiendo [41] y [132], veremos que para este caso la respuesta al problema 2 es positiva (lo mismo ocurre para el problema de finitud de componentes conexas del complemento módulo la acción: vea [42]).

Sea $P=\left(p_{i j}\right)$ una matriz de incidencia de orden $k \times k$ (i.e., una matriz con entradas 0 y 1$)$. Consideremos el espacio $\Omega=\{1, \ldots, k\}^{\mathbb{N}}$ y el subespacio $\Omega^{*}$ de las sucesiones admisibles, es decir, de los elementos $\omega=\left(i_{1}, i_{2}, \ldots\right) \in \Omega$ tales que $p_{i_{j} i_{j+1}}=1$ para todo $j \in \mathbb{N}$. Sobre este último espacio (provisto de la topología natural) podemos considerar la dinámica de la aplicación desplazamiento $\sigma$.

Definición 3.57. Sea $\mathcal{G}=\left\{g_{1}, \ldots, g_{k}\right\}$ una familia finita de homeomorfis$\operatorname{mos} g_{i}: \operatorname{dom}\left(g_{i}\right) \rightarrow \operatorname{ran}\left(g_{i}\right)$ definidos sobre intervalos abiertos y acotados. Si $\left\{I_{1}, \ldots, I_{k}\right\}$ es una familia de intervalos cerrados tal que $I_{j} \subset \operatorname{ran}\left(g_{j}\right)$ para todo $j \in\{1, \ldots, k\}$, entonces decimos que $\mathcal{S}=\left(\left\{I_{1}, \ldots, I_{k}\right\}, \mathcal{G}, P\right)$ es un sistema markoviano para el pseudo-grupo markoviano $\Gamma_{\mathcal{S}}$ generado por los $g_{i}$ si las siguientes propiedades son satisfechas: 
(i) $\operatorname{ran}\left(g_{i}\right) \cap \operatorname{ran}\left(g_{j}\right)=\emptyset$ para todo $i \neq j$,

(ii) si $p_{i j}=1$ (resp. $p_{i j}=0$ ) entonces $I_{j} \subset \operatorname{dom}\left(g_{i}\right)$ y $g_{i}\left(I_{j}\right) \subset I_{i}$ (resp. $\left.I_{j} \cap \operatorname{dom}\left(g_{i}\right)=\emptyset\right)$.

Para cada sucesión admisible $\omega=\left(i_{1}, i_{2}, \ldots\right)$ y cada $n \in \mathbb{N}$, la transformación $\bar{h}_{n}(\omega)=g_{i_{1}} \cdots g_{i_{n}}$ está definida sobre el intervalo $g_{i_{n}}^{-1}\left(I_{i_{n}}\right)$. Denotemos entonces $I_{n}(\omega)=\bar{h}_{n}(\omega)\left(g_{i_{n}}^{-1}\left(I_{i_{n}}\right)\right)$ y

$$
\Lambda_{\mathcal{S}}=\cap_{n \in \mathbb{N}} \cup_{\omega \in \Omega^{*}} I_{n}(\omega) .
$$

Observemos que si $T: \cup_{i=1}^{k} \operatorname{ran}\left(g_{i}\right) \rightarrow \cup_{i=1}^{k} \operatorname{dom}\left(g_{i}\right)$ es la aplicación cuya restricción a cada conjunto $\operatorname{ran}\left(g_{i}\right)$ coincide con $g_{i}^{-1}$, entonces la restricción de $T$ a $\Lambda_{\mathcal{S}}$ es naturalmente semiconjugada al desplazamiento $\sigma: \Omega^{*} \rightarrow \Omega^{*}$.

Definición 3.58. Un conjunto $\Lambda$ invariante por la acción de un pseudogrupo es dicho markoviano si existe un intervalo abierto $L$ que intersecta a $\Lambda$ de modo que $L \cap \Lambda$ es igual a $\Lambda_{\mathcal{S}}$ para un sistema markoviano $\mathcal{S}$ definido sobre $L$. A dicho conjunto asociaremos la clase de diferenciabilidad del sistema de generadores del pseudo-grupo subyacente en $\mathcal{S}$.

Dentro de este capítulo ya hemos estudiado un ejemplo de pseudo-grupo markoviano: se trata de aquél ilustrado por la figura 16 (las propiedades de la definición son satisfechas para $g_{1}=f, g_{2}=g, I_{1}=[a, b]$ e $I_{2}=[c, d]$, considerando como dominio de definición para $g_{1}$ y $g_{2}$ un intervalo abierto ligeramente más grande que $[a, d]$; se tiene además $\Omega^{*}=\Omega$ ).

Teorema 3.59. Si $\Lambda$ es un conjunto excepcional local para un pseudo-grupo de difeomorfismos unidimensionales de clase $\mathrm{C}^{1+l i p} y$ de tipo markoviano, entonces la medida de Lebesgue de $\Lambda$ es nula.

En el caso en que las aplicaciones $h_{i}$ puedan ser escogidas uniformemente diferenciablemente contractantes, el resultado precedente es aún válido en clase $\mathrm{C}^{1+\tau}$ (compare con el teorema 3.62), pero no en clase $\mathrm{C}^{1}$ (vea el ejemplo 3.63). Para el caso $\mathrm{C}^{1+\tau}$ contractante vale en realidad un resultado mucho más fino: la dimensión de Hausdorff de $\Lambda$ es inferior a 1 [163]. Sin embargo, esto deja de ser válido en el caso markoviano no contractante, incluso en el contexto real-analítico [71].

Daremos la demostración del teorema 3.59 sólo para el caso particular del sistema markoviano discutido más arriba y representado por la figura 16. El lector no debiese tener problemas en adaptar los argumentos dados a continuación para el caso general (módulo pequeños detalles de índole combinatorio). Comenzamos con un lema de interés general similar al de Schwartz (para una aplicación interesante, vea los ejercicios 3.67 y 3.68). Por simplicidad, daremos una versión simplificada del lema (admitiendo una hipótesis sobre los dominios de definición de los generadores) que será suficiente para nuestros propósitos; sin embargo, el lector no debiese tener problemas en dar argumentos que permitan suprimirla. 
Lema 3.60. Sea $\Gamma$ un semigrupo de difeomorfismos de clase $C^{1+l i p}$ de una variedad unidimensional. Supongamos que $\Gamma$ sea generado por una familia finita $\mathcal{G}$ de elementos cuyos dominios de definición contienen un intervalo compacto $L$ tal que $g(L) \subset L$ para todo $g \in \Gamma$. Supongamos además que para un punto $x_{0} \in L$ se tenga $\sum_{g \in \Gamma} g^{\prime}\left(x_{0}\right) \leq S<\infty$. Si $C$ designa una constante de lipschitzianidad para el logaritmo de la derivada de los elementos de $\mathcal{G}$ sobre $L$, entonces para $\ell=\log (2) / 2 C S$ se tiene que para todo $g \in \Gamma$ y todo punto $x \in L$ en la $\ell$-vecindad de $x_{0}$ se cumple

$$
g^{\prime}(x) / 2 \leq g^{\prime}\left(x_{0}\right) \leq 2 g^{\prime}(x) .
$$

Demostración. Razonaremos por inducción sobre la longitud del elemento $g \in \Gamma$ (i.e., el número mínimo de factores en $\mathcal{G}$ necesarios para escribir $g$ como un producto; vea el apéndice para una discusión de esta noción en el caso de grupos). La afirmación es evidente si long $(g)=0$ (es decir, si $g$ es la identidad). Supongamos que ella sea válida para elementos de longitud menor a $n \in \mathbb{N}$, y sea $g \in \Gamma$ tal que $\operatorname{lon} g(g)=n$. Podemos entonces escribir $g=g_{n} \cdots g_{1}$, donde cada $g_{i}$ pertenece a $\mathcal{G}$. Por la hipótesis de inducción tenemos, para todo $x \in I \cap\left[x_{0}-\ell, x_{0}+\ell\right]$ y todo $i \in\{0, \ldots, n-1\}$,

$\left|g_{i} \cdots g_{1}(x)-g_{i} \cdots g_{1}\left(x_{0}\right)\right| \leq \sup _{y \in I}\left(g_{i} \cdots g_{1}\right)^{\prime}(y)\left|x-x_{0}\right| \leq\left(g_{i} \cdots g_{1}\right)^{\prime}\left(x_{0}\right) \frac{\log (2)}{C S}$.

Luego,

$$
\begin{aligned}
\left|\log \left(\frac{g^{\prime}(x)}{g^{\prime}\left(x_{0}\right)}\right)\right| & =\left|\log \left(\frac{g_{n}^{\prime}\left(g_{n-1} \cdots g_{1}(x)\right) \cdots g_{1}^{\prime}(x)}{g_{n}^{\prime}\left(g_{n-1} \cdots g_{1}\left(x_{0}\right)\right) \cdots g_{1}^{\prime}\left(x_{0}\right)}\right)\right| \\
& \leq \sum_{i=0}^{n-1}\left|\log \left(g_{i+1}^{\prime}\left(g_{i} \cdots g_{1}(x)\right)\right)-\log \left(g_{i+1}^{\prime}\left(g_{i} \cdots g_{1}\left(x_{0}\right)\right)\right)\right| \\
& \leq \sum_{i=0}^{n-1} C\left|g_{i} \cdots g_{1}(x)-g_{i} \cdots g_{1}\left(x_{0}\right)\right| \\
& \leq \sum_{i=0}^{n-1} \frac{\left(g_{i} \cdots g_{1}\right)^{\prime}\left(x_{0}\right) \log (2)}{S} \\
& \leq \log (2),
\end{aligned}
$$

lo cual prueba la estimación deseada para $g \in \Gamma$.

Estamos ahora en condiciones de proceder a la demostración del teorema 3.59 (al menos para el pseudo-grupo representado por la figura 16). Notemos primeramente que, para todo $x \in I=[b, c]$ y todo $g=g_{i_{n}} \cdots g_{i_{1}} \in \Gamma$ (donde cada $g_{i_{j}}$ pertenece a $\mathcal{G}=\left\{g_{1}=f, g_{2}=g\right\}$ ) se tiene

$\left|\log \left(g^{\prime}(b)\right)-\log \left(g^{\prime}(x)\right)\right| \leq C \sum_{j=0}^{n-1}\left|g_{i_{j}} \cdots g_{i_{1}}(b)-g_{i_{j}} \cdots g_{i_{1}}(x)\right| \leq C \sum_{j=0}^{n-1}|g(I)| \leq C(d-a)$. 
Escogiendo $x \in I$ de modo que $g^{\prime}(x)=|g(I)| /|I|$, de lo anterior deducimos que $g^{\prime}(b) \leq|g(I)| e^{C(d-a)} /|I|$, por lo que

$$
\sum_{g \in \Gamma} g^{\prime}(b) \leq \frac{e^{C(d-a)}}{|I|} \sum_{g \in \Gamma}|g(I)| \leq \frac{e^{C(d-a)}(d-a)}{|I|}=S .
$$

Haciendo $\ell=\log (2) / 2 C S$, del lema precedente se desprende que para todo $g, h$ en $\Gamma$ y todo punto $x \in[c, d]$ perteneciente a la $\ell$-vecindad de $h(b)$ se tiene $g^{\prime}(x) / 2 \leq g^{\prime}(b) \leq 2 g^{\prime}(x)$, y por lo tanto

$$
\sum_{g \in \Gamma}|g([h(b)-\ell, h(b)+\ell])| \leq 2 \ell \sum_{g \in \Gamma} \sup _{x \in[h(b)-\ell, h(b)+\ell]} g^{\prime}(x) \leq 4 \ell S .
$$

Fijemos $r \in \mathbb{N}$ de modo que para todo $\left(i_{1}, \ldots, i_{r}\right) \in\{1,2\}^{r}$ se tenga

$$
\left|\left(g_{i_{1}} \cdots g_{i_{r}}\right)([a, b])\right| \leq \ell .
$$

Observe que los intervalos en cuestión cubren al minimal excepcional $\Lambda$, por lo que para cada $n \in \mathbb{N}$ lo mismo ocurre para los intervalos de la forma $g_{i_{1}} \cdots g_{i_{n}} h([a, d])$, donde $\left(i_{1}, \ldots, i_{n}\right)$ varía en $\{1,2\}^{n}$ y $h$ recorre los elementos del tipo $g_{i_{1}} \cdots g_{i_{r}}$, con $\left(i_{1}, \ldots i_{r}\right) \in\{1,2\}^{r}$. Luego,

$$
\operatorname{Leb}(\Lambda) \leq \sum_{\left(i_{1}, \ldots, i_{n}\right) \in\{1,2\}^{n}} \sum_{\left(h_{1}, \ldots, h_{r}\right) \in\left\{g_{1}, g_{2}\right\}^{r}}\left|g_{i_{1}} \cdots g_{i_{n}} h_{1} \cdots h_{r}([a, d])\right|,
$$

y como para cada $\left(h_{1}, \ldots, h_{r}\right) \in\left\{g_{1}, g_{2}\right\}^{r}$ el punto $h_{1} \cdots h_{r}(b)$ pertenece a $h_{1} \cdots h_{r}([a, d])$, de lo anterior se deduce que

$$
\operatorname{Leb}(\Lambda) \leq \sum_{\left(h_{1}, \ldots, h_{r}\right) \in\left\{g_{1}, g_{2}\right\}^{r}} \sum_{\text {long }(g)=n}\left|g\left(\left[h_{1} \cdots h_{r}(b)-\ell, h_{1} \cdots h_{r}(b)+\ell\right]\right)\right| .
$$

Haciendo tender $n$ al infinito en esta última desigualdad, de la convergencia dada por (3.49) se deduce fácilmente que $\operatorname{Leb}(\Lambda)=0$, lo cual cierra la demostración.

Referimos a [40] y [105] para la realización de pseudo-grupos markovianos como pseudo-grupos de holonomía de foliaciones de codimensión 1. Señalemos en todo caso que no es difícil construir minimales excepcionales inducidos por sistemas no markovianos; para más información en torno a esto recomendamos la lectura de la última sección de [42].

Ejercicio 3.61. Completando el ejercicio 3.46, pruebe que si un minimal excepcional local es markoviano entonces todas las órbitas contenidas en él poseen infinitos fines (vea [41] si tiene problemas con esto).

Para cerrar esta sección comentemos brevemente otro caso para el cual la respuesta al problema 2 es positiva. El lector no debiera tener problemas en adaptar la demostración del teorema 3.50 para probar el siguiente resultado (alternativamente, vea [98]). 
Teorema 3.62. Sea $\Gamma$ un subgrupo de $\mathrm{Difeo}_{+}^{1+\tau}\left(\mathrm{S}^{1}\right)$ que admite un minimal excepcional $\Lambda$. Si $\tau>0$ entonces el conjunto $\Lambda \cap E(\Gamma)$ tiene medida de Lebesgue nula.

La pertinencia de la hipótesis $\tau>0$ para este resultado queda de manifiesto en el siguiente ejemplo debido a Bowen [17].

Ejemplo 3.63. El lector verificará sin dificultad que es posible construir dos aplicaciones $f$ y $g$ que cumplen las propiedades topológicas ilustradas en la figura 16 y satisfacen además las condiciones siguientes (notemos nuevamente $g_{1}=f$, $g_{2}=g$ e $I=[b, c]$ para así situarnos en el contexto markoviano, con $a=0$ y $d=1$ ): (i) existe una sucesión de números $\ell_{n}$ (donde $n \geq 0$ ) tal que

$$
\sum_{n \geq 0} \ell_{n}<1 \quad \text { y } \quad \lim _{n \rightarrow \infty} \frac{\ell_{n+1}}{\ell_{n}}=1,
$$

de modo que $|I|=\ell_{0}$ y si para cada $n \in \mathbb{N}$ y cada $\left(g_{i_{1}}, \ldots, g_{i_{n}}\right) \in\left\{g_{1}, g_{2}\right\}^{n}$ denotamos $I_{i_{1}, \ldots, i_{n}}=g_{i_{1}} \cdots g_{i_{n}}(I)$, entonces se tiene $\left|I_{i_{1}, \ldots, i_{n}}\right|=\ell_{n} / 2^{n}$;

(ii) cada $g_{i}$ es diferenciable sobre $I$ y sobre cada $I_{i_{1}, \ldots, i_{n}}$, su derivada en los extremos de estos intervalos es igual a $1 / 2$, y se tiene

$$
\lim _{n \rightarrow \infty} \max _{\left(i_{1}, \ldots, i_{n}\right)} \sup _{x \in I_{i_{1}, \ldots, i_{n}}}\left|g_{i}^{\prime}(x)-\frac{1}{2}\right|=0
$$

(iii) cada $g_{i}$ es diferenciable sobre una vecindad de $a=0$ y $d=1$, con derivada idénticamente igual a $1 / 2$ a izquierda (resp. derecha) de $a$ (resp. $d$ ).

Con estas condiciones no es difícil ver que $g_{1}$ y $g_{2}$ son de clase $\mathrm{C}^{1}$ sobre todo el intervalo $[a, d]=[0,1]$ y que sus derivadas son idénticamente iguales a $1 / 2$ sobre el conjunto de Cantor markoviano $\Lambda$. En cuanto a la medida de Lebesgue de este conjunto se tiene

$$
\operatorname{Leb}(\Lambda)=1-|I|-\sum_{n \geq 1} \sum_{\left(i_{1}, \ldots, i_{n}\right)}\left|I_{i_{1}, \ldots, i_{n}}\right|=1-\sum_{n \geq 0} \ell_{n}>0 .
$$

Observe que, por un método análogo al de la construcción del segundo ejemplo de la sección 1 del capítulo 2, sobre la base del ejemplo precedente es posible fabricar un subgrupo a dos generadores de Difeo ${ }_{+}^{1}\left(\mathrm{~S}^{1}\right)$ que admite un minimal excepcional de medida positiva completamente contenido en el conjunto exponencial de la acción.

Ejercicio 3.64. Después de leer la sección 1.4 del capítulo 4, pruebe que para todo $\tau>0$ existen subgrupos abelianos y finitamente generados de Difeo ${ }_{+}^{1+\tau}\left(\mathrm{S}^{1}\right)$ que admiten un minimal excepcional de medida positiva. Demuestre directamente (i.e., sin utilizar el teorema 3.62) que, para dichos ejemplos, el minimal excepcional correspondiente está contenido (módulo un conjunto de medida nula) en el conjunto subexponencial de la acción.

Ejemplo 3.65. Siguiendo una idea debida a Mañé, dado $\tau<1$ consideremos un difeomorfismo de clase $\mathrm{C}^{1+\tau}$ del círculo con número de rotación irracional y admitiendo un conjunto de Cantor invariante $\Lambda_{0}$ de medida positiva (vea el ejercicio anterior). Fijemos una componente conexa $I$ del complemento de $\Lambda_{0}$, 
y sea $\bar{H}: \mathrm{S}^{1} \rightarrow \mathrm{S}^{1}$ un difeomorfismo local de grado 2 que coincide con $f$ fuera de $I$ (i.e., la aplicación $\bar{H}$ "da una vuelta extra" sobre el círculo al recorrer $I$ ). A partir de $\bar{H}$ resulta fácil construir un pseudo-grupo a dos generadores con un minimal excepcional $\Lambda$ que contiene a $\Lambda_{0}$. Como la medida de Lebesgue de $\Lambda_{0}$ es positiva, lo mismo vale para $\Lambda$.

Ejercicio 3.66. Pruebe que si $\Gamma$ es un grupo finitamente generado de difeomorfismos real-analíticos del círculo que admite un minimal excepcional $\Lambda$, entonces a excepción posible de una cantidad numerable de puntos, $S^{1} \backslash \Lambda$ está contenido en el conjunto subexponencial $S(\Gamma)$ (señalemos que es muy probable que esto sea válido en general para subgrupos de $\left.\operatorname{Difeo}_{+}^{1+\text { va }}\left(\mathrm{S}^{1}\right)\right)$.

Sugerencia. Utilice la analiticidad para verificar que, salvo a lo más un conjunto contable de puntos, si $x \in \mathrm{S}^{1} \backslash \Lambda$ entonces existe un intervalo abierto $I_{x}$ conteniendo a $x$ en su interior y tal que las imágenes de $I_{x}$ por los elementos del grupo son dos a dos disjuntas. De la desigualdad

$$
1 \geq \sum_{g \in \Gamma}\left|g\left(I_{x}\right)\right|=\sum_{g \in \Gamma} \int_{I_{x}} g^{\prime}(y) d y=\int_{I_{n}}\left(\sum_{g \in \Gamma} g^{\prime}(y)\right) d y
$$

concluya que para casi todo $y \in I_{x}$ la serie $\sum_{g \in \Gamma} g^{\prime}(y)$ converge (un tal punto $y$ pertenece obviamente a $S(\Gamma)$ ). Finalmente, pruebe que esto es válido para todo $y \in I_{x}$ mediante un argumento de control de distorsión.

Ejercicio 3.67. Siguiendo las indicaciones dadas a continuación, pruebe que si $\Gamma$ es un grupo finitamente generado de difeomorfismos de clase $\mathrm{C}^{1+l i p}$ del círculo cuya acción es minimal, entonces para todo $y \in \mathrm{S}^{1}$ la serie $\sum_{g \in \Gamma} g^{\prime}(y)$ diverge.

(i) Usando el lema 3.60 y la minimalidad de la acción, pruebe que si el conjunto $\sum(\Gamma)$ de los puntos $y \in \Gamma$ tales que la serie $\sum_{g \in \Gamma} g^{\prime}(y)$ converge no es vacío entonces coincide con todo el círculo, y existe además una constante $S$ tal que $S(y) \leq S$ para todo $y \in \mathrm{S}^{1}$.

(ii) Escogiendo un elemento $g \in \Gamma$ de orden infinito, y utilizando la igualdad

$$
\sum_{i=0}^{n-1} \int_{\mathrm{S}^{1}}\left(g^{i}\right)^{\prime}(y) d y=n,
$$

obtenga una contradicción para $n>S$.

Ejercicio 3.68. Usando el ejercicio precedente pruebe el siguiente resultado del tipo recurrencia de Poincaré: si $\Gamma$ es un grupo finitamente generado de difeomorfismos de clase $\mathrm{C}^{1+\text { lip }}$ del círculo cuya acción es minimal, entonces para todo subconjunto medible $A$ de $\mathrm{S}^{1}$ de medida de Lebesgue positiva existe $g \neq i d$ tal que $\operatorname{Leb}(A \cap g(A))>0$.

Sugerencia. En caso contrario, el conjunto $\sum(\Gamma)$ es no vacío.

\section{Conjugación diferenciable entre grupos de difeomorfismos}

El problema de la diferenciabilidad de la conjugación entre grupos de difeomorfismos del círculo es un tópico sorprendentemente extenso y com- 
plejo. Ya de una dificultad increíble es el caso de grupos actuando libremente, el cual sin embargo está en gran medida entendido gracias a trabajos señeros de Siegel, Arnold, Herman, Moser, Yoccoz, Sinai, Khanin, Katznelson y Orstein, entre otros. En dichos trabajos el ingrediente esencial para obtener la diferenciabilidad de la conjugación de un difeomorfismo $f$ de clase al menos $\mathrm{C}^{2}$ del círculo y número de rotación $\rho(f)$ irracional respecto a la rotación de ángulo $\rho(f)$ es la naturaleza diofantina de $\rho(f)$. En otros términos, dicha conjugación es diferenciable cuando las aproximaciones de $\rho(f)$ por números racionales no son "demasiado rápidas" en relación al denominador del racional considerado (i.e., $\rho(f)$ es "mal aproximado" por los racionales).

A continuación estudiaremos el caso esencialmente diferente de grupos no conjugados a grupos de rotaciones. En este contexto no se dispone de una teoría unificada, y podría decirse que los únicos resultados generales son el teorema 3.74, que estudiaremos en detalle en las dos secciones a seguir, y la proposición 4.13 del próximo capítulo. Notemos que estos dos resultados son bastante más sencillos que aquéllos de la teoría de pequeños denominadores. Otros resultados un poco más finos son válidos en casos particulares. Por ejemplo, Sullivan probó que toda conjugación topológica y absolutamente continua entre grupos fuchsianos de primera especie es real-analítica, obteniendo también resultados de regularidad sobre el minimal excepcional para conjugaciones entre grupos de segunda especie $[193,194]$. Por otra parte, sobre la base de resultados clásicos de existencia y unicidad de medidas invariantes absolutamente continuas para aplicaciones dilatantes del intervalo (vea por ejemplo [106]), el autor probó en [147] que si dos grupos de difeomorfismos de clase $\mathrm{C}^{r}$ de $\mathrm{S}^{1}$, con $r \geq 2$, satisfacen las hipótesis del teorema de Duminy (i.e., son generados por elementos cercanos a rotaciones), y si sus órbitas son densas, entonces toda conjugación topológica y absolutamente continua entre ellos es de clase $\mathrm{C}^{r}$ (vea $[173,174]$ para el caso real-analítico de este resultado).

\subsection{Linearización de Sternberg y conjugaciones $\mathrm{C}^{\mathbf{1}}$}

Sean $f$ y $g$ dos difeomorfismos de clase $\mathrm{C}^{r}$ de una vecindad del origen de la recta sobre sus imágenes respectivas. Si $f$ y $g$ fijan el origen decimos que ellos son equivalentes si existe $\varepsilon^{\prime \prime}>0$ tal que $\left.f\right|_{]-\varepsilon^{\prime \prime}, \varepsilon^{\prime \prime}[}=\left.g\right|_{]-\varepsilon^{\prime \prime}, \varepsilon^{\prime \prime}[\text {. Módulo }}$ esta relación de equivalencia, la clase de $g$ será denotada $[g]$. El conjunto de clases constituye un grupo respecto a la operación de composición de representantes, es decir, $[f][g]=[f \circ g]$. Dicho grupo, llamado grupo de gérmenes de difeomorfismos de clase $\mathrm{C}^{r}$ de la recta que fijan el origen $y$ preservan orientación, será designado por $\mathcal{G}_{+}^{r}(\mathbb{R}, 0)$.

Observe que la derivada $g^{(i)}(0)$ de orden $i \leq r$ en el origen está bien definida para todo germen $[g] \in \mathcal{G}_{+}^{r}(\mathbb{R}, 0)$. Diremos que este germen es hiperbólico si $g^{\prime}(0) \neq 1$. El siguiente lema es bien conocido. 
Lema 3.69. Si $1 \leq r<\infty y[g] \in \mathcal{G}_{+}^{r}(\mathbb{R}, 0)$ es hiperbólico, entonces existe $[h] \in \mathcal{G}_{+}^{r}(\mathbb{R}, 0)$ tal que $h^{\prime}(0)=1$ y $\left(h g h^{-1}\right)^{(i)}(0)=0$ para todo $2 \leq i \leq r$.

Demostración. Sea $g(x)=a x+a_{2} x^{2}+\ldots+a_{r} x^{r}+\mathrm{o}\left(x^{r}\right)$ el desarrollo de Taylor de $g$ en torno al origen. Escribamos formalmente

$$
\hat{h}(x)=x+b_{2} x^{2}+\ldots+b_{r} x^{r}+\ldots,
$$

y tratemos de hallar los coeficientes $b_{i}$ de modo que

$$
\hat{h} \circ g=M_{a} \circ \hat{h},
$$

donde $M_{a}$ denota la multiplicación por $a=g^{\prime}(0)$. Identificando los coeficientes de $x^{2}$ de ambos miembros de esta igualdad obtenemos $a_{2}+b_{2} a^{2}=a b_{2}$, y por lo tanto $b_{2}=a_{2} /\left(a-a^{2}\right)$. En general, suponiendo $b_{2}, \ldots, b_{i-1}$ ya calculados (con $i \leq r)$, a partir de (3.50) se obtiene la igualdad

$$
Q_{i}\left(b_{1}, \ldots, b_{i-1}, a_{1}, \ldots, a_{r}\right)+b_{i} a^{i}=a b_{i}
$$

para cierto polinomio $Q_{i}$ en $(r+i-1)$ variables, de donde

$$
b_{i}=\frac{Q_{i}\left(b_{1}, \ldots, b_{i-1}, a_{1}, \ldots, a_{r}\right)}{a-a^{i}} .
$$

Sea ahora $h$ un difeomorfismo de clase $\mathrm{C}^{r}$ de una vecindad del origen tal que $h(0)=0, h^{\prime}(0)=1$ y $h^{(i)}(0)=i$ ! $b_{i}$ para todo $2 \leq i \leq r$. Reinvirtiendo los cálculos anteriores, es fácil ver que $[h] \in \mathcal{G}_{+}^{r}(\mathbb{R}, 0)$ verifica las condiciones pedidas.

El siguiente resultado, debido esencialmente a Sternberg, ha sido transcrito de [211] (vea también [192]). Señalemos que un resultado análogo subsiste para gérmenes de difeomorfismos real-analíticos [44].

Teorema 3.70. Sea $g \in \mathcal{G}_{+}^{r}(\mathbb{R}, 0)$ un germen hiperbólico, con $2 \leq r \leq \infty$. Si denotamos $a=g^{\prime}(0)$, entonces existe un germen $h \in \mathcal{G}_{+}^{r}(\mathbb{R}, 0)$ tal que $h^{\prime}(0)=1$ y $h(g(x))=a h(x)$ para todo $x$ cercano al origen. Además, si $\left[h_{1}\right] \in \mathcal{G}_{+}^{1}(\mathbb{R}, 0)$ verifica estas últimas dos propiedades, entonces $\left[h_{1}\right]$ pertenece a $\mathcal{G}_{+}^{r}(\mathbb{R}, 0)$ y $\left[h_{1}\right]=[h]$.

Demostración. Consideremos en primer lugar el caso $r<\infty$. Por el lema anterior, para obtener la conjugación podemos suponer que $g^{(i)}(0)=0$ para todo $i \in\{2, \ldots, r\}$. Cambiando $g$ por $g^{-1}$ si es necesario, podemos suponer además que $g^{\prime}(0)=a<1$. Sea $0<\delta<1$ tal que el dominio de definición de $g$ contenga al intervalo $[-\delta, \delta]$. Definamos

$$
C(\delta)=\sup \left\{\left|g^{(r)}(t)\right|: t \in[-\delta, \delta]\right\} .
$$

Una aplicación simple del teorema del valor medio muestra que para todo $t \in[-\delta, \delta]$ y todo $i \in\{2, \ldots, r\}$ se tiene $\left|g^{(i)}(t)\right| \leq C(\delta)$ y $\left|g^{\prime}(t)\right| \leq a+C(\delta)$. 
Sea $E_{\delta}$ el espacio de las funciones $\psi$ de clase $\mathrm{C}^{r}$ de $[-\delta, \delta]$ en $\mathbb{R}$ tales que $\psi(0)=\psi^{\prime}(0)=\ldots=\psi^{(r)}(0)=0$. Dotado de la norma

$$
\|\psi\|=\sup \left\{\left|\psi^{(r)}(t)\right|: t \in[-\delta, \delta]\right\},
$$

el espacio $E_{\delta}$ es de Banach. Para $\psi \in E_{\delta}, t \in[-\delta, \delta]$ e $i \in\{0, \ldots, r\}$ se tiene

$$
\left|\psi^{(i)}(t)\right| \leq \frac{t^{r-i}}{(r-i) !}\|\psi\| .
$$

Consideremos el operador $S_{\delta}: E_{\delta} \rightarrow E_{\delta}$ definido por $S_{\delta}(\psi)=(\psi \circ g) / a$. Afirmamos que si $\delta>0$ es suficientemente pequeño entonces $S_{\delta}$ es una contracción (es decir, $\left\|S_{\delta}\right\|<1$ ). En efecto, es fácil verificar que

$$
(\psi \circ g)^{(r)}=\sum_{i=1}^{r}\left(\psi^{(i)} \circ g\right) \cdot Q_{i}\left(g^{\prime}, \ldots, g^{(r-i+1)}\right),
$$

donde $Q_{i}$ es un polinomio a coeficientes positivos en $(\mathrm{r}+1-\mathrm{i})$ variables y $Q_{r}(x)=x^{r}$. Se deduce así que

$$
\left|(\psi \circ g)^{(r)}(t)\right| \leq K(\delta)\|\psi\|
$$

para todo $t \in[-\delta, \delta]$, donde

$$
K(\delta)=(a+C(\delta))^{r}+\sum_{i=1}^{r-1} \frac{\delta^{r-i}}{(r-i) !} Q_{i}(a+C(\delta), \ldots, a+C(\delta)) .
$$

Notemos que $K(\delta)$ tiende hacia $a^{r}$ cuando $\delta$ tiende a cero. De las desigualdades $\left\|S_{\delta}\right\| \leq K(\delta) / a, \quad a<1$ y $r \geq 2$, se deduce que el valor de $\left\|S_{\delta}\right\|$ es menor que 1 para $\delta$ suficientemente pequeño.

Fijemos ahora $\delta>0$ tal que $\left\|S_{\delta}\right\|<1$. Observe que la restricción de la aplicación $x \mapsto g(x)-a x$ al intervalo $[-\delta, \delta]$ define un elemento $\psi_{1}$ de $E_{\delta}$. Como $S_{\delta}$ es una contracción, la ecuación (en la variable $\psi$ )

$$
S_{\delta}(\psi)+a^{-1} \psi_{1}=\psi
$$

posee una única solución $\psi_{0} \in E_{\delta}$. Para $h=I d+\psi_{0}$ tenemos

$$
\begin{aligned}
h(g(x)) & =g(x)+\psi_{0} \circ g(x)=\psi_{1}(x)+a x+\psi_{0} \circ g(x) \\
& =\psi_{1}(x)+a x+a S_{\delta}\left(\psi_{0}\right)(x)=a \psi_{0}(x)+a x=a h(x) .
\end{aligned}
$$

Puesto que $\psi_{0} \in E_{\delta}$, se tiene $h(0)=0$ y $h^{\prime}(0)=1$. Luego, si $r<\infty$ entonces $[h]$ es un germen en $\mathcal{G}_{+}^{r}(\mathbb{R}, 0)$ que satisface las dos condiciones requeridas.

Si $h_{1} \in \mathcal{G}_{+}^{1}(\mathbb{R}, 0)$ verifica estas mismas condiciones entonces

$$
a h h_{1}^{-1}(t)=h \circ g \circ h_{1}^{-1}(t)=h h_{1}^{-1}(a t)
$$


para todo $t$ próximo al origen. De esto se deduce que

$$
h h_{1}^{-1}(t)=\lim _{n \rightarrow \infty} \frac{h h_{1}^{-1}\left(a^{n} t\right)}{a^{n}}=t\left(h h_{1}^{-1}\right)^{\prime}(0)=t,
$$

lo cual demuestra la unicidad.

Finalmente, el caso $r=\infty$ se obtiene fácilmente por inducción en $r \geq 2$ gracias a la unicidad obtenida anteriormente.

Una consecuencia casi directa del teorema precedente es el resultado siguiente.

Corolario 3.71. Sean $g_{1}$ y $g_{2}$ elementos de $\mathcal{G}_{+}^{r}(\mathbb{R}, 0)$, con $2 \leq r \leq \infty$. Supongamos que $[\varphi] \in \mathcal{G}_{+}^{1}(\mathbb{R}, 0)$ conjuga $g_{1} y g_{2}$, es decir, sobre una vecindad del origen se tiene $\varphi \circ g_{1}=g_{2} \circ \varphi$. Si $g_{1}$ es hiperbólico entonces $[\varphi]$ es un elemento de $\mathcal{G}_{+}^{r}(\mathbb{R}, 0)$.

Demostración. Notemos en primer lugar que $\left[g_{2}\right]$ también es hiperbólico, pues $g_{2}^{\prime}(0)=g_{1}^{\prime}(0)$. Si $h$ es un germen de $\mathcal{G}_{+}^{r}(\mathbb{R}, 0)$ que conjuga $g_{2}$ a su parte lineal, entonces $h \circ \varphi$ conjuga $g_{1}$ a su parte lineal. Si $b=(h \varphi)^{\prime}(0)$ entonces $M_{1 / b} h \varphi$ conjuga aún $g_{1}$ a su parte lineal y verifica además $\left(M_{1 / b} h \varphi\right)^{\prime}(0)=1$. Por la unicidad de tal conjugación obtenemos que $\left[M_{1 / b} h \varphi\right] \in \mathcal{G}_{+}^{r}(\mathbb{R}, 0)$, y por lo tanto $[\varphi] \in \mathcal{G}_{+}^{r}(\mathbb{R}, 0)$.

Ejemplo 3.72. El teorema de linearización de Sternberg sigue siendo válido en clase $\mathrm{C}^{1+\tau}$ para todo $\tau>0$ (la demostración consiste en una variación de los argumentos dados anteriormente; vea también [46]). Sin embargo, el teorema deja de ser válido en clase $\mathrm{C}^{1}$, como lo ilustra el siguiente ejemplo debido al propio Sternberg.

Consideremos la aplicación $g$ definida sobre un intervalo abierto en torno al origen por $g(0)=0$ y $g(x)=a x(1-1 / \log (x))$ para $x \neq 0$, donde $0<a<1$. Es fácil ver que $[g]$ es un germen de difeomorfismo de clase $\mathrm{C}^{1}$ satisfaciendo $g^{\prime}(0)=a$. Pese a ello, $[g]$ no es conjugado al germen de la aplicación lineal $M_{a}$ por ningún germen de homeomorfismo lipschitziano (en particular, $[g]$ no es $\mathrm{C}^{1}$ conjugado a $\left[M_{a}\right]$ ). Para verificar esto, fijemos una constante $\bar{a} \in] 0,1\left[\right.$ de modo que $g^{\prime}(x) \geq \bar{a}$ para todo $x$ suficientemente cercano al origen, y escojamos un homeomorfismo local $h$ fijando $0 \mathrm{y}$ tal que para tales puntos $x$ se tenga $g(x)=h M_{a} h^{-1}(x)$. Para todo $k \in \mathbb{N}$ se cumple entonces $g^{k}(x)=h\left(a^{k} h^{-1}(x)\right)$, por lo que

$$
\frac{g^{k}(x)}{a^{k}}=\frac{h\left(a^{k} h^{-1}(x)\right)}{a^{k}} .
$$

Ahora bien, si $h$ fuese lipschitziano con constante de Lipschitz $\bar{C}$, entonces el miembro a derecha en la igualdad anterior estaría acotado (independientemente de $k$ ) por $C=\bar{C} h^{-1}(x)$. Por otro lado, a partir de la definición 
de $g$ se verifica rápidamente que

$$
\frac{g^{k}(x)}{a^{k}}=x \prod_{i=0}^{k-1}\left(1-\frac{1}{\log \left(g^{i}(x)\right)}\right) .
$$

Puesto que $g^{i}(x) \geq \bar{a}^{i} x$, existe un entero $i_{0} \geq 1$ tal que para cierta constante positiva $c$ y todo $i \geq i_{0}$ se tiene

$$
\log \left(g^{i}(x)\right) \geq i \log (\bar{a})+\log (x) \geq-\frac{i}{c} .
$$

De esto se deduce que

$$
\prod_{i=i_{0}}^{k-1}\left(1-\frac{1}{\log \left(g^{i}(x)\right)}\right) \geq \prod_{i=i_{0}}^{k-1}\left(1+\frac{c}{i}\right) .
$$

Como este último producto diverge cuando $k$ tiene al infinito (debido a la divergencia de la serie armónica), concluimos que el miembro a derecha de (3.51) no es acotado, lo cual nos da una contradicción.

Ejercicio 3.73. Verifique que el germen al origen del ejemplo 3.63 es (hiperbólico y) no linearizable por un germen de clase $\mathrm{C}^{1}$.

Como una aplicación del teorema de linearización y del corolario 3.19, probaremos el siguiente resultado de [79].

Teorema 3.74. Sean $\Phi_{1}$ y $\Phi_{2}$ dos representaciones de un grupo $\Gamma$ en Difeo $_{+}^{r}\left(\mathrm{~S}^{1}\right)$, con $2 \leq r \leq \infty$. Supongamos que las órbitas de ellas son densas y que $\Phi_{1}(\Gamma)$ no es topológicamente conjugado a un grupo de rotaciones. Si un difeomorfismo $\varphi$ de clase $\mathrm{C}^{1}$ de $\mathrm{S}^{1}$ conjuga $\Phi_{1} y \Phi_{2}$, entonces $\varphi$ es un difeomorfismo de clase $\mathrm{C}^{r}$.

Demostración. Por el corolario 3.19, existen un elemento $g \in \Gamma$ y un punto $x \in \mathrm{S}^{1}$ tales que $\Phi_{1}(g)(x)=x$ y $\Phi_{1}(g)^{\prime}(x) \neq 1$. En coordenadas locales obtenemos un germen hiperbólico de un difeomorfismo que fija el origen. El punto $\varphi(x) \in \mathrm{S}^{1}$ es un punto fijo por $\Phi_{2}(g)=\varphi \circ \Phi_{1}(g) \circ \varphi^{-1} \in \Gamma_{2}$. Luego, $\varphi$ induce una conjugación de gérmenes de difeomorfismos hiperbólicos. $\mathrm{El}$ corolario 3.71 implica entonces que $\varphi$ es de clase $\mathrm{C}^{r}$ en una vecindad de $x$. De la hipótesis de conjugación, a saber, $\varphi \circ \Phi_{1}(h)=\Phi_{2}(h) \circ \varphi$ para todo $h \in \Gamma$, se concluye que el conjunto de puntos en torno a los cuales $\varphi$ es de clase $\mathrm{C}^{r}$ es $\Phi_{1}(\Gamma)$-invariante. Puesto que las órbitas de $\Phi_{1}(\Gamma)$ son densas, $\varphi$ es de clase $\mathrm{C}^{r}$ en todo el círculo. Para probar que $\varphi^{-1}$ es de clase $\mathrm{C}^{r}$ en todo $\mathrm{S}^{1}$ basta invertir los roles de $\Phi_{1}$ y $\Phi_{2}$.

Observación 3.75. El teorema precedente sigue siendo válido para conjugaciones bilipschitzianas, al menos si se asume a priori que la acción es ergódica (vea el teorema 3.77). Por otra parte, el teorema permanece también válido para conjugaciones entre grupos de difeomorfismos de clase $\mathrm{C}^{1+\tau}$ del círculo (que son no abelianos y actúan minimalmente). 
Ejercicio 3.76. Pruebe que el teorema 3.74 vale también (ya sea en clase $\mathrm{C}^{2}$ o $\mathrm{C}^{1+\tau}$ ) para grupos que admiten un minimal excepcional (y no son semiconjugados a grupos de rotaciones).

Sugerencia. Aplique el mismo argumento recordando que toda órbita se acumula sobre el conjunto de Cantor minimal.

Observación. Este último resultado no se generaliza para conjugaciones bilipschitzianas: vea el teorema 3.81 .

\subsection{El caso de las conjugaciones bilipschitzianas}

El primer objetivo de esta sección consiste en extender el teorema 3.74 para conjugaciones bilipschitzianas bajo una hipótesis de ergodicidad (la cual de acuerdo a lo discutido en la sección 5.1 de este capítulo pareciera siempre ser satisfecha).

Teorema 3.77. Sean $\Phi_{1}$ y $\Phi_{2}$ dos representaciones de un grupo finitamente generado $\Gamma$ en Difeo $_{+}^{r}\left(\mathrm{~S}^{1}\right)$, con $2 \leq r \leq \infty$. Supongamos que sus órbitas sean densas y que la acción de $\Phi_{1}(\Gamma)$ sea ergódica. Si un homeomorfismo bilipschitziano de $\mathrm{S}^{1}$ conjuga $\Phi_{1}$ y $\Phi_{2}$, entonces $\varphi$ es un difeomorfismo de clase $\mathrm{C}^{1}$. Además, si $\Phi_{1}(\Gamma)$ no es topológicamente conjugado a un grupo de rotaciones, entonces $\varphi$ es un difeomorfismo de clase $\mathrm{C}^{r}$.

La demostración de este resultado utiliza una versión equivariante de un clásico lema de índole cohomológico debido a Gottschalk y Hedlund. Para formular esta versión consideremos un espacio métrico M y un grupo $\Gamma$ actuando sobre $\mathrm{M}$ por homeomorfismos. Un cociclo asociado a esta acción (compare con la sección 2.1 del último capítulo) es una función $c: \Gamma \times X \rightarrow \mathbb{R}$ tal que para todo elemento $f \in \Gamma$ la función $x \mapsto c(f, x)$ es continua, y tal que para todo $f, g$ en $\Gamma$ y todo $x \in X$ se tiene

$$
c(f g, x)=c(g, x)+c(f, g(x)) .
$$

Con estas definiciones el lema de Gottschalk-Hedlund equivariante se enuncia de la siguiente manera.

Lema 3.78. Si $\Gamma$ es finitamente generado y su acción sobre $\mathrm{M}$ es minimal, entonces las dos condiciones siguientes son equivalentes:

(i) existen $x_{0} \in \mathrm{M} y C>0$ tales que $\left|c\left(f, x_{0}\right)\right| \leq C$ para todo $f \in \Gamma$,

(ii) existe una función continua $\phi: X \rightarrow \mathbb{R}$ tal que $c(f, x)=\phi(f(x))-\phi(x)$ para todo $f \in \Gamma$ y todo $x \in X$.

Demostración. Si la segunda condición es satisfecha entonces

$$
\left|c\left(f, x_{0}\right)\right| \leq\left|\phi\left(f\left(x_{0}\right)\right)\right|+\left|\phi\left(x_{0}\right)\right| \leq 2\|\phi\|_{C(\mathrm{M})},
$$

lo cual prueba la validez de la condición (i).

Supongamos ahora que la primera condición se satisfaga, y para cada elemento $f \in \Gamma$ consideremos el homeomorfismo $\hat{f}$ de $\mathrm{M} \times \mathbb{R}$ definido por 
$\hat{f}(x, t)=(f(x), t+c(f, x))$. Se verifica rápidamente que la relación de cociclo (3.52) implica que esta asignación define una acción del grupo $\Gamma$ sobre $\mathrm{M} \times \mathbb{R}$, en el sentido que para todo $f, g$ en $\Gamma$ se tiene $\hat{f} \hat{g}=\widehat{f g}$. Además, la condición (i) implica que la órbita del punto $\left(x_{0}, 0\right)$ bajo esta acción es acotada; en particular, su clausura es un conjunto (no vacío e) invariante por $\Gamma$. Valiéndose del lema de Zorn se deduce entonces la existencia de un subconjunto $\Lambda$ de $\mathrm{M}$ que es compacto, no vacío, invariante por $\Gamma$, y minimal para estas propiedades.

Afirmamos ahora que el conjunto $\Lambda$ es el gráfico de una función (real y) continua definida en M. Para verificar esto notemos en primer lugar que, como la acción de $\Gamma$ sobre $M$ es minimal, la proyección de $\Lambda$ sobre $M$ coincide con todo el espacio. Por otro lado, si $\left(\bar{x}, t_{1}\right)$ y $\left(\bar{x}, t_{2}\right)$ pertenecieran a $\Lambda$ para algún $\bar{x} \in \mathrm{M}$ y dos reales distintos $t_{1}$ y $t_{2}$, entonces esto implicaría que $\Lambda \cap \Lambda_{t} \neq \emptyset$, donde $t=t_{2}-t_{1} \neq 0$ y $\Lambda_{t}=\{(x, s+t):(x, s) \in \Lambda\}$. Ahora bien, como la acción de $\Gamma$ sobre $\mathrm{M} \times \mathbb{R}$ conmuta con la aplicación $(x, s) \mapsto(x, s+t)$, el conjunto $\Lambda_{t}$ también sería invariante, y como $\Lambda$ es minimal esto implicaría que $\Lambda=\Lambda_{t}$. Se tendría entonces

$$
\Lambda=\Lambda_{t}=\Lambda_{2 t}=\Lambda_{3 t}=\ldots,
$$

lo cual es imposible dada la compacidad de $\Lambda$. Concluimos así que para cada $x \in \mathrm{M}$ el conjunto $\Lambda$ contiene exactamente un punto de la forma $(x, t)$. Haciendo $\phi(x)=t$ obtenemos entonces una función de $\mathrm{M}$ en $\mathbb{R}$ cuyo gráfico coincide con $\Lambda$. El que esta función sea continua se desprende del hecho de que su gráfico es compacto.

Para concluir la demostración notemos que, como el gráfico de $\phi$ es invariante por la acción de $\Gamma$, para cada $f \in \Gamma$ y cada $x \in \mathrm{M}$ el punto $\hat{f}(x, \phi(x))=(f(x), \phi(x)+c(f, x))$ debe ser de la forma $(f(x), \phi(f(x)))$, lo cual implica que $c(f, x)=\phi(f(x))-\phi(x)$.

Para probar el teorema 3.77 necesitaremos la siguiente "versión medible" del lema anterior.

Lema 3.79. Sean $\mathrm{M}$ un espacio métrico compacto y $\Gamma$ un grupo finitamente generado actuando sobre él por homeomorfismos. Supongamos que la acción sea minimal y ergódica respecto a alguna medida de probabilidad $\mu$ sobre los borelianos de M, y sea c un cociclo asociado a esta acción. Si $\phi$ es una función de $\mathcal{L}_{\mathbb{R}}^{\infty}(\mathrm{M}, \mu)$ tal que para todo $f \in \Gamma$ y $\mu$-casi todo $x \in \mathrm{M}$ se tiene

$$
c(f, x)=\phi(f(x))-\phi(x),
$$

entonces existe una función continua $\tilde{\phi}: \mathrm{M} \rightarrow \mathbb{R}$ que coincide en $\mu$-casi todo punto con $\phi$ y tal que para todo $f \in \Gamma$ y todo $x \in \mathrm{M}$ se tiene

$$
c(f, x)=\tilde{\phi}(f(x))-\tilde{\phi}(x) .
$$


Demostración. Sea $\mathrm{M}_{0}$ el conjunto de los puntos en los cuales la igualdad (3.53) no es verificada para algún $f \in \Gamma$. Como $\Gamma$ es finitamente generado, $\mu\left(\mathrm{M}_{0}\right)=0$. Sea $\mathrm{M}_{1}^{\prime}$ el complemento del soporte esencial de $\phi$, y sea $\mathrm{M}_{1}=\cup_{f \in \Gamma} f\left(\mathrm{M}_{1}^{\prime}\right)$. Fijemos un punto $x_{0}$ en el conjunto de medida total $\mathrm{M} \backslash\left(\mathrm{M}_{0} \cup \mathrm{M}_{1}\right)$. La igualdad (3.53) implica que $\left|c\left(f, x_{0}\right)\right| \leq 2\|\phi\|_{\mathcal{L}^{\infty}}$ para todo $f \in \Gamma$. Por el lema precedente, existe una función continua $\bar{\phi}: \mathrm{M} \rightarrow \mathbb{R}$ tal que, para todo $x$ y todo $f$,

$$
c(f, x)=\bar{\phi}(f(x))-\bar{\phi}(x) .
$$

Esto implica que en $\mu$-casi todo punto se tiene

$$
\bar{\phi} \circ f-\bar{\phi}=\phi \circ f-\phi,
$$

es decir,

$$
\bar{\phi}-\phi=(\bar{\phi}-\phi) \circ f .
$$

Como asumimos que la acción de $\Gamma$ es ergódica respecto a $\mu$, existe $C \in \mathbb{R}$ tal que la diferencia $\bar{\phi}-\phi$ es igual a $C$ en $\mu$-casi todo punto (pues los conjuntos de nivel $(\bar{\phi}-\phi)^{-1}(c)$ son invariantes por $\Gamma$ ). Para finalizar la demostración basta definir $\tilde{\phi}=\bar{\phi}-C$.

Estamos ahora en condiciones de probar el teorema 3.77. Para ello notemos que si $\varphi$ es un homeomorfismo bilipschitziano de $\mathrm{S}^{1}$ entonces $\varphi$ y $\varphi^{-1}$ son diferenciables en casi todo punto, y las derivadas correspondientes son funciones pertenecientes a $\mathcal{L}_{\mathbb{R}}^{\infty}\left(\mathrm{S}^{1}, L e b\right)$. Por lo tanto, $\phi=-\log \left(\varphi^{\prime}\right)$ también pertenece a $\mathcal{L}_{\mathbb{R}}^{\infty}\left(\mathrm{S}^{1}, L e b\right)$. Si $\varphi$ conjuga $\Phi_{1}$ y $\Phi_{2}$ entonces de la igualdad $\Phi_{1}(f)=\varphi^{-1} \circ \Phi_{2}(f) \circ \varphi$ se deduce que en casi todo punto se tiene

$$
\log \left(\Phi_{1}(f)^{\prime}(x)\right)=\log \left(\varphi^{\prime}(x)\right)-\log \left(\varphi^{\prime}\left(\Phi_{1}(f)(x)\right)\right)+\log \left(\Phi_{2}(f)^{\prime}(\varphi(x))\right) .
$$

Haciendo $c(f, x)=\log \left(\Phi_{1}(f)^{\prime}(x)\right)-\log \left(\Phi_{2}(f)^{\prime}(\varphi(x))\right)$ esto implica que, para todo $f \in \Gamma$ y casi todo $x \in \mathrm{S}^{1}$,

$$
c(f, x)=\phi\left(\Phi_{1}(f)(x)\right)-\phi(x) .
$$

Dejamos a cargo del lector la tarea de verificar la relación de cociclo

$$
c(f g, x)=c(g, x)+c\left(f, \Phi_{1}(g)(x)\right) .
$$

Como estamos suponiendo que la acción $\Phi_{1_{\sim}}$ es ergódica, el lema 3.79 nos brinda la existencia de una función continua $\tilde{\phi}$ que coincide casi ciertamente con $\phi$ (y para la cual la relación (3.54) es satisfecha para todo $x \in \mathrm{S}^{1}$ y todo $f \in \Gamma$ ). Por simple integración se concluye que la derivada de $\varphi$ está bien definida en todo punto y coincide con la función $\exp (-\tilde{\phi})$. En particular, $\varphi$ es diferenciable (con derivada continua), e invirtiendo los roles de $\Phi_{1}$ y $\Phi_{2}$ se deduce que $\varphi$ es un difeomorfismo de clase $\mathrm{C}^{1}$. Finalmente, para probar que $\varphi$ es un difeomorfismo de clase $\mathrm{C}^{r}$ en el caso de acciones no conjugadas a acciones por rotaciones, basta con aplicar el teorema 3.74. 
Ejercicio 3.80. Sean $\Gamma$ un grupo de difeomorfismos de clase $\mathrm{C}^{1}$ del círculo y $\varphi$ un homeomorfismo bilipschitziano de $\mathrm{S}^{1}$. Suponga que para todo $f \in \Gamma$ y casi todo $x \in \mathrm{S}^{1}$ se cumple la igualdad

$$
\log \left(f^{\prime}(x)\right)-\log \left(f^{\prime}(\varphi(x))\right)=\log \left(\varphi^{\prime}(x)\right)-\log \left(\varphi^{\prime}(f(x))\right) .
$$

Pruebe que $\varphi$ centraliza a $\Gamma$, es decir, todos los elementos de $\Gamma$ conmutan con $\varphi$.

Notemos que una conjugación de una acción consigo misma corresponde a una aplicación (invertible y regular) que centraliza a la acción, es decir, que conmuta con todos los elementos del grupo en cuestión. Además, si $\Phi_{1}$ y $\Phi_{2}$ son dos acciones por difeomorfismos de clase $\mathrm{C}^{r}$ del círculo que son conjugadas por algún difeomorfismo $\varphi_{0}$ de clase $\mathrm{C}^{r}$, y si $\varphi$ es otro homeomorfismo bilipschitziano que las conjuga, entonces el homeomorfismo (bilipschitziano) $\varphi_{0}^{-1} \varphi$ centraliza la acción $\Phi_{1}$. Ésta es la razón por la cual resulta tan importante el estudio de los centralizadores previo al tratamiento general de las conjugaciones (vea sin embargo el ejercicio 3.82). En este contexto puede probarse que el teorema 3.77 dista muchísimo de ser verdad para acciones no minimales.

Teorema 3.81. Sea $\Gamma$ un grupo finitamente generado de difeomorfismos de clase $\mathrm{C}^{1+l i p}$ del círculo cuya acción no es minimal. Si los estabilizadores de puntos son triviales o bien cíclicos infinitos, entonces existen homeomorfismos bilipschitzianos que no son de clase $\mathrm{C}^{1}$ y que conmutan con todos los elementos de $\Gamma$. Además, tales homeomorfismos pueden ser escogidos siendo no diferenciables sobre todo abierto no vacío de $\mathrm{S}^{1}$.

El teorema 3.81 se basa en una sencilla construcción relacionada con las técnicas del capítulo siguiente; es por ello que no daremos la demostración hasta la sección 1.1 de dicho capítulo. Por lo pronto, limitémosnos a comentar que la hipótesis sobre los estabilizadores no es muy fuerte. Por ejemplo, ella siempre es satisfecha en el caso real-analítico (este resultado, debido a Hector, aparece desarrollado en el apéndice de [144]); por supuesto, ella es verificada por muchas otras acciones no analíticas interesantes. No es difícil ver que, sin una hipótesis de este tipo, en muchos casos las conjugaciones bilipschitzianas son necesariamente diferenciables.

Ejercicio 3.82. Dé ejemplos de grupos (finitamente generados) de difeomorfismos de clase $\mathrm{C}^{\infty}$ del círculo que sean conjugados por algún homeomorfismo bilipschitziano pero para los cuales no exista ninguna conjugación diferenciable. Observación. Los ejemplos pedidos pueden ser construidos tanto con órbitas finitas como con un minimal excepcional. Ignoramos sin embargo si existen ejemplos de grupos de difeomorfismos real-analíticos de $\mathrm{S}^{1}$ verificando la propiedad exigida. 


\section{Capítulo 4}

\section{Estructura Algebraica y Rigidez: Métodos Dinámicos}

\section{Grupos abelianos de difeomorfismos}

\section{$1.1 \quad$ El lema de Kopell}

Para un grupo de homeomorfismos de un intervalo, del círculo o de la recta, diremos que $] a, b[$ es una componente irreducible para la acción si es invariante y no contiene (estrictamente) ningún subintervalo invariante. Denotemos por Difeo ${ }_{+}^{1+\text { va }}([0,1[)$ al grupo de los difeomorfismos de clase $\mathrm{C}^{1+\text { va }}$ del intervalo [0,1[, es decir, al grupo de los difeomorfismos $f$ de clase $\mathrm{C}^{1}$ de $[0,1$ t tales que la variación del logaritmo de su derivada es finita sobre cada subintervalo compacto $[a, b] \subset[0,1[$. Recordemos que esta variación es denotada $V(f ;[a, b])$, es decir,

$$
V(f ;[a, b])=\sup _{a=a_{0}<a_{1}<\cdots<a_{n}=b} \sum_{i=1}^{n}\left|\log \left(f^{\prime}\right)\left(a_{i}\right)-\log \left(f^{\prime}\right)\left(a_{i-1}\right)\right| .
$$

De manera análoga se define el grupo Difeo $\left.\left.{ }_{+}^{1+v a}(] 0,1\right]\right)$. Observe que todo elemento $f$ de $\operatorname{Difeo}_{+}^{2}([0,1])$ pertenece a Difeo ${ }_{+}^{1+v a}([0,1[)$. En efecto, para $0 \leq a<b<1$ se tiene

$$
V(f ;[a, b])=\int_{a}^{b}\left|\left(\log \left(f^{\prime}\right)\right)^{\prime}(s)\right| d s=\int_{a}^{b}\left|\frac{f^{\prime \prime}(s)}{f^{\prime}(s)}\right| d s .
$$

Enunciamos el importantísimo resultado siguiente como un teorema, a pesar de que es ampliamente conocido (y a veces nos referiremos a él) como el 
"lema de Kopell" por tratarse del primer lema de la tesis de Kopell [118]. La demostración que presentamos es sin embargo bastante más sencilla que la que aparece en [118].

Teorema 4.1. Sean $f$ y $g$ dos difeomorfismos del intervalo $[0,1[$ o de $] 0,1]$ que conmutan, es decir, tales que $f \circ g=g \circ f$. Supongamos que $f$ sea de clase $\mathrm{C}^{1+\mathrm{va}}$ y $g$ de clase $\mathrm{C}^{1}$. Si $f$ no posee puntos fijos en $] 0,1[$ y g tiene (al menos) un punto fijo en $] 0,1[$, entonces $g$ es la identidad.

Demostración. Daremos la prueba sólo para el caso del intervalo [0, 1 , pues el caso del intervalo ]0,1] es análogo. Reemplazando $f$ por $f^{-1}$ si es necesario, podemos suponer que $f(x)<x$ para todo $x \in] 0,1[$. Sea $b \in] 0,1[$ uno de los puntos fijos de $g$. Para cada $n \in \mathbb{Z}$ denotemos $b_{n}=f^{n}(b)$, y denotemos $a=b_{1}=f(b)$. Puesto que $g$ fija cada intervalo $\left[b_{n+1}, b_{n}\right]$, para cada $n \in \mathbb{N}$ existe un punto $c_{n} \in\left[b_{n}, b_{n+1}\right]$ tal que $g^{\prime}\left(c_{n}\right)=1$. Como $c_{n}$ tiende al origen y $g$ es de clase $\mathrm{C}^{1}$, se tiene necesariamente $g^{\prime}(0)=1$. Sea $\delta=V(f ;[0, b])$. Si $u$ y $v$ pertenecen a $[a, b]$ entonces

$$
\left|\log \left(\frac{\left(f^{n}\right)^{\prime}(v)}{\left(f^{n}\right)^{\prime}(u)}\right)\right| \leq \sum_{i=1}^{n}\left|\log \left(f^{\prime}\right)\left(f^{i-1}(v)\right)-\log \left(f^{\prime}\right)\left(f^{i-1}(u)\right)\right| \leq \delta .
$$

Haciendo $u=x \in[a, b]$ y $v=f^{-n} g f^{n}(x)=g(x) \in[a, b]$, utilizando la igualdad

$$
g^{\prime}(x)=\frac{\left(f^{n}\right)^{\prime}(x)}{\left(f^{n}\right)^{\prime}\left(f^{-n} g f^{n}(x)\right)} g^{\prime}\left(f^{n}(x)\right)=\frac{\left(f^{n}\right)^{\prime}(x)}{\left(f^{n}\right)^{\prime}(g(x))} g^{\prime}\left(f^{n}(x)\right),
$$

y pasando al límite cuando $n$ tiende al infinito, obtenemos la desigualdad $\sup _{x \in[a, b]} g^{\prime}(x) \leq e^{\delta}$. Notemos sin embargo que lo anterior permanece válido al reemplazar $g$ por $g^{j}$, cualquiera sea $j \in \mathbb{N}$ (esto se debe a que la constante $\delta$ depende solamente de $f$ ). Se tiene entonces

$$
\sup _{x \in[a, b]}\left(g^{j}\right)^{\prime}(x) \leq e^{\delta} .
$$

Puesto que $g$ fija $a$ y $b$, esto no es posible a menos que la restricción de $g$ a $[a, b]$ sea la identidad. Finalmente, como $f$ y $g$ conmutan, se concluye que $g$ es la identidad sobre todo el intervalo $[0,1[$.

El teorema precedente permite concluir que para todo $f \in \operatorname{Difeo}_{+}^{1+v a}([0,1[)$ sin punto fijo sobre ]0, 1 [, su centralizador en Difeo ${ }_{+}^{1}([0,1[)$ actúa libremente sobre ]0,1[. El teorema de Hölder implica entonces que dicho centralizador es semiconjugado a un grupo de traslaciones. De hecho, si dicho grupo de traslaciones es denso entonces la semiconjugación es en realidad una conjugación topológica, tal como queda estipulado en la proposición siguiente.

Proposición 4.2. Sea $\Gamma$ un subgrupo de Difeo ${ }_{+}^{1}([0,1[)$ semiconjugado a un subgrupo denso del grupo de las traslaciones. Si $\Gamma$ contiene un elemento de clase $\mathrm{C}^{1+\mathrm{va}}$ sin punto fijo sobre ]0,1[, entonces la semiconjugación es una conjugación topológica. 
Demostración. Supongamos que $\Gamma$ sea un subgrupo de Difeo ${ }_{+}^{1}([0,1[)$ que es semiconjugado a un subgrupo denso del grupo de traslaciones sin serle conjugado. Fijemos el elemento $f \in \Gamma$ dado por la hipótesis. Sin pérdida de generalidad, supongamos que $f$ es enviado sobre la traslación $T_{-1}: x \mapsto x-1$ por el homomorfismo inducido por la semiconjugación. En particular, se tiene $f(x)<x$ para todo $x \in] 0,1[$. Fijemos un intervalo $[a, b]$ no reducido a un punto que sea enviado sobre un único punto por la semiconjugación, y que sea maximal para esta propiedad. Dada la definición de $[a, b]$, existe una sucesión creciente $\left(n_{i}\right)$ de enteros positivos tal que para todo $i \in \mathbb{N}$ existe $\bar{f}_{i} \in \Gamma$ verificando, para todo $n \in \mathbb{N}$,

$$
\begin{array}{rlrl}
\bar{f}_{i}^{n_{i}}\left(f^{n}(a)\right) & \geq f^{n+1}(a), & & \bar{f}_{i}^{n_{i}+1}\left(f^{n}(a)\right)<f^{n+1}(a), \\
\bar{f}_{i}^{n_{i}}\left(f^{n}(b)\right) \geq f^{n+1}(b), & \bar{f}_{i}^{n_{i}+1}\left(f^{n}(b)\right)<f^{n+1}(b) .
\end{array}
$$

Denotemos $a_{n}=f^{n}(a)$ y $b_{n}=f^{n}(b)$. Pasando al límite cuando $n$ tiende al infinito en las desigualdades

$$
\frac{\bar{f}_{i}^{n_{i}+1}\left(a_{n}\right)}{a_{n}}<\frac{f\left(a_{n}\right)}{a_{n}} \leq \frac{\bar{f}_{i}^{n_{i}}\left(a_{n}\right)}{a_{n}}
$$

obtenemos

$$
\left(\bar{f}_{i}^{\prime}(0)\right)^{n_{i}+1} \leq f^{\prime}(0) \leq\left(\bar{f}_{i}^{\prime}(0)\right)^{n_{i}} .
$$

Para $n \geq 0$ los intervalos $f^{n}(] \bar{f}_{i}(a), b[)$ son dos a dos disjuntos. Si denota$\operatorname{mos} \delta=V(f ;[0, b])$, entonces para todo $u$ y $v$ pertenecientes a $] \bar{f}_{i}(a), b[$ se verifica

$$
\left|\log \left(\frac{\left(f^{n}\right)^{\prime}(v)}{\left(f^{n}\right)^{\prime}(u)}\right)\right| \leq \sum_{i=1}^{n}\left|\log \left(f^{\prime}\right)\left(f^{i-1}(v)\right)-\log \left(f^{\prime}\right)\left(f^{i-1}(u)\right)\right| \leq \delta .
$$

Pasando al límite cuando $n$ tiende al infinito en la desigualdad

$$
\left|\bar{f}_{i}(a, b)\right|=\left|f^{-n} \bar{f}_{i} f^{n}([a, b])\right| \geq \frac{\inf _{u \in\left[\bar{f}_{i}(a), b\right]}\left(f^{n}\right)^{\prime}(u)}{\sup _{v \in\left[\bar{f}_{i}(a), b\right]}\left(f^{n}\right)^{\prime}(v)} \cdot \inf _{x \in\left[f^{n}(a), f^{n}(b)\right]} \bar{f}_{i}^{\prime}(x) \cdot|[a, b]|,
$$

y utilizando las estimaciones (4.1) y (4.2) obtenemos, para cierta constante positiva $C$ y todo $i \in \mathbb{N}$,

$$
\left|\bar{f}_{i}([a, b])\right| \geq e^{-\delta}\left(f^{\prime}(0)\right)^{1 / n_{i}} \cdot|[a, b]| \geq C .
$$

Sin embargo, esta última desigualdad es absurda, pues $\left|\bar{f}_{i}([a, b])\right|$ converge evidentemente a cero cuando $i$ tiende al infinito.

Ejercicio 4.3. Usando el teorema de Denjoy, pruebe que la proposición precedente vale para subgrupos finitamente generados de Difeo ${ }_{+}^{1+v a}(] 0,1[)$ conteniendo un elemento central sin puntos fijos.

De la proposición precedente se deduce inmediatamente lo siguiente. 
Corolario 4.4. Si $f$ es un elemento de $\mathrm{Difeo}_{+}^{1+\mathrm{va}}([0,1[)$ sin puntos fijos en $] 0,1\left[\right.$, entonces su centralizador Cent $(f)$ en Difeo $_{+}^{1}([0,1[)$ es topológicamente conjugado a un grupo de traslaciones de la recta.

Ejercicio 4.5. Pruebe la "versión fuerte" del lema de Kopell (en clase $\mathrm{C}^{1+l i p}$ ) dada por la siguiente proposición (si tiene problemas para esto vea [57]).

Proposición 4.6. Sea $f:\left[0,1\left[\rightarrow\left[0,1\left[\right.\right.\right.\right.$ un difeomorfismo de clase $\mathrm{C}^{1+l i p}$ tal que $f(x)<x$ para todo $x \in] 0,1[$. Fijemos un punto $a \in] 0,1[$, y para cada $n \in \mathbb{N}$ consideremos un difeomorfismo $g_{n}:[f(a), a] \rightarrow[f(a)$, a] tangente a la identidad en los extremos. Si $g:[0,1] \rightarrow] 0,1]$ es tal que su restricción a $\left[f^{n+1}(a), f^{n}(a)\right]$ coincide con $f^{n} g_{n} f^{-n}$ para todo $n \in \mathbb{Z}$, entonces $g$ se extiende a un difeomorfismo de clase $\mathrm{C}^{1}$ de $\left[0,1\left[\right.\right.$ si y sólo si $\left(g_{n}\right)$ converge a la identidad respecto a la topología $\mathrm{C}^{1}$.

Ejercicio 4.7. Pruebe la siguiente "versión real-analítica" del lema de Kopell: si $f$ y $g$ son difeomorfismos real-analíticos de una vecindad del origen que se escriben de la forma $f(x)=x+a_{i} x^{i}+\cdots$ y $g(x)=x+b_{j} x^{j}+\cdots$ para $|x| \leq \varepsilon$, con $j>i$ y $f(x)<x$ para todo $x$ positivo y suficientemente pequeño, entonces la sucesión $f^{-n} g f^{n}$ converge uniformemente a la identidad sobre un intervalo $\left[0, \varepsilon^{\prime}\right]$ (vea [144] para aplicaciones de esta afirmación).

Para cerrar esta sección nos concentraremos en el teorema 3.81 del capítulo precedente. Comencemos considerando un difeomorfismo $f$ de clase $\mathrm{C}^{1+\text { va }}$ de un intervalo $I=[a, b]$ tal que $f^{n}(x)$ converge hacia $a$ cuando $n$ tiende al infinito para todo $x \in[a, b[$. Fijemos un punto arbitrario $c \in] a, b[$, y consideremos cualquier homeomorfismo bilipschitziano $h$ de $[f(c), c]$ en sí mismo. Extendiendo $h$ a todo $] a, b[$ de modo que conmute con $f$, y luego haciendo $h(a)=a$ y $h(b)=b$, obtenemos un homeomorfismo (unívocamente definido) de $[a, b]$ (al que también denotaremos por $h$ ). Afirmamos que si $C$ es una constante de bilipschitzianidad para $h$ en $[f(c), c]$, entonces $C e^{V}$ es una constante de bilipschitzianidad para $h$ en $[a, b]$, donde $V=V(f ;[a, b])$. Para verificar esto consideremos por ejemplo un punto $x$ perteneciente a $f^{n}([f(c), c])$ para cierto $n \geq 0, \mathrm{y}$ tal que $h$ sea derivable en $f^{-n}(x) \in[f(c), c]$ con derivada menor o igual a $C$ (observe que éste es el caso para casi todo punto $\left.x \in\left[f^{n+1}(c), f^{n}(c)\right]\right)$. Debido la relación $h=f^{n} h f^{-n}$ se tiene la desigualdad

$$
h^{\prime}(x)=h^{\prime}\left(f^{-n}(x)\right) \cdot \frac{\left(f^{n}\right)^{\prime}\left(h f^{-n}(x)\right)}{\left(f^{n}\right)^{\prime}\left(f^{-n}\right)(x)} \leq C \cdot \frac{\left(f^{n}\right)^{\prime}\left(h f^{-n}(x)\right)}{\left(f^{n}\right)^{\prime}\left(f^{-n}\right)(x)} .
$$

Haciendo $y=f^{-n}(x) \in[f(c), c]$ y $z=h(y) \in[f(c), c]$, y razonando como en la demostración del lema de Kopell, obtenemos

$$
\begin{aligned}
\left|\log \left(\frac{\left(f^{n}\right)^{\prime}(z)}{\left(f^{n}\right)^{\prime}(y)}\right)\right| & =\left|\log \left(\frac{\prod_{i=0}^{n-1} f^{\prime}\left(f^{i}(z)\right)}{\prod_{i=0}^{n-1} f^{\prime}\left(f^{i}(y)\right)}\right)\right| \\
& \leq \sum_{i=0}^{n-1}\left|\log \left(f^{\prime}\left(f^{i}(z)\right)\right)-\log \left(f^{\prime}\left(f^{i}(y)\right)\right)\right| \leq V .
\end{aligned}
$$


Introduciendo esta última desigualdad en (4.3) deducimos que $h^{\prime}(x) \leq C e^{V}$. Como $x$ era un punto genérico, esto muestra que la constante de Lipschitz de $h$ está acotada por $C e^{V}$. Obviamente, un argumento análogo muestra que la misma cota vale para la constante de Lipschitz de $h^{-1}$.

Para la demostración del teorema 3.81 usaremos una construcción similar. Para simplificar la exposición, probaremos sólo la primera de las afirmaciones de dicho teorema, dejando a cargo del lector la prueba de la segunda afirmación concerniente a la existencia de centralizadores bilipschitzianos no diferenciables sobre ningún abierto.

Comencemos recordando que si $\Gamma$ es un grupo de difeomorfismos de clase $\mathrm{C}^{1+\text { lip }}$ del círculo que preserva un minimal excepcional, entonces el estabilizador de toda componente conexa $] a, b[$ del complemento de dicho minimal es no trivial (vea el ejercicio 3.20). Por la hipótesis del teorema, dicho estabilizador es cíclico infinito, digamos generado por un elemento $f$. Si la restricción de $f$ a $I$ es trivial definimos $h$ como siendo cualquier homeomorfismo bilipschitziano y no diferenciable de $I$. En caso contrario, fijemos $[\bar{a}, \bar{b}] \subset[a, b]$ tal que $f^{n}(x) \neq x$ para todo $\left.x \in\right] \bar{a}, \bar{b}[$, y tal que $f(\bar{a})=\bar{a}$ y $f(\bar{b})=\bar{b}$. Cambiando $f$ por $f^{-1}$ si es necesario, podemos asumir que $f^{n}(x)$ converge a $\bar{a}$ cuando $n$ tiende al infinito para todo $x \in[\bar{a}, \bar{b}[$. Procediendo como lo hicimos anteriormente, fijamos un punto $\bar{c} \in] \bar{a}, \bar{b}[$ y consideramos cualquier homeomorfismo bilipschitziano y no diferenciable $h$ de $[f(\bar{c}), \bar{c}]$. Este homeomorfismo se extiende de manera única a un homeomorfismo de $[a, b]$ que conmuta con la restricción de $f$ a $[\bar{a}, \bar{b}]$ y que coincide con la identidad en $I \backslash[\bar{a}, \bar{b}]$.

Dada la hipótesis sobre los estabilizadores, no es difícil comprobar que existe una única extensión de $h$ a un homeomorfismo de $\mathrm{S}^{1}$ (a la que también denotaremos por $h$ ) que conmuta con (cada elemento de) $\Gamma$ y que coincide con la identidad en el complemento de $\cup_{g \in \Gamma} g(] a, b[)$. Afirmamos que esta extensión es bilipschitziana; de manera más precisa, si fijamos un sistema finito $\mathcal{G}=\left\{g_{1}, \ldots, g_{k}\right\}$ de generadores para $\Gamma$, entonces denotando por $V$ al máximo sobre los $g_{i}$ de la variación del logaritmo de la derivada, y por $C$ a la constante de bilipschitzianidad de $h$ en $[a, b]$, el homeomorfismo $h$ globalmente definido tiene constante de bilipschitzianidad menor o igual a $C e^{k V}$. La prueba de esta afirmación es similar a la dada anteriormente para el caso del intervalo. Fijemos por ejemplo $x \in \cup_{g \in \Gamma}(g(I) \backslash I)$, y tratemos de estimar el valor de $h^{\prime}(x)$. Para esto, consideremos el mínimo $n \in \mathbb{N}$ para el cual existe un elemento $g=g_{i_{n}} \ldots g_{i_{1}} \in \Gamma$ con cada $g_{i_{j}}$ en $\mathcal{G}$ y tal que $g(x) \in I$. La minimalidad de $n$ implica que los intervalos $I, g_{i_{n}}^{-1}(I), g_{i_{n-1}}^{-1} g_{i_{n}}^{-1}(I), \ldots, g_{i_{1}}^{-1} \cdots g_{i_{n}}^{-1}(I)$ tienen interiores disjuntos. Usando la relación $h=g^{-1} h g$ obtenemos, para cada $x \in g^{-1}(I)$ genérico,

$$
h^{\prime}(x)=h^{\prime}(g(x)) \cdot \frac{g^{\prime}(x)}{g^{\prime}(h(x))} \leq C \cdot \frac{g^{\prime}(x)}{g^{\prime}(y)},
$$

donde $y=h(x) \in g^{-1}(I)$. Usando ahora sólo el hecho que la variación total 
del logaritmo de la derivada de cada $g_{i}$ está mayorado por $V$ obtenemos

$$
\begin{aligned}
\left|\log \left(\frac{g^{\prime}(x)}{g^{\prime}(y)}\right)\right| & \leq \sum_{j=0}^{n-1}\left|\log \left(g_{i_{j+1}}^{\prime}\left(g_{i_{j}} \cdots g_{i_{1}}\right)(x)\right)-\log \left(g_{i_{j+1}}^{\prime}\left(g_{i_{j}} \cdots g_{i_{1}}\right)(y)\right)\right| \\
& \leq \sum_{i=1}^{k} V\left(\log \left(g_{i}^{\prime}\right) ; \mathrm{S}^{1}\right) \leq k V .
\end{aligned}
$$

De (4.4) concluimos que $h^{\prime}(x) \leq C e^{k V}$, tal como queríamos verificar.

Para terminar consideremos el caso en que $\Gamma$ admite órbitas finitas. Si $\Gamma$ es finito entonces consideramos cualquier homeomorfismo bilipschitziano y no diferenciable que conmute con su generador. Si $\Gamma$ es infinito, el teorema de Hölder implica que la acción no puede ser libre. Fijemos un elemento no trivial $f \in \Gamma$ que posea puntos fijos, y sea $I$ una componente conexa del complemento de la unión de las órbitas finitas. Observe que $f$ debe fijar todos los puntos de dichas órbitas. Por lo tanto, si procedemos como lo hicimos anteriormente con $I$ y $f$, podemos construir un homeomorfismo bilipschitziano no diferenciable que centraliza a $\Gamma$.

\subsection{El teorema de Szekeres}

En clase $\mathrm{C}^{2}$, el homomorfismo dado por el corolario 4.4 es necesariamente sobreyectivo. Esto se desprende del resultado a seguir, debido a Szekeres [196] (vea también [211]). Dados un intervalo no vacío $[a, b[\mathrm{y}$ $2 \leq r \leq \omega$, denotemos por Difeo ${ }_{+}^{r, \Delta}\left(\left[a, b[)\right.\right.$ al subconjunto de Difeo ${ }_{+}^{r}([a, b[)$ formado por los elementos $f$ tales que $f(x) \neq x$ para todo $x \in] a, b[$. Convengamos además en que $\infty-1=\infty$ y $\omega-1=\omega$.

Teorema 4.8. Para todo $f \in \operatorname{Difeo}_{+}^{r, \Delta}([0,1[)$ existe un único campo de vectores $X_{f}$ sobre $[0,1[$ sin singularidades en $] 0,1[y$ que verifica:

(i) el campo $X_{f}$ es de clase $\mathrm{C}^{r-1}$ sobre $] 0,1\left[y\right.$ de clase $\mathrm{C}^{1}$ sobre $[0,1[$;

(ii) si $f^{\mathbb{R}}=\left\{f^{t}: t \in \mathbb{R}\right\}$ es el flujo asociado a dicho campo, entonces $f^{1}=f$;

(iii) el centralizador de $f$ en $\operatorname{Difeo}_{+}^{1}\left(\left[0,1[)\right.\right.$ es igual a $f^{\mathbb{R}}$.

Este teorema es de particular interés si el germen de $f$ en el origen no es hiperbólico. En caso contrario podemos apelar al teorema de linearización de Sternberg; de hecho, para este caso la afirmación inicial del ejemplo 3.72 permite extender el teorema 4.8 a la clase $\mathrm{C}^{1+\tau}$ para todo $\tau>0$.

Ejercicio 4.9. Dado $\lambda<0$ considere un campo de vectores $X=\varrho \partial / \partial x$ de clase $\mathrm{C}^{1}$ definido sobre $[0,1]$ y tal que para todo $x$ suficientemente pequeño se tenga $\varrho(x)=\lambda x(1-1 / \log (x))$. Pruebe que $X$ no es $C^{1}$ linearizable, en el sentido que no existe ningún difeomorfismo de clase $\mathrm{C}^{1}$ que conjugue $X$ a su parte lineal $\lambda x \frac{\partial}{\partial x}$. 


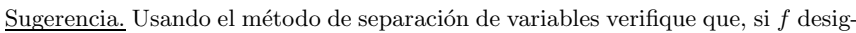
na el tiempo 1 del flujo asociado a $X$, entonces

$$
f(x)=e^{\lambda} x\left(\frac{1-\log (x)}{1-\log (f(x))}\right)
$$

para todo $x$ suficientemente cercano al origen. Usando esto pruebe que $f$ no es conjugado a su parte lineal por ningún homeomorfismo bilipschitziano.

Daremos la demostración del teorema 4.8 sólo para el caso $r=2$. Notemos en primer lugar que podemos suponer que $f(x)>x$ para todo $x \in] 0,1$ [: el campo asociado a un difeomorfismo topológicamente contractante en el origen se obtiene cambiando el signo del campo asociado a su inverso.

Lema 4.10. Sean $a \in] 0,1\left[\right.$ y $f:\left[0,1\left[\rightarrow\left[0,1\left[\right.\right.\right.\right.$ un difeomorfismo de clase $\mathrm{C}^{2}$ sin otro punto fijo que el origen, el cual es topológicamente dilatante. Si $X(x)=\varrho(x) \partial / \partial x$ es un campo de vectores de clase $\mathrm{C}^{1}$ sobre $[0,1[$, entonces $X$ está asociado a $f$ si y sólo si se cumplen las siguientes condiciones:

(i) la función @ es estrictamente positiva sobre ]0,1[,

(ii) para todo $x \in\left[0,1\left[\right.\right.$ se tiene $\varrho(f(x))=f^{\prime}(x) \varrho(x)$,

(iii) se verifica la igualdad $\int_{a}^{f(a)} \frac{d s}{\varrho(s)}=1$.

Demostración. Las dos primeras condiciones son evidentemente necesarias. Con respecto a la tercera, observe que si $X$ está asociado a $f$ entonces

$$
\frac{d}{d t} \int_{a}^{f^{t}(a)} \frac{d s}{\varrho(s)}=\frac{d f^{t}(a)}{d t} \cdot \frac{1}{\varrho\left(f^{t}(a)\right)}=1
$$

para todo $t \geq 0$. De esto se desprende que

$$
\int_{a}^{f^{t}(a)} \frac{d s}{\varrho(s)}=t
$$

lo cual implica (iii) para $t=1$. Recíprocamente, si la condición (ii) es satisfecha, se verifica fácilmente que para todo $x \in] 0,1[$ la derivada de la función $x \mapsto \int_{x}^{f(x)} \frac{d s}{\varrho(s)}$ es idénticamente nula. Luego, para todo $\left.x \in\right] 0,1[$,

$$
\int_{x}^{f(x)} \frac{d s}{\varrho(s)}=\int_{a}^{f(a)} \frac{d s}{\varrho(s)}=1 .
$$

Por lo tanto, si denotamos por $\hat{f}$ al difeomorfismo obtenido al integrar $X$ en tiempo 1 , entonces $\hat{f}(0)=f(0)=0$, y para todo $x \in] 0,1[$ se tiene

$$
1=\int_{x}^{\hat{f}(x)} \frac{d s}{\varrho(s)}=\int_{x}^{f(x)} \frac{d s}{\varrho(s)} .
$$

De lo anterior se deduce fácilmente que $\hat{f}=f$, i.e., $X$ está asociado a $f$. 
Para construir el campo $X$, comenzamos considerando la "diferencia discreta" $\Delta=\Delta_{f}$ definida por $\Delta(x)=f(x)-x$. Si bien $\Delta(f(x))$ y $\Delta(x) f^{\prime}(x)$ no son necesariamente iguales, el "error" cometido tiene una expresión agradable (y es fácilmente controlable). En efecto, si definimos

$$
\Theta(x)=\Theta_{f}(x)=\log \left(f^{\prime}(x)\right)-\log \left[\int_{0}^{1} f^{\prime}(x+t \Delta(x)) d t\right],
$$

entonces se tiene

$$
\Delta(f(x)) \exp (\Theta(x))=\Delta(x) f^{\prime}(x) .
$$

Esta igualdad se deduce inmediatamente de

$$
f^{2}(x)=f(x)+\Delta(x) \int_{0}^{1} f^{\prime}(x+t \Delta(x)) d t .
$$

Para estimaciones futuras será necesario también el desarrollo de Taylor

$$
f^{2}(x)=f(x)+\Delta(x) f^{\prime}(x)+\Delta(x)^{2} \int_{0}^{1}(1-t) f^{\prime \prime}(x+t \Delta(x)) d t,
$$

el cual permite verificar la igualdad

$$
\Theta(x)=\log \left(1-\frac{\Delta(x)^{2}}{\Delta(f(x))} \int_{0}^{1}(1-t) f^{\prime \prime}(x+t \Delta(x)) d t\right) .
$$

La función $\varrho$ correspondiente a $X$ será obtenida multiplicando $\Delta$ por la suma de los errores sucesivos bajo iteración, módulo una normalización apropiada. Para ser más precisos, definamos (por el momento formalmente)

$$
\left.\Sigma(x)=\sum_{n>0} \Theta\left(f^{-n}(x)\right), \quad x \in\right] 0,1[, \quad \Sigma(0)=0 .
$$

Esta función satisface la igualdad formal

$$
\Sigma(f(x))=\Sigma(x)+\Theta(x) .
$$

Luego, si definimos el campo $Y(x)=\Delta(x) \exp (\Sigma(x)) \partial / \partial x$, a partir de (4.6) y (4.8) se concluye que $Y(f(x))$ es igual a

$$
\Delta(f(x)) \exp (\Sigma(f(x))) \frac{\partial}{\partial x}=\frac{\Delta(x) f^{\prime}(x)}{\exp (\Theta(x))} \exp (\Theta(x)+\Sigma(x)) \frac{\partial}{\partial x}=f^{\prime}(x) Y(x) .
$$

El campo $X$ será entonces de la forma $X=c Y$ para cierta constante de normalización $c=c(f)$ para la cual se tenga

$$
\int_{a}^{f(a)} \frac{d s}{X(s)}=1
$$


Observe que la condición impuesta por esta igualdad corresponde a

$$
c=\int_{a}^{f(a)} \frac{d x}{\Delta(x) \exp (\Sigma(x))} .
$$

Supongamos que $f^{\prime}(0)>1$ y denotemos $\lambda=f^{\prime}(0)$. Cuando $a \sim 0$ se tiene $\Sigma(x) \sim 0$ para $x \leq f(a)$, mientras que

$$
\Delta(x)=x\left(\frac{f(x)}{x}-1\right) \sim x(\lambda-1) .
$$

Luego, de acuerdo a (4.9), debe satisfacerse

$$
c=\int_{a}^{f(a)} \frac{d x}{\Delta(x) \exp (\Sigma(x))} \sim \int_{a}^{f(a)} \frac{d x}{x(\lambda-1)}=\frac{\log (f(a) / a)}{\lambda-1} \sim \frac{\log (\lambda)}{\lambda-1},
$$

por lo que la elección apropiada es $c(f)=\log (\lambda) /(\lambda-1)$. Cuando $\lambda$ tiende a 1 esta última expresión converge a 1 , por lo que en el caso $f^{\prime}(0)=1$ la constante de normalización a considerar es $c(f)=1$. A continuación verificaremos que la definición que acabamos de esbozar es pertinente y origina un campo asociado a $f$.

Proposición 4.11. Sea $f$ un difeomorfismo satisfaciendo las hipótesis del lema 4.10. Si definimos $X=\varrho \partial / \partial x$ por $\varrho(x)=c(f) \Delta(x) \exp (\Sigma(x))$, entonces $X$ es un campo de clase $\mathrm{C}^{1}$ asociado a $f$.

Demostración. Debemos verificar sucesivamente tres afirmaciones: el campo $X$ está bien definido (en el sentido de que la serie correspondiente a la definición de la función $\Sigma$ converge), $X$ es de clase $\mathrm{C}^{1}$ (sobre $[0,1[$ ) y $f$ es el tiempo 1 del flujo asociado a él.

Primera parte: la convergencia de $\Sigma(x)$.

Puesto que $f$ es de clase $\mathrm{C}^{1}$, para cada $\left.x \in\right] 0,1[$ existe $y=y(x) \in[x, f(x)]$ tal que

$$
\int_{0}^{1} f^{\prime}(x+t \Delta(x)) d t=f^{\prime}(y) .
$$

De esta igualdad y de (4.5) se deduce que

$$
|\Theta(x)|=\left|\log \left(f^{\prime}(x)\right)-\log \left(f^{\prime}(y)\right)\right| \leq C|y-x| \leq C \Delta(x),
$$

donde $C$ es la constante de Lipschitz de la función $\log \left(f^{\prime}\right)$ sobre $[x, f(x)]$ (la cual es igual al supremo de la función $\left|f^{\prime \prime}\right| /\left|f^{\prime}\right|$ sobre dicho intervalo). Se deduce entonces que la serie correspondiente a $\Sigma(x)$ es convergente para todo $x \in] 0,1[$, con $|\Sigma(x)| \leq C x$. Además, la función $\Sigma$ se extiende continuamente a $[0,1[$ haciendo $\Sigma(0)=0$.

Segunda parte: la diferenciabilidad de $\varrho$. 
Para verificar que $\varrho$ es derivable en el origen basta observar que

$$
\lim _{t \rightarrow 0} \frac{\varrho(t)}{t}=c \lim _{t \rightarrow 0} \exp (\Sigma(t)) \lim _{t \rightarrow 0} \frac{\Delta(t)}{t}=c \Delta^{\prime}(0)=\log (\lambda) .
$$

La verificación de la diferenciabilidad al interior es indirecta. Denotando $L_{X}$ la derivada de Lie según el campo $X$, de la relación $\varrho(f(x))=\varrho(x) f^{\prime}(x)$ se desprende la igualdad $L_{X}\left(\Theta \circ f^{-1}\right)=\left(L_{X} \Theta\right) \circ f^{-1}$. Ahora bien, de (4.7) se concluye que, para cierta constante $C>0$,

$$
\varrho(x) \Theta^{\prime}(x) \leq C \Delta(x) .
$$

Luego, la serie $\sum_{n>0}\left(L_{X} \Theta\right) \circ f^{-n}$ converge, y su valor es igual a $L_{X}(\Sigma)(x)$. Esto prueba que $\Sigma-$ y por lo tanto $X$ - es de clase $\mathrm{C}^{1}$ sobre $] 0,1[$. Observe que lo anterior puede ser reformulado mediante la igualdad (válida para todo $x \in] 0,1[)$

$$
\varrho^{\prime}(x)=\varrho(x) \frac{\Delta^{\prime}(x)}{\Delta(x)}+\sum_{n>0} L_{X}(\varrho) \circ f^{-n}(x) .
$$

Haciendo tender $x$ al origen se deduce rápidamente de esta relación que $\varrho^{\prime}(x)$ tiende a $c \Delta^{\prime}(0)=\log (\lambda)$, lo cual muestra que $X$ es de clase $\mathrm{C}^{1}$ sobre todo el intervalo $[0,1[$.

Tercera parte: el tiempo 1 del flujo.

Debemos verificar la validez de la condición (iii) para todo $x \in] 0,1[$. Sin embargo, ya observamos anteriormente que la igualdad $\varrho(f(x))=\varrho(x) f^{\prime}(x)$ implica que la función $x \mapsto \int_{x}^{f(x)} \frac{d s}{\varrho(s)}$ es constante.

Supongamos primeramente que $f$ sea tangente a la identidad en el origen, es decir, $\lambda=1$. En tal caso se tiene $\Delta^{\prime}(0)=0$, por lo que para todo $\varepsilon>0$ existe $\delta>0$ tal que si $a<\delta$ y $t \in[a, f(a)]$ entonces

$$
|\Delta(t)-\Delta(a)|<\varepsilon(t-a) \quad \text { y } \quad 1-\varepsilon<\frac{1}{\exp (\Sigma(t))}<1+\varepsilon .
$$

La primera de estas desigualdades implica $|\Delta(t)-(f(a)-a)|<\varepsilon(b-a)$, mientras que la segunda nos da

$$
\left|\frac{\Delta(t)}{\varrho(t)}-1\right|<\varepsilon .
$$

Se obtiene así, para $a<\delta$,

$$
\begin{aligned}
& \int_{a}^{f(a)} \frac{d u}{\varrho(u)}>(1-\varepsilon) \int_{a}^{f(a)} \frac{d u}{\Delta(u)}>\frac{1-\varepsilon}{1+\varepsilon} \\
& \int_{a}^{f(a)} \frac{d u}{\varrho(u)}<(1+\varepsilon) \int_{a}^{f(a)} \frac{d u}{\Delta(u)}<\frac{1+\varepsilon}{1-\varepsilon} .
\end{aligned}
$$


Sin embargo, como la función $x \mapsto \int_{x}^{f(x)} \frac{d s}{\varrho(s)}$ es constante, haciendo tender el punto $a$ hacia el origen en conjunto con $\varepsilon$ hacia cero se deduce que dicha constante es igual a 1.

Supongamos ahora que $\lambda>1$. En este caso $\Delta^{\prime}(0)=c-1$, por lo que para $t$ pequeño se tiene

$$
|\Delta(t)-t(c-1)|<\varepsilon t \quad \text { y } \quad\left|\frac{\lambda \Delta(t)}{\varrho(f)(t)}-1\right|<\varepsilon .
$$

De esto se desprende que

$$
\begin{aligned}
\int_{a}^{f(a)} \frac{d s}{\varrho(s)} & <(1+\varepsilon) \int_{a}^{f(a)} \frac{d s}{\lambda \Delta(s)}=\frac{1+\varepsilon}{\lambda} \int_{a}^{f(a)} \frac{d s}{\Delta(s)}<\frac{1+\varepsilon}{\lambda} \int_{a}^{f(a)} \frac{d s}{(c-1-\varepsilon) s} \\
& =\frac{1+\varepsilon}{\lambda(c-1-\varepsilon)} \log \left(\frac{f(a)}{a}\right)=\frac{1+\varepsilon}{\lambda(c-1-\varepsilon)} \log \left(1+\frac{\Delta(a)}{a}\right) \\
& <\frac{1+\varepsilon}{\lambda} \cdot \frac{\log (c+\varepsilon)}{c-1-\varepsilon}=(1+\varepsilon) \frac{\log (c+\varepsilon)}{\log (c)} \cdot \frac{c-1}{c-1-\varepsilon},
\end{aligned}
$$

y pasando al límite obtenemos

$$
\int_{a}^{f(a)} \frac{d s}{\varrho(s)} \leq 1
$$

Un argumento análogo permite probar la desigualdad opuesta, completando así la demostración.

Ejercicio 4.12. Verifique que si el difeomorfismo original es de clase $\mathrm{C}^{1+l i p}$, entonces la construcción precedente origina un campo lipschitziano asociado a él.

Dejamos al lector la tarea de verificar que si el difeomorfismo original es de clase $\mathrm{C}^{r}$ para algún $r \geq 2$, entonces el campo asociado $X$ es de clase $\mathrm{C}^{r-1}$ sobre ]0, 1[. Sin embargo, el campo puede no ser dos veces diferenciable en el origen. Por otra parte, si $f$ es un difeomorfismo del intervalo $[0,1]$, el campo $X$ definido sobre $[0,1$ [ se extiende continuamente al intervalo cerrado haciendo $X(1)=0$, pero esta extensión no es diferenciable en "la mayoría" de los casos (para profundizar en torno a estos temas recomendamos la lectura de los capítulos IV y V de [211] y de las referencias allí indicadas). En todo caso, la diferenciabilidad $\mathrm{C}^{r}$ sobre $] 0,1[$ del campo asociado y la unicidad de éste implican directamente un resultado interesante de regularidad para conjugaciones entre difeomorfismos.

Proposición 4.13. Si $r \geq 2$ y $f_{1}, f_{2}$ son dos difeomorfismos de clase $\mathrm{C}^{r}$ de un intervalo cerrado (al menos por un lado) y sin punto fijo al interior, entonces la restricción al interior de todo difeomorfismo $\mathrm{C}^{1}$ que conjuga dichos difeomorfismos es de clase $\mathrm{C}^{r}$. 


\subsection{La clasificación}

Podemos dar ahora la descripción de los grupos abelianos de difeomorfismos de clase $\mathrm{C}^{1+\text { va }}$ de variedades unidimensionales. El caso del intervalo es muy simple. En efecto, debido al corolario 4.4, la restricción de un grupo tal a cada componente irreducible es conjugada a un grupo de traslaciones. Para el caso del círculo tendremos necesidad del siguiente lema de interés general (compare con la sección 3.1, capítulo 2).

Lema 4.14. Si $\Gamma$ es un subgrupo promediable de Homeo ${ }_{+}\left(\mathrm{S}^{1}\right)$, entonces o bien $\Gamma$ es semiconjugado a un grupo de rotaciones, o bien contiene un subgrupo de indice finito que admite puntos fijos.

Demostración. Como $\Gamma$ es promediable, necesariamente preserva una medida de probabilidad $\mu$ sobre el círculo. Si las órbitas por $\Gamma$ son densas, entonces el soporte de $\mu$ es total, y mediante una reparametrización se constata que $\Gamma$ es topológicamente conjugado a un grupo de rotaciones. Si existe un conjunto de Cantor invariante y minimal, entonces el soporte de $\mu$ coincide con dicho conjunto, y esto permite semiconjugar $\Gamma$ a un grupo de rotaciones. Finalmente, si hay una órbita finita, entonces los elementos de $\Gamma$ preservan el orden cíclico de los puntos de esta órbita, por lo que el estabilizador de dichos puntos es un subgrupo de índice finito de $\Gamma$ que posse puntos fijos.

Del lema precedente se desprende que si $\Gamma$ es un subgrupo abeliano de Difeo $_{+}^{1+v a}\left(\mathrm{~S}^{1}\right)$, entonces o bien es semiconjugado a un grupo de rotaciones, o bien es una extensión central finita de un subgrupo de un producto (a lo más numerable) de grupos abelianos que actúan sobre intervalos disjuntos. Por el corolario 4.4, estos últimos son conjugados a grupos de traslaciones sobre cada componente irreducible. Recuerde que para subgrupos finitamente generados de Difeo ${ }_{+}^{1+v a}\left(\mathrm{~S}^{1}\right)$, toda semiconjugación a un grupo de rotaciones es necesariamente una conjugación; sin embargo, esto no es necesariamente válido para grupos no finitamente generados (vea el ejemplo 3.7).

Ejercicio 4.15. Pruebe que todo grupo virtualmente abeliano de difeomorfismos de clase $\mathrm{C}^{1+\mathrm{va}}$ del intervalo o del círculo es abeliano.

Ejercicio 4.16. Pruebe que todo subgrupo abeliano de $\operatorname{Difeo}_{+}^{1+\mathrm{va}}(\mathbb{R})$ preserva una medida de Radon sobre los borelianos de la recta (vea la proposición 2.56 y los comentarios previos a ella).

\subsection{Contra-ejemplos de Denjoy}

La clase de diferenciabilidad $\mathrm{C}^{1+\text { lip }}$ (o bien $\mathrm{C}^{1+\text { va }}$ ) resulta esencial para los resultados dinámicos del capítulo precedente, así como para algunos resultados algebraicos del presente capítulo. Antes de pasar a la construcción de "contra-ejemplos" (llamados de esta forma por abuso de lenguaje) hagamos una breve revisión de la noción de módulo de continuidad. 
Definición 4.17. Dado un homeomorfismo $\omega:[0,1] \rightarrow[0, \omega(1)]$, decimos que una función $\psi:[0,1] \rightarrow \mathbb{R}$ es $\omega$-continua si existe $C \in \mathbb{R}$ tal que, para todo $x \neq y$ en $[0,1]$,

$$
\left|\frac{\psi(x)-\psi(y)}{\omega(|x-y|)}\right| \leq C .
$$

Denotemos por $\|\psi\|_{\omega}$ el supremo de la expresión a izquierda, y llamémoslo la $\mathrm{C}^{\omega}$-norma de $\psi$. El interés en la noción de $\omega$-continuidad se debe al hecho (evidente) de que si $\left(\psi_{n}\right)$ es una sucesión de funciones definidas en $[0,1]$ tal que

$$
\sup _{n \in \mathbb{N}}\left\|\psi_{n}\right\|_{\omega}<\infty
$$

entonces $\left(\psi_{n}\right)$ es una sucesión equicontinua.

Ejemplo 4.18. Para $\omega(s)=s^{\tau}$, con $0<\tau<1$, las nociones de $\omega$-continuidad y $\tau$-hölderianidad coinciden.

Ejemplo 4.19. Para $\omega(s)=s$, la noción de $\omega$-continuidad coincide con la de lipschitzianidad.

Ejemplo 4.20. Dado $\varepsilon \geq 0$, suponga que $\omega=\omega_{\varepsilon}$ es tal que $\omega_{\varepsilon}(s)=s[\log (1 / s)]^{1+\varepsilon}$ para $s$ pequeño. Si una aplicación es $\omega_{\varepsilon}$-continua, entonces ella es $\tau$-continua para todo $0<\tau<1$. De hecho, es fácil verificar que

$$
s \log \left(\frac{1}{s}\right)^{1+\varepsilon} \leq C_{\varepsilon, \tau} s^{\tau}, \quad \text { donde } C_{\varepsilon, \tau}=\frac{1}{e^{1+\varepsilon}}\left(\frac{1+\varepsilon}{1-\tau}\right)^{1+\varepsilon}
$$

Observe que la aplicación $s \mapsto s \log (1 / s)^{1+\varepsilon}$ no es lipschitziana. Por lo tanto, la $\omega_{\varepsilon}$-continuidad para una función no implica que dicha función sea lipschitziana.

Ejemplo 4.21. Un módulo de continuidad $\omega$ satisfaciendo $\omega(s)=1 / \log (1 / s)$ para todo $s$ suficientemente pequeño es más débil que cualquier módulo Hölder $s \mapsto s^{\tau}$, donde $\tau>0$.

Para nuestra construcción, uno de los problemas centrales consistirá en controlar el módulo de continuidad de la derivada de una aplicación obtenida al "pegar" infinitos difeomorfismos definidos parcialmente sobre subintervalos. Para esto, el lema elemental presentado a continuación será primordial.

Lema 4.22. Sea $\left\{I_{n}: n \in \mathbb{N}\right\}$ una familia de subintervalos cerrados de $[0,1]$ (resp. $\mathrm{S}^{1}$ ) con interiores disjuntos y tales que el complemento de su unión tiene medida de Lebesgue nula. Supongamos que $\varphi$ sea un homeomorfismo de $[0,1]$ tal que sus restricciones a cada intervalo $I_{n}$ sean difeomorfismos de clase $\mathrm{C}^{1+\omega}$ tangentes a la identidad en los extremos. Supongamos además que las $\mathrm{C}^{\omega}$-normas de las derivadas de estas restricciones estén uniformemente acotadas por una constante $C>0$. Entonces $\varphi$ es un difeomorfismo de clase $\mathrm{C}^{1+\omega}$ de $[0,1]$ (resp. de $\mathrm{S}^{1}$ ), y la $\mathrm{C}^{\omega}$-norma de su derivada es menor o igual que $2 C$. 
Demostración. Consideraremos sólo el caso del intervalo, pues el caso del círculo es análogo. Sean $x<y$ dos puntos de $\cup_{n \in \mathbb{N}} I_{n}$. Si ellos pertenecen a un mismo intervalo $I_{n}$ entonces, por hipótesis,

$$
\left|\frac{\varphi^{\prime}(y)-\varphi^{\prime}(x)}{\omega(y-x)}\right| \leq C .
$$

Supongamos ahora que $x \in I_{i}=\left[x_{i}, y_{i}\right]$ e $y \in I_{j}=\left[x_{j}, y_{j}\right]$, con $y_{i} \leq x_{j}$. En este caso,

$$
\left|\frac{\varphi^{\prime}(y)-\varphi^{\prime}(x)}{\omega(y-x)}\right|=\left|\frac{\left(\varphi^{\prime}(y)-1\right)+\left(1-\varphi^{\prime}(x)\right)}{\omega(y-x)}\right| \leq\left|\frac{\varphi^{\prime}(y)-\varphi^{\prime}\left(x_{j}\right)}{\omega(y-x)}\right|+\left|\frac{\varphi^{\prime}\left(y_{i}\right)-\varphi^{\prime}(x)}{\omega(y-x)}\right|,
$$

y esta última expresión está mayorada por

$$
C\left[\frac{\omega\left(y-x_{j}\right)}{\omega(y-x)}+\frac{\omega\left(y_{i}-x\right)}{\omega(y-x)}\right] \leq 2 C .
$$

La aplicación $x \mapsto \varphi^{\prime}(x)$ es por lo tanto uniformemente continua sobre el conjunto denso $\cup_{n \in \mathbb{N}} I_{n}$, por lo que se extiende a una función continua definida en $[0,1]$ y cuya derivada posee $\mathrm{C}^{\omega}$-norma acotada por $2 C$. Siendo nula la medida de $I \backslash \cup_{n \in \mathbb{N}} I_{n}$, el teorema fundamental del cálculo de Lebesgue implica que esta función continua coincide (en todo punto) con la derivada de $\varphi$.

Dado lo anterior, será útil disponer de una buena familia de difeomorfismos entre intervalos que sean tangentes a la identidad en los extremos.

Definición 4.23. Una familia $\left\{\varphi_{a, b}:[0, a] \rightarrow[0, b] ; a>0, b>0\right\}$ de homeomorfismos es equivariante si $\varphi_{b, c} \circ \varphi_{a, b}=\varphi_{a, c}$ para todo $a>0, b>0$ y $c>0$.

Dadas una familia equivariante y dos intervalos $I=\left[x_{1}, x_{2}\right]$ y $J=\left[y_{1}, y_{2}\right]$, denotaremos por $\varphi(I, J): I \rightarrow J$ al homeomorfismo definido por

$$
\varphi(I, J)(x)=\varphi_{x_{2}-x_{1}, y_{2}-y_{1}}\left(x-x_{1}\right)+y_{1} .
$$

Observe que $\varphi(I, I)$ coincide necesariamente con la aplicación identidad.

La familia equivariante más simple es aquélla de las aplicaciones afines $\varphi_{a, b}(x)=b x / a$. Sin embargo, resulta evidente que esta familia no es adecuada si se busca "pegar diferenciablemente" aplicaciones definidas en subintervalos. Para soslayar este problema, introduzcamos el siguiente procedimiento: dada una familia de homeomorfismos $\left\{\varphi_{a}:\right] 0, a[\rightarrow \mathbb{R} ; a>0\}$, definamos $\left.\varphi_{a, b}=\varphi_{b}^{-1} \circ \varphi_{a}:\right] 0, a[\rightarrow] 0, b[$; de la igualdad

$$
\varphi_{b, c} \circ \varphi_{a, b}=\left(\varphi_{c}^{-1} \circ \varphi_{b}\right) \circ\left(\varphi_{b}^{-1} \circ \varphi_{a}\right)=\varphi_{c}^{-1} \circ \varphi_{a}=\varphi_{a, c}
$$

se deduce que, al extender continuamente $\varphi_{a, b}$ a todo el intervalo $[0, a]$, se obtiene una familia equivariante. Para nosostros serán fundamentales las aplicaciones $\left.\varphi_{a}:\right] 0, a[\rightarrow \mathbb{R}$ definidas por

$$
\varphi_{a}(x)=-\frac{1}{a} \operatorname{ctg}\left(\frac{\pi x}{a}\right) .
$$


La familia equivariante asociada fue introducida por Yoccoz; sus elementos poseen propiedades notables de regularidad que pasamos a discutir.

Utilizando la notación $u=\varphi_{a}(x)$ tenemos

$$
\varphi_{a, b}^{\prime}(x)=\left(\varphi_{b}^{-1}\right)^{\prime}\left(\varphi_{a}(x)\right) \cdot \varphi_{a}^{\prime}(x)=\frac{\left(\varphi_{b}^{-1}\right)^{\prime}(u)}{\left(\varphi_{a}^{-1}\right)^{\prime}(u)}=\frac{u^{2}+1 / a^{2}}{u^{2}+1 / b^{2}} .
$$

Observe que si $x \rightarrow 0$ (resp. $x \rightarrow a$ ), entonces $u \rightarrow-\infty$ (resp. $u \rightarrow+\infty$ ) y $\varphi_{a, b}^{\prime}(x) \rightarrow 1$. Por lo tanto, la aplicación $\varphi_{a, b}$ se extiende a un difeomorfismo de clase $\mathrm{C}^{1}$ de $[0, a]$ sobre $[0, b]$ tangente a la identidad en los extremos. Además, para $a \geq b$ (resp. $a \leq b$ ), la función $u \mapsto \frac{u^{2}+1 / a^{2}}{u^{2}+1 / b^{2}}$ alcanza su valor mínimo (resp. máximo) en $u=0$, y siendo este valor igual a $b^{2} / a^{2}$, tenemos

$$
\sup _{x \in[0, a]}\left|\varphi_{a, b}^{\prime}(x)-1\right|=\left|\frac{b^{2}}{a^{2}}-1\right| .
$$

Para la segunda derivada de $\varphi_{a, b}$ tenemos

$$
\begin{aligned}
\varphi_{a, b}^{\prime \prime}(x) & =\frac{d \varphi_{a, b}^{\prime}(x)}{d u} \cdot \frac{d u}{d x}=\frac{2 u\left(u^{2}+1 / b^{2}\right)-2 u\left(u^{2}+1 / a^{2}\right)}{\left(u^{2}+1 / b^{2}\right)^{2}} \pi\left(u^{2}+1 / a^{2}\right) \\
& =\pi \frac{u^{2}+1 / a^{2}}{\left(u^{2}+1 / b^{2}\right)^{2}}\left[2 u\left(\frac{1}{b^{2}}-\frac{1}{a^{2}}\right)\right]
\end{aligned}
$$

por lo que

$$
\left|\varphi_{a, b}^{\prime \prime}(x)\right|=\pi \frac{u^{2}+1 / a^{2}}{u^{2}+1 / b^{2}} \cdot \frac{\left|2 u\left(1 / b^{2}-1 / a^{2}\right)\right|}{u^{2}+1 / b^{2}} .
$$

De esto se desprende que $\varphi_{a, b}$ es un difeomorfismo de clase $\mathrm{C}^{2}$ satisfaciendo $\varphi_{a, b}^{\prime \prime}(0)=\varphi_{a, b}^{\prime \prime}(a)=0$. La desigualdad $\frac{2|u|}{u^{2}+t^{2}} \leq \frac{1}{t}$ aplicada a $t=1 / b$ nos da

$$
\left|\varphi_{a, b}^{\prime \prime}(x)\right| \leq \pi \frac{u^{2}+1 / a^{2}}{u^{2}+1 / b^{2}}\left|\frac{1}{b^{2}}-\frac{1}{a^{2}}\right| b .
$$

Para $a \leq b$, lo anterior implica que

$$
\left|\varphi_{a, b}^{\prime \prime}(x)\right| \leq \pi \frac{b^{2}}{a^{2}}\left(\frac{b^{2}-a^{2}}{a^{2} b^{2}}\right) b=\frac{\pi b}{a^{2}}\left(\frac{b^{2}}{a^{2}}-1\right) .
$$

Luego, si $a \leq b \leq 2 a$ entonces

$$
\left|\varphi_{a, b}^{\prime \prime}(x)\right| \leq 6 \pi\left|\frac{b}{a}-1\right| \frac{1}{a} .
$$

Análogamente, si $2 b \geq a \geq b$ entonces

$$
\left|\varphi_{a, b}^{\prime \prime}(x)\right| \leq \frac{\pi}{b}\left(1-\frac{b^{2}}{a^{2}}\right) \leq 2 \pi\left|\frac{b}{a}-1\right| \frac{1}{b} \leq 4 \pi\left|\frac{b}{a}-1\right| \frac{1}{a} .
$$


Por lo tanto, en ambos casos se tiene

$$
\left|\varphi_{a, b}^{\prime \prime}(x)\right| \leq 6 \pi\left|\frac{b}{a}-1\right| \frac{1}{a} .
$$

Esta última desigualdad y el ejercicio a continuación muestran que la familia de aplicaciones $\varphi_{a, b}$ es en cierto sentido optimal.

Ejercicio 4.24. Pruebe que si $\varphi:[0, a] \rightarrow[0, b]$ es un difeomorfismo de clase $\mathrm{C}^{2}$ tal que $\varphi^{\prime}(0)=\varphi^{\prime}(a)=1$, entonces existe un punto $\left.s \in\right] 0, a[$ tal que

$$
\left|\varphi^{\prime \prime}(s)\right| \geq \frac{2}{a}\left|\frac{b}{a}-1\right| .
$$

Con excepción del ejercicio 4.29, en lo que sigue la notación $\varphi_{a, b}$ será utilizada exclusivamente para denotar las aplicaciones de la familia de Yoccoz. Sin pérdida de generalidad, supondremos que la función $s \mapsto \omega(s) / s$ es decreciente. Observe que los módulos considerados en los ejemplos 4.18, 4.20 y 4.21 pueden ser modificados (lejos de 0) con el fin de satisfacer esta condición. Bajo esta suposición se verifica lo siguiente.

Lema 4.25. Si $a>0$ y $b>0$ son tales que $a / b \leq 2, b / a \leq 2$ y

$$
\left|\frac{b}{a}-1\right| \frac{1}{\omega(a)} \leq C,
$$

entonces la $\mathrm{C}^{\omega}$-norma de $\varphi_{a, b}^{\prime}$ es menor o igual a $6 \pi C$.

Demostración. En virtud de (4.11), para todo $x \in[0, a]$ se tiene

$$
\left|\varphi_{a, b}^{\prime \prime}(x)\right| \leq \frac{6 \pi C \omega(a)}{a} .
$$

Si $y<z$ pertenecen a $[0, a]$ entonces existe un punto $x \in[y, z]$ satisfaciendo $\varphi_{a, b}^{\prime}(z)-\varphi^{\prime}(y)=\varphi_{a, b}^{\prime \prime}(x)(z-y)$. Como $s \mapsto \omega(s) / s$ es una función decreciente y $z-y \leq a$, esto implica que

$$
\left|\frac{\varphi_{a, b}^{\prime}(z)-\varphi_{a, b}^{\prime}(y)}{\omega(z-y)}\right|=\left|\varphi_{a, b}^{\prime \prime}(x)\right|\left|\frac{z-y}{\omega(z-y)}\right| \leq\left|\varphi_{a, b}^{\prime \prime}(x)\right|\left|\frac{a}{\omega(a)}\right| \leq 6 \pi C,
$$

lo cual prueba nuestra afirmación.

Sobre la base de lo discutido anteriormente podemos - de manera relativamente conceptual- dar la construcción de los llamados contra-ejemplos de Denjoy (i.e., dar una prueba del teorema 3.2). El método empleado será reutilizado más adelante para suavizar diversas acciones de grupos.

Demostración del teorema 3.2. De manera más general, para todo $\varepsilon>0$, todo ángulo irracional $\theta$ y todo $k \in \mathbb{N}$, exhibiremos un difeomorfismo de clase $\mathrm{C}^{1+\omega_{\varepsilon}}$ del círculo con número de rotación $\theta$ y cuya derivada tiene 
$\mathrm{C}^{\omega_{\varepsilon}}$-norma acotada por $2 /[\log (k)]^{\varepsilon / 2}$. Para ello retomemos la construcción de los ejemplos de Denjoy: fijemos $x_{0} \in \mathrm{S}^{1}$ y para cada $n \in \mathbb{Z}$ reemplacemos cada punto $x_{n}=R_{\theta}^{n}\left(x_{0}\right)$ de su órbita por un intervalo $I_{n}$ de longitud

$$
\ell_{n}=\frac{1}{(|n|+k)[\log (|n|+k)]^{1+\varepsilon / 2}} .
$$

La rotación original $R_{\theta}$ induce un nuevo homeomorfismo $\bar{R}_{\theta}$ de un círculo de longitud $\bar{\ell}_{k}=\sum_{n \in \mathbb{Z}} \ell_{n}$ haciendo $\bar{R}_{\theta}(x)=\varphi\left(I_{n}, I_{n+1}\right)(x)$ para $x \in I_{n}$ y extendiendo continuamente a todo $S^{1}$. Verificaremos a continuación que $\bar{R}_{\theta}$ es un difeomorfismo que satisface las propiedades pedidas. Para ello, debemos estimar el valor de expresiones del tipo

$$
\left|\frac{\ell_{n+1}}{\ell_{n}}-1\right| \frac{1}{\omega_{\varepsilon}\left(\ell_{n}\right)} .
$$

Haremos las estimaciones para el caso $n>0$, dejando el caso $n \leq 0$ a cargo del lector. La expresión considerada (4.12) es igual a

$$
\left|\frac{(n+k)[\log (n+k)]^{1+\varepsilon / 2}}{(n+k+1)[\log (n+k+1)]^{1+\varepsilon / 2}}-1\right| \frac{(n+k)[\log (n+k)]^{1+\varepsilon / 2}}{[\log (n+k)+(1+\varepsilon / 2) \log \log (n+k)]^{1+\varepsilon}},
$$

que es evidentemente menor o igual a

$$
\left|\frac{(n+k)[\log (n+k)]^{1+\varepsilon / 2}}{(n+k+1)[\log (n+k+1)]^{1+\varepsilon / 2}}-1\right| \frac{n+k}{[\log (n+k)]^{\varepsilon / 2}} .
$$

El teorema del valor medio aplicado a la función $s \mapsto s[\log (s)]^{1+\varepsilon / 2}$ implica que esta última expresión está mayorada por

$$
\frac{[\log (n+k+1)]^{1+\varepsilon / 2}+(1+\varepsilon / 2)[\log (n+k+1)]^{\varepsilon / 2}}{[\log (n+k+1)]^{1+\varepsilon / 2}[\log (n+k)]^{\varepsilon / 2}} \leq \frac{2}{[\log (n+k)]^{\varepsilon / 2}} .
$$

Ahora bien, el difeomorfismo obtenido $h$ actúa sobre un círculo de longitud $\bar{\ell}_{k} \sim 2 / \varepsilon[\log (k)]^{\varepsilon / 2}$. Por lo tanto, para obtener un difeomorfismo genuino del círculo (normalizado) debemos conjugar por una aplicación afín $\varphi$. Sin embargo, este proceso no aumenta la $C^{\omega_{\varepsilon}}$-norma para la derivada; en efecto, de la igualdad $\bar{R}_{\theta}^{\prime}=\left(\varphi \circ h \circ \varphi^{-1}\right)^{\prime}=\bar{\ell}_{k}^{-1} \cdot\left(h^{\prime} \circ \varphi^{-1}\right) \cdot L_{k}$ se deduce que

$$
\begin{aligned}
\frac{\left|\bar{R}_{\theta}^{\prime}(x)-\bar{R}_{\theta}^{\prime}(y)\right|}{\omega_{\varepsilon}(|x-y|)} & =\frac{\left|\left(\varphi \circ h \circ \varphi^{-1}\right)^{\prime}(x)-\left(\varphi \circ h \circ \varphi^{-1}\right)^{\prime}(y)\right|}{\omega_{\varepsilon}\left(\left|\varphi^{-1}(x)-\varphi^{-1}(y)\right|\right)} \cdot \frac{\omega_{\varepsilon}\left(\left|\varphi^{-1}(x)-\varphi^{-1}(y)\right|\right.}{\omega_{\varepsilon}(|x-y|)} \\
& \leq \frac{\left|h^{\prime}\left(\varphi^{-1}(x)\right)-h^{\prime}\left(\varphi^{-1}(y)\right)\right|}{\omega_{\varepsilon}\left(\left|\varphi^{-1}(x)-\varphi^{-1}(y)\right|\right)} \leq \frac{2}{[\log (k)]^{\varepsilon / 2}},
\end{aligned}
$$

donde la penúltima desigualdad se justifica por el hecho que $\omega_{\varepsilon}$ es una función creciente y $\bar{\ell}_{k}<1$.

La construcción anterior podría inducir erróneamente a pensar que existen contra-ejemplos de Denjoy para cualquier módulo de continuidad más 
débil que $\mathrm{C}^{2}$. Sin embargo, existen obstrucciones ligadas a la naturaleza diofantina del número de rotación, y que hasta hoy no han sido del todo dilucidadas. Recomendamos al respecto la lectura de la sección 4 del capítulo X de [94].

Ejercicio 4.26. Respecto a la notación del ejemplo 4.20, pruebe que no existen contra-ejemplos de Denjoy de clase $\mathrm{C}^{1+\omega_{0}}$ cuya derivada sea idénticamente igual a 1 sobre el conjunto de Cantor invariante minimal (compare con el lema $3.3 \mathrm{y}$ el argumento que le precede).

Observación. Se desconoce si la afirmación anterior sigue siendo válida si se suprime la hipótesis sobre la derivada en el conjunto de Cantor invariante.

Quisiéramos ahora aplicar la técnica precedente para construir acciones suaves de grupos abelianos de rango superior admitiendo un minimal excepcional. Sin embargo, la proposición 3.3 sugiere que debiésemos encontrar obstrucciones en clases de diferenciabilidad bastante más pequeñas que $\mathrm{C}^{1+l i p}$. Éste será el tema central a tratar en la sección siguiente, donde probaremos en particular que, para todo $d \geq 2$ y todo $\varepsilon>0$, toda acción libre de $\mathbb{Z}^{d}$ por difeomorfismos de clase $\mathrm{C}^{1+1 / d+\varepsilon}$ del círculo es minimal ${ }^{1}$. De acuerdo a [199] (vea también [200]), la hipótesis $\varepsilon>0$ debiese ser superflua para este último resultado; de manera aún más general, el teorema debiese ser aún válido para difeomorfismos cuya derivada tiene $d$-variación acotada (vea el ejercicio 4.32). Por el momento nos contentaremos con mostrar que, en regularidad inferior a $\mathrm{C}^{1+1 / d}$, el resultado deja de ser válido.

Proposición 4.27. Para todo $\varepsilon>0$ y todo entero positivo d existen acciones libres de $\mathbb{Z}^{d}$ por difeomorfismos de clase $\mathrm{C}^{1+1 / d-\varepsilon}$ del círculo admitiendo un minimal excepcional.

Demostración. Observe que el caso $d=1$ corresponde al teorema 3.2. La construcción abarca sin embargo de manera global todos los casos, generando ejemplos de grupos de difeomorfismos clase $\mathrm{C}^{1+\omega}$ respecto al módulo de continuidad $\omega(s)=s^{1 / d}[\log (1 / s)]^{1 / d+\varepsilon}$.

Comencemos fijando un subgrupo de rango $d$ de $\mathrm{SO}(2, \mathbb{R})$, y notemos $\theta_{1}, \ldots, \theta_{d}$ los ángulos de las rotaciones que lo generan. Fijemos un entero $m \geq d-1$ y un punto $p \in \mathrm{S}^{1}$, y para cada $\left(i_{1}, \ldots, i_{d}\right) \in \mathbb{Z}^{d}$ reemplacemos el punto $p_{i_{1}, \ldots, i_{d}}=R_{\theta_{1}}^{i_{1}} \cdots R_{\theta_{d}}^{i_{d}}(p)$ por un intervalo $I_{i_{1}, \ldots, i_{d}}$ de longitud

$$
\ell_{\left(i_{1}, \ldots, i_{d}\right)}=\frac{1}{\left(\left|i_{1}\right|+\cdots+\left|i_{d}\right|+m\right)^{d}\left[\log \left(\left|i_{1}\right|+\cdots+\left|i_{d}\right|+m\right)\right]^{1+\varepsilon}} .
$$

Este proceso origina un nuevo círculo (cuya longitud $\bar{\ell}_{m}$ está mayorada por $\left.2^{d} / \varepsilon[\log (m)]^{\varepsilon}(d-1) !\right)$, sobre el cual las rotaciones $R_{\theta_{j}}$ inducen homeomorfismos $f_{j}$ verificando, para todo $x \in I_{i_{1}, \ldots, i_{j}, \ldots, i_{d}}$,

$$
f_{j}(x)=\varphi\left(I_{i_{1}, \ldots, i_{j}, \ldots, i_{d}}, I_{i_{1}, \ldots, 1+i_{j}, \ldots, i_{d}}\right)(x) .
$$

\footnotetext{
${ }^{1}$ De hecho, vale un resultado más fuerte: toda acción libre por difeomorfismos del círculo del grupo $\mathbb{Z}^{d}$ con generadores $f_{i}, i \in\{1, \ldots, d\}$, respectivamente de clase $\mathrm{C}^{1+\tau_{i}}$, es necesariamente minimal si $\tau_{1}+\cdots+\tau_{d}>1$ (vea [114]).
} 
En virtud de las propiedades de equivarianza de las $\varphi(I, J)$, estos homeomorfismos $f_{j}$ conmutan entre ellos. La verificación de que cada $f_{i}$ es de clase $\mathrm{C}^{1+\omega}$ es análoga a la de la prueba del teorema 3.2 , y la dejamos a cargo del lector. Observe finalmente que, renormalizando la longitud de $\mathrm{S}^{1}$ y haciendo tender $m$ al infinito, cada aplicación converge (en topología $\left.\mathrm{C}^{1+\omega}\right)$ a la rotación $R_{\theta_{i}}$ respectiva.

Es posible fabricar contra-ejemplos al lema de Kopell análogos a los construidos precedentemente para el teorema de Denjoy. De manera aún más general, para cada entero $d \geq 2$ y cada $\varepsilon>0$ existen difeomorfismos $f_{1}, \ldots, f_{d}$ de clase $\mathrm{C}^{1+1 /(d-1)-\bar{\varepsilon}}$ de $[0,1]$ e intervalos abiertos disjuntos $I_{n_{1}, \ldots, n_{d}}$ dispuestos sobre ]0,1[ respetando su orden lexicográfico de modo que, para todo $\left(n_{1}, \ldots, n_{d}\right) \in \mathbb{Z}^{d}$ y todo $j \in\{1, \ldots, d\}$,

$$
f_{j}\left(I_{n_{1}, \ldots, n_{j}, \ldots, n_{d}}\right)=I_{n_{1}, \ldots, n_{j}-1, \ldots, n_{d}} .
$$

Para construir tales ejemplos el procedimiento natural sería el siguiente. Fijado un entero $m \geq d-1$, para cada $\left(i_{1}, \ldots, i_{d}\right) \in \mathbb{Z}^{d}$ hagamos

$$
\ell_{i_{1}, \ldots, i_{d}}=\frac{1}{\left(\left|i_{1}\right|+\cdots+\left|i_{d}\right|+m\right)^{d}\left[\log \left(\left|i_{1}\right|+\cdots+\left|i_{d}\right|+m\right)\right]^{1+\varepsilon}} .
$$

Por inducción en $j$ definamos $\ell_{i_{1}, \ldots, i_{j-1}}=\sum_{i_{j} \in \mathbb{Z}} \ell_{i_{1}, \ldots, i_{j-1}, i_{j}}$. Notemos $\left[x_{i_{1}, \ldots, i_{j}, \ldots, i_{d}}, y_{i_{1}, \ldots, i_{j}, \ldots, i_{d}}\right]$ el intervalo de extremos

$$
\begin{gathered}
x_{i_{1}, \ldots, i_{j}, \ldots, i_{d}}=\sum_{i_{1}^{\prime}<i_{1}} \ell_{i_{1}^{\prime}}+\sum_{i_{2}^{\prime}<i_{2}} \ell_{i_{1}, i_{2}^{\prime}}+\ldots+\sum_{i_{d}^{\prime}<i_{d}} \ell_{i_{1}, \ldots, i_{d}, i_{d}^{\prime}}, \\
y_{i_{1}, \ldots, i_{j}, \ldots, i_{d}}=x_{i_{1}, \ldots, i_{j}, \ldots, i_{d}}+\ell_{i_{1}, \ldots, i_{d}} .
\end{gathered}
$$

Sea entonces $f_{j}$ el difeomorfismo de $[0,1]$ cuya restricción a cada intervalo $\left[x_{i_{1}, \ldots, i_{j}, \ldots, i_{d}}, y_{i_{1}, \ldots, i_{j}, \ldots, i_{d}}\right]$ coincide con

$$
\varphi\left(\left[x_{i_{1}, \ldots, i_{j}, \ldots, i_{d}}, y_{i_{1}, \ldots, i_{j}, \ldots, i_{d}}\right],\left[x_{i_{1}, \ldots, i_{j}-1, \ldots, i_{d}}, y_{i_{1}, \ldots, i_{j}-1, \ldots, i_{d}}\right]\right) .
$$

Utilizando las estimaciones de esta sección se verifica rápidamente que los $f_{j}$ así obtenidos son de clase $\mathrm{C}^{1+\omega}$ respecto al módulo de continuidad $\omega(s)=s^{1 / d}[\log (1 / s)]^{1 / d+\varepsilon}$; en particular, se trata de difeomorfismos de clase $\mathrm{C}^{1+1 / d-\varepsilon}$. Sin embargo, de acuerdo a lo afirmado anteriormente, la regularidad alcanzada por este método no es optimal. Además, una aplicación directa de la proposición 3.3 muestra que mediante este método no se podrá alcanzar jamás la clase $\mathrm{C}^{1+1 / d}$. Para alcanzar la regularidad optimal $\mathrm{C}^{1+1 /(d-1)-\varepsilon}$ es necesario "suprimir" las tangencias a la identidad "excesivas" de los difeomorfismos construidos. Para ello utilizaremos la técnica original de Pixton [164] siguiendo la brillante presentación de Tsuboi [199].

Sea $X=\varrho \partial / \partial x$ un campo de vectores de clase $\mathrm{C}^{\infty}$ en $[0,1]$ tal que $\left|\varrho^{\prime}(x)\right| \leq 1$ para todo $x \in[0,1]$, y de modo que $\varrho(x)=x$ para todo 
$x \in[0,1 / 5]$ y $\varrho(x)=0$ para todo $x \in[1 / 2,1]$. Sea $\varphi^{t}(x)$ el flujo asociado a $X$, es decir, la solución de la ecuación diferencial

$$
\frac{d \varphi^{t}(x)}{d t}(x)=\varrho\left(\varphi^{t}(x)\right), \quad \varphi^{0}(x)=x .
$$

Para cada $a$ y $b$ positivos y cada $t \geq 0$, el difeomorfismo $x \mapsto b \varphi^{t}(x / a)$ envía el intervalo $[0, a]$ sobre $[0, b]$, con derivada igual a $b / a$ en $[a / 2, a]$ e igual a $b e^{t} / a$ en el origen. Dados $a^{\prime}<0<a$ y $b^{\prime}<0<b$ denotemos $\varphi_{b^{\prime}, b}^{a^{\prime}, a}:[0, a] \rightarrow[0, b]$ el difeomorfismo definido por

$$
\varphi_{b^{\prime}, b}^{a^{\prime}, a}(x)=b \varphi^{\log \left(b^{\prime} a / a^{\prime} b\right)}\left(\frac{x}{a}\right) .
$$

Observe que para todo $c^{\prime}<0<c$ se satisface la propiedad de equivarianza

$$
\varphi_{c^{\prime}, c}^{b^{\prime}, b} \circ \varphi_{b^{\prime}, b}^{a^{\prime}, a}=\varphi_{c^{\prime}, c}^{a^{\prime}, a} .
$$

Esta propiedad es análoga a aquélla de la definición 4.23. Sin embargo, el hecho de que en la familia $\left\{\varphi_{b^{\prime}, b}^{a^{\prime}, a}\right\}$ estén involucrados cuatro parámetros en lugar de dos permitirá obtener un mejor control del módulo de continuidad para las derivadas.

Proposición 4.28. Si $C=\sup _{y \in[0,1]} \varrho^{\prime \prime}(y)$ entonces para todo $x \in[0, a]$ se tiene la desigualdad

$$
\left|\frac{\partial}{\partial x} \log \left(\frac{\partial \varphi_{b^{\prime}, b}^{a^{\prime}, a}}{\partial x}\right)(x)\right| \leq \frac{C}{a}\left|\frac{b^{\prime} a}{a^{\prime} b}-1\right| .
$$

Demostración. De $d \varphi^{t}(x) / d t=\varrho\left(\varphi^{t}(x)\right)$ se concluye que

$$
\frac{d}{d t} \frac{\partial \varphi^{t}}{\partial x}(x)=\frac{\partial}{\partial x} \varrho\left(\varphi^{t}(x)\right)=\frac{\partial \varrho}{\partial x}\left(\varphi^{t}(x)\right) \cdot \frac{\partial \varphi^{t}}{\partial x}(x),
$$

por lo que

$$
\frac{d}{d t} \log \left(\frac{\partial \varphi^{t}}{\partial x}\right)(x)=\frac{\partial \varrho}{\partial x}\left(\varphi^{t}(x)\right) .
$$

Por otro lado, de $\left|\varrho^{\prime}(x)\right| \leq 1$ se desprende que

$$
\left|\log \left(\frac{\partial \varphi^{t}}{\partial x}\right)(x)\right|=\left|\int_{0}^{t} \frac{d}{d s} \log \left(\frac{\partial \varphi^{s}}{\partial x}\right)(x) d s\right|=\left|\int_{0}^{t} \frac{\partial \varrho}{\partial x}\left(\varphi^{s}(x)\right) d s\right| \leq \int_{0}^{t} d s=t,
$$

y por lo tanto

$$
\frac{\partial \varphi^{t}}{\partial x}(x) \leq e^{t} .
$$

Puesto que

$$
\log \left(\frac{\partial \varphi_{b^{\prime}, b}^{a^{\prime}, a}}{\partial x}\right)(x)=\log \left(\frac{b}{a}\right)+\log \left(\frac{\partial}{\partial x} \varphi^{\log \left(b^{\prime} a / a^{\prime} b\right)}\right)\left(\frac{x}{a}\right),
$$


usando (4.15) y (4.16) obtenemos

$$
\begin{aligned}
\left|\frac{\partial}{\partial x} \log \left(\frac{\partial \varphi_{b^{\prime}, b}^{a^{\prime}, a}}{\partial x}\right)(x)\right| & =\left|\frac{\partial}{\partial x} \log \left(\frac{\partial}{\partial x} \varphi^{\log \left(b^{\prime} a / a^{\prime} b\right)}\right)\left(\frac{x}{a}\right)\right| \\
& =\left|\int_{0}^{\log \left(b^{\prime} a / a^{\prime} b\right)} \frac{d}{d t}\left(\frac{\partial}{\partial x} \log \left(\frac{\partial \varphi^{t}}{\partial x}\right)\left(\frac{x}{a}\right)\right) d t\right| \\
& =\frac{1}{a}\left|\int_{0}^{\log \left(b^{\prime} a / a^{\prime} b\right)} \frac{\partial}{\partial x} \frac{d}{d t} \log \left(\frac{\partial \varphi^{t}}{\partial x}\right)\left(\frac{x}{a}\right) \cdot \frac{d \varphi^{t}}{d x}\left(\frac{x}{a}\right) d t\right| \\
& =\frac{1}{a}\left|\int_{0}^{\log \left(b^{\prime} a / a^{\prime} b\right)} \frac{\partial}{\partial x} \frac{\partial \varrho}{\partial x}\left(\varphi^{t}\left(\frac{x}{a}\right)\right) \cdot \frac{d \varphi^{t}}{d x}\left(\frac{x}{a}\right) d t\right| \\
& \leq \frac{C}{a}\left|\int_{0}^{\log \left(b^{\prime} a / a^{\prime} b\right)} e^{t} d t\right|=\frac{C}{a}\left|\frac{b^{\prime} a}{a^{\prime} b}-1\right|
\end{aligned}
$$

Observe que

$\frac{\partial \varphi_{b^{\prime}, b}^{a^{\prime}, a}}{\partial x}(0)=\frac{b}{a} \cdot \frac{\partial}{\partial x} \varphi^{\log \left(b^{\prime} a / a^{\prime} b\right)}(0)=\frac{b}{a} \cdot \exp \left(\log \left(\frac{b^{\prime} a}{a^{\prime} b}\right)\right)=\frac{b^{\prime}}{a^{\prime}}, \quad \frac{\partial \varphi_{b^{\prime}, b}^{a^{\prime}, a}}{\partial x}(a)=\frac{b}{a}$.

Por lo tanto, los difeomorfismos $\varphi_{b^{\prime}, b}^{a^{\prime}, a}$ no son en general tangentes a la identidad en los extremos. Gracias a esto podremos "pegarlos" sin la necesidad de "imponer" esta condición de tangencia, y una elección conveniente de los parámetros $a^{\prime}<0$ y $b^{\prime}<0$ permitirá incrementar la regularidad global. En lo que sigue daremos sólo un esbozo de la construcción de dos difeomorfismos conmutantes del intervalo que constituyan un contra-ejemplo de clase $\mathrm{C}^{2-\varepsilon}$ al lema de Kopell. Para el caso general de acciones de $\mathbb{Z}^{d}$ sobre el intervalo el lector puede consultar el interesantísimo trabajo [199]. Advertimos sin embargo que la elección de las longitudes de los intervalos en dicho trabajo es ligeramente diferente a la que haremos a continuación.

Fijemos $k \in \mathbb{N}$ y $\varepsilon>0$, y para cada par de enteros $m, n$ hagamos $\ell_{m, n}=1 /(|m|+|n|+k)^{2+\varepsilon}$. Sea $\left\{I_{m, n}=\left[x_{m, n}, y_{m, n}\right]\right\}$ una familia de intervalos dispuestos sobre un intervalo $I$ respetando su orden lexicográfico y de modo que su unión sea densa en $I$, con $\left|I_{m, n}\right|=\ell_{m, n}$. Definimos los homeomorfismos $f$ y $g$ de $I$ sobre sí mismo haciendo, para todo $x \in I_{m, n}$,

$$
\begin{gathered}
f(x)=\varphi_{\ell_{m-1, n-1}, \ell_{m-1, n}}^{\ell_{m, n-1}, \ell_{m, n}}\left(x-x_{m, n}\right)+x_{m-1, n}, \\
g(x)=\varphi_{\ell_{m, n-2}, \ell_{m, n-1}}^{\ell_{m, n-1}, \ell_{m, n}}\left(x-x_{m, n}\right)+x_{m, n-1} .
\end{gathered}
$$

Las propiedades (4.13) y (4.17) implican inmediatamente que tanto $f$ como $g$ son difeomorfismos conmutantes y de clase $\mathrm{C}^{1}$ del intervalo. Dejamos al lector la tarea de verificar, a partir de la desigualdad (4.14), que $f$ y $g$ son en realidad de clase $\mathrm{C}^{2-\varepsilon^{\prime}}$, donde $\varepsilon^{\prime}>0$ depende sólo de $\varepsilon>0$ y tiende 
a cero conjuntamente con $\varepsilon$ (señalemos que esto no es una tarea del todo sencilla, debido en gran medida a la carencia de un análogo del lema 4.22 en la presente situación; vea [199] para mayores detalles).

Ejercicio 4.29. Permitámosnos un pequeño abuso de notación y definamos $\varphi_{a, b}:[0, a] \rightarrow[0, b]$ haciendo $\varphi_{a, b}(x)=\varphi_{-1, b / 2}^{-1, a / 2}(x)$ y $\varphi_{a, b}(x)=b-\varphi_{-1, b / 2}^{-1, a / 2}(x)$ para $x \in[a / 2, a]$ y $x \in[0, a / 2]$ respectivamente. Verifique que $\left\{\varphi_{a, b}\right\}$ es una familia equivariante de difeomorfismos tangentes a la identidad en los extremos satisfaciendo la desigualdad (a ser comparada con (4.11))

$$
\left|\frac{\partial}{\partial x} \log \left(\frac{\partial \varphi_{a, b}}{\partial x}\right)(x)\right| \leq \frac{2 C}{a}\left|\frac{a}{b}-1\right| .
$$

\subsection{Rigidez en regularidad intermedia}

El resultado a continuación, discutido parcialmente en la sección precedente, aparece en [54]. Ignoramos si bajo las mismas hipótesis las acciones correspondientes son ergódicas (compare con la proposición 3.47).

Teorema 4.30. Para todo entero $d \geq 2$ y todo $\varepsilon>0$, toda acción libre de $\mathbb{Z}^{d}$ por difeomorfismos de clase $\mathrm{C}^{1+1 / d+\varepsilon}$ del círculo es minimal.

Antes de comenzar la demostración de este resultado quisiéramos dar la idea esencial, la cual se inspira en el famoso principio de Erdös e ilustra nuevamente el interés de la introducción de métodos probabilísticos en la teoría. Supongamos por contradicción que $I$ sea un intervalo errante para la dinámica de dos difeomorfismos $g_{1}$ y $g_{2}$ del círculo de clase $\mathrm{C}^{1+\tau}$ y que conmutan entre ellos. Notemos que el conjunto

$$
\left\{g_{1}^{m} g_{2}^{n}(I):(m, n) \in \mathbb{N}_{0} \times \mathbb{N}_{0}, m+n \leq k\right\}
$$

contiene exactamente $(k+1)(k+2) / 2$ intervalos. Puesto que ellos son dos a dos disjuntos, su "longitud típica" es del orden de $1 / k^{2}$. Por lo tanto, para una sucesión aleatoria "genérica" $I, h_{1}(I), h_{2}(I) \ldots$, donde $h_{n+1}=g_{1} h_{n}$ ó $h_{n+1}=g_{2} h_{n}$, debiésemos esperar que, para $\tau>1 / 2$,

$$
\sum_{k \geq 1}\left|h_{k}(I)\right|^{\tau} \leq C \sum_{k \geq 1} \frac{1}{k^{2 \tau}}<\infty .
$$

Ahora bien, la serie que aparece a izquierda es precisamente aquélla cuya convergencia permite controlar la distorsión de la sucesión de composiciones. De manera más precisa, si dicha suma es finita entonces el lema 3.16 debiese permitir hallar elementos que poseen puntos fijos hiperbólicos, contradiciendo así la libertad de la acción.

Para "modelar" esta demostración debemos formalizar el sentido en el que entenderemos las sucesiones típicas. Para ello, consideremos el proceso de Markov sobre $\mathbb{N}_{0} \times \mathbb{N}_{0}$ con probabilidades de transición

$$
p((m, n) \rightarrow(m+1, n))=\frac{m+1}{m+n+2}, \quad p((m, n) \rightarrow(m, n+1))=\frac{n+1}{m+n+2} .
$$


Este proceso markoviano induce una medida de probabilidad $\mathbb{P}$ en el espacio de caminos correspondiente $\Omega$. Se comprueba fácilmente que, partiendo desde el origen, la probabilidad de llegar en $k$ pasos al punto $(m, n)$ es igual a $1 /(k+1)($ resp. 0$)$ si $m+n=k$ (resp. $m+n \neq k)$.

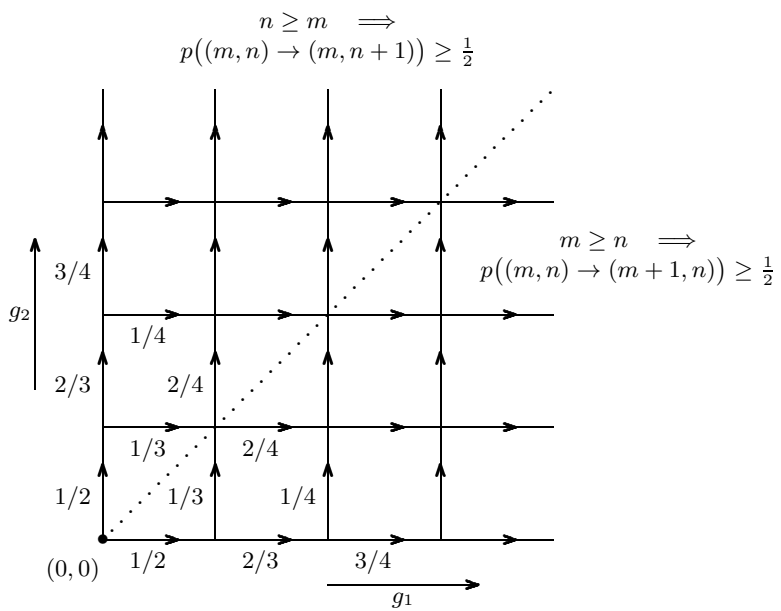

Figura 21

Para probar el teorema 4.30 en el caso $d=2$ procedemos por contradicción. Sean $g_{1}$ y $g_{2}$ dos difeomorfismos del círculo de clase $\mathrm{C}^{1+\tau}$ que conmutan. El semigrupo $\Gamma^{+}$generado por $g_{1}$ y $g_{2}$ se identifica a $\mathbb{N}_{0} \times \mathbb{N}_{0}$; por lo tanto, el proceso de Markov descrito anteriormente induce una "caminata aleatoria" sobre $\Gamma^{+}$. En lo que sigue identificaremos $\Omega$ al espacio de caminos sobre $\Gamma^{+}$correspondiente. Para cada $\omega \in \Omega$ y $n \in \mathbb{N}$, denotemos $h_{n}(\omega) \in \Gamma^{+}$el producto de las $n$ primeras coordenadas de $\omega$. En otras palabras, para $\omega=\left(g_{i_{1}}, g_{i_{2}}, \ldots\right) \in \Omega$ hagamos $h_{n}(\omega)=g_{i_{n}} \cdots g_{i_{1}}$ (donde los cada $g_{i_{j}}$ puede ser igual a $g_{1}$ o a $\left.g_{2}\right)$, y convengamos en que $h_{0}(\omega)=i d$.

Si la acción del grupo $\Gamma=\left\langle g_{1}, g_{2}\right\rangle \sim \mathbb{Z}^{2}$ es libre entonces los números de rotación $\rho\left(g_{1}\right)$ y $\rho\left(g_{2}\right)$ de $g_{1}$ y $g_{2}$ respectivamente son independientes sobre los racionales, en el sentido que para todo $\left(r_{0}, r_{1}, r_{2}\right) \in \mathbb{Q}^{3}$ distinto de $(0,0,0)$ se tiene $r_{1} \rho\left(g_{1}\right)+r_{2} \rho\left(g_{2}\right) \neq r_{0}$. En efecto, en caso contrario se podrían hallar elementos no triviales (y por lo tanto de orden infinito) con número de rotación racional; tales elementos poseerían entonces puntos periódicos, violando así la hipótesis de libertad para la acción.

Si la acción de $\Gamma$ es (libre y) no minimal, entonces existe un conjunto de Cantor invariante y minimal. Además, toda componente conexa $I$ del complemento de este conjunto es errante por la dinámica (en el sentido que sus imágenes por elementos distintos del grupo son disjuntas). 
Lema 4.31. Si $\tau>1 / 2$ entonces la serie $\sum_{n \geq 0}\left|h_{n}(\omega)(I)\right|^{\tau}$ converge para $\mathbb{P}$-casi todo $\omega \in \Omega$.

Demostración. Para cada $\omega \in \Omega$ definamos $\ell_{\tau}(\omega)=\sum_{k>0}\left|h_{k}(\omega)(I)\right|^{\tau}$. Probaremos que, si $\tau>1 / 2$, entonces la esperanza (respecto a $\mathbb{P}$ ) de la función $\ell_{\tau}$ es finita, lo cual implica evidentemente la afirmación del lema. Notemos en primer lugar que, como las probabilidades de llegada en $k$ pasos están equidistribuidas sobre los puntos a distancia simplicial $k$ del origen,

$$
\mathbb{E}\left(\ell_{\tau}\right)=\mathbb{E}\left(\sum_{k \geq 0}\left|h_{k}(\omega)(I)\right|^{\tau}\right)=\sum_{k \geq 0} \mathbb{E}\left(\left|h_{k}(\omega)(I)\right|^{\tau}\right)=\sum_{k \geq 0} \sum_{m+n=k} \frac{\left|g_{1}^{m} g_{2}^{n}(I)\right|^{\tau}}{k+1} .
$$

Ahora bien, la desigualdad de Hölder implica

$$
\sum_{m+n=k} \frac{\left|g_{1}^{m} g_{2}^{n}(I)\right|^{\tau}}{k+1} \leq\left(\sum_{m+n=k}\left|g_{1}^{m} g_{2}^{n}(I)\right|\right)^{\tau}\left((k+1) \cdot \frac{1}{(k+1)^{1 /(1-\tau)}}\right)^{1-\tau},
$$

y por lo tanto

$$
\mathbb{E}\left(\ell_{\tau}\right) \leq \sum_{k \geq 0} \frac{\left(\sum_{m+n=k}\left|g_{1}^{m} g_{2}^{n}(I)\right|\right)^{\tau}}{(k+1)^{\tau}} .
$$

Aplicando nuevamente la desigualdad de Hölder obtenemos

$$
\mathbb{E}\left(\ell_{\tau}\right) \leq\left[\sum_{(m, n) \in \mathbb{N}_{0} \times \mathbb{N}_{0}}\left|g_{1}^{m} g_{2}^{n}(I)\right|\right]^{\tau}\left[\sum_{k \geq 1}\left(\frac{1}{k^{\tau}}\right)^{\frac{1}{1-\tau}}\right]^{1-\tau} .
$$

Puesto que $\tau>1 / 2$, la serie

$$
\sum_{k \geq 1}\left(\frac{1}{k^{\tau}}\right)^{\frac{1}{1-\tau}}=\sum_{k \geq 1} \frac{1}{k^{\tau /(1-\tau)}}
$$

converge, y puesto que los intervalos del tipo $g_{1}^{m} g_{2}^{n}(I)$ son dos a dos disjuntos, esto muestra la finitud de $\mathbb{E}\left(\ell_{\tau}\right)$.

Ejercicio 4.32. Dado $\alpha>0$, una función $\psi$ posee $\alpha$-variación acotada si el valor de

$$
\sup _{a_{0}<a_{1}<\ldots<a_{n}} \sum_{i=1}^{n}\left|\psi\left(a_{i}\right)-\psi\left(a_{i-1}\right)\right|^{\alpha}
$$

es finito. Suponga que $g_{1}$ y $g_{2}$ sean difeomorfismos conmutantes del círculo que generan un grupo abeliano de rango 2 actuando de manera libre y no minimal, y fije $x$ e $y$ en una componente conexa del complemento del conjunto de Cantor invariante. Suponiendo que $g_{1}^{\prime}$ y $g_{2}^{\prime}$ poseen $\alpha$-variación acotada para algún $\alpha<2$, muestre la finitud de la esperanza de la función $V_{x, y}: \Omega \rightarrow \mathbb{R}$ definida por

$$
V_{x, y}(\omega)=\sum_{k \geq 0}\left|g_{i_{k+1}}^{\prime}\left(h_{k}(\omega)(x)\right)-g_{i_{k+1}}^{\prime}\left(h_{k}(\omega)(y)\right)\right| .
$$


Del lema precedente se deduce que si $S>0$ es suficientemente grande, entonces la probabilidad del conjunto $\Omega(S)=\left\{\omega \in \Omega: \ell_{\tau}(\omega) \leq S\right\}$ es positiva (de hecho, $\mathbb{P}[\Omega(S)]$ converge a 1 cuando $S$ tiende al infinito). Fijemos una constante tal $S$, y sea $\ell=\ell\left(\tau, S,|I| ;\left\{g_{1}, g_{2}\right\}\right)$ la constante del lema 3.16. Consideremos finalmente el intervalo abierto $L^{\prime}$ de longitud $\left|L^{\prime}\right|=\ell$ que es adyacente por la derecha a $I$. Afirmamos que

$$
\mathbb{P}\left[\omega \in \Omega: h_{n}(\omega)(I) \not \subset L^{\prime} \text { para todo } n \in \mathbb{N}\right]=0 .
$$

Para probar esta igualdad notemos en primer lugar que la acción del grupo generado por $g_{1}$ y $g_{2}$ es semiconjugada a una acción por rotaciones. Por lo tanto, si "colapsamos" cada componente conexa del complemento del conjunto de Cantor invariante y minimal $\Lambda$, entonces obtenemos un círculo topológico $\mathrm{S}_{\Lambda}^{1}$ sobre el cual $g_{1}$ y $g_{2}$ inducen dos homeomorfismos a órbitas densas. Por otra parte, el intervalo $L^{\prime}$ se transforma en un intervalo $U$ de interior no vacío en $\mathrm{S}_{\Lambda}^{1}$. Como los números de rotación de $g_{1}$ y $g_{2}$ son irracionales, existe $N \in \mathbb{N}$ tal que, tras colapsar, $g_{1}^{-1}(U), \ldots, g_{1}^{-N}(U)$ recubren el círculo topológico $\mathrm{S}_{\Lambda}^{1}$, al igual que $g_{2}^{-1}(U), \ldots, g_{2}^{-N}(U)$. Sobre el círculo original $\mathrm{S}^{1}$ esto se traduce en el hecho que, para toda componente conexa $I_{0}$ de $\mathrm{S}^{1} \backslash \Lambda$, existen $n_{1}$ y $n_{2}$ en $\{1, \ldots, N\}$ tales que $g_{1}^{n_{1}}\left(I_{0}\right) \subset L^{\prime}$ y $g_{2}^{n_{2}}\left(I_{0}\right) \subset L^{\prime}$.

De la definición de $N$ se desprende inmediatamente que, para todo entero $k \geq 0$, la probabilidad condicional del evento

$$
\left[g_{1}^{i} h_{k}(\omega)(I) \not \subset L^{\prime} \text { y } g_{2}^{i} h_{k}(\omega)(I) \not \subset L^{\prime} \text { para todo } i \in\{1, \ldots, N\}\right]
$$

dado que $h_{j}(\omega)(I) \not \subset L^{\prime}$ para todo $j \in\{1, \ldots, k\}$ es nula. Remarquemos ahora la propiedad elemental siguiente que se deduce directamente de (4.18): las probabilidades de salto hacia la derecha (resp. hacia arriba) del proceso markoviano considerado son mayores o iguales que $1 / 2$ bajo (resp. sobre) la diagonal (vea la figura 21). En conjunto con lo anterior, esto implica que

$\mathbb{P}\left[h_{k+i}(\omega)(I) \not \subset L^{\prime}, i \in\{1, \ldots, N\} \mid h_{j}(\omega)(I) \not \subset L^{\prime}, j \in\{1, \ldots, k\}\right] \leq 1-\frac{1}{2^{N}}$.

En consecuencia, para todo $r \in \mathbb{N}$,

$\mathbb{P}\left[h_{n}(\omega)(I) \not \subset L^{\prime}, n \in \mathbb{N}\right] \leq \mathbb{P}\left[h_{n}(\omega)(I) \not \subset L^{\prime}, i \in\{1, \ldots, r N\}\right] \leq\left(1-\frac{1}{2^{N}}\right)^{r}$,

de donde se deduce (4.19) haciendo tender $r$ hacia el infinito.

Para concluir la prueba del teorema 4.30 (en el caso $d=2$ ) notemos que, si $\omega \in \Omega(S)$ y $n \in \mathbb{N}$ satisfacen $h_{n}(\omega)(I) \subset L^{\prime}$, entonces el lema 3.16 permite encontrar un punto fijo hiperbólico para $h_{n}(\omega) \in \Gamma^{+}$, lo cual contradice la hipótesis de libertad de la acción. 
La demostración del teorema 4.30 para $d>2$ es análoga: asumiendo la existencia de un intervalo errante, se considera el proceso markoviano sobre $\mathbb{N}_{0}^{d}$ a probabilidades de transición

$$
p\left(\left(n_{1}, \ldots, n_{i}, \ldots, n_{d}\right) \longrightarrow\left(n_{1}, \ldots, 1+n_{i}, \ldots, n_{d}\right)\right)=\frac{1+n_{i}}{n_{1}+\cdots+n_{d}+d} .
$$

Las probabilidades de llegada en $k$ pasos se equidistribuyen sobre los puntos a distancia simplicial $k$ del origen. Esto permite nuevamente controlar la distorsión de casi toda sucesión aleatoria (es decir, un análogo del lema 4.31 es válido para $\tau>1 / d)$. Nuevamente se verifica que cada $\left(n_{1}, \ldots, n_{d}\right) \in \mathbb{N}_{0}^{d}$ es el punto de partida de al menos una semirecta tal que las probabilidades de salto entre dos vértices consecutivos es mayor o igual que $1 / d$ (basta seguir la dirección de la coordenada $i$-ésima para la cual $n_{i}$ toma el valor máximo). Esto permite obtener una desigualdad análoga a (4.20) (cuyo miembro a derecha será igual a $1-1 / d^{N}$ para cierto entero $N$ suficientemente grande). Esta desigualdad implica la validez de la propiedad (4.19), la cual gracias al control de distorsión permite utilizar el lema 3.16 para hallar elementos con puntos fijos hiperbólicos, violando así la hipótesis de libertad de la acción.

Ejercicio 4.33. Pruebe la siguiente generalización del teorema 4.30: si $\tau>1 / d$ para cierto $d \in \mathbb{N}$ y si $\Gamma$ es un subgrupo de $\operatorname{Difeo}_{+}^{1+\tau}\left(\mathrm{S}^{1}\right)$ semiconjugado a un grupo de rotaciones que contiene $d$ elementos con números de rotación independientes sobre los racionales, entonces la semiconjugación es una conjugación. En particular, si $f$ es un contra-ejemplo de Denjoy de clase $\mathrm{C}^{1+\tau}$, entonces su centralizador en Difeo ${ }_{+}^{1+\tau}\left(\mathrm{S}^{1}\right)$ no puede contener elementos $f_{1}, \ldots, f_{d-1}$ tales que $\rho\left(f_{1}\right), \ldots, \rho\left(f_{d-1}\right)$ y $\rho(f)$ sean independientes sobre los racionales.

Sugerencia. Examine atentamente la demostración del teorema 4.30 y note que, a lo largo de ella, la conmutatividad es "esencial" sólo sobre el eventual conjunto de Cantor invariante y minimal.

Al igual que el teorema de Denjoy, el lema de Kopell admite versiones más finas para grupos abelianos de difeomorfismos del intervalo de clase de diferenciabilidad intermedia.

Teorema 4.34. Sean $d \geq 2$ un entero, $\varepsilon>0, y f_{1}, \ldots, f_{d+1}$ difeomorfismos de clase $\mathrm{C}^{1}$ del intervalo [0,1[ que conmutan entre ellos. Supongamos que existan intervalos abiertos disjuntos $I_{n_{1}, \ldots, n_{d}}$ dispuestos sobre ]0,1[ respetando su orden lexicográfico y tales que $f_{i}\left(I_{n_{1}, \ldots, n_{i}, \ldots, n_{d}}\right)=I_{n_{1}, \ldots, n_{i}-1, \ldots, n_{d}} y$ $f_{d+1}\left(I_{n_{1}, \ldots, n_{d}}\right)=I_{n_{1}, \ldots, n_{d}}$ para todo $\left(n_{1}, \ldots, n_{d}\right) \in \mathbb{Z}^{d}$ y todo $i \in\{1, \ldots, d\}$. Si $f_{1}, \ldots, f_{d}$ son de clase $\mathrm{C}^{1+1 / d+\varepsilon}$, entonces la acción de $f_{d+1}$ sobre la unión de los $I_{n_{1}, \ldots, n_{d}}$ es trivial.

Demostración. Trataremos sólo el caso $d=2$, dejando al lector la tarea de adaptar los argumentos al caso general. Hagamos $\tau=1 / 2+\varepsilon$, identifiquemos el semigrupo $\Gamma^{+}$generado por los elementos $f_{1}$ y $f_{2}$ de Difeo ${ }_{+}^{1+\tau}([0,1])$ con $\mathbb{N}_{0} \times \mathbb{N}_{0}$, y consideremos el proceso markoviano utilizado precedentemente. Si fijamos el intervalo $\left.I=I_{0,0}=\right] a, b[\mathrm{y}$ para cada $\omega \in \Omega$ definimos 
$\ell_{\tau}(\omega)=\sum_{i \geq 0}\left|h_{i}(\omega)(I)\right|^{\tau}$, entonces el argumento de la prueba del lema 4.31 permite verificar que, como $\varepsilon>0$, la función $\ell_{\tau}: \Omega \rightarrow \mathbb{R}$ es casi ciertamente finita (y que de hecho su esperanza es finita).

Sea $C$ una constante de $\tau$-hölderianidad para $\log \left(f_{1}^{\prime}\right)$ y $\log \left(f_{2}^{\prime}\right)$ sobre $[0, b]$. Para cada $\omega=\left(f_{j_{1}}, f_{j_{2}}, \ldots\right)$ en $\Omega$, cada $n, k$ en $\mathbb{N}$ y cada $x \in I$, la igualdad $f_{3}^{n}=h_{k}(\omega)^{-1} \circ f_{3}^{n} \circ h_{k}(\omega)$ implica que $\log \left(\left(f_{3}^{n}\right)^{\prime}(x)\right)$ coincide con

$\log \left(\left(f_{3}^{n}\right)^{\prime}\left(h_{k}(\omega)(x)\right)\right)+\sum_{i=1}^{k}\left[\log \left(f_{j_{i}}^{\prime}\left(h_{i-1}(\omega)(x)\right)\right)-\log \left(f_{j_{i}}^{\prime}\left(f_{3}^{n} \circ h_{i-1}(\omega)(x)\right)\right)\right]$,

por lo que

$$
\begin{aligned}
\left|\log \left(\left(f_{3}^{n}\right)^{\prime}(x)\right)\right| & \leq\left|\log \left(\left(f_{3}^{n}\right)^{\prime}\left(h_{k}(\omega)(x)\right)\right)\right|+C \sum_{i=1}^{k}\left|h_{i-1}(\omega)(x)-f_{3}^{n} \circ h_{i-1}(\omega)(x)\right|^{\tau} \\
& \leq\left|\log \left(\left(f_{3}^{n}\right)^{\prime}\left(h_{k}(\omega)(x)\right)\right)\right|+C \sum_{i=1}^{k}\left|h_{i-1}(\omega)(I)\right|^{\tau} .
\end{aligned}
$$

Escogiendo $\omega \in \Omega$ tal que $\ell_{\tau}(\omega)=S$ sea finito, lo anterior implica que

$$
\left|\log \left(\left(f_{3}^{n}\right)^{\prime}(x)\right)\right| \leq\left|\log \left(\left(f_{3}^{n}\right)^{\prime}\left(h_{k}(\omega)(x)\right)\right)\right|+C S .
$$

Observe que la sucesión de los $h_{k}(\omega)(x)$ converge necesariamente a un punto fijo (parabólico) de $f_{3}$ (de hecho, para casi todo $\omega \in \Omega$ dicha sucesión converge hacia el origen). Haciendo tender $k$ al infinito deducimos que $\left|\log \left(\left(f_{3}^{n}\right)^{\prime}(x)\right)\right| \leq C S$. Por lo tanto, $\left(f_{3}^{n}\right)^{\prime}(x) \leq \exp (C S)$ para todo $x \in I$ y todo $n \in \mathbb{N}$, lo cual implica evidentemente que la restricción de $f_{3}$ al intervalo $I$ es la identidad. Por conmutatividad, esto es válido para todos los intervalos $I_{n_{1}, n_{2}}$, lo cual concluye la demostración.

Ejercicio 4.35. Pruebe que el teorema 4.34 vale aún si $f_{1}, \ldots, f_{d}$ son difeomorfismos conmutantes de clase $\mathrm{C}^{1}$ del intervalo y la $(d-\varepsilon)$-variación de la derivada de $f_{1}, \ldots, f_{d-1}$ y $f_{d}$ es acotada para algún $\varepsilon>0$ (vea el ejercicio 4.32).

Ejercicio 4.36. Análogamente al ejercicio 4.33, pruebe que si $d \geq 2$ es un entero, $\varepsilon>0$, y $f_{1}, \ldots, f_{d+1}$ son difeomorfismos de clase $\mathrm{C}^{1}$ del intervalo [0,1[ (no necesariamente conmutantes entre ellos) para los cuales existen intervalos abiertos disjuntos $I_{n_{1}, \ldots, n_{d+1}}$ dispuestos sobre ]0,1[ respetando el orden lexicográfico y tales que, para cada $\left(n_{1}, \ldots, n_{d+1}\right) \in \mathbb{Z}^{d+1}$,

$$
f_{i}\left(I_{n_{1}, \ldots, n_{i}, \ldots, n_{d+1}}\right)=I_{n_{1}, \ldots, n_{i}-1, \ldots, n_{d+1}} \quad \text { para todo } i \in\{1, \ldots, d+1\},
$$

entonces $f_{1}, \ldots, f_{d}$ no pueden ser simultáneamente de clase $\mathrm{C}^{1+1 / d+\varepsilon}$.

Sugerencia. Retome los argumentos de la demostración de dicho teorema: una vez establecido el control (genérico) para la distorsión, aplique un argumento similar al de la prueba del lema 4.51 para concluir.

El lector podría sentirse incomodado por la hipótesis combinatoria del teorema 4.34. Sin embargo, de acuerdo a [201], las acciones de $\mathbb{Z}^{d+1}$ sobre $[0,1]$ que verifican esta hipótesis son las "más interesantes" desde el 
punto de vista cohomológico. Por otra parte, no es difícil construir acciones de $\mathbb{Z}^{d+1}$ por difeomorfismos de clase $\mathrm{C}^{2-\varepsilon}$ del intervalo sin punto fijo global en el interior. Para ello, consideremos por ejemplo $d$ difeomorfismos $f_{2}, \ldots, f_{d+1}$ de un intervalo $\left.[a, b] \subset\right] 0,1[$ cuyos soportes sean disjuntos, y sea $f_{1}$ un difeomorfismo de $[0,1]$ sin punto fijo al interior y que envía ]$a, b\left[\right.$ sobre un intervalo disjunto. Extendiendo $f_{2}, \ldots, f_{d+1}$ a todo el intervalo $[0,1]$ de modo que conmuten con $f_{1}$ se obtiene una acción (efectiva) de $\mathbb{Z}^{d+1}$ por homeomorfismos del intervalo y sin punto fijo global en ]0, 1[. Evidentemente, los métodos de la sección 1.4 del capítulo 4 permiten suavizar esta acción de manera de tornarla de clase $\mathrm{C}^{2-\varepsilon}$ para todo $\varepsilon>0$.

Otro fenómeno interesante a señalar es la existencia de acciones por difeomorfismos del intervalo que al interior son libres y no conjugadas a acciones por traslaciones. De acuerdo a la proposición 4.2, dicho fenómeno no puede producirse en clase $\mathrm{C}^{1+\mathrm{va}}$. Sin embargo, usando nuevamente los métodos de la sección 1.4 del capítulo 4, es posible construir ejemplos de acciones de $\mathbb{Z}^{2}$ por difeomorfismos de clase $C^{3 / 2-\varepsilon}$ de $[0,1]$ que son libres sobre ]0, 1[ pero que admiten intervalos errantes. Nuevamente, la clase de diferenciabilidad $\mathrm{C}^{3 / 2-\varepsilon}$ es optimal para la existencia de tales ejemplos. El resultado siguiente debiese ser comparado con el corolario 4.4 o con el teorema de Szekeres ya tratado en la sección 1.2 de este capítulo.

Teorema 4.37. Sea $\Gamma$ un subgrupo de Difeo ${ }_{+}^{1+\tau}\left(\left[0,1[)\right.\right.$ isomorfo a $\mathbb{Z}^{d}$, con $\tau>1 / d$ y $d \geq 2$. Si la restricción a ]0,1[ de la acción de $\Gamma$ es libre, entonces ella es minimal y topológicamente conjugada a la acción de un grupo de traslaciones.

Demostración. Una vez más, daremos la prueba completa sólo para el caso $d=2$. Sean $f_{1}$ y $f_{2}$ los generadores de un grupo $\Gamma \sim \mathbb{Z}^{2}$ de difeomorfismos de clase $\mathrm{C}^{1+\tau}$ de $[0,1$ [ que actúa libremente sobre ]0,1[. Cambiando dichos elementos por sus inversos si es necesario, podemos suponer que contractan topológicamente hacia el origen. Supongamos que la acción de $\Gamma$ sobre ] $0,1[$ no sea conjugada a una acción por traslaciones. En tal caso, un argumento simple de control de distorsión hiperbólica muestra que todos los elementos de $\Gamma$ son tangentes a la identidad en el origen. En efecto, supongamos por contradicción que existan intervalo errantes y un elemento $f \in \Gamma$ tal que $f^{\prime}(0)<1$. Fijemos $\lambda<1$ y $\left.c \in\right] 0,1\left[\right.$ tales que $f^{\prime}(x) \leq \lambda$ para todo $x \in[0, c$ [, y fijemos un intervalo errante abierto y maximal $I=] a, b[$ contenido en $] 0, c[$. Si designamos por $L$ el intervalo $[f(b), b]$ entonces

$$
\sum_{n \geq 0}\left|f^{n}(L)\right|^{\tau} \leq|L|^{\tau} \sum_{n \geq 0} \lambda^{n \tau}=\frac{|L|^{\tau}}{1-\lambda^{\tau}}=\bar{S} .
$$

En consecuencia, $\left(f^{n}\right)^{\prime}(x) /\left(f^{n}\right)^{\prime}(y) \leq \exp (C \bar{S})$ para todo $x, y$ en $L$, donde $C>0$ es una constante de $\tau$-hölderianidad para $\log \left(f^{\prime}\right)$ sobre $[0, c]$. Esta última estimación permite aplicar los argumentos de la prueba de la proposi- 
ción 4.2 , llegando así a una contradicción ${ }^{2}$.

Identifiquemos ahora el semigrupo $\Gamma^{+}$generado por $f_{1}$ y $f_{2}$ a $\mathbb{N}_{0} \times \mathbb{N}_{0}$, y consideremos una vez más nuestro proceso markoviano sobre él. Si $\tau>1 / 2$ entonces la prueba del lema 4.31 muestra la finitud de la esperanza de la función

$$
\omega \mapsto \ell_{\tau}(\omega)=\sum_{k \geq 0}\left|h_{k}(\omega)(I)\right|^{\tau} .
$$

Escojamos una constante $S>0$ lo suficientemente grande de modo que el conjunto $\Omega(S)=\left\{\omega \in \Omega: \ell_{\tau}(\omega) \leq S\right\}$ tenga probabilidad positiva, y hagamos $\bar{\ell}=|I| / \exp \left(2^{\tau} C S\right)$. La primera parte de la prueba del lema 3.16 muestra que si $I^{\prime \prime}$ designa al intervalo adyacente por la izquierda a $I$ de longitud $\bar{\ell}$, entonces para todo $x$ e $y$ pertenecientes a $J=\bar{I}^{\prime \prime} \cup \bar{I}$, todo $\omega \in \Omega(S)$ y todo $n \in \mathbb{N}$,

$$
\frac{h_{n}(\omega)^{\prime}(x)}{h_{n}(\omega)^{\prime}(y)} \leq \exp \left(2^{\tau} C S\right) .
$$

Como el intervalo $I$ no está contenido en ningún otro intervalo abierto y errante, existe $h \in \Gamma$ tal que $h(I) \subset I^{\prime \prime}$ y $|h(I)|<|I| \exp \left(-2^{\tau} C S\right)$. Fijemos un punto arbitrario $y$ de $I$. Puesto que $h^{\prime}(0)=1$ y que $h_{n}(\omega)(y)$ tiende al origen para todo $\omega \in \Omega(S)$, a partir de la igualdad

$$
h^{\prime}(y)=\frac{h_{n}(\omega)^{\prime}(y)}{h_{n}(\omega)^{\prime}(h(y))} h^{\prime}\left(h_{n}(\omega)(y)\right)
$$

y de (4.21) se deduce que $h^{\prime}(y) \geq \exp \left(-2^{\tau} C S\right)$. Integrando esta última desigualdad obtenemos $|h(I)| \geq|I| \exp \left(-2^{\tau} C S\right)$, lo cual está en contradicción con la elección de $h$.

Observación 4.38. Sean $f_{1}, \ldots, f_{d}$ los generadores de un grupo $\Gamma \sim \mathbb{Z}^{d}$ actuando libremente por homeomorfismos de ]0,1[ de modo que la acción no es conjugada a una acción por traslaciones. Tras identificar los puntos de las órbitas de $f_{1}$, las transformaciones $f_{2}, \ldots, f_{d}$ devienen los generadores de un grupo abeliano de rango $d-1$ que actúa libremente por homeomorfismos del círculo y que no es conjugado a un grupo de rotaciones: el teorema 4.30 implica entonces que los $f_{i}$ no pueden ser todos de clase $\mathrm{C}^{1 /(d-1)+\varepsilon}$. Notemos que este argumento sólo utiliza la regularidad de los $f_{i}$ al interior; en tal contexto, la obstrucción aparece sólo en clase $\mathrm{C}^{1 /(d-1)+\varepsilon}$. Sin embargo, de acuerdo al teorema 4.37, para difeomorfismos del intervalo $\left[0,1\left[\right.\right.$ la obstrucción ya aparace en clase $\mathrm{C}^{1 / d+\varepsilon}$. Obviamente, la diferencia radica en la diferenciabilidad de las transformaciones en el origen, la cual de hecho juega un rol primordial a lo largo de la demostración de dicho teorema.

Ejercicio 4.39. Extienda el teorema 4.37 para subgrupos de $\operatorname{Difeo}_{+}^{1+\tau}([0,1[)$ que son semiconjugados (sobre $] 0,1[$ ) a grupos de traslaciones pero no actúan libremente en el interior (compare con los ejercicios 4.33 y 4.36).

\footnotetext{
${ }^{2}$ Observemos que en esta parte de la prueba sólo hemos utilizado la hipótesis $\tau>0$. Para explicar esto recordemos que el teorema de linearización de Sternberg sigue siendo válido en clase $\mathrm{C}^{1+\tau}$ (vea la observación 3.75). Ahora bien, como el centralizador de un germen lineal no trivial es el grupo a un parámetro de gérmenes lineales, esto impide la existencia de intervalos errantes para la dinámica en consideración.
} 


\section{Grupos nilpotentes de difeomorfismos}

\subsection{Los teoremas de Plante y Thurston}

Dado un grupo nilpotente $\Gamma$, denotemos

$$
\{i d\}=\Gamma_{k}^{\mathrm{nil}} \triangleleft \Gamma_{k-1}^{\mathrm{nil}} \triangleleft \ldots \triangleleft \Gamma_{1}^{\mathrm{nil}} \triangleleft \Gamma_{0}^{\mathrm{nil}}=\Gamma
$$

la serie central de $\Gamma$, es decir, $\Gamma_{i+1}^{\text {nil }}=\left[\Gamma, \Gamma_{i}^{\text {nil }}\right]$ y $\Gamma_{k-1}^{\text {nil }} \neq\{i d\}$. Notemos que $\Gamma_{k-1}^{\text {nil }}$ es un subgrupo normal contenido en el centro de $\Gamma$. El resultado siguiente corresponde a (una versión muy particular de) un teorema debido a Plante y Thurston $[167,168]$.

Teorema 4.40. Todo subgrupo nilpotente de Difeo ${ }_{+}^{1+\mathrm{va}}([0,1[)$ es abeliano.

Demostración. Sin pérdida de generalidad, podemos suponer que ]0,1[ es una componente irreducible para la acción de $\Gamma$. Probaremos que la restricción de $\Gamma$ a ]0,1[ es libre, lo cual permite concluir la demostración gracias al teorema de Hölder. Supongamos por contradicción que existe $f$ en $\Gamma$ cuyo conjunto de puntos fijos tiene frontera no vacía en $] 0,1[$, y denotemos por $\left.x_{0} \in\right] 0,1[$ a uno de los puntos de dicha frontera. Fijemos un elemento no trivial $g$ perteneciente al centro de $\Gamma$. Afirmamos que $g\left(x_{0}\right)=x_{0}$. En efecto, en caso contrario, módulo reemplazar $g$ por su inversa se tiene $g\left(x_{0}\right)<x_{0}$. Definamos

$$
a^{\prime}=\lim _{n \rightarrow+\infty} g^{n}\left(x_{0}\right), \quad b^{\prime}=\lim _{n \rightarrow-\infty} g^{n}\left(x_{0}\right) .
$$

Observemos que $\left[a^{\prime}, b^{\prime}[\subset[0,1[\right.$. Además, la restricción de $g$ al intervalo ]$a^{\prime}, b^{\prime}\left[\right.$ no posee puntos fijos. Como $f$ y $g$ conmutan, cada $g^{n}\left(x_{0}\right)$ es un punto fijo de $f$, por lo que $f$ fija $a^{\prime}$ y $b^{\prime}$. Obtenemos entonces una contradicción al aplicar el lema de Kopell a las restricciones de $f$ y $g$ al intervalo $\left[a^{\prime}, b^{\prime}[\right.$, Luego, $g\left(x_{0}\right)=x_{0}$.

Como $g$ era no trivial y $g\left(x_{0}\right)=x_{0}$, la frontera en $] 0,1[$ del conjunto de los puntos fijos de $g$ es no vacía. Fijemos un punto $\left.x_{1} \in\right] 0,1[$ en dicha frontera. Sea $h$ un elemento arbitrario en $\Gamma$. Puesto que $g\left(x_{1}\right)=x_{1}$ y $g h=h g$, el mismo argumento utilizado anteriormente prueba que $h\left(x_{1}\right)=x_{1}$. Por lo tanto, los intervalos $\left[a^{\prime}, x_{1}\left[\mathrm{y}\left[x_{1}, b^{\prime}\right.\right.\right.$ [ son invariantes por $\Gamma$, y esto contradice el hecho que $] 0,1[$ es una componente irreducible.

Ejercicio 4.41. Pruebe que el teorema 4.40 vale también para grupos nilpotentes de gérmenes de difeomorfismos unidimensionales de clase $\mathrm{C}^{1+\mathrm{va}}$.

Consideremos ahora el caso del círculo. Si $\Gamma$ es un subgrupo nilpotente de $\operatorname{Difeo}_{+}^{1+\mathrm{va}}\left(\mathrm{S}^{1}\right)$ entonces, por ser promediable, preserva una medida de probabilidad $\mu$ sobre $\mathrm{S}^{1}$. La función número de rotación $g \mapsto \rho(g)$ es en tal caso un homomorfismo de grupos entre $\Gamma$ y $\mathbb{T}^{1}$ (vea la sección 2.2 del capítulo 2). Si el número de rotación de un elemento $g$ de $\Gamma$ es irracional, 
entonces $\mu$ es conjugada a la medida de Lebesgue. El grupo $\Gamma$ es por lo tanto conjugado a un grupo de rotaciones; en particular, $\Gamma$ es abeliano.

Supongamos ahora que el número de rotación de cada elemento $g$ de $\Gamma$ sea racional. En tal caso, el soporte de $\mu$ está contenido en la intersección del conjunto de los puntos periódicos de dichos elementos. Si $\Gamma$ no fuese abeliano entonces es fácil verificar la existencia de elementos $f$ y $g$ en $\Gamma$ tales que $h=[f, g]$ es un elemento no trivial del centro de $\Gamma$. Notemos que $\rho(h)=0$, por lo que $h$ possee puntos fijos. De la igualdad $f g f^{-1} g^{-1}=h$ se obtiene $f g f^{-1}=h g$, por lo que $f^{n} g f^{-n}=h^{n} g$ para todo $n \in \mathbb{N}$. Se tiene entonces $g f^{-n} g^{-1}=f^{-n} h^{n}$, y conjugando sucesivamente por $g$ se comprueba que $g^{m} f^{-n} g^{-m}=f^{-n} h^{m n}$ para todo $m \in \mathbb{N}$. A partir de esto se concluye que

$$
h^{m n}=f^{n} g^{m} f^{-n} g^{-m} \text {. }
$$

Sean $x_{0}$ un punto del soporte de $\mu$ y $m, n$ enteros positivos tales que $f^{m}\left(x_{0}\right)=g^{n}\left(x_{0}\right)=x_{0}$. Notemos que $h\left(x_{0}\right)=x_{0}$. Consideremos la restricción al intervalo $\left[x_{0}, x_{0}+1\right.$ [ del grupo generado por $f^{n}, g^{m}$ y $h$. Como este grupo es nilpotente, dicha restricción debe ser abeliana, por lo que de (4.22) se concluye que $h^{m n}$ es la identidad sobre $\left[x_{0}, x_{0}+1[\right.$. Se concluye así que $h$ es la identidad, lo cual es una contradicción. Hemos probado entonces el resultado siguiente.

Teorema 4.42. Todo subgrupo nilpotente de $\operatorname{Difeo}_{+}^{1+\mathrm{va}}\left(\mathrm{S}^{1}\right)$ es abeliano.

Finalmente, para el caso de la recta vale el siguiente resultado (recuerde que un grupo $\Gamma$ es metabeliano si su primer grupo derivado $\Gamma^{\prime}=[\Gamma, \Gamma]$ es abeliano).

Teorema 4.43. Todo subgrupo nilpotente de $\operatorname{Difeo}_{+}^{1+\mathrm{va}}(\mathbb{R})$ es metabeliano.

Este teorema es una consecuencia directa del corolario 2.49 y de la proposición siguiente.

Proposición 4.44. Sea $\Gamma$ un subgrupo nilpotente de $\operatorname{Difeo}_{+}^{1+v a}(\mathbb{R})$. Si cada elemento de $\Gamma$ fija al menos un punto de la recta, entonces $\Gamma$ es abeliano.

Demostración. Fijemos un elemento no trivial $g$ del centro de $\Gamma$, y sea $b$ un punto de la frontera del conjunto de los puntos fijos de $g$. Afirmamos que $b$ es fijo por todo elemento de $\Gamma$. En efecto, supongamos por contradicción que $f \in \Gamma$ es tal que $f(b) \neq b$. Reemplazando $f$ por $f^{-1}$ si es necesario, podemos suponer que $f(b)<b$. Al menos una de las sucesiones $\left(f^{n}(b)\right)$ o $\left(f^{-n}(b)\right)$ converge a un punto fijo $a \in \mathbb{R}$ de $f$. Siendo ambos casos análogos, supongamos el primero de ellos. Observe que $f^{n}(b)$ es un punto fijo de $g$ para todo $n \in \mathbb{N}$, por lo que $g(a)=a$. Por otra parte, es fácil ver que $f$ no posee puntos fijos en $] a, b\left[\right.$. Si hacemos $a^{\prime}=\lim _{n \rightarrow \infty} f^{-n}(b)$, entonces obtenemos una contradicción al aplicar el lema de Kopell a $f$ y $g$ sobre el intervalo $\left[a, a^{\prime}[\right.$. 
Por lo anterior, cada elemento $f \in \Gamma$ fija $]-\infty, b]$ y $[b, \infty[$. La conclusión de la proposición se obtiene entonces utilizando el teorema 4.40.

Es importante señalar que el teorema 4.43 no significa que el orden de nilpotencia de todo subgrupo nilpotente de $\operatorname{Difeo}_{+}^{1+\text { va }}(\mathbb{R})$ sea menor o igual que 2. De hecho, $\operatorname{Difeo}_{+}^{\infty}(\mathbb{R})$ contiene subgrupos nilpotentes de longitud de nilpotencia arbitraria. En la construcción del siguiente ejemplo (tomado de [63]) se utilizan ideas similares a las de un ejemplo contenido en [166].

Ejemplo 4.45. Consideremos un difeomorfismo $g:[0,1] \rightarrow[0,1]$ de clase $\mathrm{C}^{\infty}$ que fije los extremos del intervalo y sea infinitamente tangente a la identidad en dichos puntos. En otras palabras, $g^{\prime}(0)=g^{\prime}(1)=1$ y todas las derivadas de orden superior en 0 y 1 son nulas. Sea $f$ la traslación de la recta dada por $f(x)=x-1$. Comencemos definiendo el difeomorfismo $h_{0}$ de la recta haciendo $h_{0}(x)=g(x-m)+m$ para $x \in[m, m+1$ [. Consideremos ahora para cada entero $n \geq 1$ una sucesión $(k(n, m))_{m \in \mathbb{Z}}$ satisfaciendo:

(i) $k(1, m)=m$ para todo $m \in \mathbb{Z}$,

(ii) $k(n, 0)=0$ para todo $n \geq 2$,

(iii) $k(n, m)-k(n, m-1)=k(n-1, m)$.

Es fácil ver que estas propiedades determinan unívocamente el valor de $k(n, m)$. Por ejemplo, si $m \geq 0$ entonces $k(2, m)=m(m+1) / 2$. Definamos ahora el difeomorfismo $h_{n}$ de la recta haciendo

$$
h_{n}(x)=g^{k(n, m)}(x-m)+m \text { para } x \in[m, m+1[.
$$

Dejamos al lector la tarea de verificar que los $h_{n}$ conmutan entre ellos. Además, la propiedad (iii) implica que $\left[f, h_{n}\right]=f^{-1} h_{n}^{-1} f h_{n}=h_{n-1}$ para todo $n \geq 1$, mientras que $\left[f, h_{0}\right]=I d$. A partir de ello se deduce fácilmente que el grupo $\Gamma_{n}$ generado por $f$ y $h_{0}, \ldots, h_{n}$ es nilpotente de grado de nilpotencia igual a $n+1$.

Ejercicio 4.46. Usando el teorema 4.43, pruebe directamente la siguiente versión débil del teorema 2.32: para $n \geq 4$, toda acción de un subgrupo de índice finito de $\operatorname{SL}(n, \mathbb{Z})$ por difeomorfismos y de clase $\mathrm{C}^{1+\text { va }}$ de la recta es trivial.

\subsection{Crecimiento de grupos de difeomorfismos}

Un enfoque más moderno del teorema de Plante y Thurston es aquél centrado en la noción de crecimiento de un grupo. Recuerde que dado un grupo provisto de un sistema finito de generadores, la función de crecimiento es aquélla que asigna, a cada entero positivo $n$, el número de elementos del grupo que pueden ser escritos como producto de no más de $n$ generadores o sus inversos. Se dice que el grupo tiene crecimiento polinomial, exponencial o intermedio, si su función de crecimiento tiene el comportamiento asintótico correspondiente. Estas nociones no dependen de la elección del sistema (finito) de generadores.

Ejercicio 4.47. Pruebe que si un grupo contiene un semigrupo libre a dos generadores, entonces dicho grupo tiene crecimiento exponencial.

Observación. Existen grupos de crecimiento exponencial sin semigrupos libres a dos generadores: vea por ejemplo [159]. 
Ejemplo 4.48. La construcción de grupos de crecimiento intermedio es un tópico apasionante que dista mucho de ser sencillo. Los primeros ejemplos fueron introducidos por Grigorchuk en [84]. Uno de ellos (a saber, el primer grupo de Grigorchuk $\hat{\mathrm{H}}$ ) puede ser pensado ya sea como un grupo de automorfismos del árbol binario enraizado $\mathcal{T}_{2}$, o equivalentemente como un grupo de isometrías del conjunto de Cantor $\{0,1\}^{\mathbb{N}}$ (observe que el borde al infinito de $\mathcal{T}_{2}$ se identifica con $\left.\{0,1\}^{\mathbb{N}}\right)$. Para referencia futura, presentamos la definición a continuación; para mayores detalles, recomendamos la lectura del último capítulo de [90].

Conveniendo en que $\left(x_{1},\left(x_{2}, x_{3}, \ldots\right)\right)=\left(x_{1}, x_{2}, x_{3}, \ldots\right)$ para $x_{i} \in\{0,1\}$, los generadores de $\hat{\mathrm{H}}$ son elementos $\hat{a}, \hat{b}, \hat{c}$ y $\hat{d}$ cuya acción sobre $\{0,1\}^{\mathbb{N}}$ viene definida inductivamente por $\hat{a}\left(x_{1}, x_{2}, x_{3}, \ldots\right)=\left(1-x_{1}, x_{2}, x_{3}, \ldots\right)$ y

$$
\begin{aligned}
& \hat{b}\left(x_{1}, x_{2}, x_{3}, \ldots\right)= \begin{cases}\left(x_{1}, \hat{a}\left(x_{2}, x_{3}, \ldots\right)\right), x_{1}=0, \\
\left(x_{1}, \hat{c}\left(x_{2}, x_{3}, \ldots\right)\right), x_{1}=1,\end{cases} \\
& \hat{c}\left(x_{1}, x_{2}, x_{3}, \ldots\right)= \begin{cases}\left(x_{1}, \hat{a}\left(x_{2}, x_{3}, \ldots\right)\right), & x_{1}=0, \\
\left(x_{1}, \hat{d}\left(x_{2}, x_{3}, \ldots\right)\right), & x_{1}=1,\end{cases} \\
& \hat{d}\left(x_{1}, x_{2}, x_{3}, \ldots\right)= \begin{cases}\left(x_{1}, x_{2}, x_{3}, \ldots\right), & x_{1}=0, \\
\left(x_{1}, \hat{b}\left(x_{2}, x_{3}, \ldots\right)\right), & x_{1}=1 .\end{cases}
\end{aligned}
$$

De esta forma, la acción sobre $\mathcal{T}_{2}$ del elemento $\hat{a} \in \hat{\mathrm{H}}$ consiste en permutar las dos primeras aristas (conjuntamente con los árboles enraizados en los vértices finales de ellos). Los elementos $\hat{b}, \hat{c}$ y $\hat{d}$ fijan estas dos primeras aristas, y sus acciones sobre los niveles superiores de $\mathcal{T}_{2}$ quedan ilustrados en la siguiente figura.
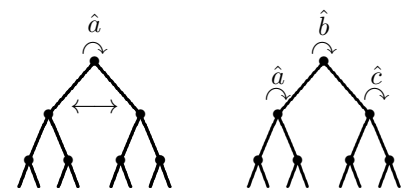
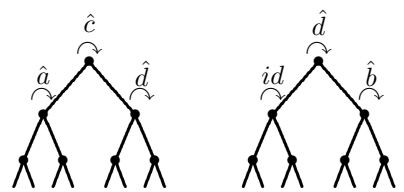

Figura 22

Un célebre teorema de Gromov estipula que un grupo es virtualmente nilpotente si y sólo si su crecimiento es polinomial. Debido a esto y al ejercicio 4.15, el teorema de Plante y Thurston puede ser reformulado diciendo que todo subgrupo de crecimiento polinomial de $\mathrm{Difeo}_{+}^{1+\mathrm{va}}([0,1[)$ es abeliano (ésta era de hecho la versión original del teorema, demostrada previamente a la aparición del trabajo de Gromov; vea el ejercicio 4.54). Siguiendo [142], en lo que sigue extenderemos este hecho para grupos de crecimiento subexponencial, y de manera más general para grupos sin semigrupos libres a dos generadores.

Teorema 4.49. Todo subgrupo finitamente generado de Difeo ${ }_{+}^{1+\mathrm{va}}([0,1[)$ sin semigrupos libres a dos generadores es abeliano. 
Ejemplo 4.50. El producto en corona $\Gamma=\mathbb{Z} \imath \mathbb{Z}=\mathbb{Z} \ltimes \oplus_{\mathbb{Z}} \mathbb{Z}$ actúa de manera natural sobre el intervalo: basta identificar uno de los generadores canónicos de $\Gamma$ con un homeomorfismo $f$ de $[0,1]$ satisfaciendo $f(x)<x$ para todo $x \in] 0,1$, y el otro generador con un homeomorfismo $g$ satisfaciendo $g(x) \neq x$ para todo $x \in] f(a), a[$ y $g(x)=x$ para todo $x \in[0,1] \backslash[f(a), a]$, donde $a$ es un punto de ] 0,1 . Esta acción puede ser suavizada hasta alcanzar la clase $\mathrm{C}^{\infty}$ (vea el ejemplo 4.65 para mayores detalles sobre esta construcción). Observe que $\Gamma$ es un grupo metabeliano que no es virtualmente nilpotente. De acuerdo a un resultado clásico de Rosenblatt [178] (el cual generaliza resultados previos de Milnor [134] y Wolf [209]; vea también [18]), todo grupo soluble que no es virtualmente nilpotente contiene semigrupos libres a dos generadores. Por lo tanto, $\Gamma$ contiene tales semigrupos. El lector debiera tratar de probar que de hecho $f$ y $g$ generan un semigrupo libre analizando su acción sobre el intervalo.

Para probar el teorema 4.49 necesitaremos una versión del lema de Kopell que no utilice la conmutatividad de las aplicaciones de manera muy fuerte. Esta versión será también esencial para el estudio de los grupos solubles de difeomorfismos del intervalo de la sección 3 de este capítulo. La demostración que nosotros presentamos ha sido tomada de [39].

Lema 4.51. Sean $h_{1} y h_{2}$ dos difeomorfismos del intervalo $[0,1[$ sobre sus imágenes que fijan el origen. Supongamos que $h_{1}$ sea de clase $\mathrm{C}^{1+\mathrm{va}} y$ $h_{2}$ de clase $\mathrm{C}^{1}$. Supongamos también que $h_{1}(x)<x$ para todo $\left.x \in\right] 0,1[$, que $h_{2}\left(x_{0}\right)=x_{0}$ para cierto $\left.x_{0} \in\right] 0,1[$, y que para cada $n \in \mathbb{N}$ el punto $x_{n}=h_{1}^{n}\left(x_{0}\right)$ sea fijo por $h_{2}$. Supongamos finalmente que $h_{2}(y) \geq z>y$ (resp. $h_{2}(y) \leq z<y$ ) para ciertos $y, z$ en $] x_{1}, x_{0}[$. Entonces existe $N \in \mathbb{N}$ tal que $h_{2}\left(h_{1}^{n}(y)\right)<h_{1}^{n}(z)$ (resp. $\left.h_{2}\left(h_{1}^{n}(y)\right)>h_{1}^{n}(z)\right)$ para todo $n \geq N$.

Demostración. Observemos que la sucesión $\left(x_{n}\right)$ tiende a cero cuando $n \in \mathbb{N}$ tiende al infinito. Sea $\delta=V\left(h_{1} ;\left[0, x_{0}\right]\right)$. Para todo $u, v$ en $\left[x_{1}, x_{0}\right]$ y todo $n \in \mathbb{N}$ se tiene (compare con la desigualdad (4.2))

$$
\left|\log \left(\frac{\left(h_{1}^{n}\right)^{\prime}(u)}{\left(h_{1}^{n}\right)^{\prime}(v)}\right)\right| \leq \delta .
$$

Puesto que $h_{2}$ fija cada punto $x_{n}$, se tiene necesariamente $h_{2}^{\prime}(0)=1$. Supongamos que la afirmación del lema no sea satisfecha para ciertos $y, z$ en $] x_{1}, x_{0}[$, y sea $\kappa$ una constante tal que

$$
1<\kappa<1+\frac{z-y}{e^{\delta}\left(y-x_{1}\right)}
$$

Fijemos $N_{0} \in \mathbb{N}$ suficientemente grande de manera que

$$
h_{2}^{\prime}(w) \leq \kappa \quad \text { y } \quad\left(h_{2}^{-1}\right)^{\prime}(w) \leq \kappa \text { para todo } w \in\left[x_{N+1}, x_{N}\right] .
$$

Para cierto $n \geq N_{0}$ tenemos $h_{2}\left(h_{1}^{n}(y)\right) \geq h_{1}^{n}(z)$ o bien $h_{2}\left(h_{1}^{n}(z)\right) \leq h_{1}^{n}(y)$. Consideraremos sólo el primer caso, pues el segundo puede ser reducido a 
éste cambiando $h_{2}$ por $h_{2}^{-1}$. Definamos $y_{n}=h_{1}^{n}(y)$ y $z_{n}=h_{1}^{n}(z)$. Por el teorema del valor medio, existen puntos $u, v$ en $\left[x_{1}, x_{0}\right]$ tales que

$$
\frac{h_{2}\left(y_{n}\right)-y_{n}}{y_{n}-x_{n+1}} \geq \frac{z_{n}-y_{n}}{y_{n}-x_{n+1}}=\frac{\left(h_{1}^{n}\right)^{\prime}(u) \cdot(z-y)}{\left(h_{1}^{n}\right)^{\prime}(v) \cdot\left(y-x_{1}\right)} .
$$

De la desigualdad (4.23) se concluye

$$
\frac{h_{2}\left(y_{n}\right)-y_{n}}{y_{n}-x_{n+1}} \geq \frac{z-y}{e^{\delta}\left(y-x_{1}\right)}>\kappa-1 .
$$

Se deduce así que, para cierto $w \in\left[x_{n+1}, y_{n}\right]$,

$$
h_{2}^{\prime}(w)=\frac{h_{2}\left(y_{n}\right)-h_{2}\left(x_{n+1}\right)}{y_{n}-x_{n+1}}=\frac{h_{2}\left(y_{n}\right)-x_{n+1}}{y_{n}-x_{n+1}}>\kappa,
$$

lo cual contradice (4.24).

En la sección 2.5 del capítulo 2 ya introdujimos un criterio simple (dado por el lema 2.53) para detectar semigrupos libres de grupos de homeomorfismos del intervalo. Presentamos ahora otro criterio para obtener semigrupos libres de Difeo ${ }_{+}^{1+\text { va }}([0,1[)$, el cual utiliza de manera esencial la hipótesis de diferenciabilidad y será fundamental para la demostración del teorema 4.49. El ejemplo 4.50 permite entender la naturalidad del lema a seguir.

Lema 4.52. Sean $f$ y $g$ elementos de Homeo $+([0,1[)$ tales que $f(x)<x$ para todo $x \in] 0,1[$. Supongamos que exista un intervalo $[a, b]$ contenido en ]0, $1[$ tal que $g(x)<x$ para todo $x \in] a, b[, g(a)=a, g(b)=b$ y $f(b) \leq a$. Sea $[y, z] \subset] a, b[$ un intervalo tal que $g(y) \geq z$ o bien $g(z) \leq y$. Supongamos que $g$ fije todos los intervalos $f^{n}([a, b])$, y que exista $N_{0} \in \mathbb{N}$ tal que g posee puntos fijos dentro de $f^{n}(] a, b[)$ para todo $n \geq N_{0}$. Si el grupo generado por $f$ y $g$ no tiene elementos entrecruzados, entonces el semigrupo generado por dichos elementos es libre.

Demostración. Cambiando $[a, b]$ por alguna de sus imágenes bajo un iterado positivo de $f$, podemos asumir que $g$ no tiene puntos fijos en $] a, b[$, pero que sí los posee sobre cada intervalo $f^{n}(] a, b[)$ para $n \geq 1$. Consideremos dos palabras distintas en potencias positivas de $f$ y $g$, y tratemos de verificar que ellas representan homeomorfismos diferentes. Módulo conjugación, podemos suponer que dichas palabras son de la forma $P_{1}=f^{n} g^{m_{r}} f^{n_{r}} \cdots g^{m_{1}} f^{n_{1}} \quad$ y $P_{2}=g^{q} f^{p_{s}} g^{q_{s}} \cdots f^{p_{1}} g^{q_{1}}$, donde $m_{j}, n_{j}$, $p_{j}, q_{j}$ son enteros positivos, $n \geq 0$ y $q \geq 0$ (con $n>0$ si $r=0$, y con $p>0$ si $s=0$ ). Como el grupo generado por $f$ y $g$ no posee elementos entrecruzados, de acuerdo al teorema 2.54 dicho grupo debe preservar una medida de Radon $v$ sobre $] 0,1[$. Notemos ahora que

$$
\tau_{v}\left(P_{1}\right)=\left(n_{1}+\ldots+n_{r}+n\right) \tau_{v}(f) \quad \text { y } \quad \tau_{v}\left(P_{2}\right)=\left(p_{1}+\ldots+p_{s}\right) \tau_{v}(f) .
$$


Como $\tau_{v}(f) \neq 0$, si los valores de $\left(n_{1}+\ldots+n_{r}+n\right)$ y $\left(p_{1}+\ldots+p_{s}\right)$ son diferentes entonces $P_{1} \neq P_{2}$. Asumamos ahora que dichos valores coinciden con algún $N \in \mathbb{N}$. Tenemos entonces

$$
\begin{aligned}
& f^{-N} P_{1}=f^{-N} f^{n} g^{m_{r}} f^{n_{r}} \cdots g^{m_{1}} f^{n_{1}} \\
& =f^{-N} f^{n} g^{m_{r}} f^{n_{r}} \cdots g^{m_{2}} f^{n_{1}+n_{2}}\left(f^{-n_{1}} g^{m_{1}} f^{n_{1}}\right) \\
& =f^{-N} f^{n} g^{m_{r}} f^{n_{r}} \cdots g^{m_{3}} f^{n_{1}+n_{2}+n_{3}}\left(f^{-\left(n_{1}+n_{2}\right)} g^{m_{2}} f^{n_{1}+n_{2}}\right)\left(f^{-n_{1}} g^{m_{1}} f^{n_{1}}\right) \\
& =\left(f^{-(N-n)} g^{m_{r}} f^{N-n}\right) \cdots\left(f^{-\left(n_{1}+n_{2}\right)} g^{m_{2}} f^{n_{1}+n_{2}}\right)\left(f^{-n_{1}} g^{m_{1}} f^{n_{1}}\right) \\
& f^{-N} P_{2}=f^{-N} g^{q} f^{p_{s}} g^{q_{s}} \ldots f^{p_{1}} g^{q_{1}} \\
& =f^{-N} g^{q} f^{p_{s}} g^{q_{s}} \cdots f^{p_{3}} g^{q_{3}} f^{p_{1}+p_{2}}\left(f^{-p_{1}} g^{q_{2}} f^{p_{1}}\right) g^{q_{1}} \\
& =\left(f^{-N} g^{q} f^{N}\right) \cdots\left(f^{-p_{1}} g^{q_{2}} f^{p_{1}}\right) g^{q_{1}} .
\end{aligned}
$$

Como el grupo generado por $f$ y $g$ no posee elementos entrecruzados, y como cada aplicación $\left(f^{-(N-n)} g^{m_{r}} f^{N-n}\right), \ldots,\left(f^{-\left(n_{1}+n_{2}\right)} g^{m_{2}} f^{n_{1}+n_{2}}\right)$, $\left(f^{-n_{1}} g^{m_{1}} f^{n_{1}}\right)$ y $\left(f^{-N} g^{q} f^{N}\right), \ldots,\left(f^{-p_{1}} g^{q_{2}} f^{p_{1}}\right)$ tiene puntos fijos dentro de $] a, b[$, ellas deben fijar $] a, b\left[\right.$. Por otro lado, $g^{q_{1}}$ fija el intervalo $] a, b[$, pero carece de puntos fijos en su interior. Por lo tanto, si $\bar{v}$ es una medida de Radon sobre $] a, b[$ que es invariante por el grupo generado por (las restricciones a $] a, b\left[\right.$ de) todas estas aplicaciones (incluyendo a $g^{q_{1}}$ ), entonces $\tau_{\bar{v}}\left(f^{-N} P_{1}\right)=0$ y $\tau_{\bar{v}}\left(f^{-N} P_{2}\right)=\tau_{\bar{v}}\left(g^{q_{1}}\right) \neq 0$, lo cual muestra que $f^{-N} P_{1} \neq f^{-N} P_{2}$, y por lo tanto $P_{1} \neq P_{2}$.

Podemos abordar ahora la demostración del teorema 4.49. Sea entonces $\Gamma$ un subgrupo finitamente generado de Difeo ${ }_{+}^{1+v a}([0,1[)$ sin semigrupos libres a dos generadores. Para probar que $\Gamma$ es abeliano, sin perder en generalidad podemos suponer que $\Gamma$ no admite punto fijo global en $] 0,1[$. Por el teorema 2.54, $\Gamma$ preserva una medida de Radon $v$ sobre $] 0,1[$; además, la prueba de dicho teorema nos da la existencia de un elemento $f \in \Gamma$ tal que $f(x)<x$ para todo $x \in \mathbb{R}$. Sea $\Lambda$ el conjunto de los puntos de $] 0,1[$ que son globalmente fijos por la acción del grupo derivado $\Gamma^{\prime}=[\Gamma, \Gamma]$. El conjunto $\Lambda$ es no vacío, pues contiene al soporte de $v$. Si $\Lambda$ coincide con $] 0,1[$ entonces $\Gamma$ es abeliano. Supongamos ahora que $\Lambda$ esté estrictamente contenido en ]0,1[ y que la restricción de $\Gamma^{\prime}$ a cada una de las componentes conexas de ] $0,1\left[\backslash \Lambda\right.$ sea libre. Por el teorema de Hölder, la restricción de $\Gamma^{\prime}$ a cada una de esas componentes conexas es abeliana, por lo que $\Gamma$ es metabeliano. Por el teorema de Rosenblatt $[18,178], \Gamma$ es virtualmente nilpotente, y por el teorema de Plante y Thurston, $\Gamma$ es virtualmente abeliano. Finalmente, del ejercicio 4.15 se concluye que, en este caso, $\Gamma$ es abeliano.

Resta el caso en que la acción de $\Gamma^{\prime}$ sobre alguna componente conexa I del complemento de $\Lambda$ no es libre. La demostración del teorema 4.49 
quedará concluida al verificar que, bajo estas condiciones, $\Gamma$ contiene semigrupos libres a dos generadores. Para ello fijemos un elemento $h \in \Gamma^{\prime}$ y un intervalo ] $y, z$ [ estrictamente contenido en $I$ de modo que $h$ fije ] $y, z$ [ pero ningún punto dentro de $] y, z[$ sea fijo por $h$. Afirmamos que debe existir un elemento $g \in \Gamma^{\prime}$ que envía ] $y, z$ [ en un intervalo disjunto (contenido en $I)$. En efecto, si éste no fuera el caso entonces, como $\Gamma$ no tiene elementos entrecruzados, todo elemento de $\Gamma^{\prime}$ tendría que fijar ]y, $z$, por lo que los puntos y y $z$ estarían contenidos en $\Lambda$, contradiciendo el hecho que $] y, z[$ está estrictamente contenido en la componente conexa $I$ de $] 0,1[\backslash \Lambda$.

El elemento $g \in \Gamma^{\prime}$ fija la componente conexa $f^{n}(I)$ de $] 0,1[\backslash \Lambda$ para todo $n \geq 0$. Además, como $\Gamma$ no posee elementos entrecruzados, para cada $n \geq 0$ los intervalos $f^{n}(] y, z[)$ y $g f^{n}(] y, z[)$ coinciden o son disjuntos. El lema 4.51 (aplicado a $h_{1}=f, h_{2}=g$, con $x_{0}$ siendo la extremidad derecha de $I$ ) implica entonces la existencia de un entero $N_{0} \in \mathbb{N}$ tal que $g$ fija el intervalo $f^{n}(] y, z\left[\right.$ ) para todo $n \geq N_{0}$ (vea la figura 23). Definiendo el intervalo $] a, b[$ como la clausura convexa (abierta) de la unión $\cup_{n \in \mathbb{Z}} g^{n}(] y, z[)$, del lema 4.52 se deduce rápidamente que el semigrupo generado por $f$ y $g$ es libre, lo cual concluye la demostración del teorema 4.49.

Ejercicio 4.53. Generalizando los teoremas 4.42 y 4.43 , pruebe que todo subgrupo de $\mathrm{Difeo}_{+}^{1+\text { va }}\left(\mathrm{S}^{1}\right)$ (resp. de $\operatorname{Difeo}_{+}^{1+\text { va }}(\mathbb{R})$ ) sin semigrupos libres a dos generadores es abeliano (resp. metabeliano).

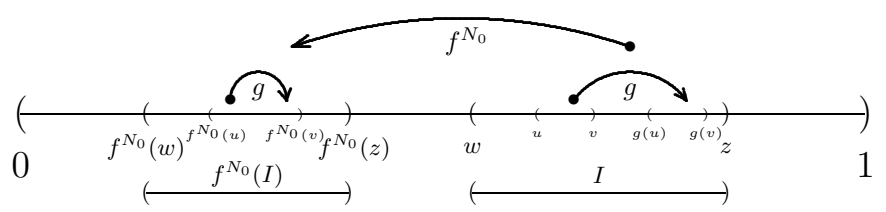

Figura 23

Ejercicio 4.54. A lo largo de este ejercicio, $\Gamma$ será siempre un grupo finitamente generado de crecimiento polinomial de grado $k$.

(i) Pruebe que todo subgrupo finitamente generado de $\Gamma$ tiene crecimiento polinomial de grado inferior o igual a $k$.

(ii) Demuestre que si $\Gamma_{n} \subset \Gamma_{n-1} \subset \ldots \subset \Gamma_{0}=\Gamma$ es una cadena de subgrupos tal que para todo $i \in\{0, \ldots, n-1\}$ existe un homomorfismo $\phi_{i}: \Gamma_{i} \rightarrow(\mathbb{R},+)$ satisfaciendo $\phi_{i}\left(\Gamma_{i+1}\right)=\{0\}$, entonces $n \leq k$.

Sugerencia. Para cada $i \in\{0, \ldots, n-1\}$ escoja un elemento $g_{i} \in \Gamma_{i}$ tal que $\widehat{\phi_{i}\left(g_{i}\right) \neq 0}$ y considere las palabras de la forma $P=g_{1}^{m_{1}} g_{2}^{m_{2}} \cdots g_{n}^{m_{n}}$.

(iii) Suponga que $\Gamma$ sea un subgrupo de $\mathrm{Homeo}_{+}([0,1])$. Usando (ii) y el homomorfismo número de traslación, pruebe que $\Gamma$ es soluble de orden de solubilidad menor o igual a $k$. Aplicando el teorema de Milnor y Wolf [134, 209], deduzca que $\Gamma$ es virtualmente nilpotente.

(iv) Suponga ahora que $\Gamma$ sea un subgrupo de Difeo ${ }_{+}^{1+\text { va }}([0,1[)$. Usando el teorema 4.40 y el ejercicio 4.15 , concluya que $\Gamma$ es abeliano.

(v) Pruebe que (iv) vale aún cuando $\Gamma$ es un subgrupo de $\mathcal{G}_{+}^{1+\text { va }}(\mathbb{R}, 0)$. 


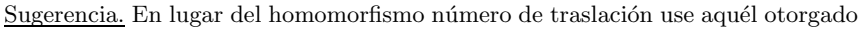
por el teorema de estabilidad de Thurston (vea la sección 1 del último capítulo).

Ejercicio 4.55. La afirmación del ítem (ii) a continuación puede también ser demostrada usando el lema del ping-pong positivo (vea [18]).

(i) Bajo las hipótesis del lema 4.52, pruebe que el grupo generado por la familia de elementos $\left\{f^{n} g f^{-n}: n \in \mathbb{Z}\right\}$ no es finitamente generado.

(ii) Pruebe que si $f$ y $g$ son los generadores de un grupo $\Gamma$ de modo que el subgrupo generado por $\left\{f^{n} g f^{-n}: n \in \mathbb{Z}\right\}$ no es finitamente generado, entonces el semigrupo generado por $f$ y $g f g^{-1}$ es libre.

Sugerencia. Suponga que existen dos palabras diferentes en potencias positivas de $f \mathrm{y} g \mathrm{fg}^{-1}$, y verifique que esto permite hallar un número finito de generadores para el grupo generado por $\left\{f^{n} g f^{-n}: n \in \mathbb{Z}\right\}$.

(iii) Use (ii) para dar una demostración alternativa del teorema 4.49.

Observación 4.56. Ignoramos si el teorema 4.49 se extiende (al menos en el caso de crecimiento subexponencial) para grupos de gérmenes de difeomorfismos de clase $\mathrm{C}^{1+\text { va }}$ (compare con el ejercicio 4.41).

\subsection{Nilpotencia, crecimiento y regularidad intermedia}

Tal como sucede para el teorema de Denjoy y el lema de Kopell, los teoremas de las dos secciones precedentes dejan de ser válidos para grupos de difeomorfismos de regularidad inferior a $\mathrm{C}^{1+l i p}$. En efecto, utilizando la primera de las técnicas de la sección 1.4 de este capítulo, Farb y Franks probaron en [63] que para todo $n \in \mathbb{N}$ el grupo $\mathrm{N}_{n}$ introducido en el ejercicio 2.34 se incrusta en Difeo ${ }_{+}^{1}\left([0,1]\right.$ ) (observe que $\mathrm{N}_{n}$ es nilpotente para todo $n$ y no abeliano para $n \geq 3$ ). Por otra parte, de acuerdo a un importante resultado de Malcev (vea [172]), todo grupo nilpotente finitamente generado y sin torsión se incrusta en algún $\mathrm{N}_{n}$. Todo esto muestra la existencia de una gran variedad de contra-ejemplos de clase $\mathrm{C}^{1}$ al teorema de Plante y Thurston. De hecho, usando los métodos introducidos al final de la sección anteriormente referida, es posible probar que la acción canónica de $N_{n}$ sobre el intervalo puede realizarse con cuaquier clase de diferenciabilidad inferior a $\mathrm{C}^{1+1 /(n-2)}$. Por otro lado, el teorema 4.34 muestra que esta inclusión no puede alcanzar ninguna regularidad superior a $\mathrm{C}^{1+1 /(n-2)}$. Sería interesante dar una versión de este fenómeno que sea independiente del modelo topológico impuesto a la acción. De manera más precisa, es muy probable que un enunciado del siguiente tipo sea cierto: para cada grupo $\Gamma$ nilpotente, finitamente generado y sin torsión, existe una constante $\tau(\Gamma) \in] 0,1]$ tal que si $\Phi: \Gamma \rightarrow \operatorname{Difeo}_{+}^{1+\tau}\left(\mathrm{S}^{1}\right)$ es una representación efectiva y sin punto fijo global al interior, entonces $\tau<\tau(\Gamma)$ (y tal que para todo $\tau<\tau(\Gamma)$ existen representaciones efectivas para las cuales ]0,1[ es una componente irreducible). Sería aún más interesante si el valor de dicha constante pudiese ser explicitado (es probable que éste sea igual a $1 /(k-1)$, donde $k$ es el grado de nilpotencia del grupo en cuestión). 
Observación 4.57. Para cada par de enteros positivos $m<n$, el grupo nilpotente $\mathrm{N}_{m}$ se incrusta canónicamente en $\mathrm{N}_{n}$. Si denotamos por $\mathrm{N}$ a la reunión correspondiente, entonces se comprueba fácilmente que $\mathrm{N}$ es un grupo ordenable, numerable y no finitamente generado. Además, $\mathrm{N}$ contiene a todos los grupos nilpotentes finitamente generados y sin torsión. Sería muy interesante verificar si los métodos de la sección 1.4 de este capítulo permiten probar que $\mathrm{N}$ es isomorfo a un grupo de difeomorfismos de clase $\mathrm{C}^{1}$ del intervalo.

Resumiendo lo anterior, todo grupo nilpotente finitamente generado y sin torsión puede ser incrustado en $\operatorname{Difeo}_{+}^{1+\tau}([0,1])$ para algún $\tau<1$ suficientemente pequeño. Resulta natural preguntarse si otros grupos de crecimiento subexponencial pueden aparecer como grupos de difeomorfismos de clase $\mathrm{C}^{1+\tau}$ del intervalo. De acuerdo al ejercicio 4.47 , el resultado presentado a continuación, el cual fue obtenido por el autor en [142], da una respuesta negativa a esta interrogante.

Teorema 4.58. Para todo $\tau>0$, todo grupo de difeomorfismos de clase $\mathrm{C}^{1+\tau}$ del intervalo [0,1[ que sea finitamente generado y que no contenga semigrupos libres a dos generadores es virtualmente nilpotente.

Observación 4.59. Un enorme interés ha suscitado recientemente una noción más fina de crecimiento para grupos, a saber, la del crecimiento exponencial uniforme. Se dice que un grupo finitamente generado $\Gamma$ tieme crecimiento exponencial uniforme si existe una constante $\lambda>1$ tal que para todo sistema finito (y simétrico) de generadores $\mathcal{G}$ de $\Gamma$, el número $L_{\mathcal{G}}(n)$ de elementos de $\Gamma$ que pueden ser expresados como producto de a lo más $n$ elementos de $\mathcal{G}$ satisface

$$
\lim _{n \rightarrow \infty} \frac{\log \left(L_{\mathcal{G}}(n)\right)}{n} \geq \lambda .
$$

Si bien en muchas situaciones los grupos de crecimiento exponencial tienen automáticamente crecimiento uniformemente exponencial (vea por ejemplo $[18,62$, 161]), existen grupos que no comparten ambas propiedades [206]. Ignoramos si esto puede suceder para un grupo de difeomorfismos de clase $\mathrm{C}^{1+\tau}$ de $[0,1]$.

Una observación análoga puede hacerse en torno a la alternativa de Tits. Un grupo satisface una alternativa de Tits uniforme si todo subgrupo no virtualmente soluble contiene elementos que generan un grupo libre y que pueden ser expresados como un producto de a lo más $N$ generadores del subgrupo, donde $N$ es una constante que sólo depende del grupo original y no del subgrupo en consideración ni del sistema de generadores allí elegido. Por ejemplo, los grupos "lineales" satisfacen la altenativa de Tits uniforme [20]. Ignoramos a este respecto si puede darse una versión uniforme del resultado de la sección 3.1 del capítulo 2. El tratamiento específico de este problema para el caso real-analítico o para el grupo de Thompson $\mathrm{G}$ reviste desde ya un interés no despreciable.

La condición de positividad de $\tau$ para el teorema 4.58 es esencial. Para ilustrar este aspecto, a continuación esbozaremos la construcción de un subgrupo de Difeo ${ }_{+}^{1}([0,1])$ de crecimiento intermedio. Este subgrupo está relacionado con el grupo de crecimiento intermedio $\hat{\mathrm{H}}$ presentado en el ejemplo 4.48. En efecto, si bien no es difícil verificar que $\hat{\mathrm{H}}$ es un grupo 
de torsión para el cual el orden de cada elemento es una potencia de 2 (vea el último capítulo de [90]), es posible generar a partir de él un grupo sin torsión y de crecimiento intermedio. Este grupo fue introducido en [83]. Geométricamente, la idea consiste en reemplazar $\mathcal{T}_{2}$ por un árbol enraizado cuyos vértices tienen valencia infinita (numerable). En términos más precisos, consideramos el grupo $\overline{\mathrm{H}}$ actuando en el espacio $\Omega=\mathbb{Z}^{\mathbb{N}}$ generado por los elementos $\bar{a}, \bar{b}, \bar{c}$ y $\bar{d}$ definidos inductivamente por $\bar{a}\left(x_{1}, x_{2}, x_{3}, \ldots\right)=\left(1+x_{1}, x_{2}, x_{3}, \ldots\right)$ y

$$
\begin{aligned}
& \bar{b}\left(x_{1}, x_{2}, x_{3}, \ldots\right)=\left\{\begin{array}{l}
\left(x_{1}, \bar{a}\left(x_{2}, x_{3}, \ldots\right)\right), x_{1} \text { par }, \\
\left(x_{1}, \bar{c}\left(x_{2}, x_{3}, \ldots\right)\right), x_{1} \text { impar },
\end{array}\right. \\
& \bar{c}\left(x_{1}, x_{2}, x_{3}, \ldots\right)=\left\{\begin{array}{l}
\left(x_{1}, \bar{a}\left(x_{2}, x_{3}, \ldots\right)\right), x_{1} \text { par, } \\
\left(x_{1}, \bar{d}\left(x_{2}, x_{3}, \ldots\right)\right), x_{1} \text { impar },
\end{array}\right. \\
& \bar{d}\left(x_{1}, x_{2}, x_{3}, \ldots\right)= \begin{cases}\left(x_{1}, x_{2}, x_{3}, \ldots\right), & x_{1} \text { par }, \\
\left(x_{1}, \bar{b}\left(x_{2}, x_{3}, \ldots\right)\right), & x_{1} \text { impar. }\end{cases}
\end{aligned}
$$

El grupo $\overline{\mathrm{H}}$ preserva el orden lexicográfico en $\Omega$, de donde se concluye que es ordenable, por lo que puede ser visto como un grupo de homeomorfismos del intervalo (compare con [85]). Para clarificar este punto, daremos a continuación una realización elemental de $\overline{\mathrm{H}}$ como un grupo de homeomorfismos bilipschitzianos de [0,1] (compare con la proposición 2.87).

Ejemplo 4.60. Fijemos una sucesión $\left(\ell_{i}\right)_{i \in \mathbb{Z}}$ de reales positivos tales que

$$
\sum \ell_{i}=1 \quad \text { y } \quad \max \left\{\frac{\ell_{i+1}}{\ell_{i}}, \frac{\ell_{i}}{\ell_{i+1}}\right\} \leq C<\infty \text { para todo } i \in \mathbb{Z} .
$$

Denotemos por $I_{i}$ al intervalo $] \sum_{j<i} \ell_{j}, \sum_{j \leq i} \ell_{j}[$. Sea $f:[0,1] \rightarrow[0,1]$ el homeomorfismo que lleva cada intervalo $I_{i}$ sobre $I_{i+1}$ de manera afín. Designemos por $g$ al homeomorfismo afín que lleva $[0,1]$ sobre $\bar{I}_{0}$, y por $\lambda=1 / \ell_{0}$ al valor (constante) de su derivada. Consideremos las aplicaciones $a, b, c$ y $d$ definidas inductivamente en un subconjunto denso de $[0,1]$ por $a(x)=f(x)$ y, para $x \in I_{i}$,

$$
\begin{aligned}
& b(x)= \begin{cases}f^{i} g a g^{-1} f^{-i}(x), & i \text { par, } \\
f^{i} g c g^{-1} f^{-i}(x), & i \text { impar, }\end{cases} \\
& c(x)= \begin{cases}f^{i} g a g^{-1} f^{-i}(x), & i \text { par, } \\
f^{i} g d g^{-1} f^{-i}(x), & i \text { impar, }\end{cases} \\
& d(x)= \begin{cases}x, & i \text { par, } \\
f^{i} g b g^{-1} f^{-i}(x), & i \text { impar. }\end{cases}
\end{aligned}
$$

Afirmamos que $a, b, c$ y $d$ son homeomorfismos bilipschitzianos con constante de Lipschitz mayorada por $C$ (note que $C$ puede ser tomada tan cercana a 1 como se quiera). En efecto, esto es evidente para $a$, mientras que para $b, c$ y $d$, esto es verificable por inducción. Por ejemplo, si $x \in I_{i}$ para un entero impar $i$, entonces

$$
b^{\prime}(x)=\frac{\left(f^{i}\right)^{\prime}\left(g a g^{-1} f^{-i}(x)\right)}{\left(f^{i}\right)^{\prime}\left(f^{-i}(x)\right)} \cdot \frac{g^{\prime}\left(a g^{-1} f^{-i}(x)\right)}{g^{\prime}\left(g^{-1} f^{-i}(x)\right)} \cdot a^{\prime}\left(g^{-1} f^{-i}(x)\right),
$$

y como $\left.g^{\prime}\right|_{[0,1]} \equiv \lambda$ y $\left.\left(f^{i}\right)^{\prime}\right|_{I_{0}} \equiv \ell_{i} / \ell_{0}$, tenemos $b^{\prime}(x)=a^{\prime}\left(g^{-1} f^{-i}(x)\right) \leq C$. Resulta geométricamente claro que el grupo generado por $a, b, c$ y $d$ es isomorfo a $\overline{\mathrm{H}}$. 
Ejercicio 4.61. Dé ejemplos de grupos (finitamente generados) de homeomorfismos del intervalo y del círculo para los cuales exista un sistema finito de generadores y una constante $\delta>0$ de modo que, para toda conjugación topológica a un grupo de homeomorfismos lipschitzianos, al menos uno de estos generadores (o su inverso) tiene constante de Lipschitz mayor a $1+\delta$.

La idea precedente es inapropiada si se busca obtener una incrustación de $\overline{\mathrm{H}}$ en $\operatorname{Difeo}_{+}^{1}([0,1])$, pues aparecen discontinuidades para las derivadas en cada "nivel" de la acción de H. Daremos entonces otra realización, a lo largo de la cual será esencial el "renormalizar" la geometría en cada paso. Comencemos por fijar cualquier familia equivariante de homeomorfismos $\{\varphi([0, u],[0, v]):[0, u] \rightarrow[0, v] ; u>0, v>0\}$. Para cada $n \in \mathbb{N}$ y cada $\left(x_{1}, \ldots, x_{n}\right) \in \mathbb{Z}^{n}$, consideremos un intervalo cerrado (no reducido a un punto) $I_{x_{1}, \ldots, x_{n}}=\left[u_{x_{1}, \ldots, x_{n}}, w_{x_{1}, \ldots, x_{n}}\right]$ y un intervalo (posiblemente degenerado) $J_{x_{1}, \ldots, x_{n}}=\left[v_{x_{1}, \ldots, x_{n}}, w_{x_{1}, \ldots, x_{n}}\right]$, ambos contenidos en algún intervalo $[0, T]$. Supongamos cumplidas las siguientes condiciones (vea la figura 24):

(i) $\sum_{x_{1} \in \mathbb{Z}}\left|I_{x_{1}}\right|=T$,

(ii) $u_{x_{1}, \ldots, x_{n}}<v_{x_{1}, \ldots, x_{n}} \leq w_{x_{1}, \ldots, x_{n}}$ (y por lo tanto $J_{x_{1}, \ldots, x_{n}} \subset I_{x_{1}, \ldots, x_{n}}$ ),

(iii) $w_{x_{1}, \ldots, x_{n-1}, x_{n}}=u_{x_{1}, \ldots, x_{n-1}, 1+x_{n}}$,

(iv) $\lim _{x_{n} \rightarrow-\infty} u_{x_{1}, \ldots, x_{n-1}, x_{n}}=u_{x_{1}, \ldots, x_{n-1}}$,

(v) $\lim _{x_{n} \rightarrow \infty} u_{x_{1}, \ldots, x_{n-1}, x_{n}}=v_{x_{1}, \ldots, x_{n-1}}$,

(vi) $\lim _{n \rightarrow \infty} \sup _{\left(x_{1}, \ldots, x_{n}\right) \in \mathbb{Z}^{n}}\left|I_{x_{1}, \ldots, x_{n}}\right|=0$.

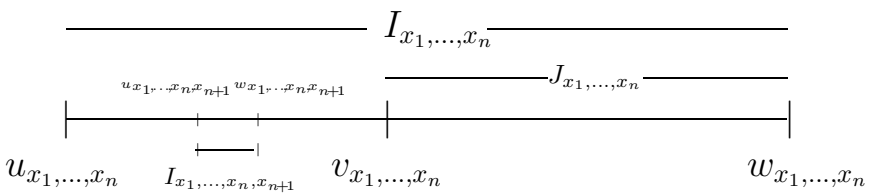

Figura 24

Observe que

$$
\left|J_{x_{1}, \ldots, x_{n}}\right|+\sum_{x_{n+1} \in \mathbb{Z}}\left|I_{x_{1}, \ldots, x_{n}, x_{n+1}}\right|=\left|I_{x_{1}, \ldots, x_{n}}\right| .
$$

Denotemos por $\overline{\mathrm{H}}_{n}$ el estabilizador en del nivel $n$ del árbol $\mathcal{T}_{\infty}$. Para cada $n \in \mathbb{N}$ definiremos homeomorfismos $a_{n}, b_{n}, c_{n}$ y $d_{n}$ que generen un grupo isomorfo a $\overline{\mathrm{H}} / \overline{\mathrm{H}}_{n}$. Para esto, consideremos los homomorfismos $\phi_{0}$ y $\phi_{1}$ del subgrupo de $\overline{\mathrm{H}}$ generado por $\bar{b}, \bar{c}$ y $\bar{d}$ hacia $\overline{\mathrm{H}}$ definidos por $\phi_{0}(\bar{b})=\bar{a}$, $\phi_{0}(\bar{c})=\bar{a}, \phi_{0}(\bar{d})=i d$, y $\phi_{1}(\bar{b})=\bar{c}, \phi_{1}(\bar{c})=\bar{d}, \phi_{1}(\bar{d})=\bar{b}$.

Definición de $a_{n}$ :

- Si $p \in J_{x_{1}, \ldots, x_{i}}$ para algún $i<n$, sea $a_{n}(p)=\varphi\left(J_{x_{1}, x_{2}, \ldots, x_{i}}, J_{1+x_{1}, x_{2}, \ldots, x_{i}}\right)(p)$.

- Si $p \in I_{I_{x_{1}, \ldots, x_{n}}}$, sea $a_{n}(p)=\varphi\left(I_{x_{1}, x_{2}, \ldots, x_{n}}, I_{1+x_{1}, x_{2}, \ldots, x_{n}}\right)(p)$. 
Definición de $b_{n}$ :

Supongamos que $p \in] 0,1\left[\right.$ pertenezca al intervalo $I_{x_{1}, \ldots, x_{n}}$, y denotemos por $\left(\bar{x}_{1}, \ldots, \bar{x}_{n}\right) \in\{0,1\}^{n}$ la sucesión obtenida al reducir módulo 2 .

- Si $\phi_{\bar{x}_{1}}(\bar{b}), \phi_{\bar{x}_{2}} \phi_{\bar{x}_{1}}(\bar{b}), \ldots, \phi_{\bar{x}_{n}} \ldots \phi_{\bar{x}_{2}} \phi_{\bar{x}_{1}}(\bar{b})$ están bien definidos, hagamos $b_{n}(p)=p$.

- En caso contrario, denotemos por $i=i(p) \leq n$ al menor entero tal que $\phi_{\bar{x}_{i}} \ldots \phi_{\bar{x}_{2}} \phi_{\bar{x}_{1}}(\bar{b})$ no esté definido y hagamos

$b_{n}(p)= \begin{cases}p, & p \in J_{x_{1}, \ldots, x_{j}}, j<i, \\ \varphi\left(J_{x_{1}, \ldots, x_{i}, \ldots, x_{j}}, J_{x_{1}, \ldots, 1+x_{i}, \ldots, x_{j}}\right)(p), & p \in J_{x_{1}, \ldots, x_{i}, \ldots, x_{j}}, i \leq j<n, \\ \varphi\left(I_{x_{1}, \ldots, x_{i}, \ldots, x_{n}}, I_{x_{1}, \ldots, 1+x_{i}, \ldots, x_{n}}\right)(p), & p \in I_{x_{1}, \ldots, x_{n} .} .\end{cases}$

Las definiciones de $c_{n}$ y $d_{n}$ son similares a la de $b_{n}$. Claramente, las aplicaciones $a_{n}, b_{n}, c_{n}$ y $d_{n}$ se extienden en homeomorfismos de $[0, T]$. El hecho que ellos generan un grupo isomorfo a $\overline{\mathrm{H}} / \overline{\mathrm{H}}_{n}$ se desprende de las propiedades de equivarianza de las aplicaciones $\varphi(I, J)$ (dejamos la verificación a cargo del lector). La condición (vi) implica además que las sucesiones de aplicaciones $a_{n}, b_{n}, c_{n}$ y $d_{n}$ convergen a homeomorfismos $a, b, c \mathrm{y}$ $d$ respectivamente, los cuales generan un grupo isomorfo a $\overline{\mathrm{H}}$.

Ejemplo 4.62. Dada una sucesión $\left(\ell_{i}\right)_{i \in \mathbb{Z}}$ de reales positivos tales que $\sum \ell_{i}=1$, definamos $\left|I_{x_{1}, \ldots, x_{n}}\right| \mathrm{y}\left|J_{x_{1}, \ldots, x_{n}}\right|$ por

$$
\left|J_{x_{1}, \ldots, x_{n}}\right|=0 \quad \text { y } \quad\left|I_{x_{1}, \ldots, x_{n}}\right|=\ell_{x_{1}} \ldots \ell_{x_{n}} .
$$

Si efectuamos la construcción precedente (para $T=1$ ) usando la familia equivariante de las aplicaciones afines $\varphi([0, u],[0, v])(x)=v x / u$, entonces reobtenemos la inclusión de $\overline{\mathrm{H}}$ en el grupo de los homeomorfismos bilipschitzianos del intervalo del ejemplo 4.60 (manteniendo siempre las hipótesis $\ell_{i+1} / \ell_{i} \leq C$ y $\ell_{i} / \ell_{i+1} \leq C$ para todo $i \in \mathbb{Z})$.

La verificación de los detalles del resto de la construcción quedará a cargo del lector (referimos a [142] en caso de dificultades). Sea $\omega$ un módulo de continuidad satisfaciendo $\omega(s)=1 / \log (1 / s)$ para $s \leq 1 / e$, y tal que la aplicación $s \mapsto \omega(s) / s$ sea decreciente. Fijemos una constante $C>0 \mathrm{y}$ para cada $k \in \mathbb{N}$ definamos $T_{k}=\sum_{i \in \mathbb{Z}} \frac{1}{(|i|+k)^{2}}$. Consideremos una sucesión creciente $\left(k_{n}\right)$ de enteros positivos, y para $n \in \mathbb{N}$ y $\left(x_{1}, \ldots, x_{n}\right) \in \mathbb{Z}^{n}$ hagamos

$$
\left|I_{x_{1}, \ldots, x_{n}}\right|=\frac{1}{\left(\left|x_{1}\right|+\ldots\left|x_{n}\right|+k_{n}\right)^{2 n}} .
$$

Valiéndose de la familia equivariante inducida por (4.10), nuestro método general origina subgrupos de Difeo ${ }_{+}^{1}\left(\left[0, T_{k_{1}}\right]\right)$ isomorfos a $\overline{\mathrm{H}} / \overline{\mathrm{H}}_{n}$ generados por elementos $a_{n}, b_{n}, c_{n}$ y $d_{n}$. El objetivo consiste entonces en controlar la norma $\mathrm{C}^{\omega}$ para la derivada de estos difeomorfismos. Ahora bien, si la sucesión $\left(k_{n}\right)$ satisface ciertas "condiciones de crecimiento rápido" (que dependen de $C$ ), no es difícil verificar que dichas normas están acotadas superiormente por $C$ para todo $n \in \mathbb{N}$ (y que lo mismo ocurre para las derivadas 
de los inversos de dichos difeomorfismos). Las sucesiones correspondientes son por lo tanto equicontinuas, y es fácil ver que ellas convergen a aplicaciones $\omega$-continuas con $\mathrm{C}^{\omega}$-norma acotada superiormente por $C$. Estas aplicaciones corresponden a las derivadas de difeomorfismos $a, b, c, d$ (y sus inversos) de clase $\mathrm{C}^{1+\omega}$, quienes generan un grupo isomorfo a $\overline{\mathrm{H}}$. Como este grupo actúa sobre el intervalo $\left[0, T_{k_{1}}\right]$, para obtener una acción sobre $[0,1]$ debemos conjugar por la aplicación afín $g:[0,1] \rightarrow\left[0, T_{k_{1}}\right]$. Puesto que para $k_{1}$ suficientemente grande se tiene $T_{k_{1}} \leq 1$, este procedimiento de conjugación no aumenta la $\mathrm{C}^{\omega}$-norma para las derivadas. Observe finalmente que, como para todo difeomorfismo $f$ de $[0,1]$ existe un punto con derivada 1 , si la $C^{\omega}$-norma de $f^{\prime}$ está acotada por $C$ entonces

$$
\sup _{x \in[0,1]}\left|f^{\prime}(x)-1\right| \leq C \omega(1) .
$$

En consecuencia, para $C$ pequeño la realización precedente de $\overline{\mathrm{H}}$ tiene a sus generadores cercanos a la identidad respecto a la topología $\mathrm{C}^{1+\omega}$.

Ejercicio 4.63. Dentro del grupo de Grigorchuk-Maki $\bar{H}$ existen "muchos" elementos que conmutan. De manera más precisa, para cada $d \in \mathbb{N}$ pueden ser escogidos $d+1$ elementos de $\bar{H}$ satisfaciendo una condición combinatoria del tipo semejante a la del teorema 4.34 (considere por ejemplo $f_{1}=\bar{a}^{-2}, f_{2}=\bar{b}^{-2}$, $f_{3}=\bar{a}^{-1} \bar{b}^{-2} \bar{a}$, etc). De esta forma, para verificar que la acción canónica de $\bar{H}$ no es semiconjugada a una acción por difeomorfismos de clase $\mathrm{C}^{1+\tau}$ basta con aplicar el lema de Kopell generalizado (i.e., el teorema 4.34) para $d>1 / \tau$. Los ítemes a continuación permiten probar la misma afirmación usando métodos "elementales" (vea [142] para mayores detalles).

(i) Pruebe que si $h$ es un difeomorfismo de clase $\mathrm{C}^{1+\tau}$ de un intervalo cerrado $[u, v]$, y si $C$ denota la constante de hölderianidad correspondiente para $h^{\prime}$, entonces para todo $x \in[u, v]$ se tiene $|h(x)-x| \leq C|v-u|^{1+\tau}$.

(ii) Usando (i) pruebe directamente (i.e., sin usar ningún argumento probabilístico) que el teorema (4.34) es válido para $d \geq d(\alpha)$, donde $d(\alpha)$ es el mínimo entero mayor o igual a 2 para el cual se verifica la desigualdad $\alpha(1+\tau)^{d-2} \geq 1$.

(iii) Usando (ii) concluya que la acción canónica de $\bar{H}$ no es semiconjugada a una acción por difeomorfismos de clase $\mathrm{C}^{1+\tau}$ para ningún $\tau>0$.

Para cerrar esta sección resulta interesante observar una vez más que el elemento $\bar{a}^{2}$ pertenece al centro de $\overline{\mathrm{H}}$. Teniendo en mente la realización de $\overline{\mathrm{H}}$ como un grupo de difeomorfismos de clase $\mathrm{C}^{1}$, el estudio de los centralizadores en $\operatorname{Difeo}_{+}^{1}([0,1])$ de difeomorfismos (u homeomorfismos) del intervalo se torna natural. La siguiente sencilla pero interesantísima proposición, debida a Bonatti, Crovisier y Wilkinson, podría resultar fundamental para este estudio.

Proposición 4.64. Si h es un homeomorfismo de [0,1[ sin puntos fijos en $] 0,1\left[\right.$, entonces el grupo de los difeomorfismos de clase $\mathrm{C}^{1}$ de $[0,1[$ que conmutan con h no contiene elementos entrecruzados. 
Demostración. Supongamos por contradicción que $f$ y $g$ sean difeomorfismos de clase $\mathrm{C}^{1}$ de $[0,1[$ que conmutan con $h$, y que ellos estén entrecruzados sobre cierto intervalo $[x, y] \subset[0,1]$. Como en el curso de la prueba del lema 2.53, podemos restringirnos al caso en que $f(x)=x$, $f(y) \in] x, y[, g(x) \in] x, y[$ y $g(y)=y$. Además, cambiando $f$ y $g$ por alguno de sus iterados, podemos suponer que $g(x)>f(y)$. Todas estas condiciones son preservadas por conjugación topológica, lo que es equivalente a decir que $f$ y $g$ satisfacen $\left.f\left(h^{n}(x)\right)=h^{n}(x), f\left(h^{n}(y)\right) \in\right] h^{n}(x), h^{n}(y)[$, $\left.g\left(h^{n}(x)\right) \in\right] h^{n}(x), h^{n}(y)\left[, g\left(h^{n}(y)\right)=h^{n}(y)\right.$ y $g\left(h^{n}(x)\right)>f\left(h^{n}(y)\right)$ para todo $n \in \mathbb{Z}$. Si $h(z)<z($ resp. si $h(z)>z)$ para todo $z \in] 0,1[$, entonces las sucesiones $\left(h^{n}(x)\right)$ y $\left(h^{n}(y)\right)\left(\right.$ resp. $\left(h^{-n}(x)\right)$ y $\left.\left(h^{-n}(y)\right)\right)$ convergen al origen. Como $f$ y $g$ son de clase $\mathrm{C}^{1}$, esto implica que $f^{\prime}(0)=g^{\prime}(0)=1$. Sin embargo, puesto que $g\left(h^{n}(x)\right)>f\left(h^{n}(y)\right)$, debe existir una sucesión de puntos $\left.z_{n} \in\right] x_{n}, y_{n}$ [ tal que para cada $n \in \mathbb{N}$ se tiene $f^{\prime}\left(z_{n}\right)<1 / 2$ ó $g^{\prime}\left(z_{n}\right)<1 / 2$, y esto está en contradicción con la continuidad de la derivada de $f$ y $g$ en el origen.

\section{Grupos solubles de difeomorfismos}

\subsection{Ejemplos y formulación de resultados}

A diferencia del caso nilpotente, dentro del grupo de los difeomorfismos de clase $\mathrm{C}^{2}$ del intervalo existe una gran variedad de subgrupos solubles. Notemos en primer lugar que el grupo afín es conjugado a un subgrupo de $\operatorname{Difeo}_{+}^{\infty}([0,1])$. Para comprobar esto nos valdremos de la linearización introducida en el ejercicio 5.11. Fijemos una constante $0<\varepsilon<1 / 2$ y consideremos dos difeomorfismos $\left.\varphi_{1}:\right] 0,1\left[\rightarrow \mathbb{R}\right.$ y $\left.\varphi_{2}:\right] 0,1[\rightarrow] 0,1[$ de clase $\mathrm{C}^{\infty}$ tales que

$$
\begin{gathered}
\left.\left.\varphi_{1}(x)=-\frac{1}{x} \quad \text { y } \quad \varphi_{2}(x)=\exp \left(-\frac{1}{x}\right) \text { para } x \in\right] 0, \varepsilon\right], \\
\varphi_{1}(x)=\frac{1}{1-x} \quad \text { y } \quad \varphi_{2}(x)=1-\exp \left(\frac{1}{x-1}\right) \text { para } x \in[1-\varepsilon, 1[.
\end{gathered}
$$

Consideremos ahora los campos de vectores sobre la recta $Y_{1}=\frac{\partial}{\partial x}$ e $Y_{2}=x \frac{\partial}{\partial x}$, los cuales generan el álgebra de Lie del grupo afín. Se verifica fácilmente que los campos $X_{j}=\varphi^{*}\left(Y_{j}\right)$, donde $j \in\{1,2\}$ y $\varphi=\varphi_{1} \circ \varphi_{2}^{2}$, se extienden a campos de clase $\mathrm{C}^{\infty}$ de $[0,1]$ que son nulos e infinitamente planos en los extremos. Por esta razón, para cada $g \in \mathrm{Af}_{+}(\mathbb{R})$ se tiene que $\varphi \circ g \circ \varphi^{-1}$ es un difeomorfismo de clase $\mathrm{C}^{\infty}$ de $[0,1]$ infinitamente tangente a la identidad en los extremos.

De la construcción anterior surgen inmediatamente algunas preguntas: ¿todo subgrupo soluble y finitamente generado de $\operatorname{Difeo}_{+}^{1+v a}([0,1[)$ que no posee puntos fijos globales en $] 0,1[$ es semiconjugado a un subgrupo del 
grupo afín?; ¿todo subgrupo soluble de Difeo ${ }_{+}^{1+v a}([0,1[)$ tiene orden de solubilidad inferior o igual a 2? Los ejemplos a continuación (inspirados del capítulo $\mathrm{V}$ de [80]) muestran sin embargo que es posible fabricar grupos solubles de difeomorfismos del intervalo de orden de solubilidad arbitrario y dinámica muy diferente a la del grupo afín. Para ello, basta tomar extensiones sucesivas por $\mathbb{Z}$ de manera apropiada.

Ejemplo 4.65. Sea $f:[0,1] \rightarrow[0,1]$ un difeomorfismo de clase $\mathrm{C}^{\infty}$ sin otro punto fijo que 0 y 1 , y que es topológicamente contractante en 0 . Supongamos que $f$ sea el tiempo 1 del flujo asociado a un campo de vectores de clase $\mathrm{C}^{\infty}$ de $[0,1]$ que es infinitamente plano en 0 y 1 . Fijemos $a \in] 0,1[$ y consideremos un campo de vectores $X$ sobre $[f(a), a]$ cuyas únicas singularidades sean $f(a)$ y $a$, siendo $X$ infinitamente plano en dichos puntos. Extendamos esta definición haciendo $X(x)=0$ para $x \in[0,1] \backslash[f(a), a]$. Obtenemos así un campo de vectores de clase $\mathrm{C}^{\infty}$ sobre $[0,1]$ que es infinitamente plano en 0 y 1.

Sea $g$ el difeomorfismo de clase $\mathrm{C}^{\infty}$ obtenido al integrar el campo $X$ (en tiempo $1)$, y sea $\Gamma$ el grupo generado por $f$ y $g$. Evidentemente, la restricción de $\Gamma$ a ]0,1[ no es semiconjugada a un subgrupo del grupo afín, y $\Gamma$ no posee punto fijo sobre $] 0,1[$. Afirmamos sin embargo que $\Gamma$ es soluble de orden de solubilidad igual a 2. En efecto, sea $\Gamma^{*}$ el subgrupo abeliano de $\Gamma$ formado por los elementos que fijan los puntos $f^{n}(a)$ (con $n \in \mathbb{Z}$ ), de modo que sus restricciones a cada intervalo $\left[f^{n+1}(a), f^{n}(a)\right]$ estén contenidas en el grupo generado por la restricción del elemento $f^{n} g f^{-n}$ a dicho intervalo. Evidentemente, $\Gamma^{*}$ es un subgrupo normal de $\Gamma$ que contiene al grupo derivado $\Gamma^{\prime}$. Además, el cuociente $\Gamma / \Gamma^{*}$ se identifica a $(\mathbb{Z},+)$. A partir de esto se concluye fácilmente la afirmación.

A continuación aplicaremos sucesivamente la idea precedente para obtener, para cada $k \geq 2$, ejemplos de grupos solubles $\bar{\Gamma}_{k}$ de difeomorfismos de clase $\mathrm{C}^{\infty}$ del intervalo $[2-k, k-1]$ con orden de solubilidad igual a $k$ y generados por elementos $f_{1, k}, \ldots, f_{k, k}$ que son cada uno el tiempo 1 del flujo asociado a un campo de vectores de clase $\mathrm{C}^{\infty}$ e infinitamente plano en $2-k$ y $k-1$. Para ello razonamos por inducción. Para $k=2$ hacemos $\bar{\Gamma}_{2}=\Gamma$, donde $f_{1,2}=g$ y $f_{2,2}=f$. Supongamos ahora que ya ha sido construido el grupo $\Gamma_{k}$, y consideremos los campos de vectores $X_{i, k}:[2-k, k-1] \rightarrow \mathbb{R}$ correspondientes a los $f_{i, k}$. Consideremos un campo $X_{k+1, k+1}$ definido sobre $[1-k, k]$ que sea de la forma $\varrho \partial / \partial x$ para cierta función $\varrho:[1-k, k] \rightarrow \mathbb{R}$ de clase $\mathrm{C}^{\infty}$, negativa en el interior e infinitamente plana en los extremos. Multiplicando este campo por un factor constante si es necesario, podemos suponer que el tiempo 1 del flujo asociado es un difeomorfismo $f_{k+1, k+1}$ de $[1-k, k]$ que verifica $f_{k+1, k+1}(k-1)=2-k$.

Para $i \in\{1, \ldots, k\}$ extendemos el campo $X_{i, k}$ en un campo $X_{i, k+1}$ haciendo $X_{i, k+1}(x)=0$ para todo punto $x \in[1-k, k] \backslash[2-k, k-1]$. Los campos así obtenidos son de clase $\mathrm{C}^{\infty}$ sobre $[1-k, k]$ e infinitamente planos en $1-k$ y $k$. Sean $f_{i, k+1}, i \in\{1, \ldots, k\}$, los tiempos 1 de los flujos asociados. Afirmamos que el grupo $\bar{\Gamma}_{k+1}$ generado por los $f_{i, k+1}, i \in\{1, \ldots, k+1\}$, es soluble de orden de solubilidad igual a $k+1$. En efecto, el estabilizador en $\Gamma$ de $[2-k, k-1]$ es un subgrupo normal $\bar{\Gamma}^{*}$ que se identifica a una suma directa de grupos isomorfos a $\bar{\Gamma}_{k}$, y por la hipótesis de inducción este último grupo es soluble de orden de solubilidad $k$. Por otra parte, $\bar{\Gamma}_{k+1} / \bar{\Gamma}^{*}$ se identifica a $(\mathbb{Z},+)$, y el grupo derivado de $\Gamma$ está contenido en $\bar{\Gamma}^{*}$. A partir de esto se concluye fácilmente la afirmación. Notemos de paso que $\bar{\Gamma}_{k+1}$ no posee punto fijo al interior de $[1-k, k]$. 
Ejemplo 4.66. Mejoraremos ahora el primer paso del ejemplo precedente utilizando la construcción del inicio de esta sección; obtendremos de esta manera una familia dinámicamente más interesante de subgrupos solubles de $\operatorname{Difeo}_{+}^{\infty}([0,1])$ de orden de solubilidad igual a 3 . Consideremos para ello dos campos de vectores $X_{1}$ y $X_{2}$ definidos sobre el intervalo $[1 / 3,2 / 3]$, que sean de clase $\mathrm{C}^{\infty}$, infinitamente planos en los extremos, y cuyos flujos originen un grupo conjugado al grupo afín. Denotemos por $g$ y $h$ los difeomorfismos de clase $\mathrm{C}^{\infty}$ obtenidos al integrar en tiempo 1 dichos campos $X_{1}$ y $X_{2}$ respectivamente.

Fijemos un difeomorfismo $f:[0,1] \rightarrow[0,1]$ de clase $\mathrm{C}^{\infty}$, sin punto fijo en el interior, y que verifique $f(2 / 3)=1 / 3$. Notemos $a=2 / 3$, consideremos una sucesión (a ser fijada) de reales positivos $\left(t_{n}\right)_{n \in \mathbb{Z}}$, y extendamos inductivamente la definición de los campos $X_{j}$ haciendo

$$
\begin{gathered}
X_{j}(x)=t_{n} X_{j}\left(f^{-1}(x)\right) /\left(f^{-1}\right)^{\prime}(x), \quad x \in\left[f^{n+1}(a), f^{n}(a)\right], \quad n \geq 1, \\
X_{j}(x)=t_{n} X_{j}(f(x)) / f^{\prime}(x), \quad x \in\left[f^{n+1}(a), f^{n}(a)\right], \quad n \leq-1 .
\end{gathered}
$$

Supongamos que $\prod_{i=1}^{n} t_{i} \rightarrow 0$ cuando $n \rightarrow+\infty$, que $\Pi_{i=n}^{0} t_{i} \rightarrow 0$ cuando $n \rightarrow-\infty, \mathrm{y}$ que estas convergencias sean suficientemente rápidas (para fijar ideas, supongamos que la rapidez de convergencia sea supra-exponencial). En tal caso, no es difícil verificar que $\operatorname{los} X_{j}$ se extienden a campos de vectores de clase $\mathrm{C}^{\infty}$ en $[0,1]$ que en los extremos son nulos e infinitamente planos. Sea $\Gamma$ el grupo generado por $f, g$ y $h$. Evidentemente, la restricción de $\Gamma$ al interior de $[0,1]$ no es semiconjugada a un subgrupo del grupo afín, y $\Gamma$ no posee puntos fijos en $] 0,1[$. Afirmamos sin embargo que $\Gamma$ es soluble de orden de solubilidad igual a 3 . En efecto, sea $\Gamma^{*}$ el subgrupo metabeliano y no conmutativo de $\Gamma$ formado por los elementos que fijan los puntos $f^{n}(a)$, de manera que sus restricciones al interior de cada intervalo $\left[f^{n+1}(a), f^{n}(a)\right]$ estén contenidas en el conjugado del grupo afín generado por $X_{1}$ y $X_{2}$. Si designamos respectivamente por $g_{\left[f^{n+1}(a), f^{n}(a)\right]}^{t}$ y $h_{\left[f^{n+1}(a), f^{n}(a)\right]}^{t}$ los flujos asociados a las restricciones de $X_{1}$ y $X_{2}$ a $\left[f^{n+1}(a), f^{n}(a)\right](\operatorname{con} t \in \mathbb{R})$, entonces para todo $n \in \mathbb{N}$ se tiene

$$
f^{-1} g_{\left[f^{n+1}(a), f^{n}(a)\right]} f=g_{\left[f^{n}(a), f^{n-1}(a)\right]}^{t_{n}}, \quad f^{-1} h_{\left[f^{n+1}(a), f^{n}(a)\right]} f=h_{\left[f^{n}(a), f^{n-1}(a)\right]}^{t_{n}} .
$$

El grupo $\Gamma^{*}$ es por lo tanto un subgrupo normal de $\Gamma$. Además, el grupo cuociente $\Gamma / \Gamma^{*}$ se identifica a $(\mathbb{Z},+)$, y $\Gamma^{*}$ contiene al grupo derivado de $\Gamma$. A partir de todo esto se concluye fácilmente lo afirmado.

$\mathrm{Al}$ igual que en ejemplo precedente, para cada entero $k \geq 2$ pueden hacerse extensiones sucesivas de $\Gamma$ por $(\mathbb{Z},+)$ para construir subgrupos solubles de $\operatorname{Difeo}_{+}^{\infty}([1-k, k])$ de orden de solubilidad $k+2 \ldots$

Ejercicio 4.67. Pruebe que las convergencias $\Pi_{i=1}^{n} t_{i} \rightarrow 0$ y $\Pi_{i=n}^{0} t_{i} \rightarrow 0$ exigidas para el segundo de los ejemplo precedentes son necesarias, incluso para obtener grupos de difeomorfismos de clase $\mathrm{C}^{2}$. De manera más precisa, pruebe la validez de la siguiente proposición.

Proposición 4.68. Sean $g:\left[0,1\left[\rightarrow\left[0, g(1)\left[\right.\right.\right.\right.$ un difeomorfismo de clase $\mathrm{C}^{2}, y$ $a<b$ dos puntos fijos de $g$ en $] 0,1[$ tales que $g$ no posee puntos fijos en ] $a, b[$. Sea $f:\left[0, b\left[\rightarrow\left[0, f(b)\left[\right.\right.\right.\right.$ un difeomorfismo de clase $\mathrm{C}^{2}$ sin otro punto fijo sobre $[0, b]$ que 0 , y tal que $f(b) \leq a$. Supongamos que exista una sucesión $\left(t_{n}\right)_{n \in \mathbb{N}}$ de reales positivos tal que para todo $n \in \mathbb{N}$ se tiene $f^{-1} \circ g_{\left[f^{n}(a), f^{n}(b)[\right.} \circ f=g_{\left[f^{n-1}(a), f^{n-1}(b)[\right.}^{t_{n}}$. Entonces el valor de $\prod_{i=1}^{n} t_{i}$ converge a cero cuando $n$ tiende al infinito. 
Los ejemplos 4.65 y 4.66 son fundamentales, en el sentido que en ellos se utiliza el que es básicamente el único método de fabricación de grupos solubles de difeomorfismos del intervalo. Comencemos por dar una versión precisa de esto para el caso metabeliano.

Teorema 4.69. Si $\Gamma$ es un subgrupo metabeliano de Difeo ${ }_{+}^{1+\mathrm{va}}([0,1[)$ sin punto fijo sobre ]0,1[, entonces $\Gamma$ es o bien conjugado a un subgrupo del grupo afin, o bien un producto semidirecto entre $(\mathbb{Z},+)$ y un subgrupo de un producto (a lo más numerable) de grupos conjugados a grupos de traslaciones.

Daremos la demostración de este teorema en la sección siguiente. Consignemos inmediatamente que una clasificación completa de los subgrupos solubles de Difeo ${ }_{+}^{1+v a}([0,1[)$ puede ser dada usando las mismas ideas valiéndose de un (engorroso) argumento de inducción [148]. Para formular el teorema en el caso general, denotemos por $r(1)$ la familia de los grupos conjugados a grupos de traslaciones, y por $r(2)$ la familia de los grupos que son conjugados a subgrupos no abelianos del grupo afín, o bien un producto semidirecto entre $(\mathbb{Z},+)$ y un subgrupo de un producto (a lo más numerable) de grupos conjugados a grupos (no triviales) de traslaciones. Para $k>2$ definimos por inducción la familia $r(k)$ de los grupos que son un producto semidirecto entre $(\mathbb{Z},+)$ y un subgrupo de un producto (a lo más numerable) de grupos en $\mathcal{R}(k-1)=r(1) \cup \ldots \cup r(k-1)$, de manera que al menos uno de los factores no pertenece a $\mathcal{R}(k-2)$.

Teorema 4.70. Sea $\Gamma$ un subgrupo soluble de Difeo ${ }_{+}^{1+\mathrm{va}}([0,1[)$ sin punto fijo en $] 0,1[$. Si el orden de solubilidad de $\Gamma$ es igual a $k \geq 2$, entonces $\Gamma$ pertenece a la familia $r(k)$.

La clasificación precedente permite obtener interesantes resultados de rigidez. Por ejemplo, el normalizador de un grupo soluble de difeomorfismos del intervalo tiende a parecerse mucho al grupo original, como queda consignado en el siguiente teorema [146].

Teorema 4.71. Sea $\Gamma$ un subgrupo soluble de $\mathrm{Difeo}_{+}^{1+\mathrm{va}}([0,1[)$ de orden de solubilidad igual a $k \geq 1$ y sin punto fijo sobre $] 0,1[$. Si $\mathcal{N}(\Gamma)$ designa su normalizador en Difeo ${ }_{+}^{1}([0,1[)$, entonces se tiene una de las posibilidades siguientes:

(i) si $k>1$ entonces $\mathcal{N}(\Gamma)$ es soluble de orden de solubilidad igual a $k$;

(ii) si $k=1$ y $\Gamma$ no es cíclico infinito, entonces $\mathcal{N}(\Gamma)$ es topológicamente conjugado a un subgrupo (eventualmente no abeliano) del grupo afín;

(iii) si $k=1$ y $\Gamma$ es cíclico infinito, entonces $\mathcal{N}(\Gamma)$ es topológicamente conjugado a un subgrupo del grupo de las traslaciones.

La clasificación de los grupos solubles de difeomorfismos del $\mathrm{S}^{1}$ se reduce, gracias al lema 4.14, a aquélla dada para el caso del intervalo. La verificación del resultado siguiente a partir del teorema 4.70 es dejada al lector. 
Teorema 4.72. Sea $\Gamma$ un subgrupo soluble de $\mathrm{Difeo}_{+}^{1+\mathrm{va}}\left(\mathrm{S}^{1}\right)$. Si la longitud de solubilidad de $\Gamma$ es igual a $k+1$, entonces se tiene una de las posibilidades siguientes:

(i) $\Gamma$ es topológicamente semiconjugado a un grupo de rotaciones;

(ii) existe un subconjunto finito no vacío $F$ de $\mathrm{S}^{1}$ invariante por $\Gamma$ tal que el grupo derivado $\Gamma^{\prime}$ fija cada punto de $F$, y la restricción de $\Gamma^{\prime}$ a cada una de las componentes conexas de $\mathrm{S}^{1} \backslash F$ pertenece a la familia $\mathcal{R}(k)$.

El caso de la recta presenta complicaciones adicionales. Sin embargo, gracias a los resultados parciales de la sección 2.5 del capítulo 2 , se puede llegar a la descripción dada por el teorema enunciado a continuación.

Teorema 4.73. Sea $\Gamma$ un subgrupo soluble de $\mathrm{Difeo}_{+}^{1+\mathrm{va}}(\mathbb{R})$. Si su orden de solubilidad es igual a $k \geq 1$, entonces se tiene una de las posibilidades siguientes:

(i) $\Gamma$ es topológicamente semiconjugado a un subgrupo del grupo afín;

(ii) $\Gamma$ es un subgrupo de un producto (a lo más numerable) de grupos de la familia $\mathcal{R}(k)$, de modo que al menos uno de los factores no pertenece a $\mathcal{R}(k-1)$;

(iii) $\Gamma$ pertenece a $r(k)$ o bien a $r(k+1)$.

Observemos que si $\Gamma$ es soluble y semiconjugado a un grupo de transformaciones afines sin serle conjugado, entonces el segundo grupo derivado $\Gamma^{\prime \prime}$ actúa fijando una familia numerable de intervalos abiertos y disjuntos cuya unión es densa. Los teoremas 4.69 y 4.70 permiten entonces describir la dinámica de $\Gamma^{\prime \prime}$. Por otra parte, el que $\Gamma$ pueda pertenecer a $r(k+1)$ siendo de orden de solubilidad $k$ es muy natural, pues contrariamente al caso del intervalo, sobre la recta es posible hacer extensiones centrales de grupos no triviales, sin que por ello la clase de diferenciabilidad se vea necesariamente afectada.

\subsection{El caso metabeliano}

El siguiente lema elemental será fundamental en lo que sigue.

Lema 4.74. El normalizador en $\mathrm{Homeo}_{+}(\mathbb{R})$ de todo subgrupo denso del grupo de traslaciones está contenido en el grupo afín.

Demostración. Módulo un factor multiplicativo, la única medida de Radon sobre la recta invariante por un grupo denso de traslaciones es la de Lebesgue. El normalizador de tal grupo deja entonces quasi-invariante esta medida, por lo que el lema se deduce de la proposición 1.4.

El lema siguiente es una versión mejorada del anterior en clase $\mathrm{C}^{1+\mathrm{va}}$.

Lema 4.75. Si $\Gamma$ es un subgrupo abeliano de $\operatorname{Difeo}_{+}^{1+\mathrm{va}}([0,1[)$ sin punto fijo en $] 0,1\left[\right.$, entonces su normalizador en Difeo $_{+}^{1}([0,1[)$ es conjugado a un subgrupo del grupo afín. 
Demostración. Observemos que la hipótesis de ausencia de punto fijo en ]0,1[ equivale, por el corolario 4.4, a que $\Gamma$ está contenido en un grupo a un parámetro (topológicamente conjugado al interior a un grupo de traslaciones). Fijemos un elemento no trivial $g$ de $\Gamma$.

Si $\left\{t \in \mathbb{R}: g^{t} \in \Gamma\right\}$ es denso en $\mathbb{R}$ entonces por conjugación podemos transformar $\Gamma$ en un subgrupo denso de $(\mathbb{R},+)$. La imagen por esta conjugación del normalizador $\mathcal{N}$ de $\Gamma$ en $\operatorname{Difeo}_{+}^{1}([0,1[)$ está por lo tanto contenida en el normalizador en $\mathrm{Homeo}_{+}(\mathbb{R})$ de dicho grupo denso de traslaciones. El lema 4.74 implica entonces que esta imagen está contenida en el grupo afín.

Supongamos ahora que $\left\{t \in \mathbb{R}: g^{t} \in \Gamma\right\}$ sea cíclico infinito. Afirmamos que el normalizador $\mathcal{N}$ de $\Gamma$ en Difeo ${ }_{+}^{1}\left(\left[0,1[)\right.\right.$ es igual a $g^{\mathbb{R}}$. En efecto, si $k$ es un entero positivo tal que $g^{1 / k}$ es el generador de $\left\{t \in \mathbb{R}: g^{t} \in \Gamma\right\}$, entonces para todo $h \in \mathcal{N}$ existen enteros positivos $n, m$ tales que $h g^{1 / k} h^{-1}=\left(g^{1 / k}\right)^{n}$ y $h^{-1} g^{1 / k} h=\left(g^{1 / k}\right)^{m}$. Se tiene entonces

$$
\left(g^{1 / k}\right)^{m n}=\left(\left(g^{1 / k}\right)^{m}\right)^{n}=\left(h^{-1} g^{1 / k} h\right)^{n}=h^{-1}\left(g^{1 / k}\right)^{n} h=g^{1 / k},
$$

de donde se obtiene $m=n=1$. Esto implica que los elementos de $\mathcal{N}$ conmutan con $g^{1 / k}$, por lo que $\mathcal{N}$ está contenido en $g^{\mathbb{R}}$. Puesto que $g^{\mathbb{R}}$ normaliza a $\Gamma$, obtenemos finalmente $\mathcal{N}=g^{\mathbb{R}}$.

Pasamos ahora a la demostración del teorema 4.69. Por el corolario 4.4, si $\Gamma$ es un subgrupo conmutativo de Difeo ${ }_{+}^{1+\text { va }}([0,1[)$ entonces $\Gamma$ es un subgrupo de un producto a lo más numerable de grupos conjugados a grupos de traslaciones. Fijemos ahora un subgrupo metabeliano y no conmutativo $\Gamma$ de $\operatorname{Difeo}_{+}^{1+\text { va }}\left([0,1[)\right.$ sin punto fijo en $] 0,1\left[\right.$. Si existe $g \in \Gamma^{\prime}$ tal que las órbitas por $g$ se acumulan hacia 0 y 1 , entonces el grupo abeliano $\Gamma^{\prime}$ está contenido en un flujo topológico asociado a $g$. Por ello, $\Gamma^{\prime}$ actúa sin punto fijo en $] 0,1[$. El lema precedente implica que $\Gamma$ es conjugado a un subgrupo (no conmutativo) del grupo afín.

Supongamos en lo que sigue que todo $g \in \Gamma^{\prime}$ fija puntos de $] 0,1[$. Usando el lema de Kopell, se verifica fácilmente la existencia de puntos fijos por $\Gamma^{\prime}$ en $] 0,1[$. Sea $] a, b\left[\right.$ una componente irreducible de $\Gamma^{\prime}$. Notemos que, para todo $h \in \Gamma$, el intervalo $h(] a, b[)$ también es una componente irreducible de $\Gamma^{\prime}$. En particular, si $\left.h(] a, b[) \neq\right] a, b[$ entonces $h(] a, b[) \cap] a, b[=\emptyset$.

Si $a=0$ ó $b=1$ entonces todo $f \in \Gamma$ fija $] a, b[$, lo cual contradice la hipótesis de ausencia de punto fijo en ]0,1[. Las componentes irreducibles de $\Gamma^{\prime}$ están por lo tanto contenidas en subintervalos compactos de $] 0,1[$. Si $f \in \Gamma$ fija una de estas componentes $] a, b[$, entonces el lema 4.75 muestra que la restricción de $f$ a $] a, b\left[\right.$ es afín en las coordenadas inducidas por $\Gamma^{\prime}$. El caso de los elementos que no fijan $] a, b[$ es más interesante.

Afirmación (i): si $f$ es un elemento cualquiera de $\Gamma$ que no fija $] a, b[$ y $u$ y $v$ son los puntos fijos de $f$ a izquierda y a derecha de $] a, b[$ respectivamente, entonces el intervalo ] $u, v$ [ es una componente irreducible de $\Gamma$ (es decir, $u=0$ y $v=1$ ). 
Supongamos lo contrario y sea $\bar{f} \in \Gamma$ un elemento que no fija $] u, v[$. Reemplazando $f$ por $f^{-1}$ si es necesario, podemos suponer que $f(x)>x$ para todo $x \in] u, v[$. Se tiene entonces $f(a) \geq b$. Para $n \in \mathbb{N}$, el elemento $f^{-1} \bar{f}^{-n} f \bar{f}^{n}$ pertenece a $\Gamma^{\prime}$, por lo que fija los puntos a y $u$. Luego,

$$
f \bar{f}^{n}(u)=\bar{f}^{n} f(u)=\bar{f}^{n}(u), \quad f \bar{f}^{n}(a)=\bar{f}^{n} f(a) \geq \bar{f}^{n}(b), \quad n \in \mathbb{N} .
$$

Se tiene $\bar{f} f \bar{f}^{-1}=f \bar{g}$ para cierto $\bar{g} \in \Gamma^{\prime}$. Por otra parte, $f \bar{g}$ no posee puntos fijos en $] u, v[$, y fija $u$ y $v$. Luego, $f$ no posee puntos fijos en $] \bar{f}(u), \bar{f}(v)[$, y fija $\bar{f}(u)$ y $\bar{f}(v)$. Esto muestra que $] \bar{f}(u), \bar{f}(v)[\cap] u, v[=\emptyset$. Reemplacemos $\bar{f}$ por $\bar{f}^{-1}$ si es necesario de manera que $\bar{f}(u)<u$. Notemos que la sucesión $\left(\bar{f}^{n}(u)\right)$ tiende hacia un punto fijo de $\bar{f}$. Se verifica entonces fácilmente que las relaciones (4.26) contradicen el lema 4.51 (aplicado a los elementos $h_{1}=\bar{f}$ y $h_{2}=f$ relativamente a los puntos $\left.x_{0}=\bar{f}^{-1}(u), y=a, z=b \leq f(a)\right)$. Esto concluye la demostración de la afirmación.

Designemos por $\Gamma_{1}^{*}$ al subgrupo normal de $\Gamma$ constituido por los elementos que fijan las componentes irreducibles de $\Gamma^{\prime}$. Como la afirmación (i) es válida para toda componente irreducible $] a, b\left[\right.$ de $\Gamma^{\prime}$, un elemento de $\Gamma$ pertenece a $\Gamma_{1}^{*}$ si y solamente si dicho elemento fija al menos una componente irreducible de $\Gamma^{\prime}$. La restricción de $\Gamma_{1}^{*}$ a toda componente irreducible de $\Gamma^{\prime}$ es afín en las coordenadas inducidas. Remarquemos que $\Gamma_{1}^{*}$ puede admitir componentes irreducibles contenidas en el complemento de la unión de las componentes irreducibles de $\Gamma^{\prime}$. Sin embargo, la restricción de $\Gamma_{1}^{*}$ a una componente tal es abeliana, y por lo tanto conjugada a un subgrupo del grupo de traslaciones. Concluimos así que $\Gamma_{1}^{*}$ es un subgrupo de un producto (a lo más numerable) de grupos conjugados a grupos de transformaciones afines. Además, el grupo cuociente $\Gamma / \Gamma_{1}^{*}$ actúa de manera libre sobre el conjunto $\operatorname{Fix}\left(\Gamma^{\prime}\right)$. Fijemos una componente irreducible $] a, b\left[\right.$ de $\Gamma^{\prime}$, y definamos una relación de orden $\prec$ sobre $\Gamma / \Gamma_{1}^{*}$ por $f_{1} \Gamma_{1}^{*} \prec f_{2} \Gamma_{1}^{*}$ cuando $f_{1}(] a, b[)$ está a izquierda de $f_{2}(] a, b[)$. Esta relación es total, bi-invariante y arquimediana. El argumento de la demostración del teorema de Hölder muestra entonces la existencia de una secuencia exacta

$$
0 \longrightarrow \Gamma_{1}^{*} \longrightarrow \Gamma \longrightarrow H \subset(\mathbb{R},+) \longrightarrow 0
$$

Notemos que la imagen $H$ es no trivial, pues $\Gamma$ no fija $] a, b[$. La proposición 4.2 implica entonces la validez de la afirmación siguiente.

Afirmación (ii): el grupo $H$ es cíclico infinito.

De lo que precede concluimos la existencia de una sucesión exacta

$$
0 \longrightarrow \Gamma_{1}^{*} \longrightarrow \Gamma \longrightarrow(\mathbb{Z},+) \longrightarrow 0 .
$$

La demostración del teorema 4.69 se completa con la siguiente afirmación.

Afirmación (iii): el grupo $\Gamma_{1}^{*}$ es en realidad un subgrupo de un producto de grupos conjugados a grupos de traslaciones. 
Para verificar esto, fijemos un elemento $g \in \Gamma^{\prime}$ cuya restricción a una componente irreducible $] a, b\left[\right.$ de $\Gamma^{\prime}$ no sea trivial. Debemos mostrar que no existe ningún elemento $h \in \Gamma$ que fije $] a, b[\mathrm{y}$ tal que las restricciones de $g$ y $h$ a dicho intervalo generen un grupo no conmutativo. Supongamos lo contrario y sea $f \in \Gamma$ un elemento cuya imagen en $\Gamma / \Gamma_{1}^{*}$ genere $(\mathbb{Z},+)$. Cambiando $f$ por $f^{-1}$ si es necesario, podemos suponer que $f(b) \leq a$. Puesto que $\Gamma$ es metabeliano, para todo $n \in \mathbb{N}$ existe $t_{n} \in \mathbb{R}$ tal que las restricciones de $f^{-n} h f^{n}$ y $h g^{t_{n}}$ al intervalo $] a, b[$ coinciden. Para $\delta=V(f ;[0, b])>0$ la desigualdad (4.2) permite probar que para todo $x \in] a, b[$ se tiene

$\left(h g^{t_{n}}\right)^{\prime}(x)=\left(f^{-n} h f^{n}\right)^{\prime}(x) \leq \frac{\left(f^{n}\right)^{\prime}(x)}{\left(f^{n}\right)^{\prime}\left(f^{-n} h f^{n}(x)\right)} \sup _{y \in] 0, f^{n}(b)[} h^{\prime}(y) \leq e^{\delta} \sup _{y \in] 0, f^{n}(b)[} h^{\prime}(y)$,

así como

$$
\left(h g^{t_{n}}\right)^{\prime}(x) \geq e^{-\delta} \cdot \inf _{y \in] 0, f^{n}(b)[} h^{\prime}(y) .
$$

De (4.27) se concluye que

$$
\sup _{y \in] a, b[}\left(g^{t_{n}}\right)^{\prime}(x) \leq e^{\delta} \cdot \frac{\sup _{y \in] 0, b[} h^{\prime}(y)}{\inf _{y \in] a, b[} h^{\prime}(y)} .
$$

Esto implica de manera evidente que $\left(t_{n}\right)$ es una sucesión acotada. Tomemos una subsucesión $\left(t_{n_{k}}\right)$ que converja a un límite $T \in \mathbb{R}$. Puesto que $h$ fija cada intervalo $\left[f^{n}(a), f^{n}(b)\right]$, se tiene $h^{\prime}(0)=1$. Integrando (4.27) y (4.28) obtenemos, para todo $k$ suficientemente grande y todo $x \in] a, b[$,

$$
(x-a) / 2 e^{\delta} \leq h g^{t_{n_{k}}}(x)-a \leq 2 e^{\delta}(x-a),
$$

y pasando al límite cuando $k$ tiende al infinito se concluye que

$$
(x-a) / 2 e^{\delta} \leq h g^{T}(x)-a \leq 2 e^{\delta}(x-a) .
$$

Ahora bien, el argumento precedente sigue siendo válido cuando se reemplaza $h$ por $h^{j}$, cualquiera sea $j \in \mathbb{N}$ (ya que la constante $\delta$ depende sólo de $f$ ). Se obtiene así, para todo $x \in] a, b[$ y todo $j \in \mathbb{N}$,

$$
(x-a) / 2 e^{\delta} \leq\left(h g^{T}\right)^{j}(x)-a \leq 2 e^{\delta}(x-a),
$$

lo cual es evidentemente imposible. Esto concluye la demostración de la afirmación (iii), y por lo tanto aquélla del teorema 4.69 .

Es interesante notar que si un subgrupo soluble de Difeo ${ }_{+}^{1+v a}([0,1[)$ tiene orden de solubilidad superior a 2 entonces posee elementos no triviales con infinitos puntos fijos en cualquier vecindad de 0 . Por esta razón, dichos elementos no pueden ser difeomorfismos real-analíticos. De hecho, todo grupo soluble de difeomorfismos real-analíticos del intervalo es topológicamente conjugado a un subgrupo del grupo afín. El lector encontrará, además de éste, otros resultados interesantes al respecto en [140] (vea también 
$[32,148])$. Finalizamos esta sección observando que los resultados descritos para subgrupos solubles de Difeo ${ }_{+}^{1+v a}([0,1[)$ continúan siendo válidos (y de hecho pueden ser refinados) en el contexto de los subgrupos del grupo $\mathrm{AfP}_{+}([0,1[)$ de los homeomorfismos afines por partes del intervalo.

Ejercicio 4.76. Pruebe que si $\Gamma$ es un subgrupo no trivial de $\operatorname{AfP}_{+}([0,1[)$ que actúa libremente sobre $] 0,1[$, entonces $\Gamma$ es cíclico infinito. Usando esto, pruebe que todo subgrupo metabeliano y finitamente generado de $\operatorname{AfP}_{+}([0,1])$ es isomorfo a un producto semidirecto entre $(\mathbb{Z},+$ ) y una suma directa (a lo más numerable) de grupos isomorfos a $(\mathbb{Z},+)$ y que actúan sobre intervalos dos a dos disjuntos. Enuncie y pruebe un resultado de clasificación general para los subgrupos solubles y finitamente generados de $\operatorname{AfP}_{+}([0,1])$ (vea [146] para más detalles; vea también el capítulo 3 de [13] para un resultado interesante sobre los subgrupos no solubles de $\mathrm{AfP}_{+}([0,1])$, así como [27] y [157] para otros desenvolvimientos en la misma dirección).

Ejercicio 4.77. Pruebe que todo grupo soluble de gérmenes de homeomorfismos afines por partes del intervalo es abeliano.

\subsection{El caso de la recta}

Si $\Gamma$ es un grupo soluble de orden de solubilidad $k$, denotemos por $\{i d\}=\Gamma_{k}^{\mathrm{sol}} \triangleleft \ldots \triangleleft \Gamma_{0}^{\mathrm{sol}}=\Gamma$ su serie derivada, es decir, $\Gamma_{i}^{\mathrm{sol}}=\left[\Gamma_{i-1}^{\mathrm{sol}}, \Gamma_{i-1}^{\mathrm{sol}}\right]$ para todo $i \in\{1, \ldots, k\}$. El resultado a seguir debe ser comparado con el teorema 2.61 .

Proposición 4.78. Sea $\Gamma$ un subgrupo soluble de $\mathrm{Homeo}_{+}(\mathbb{R})$ de orden de solubilidad $k$. Si existe un indice $i \leq k$ para el cual $\Gamma_{i}^{\text {sol }}$ preserva una medida de Radon $v_{i}$ tal que $\tau_{v_{i}}\left(\Gamma_{i}^{\mathrm{sol}}\right) \neq\{0\}$, entonces existe una medida de Radon sobre la recta que es quasi-invariante por $\Gamma$.

Demostración. Sea $j<k$ el mínimo de los índices para los cuales $\Gamma_{j}^{\text {sol }}$ preserva una medida de Radon, y designemos por $v_{j}$ esta medida. Usando la hipótesis y (2.7), no es difícil verificar que $\tau_{v_{j}}\left(\Gamma_{j}^{\text {sol }}\right) \neq\{0\}$. Por el lema 2.58 se tiene $\kappa\left(\Gamma_{j-1}^{\text {sol }}\right) \neq\{1\}$, y por el lema 2.59 esto implica que $v_{j}$ es quasi-invariante por $\Gamma_{j-1}^{\mathrm{sol}}$. El lema 2.60 permite probar por inducción que $v_{j}$ es quasi-invariante por $\Gamma_{j-2}^{\mathrm{sol}}, \Gamma_{j-3}^{\mathrm{sol}}$, etc. Se concluye así que $v_{j}$ es quasiinvariante por $\Gamma$.

Daremos a continuación la demostración del teorema 4.73 (asumiendo el teorema 4.70). Fijemos un subgrupo soluble $\Gamma$ de $\operatorname{Difeo}_{+}^{1+\text { va }}(\mathbb{R})$ con orden de solubilidad $k \geq 2$. Si $\Gamma$ possee puntos fijos globales, entonces los teoremas 4.69 y 4.70 implican que $\Gamma$ es un subgrupo de un producto (a lo más numerable) de grupos de la familia $\mathcal{R}(k)$ de modo que al menos uno de los factores no pertenece a $\mathcal{R}(k-1)$. Supongamos en lo que sigue que $\Gamma$ no posea puntos fijos. Comenzamos con la siguiente proposición, la que debe ser comparada con los resultados del final de la sección 2.5 del capítulo 2 . 
Proposición 4.79. Todo subgrupo soluble de $\operatorname{Difeo}_{+}^{1+\mathrm{va}}(\mathbb{R})$ deja quasiinvariante una medida de Radon sobre la recta.

Demostración. Consideremos el índice $j$ para el cual $\Gamma_{j}^{\text {sol }}$ possee puntos fijos sin que $\Gamma_{j-1}^{\text {sol }}$ posea tales puntos. Pueden presentarse dos casos.

Primer caso: el índice $j$ es igual a $k$.

Afirmamos que $\Gamma_{k-1}^{\mathrm{sol}}$ preserva una medida de Radon tal que la función número de traslación respecto a ella no es idénticamente nula. En efecto, como $\Gamma_{k-1}^{\text {sol }}$ es conmutativo, del lema de Kopell se concluye fácilmente la existencia de elementos de $\Gamma_{k-1}^{\text {sol }}$ sin puntos fijos, por lo que la proposición 2.56 implica la existencia de una medida de Radon invariante por $\Gamma_{k-1}$ (y tal que el número de traslación asociado es un homomorfismo no trivial). La existencia de una medida de Radon $v$ sobre la recta que sea quasi-invariante por $\Gamma$ se desprende entonces de la proposición 4.78.

Segundo caso: el índice $j$ es menor a $k$.

Notemos que, en virtud de la hipótesis de ausencia de punto fijo global para la acción de $\Gamma$, este índice $j$ positivo. Fijemos una componente irreducible cualquiera $] p_{j}, q_{j}\left[\operatorname{de} \Gamma_{j}^{\mathrm{sol}}\right.$.

Afirmación (i): si $\bar{f}_{1}$ y $\bar{f}_{2}$ son elementos de $\Gamma_{j-1}^{\text {sol }}$ que poseen puntos fijos y que no fijan $] p_{j}, q_{j}[$, entonces los puntos fijos en $\mathbb{R} \cup\{-\infty,+\infty\}$ de dichos elementos (inmediatamente) a izquierda y a derecha de $] p_{j}, q_{j}$ [ coinciden.

Para probar esto, sean $p$ y $q$ los puntos fijos de $\bar{f}_{1}$ a izquierda y a derecha de $\left[p_{j}, q_{j}\right]$ respectivamente. Supongamos que $\bar{f}_{2}$ no fije $[p, q]$. Para cada $n \in \mathbb{Z}$ existe $\bar{g} \in \Gamma_{j}^{\text {sol }}$ tal que $\bar{f}_{2}^{n} \bar{f}_{1} \bar{f}_{2}^{-n}=\bar{f}_{1} \bar{g}$. Como $\bar{f}_{1} \bar{g}$ no posee puntos fijos en $] p, q\left[\right.$ y fija $p$ y $q$, el elemento $\bar{f}_{1}$ fija el intervalo $\bar{f}_{2}^{n}(] p, q[)$ y no posee puntos fijos al interior de él. Se deduce entonces que los intervalos $\bar{f}_{2}^{n}(] p, q[)(\operatorname{con} n \in \mathbb{Z})$ son dos a dos disjuntos. Cambiando $\bar{f}_{2}$ por su inverso si es necesario, podemos suponer que $\bar{f}_{2}^{n}\left(p_{j}\right)$ tiende a un punto fijo de $\bar{f}_{2}$ cuando $n$ tiende al infinito. Obtenemos así una contradicción al aplicar los argumentos de la prueba de la afirmación (i) de la sección 3.2 de este capítulo.

Definamos el intervalo $\left[p_{j-1}^{*}, q_{j-1}^{*}\right]$ como siendo igual a $\left[p_{j}, q_{j}\right]$ si todo elemento de $\Gamma_{j-1}^{\text {sol }}$ que no fija $\left[p_{j}, q_{j}\right]$ no posee punto fijo; en caso contrario, escojamos $f_{j} \in \Gamma_{j-1}^{\text {sol }}$ que posea puntos fijos y que no fije $\left[p_{j}, q_{j}\right]$, y definamos $p_{j-1}^{*}$ y $q_{j-1}^{*}$ como siendo los puntos fijos de $f_{j}$ a izquierda y a derecha de $\left[p_{j}, q_{j}\right]$ respectivamente. Denotemos finalmente por $\Gamma_{j-1}^{*}$ al estabilizador de $\left[p_{j-1}^{*}, q_{j-1}^{*}\right]$ en $\Gamma_{j-1}$. Se trata de un subgrupo normal de $\Gamma_{j-1}$ formado por los elementos de $\Gamma_{j-1}$ que poseen puntos fijos sobre la recta.

Afirmación (ii): el grupo $\Gamma_{j-1}^{\mathrm{sol}}$ preserva una medida de Radon y no posee puntos fijos.

En efecto, como la afirmación (i) es válida para toda componente irreducible de $\Gamma_{j}$, la acción de $\Gamma_{j-1}^{\text {sol }} / \Gamma_{j-1}^{*}$ sobre $\operatorname{Fix}\left(\Gamma_{j-1}^{*}\right)$ es libre. El argumento 
de la prueba del teorema de Hölder permite entonces demostrar que $\Gamma_{j-1}^{\mathrm{sol}}$ fija una medida de Radon cuyo soporte está contenido en $\operatorname{Fix}\left(\Gamma_{j-1}^{*}\right)$. El que $\Gamma_{j-1}^{\text {sol }}$ no posea puntos fijos se desprende de la definición de $j$.

Finalmente, como la función número de traslación respecto a la medida de Radon invariante por $\Gamma_{j-1}^{\text {sol }}$ no es idénticamente nula, la proposición 4.78 permite concluir la prueba de la proposición para el segundo caso.

Estamos ahora en condiciones de concluir la demostración del teorema 4.73. Para ello, fijemos una medida de Radon $v$ que sea quasi-invariante por $\Gamma$, y consideremos la relación de equivalencia $\sim$ que identifica dos puntos si ellos pertenecen a la clausura de una misma componente conexa del complemento del soporte de $v$. El espacio cuociente $\mathbb{R} / \sim$ es topológicamente una recta sobre la cual el grupo $\Gamma$ actúa de manera natural por homeomorfismos. Si la medida $v$ no posee átomos, entonces ella induce una medida de Radon $\bar{v}$ sobre $\mathbb{R} / \sim$ cuyo soporte es total, que no posee átomos, y que es quasi-invariante por la acción de $\Gamma$. La proposición 1.4 implica que esta última acción es topológicamente conjugada a aquélla de un grupo de transformaciones afines. La acción original de $\Gamma$ sobre la recta es por lo tanto semiconjugada a una acción por transformaciones afines.

Resta el caso en que $v$ posee átomos. Observemos primeramente que $\Gamma^{\prime}$ actúa preservando $v$, por lo que el segundo grupo derivado $\Gamma^{\prime \prime}$ fija cada átomo de $v$. Este argumento prueba en particular que el índice $j$ considerado en la demostración de la proposición 4.79 es (en el caso presente) igual a 1 ó a 2 . Veremos a continuación que es necesariamente igual a 1.

Denotemos por $\Gamma_{v}$ al subgrupo de $\Gamma$ formado por los elementos que fijan $v$, y designemos por $\Gamma_{v}^{\prime}$ su grupo derivado. Los elementos de $\Gamma_{v}^{\prime}$ fijan los átomos de $v$. Denotemos por $\Gamma_{v}^{*}$ al subgrupo normal de $\Gamma$ formado por los elementos que fijan las componentes irreducibles de $\Gamma_{v}^{\prime}$. Los argumentos de las demostraciones de las afirmaciones (i) y (ii) de la proposición 4.79 prueban que $\Gamma_{v} / \Gamma_{v}^{*}$ es isomorfo a un subgrupo no trivial $H$ de $(\mathbb{R},+)$. Este subgrupo $H$ no puede ser denso. En efecto, en caso contrario habrían átomos de $v$ de la misma masa acumulándose sobre ciertos puntos de la recta, contradiciendo el hecho que $v$ es una medida de Radon.

El grupo $H$ es por lo tanto infinito cíclico, y como $\Gamma$ actúa por automorfismos de $H$, sus elementos deben preservar necesariamente $v$. Por lo tanto, se tiene la igualdad $\Gamma=\Gamma_{v}$ (esto prueba que $j=1$ ). Se deduce entonces que $\Gamma$ es una extensión por $(\mathbb{Z},+)$ de un subgrupo soluble de un producto de grupos de difeomorfismos de intervalos cerrados. Se cumple así lo estipulado en la afirmación (iii) del enunciado del teorema, con lo que la prueba del teorema 4.73 está concluida. 


\section{Un paréntesis en la clasificación}

Sobre la base de las secciones precedentes, sería natural continuar la clasificación de los grupos de difeomorfismos del intervalo (y del círculo) tratando de describir de manera satisfactoria la dinámica de los grupos promediables. Sin embargo, este problema parece ser muy complejo, y no se dispone de ningún resultado realmente significativo en tal dirección. Para corroborar este punto señalemos que, de acuerdo a la sección 5.2 del primer capítulo, el grupo de Thompson F se incrusta en Difeo $\infty_{+}^{\infty}([0,1])$. Por otro lado, el problema de saber si $\mathrm{F}$ es o no promediable se ha mantenido abierto por casi 30 años. A modo sólo de completitud presentaremos a continuación algunos resultados parciales en torno al problema de la clasificación de cierta familia particular de subgrupos promediables de $\operatorname{Difeo}_{+}^{2}([0,1])$.

Recuerde que la promediabilidad es estable por operaciones elementales, es decir, ella se preserva al pasar a un subgrupo, a un cuociente, al realizar una extensión o tomar una reunión (vea el ejercicio 5.67). Siguiendo [162], denotemos por SP la familia más pequeña de grupos promediables que es cerrada respecto a estas operaciones elementales y que contiene a los grupos de crecimiento subexponencial (vea el ejercicio 5.69). Los elementos de SP no pueden aparecer como grupos de difeomorfismos real-analíticos del intervalo, salvo en casos muy particulares. La demostración (elemental) del resultado a seguir puede ser hallada en [148].

Teorema 4.80. Si un subgrupo de Difeo $_{+}^{\mathrm{w}}([0,1[)$ pertenece a la familia $\mathrm{SP}$, entonces dicho grupo es metabeliano.

Dentro de Difeo $_{+}^{\infty}([0,1])$ pueden sin embargo aparecer grupos de la familia SP de cierto interés algebraico. La siguiente construcción debiese ser comparada con la de un ejemplo que aparece en [166].

Ejemplo 4.81. Dados cuatro puntos $a<c<d<b$ en $] 0,1$ [, sea $f$ un difeomorfismo de clase $\mathrm{C}^{\infty}$ de $[a, b]$ infinitamente tangente a la identidad en los extremos y tal que $f(c)=d$. Extendamos $f$ a $[0,1]$ haciendo $f(x)=x$ para $x \notin] a, b[$. Sea $g$ un difeomorfismo de clase $\mathrm{C}^{\infty}$ de $[0,1]$ infinitamente tangente a la identidad en los extremos, con un único punto fijo en el interior, y tal que $g(c)=a$ y $g(d)=b$.

Denotemos por $\Gamma$ al subgrupo de $\operatorname{Difeo}_{+}^{\infty}([0,1])$ generado por $f$ y $g$. Para cada $n \in \mathbb{N}$, el subgrupo $\Gamma_{n}$ de $\Gamma$ generado por $\left\{f_{i}=g^{-i} f g^{i}:|i| \leq n\right\}$ es soluble de orden de solubilidad $2 n+1$. Además, el subgrupo $\Gamma^{*}=\cup_{n \in \mathbb{N}} \Gamma_{n}$ es normal en $\Gamma$, y el cuociente $\Gamma / \Gamma^{*}$ es isomorfo a $(\mathbb{Z},+)$ (su generador es $g \Gamma^{*}$ ). El grupo $\Gamma$ es por lo tanto finitamente generado y no soluble, y pertenece a la familia SP. Este grupo puede ser presentado algebraicamente como una extensión HNN de una reunión de productos en corona. Visto de esa manera, su geometría resulta ser muy especial: vea [61] por un fenómeno interesante relacionado con sus sucesiones de Følner.

Pese a lo anterior, los subgrupos de $\operatorname{Difeo}_{+}^{1+v a}([0,1[)$ que pertenecen a la familia SP pueden ser parcialmente clasificados. Para esto definamos por inducción transfinita las siguientes subfamilias $\mathrm{SP}_{\alpha}$ de $\mathrm{SP}$ : 
(i) $\mathrm{SP}_{1}$ es la familia de los grupos contables cuyos subgrupos finitamente generados tienen crecimiento subexponencial;

(ii) si $\alpha$ no es un ordinal límite, $\mathrm{SP}_{\alpha}$ es la familia de los grupos obtenidos como cuociente o subgrupo de un grupo de $\mathrm{SP}_{\alpha-1}$, o por una extensión

$$
0 \longrightarrow G \longrightarrow \Gamma \longrightarrow H \longrightarrow 0,
$$

donde $G \in \mathrm{SP}_{\alpha-1}$ y $\Gamma / G \sim H \in \mathrm{SP}_{\alpha-1}$;

(iii) si $\alpha$ es un ordinal límite, entonces $\mathrm{SP}_{\alpha}$ es la familia de los grupos obtenidos como reunión de grupos en $\cup\left\{\mathrm{SP}_{\beta}: \beta<\alpha\right\}$.

Un grupo $\Gamma$ pertenece a $\mathrm{SP}$ si y sólo si pertenece a $\mathrm{SG}_{\alpha}$ para algún ordinal $\alpha$ [162]. Por ejemplo, el grupo del ejemplo 4.81 pertenece a $\mathrm{SG}_{\alpha+1}$, donde $\alpha$ es el primer ordinal infinito. La demostración del resultado a seguir, basada sobre los resultados de la sección 3 de este capítulo, puede ser hallada en [146] (vea también [23] para una discusión más detallada del caso afín por partes).

Teorema 4.82. Todo subgrupo $\Gamma$ de $\mathrm{Difeo}_{+}^{1+\mathrm{va}}\left(\left[0,1[)\right.\right.$ perteneciente a $\mathrm{SP}_{\alpha}$ para algún $\alpha \in \mathbb{N}$ es soluble con orden de solubilidad menor o igual a $\alpha$.

Los teoremas 4.80 y 4.82 se aplican a familias especiales de grupos promediables. En efecto, de [9] se desprende que la familia SP es más pequeña que la de los grupos promediables. Se produce por lo tanto un quiebre en la clasificación algebraica de los subgrupos de Difeo ${ }_{+}^{1+\mathrm{va}}\left(\mathrm{S}^{1}\right)$. Es por ello que en el capítulo siguiente trabajaremos en una "dirección contraria" considerando acciones de grupos que, dadas sus propiedades cohomológicas, no pueden ser promediables salvo en el caso en que esas acciones sean "triviales". 


\section{Capítulo 5}

\section{Estructura Algebraicay Rigidez: Métodos Cohomológicos}

\section{El teorema de estabilidad de Thurston}

El resultado presentado a continuación corresponde a una versión parcial de un famoso teorema debido a Thurston. Para una versión más general (relacionada con el célebre teorema de estabilidad de Reeb para foliaciones) se puede consultar la referencia original [198], así como [39]. Recomendamos en todo caso al lector tratar de resolver el ejercicio 5.10.

Diremos que un grupo topológico $\Gamma$ es compactamente generado si existe una vecindad relativamente compacta $V$ de $i d \in \Gamma$ tal que todo elemento de $\Gamma$ puede ser expresado como un producto de elementos de $V$.

Teorema 5.1. Sea $\Gamma$ un grupo topológico compactamente generado. Si $\Gamma$ no admite ningún homomorfismo continuo no trivial sobre $(\mathbb{R},+)$, entonces toda representación $\Phi: \Gamma \rightarrow \operatorname{Difeo}_{+}^{1}([0,1])$ es trivial.

Para la demostración de este teorema la noción de $(B, \varepsilon)$-homomorfismo será esencial. Dados $B \subset \Gamma$ y $\varepsilon \geq 0$, un $(B, \varepsilon)$-homomorfismo (sobre $(\mathbb{R},+)$ ) es una función continua $\phi: B \rightarrow \mathbb{R}$ satisfaciendo la desigualdad $|\phi(g)+\phi(h)-\phi(g h)| \leq \varepsilon$ para todo $g, h$ en $B$ tales que $g h \in B$. Fijemos una vecindad compacta y simétrica $\mathcal{G}$ de la identidad que genere a $\Gamma$. Diremos que $\phi$ está normalizado si $\max _{g \in \mathcal{G}}|\phi(g)|=1$. Por simplicidad, utilizaremos la notación $\nabla \phi(g, h)=\phi(g)+\phi(h)-\phi(g h)$. Observe que una aplicación continua $\phi: \Gamma \rightarrow \mathbb{R}$ es un $(\Gamma, 0)$-homomorfismo si y sólo si $\nabla \phi$ es idénticamente nula, lo que es equivalente a que $\phi$ sea un homomorfismo (continuo) sobre $(\mathbb{R},+)$. Recordemos que $B_{\mathcal{G}}(k)$ designa al conjunto de los 
elementos de $\Gamma$ que pueden ser expresados como producto de (a lo más) $k$ elementos de $\mathcal{G}$.

Lema 5.2. Sea $\Gamma$ un grupo generado por una vecindad compacta de $i d \in \Gamma$. Si para cada $k \in \mathbb{N}$ existe un $\left(B_{\mathcal{G}}(k), 1 / k\right)$-homomorfismo normalizado, entonces existe un homomorfismo continuo no trivial de $\Gamma$ en $(\mathbb{R},+)$.

Demostración. Para cada $k \in \mathbb{N}$ sea $\phi_{k}$ un $\left(B_{\mathcal{G}}(k), 1 / k\right)$-homomorfismo normalizado. Es fácil probar por inducción que $\left|\phi_{k}(g)\right| \leq k(1+\varepsilon)$ para todo $g \in B_{\mathcal{G}}(k)$. En particular, por la compacidad de $\mathcal{G}$, existe una subsucesión $\left(\phi_{k}^{1}\right)$ de $\phi_{k}$ tal que $\left.\phi_{k}^{1}\right|_{B_{\mathcal{G}}(1)}$ converge (puntualmente) a una función normalizada de $B_{\mathcal{G}}(1)$ en $\mathbb{R}$ (a priori, no necesariamente continua). Procediendo inductivamente, para cada $i \in \mathbb{N}$ podemos hallar una subsucesión $\left(\phi_{k}^{i+1}\right)_{k \in \mathbb{N}}$ de $\left(\phi_{k}^{i}\right)_{k \in \mathbb{N}}$ tal que $\left.\phi_{k}^{i+1}\right|_{B_{\mathcal{G}}(i+1)}$ converge a una función normalizada de $B_{\mathcal{G}}(i+1)$ en $\mathbb{R}$.

La sucesión $\left(\phi_{k}^{k}\right)$ converge (puntualmente) a una función $\phi: \Gamma \rightarrow \mathbb{R}$. Por construcción, $\phi$ es un homomorfismo sobre $(\mathbb{R},+)$; además, este homomorfismo es no trivial, pues está normalizado. Resta entonces probar que $\phi$ es continuo. Para ello, observe que para cada $n \in \mathbb{N}$ existe una vecindad compacta $V$ del elemento neutro $i d$ en $\Gamma$ tal que $V^{n} \subset \mathcal{G}$. En particular, $|n \phi(g)|=\left|\phi\left(g^{n}\right)\right| \leq 1$ para todo $g \in V$. En otras palabras, si $g$ pertenece a $V$ entonces $|\phi(g)| \leq 1 / n$, lo cual demuestra la continuidad de $\phi$ en $i d \in \Gamma$. Puesto que $\phi$ es un homomorfismo, la continuidad en un punto nos da la continuidad global.

Sea $\Phi: \Gamma \rightarrow$ Difeo $_{+}^{1}([0,1])$ una representación continua no trivial, y sea $x \in[0,1$ [ un punto de la frontera del conjunto de puntos fijos por $\mathcal{G}$ (equivalentemente, por $\Gamma$ ). Para cada $y \in[0,1[$ que no es fijo por $\mathcal{G}$ consideramos la función

$$
\phi_{y}(g)=\frac{1}{C(y)}[\Phi(g)(y)-y]
$$

donde $C(y)=\sup _{g \in \mathcal{G}}|\Phi(g)(y)-y|$.

Lema 5.3. Aparte de las condiciones anteriores, suponga además que $\Phi(g)^{\prime}(x)=1$ para todo $g \in \Gamma$. Entonces para cada $n \in \mathbb{N}$ y cada $\varepsilon>0$ existe $\delta>0$ tal que si $|x-y|<\delta$ entonces $\left.\phi_{y}\right|_{B_{\mathcal{G}}(n)}$ es un $\left(B_{\mathcal{G}}(n), \varepsilon\right)$-homomorfismo normalizado.

Demostración. La idea de la demostración consiste en hacer notar que, para $y$ cercano a $x$ y no fijo por $\mathcal{G}$, la aplicación $\phi_{y}$ se comporta infinitesimalmente como un homomorfismo de $\Gamma$ en $(\mathbb{R},+)$.

Para $k \in \mathbb{N}$ y $\varepsilon^{\prime}>0$ definimos inductivamente

$$
\lambda_{0}\left(0, \varepsilon^{\prime}\right)=0, \quad \lambda_{0}\left(k+1, \varepsilon^{\prime}\right)=1+\lambda_{0}\left(k, \varepsilon^{\prime}\right)\left(1+\varepsilon^{\prime}\right) .
$$

Sea $\epsilon^{\prime}>0$ suficientemente pequeño de manera que $\varepsilon^{\prime} \lambda_{0}\left(n, \varepsilon^{\prime}\right) \leq \varepsilon$, y sea $\delta^{\prime}>0$ tal que $\left|\Phi(g)^{\prime}(y)-1\right| \leq \varepsilon^{\prime}$ para todo $g \in B_{\mathcal{G}}(n)$ y todo punto $y \in[0,1]$ 
que verifica $|x-y| \leq \delta^{\prime}$. Finalmente, sea $\left.\delta \in\right] 0, \delta^{\prime}[$ tal que, si $|x-y| \leq \delta$, entonces $|\Phi(g)(y)-x| \leq \delta^{\prime}$ para todo $g \in B_{\mathcal{G}}(n)$. Afirmamos que este parámetro $\delta$ verifica la afirmación del lema.

Probemos en primer lugar que, para todo $k \leq n$ y todo $g \in B_{\mathcal{G}}(k)$,

$$
\left|\phi_{y}(g)\right| \leq \lambda_{0}\left(k, \varepsilon^{\prime}\right) .
$$

En efecto, esta desigualdad es evidente para $k=0$ ó $k=1$. Asumámosla para $k=i$. Si $g$ es un elemento de $B_{\mathcal{G}}(i+1)$ entonces $g=h_{1} h_{2}$ para ciertos $h_{1} \in \mathcal{G}$ y $h_{2} \in B_{\mathcal{G}}(i)$. Luego,

$$
\left|\phi_{y}(g)\right|=\frac{1}{C(y)}|\Phi(g)(y)-y| \leq \frac{1}{C(y)}\left|\Phi\left(h_{1}\right) \Phi\left(h_{2}\right)(y)-\Phi\left(h_{2}\right)(y)\right|+\left|\phi_{y}\left(h_{2}\right)\right| .
$$

Por otra parte, de la igualdad

$$
\Phi\left(h_{1}\right) \Phi\left(h_{2}\right)(y)-\Phi\left(h_{2}\right)(y)=\int_{y}^{\Phi\left(h_{2}\right)(y)}\left[\Phi\left(h_{1}\right)^{\prime}(s)-1\right] d s+\left[\Phi\left(h_{1}\right)(y)-y\right]
$$

se deduce

$$
\frac{1}{C(y)}\left|\Phi\left(h_{1}\right) \Phi\left(h_{2}\right)(y)-\Phi\left(h_{2}\right)(y)\right| \leq \max _{|s-x| \leq \delta^{\prime}}\left|\Phi\left(h_{1}\right)^{\prime}(s)-1\right| \cdot \frac{1}{C(y)}\left|\Phi\left(h_{2}\right)(y)-y\right|+1 .
$$

De (5.2) y de la hipótesis de inducción concluimos que

$$
\left|\phi_{y}(g)\right| \leq \varepsilon^{\prime} \lambda_{0}\left(i, \varepsilon^{\prime}\right)+1+\lambda_{0}\left(i, \varepsilon^{\prime}\right)=\lambda_{0}\left(i+1, \varepsilon^{\prime}\right),
$$

lo cual cierra la demostración de (5.1).

Estimemos ahora el valor de $\nabla \phi_{y}$. Para $h_{1}, h_{2}$ en $B_{\mathcal{G}}(n)$ tales que $h_{1} h_{2}$ pertenece a $B_{\mathcal{G}}(n)$ tenemos la igualdad

$$
\nabla \phi_{y}\left(h_{1}, h_{2}\right)=\frac{1}{C(y)}\left[\Phi\left(h_{1}\right)(y)-y+\Phi\left(h_{2}\right)(y)-y-\Phi\left(h_{1} h_{2}\right)(y)+y\right],
$$

es decir

$$
\begin{aligned}
\nabla \phi_{y}\left(h_{1}, h_{2}\right) & =-\frac{1}{C(y)}\left[\Phi\left(h_{1}\right) \Phi\left(h_{2}\right)(y)-\Phi\left(h_{2}\right)(y)-\left(\Phi\left(h_{1}\right)(y)-y\right)\right] \\
& =-\frac{1}{C(y)} \int_{y}^{\Phi\left(h_{2}\right)(y)}\left[\Phi\left(h_{1}\right)^{\prime}(s)-1\right] d s,
\end{aligned}
$$

por lo que

$$
\left|\nabla \phi_{y}\left(h_{1}, h_{2}\right)\right| \leq\left|\frac{1}{C(y)}\left[\Phi\left(h_{2}\right)(y)-y\right]\right| \cdot \sup _{|s-x| \leq \delta^{\prime}}\left|\Phi\left(h_{1}\right)^{\prime}(s)-1\right| \leq \lambda_{0}\left(n, \varepsilon^{\prime}\right) \varepsilon^{\prime} \leq \varepsilon .
$$

Luego, $\left.\phi_{y}\right|_{B_{\mathcal{G}}(n)}$ es un $\left(B_{\mathcal{G}}(n), \varepsilon\right)$-homomorfismo normalizado. 
Demostración del teorema 5.1. Supongamos que $\Phi: \Gamma \rightarrow \operatorname{Difeo}_{+}^{1}([0,1])$ sea una representación continua no trivial, y sea $x \in[0,1[$ un punto de la frontera del conjunto de los puntos fijos por $\Gamma$. La acción inducida de $\Gamma$ sobre el espacio tangente (unidimensional) $T_{x}\left(\mathrm{~S}^{1}\right)$ origina de manera natural un homomorfismo (continuo) de $\Gamma$ en $(\mathbb{R},+)$. Por hipótesis, este homomorfismo debe ser trivial, por lo que estamos bajo las condiciones del lema 5.3. La conclusión de dicho lema y del anterior a él nos indican que $\Gamma$ admite un homomorfismo continuo no trivial sobre $(\mathbb{R},+)$, lo cual contradice nuestra hipótesis. Luego, $\Phi$ debe ser trivial.

Observe que no hemos utilizado la hipótesis de estar considerando una acción sobre el intervalo de manera esencial. En efecto, es fácil ver que el teorema de Thurston es aún válido (con la misma demostración) para subgrupos del grupo de gérmenes de difeomorfismos $\mathcal{G}_{+}^{1}(\mathbb{R}, 0)$.

Ejercicio 5.4. Pruebe directamente el teorema de Thurston para grupos no necesariamente finitamente generados de gérmenes de difeomorfismos real-analíticos analizando los coeficientes correspondientes a los desarrollos en serie de los gérmenes en torno al origen.

Finalizaremos esta sección con un ejemplo explícito que muestra que el teorema de estabilidad de Thurston no se extiende a acciones por homeomorfismos (vea también la observación 5.7). Sea $\bar{G}$ el grupo de presentación $\bar{G}=\left\langle f, g, h: f^{2}=g^{3}=h^{7}=f g h\right\rangle$. Dejamos al lector la tarea de verificar que todo homomorfismo de $\bar{G}$ sobre $(\mathbb{R},+)$ es trivial. Esto no es extraño, pues $\bar{G}$ es el grupo fundamental de una "esfera homológica", es decir, de una variedad compacta de dimensión tres con homología nula y no homeomorfa a la esfera (vea [198]).

Para construir una acción no trivial de $\bar{G}$ sobre $\mathrm{S}^{1}$ con un punto fijo, consideremos el "embaldosado" del disco de Poincaré por triángulos hiperbólicos de ángulos $\pi / 2, \pi / 3$ y $\pi / 7$. La imagen en $\mathrm{PSL}(2, \mathbb{R})$ del subgrupo de $\operatorname{PSL}(2, \mathbb{R})$ que preserva este embaldosado es un grupo isomorfo a $\bar{G}$. Como $\widetilde{\operatorname{PSL}}(2, \mathbb{R})$ actúa sobre $\widetilde{\mathrm{S}^{1}}=\mathbb{R}$, añadiendo un punto en el infinito a $\mathbb{R}$ obtenemos una acción efectiva de $G$ por homeomorfismos lipschitzianos de $\mathrm{S}^{1}$ que fija globalmente dicho punto. Observe que, por el teorema de Thurston, ésta no es una acción por difeomorfismos: existe una obstrucción a la diferenciabilidad en el punto del círculo que corresponde al punto del infinito de la recta.

Ejercicio 5.5. Valiéndose del hecho que el elemento $f g h$ pertenece al centro de $\bar{G}$, y utilizando el teorema 2.54 y la proposición 4.64 , pruebe que toda acción de $\bar{G}$ por difeomorfismos de clase $\mathrm{C}^{1}$ del intervalo [0,1[ es trivial sin hacer uso del teorema de estabilidad de Thurston.

Ejercicio 5.6. Siguiendo [34], considere el grupo $\hat{G}$ con presentación $\left\langle f_{1}, g_{1}, h_{1}, f_{2}, g_{2}, h_{2}: f_{1}^{2}=g_{1}^{3}=h_{1}^{7}=f_{1} g_{1} h_{1}, f_{2}^{-1} f_{1} f_{2}=f_{1}^{2}, g_{2}^{-1} g_{1} g_{2}=g_{1}^{2}, h_{2}^{-2} h_{1} h_{2}=h_{1}^{2}\right\rangle$. Observe que $\hat{G}$ contiene una copia de $\bar{G}$. Pruebe que $\hat{G}$ actúa (de manera efectiva) por homeomorfismos del círculo, pero que $\hat{G}$ no se incruta en $\operatorname{Difeo}_{+}^{1}\left(\mathrm{~S}^{1}\right)$. 
Observación 5.7. La familia de los grupos finitamente generados de homeomorfismos de la recta conteniendo subgrupos finitamente generados que no admiten homomorfismos no triviales sobre $(\mathbb{R},+)$ es bastante grande. En efecto, un resultado clásico de la teoría de grupos ordenables (vea [141] para una demostración relativamente elemental) estipula que un grupo (de cardinalidad arbitraria) $\Gamma$ es localmente indicable (i.e., todos sus subgrupos finitamente generados admiten homomorfismos no triviales sobre $(\mathbb{R},+)$ ) si y sólo si $\Gamma$ es $\mathcal{C}$-ordenable (vea la sección 2.6). Por lo tanto, si bien todos los grupos finitamente generados de homeomorfismos del intervalo son topológicamente conjugados a grupos de homeomorfismos lipschitzianos de $[0,1]$ (vea la proposición 2.87), muchos no se incrustan en Difeo $_{+}^{1}([0,1])$.

Ejercicio 5.8. Pruebe que el teorema de estabilidad de Thurston no es válido para grupos numerables no finitamente generados de difeomorfismos del intervalo. Sugerencia. Considere el grupo derivado del grupo de Thompson F y su acción por difeomorfismos de $[0,1]$ (recuerde que dicho grupo derivado es simple [37]).

Observación 5.9. El teorema 5.1 puede ser usado para estudiar acciones de grupos en dimensión mayor que 1. Por ejemplo, usando la idea de su demostración, en [35] se prueba el interesantísimo hecho que el grupo de los difeomorfismos de clase $\mathrm{C}^{1}$ del disco cerrado que fijan todos los puntos del borde es ordenable a izquierda.

Ejercicio 5.10. El objetivo de este ejercicio es dar una demostración alternativa del teorema de estabilidad de Thurston siguiendo la técnica de [175] y [181]. Consideremos un subgrupo finitamente generado $\Gamma$ de $\operatorname{Difeo}_{+}^{1}([0,1])$, y para simplificar supongamos que $\Gamma$ no posee puntos fijos al interior de $] 0,1[\mathrm{y}$ que todos sus elementos son tangentes a la identidad en el origen (como vimos anteriormente, el caso general se reduce rápidamente a éste). Sea $\mathcal{G}=\left\{h_{1}, \ldots, h_{k}\right\}$ una familia finita de generadores de $\Gamma$. Para cada $f \in \Gamma$ definamos la función desplazamiento $\Delta_{f}$ por $\Delta_{f}(x)=f(x)-x$. Observe que $\left(\Delta_{f}\right)^{\prime}(0)=0$ para todo $f \in \Gamma$.

(i) Pruebe que para todo $x \geq 0$ y todo $f, g$ en $\Gamma$ existen $y, z$ (cercanos al origen si $x$ está cerca de 0 ) tales que

$$
\begin{gathered}
\Delta_{f g}(x)=\Delta_{f}(x)+\Delta_{g}(x)+\left(\Delta_{f}\right)^{\prime}(y) \Delta_{g}(x), \\
\Delta_{f-1(x)}=-\Delta_{f}(x)-\left(\Delta_{f}\right)^{\prime}(z) \Delta_{f-1}(x) .
\end{gathered}
$$

(ii) Fijada una sucesión (estrictamente) decreciente de puntos no globalmente fijos $x_{n}$ convergiendo al origen, para cada $n \in \mathbb{N}$ escojamos $i_{n} \in\{1, \ldots, k\}$ de modo que $\left|\Delta_{h_{i_{n}}}\left(x_{n}\right)\right| \geq\left|\Delta_{h_{j}}\left(x_{n}\right)\right|$ para todo $j \in\{1, \ldots, k\}$. Pasando a una subsucesión si es necesario, podemos asumir que $i_{n}$ es constante (digamos igual a 1 tras reordenar los índices), y que cada una de las $k$ sucesiones $\left(\Delta_{h_{i}}\left(x_{n}\right) / \Delta_{h_{1}}\left(x_{n}\right)\right)$ converge a un límite $\phi_{i}$ (menor o igual que 1) cuando $n$ tiende al infinito. A partir de las igualdades del ítem (i) verifique que la asignación $h_{i} \longmapsto \phi_{i}$ se extiende a un homomorfismo (normalizado) de $\Gamma$ en $(\mathbb{R},+$ ).

Ejercicio 5.11. Siguiendo [139] y [203], considere un difeomorfismo $\varphi$ de ]0, 1 [ en sí mismo tal que $\varphi(s)=\exp (-1 / s)$ para $s>0$ suficientemente pequeño. Pruebe que si $k \geq 0$ y $f:\left[0,1\left[\rightarrow\left[0,1\left[\right.\right.\right.\right.$ es un difeomorfismo de clase $\mathrm{C}^{k}$, entonces (la extensión a $\left[0,1[\mathrm{de}) \varphi^{-1} \circ f \circ \varphi\left(\right.\right.$ resp. $\left.\varphi^{-2} \circ f \circ \varphi^{2}\right)$ es un difeomorfismo de clase $\mathrm{C}^{k}$ con derivada 1 (resp. tangente a la identidad hasta el orden $k$ ) en el origen. 


\section{Rigidez para grupos de Kazhdan}

\subsection{La propiedad (T) de Kazhdan}

Sean $\Gamma$ un grupo numerable y $\Psi: \Gamma \rightarrow U(\mathcal{H})$ una representación de $\Gamma$ por transformaciones (lineales) unitarias de un espacio de Hilbert $\mathcal{H}$ (real o complejo). Decimos que $c: \Gamma \rightarrow \mathcal{H}$ es un cociclo respecto a $\Psi$ si para todo $g_{1}, g_{2}$ en $\Gamma$ se tiene $c\left(g_{1} g_{2}\right)=c\left(g_{1}\right)+\Psi\left(g_{1}\right) c\left(g_{2}\right)$. Decimos que un cociclo $c$ es un coborde si existe $K \in \mathcal{H}$ tal que $c(g)=K-\Psi(g) K$ para todo $g \in \Gamma$. Denotamos $Z^{1}(\Gamma, \Psi)$ el espacio de los cociclos y $B^{1}(\Gamma, \Psi)$ el subespacio de los cobordes. El espacio cuociente $Z^{1}(\Gamma, \Psi) / B^{1}(G, \Psi)$ es llamado el primer espacio de cohomología de $\Gamma$ (a valores en $\Psi$ ) y designado por $H^{1}(\Gamma, \Psi)$.

Definición 5.12. Un grupo numerable $\Gamma$ posee la propiedad de Kazhdan (o propiedad $(\mathrm{T})$ ) si para toda representación unitaria $\Psi$ de $\Gamma$ el espacio $H^{1}(G, \Psi)$ es trivial.

Para entender geométricamente la definición, recordemos que una isometría $A$ de un espacio de Hilbert $\mathcal{H}$ es una aplicación $A: \mathcal{H} \rightarrow \mathcal{H}$ que verifica $\left\|A\left(K_{1}\right)-A\left(K_{2}\right)\right\|=\left\|K_{1}-K_{2}\right\|$ para todo $K_{1}, K_{2}$ en $\mathcal{H}$. Es bien sabido que que toda isometría se escribe como la composición de una transformación unitaria y una traslación. En términos algebraicos, esto equivale a que el grupo de las isometrías de $\mathcal{H}$ es el producto semidirecto entre $U(\mathcal{H})$ y $\mathcal{H}$.

Consideremos ahora una representación unitaria $\Psi: \Gamma \rightarrow U(\mathcal{H})$ y una aplicación $c: \Gamma \rightarrow \mathcal{H}$ cualquiera. Si para cada $g \in \Gamma$ definimos la isometría $A(g)=\Psi(g)+c(g)$, entonces es fácil verificar que para todo $g_{1}, g_{2}$ en $\Gamma$ se tiene la igualdad $A\left(g_{1}\right) A\left(g_{2}\right)=A\left(g_{1} g_{2}\right)$ si y sólo si $c$ es un cociclo asociado a $\Psi$. En tal caso, la correspondencia $g \mapsto A(g)$ define una acción por isometrías.

Supongamos ahora que $K \in \mathcal{H}$ sea un punto fijo de la acción por isometrías asociada a un cociclo $c: \Gamma \rightarrow \mathcal{H}$. Para todo $g \in \Gamma$ tenemos entonces

$$
\Psi(g) K+c(g)=A(g) K=K .
$$

Por lo tanto, $c(g)=K-\Psi(g) K$, es decir, $c$ es un coborde. Recíprocamente, es fácil verificar que si $c$ es un coborde entonces existe un vector invariante para la acción por isometrías asociada. Tenemos así la siguiente reinterpretación geométrica de la definición 5.12: un grupo $\Gamma$ posee la propiedad de Kazhdan si y sólo si toda acción por isometrías de $\Gamma$ en un espacio de Hilbert posee un vector invariante.

Ejemplo 5.13. Todo grupo finito posee la propiedad de Kazhdan. Para probar esto, basta observar que el promedio a lo largo de una órbita de una acción por isometrías sobre un espacio de Hilbert es un vector invariante por dicha acción.

Ejercicio 5.14. Un vector $K_{\mathcal{C}} \in \mathcal{H}$ es el centro (geométrico) de un subconjunto $\mathcal{C}$ de $\mathcal{H}$ si $K_{\mathcal{C}}$ minimiza la función $K \mapsto \sup _{\bar{K} \in \mathcal{C}}\|K-\bar{K}\|$. Pruebe que si $\mathcal{C}$ es acotado, entonces $\mathcal{C}$ posee un único centro. Deduzca que si $\mathcal{C}$ es invariante 
por una acción isométrica, entonces su centro permanece fijo por dicha acción. Concluya la validez del lema del centro de Tits: una acción isométrica posee (al menos) un punto fijo si y sólo si (todas) sus órbitas son acotadas. En particular, un grupo posee la propiedad (T) si y sólo si las órbitas asociadas a sus acciones isométricas sobre espacios de Hilbert son acotadas.

Observación. El mismo argumento se aplica sobre espacios en los que la función distancia satisface una propiedad de "convexidad", como por ejemplo los árboles simpliciales o más generalmente los espacios a curvatura no positiva [22].

Es importante señalar que todo grupo que verifica la propiedad (T) es finitamente generado, de acuerdo a un resultado del propio Kazhdan. Una demostración directa de esto puede ser hallada en el capítulo 7 de [214]. Nosotros lo probaremos indirectamente siguiendo una idea de Serre y utilizando un resultado a ser demostrado en el ejemplo 5.22.

Sean $\Gamma$ un grupo contable y $\Gamma_{1} \subset \Gamma_{2} \subset \ldots \subset \Gamma_{n} \subset \ldots$ una sucesión de subgrupos finitamente generados tales que la reunión de los $\Gamma_{n}$ es todo $\Gamma$. Consideremos el árbol $\mathcal{T}$ cuyos vértices son las clases laterales en $\Gamma$ de los distintos $\Gamma_{n}$. Entre dos vértices $[g]$ en $\Gamma / \Gamma_{n}$ y $[h]$ en $\Gamma / \Gamma_{n+1}$ tracemos una arista (orientada de $[g]$ a $[h]$ ) si $g \in[h]$. Es fácil ver que $\mathcal{T}$ es un árbol simplicial orientado sobre el cual el grupo $\Gamma$ actúa de manera natural por isometrías que preservan la orientación de las aristas. Por el ejemplo 5.22, si $\Gamma$ posee la propiedad ( $\mathrm{T}$ ) entonces existe un vértice $[g]$ que permanece fijo bajo esta acción. Si $n \in \mathbb{N}$ es tal que $[g]$ representa la clase de $g$ respecto a $\Gamma_{n}$, lo anterior implica que $\Gamma=\Gamma_{n}$. Luego, $\Gamma$ es finitamente generado.

Ejemplo 5.15. Si $\Gamma$ posee la propiedad $(\mathrm{T})$ y es promediable entonces es finito (vea el apéndice para la noción de promediabilidad y algunas notaciones). En particular, los únicos grupos abelianos que satisfacen la propiedad de Kazhdan son los grupos finitos. En efecto, veremos a continuación que todo grupo promediable satisface la propiedad de Haagerup, es decir, actúa por isometrías de un espacio de Hilbert de manera geométricamente propia (en el sentido que $\|c(g)\|_{\mathcal{H}}$ tiende al infinito si $\operatorname{long}(g)$ tiende al infinito). El lector interesado encontrará en [47] más ejemplos de grupos que verifican esta propiedad, así como una discusión de la misma.

Fijemos un sistema de generadores $\mathcal{G}$ del grupo promediable $\Gamma$ junto con una sucesión de Følner $\left(A_{n}\right)$ asociada a $\mathcal{G}$ tal que

$$
\frac{\operatorname{card}\left(\partial A_{n}\right)}{\operatorname{card}\left(A_{n}\right)} \leq \frac{1}{2^{n}}
$$

Consideremos ahora el espacio de Hilbert $\mathcal{H}=\oplus_{n \geq 1} n \ell_{\mathbb{R}}^{2}(\Gamma)$ definido por

$$
\mathcal{H}=\left\{K=\left(K_{1}, \ldots, K_{n}, \ldots\right), K_{n} \in \ell_{\mathbb{R}}^{2}(\Gamma), \sum_{n \geq 1} n^{2}\left\|K_{n}\right\|_{\ell_{\mathbb{R}}^{2}(\Gamma)}^{2}<\infty\right\} .
$$

Para $g, h$ en $\Gamma$ definimos $\Psi_{n}(g) K_{n}(h)=K_{n}(g h)$, y luego consideramos la representación regular $\Psi: \Gamma \rightarrow U(\mathcal{H})$ dada por $\Psi(g) K=\left(\Psi_{1}(g) K_{1}, \Psi_{2}(g) K_{2}, \ldots\right)$. Además, para cada $g \in \Gamma$ consideramos el vector $c(g)=\left(c(g)_{1}, c(g)_{2}, \ldots\right)$ definido por

$$
c(g)_{n}=\frac{1}{\sqrt{\operatorname{card}\left(A_{n}\right)}}\left(\mathcal{X}_{A_{n}}-\mathcal{X}_{g\left(A_{n}\right)}\right)
$$


donde $\mathcal{X}$ denota la función característica del conjunto respectivo. Para todo $g \in \mathcal{G}$ tenemos, gracias a $(5.3)$,

$$
\|c(g)\|_{\mathcal{H}}^{2}=\sum_{n \geq 1} n^{2}\left\|\frac{\mathcal{X}_{A_{n}}-\mathcal{X}_{g\left(A_{n}\right)}}{\sqrt{\operatorname{card}\left(A_{n}\right)}}\right\|_{\ell_{\mathbb{R}}^{2}(\Gamma)}^{2} \leq \sum_{n \geq 1} n^{2}\left(\frac{\operatorname{card}\left(\partial A_{n}\right)}{\operatorname{card}\left(A_{n}\right)}\right) \leq \sum_{n \geq 1} \frac{n^{2}}{2^{n}}<\infty .
$$

La relación de cociclo $c\left(g_{1} g_{2}\right)=c\left(g_{1}\right)+\Psi\left(g_{1}\right) c\left(g_{2}\right)$ es fácilmente verificable. Afirmamos finalmente que $c: \Gamma \rightarrow \mathcal{H}$ es una aplicación geométricamente propia. Para probarlo, fijemos un entero $k$ mayor o igual al diámetro de $A_{n}$. Si un elemento $g \in \Gamma$ pertenece a $B_{\mathcal{G}}(k) \backslash B_{\mathcal{G}}(k-1)$ entonces $g\left(A_{n}\right) \cap A_{n}=\emptyset$, y por lo tanto

$$
\left\|\frac{\mathcal{X}_{A_{n}}-\mathcal{X}_{g\left(A_{n}\right)}}{\sqrt{\operatorname{card}\left(A_{n}\right)}}\right\|_{\ell_{\mathbb{R}}^{2}(\Gamma)}^{2} \geq 2 .
$$

Luego, $\|c(g)\|_{\mathcal{H}}^{2} \geq 2 n^{2}$, y esto prueba nuestra afirmación.

Ejercicio 5.16. Pruebe que la imagen de un grupo de Kazhdan por un homomorfismo posee también la propiedad (T), y que lo mismo ocurre para extensiones finitas de grupos de Kazhdan.

Como consecuencia del ejercicio precedente, todo grupo de Kazhdan verifica la hipótesis del teorema de estabilidad de Thurston. En efecto, la imagen de un grupo de Kazhdan por un homomorfismo sobre $(\mathbb{R},+)$ es abeliana y posee la propiedad $(\mathrm{T})$, por lo que debe necesariamente ser finita, y por lo tanto trivial.

Ejercicio 5.17. Pruebe directamente que todo subgrupo finitamente generado de Difeo ${ }_{+}^{1+\tau}([0,1])$ que satisface la propiedad $(\mathrm{T})$ es trivial, cualquiera sea $\tau>0$. Sugerencia. Pasando a un cuociente si es necesario, se puede suponer que el grupo en cuestión $\Gamma$ no tiene punto fijo en ]0,1[. Considere la medida de Radon $\mu$ sobre ]0,1[ definida por $d \mu=d x / x$, y sea $\Psi$ la representación regular de $\Gamma$ sobre $\mathcal{H}=\mathcal{L}_{\mathbb{R}}^{2}([0,1], \mu)$, es decir

$$
\Psi(g) K(x)=K\left(g^{-1}(x)\right)\left[\frac{d g^{-1}}{d \mu}(x)\right]^{1 / 2} .
$$

Para cada $g \in \Gamma$ considere la función

$$
c\left(g^{-1}\right)=1-\left[\frac{d g}{d \mu}(x)\right]^{1 / 2} .
$$

Verifique que se satisface la relación de cociclo $c(g h)=c(g)+\Psi(g) c(h)$ (observe que $c$ es el coborde formal de la función constante igual a 1, la cual no pertenece a $\mathcal{H}$ ). Verifique además que $c(g)$ pertenece a $\mathcal{H}$ (es aquí donde se usa la hipótesis $\tau>0$ ). Pruebe que si $c$ es cohomológicamente trivial, entonces existe $K \in \mathcal{H}$ tal que la medida $\nu$ sobre $] 0,1[$ de función de densidad (respecto a $d \mu$ ) es el cuadrado de $x \mapsto 1-K(x)$ es invariante por $\Gamma$. Concluya la demostración utilizando los resultados de la sección 2.5 , capítulo 2 .

Ejercicio 5.18. Pruebe directamente que ningún subgrupo no trivial $\Gamma$ de $(\mathbb{R},+)$ posee la propiedad $(\mathrm{T})$. Para ello, considere la representación de $\Gamma$ por traslaciones de $\mathcal{L}_{\mathbb{R}}^{2}(\mathbb{R}, L e b)$ y el cociclo asociado dado por $c(g)=\mathcal{X}_{[0, \infty[}-\mathcal{X}_{[g(0), \infty[}$, donde $\mathcal{X}$ denota la función característica del intervalo en cuestión y $g \in \Gamma$. Pruebe que este cociclo no puede ser un coborde. 
Los ejemplos no triviales de grupos de Kazhdan son las redes de los grupos de Lie simples de rango real mayor o igual que 2. Recuerde que el rango real de un grupo de Lie es la dimensión de la mayor sub-álgebra abeliana de su álgebra de Lie sobre la cual la aplicación adjunta es diagonalizable sobre $\mathbb{R}$, y que un subgrupo discreto de un grupo topológico localmente compacto es una red si el espacio cuociente tiene volumen finito (respecto a la medida de Haar). Por ejemplo, $\mathrm{SL}(3, \mathbb{Z})$ posee la propiedad $(\mathrm{T})$, pues el rango de $\operatorname{SL}(3, \mathbb{R})$ es 2 y $\operatorname{SL}(3, \mathbb{Z})$ es una red en $\operatorname{SL}(3, \mathbb{R})$. Para la demostración de todo esto vea [91], y alternativamente vea el notable trabajo [186].

Numerosos otros ejemplos interesantes de grupos con la propiedad (T) han sido construidos por diversos autores. Vale destacar al respecto el extraordinario trabajo de Gromov sobre grupos aleatorios [86], donde se prueba que "genéricamente" todo grupo de presentación finita posee la propiedad de Kazhdan (vea también [215]). Por lo tanto, un teorema que es válido para grupos de Kazhdan es válido para "casi todo grupo".

Para finalizar esta sección, probaremos dos resultados sencillos de obstrucción a la propiedad $(\mathrm{T})$ válidos para grupos relacionados con nuestra discusión, a saber, los grupos de Thompson y de Neretin. El lector deseoso de avanzar rápidamente hacia el caso de los grupos de difeomorfismos del círculo puede pasar directamente a la sección siguiente.

Proposición 5.19. El grupo de Thompson G no posee la propiedad de Kazhdan.

Demostración. Daremos una prueba elemental utilizando una elegante construcción debida a Farley [64], quien demuestra que G admite una representación geométricamente propia por isometrías de un espacio de Hilbert. La presentación está inspirada de [150].

Denotemos por $G_{0}$ al subgrupo de $G$ formado por aquellos elementos cuya restricción al subintervalo $[0,1 / 2]$ de $\mathrm{S}^{1}$ es la identidad. Consideremos el espacio de Hilbert $\mathcal{H}=\ell_{\mathbb{R}}^{2}\left(\mathrm{G} / \mathrm{G}_{0}\right)$. Claramente, el grupo $\mathrm{G}$ actúa por isometrías de $\mathcal{H}$ (considere las traslaciones a izquierda). Para cada intervalo diádico $I$ escojamos un elemento $g_{I}$ de $\mathrm{G}$ tal que $g_{I}$ envíe $[0,1 / 2]$ sobre $I$ de manera afín, y denotemos por $\left[g_{I}\right]$ la clase de $g_{I}$ módulo $\mathrm{G}_{0}$. Para cada $g \in \mathrm{G}$ consideremos la función $c(g) \in \mathcal{H}$ definida por

$$
c(g)=\sum_{I}\left(\delta_{\left[g_{I}\right]}-\delta_{g\left[g_{I}\right]}\right),
$$

donde la suma se extiende sobre el conjunto de los intervalos diádicos $I$ y $\delta$ denota la delta de Dirac (es decir, $\delta_{\left[g_{I}\right]}$ es la función característica del conjunto $\left.\left\{\left[g_{I}\right]\right\}\right)$. Cada función $c(g)$ es de soporte finito, pues para todo elemento $g \in \Gamma$ se tiene que $g\left[g_{I}\right]=\left[g g_{I}\right]$ si $|I|$ es suficientemente pequeño (la restricción de $g$ a intervalos diádicos suficientemente pequeños es afín). Luego, $c(g)$ pertenece a $\mathcal{H}$. Por otra parte, no es difícil ver que $c$ satisface la igualdad de cociclo (respecto a la representación por traslaciones en $\mathcal{H}$ ). 
El cálculo de $\|c(g)\|$ es simple. En efecto, de la definición se obtiene que $\|c(g)\|^{2}$ es igual a dos veces el número de intervalos diádicos $I$ tales que o bien la restricción de $g$ a $I$ no es afín, o bien la imagen de $I$ por $g$ no es un intervalo diádico. El que $\|c(g)\|$ tiende al infinito cuando long $(g)$ tiende al infinito se deduce fácilmente de esto.

Designemos ahora por $\mathcal{T}^{p}$ al árbol simplicial homogéneo de valencia $p+1$ en cada vértice. Fijemos un vértice $\sigma$ de $\mathcal{T}^{p}$, al cual llamaremos el origen. Un sub-árbol de $\mathcal{T}^{p}$ es completo si es conexo, compacto, y cada vez que dos de sus aristas tienen un vértice en común, todas las aristas que contienen dicho vértice están incluidas en él. Observe que el complemento de un subárbol completo es la unión de una cantidad finita de árboles enraizados.

Si $\mathcal{A}, \mathcal{B}$ son sub-árboles completos de $\mathcal{T}^{p}$, denotamos por $\mathcal{N}^{p}(\mathcal{A}, \mathcal{B})$ al conjunto de las transformaciones biyectivas de $\overline{\mathcal{T}^{p} \backslash \mathcal{A}}$ sobre $\overline{\mathcal{T}^{p} \backslash \mathcal{B}}$ que envían cada componente conexa de $\overline{\mathcal{T}^{p} \backslash \mathcal{A}}$ de manera isométrica sobre una componente conexa de $\overline{\mathcal{T}^{p} \backslash \mathcal{B}}$. Si $g$ pertenece a $\mathcal{N}^{p}(\mathcal{A}, \mathcal{B})$ entonces $g$ induce un homeomorfismo de $\partial \mathcal{T}^{p}$, al que también denotaremos por $g$. Observe que $\partial \mathcal{T}^{p}$ aparece dotado de manera natural de una métrica: la distancia entre dos puntos $x, y$ de $\partial \mathcal{T}^{p}$ viene dada por $\partial$ ist $(x, y)=p^{-n}$, donde $n$ es la distancia dist (sobre $\mathcal{T}^{p}$ ) entre $\sigma$ y la geodésica que une $x$ e $y$.

Definición 5.20. El grupo de Neretin $\mathcal{N}^{p}$ es el grupo de los homeomorfismos de $\partial \mathcal{T}^{p}$ inducidos por elementos de algún conjunto del tipo $\mathcal{N}^{p}(\mathcal{A}, \mathcal{B})$.

Intuitivamente, $\mathcal{N}^{p}$ es el grupo de los "gérmenes al infinito" de isometrías de $\partial \mathcal{T}^{p}$. Es importante notar que si $g_{1} \in \mathcal{N}^{p}(\mathcal{A}, \mathcal{B})$ y $g_{2} \in \mathcal{N}^{p}\left(\mathcal{A}^{\prime}, \mathcal{B}^{\prime}\right)$ representan al mismo elemento de $\mathcal{N}^{p}$ entonces coinciden sobre la intersección de sus dominios de definición. Un representante $\tilde{g} \in \mathcal{N}^{p}(\mathcal{A}, \mathcal{B})$ de $g \in \mathcal{N}^{p}$ será dicho maximal si su dominio contiene al de cualquier otro representante de $g$. No es difícil convencerse de la existencia de un único representante maximal para cada elemento de $\mathcal{N}^{p}$. Para $g \in \mathcal{N}^{p}$ designemos por $\mathcal{A}_{g}$ (resp. $\mathcal{B}_{g}$ ) al cierre del complemento del dominio de definición de $\tilde{g}$ (resp. de $\tilde{g}^{-1}$ ). Notemos que $\mathcal{A}_{g}=\mathcal{B}_{g^{-1}}$. El grupo $\operatorname{Isom}\left(\mathcal{T}^{p}\right)$ de las (extensiones al borde de las) isometrías de $\mathcal{T}^{p}$ es un subgrupo de $\mathcal{N}^{p}$. Un elemento $g$ de $\mathcal{N}^{p}$ está contenido en $\operatorname{Isom}\left(\mathcal{T}^{p}\right)$ si y sólo si $\mathcal{A}_{g}=\mathcal{B}_{g}=\emptyset$. Observe finalmente que el grupo de Thompson $\mathrm{G}$ puede ser visto como un subgrupo de $\mathcal{N}^{2}$.

La introducción del grupo de Neretin (también llamado grupo de los esferomorfismos) no es artificial: su aparición resulta natural en el contexto $p$-ádico. En efecto, si $p$ es primo, entonces el grupo de los difeomorfismos de la recta proyectiva de $\mathbb{Q}_{p}$ (es decir, del "círculo $p$-ádico") se incrusta en $\mathcal{N}^{p}$. Para mayor información al respecto recomendamos la lectura de [151]. Nosotros nos contentaremos con demostrar el resultado siguiente.

Proposición 5.21. Sea $\Gamma$ un subgrupo del grupo de Neretin $\mathcal{N}^{p}$. Si $\Gamma$ posee la propiedad $(\mathrm{T})$ de Kazhdan, entonces existe un subgrupo de indice finito de $\Gamma$ que fija una cantidad finita de bolas del borde del árbol, actuando isométricamente sobre cada una de ellas. 
Demostración. Para cada vértice $a \neq \sigma$ designemos por $A_{a}$ al árbol enraizado en a que "apunta hacia el infinito". Fijemos uno de los $p+1$ sub-árboles de $\mathcal{T}^{p}$ enraizados sobre el origen, y (abusando de la notación) designémoslo por $A_{\sigma}$. Consideremos el grupo $\mathcal{N}_{\sigma}^{p}$ formado por los elementos $g \in \mathcal{N}^{p}$ que fijan el borde al infinito $\partial A_{\sigma}$ de $A_{\sigma}$, actuando isométricamente (respecto a la métrica $\partial$ ist) sobre él. El grupo $\mathcal{N}^{p}$ actúa unitariamente sobre el espacio de Hilbert $\mathcal{H}=\ell_{\mathbb{R}}^{2}\left(\mathcal{N}^{p} / \mathcal{N}_{\sigma}^{p}\right)$ por traslaciones a izquierda.

A cada vértice $a$ de $\mathcal{T}^{p}$ asociemos el elemento $\phi_{a} \in \mathcal{N}^{p} / \mathcal{N}_{\sigma}^{p}$ dado por $\phi_{a}=[h]$, donde $h \in \mathcal{N}^{p}$ es un elemento cuyo representante maximal envía $A_{\sigma}$ sobre $A_{a}$ isométricamente (respecto a la métrica dist), y $[h]$ es su clase en $\mathcal{N}^{p} / \mathcal{N}_{\sigma}^{p}$. Para $g \in \mathcal{N}^{p}$ definamos "formalmente" $c(g) \in \mathcal{H}$ haciendo

$$
c_{1}(g)=\sum g\left(\delta_{\phi_{a}}\right)-\sum \delta_{\phi_{b}}
$$

donde $\delta$ designa la delta de Dirac (i.e., $\delta_{\phi_{a}}$ es la función característica del conjunto $\left.\left\{\phi_{a}\right\}\right)$. La expresión "formalemente" se debe al hecho que los términos coincidentes en la definición de $c(g)$ deben simplificarse, tras lo cual "sobrevive" sólo una cantidad finita de términos (esto prueba que la función $c(g)$ pertenece a $\mathcal{H})$. Por otra parte, $c(g)$ es el cociclo formal asociado a la función $K=\sum \delta_{\phi_{a}}$ (la cual no pertenece a $\mathcal{H}$ ), lo cual prueba que $c$ es un cociclo (respecto a la representación por traslaciones $\Psi$ ).

Para $g \in \Gamma \backslash \operatorname{Isom}\left(\mathcal{T}^{p}\right)$ sea $d=d(g)$ la distancia entre $\sigma$ y $\mathcal{A}_{g}$. Consideremos el segmento geodésico $\gamma$ que une a $\sigma$ con el vértice de $\mathcal{A}_{g}$ más alejado de $\sigma$, y sean $a_{1}, a_{2}, \ldots, a_{d-1}$ los vértices del interior de $\gamma$. Se verifica fácilmente que para todo vértice $b$ de $\mathcal{T}^{p}$ y todo $i \in\{1, \ldots, d-1\}$ se tiene $g\left(\delta_{\phi_{a_{i}}}\right) \neq \delta_{b}$, de donde se deduce que $\|c(g)\|^{2} \geq d-1$. Sea ahora $g \in \Gamma \cap \operatorname{Isom}\left(\mathcal{T}^{p}\right)$. Notemos $d^{\prime}=d^{\prime}(g)$ la distancia entre $\sigma$ y $\tilde{g}(\sigma)$, y consideremos el segmento geodésico $\gamma$ que une dichos puntos. Denotemos por $a_{1}, \ldots, a_{d^{\prime}-1}$ los vértices del interior de $\tilde{g}^{-1}(\gamma)$. Es fácil ver que para todo $i \in\left\{1, \ldots, d^{\prime}-1\right\}$ y todo vértice $b$ de $\mathcal{T}^{p}$ se tiene $g\left(\delta_{\phi_{a_{i}}}\right) \neq \delta_{b}$ (pues $A_{\tilde{g}\left(a_{i}\right)}$ no es la imagen de $A_{a_{i}}$ por $\left.\tilde{g}\right)$. Se concluye así que $\|c(g)\|^{2} \geq d^{\prime}-1$.

Si $\Gamma$ posee la propiedad (T) entonces la función $g \mapsto\|c(g)\|$ debe estar uniformemente acotada sobre $\Gamma$. Existe por lo tanto un entero $N>0$ tal que para todo $g \in \Gamma \backslash \operatorname{Isom}\left(\mathcal{T}^{p}\right)$ se tiene $\operatorname{dist}\left(\sigma, \mathcal{A}_{g}\right) \leq N$ (y por lo tanto $\operatorname{dist}\left(\sigma, \mathcal{B}_{g}\right) \leq N$ para todo $\left.g \in \Gamma \backslash \operatorname{Isom}\left(\mathcal{T}^{p}\right)\right)$, y tal que para todo $g \in \Gamma \cap \operatorname{Isom}\left(\mathcal{T}^{p}\right)$ se tiene $\operatorname{dist}(\sigma, \tilde{g}(\sigma)) \leq N$. La proposición se obtiene fácilmente como consecuencia de esto.

\subsection{Enunciado del resultado}

Se tiene la impresión general de que los grupos que tienen la propiedad (T) no pueden actuar de manera no trivial sobre espacios dotados de una estructura unidimensional razonable. Por ejemplo, veremos a continuación que para toda acción por isometrías de un grupo de Kazhdan sobre un árbol simplicial existe un punto fijo global. La prueba que presentaremos es 
debida esencialmente a Haglund, Paulin y Valette. Una modificación simple de ella permite probar el mismo resultado para acciones por isometrías sobre árboles reales. En dicha generalidad, el teorema es ya clásico y fue originalmente obtenido por Alperin y Watatani [2, 205].

Ejemplo 5.22. Sea $\mathcal{T}$ un árbol simplicial, es decir, un árbol cuyas aristas (abiertas) son isométricas al intervalo ]0,1[ (no supondremos que la valencia de cada arista sea finita). Denotemos por $\overrightarrow{a r}(\mathcal{T})$ al conjunto de las aristas (abiertas) orientadas de $\mathcal{T}$. Para cada arista $\vec{\Upsilon} \in \overrightarrow{\operatorname{ar}}(\mathcal{T})$ denotemos por $\operatorname{ver}(\vec{\Upsilon})$ al conjunto de los vértices de $\mathcal{T}$ que son conectados a $\vec{\Upsilon}$ por una geodésica cuyo segmento inicial es $\vec{\Upsilon}$ (con la orientación respectiva). Para cada vértice $v$ de $\mathcal{T}$ denotemos por $\overrightarrow{\operatorname{ar}}(v)$ al conjunto de las aristas orientadas $\vec{\Upsilon}$ tales que $v \in \operatorname{ver}(\vec{\Upsilon})$.

Consideremos el espacio de Hilbert $\mathcal{H}=\ell_{\mathbb{R}}^{2}(\overrightarrow{\mathrm{ar}}(\mathcal{T}))$. Un elemento de $\mathcal{H}$ es una función $K$ que a cada arista orientada de $\mathcal{T}$ asocia un número real, de manera que el valor de la siguiente suma es finito:

$$
\sum_{\vec{\Upsilon} \in \overrightarrow{\operatorname{ar}}(\mathcal{T})} K(\vec{\Upsilon})^{2} .
$$

Sea $\Gamma$ un subgrupo del grupo de isometrías de $\mathcal{T}$. Fijemos un vértice $\sigma$ de $\mathcal{T}$ y definamos una representación unitaria $\Psi$ de $\Gamma$ sobre $\mathcal{H}$ por $\Psi(g) K(\vec{\Upsilon})=K(g(\vec{\Upsilon}))$. Para cada $g \in \Gamma$ consideremos la función $c(g): \overrightarrow{\operatorname{ar}}(\mathcal{T}) \rightarrow \mathbb{R}$ dada por

$$
c(g)=\mathcal{X}_{\overrightarrow{\mathrm{ar}}(\sigma)}-\mathcal{X}_{\overrightarrow{\mathrm{ar}}(g(\sigma))},
$$

donde $\mathcal{X}$ denota la función característica del conjunto respectivo. Es fácil ver que $c(g)$ es una función de soporte finito, y por lo tanto pertenece a $\mathcal{H}$. Además, la correspondencia $g \mapsto c(g)$ es un cociclo, pues es el coborde formal asociado a la función $\vec{\Upsilon} \mapsto \mathcal{X}_{\overrightarrow{\mathrm{ar}}(\sigma)}(\vec{\Upsilon})$.

Si $\Gamma$ posee la propiedad de Kazhdan entonces el cociclo $c$ es un coborde. En otras palabras, existe una función $K \in \mathcal{H}$ tal que para todo $g \in \Gamma$ se tiene $c(g)=K-\Psi(g) K$. Esta igualdad implica que $\|c(g)\|_{2} \leq 2\|K\|_{2}$. Por otra parte, es fácil ver que $\|c(g)\|^{2}=2 \operatorname{dist}(\sigma, g(\sigma))$. Se deduce entonces que la órbita de $\sigma$ por la acción de $\Gamma$ permanece dentro de un subconjunto compacto de $\mathcal{T}$. Dejamos al lector la tarea de probar que esto implica la existencia de al menos un punto fijo por la acción (compare con el ejercicio 5.14), lo cual a su vez implica que un vértice o el punto medio de una arista es un punto fijo. Observe que si el árbol está dotado de una orientación coherente entre las distintas aristas y la acción de $\Gamma$ preserva esta orientación, entonces necesariamente el punto fijo es un vértice. Si tiene dificultades para probar todo esto, vea [91, 185].

En la sección 2.3 del segundo capítulo vimos que ningún subgrupo de índice finito de $\mathrm{SL}(3, \mathbb{Z})$ puede actuar por homeomorfismos de la recta de manera no trivial. De acuerdo con [208] (vea también [120]), esto es cierto también para muchas redes de grupos de Lie que poseen la propiedad $(\mathrm{T})$, pero se desconoce si es válido en general para grupos de Kazhdan.

Para el caso del círculo se dispone de resultados más precisos. En particular, un teorema de Ghys (vea [73] y también [30, 208]) estipula que si $\Phi: \Gamma \rightarrow \operatorname{Difeo}_{+}^{1}\left(\mathrm{~S}^{1}\right)$ es una representación de una red $\Gamma$ de un grupo de Lie 
simple de rango real mayor o igual que 2, entonces la imagen $\Phi(\Gamma)$ es finita. Nosotros no expondremos la prueba de este resultado, pero recomendamos la lectura de la demostración dada en [73] para el caso de redes en $\operatorname{SL}(3, \mathbb{R})$.

Ejercicio 5.23. La parte esencial de la prueba del teorema de Ghys consiste en demostrar que, para toda acción por homeomorfismos del círculo de una red $\Gamma$ de un grupo de Lie simple de rango real mayor o igual que 2, existe una medida de probabilidad invariante. Demuestre que, a partir de este hecho, puede concluirse dicho teorema.

Sugerencia. Si existe una medida de probabilidad invariante entonces la función número de rotación es un homomorfismo de $\Gamma$ en el grupo $\mathbb{T}^{1}$. Como $\Gamma$ verifica la propiedad de Kazhdan, la imagen por este homomorfismo es finita. Concluya que la órbita de todo punto del soporte de la medida invariante es finita. Finalmente, aplique el teorema de estabilidad de Thurston.

Observación 5.24. No es difícil extender el teorema 2.32 y concluir que toda acción de un subgrupo de índice finito de $\operatorname{SL}(3, \mathbb{Z})$ por homeomorfismos del círculo es trivial. Este resultado, debido también a Witte, puede ser obtenido combinando la afirmación del comienzo del ejercicio precedente con el teorema 2.32, aunque originalmente fue probado valiéndose del célebre teorema de los subgrupos normales de Margulis [129]. Consignemos que la demostración es particularmente rápida en clase $\mathrm{C}^{2}$. En efecto, las redes involucradas contienen subgrupos nilpotentes no virtualmente abelianos, por lo que, de acuerdo al teorema 4.40 de Plante y Thurston, todas sus acciones por difeomorfismos de clase $\mathrm{C}^{2}$ de $\mathrm{S}^{1}$ tienen un núcleo infinito; por el teorema de los subgrupos normales de Margulis, dichas acciones deben tener imagen finita.

En lo que sigue nos proponemos demostrar una versión del teorema discutido anteriormente en clase $\mathrm{C}^{1+\tau}$ para grupos de Kazhdan (cuando $\tau>1 / 2$ ). El resultado siguiente fue obtenido por el autor en [149].

Teorema 5.25. Sea $\Phi: \Gamma \rightarrow$ Difeo $_{+}^{1+\tau}\left(\mathrm{S}^{1}\right)$ una representación de un grupo numerable, con $\tau>1 / 2$. Si $\Gamma$ posee la propiedad de Kazhdan, entonces $\Phi(\Gamma)$ es finito.

La propiedad de Kazhdan se considera también para grupos no discretos localmente compactos (en tal caso, las representaciones y los cociclos involucrados en la definición deben ser continuos). El lector observará que la técnica que emplearemos para la demostración del teorema 5.25 puede aún ser aplicada. Un nuevo grupo de Kazhdan puede aparecer entonces, a saber, $\operatorname{SO}(2, \mathbb{R})$. Esto no es de extrañar, de acuerdo al esbozo que hemos hecho en la sección 4 (capítulo 1) de la clasificación de las acciones de grupos localmente compactos no discretos en $\mathrm{S}^{1}$.

Observe que el teorema 5.25 muestra una vez más que el grupo G de Thompson no posee la propiedad (T) (vea la proposición 5.19). En efecto, de acuerdo a la última sección del primer capítulo, $\mathrm{G}$ puede ser realizado como un grupo de difeomorfismos de clase $\mathrm{C}^{\infty}$ del círculo (en realidad, basta considerar la realización de $\mathrm{G}$ en clase $\mathrm{C}^{1+\text { lip }}$ de la sección 5.1 de 
dicho capítulo para concluir). Señalemos por otra parte que, contrariamente a la mayoría de los resultados que hemos estudiado hasta ahora, se ignora si la hipótesis de diferenciabilidad del teorema 5.25 es necesaria (por ejemplo, utilizando uno de los resultados de [4] es posible extender el enunciado para representaciones de grupos de Kazhdan en Difeo ${ }_{+}^{3 / 2}\left(\mathrm{~S}^{1}\right)$ ). Un problema parcial pero desde ya muy interesante consiste en determinar si existen grupos de Kazhdan no triviales admitiendo una relación de orden total e invariante a izquierda (vea la sección 2.3, capítulo 2). Otro problema parcial igualmente interesante es el de saber si el grupo de los homeomorfismos afines por partes del círculo contiene subgrupos numerables e infinitos satisfaciendo la propiedad ( $\mathrm{T})$.

\subsection{Demostración del teorema}

Por simplicidad, denotaremos por $\bar{g}$ al difeomorfismo $\Phi\left(g^{-1}\right)$ (consideramos la inversa de $g$ para obtener más adelante una representación a izquierda). Recuerde que la medida de Liouville sobre $\mathrm{S}^{1} \times \mathrm{S}^{1}$ es aquélla cuya función densidad es $(r, s) \mapsto 1 / 4 \operatorname{sen}^{2}((r-s) / 2)$ (vea la sección 3.2 de este capítulo). Sea $\mathcal{H}=\mathcal{L}_{\mathbb{R}}^{2, \Delta}\left(\mathrm{S}^{1} \times \mathrm{S}^{1}, L v\right)$ el subespacio (cerrado) de $\mathcal{L}_{\mathbb{R}}^{2}\left(\mathrm{~S}^{1} \times \mathrm{S}^{1}, L v\right)$ de las funciones $K$ que para casi todo $(x, y) \in \mathrm{S}^{1} \times \mathrm{S}^{1}$ verifican la igualdad $K(x, y)=K(y, x)$. Consideremos la acción $\Psi$ de $\Gamma$ sobre $\mathcal{H}$ dada por

$$
\Psi(g) K(r, s)=K(\bar{g}(r), \bar{g}(s)) \cdot[\operatorname{Jac}(\bar{g})(r, s)]^{\frac{1}{2}},
$$

donde $J a c(\bar{g})(r, s)$ es el jacobiano (respecto a $L v$ ) de la transformación $(r, s) \mapsto(\bar{g}(r), \bar{g}(s))$, es decir

$$
J a c(\bar{g})(r, s)=\frac{\operatorname{sen}^{2}\left(\frac{r-s}{2}\right)}{\operatorname{sen}^{2}\left(\frac{\bar{g}(r)-\bar{g}(s)}{2}\right)} \cdot\left[\bar{g}^{\prime}(r) \bar{g}^{\prime}(s)\right] .
$$

Es fácil ver que $\Psi$ es una representación unitaria.

Para cada $g \in \Gamma$ consideremos la función (compare con (1.4))

$$
c(g)(r, s)=1-[J a c(\bar{g})(r, s)]^{\frac{1}{2}} .
$$

Se verifica fácilmente la relación de cociclo $c\left(g_{1} g_{2}\right)=\Psi\left(g_{1}\right) c\left(g_{2}\right)+c\left(g_{1}\right)$ (compare con (1.3)). En efecto, este cociclo de Liouville c es formalmente el coborde de la función constante igual a 1 , la cual no pertenece al espacio de Hilbert $\mathcal{H}=\mathcal{L}_{\mathbb{R}}^{2, \Delta}\left(\mathrm{S}^{1} \times \mathrm{S}^{1}, L v\right)$. Sin embargo, si $\Phi$ es una representación a valores en Difeo ${ }_{+}^{1+\tau}\left(\mathrm{S}^{1}\right)$ para algún $\tau>1 / 2$ entonces $c(g)$ pertenece a $\mathcal{H}$, es decir, $c(g)$ es un cociclo genuino a valores en $\mathcal{H}$. Tal es la afirmación de la proposición siguiente, debida esencialmente a Segal y Reznikov [170, 177].

Proposición 5.26. Si $\tau>1 / 2$ entonces para todo $g \in \Gamma$ la función $c(g)$ pertenece a $\mathcal{L}_{\mathbb{R}}^{2, \Delta}\left(\mathrm{S}^{1} \times \mathrm{S}^{1}, L v\right)$ 
Demostración. En primer lugar, notemos que existe una función continua $K_{1}:[0,2 \pi] \times[0,2 \pi] \rightarrow \mathbb{R}$ tal que

$$
\frac{1}{\left|\operatorname{sen}\left(\frac{r-s}{2}\right)\right|}=2\left[\frac{1}{|r-s|}+K_{1}(r, s)\right] \text {. }
$$

Luego, para probar que $c(g) \in \mathcal{L}_{\mathbb{R}}^{2, \Delta}\left(\mathrm{S}^{1} \times \mathrm{S}^{1}, L v\right)$ debemos verificar que la función

$$
(r, s) \mapsto\left[\frac{\left[\bar{g}^{\prime}(r) \bar{g}^{\prime}(s)\right]^{\frac{1}{2}}}{|\bar{g}(r)-\bar{g}(s)|}-\frac{1}{r-s}\right]
$$

pertenece a $\mathcal{L}_{\mathbb{R}}^{2, \Delta}\left(\mathrm{S}^{1} \times \mathrm{S}^{1}, L e b\right)$. Para $r, s$ en $\mathrm{S}^{1}$ tales que $|r-s|<\pi$ existe un punto $t$ en el segmento más pequeño del círculo que los une tal que $|\bar{g}(r)-\bar{g}(s)|=\bar{g}^{\prime}(t)|r-s|$. Esto nos da

$$
\begin{aligned}
\left|\frac{\left[\bar{g}^{\prime}(r) \bar{g}^{\prime}(s)\right]^{\frac{1}{2}}}{|\bar{g}(r)-\bar{g}(s)|}-\frac{1}{|r-s|}\right| & \leq \frac{1}{|r-s| \bar{g}^{\prime}(t)} \cdot\left|\left[\bar{g}^{\prime}(r) \bar{g}^{\prime}(s)\right]^{\frac{1}{2}}-\bar{g}^{\prime}(t)\right| \\
& =\frac{1}{|r-s| \bar{g}^{\prime}(t)} \cdot \frac{\left|\bar{g}^{\prime}(r) \bar{g}^{\prime}(s)-\bar{g}^{\prime}(t)^{2}\right|}{\left[\bar{g}^{\prime}(r) \bar{g}^{\prime}(s)\right]^{\frac{1}{2}}+\bar{g}^{\prime}(t)} . \\
& \leq \frac{1}{2 \inf \left(\bar{g}^{\prime}\right)^{2}|r-s|}\left[\left|\bar{g}^{\prime}(r)-\bar{g}^{\prime}(t)\right| \bar{g}^{\prime}(s)+\bar{g}^{\prime}(t)\left|\bar{g}^{\prime}(s)-\bar{g}^{\prime}(t)\right|\right] .
\end{aligned}
$$

Como $\bar{g}^{\prime}$ es $\tau$-Hölder continua, tenemos

$$
\begin{aligned}
\left|\frac{\left[\bar{g}^{\prime}(r) \bar{g}^{\prime}(s)\right]^{\frac{1}{2}}}{\bar{g}(r)-\bar{g}(s)}-\frac{1}{r-s}\right| & \leq \frac{\left|\bar{g}^{\prime}\right|_{\tau} \sup \left(\bar{g}^{\prime}\right)}{2|r-s| \inf \left(\bar{g}^{\prime}\right)^{2}}\left[|r-t|^{\tau}+|s-t|^{\tau}\right] \\
& \leq C|r-s|^{\tau-1} .
\end{aligned}
$$

Puesto que $\tau>1 / 2$, la función $(r, s) \mapsto|r-s|^{\tau-1}$ está en $\mathcal{L}_{\mathbb{R}}^{2}\left(\mathrm{~S}^{1} \times \mathrm{S}^{1}, L e b\right)$, lo cual prueba que $c(g)$ pertenece a $\mathcal{L}_{\mathbb{R}}^{2, \Delta}\left(\mathrm{S}^{1} \times \mathrm{S}^{1}, L v\right)$.

Si el grupo $\Gamma$ verifica la propiedad de Kazhdan y $\tau>1 / 2$, entonces el cociclo (5.5) es un coborde. En otras palabras, existe una función $K$ en $\mathcal{L}_{\mathbb{R}}^{2, \Delta}\left(\mathrm{S}^{1} \times \mathrm{S}^{1}, L v\right)$ tal que para todo $g \in \Gamma$ se tiene (en casi todo punto) la igualdad

$$
1-[\operatorname{Jac}(\bar{g})(r, s)]^{\frac{1}{2}}=K(r, s)-K(\bar{g}(r), \bar{g}(s)) \cdot[\operatorname{Jac}(\bar{g})(r, s)]^{\frac{1}{2}},
$$

es decir,

$$
[1-K(\bar{g}(r), \bar{g}(s))]^{2} \cdot J a c(\bar{g})(r, s)=[1-K(r, s)]^{2} .
$$

Tenemos así la proposición siguiente.

Proposición 5.27. Sea $\Phi: \Gamma \rightarrow$ Difeo $_{+}^{1+\tau}\left(\mathrm{S}^{1}\right)$ una representación, con $\tau>1 / 2$. Si $\Gamma$ tiene la propiedad $(\mathrm{T})$, entonces existe $K \in \mathcal{L}_{\mathbb{R}}^{2, \Delta}\left(\mathrm{S}^{1} \times \mathrm{S}^{1}, L v\right)$ tal que $\Gamma$ preserva la corriente geodésica $L_{K}$ dada por

$$
\frac{d L_{K}}{d L v}=[1-K(r, s)]^{2} \text {. }
$$


Puesto que $L_{K}$ es una medida absolutamente continua respecto a la medida de Lebesgue sobre $\mathrm{S}^{1} \times \mathrm{S}^{1} \backslash \Delta$, la propiedad siguiente es evidente:

$$
L_{K}([a, a] \times[b, c])=0 \text { para todo } a<b \leq c<a .
$$

Por otra parte, el hecho que $K$ sea una función de cuadrado integrable tiene por consecuencia la siguiente propiedad de $L_{K}$ :

$$
L_{K}([a, b[\times] b, c])=\infty \text { para todo } a<b<c<a .
$$

Para demostrar esta igualdad notemos que

$$
L_{K}([a, x] \times[y, c])^{\frac{1}{2}}=\left(\int_{a}^{x} \int_{y}^{c}[1-K(r, s)]^{2} d L v\right)^{\frac{1}{2}} \geq\left(\int_{a}^{x} \int_{y}^{c} \frac{d r d s}{4 \operatorname{sen}^{2}\left(\frac{r-s}{2}\right)}\right)^{\frac{1}{2}}-\|K\|_{2} .
$$

Puesto que

$$
\int_{a}^{x} \int_{y}^{c} \frac{d r d s}{4 \operatorname{sen}^{2}\left(\frac{r-s}{2}\right)}=\log \left(\left[e^{i a}, e^{i x}, e^{i y}, e^{i c}\right]\right),
$$

y puesto que el valor de la razón cruzada $\left[e^{i a}, e^{i x}, e^{i y}, e^{i c}\right]$ tiende a $\infty$ cuando $x$ e $y$ tienden hacia $b \quad($ con $a<x<b<y<c)$, la igualdad (5.7) resulta inmediatamente.

Diremos que una corriente geodésica es estable si verifica las propiedades (5.6) y (5.7). La proposición 5.27 implica directamente la siguiente.

Proposición 5.28. Sea $\Phi: \Gamma \rightarrow$ Difeo $_{+}^{1+\tau}\left(\mathrm{S}^{1}\right)$ una representación, donde $\tau>1 / 2$. Si $\Gamma$ posee la propiedad $(\mathrm{T})$ entonces existe una corriente geodésica estable invariante por $\Gamma$.

El soporte de la medida $L_{K}$ puede ser no total, es decir, puede haber intervalos $[a, b]$ y $[c, d]$ no reducidos a un punto tales que

$$
L_{K}([a, b] \times[c, d])=0 .
$$

Esto induce a pensar que los homeomorfismos de $\mathrm{S}^{1}$ que preservan la corriente geodésica $L_{K}$ no son necesariamente rígidos, en el sentido que un homeomorfismo tal podría fijar tres puntos sin ser la identidad. Sin embargo, veremos que las propiedades de estabilidad de $L_{K}$ permiten probar que esto no ocurre.

Lema 5.29. El único homeomorfismo del círculo que preserva una corriente geodésica estable y fija al menos tres puntos es la identidad.

Demostración. Supongamos que un homeomorfismo $f \neq I d$ fije al menos tres puntos y preserve una corriente geodésica estable $L$, y sea $I=] a, b[$ una 
componente conexa del complemento del conjunto de los puntos fijos de $f$. Observe que $a$ y $b$ son puntos fijos de $f$; sea $c \in] b, a[$ otro punto fijo de $f$. Como $f$ no fija ningún punto en $] a, b[$, para cada $x \in] a, b[$ la sucesión $\left(f^{i}(x)\right)$ converge ya sea a $b$ o al punto $a$. Siendo ambos casos análogos, consideremos sólo el primero. Tenemos entonces $\lim _{i \rightarrow-\infty} f^{i}(x)=a$, por lo que

$$
L([a, x] \times[b, c])=L([a, f(x)] \times[b, c]),
$$

y por lo tanto $L([x, f(x)] \times[b, c])=0$. Como $x \in] a, b[$ es arbitrario,

$$
L(] a, b[\times[b, c])=\sum_{i \in \mathbb{Z}} L\left(\left[f^{i}(x), f^{i+1}(x)\right] \times[b, c]\right)=0,
$$

lo cual contradice (5.7).

La proposición a continuación se deduce directamente de lo que precede (en lo que sigue retomamos la notación $\Phi(g)$ en lugar de $\bar{g}$ por razones que serán evidentes en la demostración de la proposición 5.31).

Proposición 5.30. Sea $\Phi: \Gamma \rightarrow$ Difeo $_{+}^{1+\tau}\left(\mathrm{S}^{1}\right)$ una representación, con $\tau>1 / 2$. Si $\Gamma$ posee la propiedad $(\mathrm{T})$ y $g \in \Gamma$ es tal que $\Phi(g)$ fija tres puntos del círculo, entonces $\Phi(g)$ es la identidad.

La ingeniosa idea de la prueba presentada a continuación fue gentilmente comunicada al autor por Witte.

Proposición 5.31. Sea $\Phi: \Gamma \rightarrow$ Difeo $_{+}^{1+\tau}\left(\mathrm{S}^{1}\right)$ una representación, con $\tau>1 / 2$. Si $\Gamma$ posee la propiedad $(\mathrm{T})$ y $g \in \Gamma$ es tal que $\Phi(g)$ fija un punto del círculo, entonces $\Phi(g)$ es la identidad.

Demostración. Consideremos el recubrimiento a tres hojas $\hat{\mathrm{S}}^{1}$ de $\mathrm{S}^{1}$. Sobre este recubrimiento actúa (por difeomorfismos de clase $\mathrm{C}^{1+\tau}$ ) una extensión central $\hat{\Gamma}$ de $\Gamma$ de la forma $0 \longrightarrow \mathbb{Z} / 3 \mathbb{Z} \longrightarrow \hat{\Gamma} \longrightarrow \Gamma \longrightarrow 0$. Puesto que $\Gamma$ posee la propiedad $(\mathrm{T})$ y $\mathbb{Z} / 3 \mathbb{Z}$ es finito, $\hat{\Gamma}$ también posee la propiedad (T). Si $g \in \Gamma$ es tal que $\Phi(g)$ fija un punto del círculo inicial, entonces una de sus preimágenes en $\hat{\Gamma}$ fija tres puntos de $\hat{\mathrm{S}}^{1}$ por la acción inducida. Como $\hat{\mathrm{S}}^{1}$ se identifica a un círculo, utilizando la proposición precedente se deduce fácilmente que $\Phi(g)$ es la identidad.

Es fácil completar ahora la prueba del teorema 5.25. En efecto, por la proposición anterior, la acción del grupo $\Phi(\Gamma)$ sobre $\mathrm{S}^{1}$ es libre, por lo que el teorema de Hölder implica que dicho grupo es abeliano. Sin embargo, $\Phi(\Gamma)$ verifica aún la propiedad de Kazhdan, por lo que debe ser finito.

Observación 5.32. Por el ejercicio 1.16, todo grupo de homeomorfismos de $\mathrm{S}^{1}$ que preserva una corriente geodésica estable es conjugado a un subgrupo del grupo de Möbius. Luego, si $\tau>1 / 2$ y $\Phi: \Gamma \rightarrow \operatorname{Difeo}_{+}^{1+\tau}\left(\mathrm{S}^{1}\right)$ es una representación cuyo cociclo asociado (5.5) es un coborde, entonces la imagen $\Phi(\Gamma)$ es topológicamente conjugada a un subgrupo de $\operatorname{PSL}(2, \mathbb{R})$. 
Esta observación permite finalizar de una manera diferente la prueba del teorema 5.25. En efecto, en el capítulo 6 de [91] se prueba que si un grupo de Kazhdan $\Gamma$ actúa por isometrías de un espacio hiperbólico real, entonces existe un punto fijo para dicha acción. Conjugando con un elemento de $\operatorname{PSL}(2, \mathbb{R})$, podemos suponer que dicho punto fijo es el origen. Obtenemos así que $\Gamma$ es conjugado a un subgrupo de $\mathrm{SO}(2, \mathbb{R})$. En particular, $\Gamma$ es abeliano, y como verifica la propiedad $(\mathrm{T})$, debe ser finito.

Observación 5.33. Una combinación del teorema 5.25 con la versión bidimensional del teorema de estabilidad de Thurston [198] permite probar que, para $\tau>1 / 2$, todo grupo numerable de difeomorfismos de clase $\mathrm{C}^{1+\tau}$ del disco o del anillo cerrados que satisface la propiedad (T) es finito. Se ignora si esto también es válido para el disco o el anillo abiertos, o bien para superficies compactas de característica de Euler no positiva.

Ejercicio 5.34. Pruebe que para toda función $K \in \mathcal{L}_{\mathbb{R}}^{2, \Delta}\left(\mathrm{S}^{1} \times \mathrm{S}^{1}, L v\right)$, el grupo $\Gamma_{L_{K}}$ de los homeomorfismos de $\mathrm{S}^{1}$ que preservan la corriente geodésica $L_{K}$ es uniformemente quasi-simétrico (vea el ejercicio 1.15; compare también con el ejercicio 1.16).

Ejercicio 5.35. Siguiendo [94], denotemos $\mathrm{D}^{\infty}=\operatorname{Difeo}_{+}^{\infty}\left(\mathrm{S}^{1}\right) \backslash \operatorname{int}\left(\rho^{-1}(\mathbb{Q} / \mathbb{Z})\right)$, donde $\rho$ designa la función número de rotación. El espacio $\mathrm{D}^{\infty}$ es por definición un cerrado de $\operatorname{Difeo}_{+}^{\infty}\left(\mathrm{S}^{1}\right)$; en particular, se trata de un espacio de Baire. Pruebe que para elementos genéricos $g \in \mathrm{D}^{\infty}$, el grupo cíclico infinito $\left\{g^{n}: n \in \mathbb{Z}\right\}$ no es uniformemente quasi-simétrico, y que en particular el conjunto $\left\{\left\|c\left(g^{n}\right)\right\|: n \in \mathbb{Z}\right\}$ no es acotado (recuerde que una propiedad atribuible a los puntos de un espacio topológico es de tipo $G_{\delta}$ si ella es satisfecha sobre un conjunto $G_{\delta}$ de puntos del espacio, y es genérica si ella se cumple sobre un conjunto $G_{\delta}$ denso; recuerde además que, para espacios de Baire, la intersección numerable de conjuntos $G_{\delta}$ densos es aún un $G_{\delta}$ denso).

Sugerencia. La propiedad $\sup _{[a, b, c, d]=2} \sup _{n \in \mathbb{N}}\left[g^{n}(a), g^{n}(b), g^{n}(c), g^{n}(d)\right]=\infty$ es de tipo $G_{\delta}$. Verifique que ella es satisfecha por todo homeomorfismo del círculo con número de rotación racional que posee al menos tres puntos periódicos y que no es conjugado a una rotación. Concluya observando que dichos homeomorfismos son densos en $\mathrm{D}^{\infty}$.

Observación. De acuerdo a la observación 3.10, para todo $g \in \mathrm{D}^{\infty}$ se tiene la convergencia (en topología $\mathrm{C}^{1}$ ) de $g^{q_{n}}$ hacia la identidad, donde $p_{n} / q_{n}$ es la $n$-ésima aproximación racional de $\rho(g)$. Ahora bien, si $k \geq 2$ entonces para un subconjunto genérico de elementos $g$ de $\mathrm{D}^{\infty}$ existe una sucesión creciente de enteros $n_{i}$ tal que $g^{n_{i}}$ converge a la identidad en topología $\mathrm{C}^{k}$. Esto último resulta del hecho que si denotamos por $d_{i s t}$ una distancia induciendo la topología $\mathrm{C}^{k}$ en Difeo ${ }_{+}^{k}\left(\mathrm{~S}^{1}\right)$, entonces la propiedad

$$
\liminf _{n>0} \operatorname{dist}_{k}\left(g^{n}, I d\right)=0
$$

es de tipo $G_{\delta}$ en $\mathrm{D}^{\infty}$, la cual es satisfecha cuando $\rho(g)$ verifica una condición diofantina $[94,110,211]$. Se concluye así que, para $g \in \mathrm{D}^{\infty}$ genérico, la sucesión $\left(\left\|c\left(g^{n}\right)\right\|\right)$ es no acotada y contiene a cero en su adherencia. Además, las órbitas de la isometría correspondiente $A(g)=\Psi(g)+c(g)$ son no acotadas y recurrentes, en el sentido que ellas coinciden con su conjunto derivado. Vea [50] para más información sobre acciones isométricas no acotadas y recurrentes. 
Ejercicio 5.36. Pruebe la validez del lema de Kopell para difeomorfismos de clase $\mathrm{C}^{1+\text { lip }}$ del intervalo utilizando el cociclo de Liouville. De manera más precisa, suponga que $f$ y $g$ sean difeomorfismos conmutantes de $\left[0,1\left[\right.\right.$ de clase $\mathrm{C}^{1+\text { lip }}$. Considere la "medida de Liouville" $\overline{L v}$ sobre $[0,1[\times[0,1[$ cuya densidad respecto a la medida de Lebesgue es la función $(r, s) \mapsto 1 /(r-s)^{2}$.

(i) Verifique que las funciones $c(f)$ y $c(g)$ definidas por

$$
c(f)(r, s)=1-[\operatorname{Jac}(\bar{f})(r, s)]^{\frac{1}{2}} \quad \mathrm{y} \quad c(g)(r, s)=1-[\operatorname{Jac}(\bar{g})(r, s)]^{\frac{1}{2}}
$$

son de cuadrado integrable sobre los compactos de $[0,1[\times[0,1[$. Observe que $c$ es un "cociclo" respecto a la "representación regular" $\Psi$ correspondiente.

(ii) Verifique que para todo $a \in] 0,1[$ existe una constante $C=C(a, f)$ tal que para todo intervalo $[b, c]$ contenido en $[0, a]$ se tiene

$$
\int_{b}^{c} \int_{b}^{c}\|c(f)\|^{2} \leq C|c-b|^{2} .
$$

(iii) Suponga que $g$ fija un punto $a \in] 0,1[$ y que $f(x)<x$ para todo $x \in] 0,1[$. Utilizando la desigualdad precedente y la relación

$$
c\left(g^{k}\right)=c\left(f^{-n} g^{k} f^{n}\right)=c\left(f^{-n}\right)+\Psi\left(f^{-n}\right) c\left(g^{k}\right)+\Psi\left(f^{-n} g\right) c\left(f^{n}\right),
$$

concluya que el valor de

$$
\int_{f^{2}(a)}^{a} \int_{f^{2}(a)}^{a}\left\|c\left(g^{k}\right)\right\|^{2}
$$

está uniformemente acotado (independientemente de $k \in \mathbb{N}$ ).

(iv) Mediante un argumento similar al de la proposición 5.30, pruebe que la restricción de $g$ a $\left[f^{2}(a), a\right]$ (y por lo tanto a todo el intervalo [0,1[) es la identidad.

Ejercicio 5.37. El borde de $\mathcal{T}^{p}$ soporta una medida natural $\mu$ de masa finita, a saber, aquélla que otorga masa $p^{-n}$ a toda bola de radio $p^{-n}$, donde $n \geq 1$. Dado un homeomorfismo $g$ de $\partial \mathcal{T}^{p}$, denotaremos por $g^{\prime}$ la derivada de Radon-Nikodym de $g$ respecto a $\mu$ (si ella está bien definida). La medida de Liouville $L v$ sobre $\partial \mathcal{T}^{p} \times \partial \mathcal{T}^{p}$ viene dada en este caso por

$$
d L v(x, y)=\frac{d \mu(x) \times d \mu(y)}{\partial \operatorname{ist}(x, y)^{2}} .
$$

Sea $\mathcal{H}=\mathcal{L}_{\mathbb{R}}^{2, \Delta}\left(\partial \mathcal{T}^{p} \times \partial \mathcal{T}^{p}, L v\right)$ el espacio de Hilbert formado por las funciones $K \in \mathcal{L}_{\mathbb{R}}^{2}\left(\partial \mathcal{T}^{p} \times \partial \mathcal{T}^{p}, L v\right)$ satisfaciendo $K(x, y)=K(y, x)$ para casi todo $(x, y)$ en $\partial \mathcal{T}^{p} \times \partial \mathcal{T}^{p}$. Considere la representación unitaria $\Psi$ de $\mathcal{N}^{p}$ sobre $\mathcal{H}$ dada por

$$
\Psi(g) K(x, y)=K\left(g^{-1}(x), g^{-1}(y)\right) \cdot\left[\operatorname{Jac}\left(g^{-1}\right)(x, y)\right]^{1 / 2},
$$

donde $J a c\left(g^{-1}\right)(x, y)$ designa el jacobiano (respecto a la medida de Liouville) de la aplicación $(x, y) \mapsto\left(g^{-1}(x), g^{-1}(y)\right)$.

(i) Pruebe que la extensión al borde de cualquier isometría $f$ de $\mathcal{T}^{p}$ verifica dist $(x, y) f^{\prime}(x) f^{\prime}(y)=\partial \operatorname{ist}(f(x), f(y))$ para todo par de puntos $x, y$ de $\partial \mathcal{T}^{p}$.

(ii) Concluya que para todo $g \in \mathcal{N}^{p}$ la función $c(g): \partial \mathcal{T}^{p} \times \partial \mathcal{T}^{p} \rightarrow \mathbb{R}$ dada por

$$
c(g)(x, y)=1-\left[\operatorname{Jac}\left(g^{-1}\right)(x, y)\right]^{1 / 2}
$$

pertenece a $\mathcal{H}$. 
(iii) Verifique que la aplicación $c$ satisface la relación de cociclo respecto a $\Psi$.

(iv) Pruebe que si $\Gamma$ es un subgrupo de $\mathcal{N}^{p}$ que satisface la propiedad (T), entonces existe $K \in \mathcal{H}$ tal que $\Gamma$ preserva la corriente geodésica

$$
L_{K}=[1-K(x, y)]^{2} d L v .
$$

(v) Concluya que existe un subconjunto compacto $\mathcal{C}=\mathcal{C}(K)$ de $\mathcal{T}^{p}$ tal que para todo elemento $g \in \Gamma \backslash \operatorname{Isom}\left(\mathcal{T}^{p}\right)$ se cumple $\mathcal{A}_{g} \cap \mathcal{C} \neq \emptyset$ o bien $\mathcal{B}_{g} \cap \mathcal{C} \neq \emptyset$.

Observación. Ignoramos si a través del método introducido en este ejercicio es posible redemostrar la proposición 5.21.

\subsection{Propiedad $(\mathrm{T})$ relativa y propiedad de Haagerup}

Una interrogante que se plantea de manera natural tras la lectura de las secciones precedentes consiste en saber si los subgrupos finitamente generados de Difeo ${ }_{+}^{1+\tau}\left(\mathrm{S}^{1}\right)$ verifican necesariamente la propiedad de Haagerup cuando $\tau>1 / 2$. (vea el ejemplo 5.15). Tal es el caso de los subgrupos no finitos de $\operatorname{PSL}(2, \mathbb{R})$ (vea [47]) y de los grupos de Thompson (vea la prueba de la proposición 5.19). Una de las dificultades de esta pregunta consiste en que se conocen pocos ejemplos de grupos que no verifican ni la propiedad de Haagerup ni la de Kazhdan simultáneamente. De hecho, una de las pocas obstrucciones conocidas a la propiedad de Haagerup es una forma débil de la propiedad $(\mathrm{T})$, a saber, la propiedad $(\mathrm{T})$ relativa.

Definición 5.38. Si $\Gamma$ es un grupo localemente compacto y $\Gamma_{0}$ un subgrupo de $\Gamma$, entonces el par $\left(\Gamma, \Gamma_{0}\right)$ posee la propiedad $(\mathrm{T})$ relativa si para toda representación isométrica de $\Gamma$ sobre un espacio de Hilbert, existe un vector invariante por $\Gamma_{0}$.

Un ejemplo no trivial de un par que satisface la propiedad $(\mathrm{T})$ relativa es $\left(\mathbb{Z}^{2} \rtimes \mathrm{SL}(2, \mathbb{Z}), \mathbb{Z}^{2}\right)$. El lector hallará más ejemplos, así como referencias sobre el tema, en [47] y [91]. Señalemos que en la mayoría de los ejemplos conocidos, si tanto $\Gamma$ como $\Gamma_{0}$ no poseen la propiedad $(\mathrm{T})$, entonces $\Gamma_{0}$ (contiene un subgrupo cocompacto que) es normal en $\Gamma$ (vea sin embargo el reciente trabajo [49]). Bajo una hipótesis de este tipo el resultado siguiente puede ser considerado como una pequeña generalización del teorema 5.25.

Teorema 5.39. Sea $\Gamma$ un subgrupo de $\operatorname{Difeo}_{+}^{1+\tau}\left(\mathrm{S}^{1}\right)$, con $\tau>1 / 2$. Supongamos que $\Gamma$ posea un subgrupo normal $\Gamma_{0}$ tal que el par $\left(\Gamma, \Gamma_{0}\right)$ satisface la propiedad $(\mathrm{T})$ relativa. Entonces $\Gamma$ es topológicamente conjugado a un grupo de rotaciones, o bien $\Gamma_{0}$ es finito.

Demostración. Retomemos la técnica de demostración de la sección precedente. El cociclo de Liouville induce una representación isométrica de $\Gamma$ sobre $\mathcal{L}_{\mathbb{R}}^{2, \Delta}\left(\mathrm{S}^{1} \times \mathrm{S}^{1}, L v\right)$. Si $\left(\Gamma, \Gamma_{0}\right)$ posee la propiedad $(\mathrm{T})$ relativa, entonces esta representación admite un vector invariante por $\Gamma_{0}$, y los argumentos de la sección precedente (vea la observación 5.32) prueban que $\Gamma_{0}$ es topológicamente conjugado a un subgrupo del grupo de Möbius. 
La propiedad $(\mathrm{T})$ relativa también es estable por extensiones centrales finitas. Gracias a esto, y mediante el argumento de paso a un recubrimiento a tres hojas de la demostración de la proposición 5.31, se verifica que $\Gamma_{0}$ es en realidad topológicamente conjugado a un subgrupo del grupo de rotaciones (vea la observación 5.32).

Si el grupo $\Gamma_{0}$ no es finito entonces él es topológicamente conjugado a un grupo denso de rotaciones. Ahora bien, es fácil comprobar que el normalizador en $\mathrm{Homeo}_{+}\left(\mathrm{S}^{1}\right)$ de todo subgrupo denso de $\mathrm{SO}(2, \mathbb{R})$ coincide con el grupo de rotaciones. Por lo tanto, $\Gamma$ es topológicamente conjugado a un subgrupo de $\mathrm{SO}(2, \mathbb{R})$, lo cual termina la demostración.

\section{Supra-rigidez para redes de rango superior}

\subsection{Presentación del resultado}

En [76], Ghys no sólo prueba el teorema de rigidez para acciones de redes de grupos de Lie simples de rango superior por difeomorfismos del círculo, sino que también considera el caso de redes en grupos de Lie semisimples. El resultado a seguir se encuentra en dicho artículo; otros resultados relacionados debidos a Burger y Monod pueden ser hallados en [29, 30]. Recordemos que una red $\Gamma$ de un grupo de Lie $G$ es dicha irreducible si es imposible hallar dos subgrupos normales $G_{1}$ y $G_{2}$ que engendren $G$ de modo que $G_{1} \cap G_{2}$ esté contenido en el centro de $G$ (al que supondremos finito) y tales que $\left(\Gamma \cap G_{1}\right) \cdot\left(\Gamma \cap G_{2}\right)$ sea de índice finito en $\Gamma$.

Teorema 5.40. Sean $G$ un grupo de Lie semisimple conexo de rango real superior o igual a 2 y $\Gamma$ una red irreducible de $G$. Si $\Phi$ es un homomorfismo de $\Gamma$ hacia el grupo de los difeomorfismos de clase $\mathrm{C}^{1}$ del círculo, entonces $\Phi$ tiene imagen finita, o bien $\Phi$ es semiconjugado a un recubrimiento finito de un homomorfismo obtenido como composición de las operaciones siguientes:

- la inclusión de $\Gamma$ en $G$,

- un homomorfismo sobreyectivo de $G$ en $\operatorname{PSL}(2, \mathbb{R})$,

- la acción proyectiva de $\operatorname{PSL}(2, \mathbb{R})$ sobre el círculo.

Para la demostración de este resultado, Ghys comienza por examinar el caso de las redes de grupos de Lie semisimples "clásicos" $(\mathrm{SL}(n, \mathbb{R})$, $\mathrm{Sp}(2 r, \mathbb{R}), \mathrm{SO}(2, q), \mathrm{SU}(2, q)$ y $\operatorname{PSL}(2, \mathbb{R}) \times \operatorname{PSL}(2, \mathbb{R}))$, para luego finalizar la prueba utilizando algunos aspectos de la teoría de clasificación de los grupos de Lie semisimples [115]. Observemos que los cuatro primeros casos corresponden a grupos de Lie simples de rango real superior o igual a 2 (las redes correspondientes satisfacen por lo tanto la propiedad (T) de Kazhdan...). Para el último caso (que dinámicamente es el más interesante), Ghys demuestra que todo homomorfismo $\Phi: \Gamma \rightarrow \operatorname{Difeo}_{+}^{1}\left(\mathrm{~S}^{1}\right)$ transita, módulo semiconjugación topológica y recubrimiento finito, por la proyección de $\Gamma$ sobre uno de los factores, y luego por la acción proyectiva de 
dicho factor sobre el círculo. Para entender mejor este último punto, se debe tener en mente como ejemplo fundamental la incrustación de $\operatorname{PSL}(2, \mathbb{Z}(\sqrt{2}))$ como red en $\operatorname{PSL}(2, \mathbb{R}) \times \operatorname{PSL}(2, \mathbb{R})$ a través de la aplicación

$\left(\begin{array}{ll}a_{1}+b_{1} \sqrt{2} & a_{2}+b_{2} \sqrt{2} \\ a_{3}+b_{3} \sqrt{2} & a_{4}+b_{4} \sqrt{2}\end{array}\right) \longmapsto\left(\left(\begin{array}{cc}a_{1}+b_{1} \sqrt{2} & a_{2}+b_{2} \sqrt{2} \\ a_{3}+b_{3} \sqrt{2} & a_{4}+b_{4} \sqrt{2}\end{array}\right),\left(\begin{array}{cc}a_{1}-b_{1} \sqrt{2} & a_{2}-b_{2} \sqrt{2} \\ a_{3}-b_{3} \sqrt{2} & a_{4}-b_{4} \sqrt{2}\end{array}\right)\right)$.

Para generalizar el teorema 5.40, se debe hacer frente al problema de definir la noción de rango real para un grupo arbitrario. Si bien muchas tentativas han sido hechas en tal dirección (vea por ejemplo [7]), nosotros hemos preferido seguir una idea bastante simple qua ha sido introducida por Shalom en [187]. Lo esencial de su punto de vista consiste en utilizar la conmutatividad de los factores del grupo ambiente, vista como una hipótesis de rango superior. Así, el "cuadro general" que consideraremos (y que coincide con aquél de [187]) es el siguiente:

- $G=G_{1} \times \cdots \times G_{k}$ es un grupo topológico compactamente generado, con $k \geq 2$, y $\Gamma$ es una red finitamente generada y uniforme (es decir, un subgrupo discreto y cocompacto) de $G$;

- las proyecciones de $\Gamma$ sobre cada factor $G_{i}$ son densas (denotaremos por $p r_{i}$ la proyección de $G$ sobre $G_{i}$ );

- en el caso en que cada $G_{i}$ sea un grupo algebraico lineal sobre un cuerpo local [129], aceptaremos también la posibilidad de que $\Gamma$ sea una red no cocompacta en $G$.

Notemos que en el último caso, la red $\Gamma$ es siempre finitamente generada. Esto se desprende de importantes resultados debidos a Kazhdan y Margulis [129]. Por otra parte, la segunda condición corresponde a una hipótesis de irreducibilidad análoga a aquélla verificada por las redes consideradas en el teorema de Ghys.

En la introducción de [187] el lector podrá hallar otras motivaciones -además de referencias significativas- en torno al cuadro general considerado. Mencionemos en todo caso que algunos ejempos de redes "no lineales" satisfaciendo las dos primeras propiedades han sido construidos en $[12,31$, 176]. Para tales redes, así como para las redes irreducibles lineales (i.e., que se incrustan en $\mathrm{GL}(n, \mathbb{K})$ para algún cuerpo $\mathbb{K})$, el siguiente resultado de supra-rigidez fue obtenido por el autor en [145] (vea también [143]).

Teorema 5.41. En el contexto precedente, sea $\Phi: \Gamma \rightarrow \operatorname{Difeo}_{+}^{1+\tau}\left(\mathrm{S}^{1}\right)$ un homomorfismo tal que $\tau>1 / 2$. Si $\Phi(\Gamma)$ no preserva ninguna medida de probabilidad sobre el círculo, entonces $\Phi(\Gamma)$ es topológicamente conjugado a un subgrupo de $\operatorname{PSL}(2, \mathbb{R})$, o bien $\Phi$ es semiconjugado a un recubrimiento finito de un homomorfismo obtenido al componer las siguientes operaciones: - la inclusión de $\Gamma$ en $G$,

- la proyección de $G$ sobre uno de los factores $G_{i}$,

- una acción $\Phi$ de $G_{i}$ por homeomorfismos del círculo. 
La hipótesis según la cual $\Phi(\Gamma)$ no fija ninguna medida de probabilidad sobre $\mathrm{S}^{1}$ puede ser suprimida cuando el primer espacio de cohomología a valores reales de todo subgrupo normal y de índice finito de $\Gamma$ es trivial. Notemos que por [187], esta última condición se verifica cuando $\mathrm{H}^{1}(G, \mathbb{R})$ est trivial (tal es el caso por ejemplo si los $G_{i}$ son grupos lineales algebraicos semisimples sobre cuerpos locales [129]).

Corolario 5.42. Sean $\Gamma$ una red satisfaciendo las hipótesis del cuadro general y $\Phi: \Gamma \rightarrow$ Difeo $_{+}^{1+\tau}\left(\mathrm{S}^{1}\right)$ un homomorfismo, con $\tau>1 / 2$. Si $\mathrm{H}^{1}\left(\Gamma_{0}, \mathbb{R}\right)=\{0\}$ para todo subgrupo $\Gamma_{0}$ normal $y$ de indice finito en $\Gamma$, entonces se verifica la conclusión del teorema 5.41 .

Dado lo anterior, para entender las acciones de redes irreducibles de rango superior por difeomorfismos del círculo podemos valernos de la clasificación dada en la sección 4 del primer capítulo. Obtenemos así una versión refinada de los resultados anteriores bajo cualquiera de las hipótesis siguientes:

(i) el núcleo de $\Phi$ es finito y las órbitas de $\Phi(\Gamma)$ son densas,

(ii) el núcleo de $\Phi$ es finito y $\Phi$ toma valores en el grupo Difeow ${ }_{+}\left(S^{1}\right)$ de los difeomorfismos real-analíticos del círculo,

(iii) los subgrupos normales de $\Gamma$ son finitos o de índice finito (es decir, $\Gamma$ satisface el teorema de los subgrupos normales de Margulis [129]).

Teorema 5.43. Supongamos que las hipótesis del corolario sean satisfechas, que cada grupo $G_{i}$ sea no discreto, y que al menos una de las hipótesis anteriores (i), (ii) o (iii) se cumpla. Si la imagen $\Phi(\Gamma)$ no es finita entonces, módulo una semiconjugación topológica y un recubrimiento finito, $\Phi(\Gamma)$ es un subgrupo no metabeliano de $\operatorname{PSL}(2, \mathbb{R})$.

Asumimos al menos una de las hipótesis (i), (ii) o (iii) para evitar el caso degenerado de un grupo de Lie con infinitas componentes conexas cuya acción sobre el círculo transite, módulo semiconjugación topológica, por el cuociente respecto a la componente conexa de la identidad. Señalemos que la hipótesis (iii) es satisfecha por las redes de grupos algebraicos, así como por las construidas en [31] (vea [6] para una versión general de este hecho).

Las demostraciones de los resultados precedentes utilizarán como herramienta esencial un teorema de supra-rigidez para acciones isométricas sobre espacios de Hilbert debido a Shalom, el cual será discutido en la sección siguiente. Señalemos que este teorema ha sido recientemente generalizado en [4] para acciones sobre espacios $\mathcal{L}^{p}$. Esto permite obtener versiones en clase $\mathrm{C}^{1+\tau}$ de los resultados precedentes para todo $\tau>0$. Sin embargo, para no oscurecer nuestra presentación, no ahondaremos más en este punto.

Observación 5.44. Algunos de los resultados presentados en esta sección han sido recientemente generalizados (para acciones por homeomorfismos) por Bader, Furman y Shaker en [5] mediante métodos ligados a la "teoría de bordes". 


\subsection{Supra-rigidez cohomológica}

Consideramos nuevamente una representación unitaria $\Psi$ de $\Gamma$ sobre un espacio de Hilbert $\mathcal{H}$. El grupo $\Gamma$ puede ahora ser no discreto, pero es supuesto localmente compacto y generado por un subconjunto compacto.

Definición 5.45. La representación unitaria $\Psi$ casi posee vectores invariantes si existe una sucesión de vectores unitarios $K_{n} \in \mathcal{H}$ tal que, para todo subconjunto compacto $\mathcal{C}$ de $\Gamma$, el valor de $\sup _{g \in \mathcal{C}}\left\|K_{n}-\Psi(g) K_{n}\right\|$ converge a cero cuando $n$ tiende al infinito.

Definición 5.46. Un cociclo $c: \Gamma \rightarrow \mathcal{H}$ asociado a $\Psi$ es un casi coborde (o es casi cohomológicamente trivial) si existe una sucesión de cobordes $c_{n}$ tal que $\sup _{g \in \mathcal{C}}\left\|c_{n}(g)-c(g)\right\|$ converge a cero cuando $n$ tiende al infinito para todo subconjunto compacto $\mathcal{C}$ de $\Gamma$.

Ejercicio 5.47. Pruebe que el cociclo del ejemplo 5.17 es casi cohomológicamente trivial para todo subgrupo finitamente generado de $\mathrm{Difeo}_{+}^{1+\tau}([0,1])$ cuyos elementos son tangentes a la identidad en los extremos (con $\tau>0$ arbitrario).

Sugerencia. Considere el coborde $c_{n}$ asociado a la función $K_{n}(x)=\mathcal{X}_{[1 / n, 1]}(x)$.

El lema elemental que presentamos a continuación, debido a Delorme [91], resulta fundamental para el estudio de la noción de casi cobordes.

Lema 5.48. Si $\Psi$ no casi posee vectores invariantes, entonces todo cociclo que es casi cohomológicamente trivial es cohomológicamente trivial.

Demostración. Sea $\mathcal{G}$ una parte generadora compacta de $\Gamma$. Por hipótesis, existe una constante $\varepsilon>0$ tal que, para todo $K \in \mathcal{H}$,

$$
\sup _{h \in \mathcal{G}}\|K-\Psi(h) K\| \geq \varepsilon\|K\| .
$$

Puesto que $c$ es un casi coborde, existe una sucesión $\left(K_{n}\right)$ en $\mathcal{H}$ tal que para todo $g \in \Gamma$ se tiene la igualdad $c(g)=\lim _{n \rightarrow+\infty}\left(K_{n}-\Psi(g) K_{n}\right)$. La desigualdad (5.8) nos da $M=\sup _{h \in \mathcal{G}}\|c(h)\| \geq \varepsilon \lim \sup _{n}\left\|K_{n}\right\|$, por lo que $\lim \sup _{n}\left\|K_{n}\right\| \leq M / \varepsilon$. Luego, $\|c(g)\| \leq \lim \sup _{n}\left(\left\|K_{n}\right\|+\left\|\Psi(g) K_{n}\right\|\right) \leq 2 M / \varepsilon$ para todo $g \in \Gamma$. El cociclo $c$ es entonces uniformemente acotado, por lo que el lema del centro de Tits implica que es cohomológicamente trivial (vea el ejercicio 5.14).

Presentamos a continuación una versión del teorema de supra-rigidez cohomológica obtenido por Shalom en [187]. Señalemos en todo caso que nosotros no utilizaremos este resultado en toda su generalidad. En efecto, en nuestras aplicaciones nos reduciremos rápidamente al caso en que la representación unitaria correspondiente no casi posee vectores invariantes. En dicho caso, la cohomología reducida coincide con la usual, y el teorema de supra-rigidez resulta muchísimo más elemental. 
Teorema 5.49. Sean $G=G_{1} \times \cdots \times G_{k}$ un grupo topológico compactamente generado y $\Gamma$ una red en $G$ satisfaciendo las hipótesis del cuadro general. Sea $\Psi: \Gamma \rightarrow U(\mathcal{H})$ una representación unitaria que no casi posee vectores invariantes. Si c es un cociclo asociado a $\Psi$ que no es casi cohomológicamente trivial, entonces c es cohomólogo a un cociclo $c_{1}+\ldots+c_{k}$ tal que cada $c_{i}$ es un cociclo que toma valores en un subespacio $\Psi(\Gamma)$-invariante $\mathcal{H}_{i}$ sobre el cual la acción isométrica $\Psi+c_{i}$ se extiende continuamente a una acción isométrica que se factoriza sobre $G_{i}$.

Este resultado notable fue obtenido por Shalom inspirándose en la demostración del teorema de los subgrupos normales de Margulis. El principio sobre el cual se basa consiste en que las isometrías de un espacio de Hilbert que conmutan deben ser de cierta manera "degeneradas" (los ejercicios 5.53 y 5.54 ilustran este hecho). En lugar de dar su demostración (la que puede ser hallada en [187]), hemos preferido incluir algunos ejemplos que permiten visualizar en parte sus alcances (vea los ejercicios 5.51 y 5.52). Para el primero de ellos presentamos un lema que será utilizado constantemente para extender homomorfismos. Para formularlo, recordemos que un grupo topológico $H$ es secuencialemente completo si toda sucesión $\left(h_{n}\right)$ en $H$ que verifica $\lim _{m, n \rightarrow+\infty} h_{m}^{-1} h_{n}=i d_{H}$ converge a un límite en $H$.

Lema 5.50. Sean $G$ y $\Gamma$ dos grupos satisfaciendo las hipótesis del cuadro general. Sea $\Phi: \Gamma \rightarrow H$ un homomorfismo, donde $H$ es un grupo topológico Hausdorff secuencialmente completo. Supongamos que exista $i \in\{1, \ldots, k\}$ tal que para toda sucesión $\left(g_{n}\right)$ en $\Gamma$ satisfaciendo $\lim _{n \rightarrow+\infty} \operatorname{pr}_{i}\left(g_{n}\right)=i d_{G_{i}}$, se tiene $\lim _{n \rightarrow+\infty} \Phi\left(g_{n}\right)=i d_{H}$. Entonces $\Phi$ se extiende continuamente en un homomorfismo de $G$ hacia $H$ que se factoriza sobre $G_{i}$.

La demostración del lema es elemental. Para $g \in G_{i}$ tomamos una sucesión arbitraria $\left(g_{n}\right)$ en $\Gamma$ tal que $p r_{i}\left(g_{n}\right)$ converja a $g$. Por hipótesis, la sucesión $\left(h_{n}\right)$ en $H$ definida por $h_{n}=\Phi\left(g_{n}\right)$ verifica $\lim _{m, n \rightarrow+\infty} h_{m}^{-1} h_{n}=i d_{H}$. Se define entonces $\hat{\Phi}(g)=\lim _{n \rightarrow+\infty} h_{n}$. Esta definición es plausible, dada la hipótesis hecha sobre la topología de $H$. Además, ella no depende de la sucesión escogida, y es fácil verificar que la aplicación $\hat{\Phi}$ así definida es un homomorfismo continuo de $G$ que se factoriza sobre $G_{i}$.

Ejercicio 5.51. Asumiendo el teorema 5.49 y siguiendo los pasos indicados más adelante, pruebe el siguiente teorema de supra-rigidez para acciones sobre árboles: si $\Gamma$ es una red satisfaciendo las condiciones del "cuadro general" y $\Phi$ es una acción no elemental de $\Gamma$ por isometrías de un árbol simplicial $\mathcal{T}$, entonces existe un subárbol invariante por $\Gamma$ sobre el cual la acción se extiende a $G$ factorizándose sobre uno de $\operatorname{los} G_{i}$ (recordemos que la acción es no elemental si no fija ningún vértice, arista o punto en el infinito). Consignemos en todo caso que este resultado, contenido en [187], es válido en general para redes irreducibles no necesariamente cocompactas. La prueba para este caso aparece en [137] y utiliza cohomología acotada. 
(i) Mediante una subdivisión baricéntrica reduzca el caso general a aquél de una acción sin inversión de aristas.

(ii) Suponga que existan un vértice $v_{0}$ de $\mathcal{T}$ y un índice $i \in\{1, \ldots, k\}$ verificando lo siguiente: para toda sucesión $\left(g_{n}\right)$ en $\Gamma$ tal que $\lim _{n \rightarrow+\infty} p r_{i}\left(g_{n}\right)=i d_{G_{i}}$, dicho vértice $v_{0}$ es un punto fijo de $\Phi\left(g_{n}\right)$ para todo $n$ suficientemente grande. Pruebe que en este caso la afirmación del teorema es satisfecha.

Sugerencia. El conjunto de los vértices que verifica la condición precedente está contenido en un árbol invariante por $\Gamma$ sobre el cual se puede aplicar el lema 5.50.

(iii) Usando el lema de Delorme, demuestre que la hipótesis de no elementalidad de la acción implica que la representación regular asociada $\Psi$ de $\Gamma$ sobre $\mathcal{H}=\ell_{\mathbb{R}}^{2}(\overrightarrow{\operatorname{ar}}(\mathcal{T}))$ no casi posee vectores invariantes (vea el ejemplo 5.22).

(iv) Considere el subespacio $\mathcal{H}_{i}$ dado por el teorema 5.49. Defina $\operatorname{ar}(\mathcal{T})$ como el conjunto de aristas de $\mathcal{T}$ módulo la relación de equivalencia que identifica $\vec{\Upsilon}_{1}$ con $\vec{\Upsilon}_{2}$ si $\psi\left(\vec{\Upsilon}_{1}\right)=\psi\left(\vec{\Upsilon}_{2}\right)$ para toda función $\psi \in \mathcal{H}_{i}$. Considere el subconjunto $\tilde{\operatorname{ar}}_{0}(\mathcal{T})$ de las clases en $\operatorname{ar}(\mathcal{T})$ sobre las cuales al menos una de las funciones en $\mathcal{H}_{i}$ es no nula. Verifique que cada clase en $\tilde{a r}_{0}(\mathcal{T})$ es finita.

(v) Fije una de las clases precedentes $[\vec{\Upsilon}] \in \tilde{a r}_{0}(\mathcal{T})$ y designe por $\Gamma^{*}$ su estabilizador en $\Gamma$. El subgrupo $\Gamma^{*}$ fija el centro geométrico del conjunto de aristas subyacente en $[\vec{\Upsilon}]$. Por (i), $\Gamma^{*}$ fija un vértice $v_{0}$. Pruebe que $v_{0}$ satisface la condición de (ii).

Sugerencia. Dada una sucesión $\left(g_{n}\right)$ en $\Gamma$ tal que $\lim _{n \rightarrow+\infty} p r_{i}\left(g_{n}\right)=i d_{G_{i}}$, pruebe que $g_{n}$ pertenece a $\Gamma^{*}$ para todo $n$ suficientemente grande. Para ello razone por contradicción y considere por separado los casos en que las clases respecto a $\Gamma^{*}$ de $\operatorname{los} g_{n}$ son iguales o distintas, recordando siempre que las funciones de $\mathcal{H}_{i}$ son de cuadrado integrable y no nulas sobre las aristas de $[\vec{\Upsilon}]$.

Ejercicio 5.52. De acuerdo a la sección 1.4 del capítulo 4, existen difeomorfismos conmutantes $f$ y $g$ del intervalo $[0,1]$ que son no triviales y de clase $\mathrm{C}^{1+\tau}$, con $1 / 2<\tau<1$, y tales que el conjunto de puntos fijos de $f$ (resp. de $g$ ) en $] 0,1[$ es vacío (resp. no vacío y discreto). Pruebe que la restricción del cociclo de Liouville $c: \Gamma \rightarrow \mathcal{L}_{\mathbb{R}}^{2, \Delta}([0,1] \times[0,1], \overline{L v})$ al grupo $\Gamma \sim \mathbb{Z}^{2}$ generado por $f$ y $g$ es trivial en cohomología reducida (vea el ejercicio 5.36).

Sugerencia. Suponiendo lo contrario el teorema de Shalom nos brinda la existencia de un vector unitario $K$ en el espacio de Hilbert subyacente que es invariante ya sea por $\Psi(f)$ o por $\Psi(g)$.

(i) Sea $\mu_{K}$ la medida de probabilidad sobre $[0,1]$ definida por

$$
\mu_{K}(A)=\int_{0}^{1} \int_{A}|K(x, y)|^{2} d \overline{L v}
$$

donde $A$ es un subconjunto medible arbitrario de $[0,1]$. Verifique que esta medida es invariante por $f$ o por $g$.

(ii) Usando el hecho que $f$ no admite puntos fijos en el interior, pruebe que $g$ preserva $\mu_{K}$ y que dicha medida está soportada en el conjunto de puntos fijos de $g$. Finalmente, utilice el hecho de que este último conjunto es numerable para obtener una contradicción.

Observación. Sería muy interesante poder exhibir explícitamente una sucesión de cobordes convergiendo al cociclo correspondiente. 
Ejercicio 5.53. Sean $G=G_{1} \times G_{2}$ un producto de grupos topológicos compactamente generados y $A=\Psi+c$ una representación de $G$ por isometrías de un espacio de Hilbert $\mathcal{H}$. Suponga que la acción isométrica de $G_{1}$ no admita vectores invariantes. Pruebe que la representación unitaria correspondiente de $G_{2}$ casi posee vectores invariantes.

Sugerencia. Considere una sucesión $\left(g_{n}\right)$ en $G_{1}$ tal que $\left\|c\left(g_{n}\right)\right\|$ tienda al infinito. Usando la conmutatividad entre $G_{1}$ y $G_{2}$, verifique que la sucesión de vectores unitarios $c\left(g_{n}\right) /\left\|c\left(g_{n}\right)\right\|$ es casi invariante por $\Psi\left(G_{2}\right)$.

Ejercicio 5.54. Sean $\Psi: \mathbb{Z}^{2} \rightarrow U(\mathcal{H})$ una representación unitaria y $c: \mathbb{Z}^{2} \rightarrow \mathcal{H}$ un cociclo asociado. Pruebe que, si tanto $\Psi((1,0))$ como $\Psi((0,1))$ no casi poseen vectores invariantes, entonces $c$ es cohomológicamente nulo.

Sugerencia. Denote por $A_{1}=\Psi_{1}+c_{1}$ y $A_{2}=\Psi_{2}+c_{2}$ las isometrías de $\mathcal{H}$ asociadas a los generadores de $\mathbb{Z}^{2}$ en cuestión. Verifique que para $i \in\{1,2\}$, para todo $K \in \mathcal{H}$ y todo $n \in \mathbb{Z}$, se tiene la igualdad

$$
\left(I d-\Psi_{i}\right) A_{i}^{n}(K)=\Psi_{i}^{n}(K)-\Psi_{i}^{1+n}(K)-\Psi_{i}^{n}\left(c_{i}\right)+c_{i} .
$$

Usando esto y la conmutatividad entre $A_{1}$ y $A_{2}$, encuentre una cota superior uniforme para la norma de $\left(I d-\Psi_{1}\right)\left(I d-\Psi_{2}\right) A_{1}^{n_{1}} A_{2}^{n_{2}}(K)$. Del hecho que $\Psi_{1}$ y $\Psi_{2}$ no casi poseen vectores invariantes concluya que las órbitas por la acción isométrica de $\mathbb{Z}^{2}$ son acotadas. Finalmente, aplique el lema del centro de Tits.

Ejercicio 5.55. Dados $C \geq 0$ y un espacio métrico $\mathrm{M}$, un subconjunto $\mathrm{M}_{0}$ de M es $C$-denso si para todo $K \in \mathrm{M}$ existe $K_{0} \in \mathrm{M}_{0}$ tal que $\operatorname{dist}\left(K_{0}, K\right) \leq C$.

(i) Pruebe que no existen isometrías de espacios de Hilbert de dimensión mayor que 1 que tengan órbitas $C$-densas (compare con la observación del ejercicio 5.35). Sugerencia. Suponga que una isometría $A=\Psi+c$ de un espacio de Hilbert $\mathcal{H}$ admita una órbita $C$-densa $\left\{A^{n}\left(K_{0}\right): n \in \mathbb{Z}\right\}$. Usando la igualdad

$$
A^{n}\left(K_{0}\right)=\Psi^{n}\left(K_{0}\right)+\sum_{i=0}^{n-1} \Psi^{i}(c),
$$

verifique que el conjunto $\left\{(I d-\Psi) A^{n}\left(K_{0}\right): n \in \mathbb{Z}\right\}$ es $2 C$-denso en el espacio $(I d-\Psi) \mathcal{H}$. De la relación

$$
(I d-\Psi) A^{n}\left(K_{0}\right)=\Psi^{n}\left(K_{0}\right)-\Psi^{1+n}\left(K_{0}\right)-\Psi^{n}(c)+c
$$

concluya que las normas de los vectores de dicho espacio están acotadas por $2\left(\left\|K_{0}\right\|+\|c\|\right)$, y que por lo tanto $\Psi=I d$. Luego, $A$ es la traslación por el vector $c$, y esta isometría admite órbitas $C$-densas si y sólo si el espacio subyacente tiene dimensión cero o bien es unidimensional y $c$ no es el vector nulo.

(ii) Generalizando el ítem (i), pruebe que para ninguna acción de un grupo abeliano de rango finito por isometrías de un espacio de Hilbert de dimensión infinita existen órbitas $C$-densas.

Sugerencia. Sean $A_{i}=\Psi_{i}+c_{i}$ isometrías conmutantes de un espacio de Hilbert $\mathcal{H}$ de dimensión infinita, donde $i \in\{1, \ldots, k\}$. Suponiendo que la órbita de $K_{0} \in \mathcal{H}$ por el grupo generado por ellas es $C$-densa, verifique que el conjunto

$$
\left\{\left(I d-\Psi_{1}\right) \cdots\left(I d-\Psi_{k}\right) A_{k}^{n_{k}} \cdots A_{1}^{n_{1}}\left(K_{0}\right): n_{i_{j}} \in \mathbb{Z}\right\}
$$


es $2^{k} C$-denso en el espacio $\left(I d-\Psi_{1}\right) \cdots\left(I d-\Psi_{k}\right) \mathcal{H}$. Usando un argumento similar al del ejercicio 5.54, compruebe que dicho conjunto está contenido en la bola de $\mathcal{H}$ con centro en el origen y radio $2^{k}\left(\left\|K_{0}\right\|+\sum_{i=1}^{k}\left\|c_{i}\right\|\right)$. Concluya entonces que el conjunto de los vectores invariantes por al menos una de las $\Psi_{i}$ (digamos $\Psi_{k}$ ) es un subespacio de dimensión infinita $\overline{\mathcal{H}}$ de $\mathcal{H}$. Denotando por $\bar{c}_{k}$ la proyección de $c_{k}$ sobre $\overline{\mathcal{H}}$, pruebe que al proyectar ortogonalmente sobre el espacio $\mathcal{H}^{*}=\left\langle\bar{c}_{k}\right\rangle^{\perp} \cap \overline{\mathcal{H}}$ se inducen $k-1$ isometrías conmutantes que generan un grupo admitiendo órbitas $C$-densas. Concluya la demostración mediante un razonamiento inductivo.

Observación. No es difícil fabricar isometrías de un espacio de Hilbert de dimensión infinita que generen un grupo libre para el cual todas las órbitas sean densas. El problema de determinar cuáles son los grupos finitamente generados que pueden actuar de manera minimal por isometrías de un espacio de Hilbert de dimensión infinita pareciera ser interesante. Debido al ítem (ii) de este ejercicio, un tal grupo no puede ser abeliano, y una argumentación un poco más fina permite concluir que tampoco puede ser nilpotente. Invitamos al lector a probar este hecho en el próximo ejercicio (vea también [51]).

Ejercicio 5.56. Suponga que existan grupos nilpotentes y finitamente generados admitiendo acciones isométricas sobre espacios de Hilbert de dimensión infinita con órbitas $C$-densas para algún $C>0$. Fije un grupo tal $\Gamma$ con el menor índice de nilpotencia posible, y considere la acción afín $A=\Psi+c$ correspondiente. (i) Pruebe que $\Psi(h) v=v$ para todo $h$ perteneciente al centro $\mathrm{H}$ de $\Gamma$ y todo $v$ en el espacio de Hilbert subyacente $\mathcal{H}$.

Sugerencia. Considere $p \in \mathcal{H}$ de órbita $C$-densa, y para cada $v \in \mathcal{H}$ fije $g \in \Gamma$ tal que $\|A(g)(p)-v\| \leq C$. Para todo $h \in \mathrm{H}$ se tiene

$$
\begin{aligned}
\|A(h)(v)-v\| & \leq\|A(h)(v)-A(h g)(p)\|+\|A(h g)(p)-A(g)(p)\|+\|A(g)(p)-v\| \\
& \leq 2 C+\|A(g h)(p)-A(g)(p)\|=2 C+\|A(h)(p)-p\| .
\end{aligned}
$$

En particular, si $v \neq 0$ entonces reemplazando $v$ por $\lambda v$ en la desigualdad precedente, y haciendo tender $\lambda$ al infinito, concluimos que $v$ es invariante por $\mathrm{H}$.

(ii) Por el ítem (i), para todo $h \in \mathrm{H}$ la isometría $A(h)$ es una traslación, digamos por un vector $c_{h}$. Verifique que $c_{h}$ es invariante por $\Psi(\Gamma)$. Concluya que el subespacio $\mathcal{H}_{0}$ de los vectores $\Psi(\Gamma)$ invariantes no se reduce al vector cero (observe que la acción de $H$ no puede ser trivial). Proyecte ortogonalmente sobre $\mathcal{H}_{0}$ y su complemento ortogonal $\mathcal{H}_{0}^{\perp}$ para obtener representaciones isométricas $A_{0}$ y $A_{0}^{\perp}$ con órbitas $C$-densas. Usando el hecho que la parte lineal de $A_{0}$ es trivial (es decir,que $A_{0}$ es una representación por traslaciones) y que $\Gamma$ es finitamente generado, concluya que $A_{0}^{\perp}$ tiene dimensión infinita. Obtenga una contradicción notando que sobre $\mathcal{H}_{0}^{\perp}$ la acción afín de $\mathrm{H}$ es trivial, y que por lo tanto $A_{0}^{\perp}$ induce una acción afín del grupo cuociente $\Gamma / H$, cuyo grado de nilpotencia es menor al de $\Gamma$.

Ejercicio 5.57. Dé ejemplos de acciones isométricas minimales sobre espacios de Hilbert de dimensión infinita para grupos solubles finitamente generados.

\subsection{Supra-rigidez para acciones sobre el círculo}

Sean $\tau>1 / 2$ y $\Gamma$ un subgrupo de Difeo ${ }_{+}^{1+\tau}\left(\mathrm{S}^{1}\right)$. De acuerdo a la sección 2.3 de este capítulo (vea la observación 5.32), si la restricción del cociclo de 
Liouville a $\Gamma$ es cohomológicamente trivial, entonces $\Gamma$ es topológicamente conjugado a un subgrupo de $\operatorname{PSL}(2, \mathbb{R})$. Con la ayuda del lema de Delorme estudiaremos el caso en que dicho cociclo es casi cohomológicamente trivial. El lema a continuación debiera ser comparado con el ítem (iii) del ejercicio 5.51 .

Lema 5.58. Supongamos que el cociclo de Liouville restringido a $\Gamma$ sea un casi coborde no nulo cohomológicamente. Entonces existe una medida de probabilidad sobre el círculo que es invariante por $\Gamma$.

Demostración. Por el lema de Delorme, si $c$ es un casi coborde cohomológicamente no nulo, entonces $\Psi$ casi posee vectores invariantes. Por lo tanto, existe una sucesión $\left(K_{n}\right)$ de vectores unitarios de $\mathcal{L}_{\mathbb{R}}^{2, \Delta}\left(\mathrm{S}^{1} \times \mathrm{S}^{1}, L v\right)$ tal que, para todo $g \in \Gamma$, el valor de $\left\|K_{n}-\Psi(g) K_{n}\right\|$ converge a cero cuando $n$ tiende a infinito. Definamos las medidas de probabilidad $\left(\mu_{n}\right)$ sobre $\mathrm{S}^{1}$ por

$$
\mu_{n}(A)=\int_{\mathrm{S}^{1}} \int_{A} K_{n}^{2}(x, y) d L v .
$$

Para toda función continua $\varphi: \mathrm{S}^{1} \rightarrow \mathbb{R}$ se tiene

$$
\begin{aligned}
\left|\mu_{n}(\varphi)-\phi(g)\left(\mu_{n}\right)(\varphi)\right| & \leq\|\varphi\|_{\mathcal{L}^{\infty}} \int_{\mathrm{S}^{1}} \int_{\mathrm{S}^{1}}\left|K_{n}^{2}-\left(\Psi(g) K_{n}\right)^{2}\right| d L v \\
& \leq\|\varphi\|_{\mathcal{L}^{\infty}}\left\|K_{n}+\Psi(g) K_{n}\right\|_{\mathcal{L}^{2}}\left\|K_{n}-\Psi(g) K_{n}\right\|_{\mathcal{L}^{2}} \\
& \leq 2\|\varphi\|_{\mathcal{L}^{\infty}}\left\|K_{n}-\Psi(g) K_{n}\right\|_{\mathcal{L}^{2}}
\end{aligned}
$$

Se deduce entonces que $\left|\mu_{n}(\varphi)-\phi(g)\left(\mu_{n}\right)(\varphi)\right|$ tiende a cero cuando $n$ tiende al infinito. Luego, si $\mu$ es un punto de adherencia de $\left(\mu_{n}\right)$, entonces $\mu$ es una medida de probabilidad sobre $\mathrm{S}^{1}$ invariante por $\Gamma$.

Demostración del teorema 5.41. Para cada $K \in \mathcal{H}=\mathcal{L}_{\mathbb{R}}^{2, \Delta}\left(\mathrm{S}^{1} \times \mathrm{S}^{1}, L v\right)$ de norma 1 denotemos por $\mu_{K}$ la medida de probabilidad sobre $\mathrm{S}^{1}$ obtenida al proyectar sobre la primera coordenada. En términos más precisos,

$$
\mu_{K}(A)=\int_{\mathrm{S}^{1}} \int_{A} K^{2}(x, y) d L v .
$$

Designemos por $\operatorname{prob}$ la aplicación $\operatorname{prob}(K)=\mu_{K}$ definida sobre la esfera unidad de $\mathcal{H}$ y a valores en el espacio de las medidas de probabilidad del círculo absolutamente continuas respecto a la medida de Lebesgue. Esta aplicación prob es equivariante respecto a $\Gamma$, en el sentido que para todo $g \in \Gamma$ y toda función $K \in \mathcal{L}_{\mathbb{R}}^{2, \Delta}\left(\mathrm{S}^{1} \times \mathrm{S}^{1}, L v\right)$ de norma 1 ,

$$
\operatorname{prob}(\Psi(g) K)=\Phi(g)(\operatorname{prob}(K)) .
$$

Supongamos que $\Phi(\Gamma)$ no fije ninguna medida de probabilidad sobre $\mathrm{S}^{1} \mathrm{y}$ que $\Phi(\Gamma)$ no sea conjugado a un subgrupo de $\operatorname{PSL}(2, \mathbb{R})$. En tal caso, el lema 
5.58 y el teorema de supra-rigidez cohomológica nos brindan una familia $\left\{\mathcal{H}_{1}, \ldots, \mathcal{H}_{k}\right\}$ de subespacios $\Psi(\Gamma)$-invariantes de $\mathcal{H}=\mathcal{L}_{\mathbb{R}}^{2, \Delta}\left(\mathrm{S}^{1} \times \mathrm{S}^{1}, L v\right)$, además de cociclos $c_{i}: \Gamma \rightarrow \mathcal{H}_{i}$, tales que al menos uno de entre ellos no es idénticamente nulo, y tales que sobre cada $\mathcal{H}_{i}$ la representación isométrica asociada a $c_{i}$ se extiende continuamente a $G$ y se factoriza sobre $G_{i}$. Fijemos un índice $i \in\{1, \ldots, k\}$ tal que $\mathcal{H}_{i}$ sea no trivial. Afirmamos que la imagen de la esfera unitaria de $\mathcal{H}_{i}$ por la aplicación prob consiste de al menos dos medidas distintas. En efecto, si esta imagen fuese idénticamente igual a una medida $\operatorname{prob}(K)$ entonces, debido a la propiedad de equivarianza (5.9) y al hecho que $\mathcal{H}_{i}$ es un subespacio $\Psi(\Gamma)$-invariante, $\operatorname{prob}(K)$ sería una medida de probabilidad sobre el círculo invariante por $\Gamma$, contradiciendo nuestra hipótesis. Fijemos una base ortonormal $\left\{K_{1}, K_{2}, \ldots\right\}$ de $\mathcal{H}_{i}$ y definamos

$$
\bar{K}=\sum_{n \geq 0} \frac{\left|K_{n}\right|}{2^{n}}, \quad K=\frac{\bar{K}}{\|\bar{K}\|} .
$$

La medida $\mu_{K}$ es una "medida a soporte maximal" entre las medidas obtenidas al proyectar funciones de $\mathcal{H}_{i}$. Ella no posee átomos y es absolutamente continua respecto a la medida de Lebesgue. Si designamos por $\Lambda$ al cierre del soporte de $\mu_{K}$, entonces $\Lambda$ es un conjunto compacto sin puntos aislados. Además, dado que $\mathcal{H}_{i}$ es $\Psi(\Gamma)$-invariante, $\Lambda$ es invariante por $\Gamma$, y puesto que $\Phi(\Gamma)$ no fija ninguna medida de probabilidad del círculo, $\Lambda$ no se reduce a una unión finita y disjunta de subintervalos cerrados de $\mathrm{S}^{1}$.

Si $\Lambda$ no es todo el círculo retiremos cada componente conexa de $\mathrm{S}^{1} \backslash \Lambda$, y luego identifiquemos sus extremidades. Mediante este procedimiento se obtiene un círculo topológico $\mathrm{S}_{\Lambda}^{1}$, sobre el cual la acción original $\Phi$ induce una acción por homeomorfismos $\Phi_{\Lambda}$. Señalemos sin embargo que las órbitas de esta acción inducida no son necesariamente densas, pues $\Lambda$ no coincide necesariamente con el minimal excepcional de la acción original. Cuando $\Lambda$ sea todo el círculo hagamos $\mathrm{S}_{\Lambda}^{1}=\mathrm{S}^{1}$. Cualquiera sea el caso, $\mathrm{S}_{\Lambda}^{1}$ hereda una métrica natural, pues se le puede parametrizar utilizando la medida $\mu_{K}$.

Fijemos una función $K^{\prime}$ de la esfera unidad de $\mathcal{H}_{i}$ tal que la medida $\mu_{K^{\prime}}$ sea distinta de $\mu_{K}$, y designemos por $\Gamma_{\mu_{K}}$ (resp. $\Gamma_{\mu_{K^{\prime}}}$ ) al grupo de los homeomorfismos de $\mathrm{S}_{\Lambda}^{1}$ que preservan la medida (inducida sobre $\mathrm{S}_{\Lambda}^{1}$ por) $\mu_{K}$ (resp. $\mu_{K^{\prime}}$ ). Notemos que $\Gamma_{\mu_{K}}$ es topológicamente conjugado al grupo de las rotaciones. Si $\left(g_{n}\right)$ es una sucesión de elementos de $\Gamma$ tal que $\lim _{n \rightarrow+\infty} p r_{i}\left(g_{n}\right)=i d_{G_{i}}$, entonces $\left\|\Psi\left(g_{n}\right) K-K\right\|$ converge a cero cuando $n$ tiende al infinito, y lo mismo ocurre para $\left\|\Psi\left(g_{n}\right) K^{\prime}-K^{\prime}\right\|$. Un argumento análogo al de la prueba del lema 5.58 muestra que $\left(\Phi\left(g_{n}\right)\right)_{*}\left(\mu_{K}\right)$ (resp. $\left.\left(\Phi\left(g_{n}\right)\right)_{*}\left(\mu_{K^{\prime}}\right)\right)$ tiende a $\mu_{K}\left(\right.$ resp. $\left.\mu_{K^{\prime}}\right)$ cuando $n$ tiende al infinito. A partir de esto se deduce primeramente que la sucesión $\left(\Phi_{\Lambda}\left(g_{n}\right)\right)$ possee puntos de adherencia en $\mathrm{Homeo}_{+}\left(\mathrm{S}_{\Lambda}^{1}\right)$, y luego se concluye que todos los homeomorfismos límite están contenidos en $\Gamma_{\mu_{K}} \cap \Gamma_{\mu_{K^{\prime}}}$. Como $\mu_{K^{\prime}}$ es distinta de $\mu_{K}$ y su soporte está contenido en el de $\mu_{K}$, el grupo $\Gamma_{\mu_{K}} \cap \Gamma_{\mu_{K^{\prime}}}$ 
está estrictamente contenido en $\Gamma_{K}$. Puesto que $\Gamma_{\mu_{K}} \cap \Gamma_{\mu_{K^{\prime}}}$ es cerrado en $\mathrm{Homeo}_{+}\left(\mathrm{S}_{\Lambda}^{1}\right)$, y puesto que todo subgrupo no denso del grupo de las rotaciones es finito, concluimos que $\Gamma_{\mu_{K}} \cap \Gamma_{\mu_{K^{\prime}}}$ debe necesariamente ser finito.

Sea $H$ el conjunto de los $h \in$ Homeo $_{+}\left(\mathrm{S}_{\Lambda}^{1}\right)$ tales que $h=\lim _{n \rightarrow+\infty} \Phi_{\Lambda}\left(g_{n}\right)$ para alguna sucesión $\left(g_{n}\right)$ en $\Gamma$ satisfaciendo $\lim _{n \rightarrow+\infty} p r_{i}\left(g_{n}\right)=i d_{G_{i}}$. Por definición, $H$ es un subgrupo cerrado de $\mathrm{Homeo}_{+}\left(\mathrm{S}_{\Lambda}^{1}\right)$. Además, el argumento del párrafo precedente prueba que $H$ está contenido en $\Gamma_{\mu_{K}} \cap \Gamma_{\mu_{K^{\prime}}}$. Se trata entonces de un grupo finito. Denotemos por $d$ el orden de $H$ y, en el caso en que $d>1$, fijemos un generador $h$ de $H$. Observemos que $\rho(h) \neq 0$ (donde $\rho$ designa el número de rotación). Fijemos una sucesión $\left(g_{n}\right)$ de $\Gamma$ tal que $\lim _{n \rightarrow+\infty} p r_{i}\left(g_{n}\right)=i d_{G_{i}}$ y $h=\lim _{n \rightarrow+\infty} \Phi_{\Lambda}\left(g_{n}\right)$.

Probaremos ahora que $H$ está contenido en el centralizador de $\Phi_{\Lambda}(\Gamma)$ en Homeo $_{+}\left(\mathrm{S}_{\Lambda}^{1}\right)$. Para ello, notemos que para cada $g \in \Gamma$ la sucesión de transformaciones $\operatorname{pr}_{i}\left(g^{-1} g_{n} g\right)$ también tiende a $i d_{G_{i}}$ cuando $n$ tiende al infinito. Por definición, la sucesión $\left(\Phi_{\Lambda}\left(g^{-1} g_{n} g\right)\right)$ tiende a un elemento de $H$, es decir a $h^{j}$ para algún $j \in\{1, \ldots, d\}$. A partir de la igualdad $\rho\left(\Phi_{\Lambda}\left(g^{-1} g_{n} g\right)\right)=\rho\left(g^{-1} g_{n} g\right)=\rho\left(g_{n}\right)=\rho\left(\Phi_{\Lambda}\left(g_{n}\right)\right)$ (válida para todo $\left.n \in \mathbb{N}\right)$, se concluye fácilmente que $j=1$, lo cual implica que $\Phi_{\Lambda}(g)$ conmuta con $h$. Como $g \in \Gamma$ era un elemento arbitrario, el grupo $H$ centraliza a $\Phi_{\Lambda}(\Gamma)$.

Designemos por $\mathrm{S}_{\Lambda}^{1} / \sim$ al círculo topológico obtenido al identificar los puntos de $\mathrm{S}_{\Lambda}^{1}$ que están sobre una misma órbita por $H$. El círculo $\mathrm{S}_{\Lambda}^{1}$ es un recubrimiento finito de grado $d$ de $S_{\Lambda}^{1} / \sim$. Además, $\Phi_{\Lambda}: \Gamma \rightarrow \operatorname{Homeo}_{+}\left(\mathrm{S}_{\Lambda}^{1}\right)$ induce de manera natural otra representación $\tilde{\Phi}: \Gamma \rightarrow$ Homeo $_{+}\left(\mathrm{S}_{\Lambda}^{1} / \sim\right)$ tal que, si $\left(g_{n}\right)$ es una sucesión de $\Gamma$ tal que $p r_{i}\left(g_{n}\right)$ tiende hacia $i d_{G_{i}}$, entonces $\tilde{\Phi}\left(g_{n}\right)$ tiende a la identidad (en $\left.\mathrm{S}_{\Lambda}^{1} / \sim\right)$. Luego, del lema 5.50 se concluye que $\tilde{\Phi}$ se extiende a una representación $\hat{\Phi}: G \rightarrow \operatorname{Homeo}_{+}\left(\mathrm{S}_{\Lambda}^{1} / \sim\right)$ que se factoriza sobre $G_{i}$. Esta representación $\hat{\Phi}$ es aquélla que extiende $\Phi$ modulo una semiconjugación topológica y un recubrimiento finito, concluyendo así la demostración del teorema 5.41.

Recordemos ahora que los subgrupos finitos de Homeo+ $\left(\mathrm{S}^{1}\right)$ son topológicamente conjugados a grupos de rotaciones, y por lo tanto a subgrupos de $\operatorname{PSL}(2, \mathbb{R})$. Debido a esto, para demostrar el corolario del teorema B basta probar que si $\Phi(\Gamma)$ preserva una medida de probabilidad sobre el círculo y si $H^{1}\left(\Gamma_{0}, \mathbb{R}\right)=\{0\}$ para todo subgrupo normal y de índice finito $\Gamma_{0}$ de $\Gamma$, entonces $\Phi(\Gamma)$ es finito. Observe para ello que si la medida invariante no posee átomos entonces $\Phi(\Gamma)$ es semiconjugado a un grupo de rotaciones. En caso contrario, $\Phi(\Gamma)$ posse una órbita finita. Puesto que $\Gamma$ y $\Gamma_{0}$ son finitamente generados, esto implica que $\Phi(\Gamma)$ es finito. En el caso de una órbita finita esto se deduce del teorema de estabilidad de Thurston, mientras que en el caso de una semiconjugación a un grupo de rotaciones esto es relativamente evidente. La demostración del corolario 5.42 queda así concluida. 
Demostración del teorema 4.43. Siendo $G$ un grupo de Lie conexo, su acción $\hat{\Phi}$ sobre el círculo $\mathrm{S}_{\Lambda}^{1} / \sim$ transita por homomorfismos sobre $(\mathbb{R},+)$, $\mathrm{Aff}_{+}(\mathbb{R}), \mathrm{SO}(2, \mathbb{R}), \widetilde{\mathrm{PSL}}(2, \mathbb{R})$ o $\operatorname{PSL}_{k}(2, \mathbb{R})$ para algún $k \geq 1$ (vea la sección 4 del primer capítulo). Supongamos en lo que sigue que $\Phi(\Gamma)$ no sea finito, lo que de acuerdo a la prueba del corolario 5.42 equivale a que $\Phi(\Gamma)$ no preserva ninguna medida de probabilidad sobre $\mathrm{S}^{1}$.

Consideremos primeramente el caso de la hipótesis (i). El círculo $\mathrm{S}_{\Lambda}^{1}$ se identifica entonces al círculo original $\mathrm{S}^{1}$. Del hecho que el núcleo de $\Phi$ sea finito se concluye la existencia de sucesiones $\left(g_{n}\right)$ en $\Gamma$ tales que $p r_{i}\left(g_{n}\right)$ converge a $i d_{G_{i}}$ y los $\hat{\Phi}\left(g_{n}\right)$ son dos a dos distintos. Esto implica que el grupo de Lie $\hat{\Phi}\left(G_{i}\right)$ es no discreto. Dada la clasificación recordada precedentemente, la componente conexa de la identidad de dicho grupo $\hat{\Phi}\left(G_{i}\right)_{0}$ corresponde ya sea a $\mathrm{SO}(2, \mathbb{R})$, a un subgrupo de un producto de grupos de traslaciones, de grupos afines y de grupos conjugados a $\widetilde{\operatorname{PSL}}(2, \mathbb{R})$ actuando sobre intervalos abiertos dos a dos disjuntos, o bien a $\operatorname{PSL}_{k}(2, \mathbb{R})$ para algún $k \geq 1$. El primer caso no puede producirse, pues $\phi(\Gamma)$ no fija ninguna medida de probabilidad sobre $\mathrm{S}^{1}$. El segundo caso tampoco puede producirse, ya que las órbitas por $\Phi(\Gamma)$ son densas y $\hat{\Phi}\left(G_{i}\right)_{0}$ es normal en $\hat{\Phi}\left(G_{i}\right)$ (el conjunto de los intervalos fijos por $\hat{\Phi}\left(G_{i}\right)_{0}$ debe ser preservado por $\Gamma)$. El grupo $\hat{\Phi}\left(G_{i}\right)_{0}$ es por lo tanto conjugado a $\operatorname{PSL}_{k}(2, \mathbb{R})$ para algún $k \geq 1$, y como $\operatorname{PSL}_{k}(2, \mathbb{R})$ es normal en el grupo de los homeomorfismos del círculo, lo mismo vale para $\hat{\Phi}\left(G_{i}\right)$.

Supongamos válida ahora la hipótesis (ii), según la cual $\Phi$ toma valores en $\operatorname{Difeo}_{+}^{\mathrm{w}}\left(\mathrm{S}^{1}\right)$. Ya hemos observado que las órbitas de la acción de $\Gamma$ sobre $\mathrm{S}_{\Lambda}^{1}$ no son necesariamente densas. Sea $\dot{\Lambda}$ el conjunto cerrado no vacío invariante y minimal de esta última acción, y sea $\dot{\Phi}: \Gamma \rightarrow \operatorname{Homeo}\left(\mathrm{S}_{\dot{\Lambda}}^{1}\right)$ la acción inducida sobre el círculo topológico $\mathrm{S}_{\dot{\Lambda}}^{1}$ obtenido al colapsar las componentes conexas de $S_{\Lambda}^{1} \backslash \dot{\Lambda}$. Las órbitas por $\dot{\Phi}$ son densas. Para poder aplicar los argumentos de la primera parte de la demostración, basta probar que el núcleo de $\dot{\Phi}$ es finito. Pero esto es evidente, dado que los difeomorfismos de $\Phi(\Gamma)$ son real-analíticos, por lo que sus puntos fijos son aislados (el núcleo de la restricción de $\dot{\Phi}$ a $\Gamma$ coincide por lo tanto con el núcleo de $\Phi)$.

Finalmente, asumamos la hipótesis (iii) según la cual $\Gamma$ satisface el teorema de los subgrupos normales de Margulis. Nuevamente, debemos probar que el núcleo de $\dot{\Phi}$ es finito. Ahora bien, si éste no fuese el caso, entonces dicho núcleo sería de índice finito en $\Gamma$. Esto implicaría que las órbitas de los puntos de $\dot{\Lambda}$ son finitas, lo cual es absurdo, pues todas las órbitas por $\dot{\Phi}$ son densas. La demostración del teorema 5.43 queda así concluida. 


\section{Apéndice}

\section{Algunos conceptos algebraicos básicos}

Sean $\Gamma_{1}$ y $\Gamma_{2}$ dos subgrupos de un grupo $\Gamma$. Denotamos $\left[\Gamma_{1}, \Gamma_{2}\right]$ al grupo generado por los elementos de la forma $[f, g]=f^{-1} g^{-1} f g$, con $f \in \Gamma_{1}$ y $g \in \Gamma_{2}$. El grupo $\Gamma^{\prime}=[\Gamma, \Gamma]$ es llamado el grupo de los conmutadores (o grupo derivado) de $\Gamma$.

Decimos que $\Gamma$ es abeliano si $[\Gamma, \Gamma]=\{i d\}$, metabeliano si $[\Gamma, \Gamma]$ es abeliano, y perfecto si $[\Gamma, \Gamma]=\Gamma$. Un subgrupo $\Gamma_{0}$ de $\Gamma$ es normal si para todo $h \in \Gamma_{0}$ y todo $g \in \Gamma$ se tiene $g h g^{-1} \in \Gamma_{0}$. El grupo $\Gamma$ es simple si sus únicos subgrupos normales son $\{i d\}$ y $\Gamma$. Observe que el subgrupo $[\Gamma, \Gamma]$ es normal en $\Gamma$, por lo que si $\Gamma$ es simple entonces $\Gamma$ es abeliano o perfecto. El centro de $\Gamma$ es el subgrupo de $\operatorname{los} g \in \Gamma$ que conmutan con todos los elementos de $\Gamma$.

Para un grupo $\Gamma$ cualquiera definimos por inducción los grupos

$$
\begin{aligned}
\Gamma_{0}^{\mathrm{nil}}=\Gamma, \quad \Gamma_{0}^{\mathrm{sol}}=\Gamma, \\
\Gamma_{i+1}^{\mathrm{nil}}=\left[\Gamma, \Gamma_{i}^{\mathrm{nil}}\right], \quad \Gamma_{i+1}^{\mathrm{sol}}=\left[\Gamma_{i}^{\mathrm{sol}}, \Gamma_{i}^{\mathrm{sol}}\right] .
\end{aligned}
$$

El grupo $\Gamma$ es soluble (resp. nilpotente) si existe $n \in \mathbb{N}$ tal que $\Gamma_{n}^{\text {sol }}=\{i d\}$ (resp. $\Gamma_{n}^{\text {nil }}=\{i d\}$ ). El mínimo entero $n$ para el cual esto es válido es el grado, orden o longitud de solubilidad (resp. grado, orden o longitud de nilpotencia) del grupo $\Gamma$. De las definiciones se deduce fácilmente que todo grupo nilpotente es soluble. Un grupo es virtualmente soluble (resp. virtualmente nilpotente) si contiene un subgrupo soluble (resp. nilpotente) de índice finito.

Observe que cada subgrupo $\Gamma_{i+1}^{\text {sol }}$ es normal en $\Gamma_{i}^{\text {sol }}$ y cada cuociente $\Gamma_{i}^{\text {sol }} / \Gamma_{i+1}^{\text {sol }}$ es abeliano. En general, una serie $\{i d\}=\Gamma_{n} \subset \Gamma_{n-1} \subset \ldots \subset \Gamma_{0}=\Gamma$ de subgrupos de $\Gamma$ es central si cada $\Gamma_{i+1}$ es normal en $\Gamma_{i}$ y $\Gamma_{i} / \Gamma_{i+1}$ es abeliano. Un grupo es soluble si y sólo si admite una serie central.

Sea $\mathrm{P}$ una propiedad atribuible a un grupo. Por ejemplo, $\mathrm{P}$ puede ser la propiedad de solubilidad, nilpotencia, finitud, etc. Se dice que un grupo $\Gamma$ posee residualmente la propiedad $\mathrm{P}$ si para todo elemento $g \in \Gamma$ existe un grupo $\Gamma_{g}$ que verifica $\mathrm{P}$ y un homomorfismo de $\Gamma$ en $\Gamma_{g}$ de manera que 
la imagen de $g$ no sea el elemento neutro de $\Gamma_{g}$. De esta manera quedan definidas las nociones de grupo residualmente soluble, residualmente nilpotente, residualmente finito, etc.

Ejercicio 5.59. Pruebe que el centro de todo grupo no trivial y nilpotente es no trivial. Pruebe que cada uno de los grupos $\Gamma_{i}^{\text {sol }}(\Gamma)$ y $\Gamma_{i}^{\text {nil }}(\Gamma)$ es normal en $\Gamma$. Concluya que todo grupo soluble contiene al menos un subgrupo abeliano normal.

\section{Medidas invariantes y promediabilidad}

Un teorema básico en teoría ergódica, debido a Bogolioubov y Krylov, estipula que para todo homeomorfismo de un espacio métrico compacto existe (al menos) una medida de probabilidad invariante. Este resultado deja de ser válido para el caso general de acciones de grupos, como lo muestra el importante ejemplo siguiente.

Ejemplo 5.60. La acción natural del grupo

$$
\mathrm{SL}(n, \mathbb{R})=\{M \in \mathrm{M}(n \times n, \mathbb{R}): \operatorname{det}(M)=1\}
$$

sobre el espacio proyectivo $\mathbb{R P}^{n-1}$ no admite medida invariante $(n \geq 2)$. Para demostrar esta afirmación consideramos una sucesión $\left(g_{k}\right)$ de elementos de $\operatorname{SL}(n, \mathbb{R})$ que escapa de todo compacto. Cada $g_{k}$ puede ser representado por una matriz $M_{k}$ tal que $\left\|M_{k}\right\|=1$, donde $\|\cdot\|$ es una norma completa en el espacio de matrices $n \times n$. Pasando a una subsucesión podemos suponer que $M_{k}$ converge a cierta matriz $M$, la cual será necesariamente no invertible (pues en caso contrario $\left(g_{k}\right)$ no escaparía de los compactos). Sea $E$ la imagen de $\mathbb{R}^{n-1}$ por la aplicación lineal correspondiente a $M$.

Supongamos que $\mu$ sea una medida de probabilidad sobre $\mathbb{R P}^{n-1}$ invariante por $\operatorname{SL}(n, \mathbb{R})$. Dejamos al lector verificar, a partir de las igualdades $M_{k}(\mu)=\mu$, que $M(\mu)=\mu$. Esto último implica que el soporte de $\mu$ está contenido en $E$ (identificamos un espacio vectorial a su imagen en $\mathbb{R} \mathbb{P}^{n-1}$ ).

Sea $h \in \operatorname{SL}(n, \mathbb{R})$ tal que $\operatorname{dim}(E \cap h(E))<\operatorname{dim}(E)$. De la igualdad $h(\mu)=\mu$ se concluye que $\operatorname{sop}(\mu) \subset E \cap h(E)$. Procediendo inductivamente concluimos que $\mu$ es una medida cuyo soporte es un espacio vectorial de dimensión uno (i.e., un punto en $\left.\mathbb{R} \mathbb{P}^{n-1}\right)$, lo cual es absurdo pues la acción de $\operatorname{SL}(n, \mathbb{R})$ en $\mathbb{R} \mathbb{P}^{n-1}$ es transitiva.

La importancia de la existencia de medidas invariantes para acciones de grupos amerita la siguiente definición.

Definición 5.61. Un grupo $\Gamma$ es promediable si toda acción de $\Gamma$ por homeomorfismos de un espacio métrico compacto admite una medida de probabilidad invariante.

Existen muchas caracterizaciones de los grupos promediables. Nosotros nos concentraremos en el caso de grupos finitamente generados. Recordemos en primer lugar la estrategia de la prueba del teorema de Bogolioubov 
y Krylov. Dado un homeomorfismo $g: \mathrm{M} \rightarrow \mathrm{M}$ definido en un espacio métrico compacto $\mathrm{M}$, fijamos una medida de probabilidad $\mu$ sobre $\mathrm{M}$ y consideramos la sucesión de medidas $\left(\nu_{n}\right)$ dada por

$$
\nu_{n}=\frac{1}{n}\left[\mu+g(\mu)+g^{2}(\mu)+\ldots+g^{n-1}(\mu)\right] .
$$

Puesto que el espacio de medidas de probabilidad sobre $\mathrm{M}$ es compacto para la topología débil estrella, existe una subsucesión $\left(\nu_{n_{k}}\right)$ de $\left(\nu_{n}\right)$ que converge débilmente a una medida de probabilidad $\nu$. Vemos entonces que $\nu$ es una medida invariante, pues para todo $k$ tenemos

$$
g\left(\nu_{n_{k}}\right)=\nu_{n_{k}}+\frac{1}{n_{k}}\left[g^{n_{k}}(\mu)-\mu\right],
$$

lo cual implica que

$g(\nu)=g\left(\lim _{k \rightarrow \infty} \nu_{n_{k}}\right)=\lim _{k \rightarrow \infty} g\left(\nu_{n_{k}}\right)=\lim _{k \rightarrow \infty} \nu_{n_{k}}+\lim _{k \rightarrow \infty} \frac{1}{n_{k}}\left[g^{n_{k}}(\mu)-\mu\right]=\lim _{k \rightarrow \infty} \nu_{n_{k}}=\nu$.

Tratemos ahora de repetir este argumento para un grupo $\Gamma$ generado por una familia simétrica y finita de elementos $\mathcal{G}=\left\{g_{1}, \ldots, g_{m}\right\}$ (recuerde que el vocablo simétrica significa que si $g \in \mathcal{G}$ entonces $g^{-1} \in \mathcal{G}$ ). Para cada $g \in \Gamma$ definimos la longitud de $g$ como el número mínimo de elementos (no necesariamente distintos) de $\mathcal{G}$ que son necesarios para representar $g$. En términos más precisos,

$$
\operatorname{long}(g)=\min \left\{n \in \mathbb{N}: g=g_{i_{n}} g_{i_{n-1}} \cdots g_{i_{1}}, \quad g_{i_{j}} \in \mathcal{G}\right\}
$$

Llamamos bola de radio $n$ (respecto a $\mathcal{G}$ ) al conjunto $B_{\mathcal{G}}(n)$ de los elementos de $\Gamma$ de longitud menor o igual a $n$, y denotamos por $L_{\mathcal{G}}(n)$ su cardinalidad.

Supongamos ahora que tenemos una acción de $\Gamma$ sobre un espacio compacto M y fijemos una medida de probabilidad $\mu$ sobre M. Para cada $n \in \mathbb{N}$ consideramos la medida

$$
\nu_{n}=\frac{1}{L_{\mathcal{G}}(n-1)} \sum_{g \in B_{\mathcal{G}}(n-1)} g(\mu) .
$$

Pasando a una subsucesión tenemos que $\lim \nu_{n_{k}}=\nu$ para cierta medida de probabilidad $\nu$. El problema que se presenta es que $\nu$ no es necesariamente una medida invariante. En efecto, si tratásemos de repetir el argumento de la igualdad (5.10) entonces deberíamos estimar una expresión del tipo

$$
\frac{1}{L_{\mathcal{G}}\left(n_{k}\right)} \sum_{\substack{\operatorname{long}(g)=n_{k}, g_{i} \in \mathcal{G}}} g_{i} g(\mu) .
$$


Sin embargo, esta expresión no necesariamente converge a cero, pues puede ocurrir que la cantidad de elementos en el conjunto $B_{\mathcal{G}}\left(n_{k}\right) \backslash B_{\mathcal{G}}\left(n_{k}-1\right)$ sea grande respecto a $L_{\mathcal{G}}\left(n_{k}\right)$. Las siguientes definiciones resultan entonces naturales.

Definición 5.62. Dado un subconjunto $A \subset \Gamma$, definimos el borde geométrico de $A$ como el conjunto

$$
\partial A=\bigcup_{g \in \mathcal{G}}(A \Delta g(A)),
$$

donde $\Delta$ denota la diferencia simétrica de los conjuntos respectivos.

Definición 5.63. Una sucesión de Følner para $\Gamma$ es una sucesión $\left(A_{n}\right)$ de subconjuntos finitos de $\Gamma$ tales que

$$
\lim _{n \rightarrow \infty} \frac{\operatorname{card}\left(\partial A_{n}\right)}{\operatorname{card}\left(A_{n}\right)}=0 .
$$

Utilizando el argumento de Bogolioubov y Krylov, no es difícil verificar que si $\Gamma$ admite una sucesión de Følner entonces toda acción de $\Gamma$ por homeomorfismos de un espacio compacto admite una medida invariante. El panorama completo queda aclarado entonces por el siguiente teorema debido a Følner.

Teorema 5.64. Un grupo finitamente generado es promediable si y sólo si admite una sucesión de Følner.

Es importante remarcar que esta caracterización es independiente del sistema de generadores. En efecto, no es difícil verificar que el cuociente de las funciones longitud de un elemento $g \in \Gamma$ con respecto a dos sistemas finitos de generadores está acotado por una constante que depende de ambos sistemas y es independiente de $g$. Por lo tanto una sucesión de Følner respecto a un sistema origina una sucesión de Følner respecto al otro sistema.

Ejercicio 5.65. Pruebe que si un grupo discreto posee un subgrupo libre a dos generadores entonces dicho grupo no es promediable.

Ejercicio 5.66. Pruebe que todo grupo abeliano es promediable.

Ejercicio 5.67. Pruebe que la propiedad de promediabilidad es estable por operaciones elementales, es decir:

(i) todo subgrupo de un grupo promediable es promediable,

(ii) toda extensión finita de un grupo promediable es promediable,

(iii) el cuociente de un grupo promediable es promediable,

(iv) el producto semidirecto de dos grupos promediables es promediable.

Concluya que todo grupo virtualmente soluble es promediable. Si tiene problemas para probar estas afirmaciones, vea el capítulo 3 de [214]. 
Ejercicio 5.68. Un grupo finitamente generado $\Gamma$ es de crecimiento polinomial si existe un polinomio real $Q$ tal que $L_{\mathcal{G}}(n) \leq Q(n)$ para todo $n \in \mathbb{N}$. Pruebe que todo grupo de crecimiento polinomial es promediable.

Observación. Un célebre teorema de Gromov estipula que un grupo finitamente generado es de crecimiento polinomial si y solamente si dicho grupo es virtualmente nilpotente (vea [87]). Señalemos que la implicación "sencilla" de este teorema (i.e., el hecho que el crecimiento de los grupos nilpotentes es polinomial) es debida a Bass y Guivarch.

Ejercicio 5.69. Un grupo $\Gamma$ es de crecimiento subexponencial si para todo $C>0$ se tiene

$$
\liminf _{n \rightarrow \infty} \frac{L_{\mathcal{G}}(n)}{\exp (C n)}=0 .
$$

Generalizando el ejercicio anterior, pruebe que todo grupo finitamente generado y de crecimiento subexponencial es promediable. 


\section{Bibliografía}

[1] L. Ahlfors, Finitely generated Kleinian groups, Amer. Journal of Math. 86, 413-429 (1964), 87, 759 (1965).

[2] R. Alperin, Locally compact groups acting on trees and property (T), $M h$. Math. 93, 261-265 (1982).

[3] V. Antonov, Modeling of processes of cyclic evolution type. Synchronization by a random signal, Vestnik Len. Univ. Mat. Mekh. Astr. 1984:2, 67-76 (1984).

[4] U. Bader, A. Furman, T. Gelander \& N. Monod, Property (T) and actions on $L_{p}$, Acta Math. 198, 57-105 (2007).

[5] U. Bader, A. Furman \& A. Shaker, Lattices, commensurators and continuous actions on the circle, Prepublicación (2006).

[6] U. Bader \& Y. Shalom, Factor and normal subgroups theorems for lattices in products of groups, Invent. Math. 163, 415-464 (2006).

[7] W. Ballmann \& P. Eberlein, Fundamental groups of manifolds of nonpositive curvature, J. Diff. Geometry 25, 1-22 (1987).

[8] W. Ballman, M. Gromov \& V. Schroeder, Manifolds of nonpositive curvature, Progress in Mathematics, Birkhäuser (1985).

[9] L. Bartholdi \& B. Virag, Amenability via random walks, Duke Math. J. 130, 39-56 (2005).

[10] A. Beardon, The geometry of discrete groups, Springer-Verlag (1983).

[11] L. Beklaryan, On analogues of the Tits alternative for groups of homeomorphisms of the circle and the line, Math. Notes 71, 305-315 (2002).

[12] N. Benakli \& Y. Glasner, Automorphisms groups of trees acting locally with affine permutations, Geom. Dedicata 89, 1-24 (2002).

[13] C. Bleak, Solvability in groups of piecewise-linear homeomorphisms of the unit interval, Tesis doctoral, Univ. de Binghamton (2005).

[14] F. Bonahon, The geometry of Teichmüller space via geodesic currents, Invent. Math. 92, 139-162 (1988).

[15] C. Bonatti, Un point fixe commun pour des difféomorphismes commutants de $\mathrm{S}^{2}$, Annals of Math. 129, 61-69 (1989). 
[16] M. Boshernitzan, Dense orbits of rationals, Proc. of the AMS 117, 12011203 (1993).

[17] R. Bowen, A horseshoe with positive measure, Invent. Math. 29, 203-204 (1975).

[18] E. Breuillard, On uniform exponential growth for solvable groups, Pure and Applied Math. Quaterly 3, 949-967 (2007).

[19] E. Breuillard \& T. Gelander, A topological version of the Tits alternative, A ser publicado en Annals of Math.

[20] E. Breuillard \& T. Gelander, Cheeger constant and algebraic entropy for linear groups, Int. Math. Res. Not. 56, 3511-3523 (2005).

[21] E. Breuillard \& T. Gelander, On dense free groups of Lie groups, J. Algebra 261, 448-467 (2003).

[22] M. Bridson \& A. Haefliger, Metric spaces of non-positive curvature, Grundlehren der Mathematischen Wissenschaften 319, Springer-Verlag, Berlin (1999).

[23] M. Brin, Elementary amenable subgroups of R. Thompson's group F, Intl. J. Alg. and Comp. 15, 619-642 (2005).

[24] M. Brin, The ubiquity of Thompson's group F in groups of piecewise linear homeomorphisms of the unit interval, J. London Math. Soc. 60, 449-460 (1999).

[25] M. Brin, The chameleon groups of Richard J. Thompson: automorphisms and dynamics, Publ. Math. de l'IHES 84, 5-33 (1996).

[26] M. Brin \& F. Guzmán, Automorphisms of generalized Thompson groups, $J$. Algebra 203, 285-348 (1998).

[27] M. Brin \& C. Squier, Presentations, conjugacy, roots, and centralizers in groups of piecewise linear homeomorphisms of the real line, Comm. Algebra 29, 4557-4596 (2001).

[28] M. Brin \& C. Squier, Groups of piecewise linear homeomorphismsof the real line, Invent. Math. 79, 485-498 (1985).

[29] M. Burger \& N. Monod, Continuous bounded cohomology and applications to rigidity theory, Geom. and Functional Analysis 12, 219-280 (2002).

[30] M. Burger \& N. Monod, Bounded cohomology of lattices in higher rank Lie groups, J. Eur. Math. Soc. (JEMS) 1, 199-235 (1999).

[31] M. Burger \& S. Mozes, Lattices in product of trees, Publ. Math. de l'IHES 92, 151-194 (2000).

[32] L. Bursler \& A. Wilkinson, Global rigidity of solvable group actions on $\mathrm{S}^{1}$, Geometry and Topology 8, 877-924 (2004).

[33] D. Calegari, Denominator bounds in Thompson-like groups and flows, Groups, geometry, and dynamics 1, 101-109 (2007).

[34] D. Calegari, Dynamical forcing of circular groups, Trans. of the AMS 358, 3473-3491 (2006). 
[35] D. Calegari, Circular groups, planar groups, and the Euler class, Geom. Topol. Monogr. 7, 431-491 (2004).

[36] D. Calegari \& N. Dunfield, Laminations and groups of homeomorphisms of the circle, Invent. Math. 152, 149-204 (2003).

[37] J. Cannon, W. Floyd \& W. Parry, Introductory notes on Richard Thompson's groups, L'Einseignement Mathématique 42, 215-256 (1996).

[38] A. Candel, The harmonic measures of Lucy Garnett, Adv. in Math. 176, 187-247 (2003).

[39] A. Candel \& L. Conlon, Foliations I and II, Graduate Studies in Mathematics 23 and 60, Amer. Math. Soc. (2000) and (2004).

[40] J. Cantwell \& L. Conlon, Endsets of exceptional leaves: a theorem of G. Duminy, en Foliations: geometry and dynamics, World Sci. Publishing, River Edge, Warsaw, 225-261 (2002).

[41] J. Cantwell \& L. Conlon, Leaves of Markov local minimal sets infoliations of codimension one, Publ. Mat. 33, 461-484 (1989).

[42] J. Cantwell \& L. Conlon, Foliations and subshifts, Tohoku Math. J. 40, 165-187 (1988)

[43] J. Cantwell \& L. Conlon, Smoothability of proper foliations, Annales de l'Institut Fourier (Grenoble) 38, 219-244 (1988).

[44] L. Carleson \& T. Gamelin, Complex dynamics, Universitext: Tracts in Mathematics, Springer Verlag (1993).

[45] A. Casson \& D. Jungreis, Convergence groups and Seifert fibered 3manifolds, Invent. Math. 118, 441-456 (1994).

[46] M. Chaperon, Invariant manifolds revisited, Proceedings of the Steklov Institute 236, 415-433 (2002).

[47] P. Chérix, M. Cowling, P. Jolissaint, P. Julg \& A. Valette, Groups with the Haagerup property. Gromov's a-T-menability, Progress in Mathematics 197, Birkhäuser (2001).

[48] P. Conrad, Right-ordered groups, Michigan Math. J. 6, 267-275 (1959).

[49] Y. de Cornulier, Relative Kazhdan property, Annales Sci. de l'École Normale Supérieure 39, 301-333 (2006).

[50] Y. de Cornulier, R. Tessera \& A. Valette, Isometric group actions on Hilbert spaces: growth of cocycles, Geom. and Functional Analysis 17, 770-792 (2007).

[51] Y. de Cornulier, R. Tessera \& A. Valette, Isometric group actions on Hilbert spaces: structure of orbits, A ser publicado en Canadian Math. J.

[52] A. Denjoy, Sur les courbes définies par des équations différentielles à la surface du tore, J. Math. Pure et Appl. 11, 333-375 (1932).

[53] B. Deroin \& V. Kleptsyn, Random conformal dynamical systems, A ser publicado en Geom. and Functional Analysis. 
[54] B. Deroin, V. Kleptsyn \& A. Navas, Sur la dynamique unidimensionnelle en régularité intermédiaire, Acta Math. 199, 199-262 (2007).

[55] P. Dippolito, Codimension one foliations of closed manifolds, Annals of Math. 107, 403-453 (1978).

[56] S. Druck, F. Fang \& S. Firmo, Fixed points of discrete nilpotent group actions on $\mathrm{S}^{2}$, Annales de l'Institut Fourier (Grenoble) 52, 1075-1091 (2002).

[57] S. Druck \& S. Firmo, Periodic leaves for diffeomorphisms preserving codimension one foliations, J. Math. Soc. Japan 55, 13-37 (2003).

[58] T. Dubrovina \& M. Dubrovin, On braid groups. Sbornik Mathematics 192, 693-703 (2001).

[59] S. Egashira, Qualitative theory and expansion growth of transversely piecewise-smooth foliated $\mathrm{S}^{1}$-bundles, Erg. Theory and Dynam. Systems 17, 331-347 (1997).

[60] Y. Eliashberg \& W. Thurston, Confoliations, University Lecture Series, Amer. Math. Soc. (1998).

[61] A. Erschler, On isoperimetric profiles of finitely generated groups, Geom Dedicata 100, 157-171 (2003).

[62] A. Eskin, S. Mozes \& H. Oh, On uniform exponential growth for linear groups, Invent. Math. 160, 1-30 (2005).

[63] B. Farb \& J. Franks, Groups of homeomorphisms of one-manifolds III: Nilpotent subgroups, Erg. Theory and Dynam. Systems 23, 1467-1484 (2003)

[64] D. Farley, A proper isometric action of Thompson group V on Hilbert space, Int. Math. Res. Not. 45, 2409-2414 (2003).

[65] A. Fathi \& M. Herman, Existence de difféomorphismes minimaux, Astérisque 49, 37-59 (1977).

[66] A. Furman, Random walks on groups and random transformations, En Handbook of dynamical systems Vol. 1A, North-Holland, Amsterdam 9311014 (2002).

[67] H. Furstenberg, Boundary theory and stochastic processes on homogeneus spaces, Proc. Sympos. Pure Math. 26, 193-229 (1973).

[68] D. Gabai, Convergence groups are Fuchsian groups, Annals of Math. 136, 447-510 (1992).

[69] L. Garnett, Foliations, the ergodic theorem and Brownian motion, J. Funct. Anal. 51, 285-311 (1983).

[70] F. Gëhring \& G. Martin, Discrete quasiconformal groups I, Proc. London Math. Soc. 51, 331-358 (1987).

[71] K. Gelfert \& M. Rams, Geometry of limit sets for expansive Markov systems, Prepublicación (2007)

[72] É. Ghys, Groups acting on the circle, L'Enseignement Mathématique 47, 329-407 (2001). 
[73] É. Ghys, Actions des réseaux sur le cercle, Invent. Math. 137, 199-231 (1999).

[74] É. Ghys, Sur l'uniformisation des laminations paraboliques, Progr. in Math. 145, Birkhäuser, 73-91 (1997).

[75] É. Ghys, Topologie des feuilles génériques, Annals of Math. 141, 387-422 (1995).

[76] É. Ghys, Sur les groupes engendrés par des difféomorphismes proches de l'identité, Bol. Soc. Brasileira Mat. 24, 137-178 (1993).

[77] É. Ghys, Classe d'Euler et minimal exceptionnel, Topology 26, 93-105 (1987).

[78] É. Ghys \& V. Sergiescu, Sur un groupe remarquable de difféomorphismes du cercle, Comment. Math. Helvetici 62, 185-239 (1987).

[79] É. Ghys \& T. Tsuboi, Différentiabilité des conjugaisons entre systèmes dynamiques de dimension 1, Annales de l'Institut Fourier (Grenoble) 38, 215244 (1988).

[80] C. Godbillon, Feuilletages. Études géométriques, Progress in Mathematics 98, Birkhäuser (1991).

[81] C. Goffman, T. Nishiura \& D. Waterman, Homeomorphisms in Analysis, Math. Surveys and Monographs 54, Amer. Math. Soc. (1997).

[82] X. Gómez-Mont \& L. Ortiz-Bobadilla, Sistemas dinámicos holomorfos en superficies, Aportaciones Matemáticas, Sociedad Matemática Mexicana (1989).

[83] R. Grigorchuk, On degrees of growth of $p$-groups and torsion-free groups, Mat. Sbornik 126, 194-214 (1985).

[84] R. Grigorchuk, Degrees of growth of finitely generated groups and the theory of invariant means, Izv. Akad. Nauka 48, 939-985 (1984).

[85] R. Grigorchuk \& A. Maki, On a group of intermediate growth that acts on a line by homeomorphisms, Math. Notes 53, 146-157 (1993).

[86] M. Gromov, Random walk in random groups, Geom. and Functional Analysis 13, 73-146 (2003).

[87] M. Gromov, Groups of polynomial growth and expanding maps, Publ. Math. de l'IHES 53, 53-73 (1981).

[88] G. Hall, A C ${ }^{\infty}$ Denjoy counterexample, Erg. Theory and Dynam. Systems 1, 261-272 (1983).

[89] G. Hardy \& E. Wright, An introduction to the theory of numbers, The Clarendon Press, Oxford University Press, New York (1979).

[90] P. de la Harpe, Topics in geometric group theory, Univ. of Chicago Press (2000).

[91] P. de la Harpe \& A. Valette, La propriété (T) de Kazhdan pour les groupes localement compacts, Astérisque 175 (1989). 
[92] J. Harrison, Dynamics of Ahlfors quasi-circles, Proc. Indian Acad. Sci. Math. 99, 113-122 (1989).

[93] J. Harrison, Unsmoothable diffeomorphisms on higher dimensional manifolds, Proc. of the AMS 73, 249-255 (1979).

[94] M. Herman, Sur la conjugaison différentiable des difféomorphismes du cercle à des rotations, Publ. Math. de l'IHES 49, 5-234 (1979).

[95] A. Hinkkanen, Abelian and non discrete convergence groups of the circle, Trans. of the AMS 318, 87-121 (1990).

[96] M. Hirsch, A stable analytic foliation with only exceptional minimal set, Lecture Notes in Math. 468, 9-10 (1965).

[97] J. Hu \& D. Sullivan, Topological conjugacy of circle diffeomorphisms, Erg. Theory and Dynam. Systems 17, 173-186 (1997).

[98] S. Hurder, Exceptional minimal sets and the Godbillon-Vey class, A ser publicado en Annales de l'Institut Fourier (Grenoble).

[99] S. Hurder, Dynamics of expansive group actions on the circle, A ser publicado en Geom. Dedicata.

[100] S. Hurder, Entropy and dynamics of $\mathrm{C}^{1}$ foliations, Prepublicación (2004).

[101] S. Hurder, Exceptional minimal sets of $\mathrm{C}^{1+\alpha}$-group actions on the circle, Erg. Theory and Dynam. Systems 11, 455-467 (1991).

[102] S. Hurder, Ergodic theory of foliations and a theorem of Sacksteder, Lecture Notes in Math. 1342, 291-328 (1988).

[103] Y. Ilyashenko, The density of an individual solution and the ergodicity of the family of solutions of the equation $d \eta / d \xi=P(\xi, \eta) / Q(\xi, \eta)$, Mat. Zametki 4, 741-750 (1968).

[104] H. Imanishi, On the theorem of Denjoy-Sacksteder for codimension one foliations without holonomy, J. Math. Kyoto Univ. 14, 607-634 (1974).

[105] T. Inaba, Examples of exceptional minimal sets, En A fête of topology, Academic Press, Boston, 95-100 (1988).

[106] G. Iommi, A. Navas \& S. Plaza, Operador de Perron-Fröbenius y medidas invariantes para aplicaciones del intervalo, Monografías del Instituto de Matemática y Ciencias Afines 12, IMCA, Lima (2000).

[107] V. Kaimanovich, The Poisson boundary of polycyclic groups, En Probability measures on groups and related structures XI (Oberwolfach, 1994). World Sci. Publishing, River Edge, NJ, 182-195 (1995).

[108] A. Katok \& A. Mezhirov, Entropy and growth of expanding periodic orbits for one-dimensional maps, Fund. Math. 157, 245-254 (1998).

[109] S. Katok, Fushian Groups, Chicago Lectures in Mathematics (1984).

[110] Y. Katznelson \& D. Ornstein, The differentiability of the conjugation of certain diffeomorphisms of the circle, Erg. Theory and Dynam. Systems $\mathbf{9}$, 643-680 (1989). 
[111] Y. Kifer, Ergodic theory of random transformations, Progress in Prob. and Stat. 10, Birkhäuser (1986)

[112] V. Kleptsyn, Sur une interprétation algorithmique du groupe de Thompson, Prepublicación (2004).

[113] V. Kleptsyn \& M. Nalsky, Convergence of orbits in random dynamical systems on a circle, Funct. Anal. Appl. 38, 267-282 (2004).

[114] V. Kleptsyn \& A. Navas, A Denjoy type theorem for commuting circle diffeomorphisms with derivatives having different Hölder differentiability classes, A ser publicado en Moscow Math. Journal.

[115] A. Knapp, Lie groups beyond an introduction, Progress in Mathematics 140 Birkhäuser (2002).

[116] V. Kopitov \& N. Medvedev, Right ordered groups, Siberian School of Algebra and Logic, Plenum Publ. Corp., New York (1996).

[117] B. Kolev, Note sur les groupes compacts d'homéomorphismes de la sphère, Prepublicación (2006).

[118] N. Kopell, Commuting diffeomorphisms, En Global Analysis, Proc. Sympos. Pure Math. XIV, Berkeley, Calif., 165-184 (1968).

[119] N. Kovačević, Examples of Möbius-like groups wich are not Möbius groups, Trans. of the AMS 351, 4823-4835 (1999).

[120] L. Lifschitz \& D. Witte-Morris, Isotropic nonarchimedean $S$-arithmetic groups are not left orderable, C. R. Acad. Sci. Paris Sér. I Math. 339, 417-420 (2004).

[121] P. Linnell, The topology on the space of left orderings of a group, Prepublicación (2006)

[122] P. Linnell, Left ordered groups with no nonabelian free subgroups, J. Group Theory 4, 153-168 (2001).

[123] I. Liousse, Trabajo en preparación.

[124] I. Liousse, Nombre de rotation, mesures invariantes et ratio set des homéomorphismes par morceaux du cercle, Annales de l'Institut Fourier (Grenoble) 55, 431-482 (2005).

[125] D. McDuff, $\mathrm{C}^{1}$-minimal sets of the circle, Annales de l'Institut Fourier (Grenoble) 31, 177-193 (1981)

[126] R. Mañé, Introdução à teoria ergódica, Projeto Euclides (1983)

[127] A. Marden, Universal properties of Fuchsian groups, Ann. of Math. Studies 79, 315-339 (1974).

[128] G. Margulis, Free subgroups of the homeomorphism group of the circle, $C$. R. Acad. Sci. Paris Sér. I Math. 331, 669-674 (2000).

[129] G. Margulis, Discrete subgroups of semisimple Lie groups, Springer Verlag (1991).

[130] V. Markovic, Classification of continuously transitive circle groups, Geometry and Topology 10, 1319-1346 (2006). 
[131] V. Markovic, Uniformly quasisymmetric groups, Journal of the AMS 19, 673-715 (2006).

[132] S. Matsumoto, Measures of exceptional minimal sets of codimension one foliations, En A fête of topology. Academic Press, Boston, 81-94 (1988).

[133] W. de Melo \& S. van Strien, One-dimensional dynamics, Springer Verlag (1993).

[134] J. Milnor, Growth of finitely generated solvable groups, J. Diff. Geometry 2, 447-449 (1968).

[135] H. Minakawa, Classification of exotic circles of $P L_{+}\left(\mathrm{S}^{1}\right)$, Hokkaido Math. J. 26, 685-697 (1997).

[136] H. Minakawa, Exotic circles of $P L_{+}\left(\mathrm{S}^{1}\right)$, Hokkaido Math. J. 24, 567-573 (1995).

[137] N. Monod \& Y. Shalom, Cocycle superrigidity and bounded cohomology for negatively curved spaces, J. Diff. Geometry 67, 1-61 (2004).

[138] D. Montgomery \& L. Zippin, Topological Transformation Groups, Interscience Publ. (1955).

[139] M. Muller, Sur l'approximation et l'instabilité des feuilletages, Texto no publicado (1982).

[140] I. Nakai, Separatrices for non solvable dynamics on ( $\mathbb{C}, 0)$, Annales de l'Institut Fourier (Grenoble) 44, 569-599 (1994).

[141] A. Navas, On the dynamics of (left) orderable groups, Prepublicación (2007)

[142] A. Navas, Growth of groups and diffeomorphisms of the interval, A ser publicado en Geom. and Functional Analysis.

[143] A. Navas, Reduction of cocycles and groups of diffeomorphisms of the circle, Bull. Belg. Math. Soc. Simon Stevin 13, 193-205 (2006).

[144] A. Navas, On uniformly quasisymmetric groups of circle diffeomorphisms, An. Acad. Sci. Fenn. Math. 31, 437-462 (2006).

[145] A. Navas, Quelques nouveaux phénomènes de rang 1 pour les groupes de difféomorphismes du cercle, Comment. Math. Helvetici 80, 355-375 (2005).

[146] A. Navas, Quelques groupes moyennables de difféomorphismes de l'intervalle, Bol. Soc. Mat. Mexicana 10, 219-244 (2004).

[147] A. Navas, Sur les groupes de difféomorphismes du cercle engendrés par des éléments proches des rotations, L'Enseignement Mathématique 50, 29-68 (2004).

[148] A. Navas, Groupes résolubles de difféomorphismes de l'intervalle, du cercle et de la droite, Bull. of the Brazilian Math. Society 35, 13-50 (2004).

[149] A. Navas, Actions de groupes de Kazhdan sur le cercle, Annales Sci. de l'École Normale Supérieure 35, 749-758 (2002).

[150] A. Navas, Groupes de Neretin et propriété (T) de Kazhdan, C. R. Acad. Sci. Paris Sér. I Math. 335, 789-792 (2002). 
[151] Y. Neretin, Groups of hierarchomorphisms of trees and related Hilbert spaces, J. Funct. Anal. 200, 505-535 (2003).

[152] M. Newman, A theorem on periodic transformations on spaces, Quat. J. Math. 2, 1-8 (1931).

[153] P. Nicholls, The ergodic theory of discrete groups, London Mathematical Society Lecture Note Series 143, Cambridge University Press (1989).

[154] A. Norton, Denjoy minimal sets are far from affine, Erg. Theory and Dynam. Systems 22, 1803-1812 (2002).

[155] A. Norton, An area approach to wandering domains for smooth surface diffeomorphisms, Erg. Theory and Dynam. Systems 11, 181-187 (1991).

[156] A. Norton \& B. Tandy, Cantor sets, binary trees and Lipschitz circle homeomorphisms, Michigan Math. J. 46, 29-38 (1999).

[157] C. Oikonomides, Tesis doctoral, Univ. de Tokyo (2001).

[158] F. Oliveira \& F. da Rocha, Minimal non-ergodic $\mathrm{C}^{1}$-diffeomorphisms of the circle, Erg. Theory and Dynam. Systems 21, 1843-1854 (2001).

[159] Y. Ol'shanskii, On the question of the existence of an invariant mean on a group, Uspekhi Mat. Nauka 35, 199-200 (1980).

[160] Y. Ol'shanski \& M. Sapir, Non-amenable finitely presented torsion-by-cyclic groups, Publ. Math. de l'IHES 96, 43-169 (2002).

[161] D. Osin, The entropy of solvable groups, Erg. Theory and Dynam. Systems 23, $907-918$ (2003).

[162] D. Osin, Elementary classes of groups, Math. Notes 72, 75-82 (2002).

[163] J. Palis \& F. Takens, Hyperbolicity and sensitive chaotic dynamics at homoclinic bifurcations. Fractal dimensions and infinitely many attractors, Cambridge Studies in Advanced Mathematics 35, Cambridge University Press (1993).

[164] D. Pixton, Nonsmoothable, unstable group actions, Trans. of the AMS 229, 259-268 (1977).

[165] J. Plante, Subgroups of continuous groups acting differentiably on the half line, Annales de l'Institut Fourier (Grenoble) 34, 47-56 (1984).

[166] J. Plante, Solvable groups acting on the line, Trans. of the AMS 278, 401414 (1983).

[167] J. Plante, Foliations with measure preserving holonomy, Annals of Math. 102, 327-361 (1975)

[168] J. Plante \& W. Thurston, Polynomial growth in holonomy groups of foliations, Comment. Math. Helvetici 51, 567-584 (1976).

[169] V. Pliss, On a conjecture of Smale, Differencialcnye Uravnenija 8, 268-282 (1972).

[170] A. Pressley \& G. Segal, Loop groups, Oxford Mathematical Monographs (1986). 
[171] A. Quas, Non-ergodicity for $\mathrm{C}^{1}$ expanding maps and $g$-measures, Erg. Theory and Dynam. Systems 16, 531-543 (1996).

[172] M. Raghunathan, Discrete subgroups of Lie groups, Ergebnisse der Mathematik und ihrer Grenzgebiete, Band 68, Springer-Verlag, New YorkHeidelberg (1972).

[173] J. Rebelo, A theorem of measurable rigidity in $\operatorname{Diff}^{w}\left(\mathrm{~S}^{1}\right)$, Erg. Theory and Dynam. Systems 21, 1525-1561 (2001).

[174] J. Rebelo, Ergodicity and rigidity for certain subgroups of Diff ${ }^{w}\left(\mathrm{~S}^{1}\right), A n$ nales Sci. de l'École Normale Supérieure 32, 433-453 (1999).

[175] G. Reeb \& P. Schweitzer, Un théorème de Thurston établi au moyen de l'analyse non standard, Lecture Notes in Math. 652, 138 (1978).

[176] B. Rémy, Construction de réseaux en théorie de Kac-Moody, C. R. Acad. Sci. Paris Sér. I Math. 6, 475-478 (1999).

[177] A. Reznikov, Analytic topology of groups, actions, strings and varietes, Chapter 2: A theory of groups acting in the circle, Prepublicación (1999).

[178] J. Rosenblatt, Invariant measures and growth conditions, Trans. of the AMS 197, 33-53 (1974).

[179] R. Sacksteder, Foliations and pseudogroups, Amer. Journal of Math. 87, 79-102 (1965).

[180] R. Sacksteder, On the existence of exceptional leaves in foliations of codimension one, Annales de l'Institut Fourier (Grenoble) 14, 221-225 (1964).

[181] W. Schachermayer, Addendum: Une modification standard de la démonstration non standard de Reeb et Schweitzer "Un théorème de Thurston établi au moyen de l'analyse non standard", Lecture Notes in Math. 652, 139-140 (1978).

[182] A. Schwartz, A generalization of Poincaré-Bendixon theorem to closed two dimensional manifolds, Amer. J. Math. 85, 453-458 (1963).

[183] F. Sergeraert, Feuilletages et difféomorphismes infiniment tangents à l'identité, Invent. Math. 39, 253-275 (1977).

[184] V. Sergiescu, Versions combinatoires de Diff $\left(\mathrm{S}^{1}\right)$, Groupes de Thompson. Prepublicación (2003).

[185] J.P. Serre, Arbres, amalgames, $\mathrm{SL}_{2}$. Astérisque 46 (1977).

[186] Y. Shalom, Bounded generation and Kazhdan's property (T), Publ. Math. de l'IHES 90, 145-168 (2001).

[187] Y. Shalom, Rigidity of commensurators and irreducible lattices, Invent. Math. 141, 1-54 (2000).

[188] M. Shub \& D. Sullivan, Expanding endomorphisms of the circle revisited, Erg. Theory and Dynam. Systems 5, 285-289 (1985).

[189] A. Sikora, Topology on the spaces of orderings of groups, Bull. London Math. Soc. 36, 519-526 (2004). 
[190] V. Solodov, Homeomorphisms of a straight line and foliations, Izv. Akad. Nauka SSSR Ser. Mat. 46, 1047-1061 (1982).

[191] M. Stein, Groups of piecewise linear homeomorphisms, Trans. of the AMS 332, 477-514 (1992).

[192] S. Sternberg, Local $\mathrm{C}^{n}$-transformations of the real line, Duke Math. J. 24, 97-102 (1957).

[193] D. Sullivan, Discrete conformal groups and measurable dynamics, Bull. of the AMS 6, 57-73 (1982).

[194] D. Sullivan, On the ergodic theory at infinity of an arbitrary discrete group of hyperbolic motions. En Riemann surfaces and related topics, Proceedings of the 1978 Stony Brook conference, Princeton University Press, 465-496 (1980).

[195] D. Sullivan, Cycles for the dynamical study of foliated manifolds and complex manifolds, Invent. Math. 36, 225-255 (1976).

[196] G. Szekeres, Regular iteration of real and complex functions, Acta Math. 100, 203-258 (1958).

[197] W. Thurston, Three dimensional geometry and topology, Princeton University Press, Princeton, New Jersey (1997).

[198] W. Thurston, A generalization of Reeb stability theorem, Topology 13, 347352 (1974).

[199] T. Tsuboi, Homological and dynamical study on certain groups of Lipschitz homeomorphisms of the circle, J. Math. Soc. Japan 47, 1-30 (1995).

[200] T. Tsuboi, Area functionals and Godbillon-Vey cocycles, Annales de l'Institut Fourier (Grenoble) 42, 421-447 (1992).

[201] T. Tsuboi, On the foliated products of class $\mathrm{C}^{1}$, Annals of Math. 130, 227271 (1989).

[202] T. Tsuboi, Examples of nonsmoothable actions on the interval, J. Fac. Sci. Univ. Tokyo Sect. IA Math. 34, 271-274 (1987).

[203] T. Tsuboi, $\Gamma_{1}$-structures avec une seule feuille, Astérisque 116, 222-234 (1984).

[204] P. Tukia, Homeomorphic conjugates of Fuchsian groups, J. für Reine und Angew. Math. 391, 1-54 (1989).

[205] Y. Watatani, Property (T) of Kazhdan implies property FA of Serre, Math. Japonica 27, 97-103 (1981).

[206] J. Wilson, On exponential growth and uniform exponential growth for groups, Invent. Math. 155, 287-303 (2004).

[207] D. Witte-Morris, Amenable groups that act on the line, Alg. Geom. Top. 6, 2509-2518 (2006).

[208] D. Witte-Morris, Arithmetic groups of higher $\mathbb{Q}$-rank cannot act on 1manifolds, Proc. of the AMS 122, 333-340 (1994). 
[209] J. Wolf, Growth of finitely generated solvable groups and curvature of Riemanniann manifolds, J. Diff. Geometry 2, 421-446 (1968).

[210] A. Yamada, On Marden's universal constant for fuchsian groups, Kodai. Math. J. 4, 266-277 (1981).

[211] J.C. Yoccoz, Centralisateurs et conjugaison différentiable des difféomorphismes du cercle, Astérisque 231, 89-242 (1995)

[212] J.C. Yoccoz, Il n'y a pas de contre-exemple de Denjoy analytique, $C$. $R$. Acad. Sci. Paris Sér. I Math. 298, 141-144 (1984).

[213] H. Yosida, Functional analysis, Classics in Mathematics. Springer-Verlag, Berlin (1995).

[214] R. Zimmer, Ergodic theory of semisimple groups, Monographs in Mathematics, Birkhäuser (1984)

[215] A. Żuk, Property (T) and Kazhdan constants for discrete groups, Geom. and Functional Analysis 13, 643-670 (2003).

Andrés Navas

Universidad de Santiago de Chile

Alameda 3363, Estación Central

Santiago, Chile

andnavas@uchile.cl 